\title{
Cleanup Verification Package for the 116-K-2 Effluent Trench
}

Prepared for the U.S. Department of Energy by Washington Closure Hanford 


\section{EXECUTIVE SUMMARY}

This cleanup verification package documents completion of remedial action for the 116-K-2 effluent trench, also referred to as the 116-K-2 mile-long trench and the 116-K-2 site. This site is located in the 100-KR-1 Operable Unit in the 100-K Area of the Hanford Site in southeastern Washington State. During its period of operation from 1955 to 1971 , the 116-K-2 site was used to dispose of cooling water effluent from the 105-KE and 105-KW Reactors by percolation into the soil. The site also received mixed liquid wastes from the 105-KW and 105-KE fuel storage basins, reactor floor drains, and miscellaneous decontamination activities.

Site excavation and waste disposal are complete, and the exposed surfaces have been sampled and analyzed to verify attainment of the remedial action goals. Results of the sampling, laboratory analyses, and data evaluations for the 116-K-2 site indicate that all remedial action objectives for direct exposure, protection of groundwater, and protection of the Columbia River have been met (see Table ES-1).

The site meets cleanup standards and has been reclassified as "interim closed out" in accordance with the Hanford Federal Facility Agreement and Consent Order (Ecology et al. 1989) and the Waste Site Reclassification Guideline TPA-MP-14 (RL-TPA-90-0001) (DOE-RL 1998). A copy of the waste site reclassification form is included as Attachment ES-1. 
CVP-2006-00001

Rev. 0

Table ES-1, Summary of Cleanup Verification Results for the 116-K-2 Waste Site. (2 Pages)

\begin{tabular}{|c|c|c|c|c|}
\hline $\begin{array}{l}\text { Regulatory } \\
\text { Requirement }\end{array}$ & Remedial Action Goals & Results & $\begin{array}{l}\text { Remedial } \\
\text { Action } \\
\text { Objectives } \\
\text { Attained? }\end{array}$ & Ref. \\
\hline $\begin{array}{l}\text { Direct Exposure - } \\
\text { Radionuclides }\end{array}$ & $\begin{array}{l}\text { 1. Attain } 15 \mathrm{mrem} / \mathrm{yr} \text { dose rate } \\
\text { above background over } \\
1,000 \text { years. }\end{array}$ & $\begin{array}{l}\text { 1. Maximum dose rate calculated by } \\
\text { RESRAD is } 7.77 \mathrm{mrem} / \mathrm{yr} \text { (not } \\
\text { accounting for clean backfill). }\end{array}$ & Yes & $a, b$ \\
\hline $\begin{array}{l}\text { Direct Exposure - } \\
\text { Nonradionuclides }\end{array}$ & 1. Attain individual COC RAGs. & $\begin{array}{l}\text { 1. The concentration for hexavalent } \\
\text { chromium (the only nonradionuclide } \\
\text { COC) is below the direct exposure } \\
\text { RAG. }\end{array}$ & Yes & $c, d$ \\
\hline \multirow{4}{*}{$\begin{array}{l}\text { Meet } \\
\text { Nonradionuclide Risk } \\
\text { Requirements }\end{array}$} & $\begin{array}{l}\text { 1. Hazard quotient of }<1 \text { for } \\
\text { noncarcinogens. }\end{array}$ & $\begin{array}{l}\text { 1. Individual hazard quotients for the } \\
116-K-2 \text { site are less than } 1 \text {. }\end{array}$ & \multirow{4}{*}{ Yes } & $c, d$ \\
\hline & $\begin{array}{l}\text { 2. Cumulative hazard quotient of } \\
<1 \text { for noncarcinogens. }\end{array}$ & $\begin{array}{l}\text { 2. Cumulative hazard quotients for the } \\
116-\mathrm{K}-2 \text { site are less than } 1 \text {. }\end{array}$ & & $c, d$ \\
\hline & $\begin{array}{l}\text { 3. Excess cancer risk of }<1 \times 10^{-6} \text { for } \\
\text { individual carcinogens. }\end{array}$ & $\begin{array}{l}\text { 3. Individual excess carcinogenic risk } \\
\text { values for the } 116-\mathrm{K}-2 \text { site are less } \\
\text { than } 1 \times 10^{-6} \text {. }\end{array}$ & & $c, d$ \\
\hline & $\begin{array}{l}\text { 4. Attain a total excess cancer risk of } \\
<1 \times 10^{-5} \text { for carcinogens. }\end{array}$ & $\begin{array}{l}\text { 4. Cumulative excess carcinogenic risk } \\
\text { values for the } 116-\mathrm{K}-2 \text { site are less } \\
\text { than } 1 \times 10^{-5} \text {. }\end{array}$ & & $c, d$ \\
\hline \multirow[t]{4}{*}{$\begin{array}{l}\text { Groundwater/River } \\
\text { Protection - } \\
\text { Radionuclides }\end{array}$} & $\begin{array}{l}\text { 1. Attain single-COC groundwater } \\
\text { and river protection RAGs. }\end{array}$ & $\begin{array}{l}\text { 1. Radionuclide COCs are not } \\
\text { predicted to migrate to groundwater } \\
\text { or the Columbia River within } \\
1,000 \text { years. }\end{array}$ & \multirow{3}{*}{ Yes } & \multirow{3}{*}{$a, b$} \\
\hline & $\begin{array}{l}\text { 2. Attain National Primary Drinking } \\
\text { Water Standards: } 4 \mathrm{mrem} / \mathrm{yr} \\
\text { (beta/gamma) dose rate to target } \\
\text { receptor/organs. }\end{array}$ & $\begin{array}{l}\text { 2. Radionuclide COCs are not } \\
\text { predicted to migrate to groundwater } \\
\text { or the Columbia River within } \\
1,000 \text { years. }\end{array}$ & & \\
\hline & $\begin{array}{l}\text { 3. Meet drinking water standards for } \\
\text { alpha emitters: the more stringent } \\
\text { of the } 15 \mathrm{pCi} / \mathrm{L} \mathrm{MCL} \text { or } 1 / 25 \text { th of } \\
\text { the derived concentration guide } \\
\text { per DOE Order } 5400.5 \text {. }\end{array}$ & $\begin{array}{l}\text { 3. Radionuclide COCs are not } \\
\text { predicted to migrate to groundwater } \\
\text { or the Columbia River within } \\
1,000 \text { years. }\end{array}$ & & \\
\hline & $\begin{array}{l}\text { 4. Meet total uranium standard of } \\
21.2 \mathrm{pCi} / \mathrm{L} \text {. }^{\mathrm{e}}\end{array}$ & $\begin{array}{l}\text { 4. Uranium was not identified as a } \\
\text { COC for the } 116-\mathrm{K}-2 \text { waste site. }\end{array}$ & NA & \\
\hline $\begin{array}{l}\text { Groundwater/River } \\
\text { Protection - } \\
\text { Nonradionuclides }\end{array}$ & $\begin{array}{l}\text { 1. Attain individual nonradionuclide } \\
\text { groundwater and river cleanup } \\
\text { requirements. }\end{array}$ & $\begin{array}{l}\text { 1. Residual hexavalent chromium } \\
\text { concentrations are not predicted to } \\
\text { leach to groundwater or the } \\
\text { Columbia River at concentrations } \\
\text { exceeding the applicable water } \\
\text { quality criteria. }\end{array}$ & Yes & $\begin{array}{l}a, b, \\
c, d\end{array}$ \\
\hline
\end{tabular}


CVP-2006-00001

Rev. 0

\section{Table ES-1. Summary of Cleanup Verification Results for the 116-K-2 Waste Site. (2 Pages)}

\begin{tabular}{|c|c|c|c|c|}
\hline $\begin{array}{c}\text { Regulatory } \\
\text { Requirement }\end{array}$ & Remedial Action Goals & Results & $\begin{array}{l}\text { Remedial } \\
\text { Action } \\
\text { Objectives } \\
\text { Attained? }\end{array}$ & Ref. \\
\hline \multirow{5}{*}{$\begin{array}{l}\text { Other supporting } \\
\text { Information }\end{array}$} & \multicolumn{3}{|c|}{ 1. 116-K-2 (east) sample variance calculation (Appendix C) } & f \\
\hline & \multicolumn{3}{|c|}{ 2. 116-K-2 (west) and overburden sample variance calculation (Appendix C) } & $\mathrm{g}$ \\
\hline & \multicolumn{3}{|c|}{ 3. 116-K-2 overburden sample variance calculation (Appendix C) } & $\mathrm{h}$ \\
\hline & \multicolumn{3}{|c|}{ 4. 116-K-2 (east) sample location design (Appendix C) } & $\mathrm{i}$ \\
\hline & \multicolumn{3}{|c|}{ 5. 116-K-2 (west) and overburden sample location design (Appendix C) } & j \\
\hline
\end{tabular}

a 116-K-2 Trench (East End) RESRAD Calculation, 0100K-CA-V0058, Rev. 0, Washington Closure Hanford, Richland, Washington.

b 116-K-2 Trench (West End) RESRAD Calculation, 0100K-CA-V0063, Rev. 0, Washington Closure Hanford, Richland, Washington.

c 116-K-2 Trench (East End) Cleanup Verification 95\% UCL Calculations, 0100K-CA-V0056, Rev. 1, Washington Closure Hanford, Richland, Washington.

d 116-K-2 Trench (West End) Cleanup Verification 95\% UCL Calculations, 0100K-CA-V0061, Rev. 0, Washington Closure Hanford, Richland, Washington.

e Uranium limits selected in the Amendment to the Interim Action Record of Decision for the 100-BC-1, 100-DR-1, and

100-HR-1 Operable Units, Hanford Site, Benton County, Washington (ROD) (EPA 1997) and Remedial Design Report/Remedial Action Work Plan for the 100 Area (DOE-RL 2005b) were based on 1/25th of the derived concentration guidelines from DOE Order 5400.5 . Since the time of ROD signature, the U.S. Environmental Protection Agency has promulgated a more restrictive MCL of $30 \mu \mathrm{g} / \mathrm{L}$ for total uranium (65 Federal Register 76708). Based on the isotopic distribution of uranium in the 100 Areas, the $30 \mu \mathrm{g} / \mathrm{L} \mathrm{MCL}$ corresponds to $21.2 \mathrm{pCi} / \mathrm{L}$. Concentration-to-activity calculations are documented in Calculation of Total Uranium Activity Corresponding to a Maximum Contaminant Level for Total Uranium of 30 Micrograms per Liter in Groundwater, 0100X-CA-V0038 (BHI 2001a).

116-K-2 Mile Long Trench Variance Calculation, 0100K-CA-V0055, Rev. 0, Washington Closure Hanford, Richland, Washington.

9 116-K-2 Mile Long Trench Variance Calculation (Phase 2, West), 0100K-CA-V0064, Rev. 0, Washington Closure Hanford, Richland, Washington.

h 116-K-2 Overburden Variance Calculation, 0100K-CA-V0065, Rev. 0, Washington Closure Hanford, Richland, Washington.

' 116-K-2 (East End) Trench Shallow \& Deep Zone Sampling Plan, 0100K-CA-V0057, Rev. 0, Washington Closure Hanford, Richland, Washington.

116-K-2 (West End) Trench Shallow, Deep Zone and Overburden Sampling Plan, 0100K-CA-V0062, Rev. 0, Washington Closure Hanford, Richland, Washington.

$\mathrm{COC}=$ contaminant of concern

NA = not applicable

RAG = remedial action goal

$\mathrm{MCL} \quad=$ maximum contaminant level (drinking water standard)

RESRAD = RESidual RADioactivity (dose model)

UCL = upper confidence limit 
CVP-2006-00001

Rev. 0

ES-4 
CVP-2006-00001

Rev. 0

\section{Attachment ES-1 \\ Waste Site Reclassification Form}

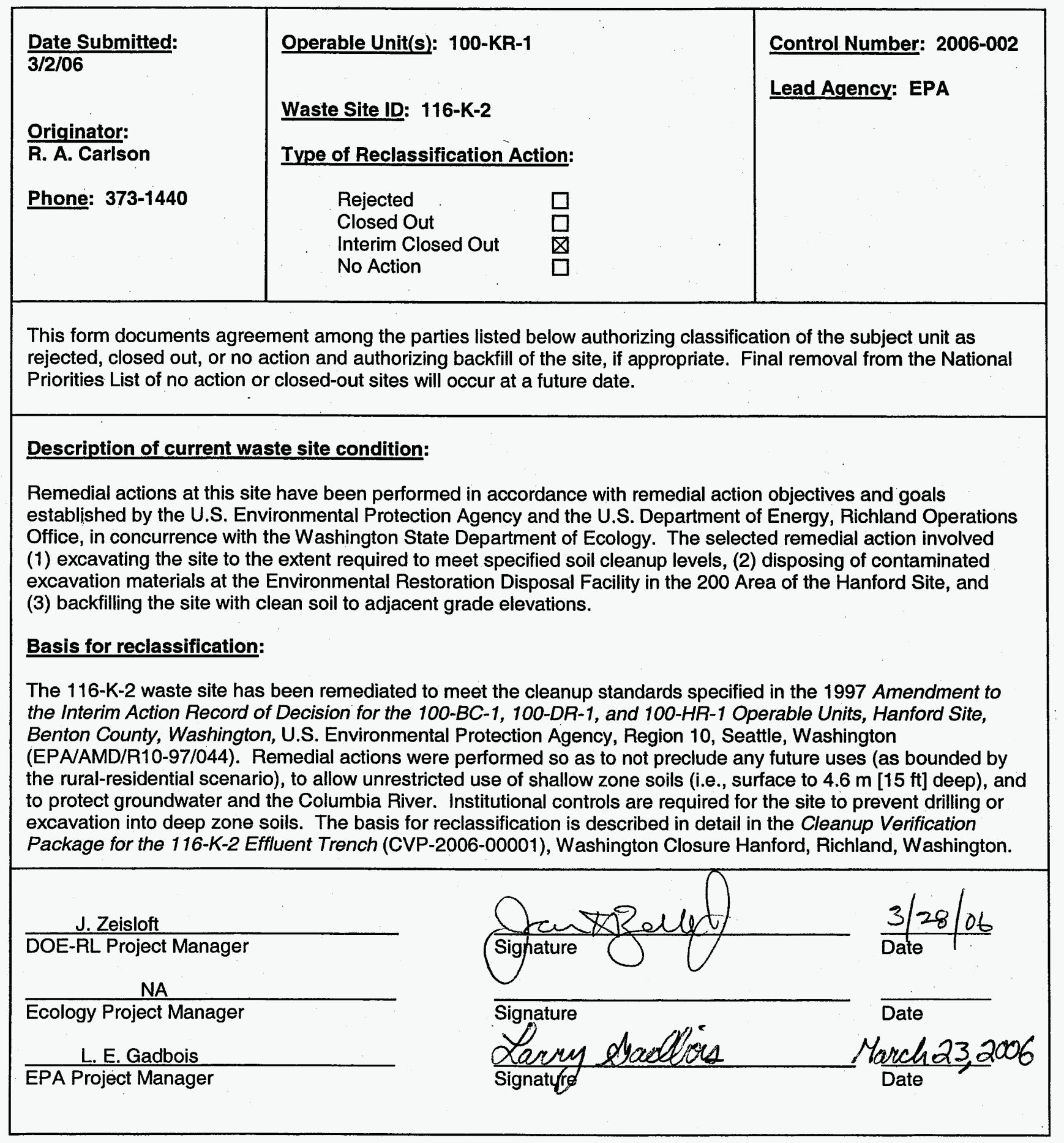


CVP-2006-00001

Rev. 0

ES-6 


\section{CONTENTS}

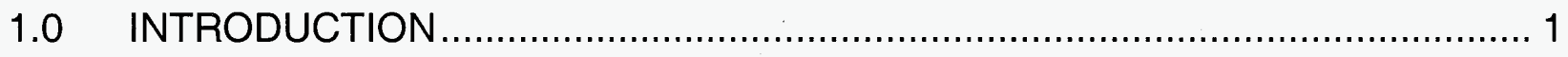

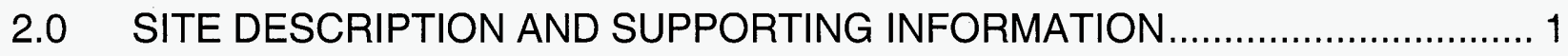

3.0 REMEDIAL ACTION FIELD ACTIVITIES ................................................... 4

3.1 EXCAVATION AND DISPOSAL ................................................... 4

3.2 FIELD SCREENING AND VARIANCE SAMPLING ............................. 4

3.3 CLEANUP VERIFICATION SAMPLING AND ANALYSIS ..................... 9

4.0 CLEANUP VERIFICATION DATA EVALUATION ........................................ 10

$4.1 \quad$ DATA QUALITY ASSESSMENT PROCESS ..................................... 10

4.2 CONTAMINANTS OF CONCERN 95\% UPPER CONFIDENCE LIMIT ... 10

4.3 SITE-SPECIFIC CLEANUP VERIFICATION MODEL ......................... 12

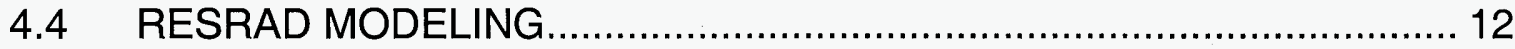

5.0 EVALUATION OF REMEDIAL ACTION GOAL ATTAINMENT ........................ 13

5.1 DIRECT EXPOSURE SOIL REMEDIAL ACTION GOALS ATTAINED.... 13

5.1.1 Radionuclides ................................................................... 13

5.1.2 Nonradionuclides .............................................................. 15

5.2 GROUNDWATER REMEDIAL ACTION GOALS ATTAINED ................ 16

5.2.1 Radionuclides .................................................................... 16

5.2.2 Nonradionuclides ............................................................. 16

5.3 COLUMBIA RIVER REMEDIAL ACTION GOALS ATTAINED ............... 18

5.3.1 Radionuclides ..................................................................... 18

5.3.2 Nonradionuclides ................................................................ 18

5.4 WAC 173-340 THREE-PART TEST FOR NONRADIONUCLIDES ......... 18

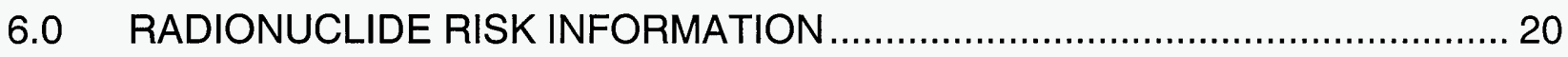

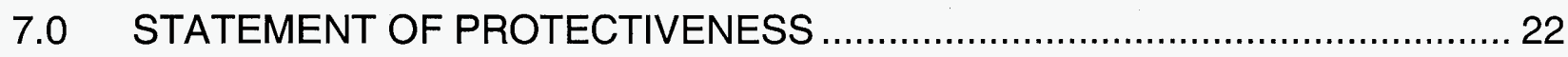

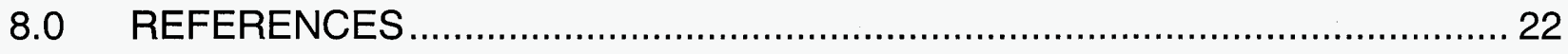




\section{APPENDICES}

A SUMMARY OF VERIFICATION SOIL SAMPLING AND ANALYTICAL RESULTS

B DATA QUALITY ASSESSMENT

C RESRAD INPUT PARAMETERS AND CALCULATION BRIEF

EXCERPTS

C1 116-K-2 (East End) Trench Shallow \& Deep Zone Sampling Plan (0100K-CA-V0057)

C2 116-K-2 Mile Long Trench Variance Calculation

(0100K-CA-V0055)

C3 116-K-2 Trench (East End) Cleanup Verification 95\% UCL Calculations (0100K-CA-V0056)

C4 116-K-2 Trench (East End) RESRAD Calculation (0100K-CA-V0058)

C5 116-K-2 (West End) Trench Shallow, Deep Zone and Overburden Sampling Plan (0100K-CA-V0062)

C6 116-K-2 Mile Long Trench Variance Calculation (Phase 2, West) (0100K-CA-V0064)

C7 116-K-2 Overburden Variance Calculation (0100K-CA-V0065)............ -163

C8 116-K-2 Trench (West End) Cleanup Verification 95\% UCL Calculations (0100K-CA-V0061)

C9 116-K-2 Trench (West End) RESRAD Calculation (0100K-CA-V0063) .

\section{FIGURES}

1. Hanford Site Map and Location of the 116-K-2 Waste Site................................. 3

2. Pre-Remediation Topographic Plan for the Western Portion of the 116-K-2 Waste Site.

3. Pre-Remediation Topographic Plan for the Eastern Portion of the 116-K-2 Waste Site.

4. Post-Remediation Topographic Plan for the Western Portion of the 116-K-2 Waste Site. 7

5. Post-Remediation Topographic Plan for the Eastern Portion of the 116-K-2 Waste Site.

6. Combined Shallow and Deep Zone Dose Rate Estimates for the Eastern 116-K-2 Site (All Radionuclides, All Pathways).

7. Combined Shallow and Deep Zone Dose Rate Estimates for the Western 116-K-2 Site (All Radionuclides, All Pathways).

8. Overburden Dose Rate Estimates for the 116-K-2 Site (All Radionuclides, All Pathways). 
9. Combined Radionuclide Excess Lifetime Cancer Risk for the Eastern 116-K-2 Shallow and Deep Zone Decision Units........................................... 20

10. Combined Radionuclide Excess Lifetime Cancer Risk for the Western 116-K-2 Shallow and Deep Zone Decision Units........................................... 21

11. Radionuclide Excess Lifetime Cancer Risk for the 116-K-2 Overburden Material. 21

\section{TABLES}

1. 116-K-2 Remedial Action Goals. ...................................................................... 2

2. 116-K-2 Cleanup Verification Data Set........................................................ 11

3. Attainment of Nonradionuclide Direct Exposure Standards............................. 16

4. Attainment of Nonradionuclide Groundwater and River Protection Standards. .. 17

5. Application of the WAC 173-340 Three-Part Test. ......................................... 19 
CVP-2006-00001

Rev. 0 


\section{ACRONYMS AND ABBREVIATIONS}

$\begin{array}{ll}\text { COC } & \text { contaminant of concern } \\ \text { CVP } & \text { cleanup verification package } \\ \text { DQA } & \text { data quality assessment } \\ \text { EPA } & \text { U.S. Environmental Protection Agency } \\ \text { ERDF } & \text { Environmental Restoration Disposal Facility } \\ \text { MTCA } & \text { Model Toxics Control Act Cleanup Regulation } \\ \text { Nal } & \text { sodium iodide } \\ \text { RAG } & \text { remedial action goal } \\ \text { RAO } & \text { remedial action objective } \\ \text { RDR/RAWP } & \text { remedial design report/remedial action work plan } \\ \text { RESRAD } & \text { RESidual RADioactivity (dose assessment model) } \\ \text { ROD } & \text { record of decision } \\ \text { SAP } & \text { sampling and analysis plan } \\ \text { UCL } & \text { upper confidence limit } \\ \text { WAC } & \text { Washington Administrative Code }\end{array}$


CVP-2006-00001

Rev. 0 
CVP-2006-00001

Rev. 0

\subsection{INTRODUCTION}

This cleanup verification package (CVP) documents that the 116-K-2 waste site was remediated in accordance with the Amendment to the Interim Action Record of Decision for the 100-BC-1, 100-DR-1, and 100-HR-1 Operable Units, Hanford Site, Benton County, Washington (hereafter referred to as the ROD Amendment) (EPA 1997). Remedial action objectives (RAOs) and remedial action goals (RAGs) for this site are documented in the Interim Action Record of Decision for the 100-BC-1, 100-DR-1, and 100-HR-1 Operable Units, Hanford Site, Benton County, Washington (hereafter referred to as the ROD) (EPA 1995) and the Remedial Design Report/Remedial Action Work Plan for the 100 Area (RDR/RAWP) (DOE-RL 2005b). The ROD provides the U.S. Department of Energy, Richland Operations Office the authority, guidance, and objectives to conduct this remedial action.

The remedy specified in the ROD Amendment and conducted for the 116-K-2 waste site included (1) excavating the site to the extent required to meet specified soil cleanup levels, (2) disposing of contaminated excavation materials at the Environmental Restoration Disposal Facility (ERDF) in the 200 Area of the Hanford Site, and (3) backfilling the site with clean soil to average adjacent grade elevation. Excavation was driven by RAOs for direct exposure, protection of groundwater, and protection of the Columbia River. For the respective points of compliance, RAGs summarized in Table 1 were established for the contaminants of concern (COCs) in the RDR/RAWP (DOE-RL 2005b). The waste site COCs presented in Table 1 were refined from the list presented in the 100 Area Remedial Action Sampling and Analysis Plan (SAP) (DOE-RL 2001) for the 116-K-2 waste site (BHI 2004).

Soil cleanup levels were established in the interim action ROD based on a limited ecological risk assessment. A baseline risk assessment for the river corridor portion of the Hanford Site began in 2004, which includes a more complete quantitative ecological risk assessment. That baseline risk assessment will be used as part of the final ROD for this site.

\subsection{SITE DESCRIPTION AND SUPPORTING INFORMATION}

The 116-K-2 waste site is part of the 100-KR-1 Operable Unit in the 100-K Area. The site consists of a former radioactive liquid effluent disposal trench, approximately $1,200 \mathrm{~m}(4,000 \mathrm{ft})$ long, $14 \mathrm{~m}(45 \mathrm{ft})$ wide at the top, and $4.6 \mathrm{~m}(15 \mathrm{ft})$ deep. The site is located immediately northeast of the former $116-\mathrm{K}-1 \mathrm{crib}$ and runs parallel to the Columbia River (Figure 1).

The 116-K-2 trench was excavated in 1955 to replace the 116-K-1 Crib and received mixed liquid waste effluent until the last of the 100-K Area reactors was shut down in 1971 (Carpenter and Cote 1994). Liquid waste was discharged to the trench by the retention basin drain system (100-K-55 and 100-K-56 pipelines) and consisted of 
CVP-2006-00001

Rev. 0

Table 1. 116-K-2 Remedial Action Goals.

\begin{tabular}{|c|c|c|c|}
\hline cocs & $\begin{array}{l}\text { Direct Exposure } \\
\text { RAG }^{\mathrm{a}}\end{array}$ & $\begin{array}{l}\text { Groundwater } \\
\text { Protection RAG } \\
(\mathrm{pCi} / \mathrm{L})^{\mathrm{b}}\end{array}$ & $\begin{array}{l}\text { Columbia River } \\
\text { Protection RAG } \\
(\mathrm{pCi} / \mathrm{L})^{\mathrm{b}}\end{array}$ \\
\hline Carbon-14 & \multirow{8}{*}{$\begin{array}{c}15 \text { mrem/yr } \\
\text { (cumulative) }^{\mathrm{a}}\end{array}$} & \multirow{6}{*}{$\begin{array}{c}4 \mathrm{mrem} / \mathrm{yr} \\
\text { (cumulative) }^{\mathrm{b}}\end{array}$} & \multirow{6}{*}{$\begin{array}{c}4 \mathrm{mrem} / \mathrm{yr} \\
\text { (cumulative) }^{\mathrm{b}}\end{array}$} \\
\hline Cesium-137 & & & \\
\hline Cobalt-60 & & & \\
\hline Europium-152 & & & \\
\hline Europium-154 & & & \\
\hline Nickel-63 & & & \\
\hline Plutonium-239/240 & & 1.2 & 1.2 \\
\hline Strontium-90 & & $8^{\mathrm{c}, \mathrm{d}}$ & $8^{\mathrm{c}, \mathrm{d}}$ \\
\hline $\mathrm{COC}$ & $\begin{array}{c}\text { Direct Exposure } \\
\text { RAG } \\
(\mathbf{m g} / \mathbf{k g})\end{array}$ & $\begin{array}{c}\text { Soil RAG for } \\
\text { Groundwater Protection } \\
(\mathrm{mg} / \mathrm{kg})\end{array}$ & $\begin{array}{l}\text { Soil RAG for Columbia } \\
\text { River Protection } \\
\text { (mg/kg) }\end{array}$ \\
\hline Hexavalent chromium & $\begin{array}{l}2.1^{\theta} \\
240^{f}\end{array}$ & $4.8^{\mathrm{g}}$ & $2^{\mathrm{h}}$ \\
\hline
\end{tabular}

${ }^{a}$ Lookup values that correspond to the $15 \mathrm{mrem} / \mathrm{yr}$ dose rate are based on a generic site model and are presented in the Remedial Design Report/Remedial Action Work Plan for the 100 Area (DOE-RL 2005b).

${ }^{b}$ Lookup values that correspond to the individual radionuclide $4 \mathrm{mrem} / \mathrm{yr}$ dose rate equivalent for beta- and gamma-emitter RAGs per National Drinking Water Standards are presented in the Remedial Design

Report/Remedial Action Work Plan for the 100 Area (DOE-RL 2005b). Non-uranium alpha emitters must meet drinking water standards for alpha emitters based on the more stringent of the $15 \mathrm{pCi} / \mathrm{L} \mathrm{MCL}$ or $1 / 25$ th of the derived concentration guide per DOE Order 5400.5 .

${ }^{c}$ Strontium-90 also contributes to the $4 \mathrm{mrem} / \mathrm{yr}$ (cumulative) dose rate for groundwater and river protection.

'Promulgated groundwater protection standard (40 CFR 141).

e WAC 173-340-750(3) Method B carcinogenic cleanup limit based on the inhalation exposure pathway, per Calculation of Hexavalent Chromium Carcinogenic Risk (BHI 2000a).

${ }^{\dagger}$ WAC 173-340-740(3) Method B noncarcinogenic cleanup limit.

g Soil RAG based on "100 times groundwater cleanup" rule.

${ }^{\mathrm{h}}$ Soil RAG based on 100 times dilution attenuation factor times surface water quality standard as presented in the Remedial Design Report/Remedial Action Work Plan for the 100 Area (DOE-RL 2005b).

CFR $=$ Code of Federal Regulations

$\mathrm{COC}=$ contaminant of concern

$\mathrm{MCL}=$ maximum contaminant level (drinking water standard)

RAG $=$ remedial action goal

WAC $=$ Washington Administrative Code

reactor cooling water effluent, overflow from the 105-KW and 105-KE Reactor Building fuel storage basins, contaminated liquid from the 105-KW and 105-KE Reactor Building floor drains, dummy decontamination waste, process cooling water following fuel cladding failures, and some special disposal (DOE-RL 1994). Up to 76,000 L/min $(20,000 \mathrm{gal} / \mathrm{min})$ of leakage through the $100-\mathrm{K}$ retention basin butterfly valves was also discharged to the 116-K-2 trench. The 100-K Area was deactivated in 1971 and 1972, at which time the 116-K-2 trench was backfilled with the soil from the original excavation. A small construction tractor and all (empty) 100-K Area hydride tanks were also disposed in the trench during deactivation (Carpenter and Cote 1994). 
Figure 1. Hanford Site Map and Location of the 116-K-2 Waste Site.

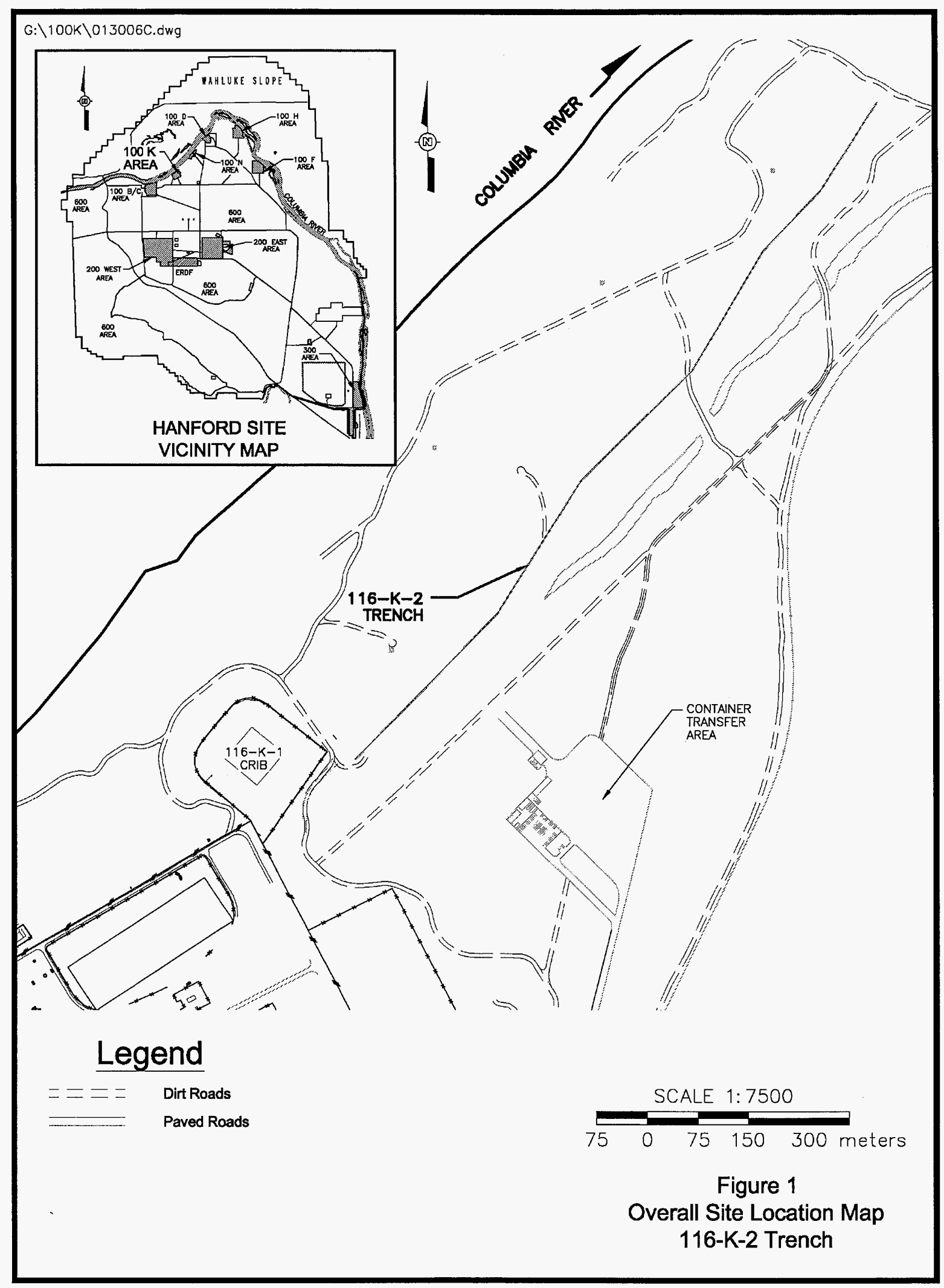


CVP-2006-00001

Rev. 0

\subsection{REMEDIAL ACTION FIELD ACTIVITIES}

\subsection{EXCAVATION AND DISPOSAL}

Remedial action activities at the 116-K-2 waste site were conducted from February 17, 2004 , to October 27, 2005. Remediation involved excavation and staging of clean overburden material and removal of contaminated soil to the extent required to satisfy the RAOs and corresponding RAGs. The previously disposed construction tractor and a small amount of concrete and steel debris were also excavated at the western end of the former trench. Contaminated materials were disposed at the ERDF.

Pre- and post-remediation topographic maps are shown in Figures 2, 3, 4, and 5. Approximately $55,540 \mathrm{~m}^{2}\left(597,830 \mathrm{ft}^{2}\right)$ of plan area was excavated, including excavation within the deep zone (greater than $4.6 \mathrm{~m}$ [15 ft] below ground surface) up to $7.6 \mathrm{~m}$ (25 ft) below ground surface. Approximately 410,000 metric tons (451,900 U.S. tons) of material from the site was removed and disposed at the ERDF.

\subsection{FIELD SCREENING AND VARIANCE SAMPLING}

Radiological field screening was conducted during the site remedial actions as specified in the SAP (DOE-RL 2001). Field screening was used to guide the excavation to quickly assess the presence and level of contamination. Field screening at the site included using a radiological data mapping system survey, hand-held sodium iodide (Nal) detectors, and gamma energy analyses of grab samples. The radiological mapping survey was performed over more than $50 \%$ of the site excavation surface area. The hand-held Nal detectors were used to screen excavated waste material and to screen the excavation wall and floor for potential hot spots. Gamma energy analyses were used to support waste characterization and to corroborate the radiological mapping survey and hand-held $\mathrm{Nal}$ detector data.

Variance analysis was performed following field screening. Because of the size of the 116-K-2 waste site, variance analysis was performed separately for overburden material and the eastern and western portions of the site. The variance analysis quantifies the variability of residual contamination (see calculation briefs in Appendix C). This information was used to determine the site-specific number of final cleanup verification samples to be collected. Initial analytical results indicated areas of excessive residual radionuclide contamination in areas $\mathrm{A} 1, \mathrm{~A} 2, \mathrm{~A} 3$, and $\mathrm{B} 7$ of the eastern $116-\mathrm{K}-2$ remediation footprint. Additional remediation and sampling was performed within these areas, pursuant to the SAP (DOE-RL 2005a). The analytical results for subsequent samples replaced previous samples for the purposes of variance calculations (Appendix C) as follows:

- Sampling node S-A1-3: Sample J103C1 replaced three previous sequential samples at the node (samples J03CT0, J03W01, and J03W11)

- Sampling node S-A1-10: Sample J03W02 replaced sample J03CT2 
Figure 2. Pre-Remediation Topographic Plan for the Western Portion of the 116-K-2 Waste Site.

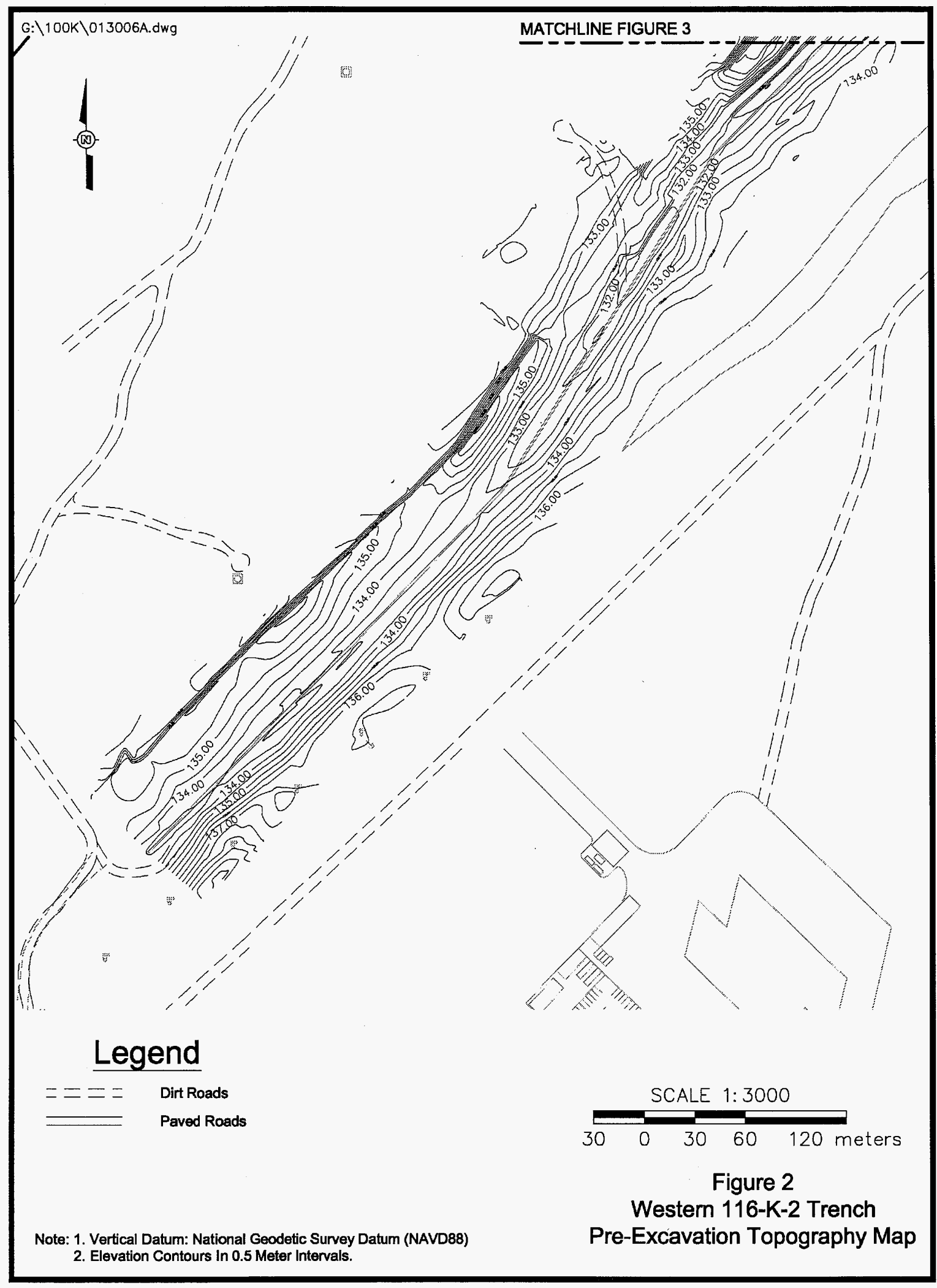


Figure 3. Pre-Remediation Topographic Plan for the Eastern Portion of the 116-K-2 Waste Site.

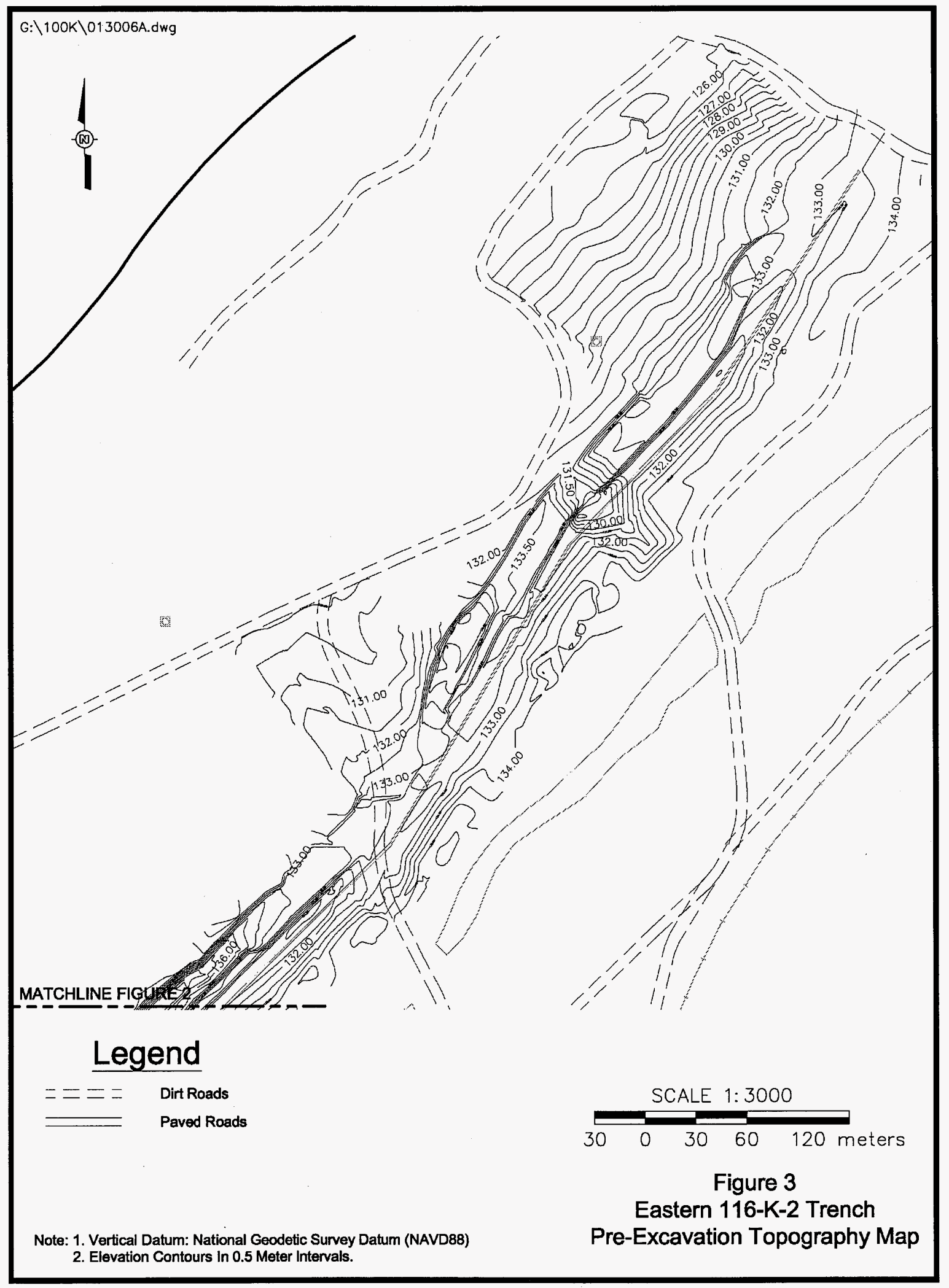


Figure 4. Post-Remediation Topographic Plan for the Western Portion of the 116-K-2 Waste Site.

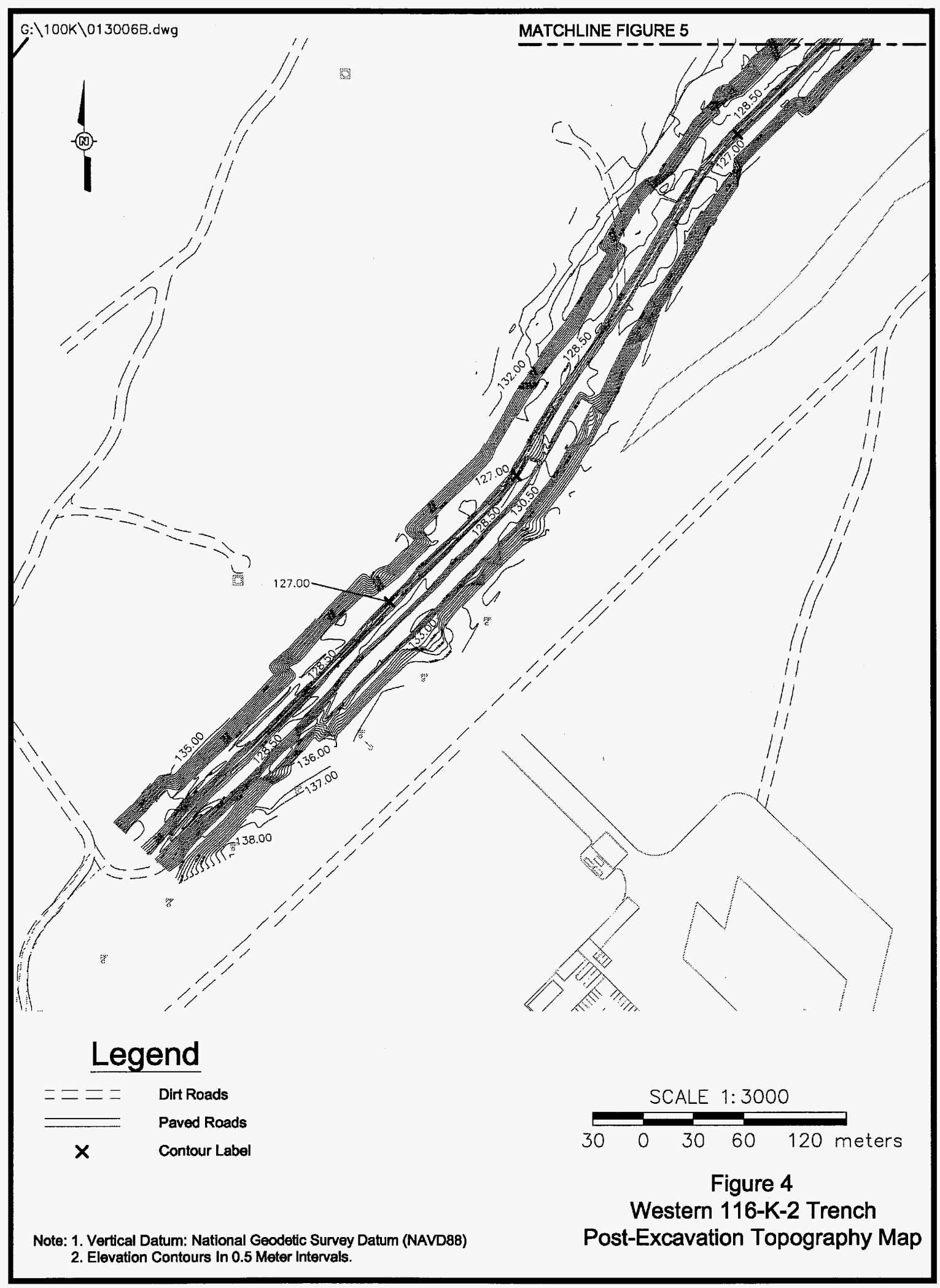


Figure 5. Post-Remediation Topographic Plan for the Eastern Portion of the 116-K-2 Waste Site.

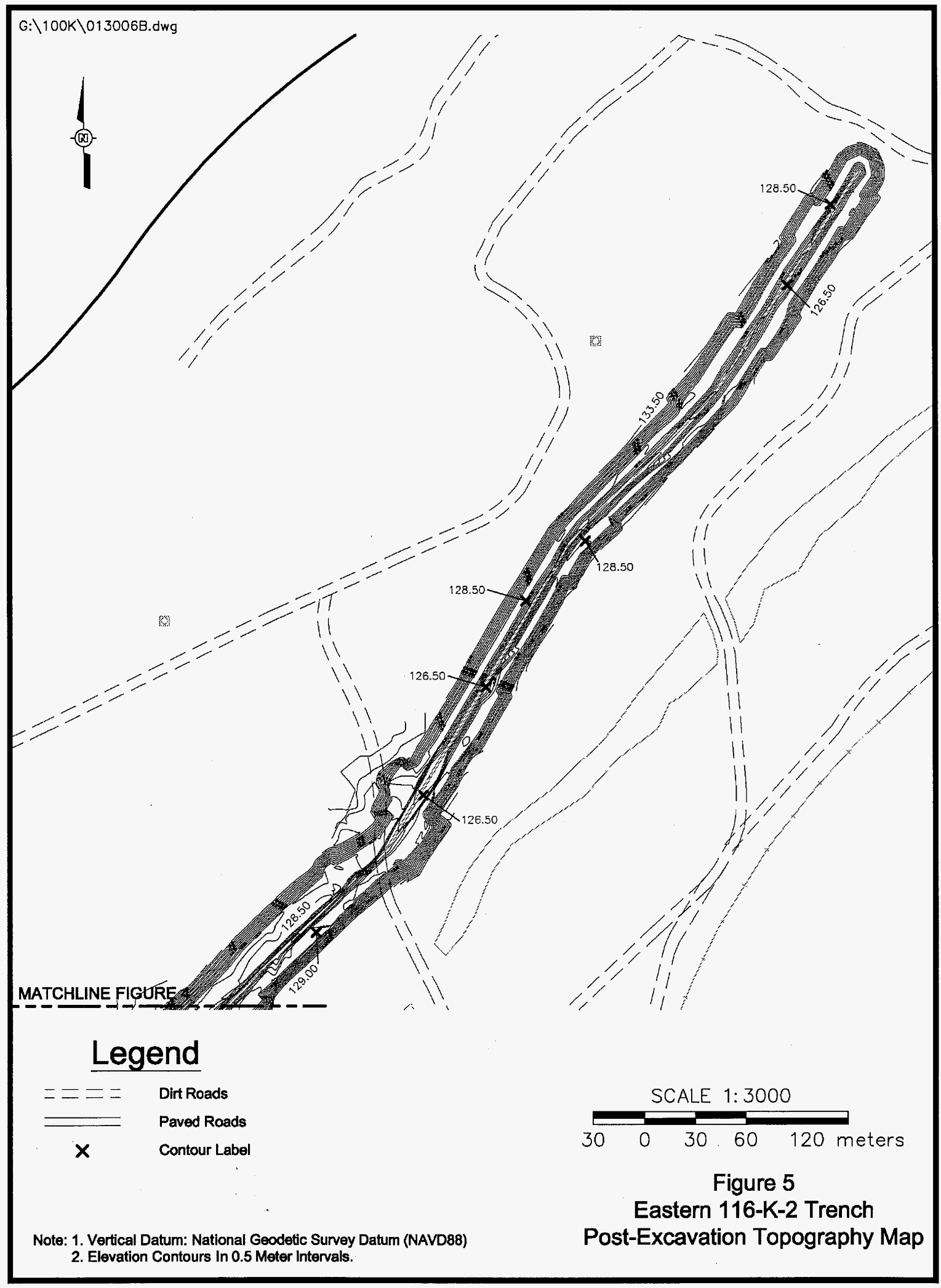


- Sampling node S-A2-15: Sample J03W03 replaced sample J03CV0

- Sampling node S-A3-1: Sample J03W04 replaced sample J03CV1

- Sampling node S-A3-9: Sample J03W05 replaced sample J03CV5

- Sampling node S-A3-11: Sample J03W06 replaced sample J03CV6

- Sampling node S-B7-3: Sample J03W07 replaced sample J03CX6.

The results of the variance analyses indicated that the number of verification samples to be taken for each shallow zone and overburden decision subunit of the 116-K-2 site was less than the default number of four specified in the SAP (DOE-RL 2005a); therefore, four final verification samples were collected from each shallow zone and overburden decision subunit. The default number of three verification samples (DOE-RL 2005a) was collected from each deep zone decision subunit.

\subsection{CLEANUP VERIFICATION SAMPLING AND ANALYSIS}

Final cleanup verification sampling was conducted from July 6,2005 , to November 2 , 2005 (BHI 2005), following variance analyses. The final verification samples were submitted to offsite laboratories for analysis using approved U.S. Environmental Protection Agency (EPA) analytical methods as required per the SAP (DOE-RL 2005a). Each verification sample was composed of a composite sample formed by combining soil collected at the required number of randomly selected locations within each sampling area (excluding the quality assurance/quality control samples).

The division of the 116-K-2 site excavation into decision units (i.e., shallow zone and deep zone) as shown on the sample design figures (Appendix $\mathrm{C}$ ) is a function of the applicable RAGs. The direct exposure, groundwater protection, and river protection

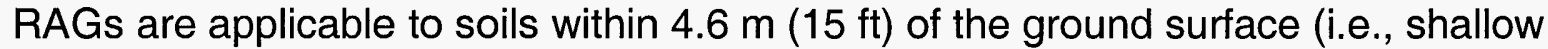
zone and overburden soil). The groundwater protection and river protection RAGs are applicable to soils greater than $4.6 \mathrm{~m}$ (15 ft) below the ground surface (i.e., deep zone).

The 116-K-2 waste site consisted of shallow zone, deep zone, and overburden decision units. The shallow zone consisted of the excavation sidewalls and floors that were less than $4.6 \mathrm{~m}(15 \mathrm{ft})$ below ground surface. The deep zone consisted of the portions of the excavation sidewalls and excavation floor that were more than $4.6 \mathrm{~m}(15 \mathrm{ft})$ below ground surface. The shallow zone decision unit for the 116-K-2 waste site contained six decision subunits, divided into four sampling areas per decision subunit. The deep zone decision unit for the site contained eight decision subunits, divided into three sampling areas per decision subunit. The overburden decision unit for the site contained two subunits, divided into four sampling areas per decision subunit. All sampling areas were further divided into 16 sampling nodes each as shown in the sample design methodology and sample location figures presented in the calculation briefs in Appendix C. Initial analytical results indicated areas of excessive residual contamination in sampling 
areas $\mathrm{A} 1$ and $\mathrm{A} 3$ of the eastern shallow zone decision unit. Additional remediation was performed and additional samples collected at the sampling nodes pursuant to the SAP (DOE-RL 2005a). The analytical results for the initial samples were replaced with those for the subsequent samples for the purposes of statistical calculations, as described in the cleanup verification calculation brief (Appendix C).

\subsection{CLEANUP VERIFICATION DATA EVALUATION}

This section presents the evaluation and modeling of the 116-K-2 cleanup verification data for comparison with the data quality criteria and RAGs.

\subsection{DATA QUALITY ASSESSMENT PROCESS}

A data quality assessment (DQA) is performed to compare the verification sampling approach and resulting analytical data with the sampling and data quality requirements specified by the project objectives and performance specifications.

The DQA for the 116-K-2 waste site determined that the data are of the right type, quality, and quantity to support site verification decisions within specified error tolerances. All analytical data were found to be acceptable for decision-making purposes. The evaluation also verified that the sample design was sufficient to support clean site verification. The cleanup verification sample analytical data are stored in the Hanford Environmental Information System and are summarized in Appendix A. The detailed DQA is presented in Appendix B.

\subsection{CONTAMINANTS OF CONCERN 95\% UPPER CONFIDENCE LIMIT}

The primary statistical calculation to support cleanup verification is the $95 \%$ upper confidence limit (UCL) on the arithmetic mean of the data. The $95 \%$ UCL values for each COC are computed for each decision unit (i.e., shallow zone, deep zone, and overburden). Prior to calculating the $95 \%$ UCL, the individual sample results are reviewed and, as appropriate, adjusted per the SAP (DOE-RL 2005a). This process is summarized below.

Verification sampling summary statistics (95\% UCL values) for the $116-\mathrm{K}-2$ waste site are listed in Table 2. Individual sample cleanup verification results are presented in Appendix A. In the interest of expediting field operations at the 116-K-2 waste site, statistical analysis of verification data sets was performed separately for the eastern and western portions of the shallow and deep zone decision units. 
CVP-2006-00001

Rev. 0

Table 2. 116-K-2 Cleanup Verification Data Set.

\begin{tabular}{|c|c|c|c|c|c|c|c|}
\hline \multirow{2}{*}{ cocs } & \multicolumn{3}{|c|}{$\begin{array}{l}\text { 95\% UCL Statistical Values } \\
(\mathrm{pCi} / \mathrm{g})\end{array}$} & \multirow{2}{*}{$\begin{array}{l}\text { Hanford Site } \\
\text { Background } \\
\text { (pCi/g) }\end{array}$} & \multicolumn{3}{|c|}{$\begin{array}{c}\text { Cleanup Verification Data Set } \\
(\mathrm{pCi} / \mathrm{g})\end{array}$} \\
\hline & $\begin{array}{l}\text { Shallow } \\
\text { Zone }\end{array}$ & $\begin{array}{l}\text { Deep } \\
\text { Zone }\end{array}$ & Overburden $^{a}$ & & $\begin{array}{l}\text { Shallow } \\
\text { Zone }\end{array}$ & $\begin{array}{l}\text { Deep } \\
\text { Zone }\end{array}$ & Overburden \\
\hline \multicolumn{8}{|c|}{$116-K-2\left(\right.$ East) ${ }^{d}$} \\
\hline Carbon-14 & 0.70 & 1.26 & 0.68 (ND) & NA & 0.70 & 1.26 & 0.68 (ND) \\
\hline Cesium-137 & 0.56 & 130 & 0.083 & 1.1 & 0.56 & 130 & $0(<B G)$ \\
\hline Cobalt-60 & $\begin{array}{l}0.018 \\
\text { (ND) }\end{array}$ & 2.48 & 0.020 (ND) & 0.008 & $\begin{array}{l}0.018 \\
\text { (ND) }\end{array}$ & 2.48 & $0.012(\mathrm{ND})$ \\
\hline Europium-152 & 0.153 & 62.7 & 0.17 & NA & 0.153 & 62.7 & 0.17 \\
\hline Europium-154 & $\begin{array}{c}0.056 \\
\text { (ND) }\end{array}$ & 5.4 & 0.068 (ND) & 0.033 & $\begin{array}{l}0.056 \\
\text { (ND) }\end{array}$ & 5.4 & 0.035 (ND) \\
\hline Nickel-63 & 3.56 & 880 & 0.689 (ND) & NA & 3.56 & 880 & 0.689 (ND) \\
\hline $\begin{array}{l}\text { Plutonium- } \\
239 / 240\end{array}$ & $\begin{array}{l}0.023 \\
(\mathrm{ND})\end{array}$ & 6.9 & 0.031 (ND) & 0.025 & $\begin{array}{l}0.023 \\
\text { (ND) }\end{array}$ & 6.9 & 0.006 (ND) \\
\hline Strontium-90 & 0.143 & 7.12 & 0.062 (ND) & 0.18 & 0.143 & 7.12 & $0(<B G)$ \\
\hline \multicolumn{8}{|c|}{$116-K-2(\text { West })^{d}$} \\
\hline Carbon-14 & 0.29 & 1.44 & $0.68(\mathrm{ND})$ & $\mathrm{NA}$ & 0.29 & 1.44 & 0.68 (ND) \\
\hline Cesium-137 & 1.10 & 117 & 0.083 & 1.1 & 1.10 & 117 & $0(<B G)$ \\
\hline Cobalt-60 & $\begin{array}{l}0.023 \\
(\mathrm{ND})\end{array}$ & 4.23 & 0.020 (ND) & 0.008 & $\begin{array}{l}0.023 \\
(\mathrm{ND})\end{array}$ & 4.23 & 0.012 (ND) \\
\hline Europium-152 & 0.626 & 76 & 0.17 & NA & 0.626 & 76 & 0.17 \\
\hline Europium-154 & 0.129 & 7.2 & 0.068 (ND) & 0.033 & 0.129 & 7.2 & 0.035 (ND) \\
\hline Nickel-63 & 1.77 & 650 & 0.689 (ND) & NA & 1.77 & 650 & 0.689 (ND) \\
\hline $\begin{array}{l}\text { Plutonium- } \\
239 / 240\end{array}$ & $\begin{array}{c}0.024 \\
(\mathrm{ND})\end{array}$ & 4.7 & 0.031 (ND) & 0.025 & $\begin{array}{l}0.024 \\
(\mathrm{ND})\end{array}$ & 4.7 & 0.006 (ND) \\
\hline Strontium-90 & 0.201 & 6.3 & 0.062 (ND) & 0.18 & 0.201 & 6.3 & $0(<B G)$ \\
\hline \multicolumn{8}{|c|}{$116-K-2$ (East) $^{d}$} \\
\hline $\begin{array}{l}\text { Hexavalent } \\
\text { chromium }\end{array}$ & 0.39 & 5.3 & 0.27 & NA & 0.39 & 5.3 & 0.27 \\
\hline \multicolumn{8}{|c|}{$116-K-2(W e s t)^{d}$} \\
\hline $\begin{array}{l}\text { Hexavalent } \\
\text { chromium }\end{array}$ & 0.23 & 2.4 & 0.27 & NA & 0.23 & 2.4 & 0.27 \\
\hline
\end{tabular}

${ }^{a}$ Overburden material from the eastern and western portions of the $116-\mathrm{K}-2$ waste site was considered within the same decision unit.

${ }^{\mathrm{b}}$ Represents the 90th percentile of the lognormal distribution (DOE-RL 1996).

${ }^{c}$ For overburden, anthropogenic background (DOE-RL 1996) and naturally occurring background is subtracted from all radionuclides. For other decision units (i.e., shallow zone and deep zone), only naturally occurring background (uranium) is subtracted. Refer to the $95 \%$ UCL calculation brief in Appendix $C$ for additional details on determination of statistical values.

${ }^{d}$ Laboratory data, including the minimum detectable activities for the individual cleanup verification samples, are included in Appendix A and the 95\% UCL calculation briefs in Appendix C.

$B G=$ background

COC = contaminant of concern

ND = not detected (in all samples in the data set)

NA = not applicable

$\mathrm{UCL}=$ upper confidence limit 
For radionuclides, the laboratory-reported value is used in the calculation of the $95 \%$ UCL. In cases where the laboratory does not report a value for data qualified with a "U" (i.e., less than the detection limit), one-half of the minimum detectable activity is used in the calculation of the $95 \%$ UCL. For nonradionuclides, a value equal to one-half the practical quantitation limit is used for data flagged with a "U" (i.e., less than the detection limit) in the calculation of the $95 \% \mathrm{UCL}$, as required by Washington Administrative Code (WAC) 173-340-740[7][g].

Statistical calculations for $116-\mathrm{K}-2$ verification data sets are presented in the $95 \% \mathrm{UCL}$ calculation briefs (Appendix C), with results shown in Table 2. The columns on the left side of Table 2 are the COCs and the 95\% statistical values before subtraction of background. The fifth column of Table 2 presents the background where values exist, and the last three columns present the statistical values adjusted for background, if appropriate, which becomes the cleanup verification data set used for RESidual RADioactivity (RESRAD) modeling.

\subsection{SITE-SPECIFIC CLEANUP VERIFICATION MODEL}

The statistical values summarized in Table 2 were evaluated and used to develop site-specific cleanup verification models. The 116-K-2 site cleanup verification models comprise three depth intervals: (1) the shallow zone and overburden, (2) the contaminated deep zone, and (3) the uncontaminated vadose (deep) zone. Based on the conservative assumption that residual contaminant levels in the deep zone data set extend uniformly to groundwater (as discussed in the RDR/RAWP [DOE-RL 2005b]), residual soil activities of nickel-63 would result in prediction of a groundwater concentration exceeding the RAG. Because this approach is overly conservative, test pit data from the analogous 116-C-1 Process Effluent Trench were used to develop a refined model of the deep zone, including an underlying portion of uncontaminated vadose zone. Schematic cross sections of this site-specific cleanup verification model are included in the RESRAD calculations in Appendix C. The elements of the RESRAD modeling are described in Section 4.4.

\subsection{RESRAD MODELING}

The individual radionuclide cleanup verification statistical values (Table 2) were entered into the RESRAD computer code, Version 6.3 (ANL 2005), to estimate the residential dose rate and predict the impact on groundwater and the Columbia River from residual radionuclide $\mathrm{COC}$ concentrations. The direct radiation exposure dose rate to the resident living in his or her basement (rural-residential scenario) was conservatively estimated by substituting (for analysis purposes) a case where the resident is standing on level ground with the soil containing concentrations representative of residual (i.e., post-cleanup) shallow zone soils. This is conservative because it ignores the potential shielding effects of concrete basement walls and any clean backfill between residual soils and the basement walls. Following the separate statistical analyses of verification 
data for the eastern and western portions of the 116-K-2 site, RESRAD modeling was performed separately for each portion of the trench using the applicable data set.

The RESRAD modeling methodologies, results, input values, and the site-specific cleanup verification model are included in the RESRAD calculation briefs (Appendix C). Specific results from the calculations are discussed as part the RAG-attainment evaluation (Section 5.0).

\subsection{EVALUATION OF REMEDIAL ACTION GOAL ATTAINMENT}

This section demonstrates that remedial action at the 116-K-2 waste site has achieved the applicable RAGs. Sections 5.1,5.2, and 5.3 address attainment of direct exposure RAGs, groundwater protection RAGs, and Columbia River protection RAGs, respectively. Section 5.4 documents application of the WAC 173-340-740(7)(e) three-part test, which is required for nonradionuclide COCs only.

\subsection{DIRECT EXPOSURE SOIL REMEDIAL ACTION GOALS ATTAINED}

\subsubsection{Radionuclides}

The results of the combined RESRAD dose rate estimates for the 116-K-2 site shallow and deep zone all-pathways scenarios are presented in Figures 6 and 7 . These dose rates represent the contributions from soils at relevant time periods. The results of the RESRAD dose rate estimate for overburden material under the all-pathways scenario is presented in Figure 8. The dose rates for the eastern and western portions of the 116-K-2 site are largest at present (year 2006), at $4.62 \mathrm{mrem} / \mathrm{yr}$ and $7.77 \mathrm{mrem} / \mathrm{yr}$, respectively, decreasing to $9.99 \times 10^{-4} \mathrm{mrem} / \mathrm{yr}$ and $7.39 \times 10^{-4} \mathrm{mrem} / \mathrm{yr}$, respectively, in 1,000 years for the shallow zone and deep zone. The estimated dose rate in the year 2018 is $1.83 \mathrm{mrem} / \mathrm{yr}$ for the eastern portion of the $116-\mathrm{K}-2$ site and $4.33 \mathrm{mrem} / \mathrm{yr}$ for the western portion. The 2018 date corresponds to the original 30-year site cleanup schedule of the Hanford Federal Facility Agreement and Consent Order (Ecology et al. 1989). The dose rate for overburden soils from the site is largest at present (year 2006), at $0.740 \mathrm{mrem} / \mathrm{yr}$, decreasing to $0.376 \mathrm{mrem} / \mathrm{yr}$ in 2018 and to $2.61 \times 10^{-16}$ $\mathrm{mrem} / \mathrm{yr}$ in 1,000 years. All dose rate estimates are less than the $15 \mathrm{mrem} / \mathrm{yr}$ RAG. The RESRAD computations are presented in detail in the RESRAD calculation briefs summarized in Appendix C. 
Figure 6. Combined Shallow and Deep Zone Dose Rate Estimates for the Eastern 116-K-2 Site (All Radionuclides, All Pathways).

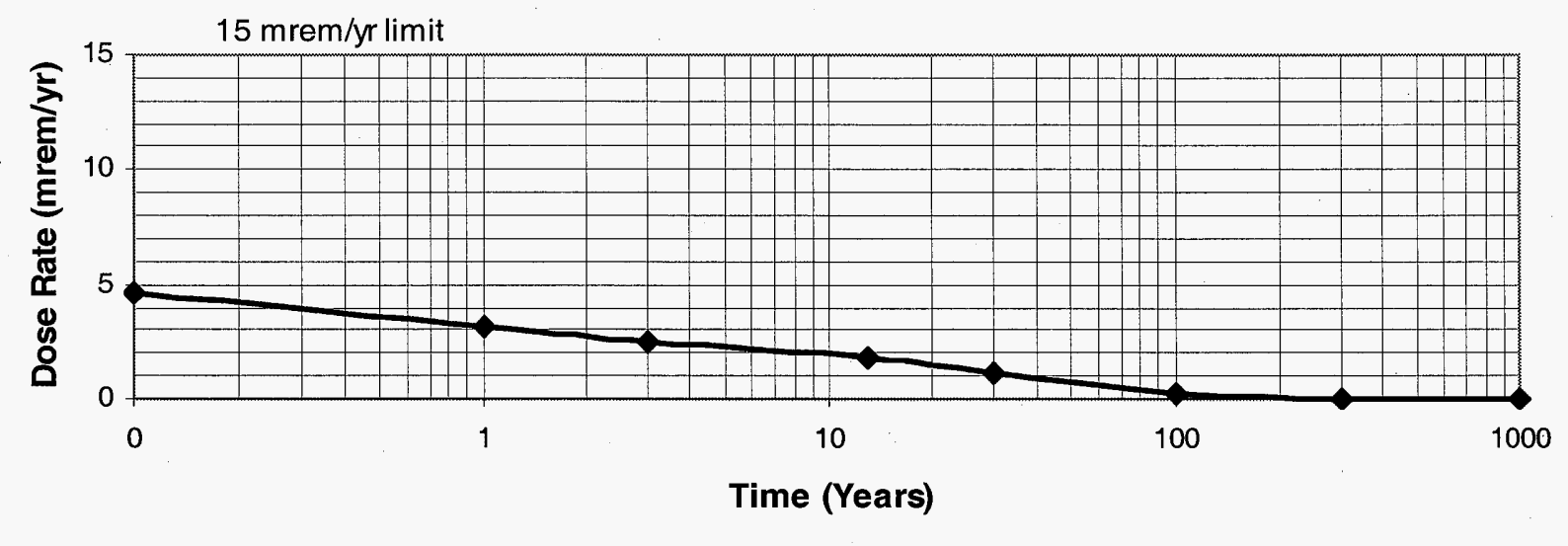

Figure 7. Combined Shallow and Deep Zone Dose Rate Estimates for the Western 116-K-2 Site (All Radionuclides, All Pathways).

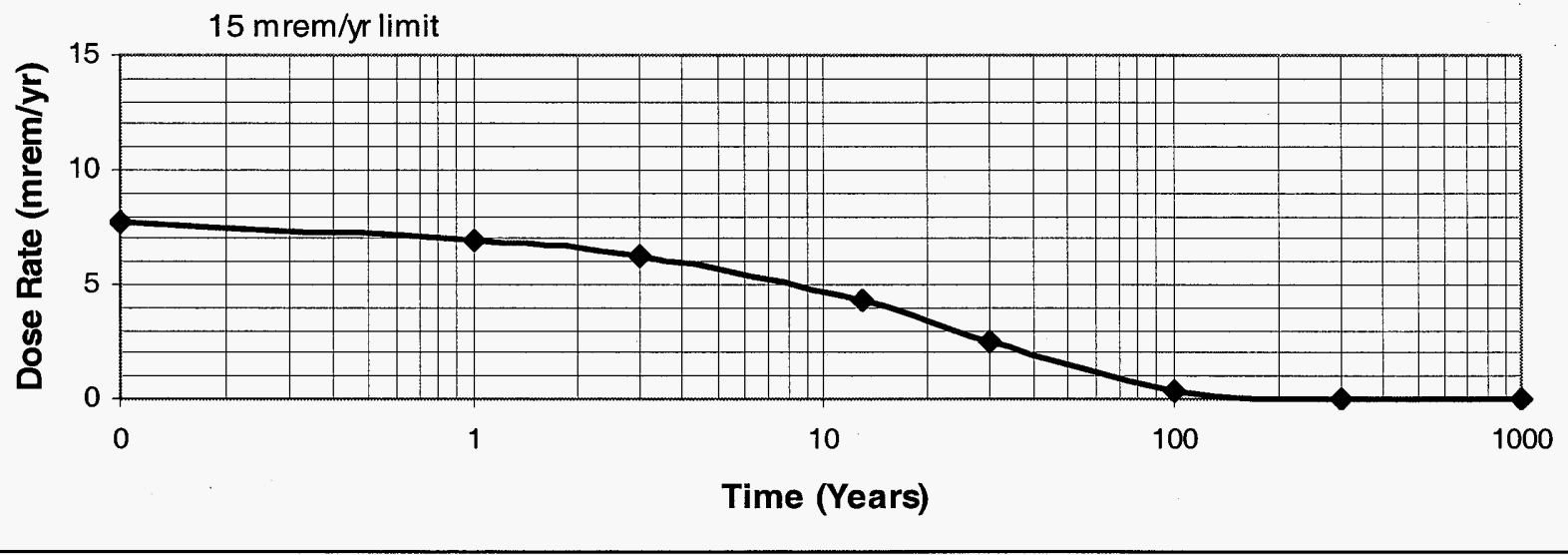


Figure 8. Overburden Dose Rate Estimates for the 116-K-2 Site (All Radionuclides, All Pathways).

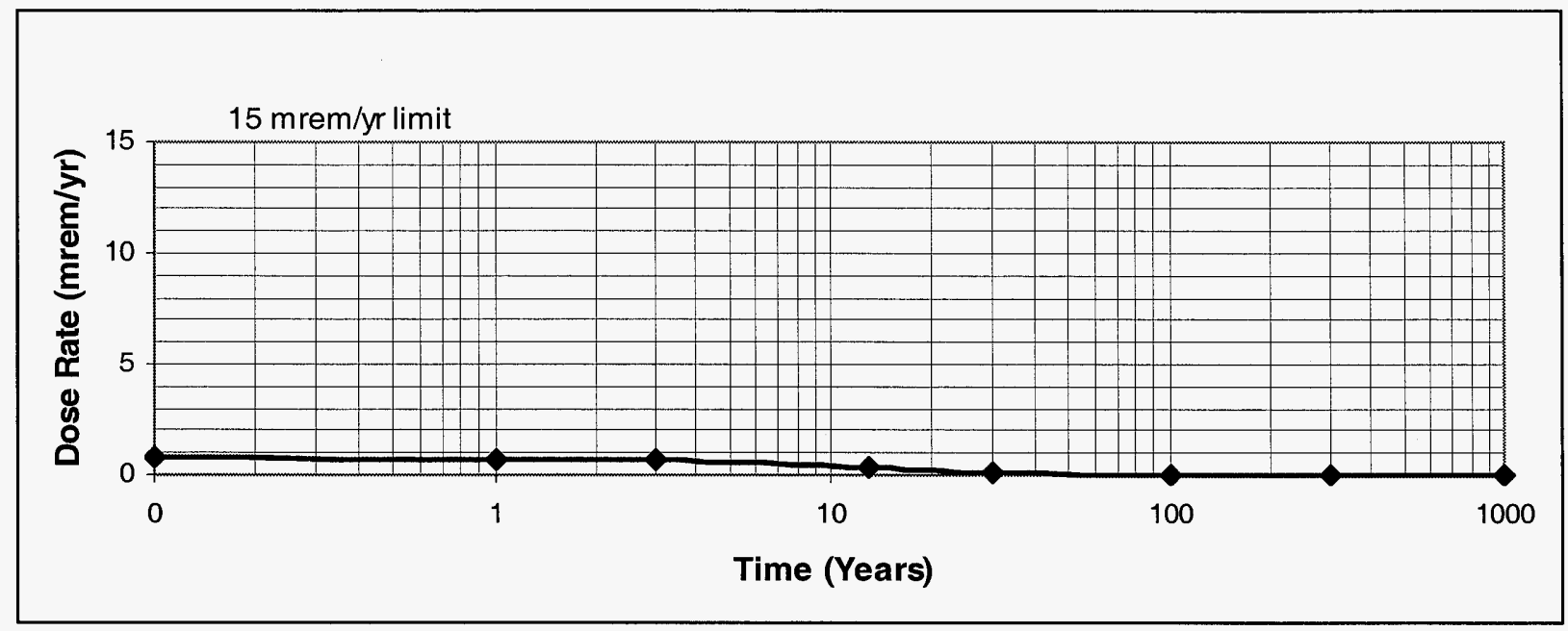

\subsubsection{Nonradionuclides}

5.1.2.1 Direct Comparison to RAGs. Table 3 compares the shallow zone nonradionuclide cleanup verification statistical values presented in Table 2 to the direct exposure RAG presented in Table 1. Residual concentrations of hexavalent chromium, the sole nonradionuclide $\mathrm{COC}$ for the $116-\mathrm{K}-2$ waste site, are less than the direct exposure RAG.

5.1.2.2 Noncarcinogenic Hazard Quotient RAG Attained. For noncarcinogenic COCs, WAC 173-340-740(5)(a) and (b) specify the evaluation of the hazard quotient, which is given as daily intake divided by a reference dose (DOE-RL 2005b). This evaluation is shown in the $95 \%$ UCL calculation briefs (Appendix C). The calculated hazard quotients for statistical residual hexavalent chromium concentrations (the only nonradionuclide $\mathrm{COC}$ ) at the eastern and western portions of the 116-K-2 site and overburden are $1.6 \times 10^{-3}, 9.6 \times 10^{-4}$, and $1.1 \times 10^{-3}$, respectively. These values are below the individual and cumulative RAGs (a hazard quotient of 1.0 in both cases).

5.1.2.3 Carcinogenic Risk RAG Attained. For individual nonradionuclide carcinogenic COCs, the WAC 173-340-700(3) Method B cleanup limits are based on an incremental cancer risk of $1 \times 10^{-6}$. The cumulative excess cancer risk for all nonradionuclide carcinogenic COCs must be less than $1 \times 10^{-5}$. The only nonradionuclide COC at the 116-K-2 site, hexavalent chromium, is a carcinogen in the inhalation exposure pathway. The excess lifetime cancer risk estimate for residual concentrations of this $\mathrm{COC}$ at the eastern and western portions of the site and overburden are $1.9 \times 10^{-7}, 1.1 \times 10^{-7}$, and $1.3 \times 10^{-7}$, respectively. These values are below the risk limit for individual COCs $\left(1 \times 10^{-6}\right)$ and the cumulative excess carcinogenic risk RAG of $1 \times 10^{-5}$. 
Table 3. Attainment of Nonradionuclide Direct Exposure Standards.

\begin{tabular}{|c|c|c|c|}
\hline COC & $\begin{array}{c}\text { Direct Exposure RAG } \\
\text { (mg/kg) }\end{array}$ & $\begin{array}{c}\text { Cleanup } \\
\text { Verification Data } \\
\text { Set } \\
(\mathbf{m g} / \mathbf{k g})\end{array}$ & $\begin{array}{c}\text { Direct Exposure } \\
\text { RAG Attained? }\end{array}$ \\
\hline \multicolumn{4}{|c|}{$116-\mathrm{K}-2$ (East) } \\
\hline Hexavalent chromium & $\begin{array}{l}2.1^{\mathrm{b}} \\
240^{\mathrm{c}}\end{array}$ & 0.39 & Yes \\
\hline \multicolumn{5}{|c|}{$116-\mathrm{K}-2$ (West) } \\
\hline Hexavalent chromium & $\begin{array}{l}2.1^{\mathrm{b}} \\
240^{\mathrm{c}}\end{array}$ & 0.23 & Yes \\
\hline \multicolumn{5}{|c|}{ Overburden } \\
\hline Hexavalent chromium & $\begin{array}{l}2.1^{\mathrm{b}} \\
240^{\mathrm{c}}\end{array}$ & 0.27 & Yes \\
\hline
\end{tabular}

${ }^{\text {a }}$ Criterion is comparison to direct exposure RAG.

${ }^{\mathrm{b}}$ WAC 173-340-750(3) Method B carcinogenic cleanup limit based on the inhalation exposure pathway, per Calculation of Hexavalent Chromium Carcinogenic Risk (BHI 2000a).

${ }^{c}$ WAC 173-340-740(3) Method B noncarcinogenic cleanup limit.

$\mathrm{COC}=$ contaminant of concern

$\mathrm{RAG}=$ remedial action goal

WAC $=$ Washington Administrative Code

\subsection{GROUNDWATER REMEDIAL ACTION GOALS ATTAINED}

\subsubsection{Radionuclides}

Radionuclide COCs associated with the 116-K-2 site are not predicted to reach groundwater within 1,000 years, based on the model described in Section 4.3 and the RESRAD calculation briefs (Appendix $\mathrm{C}$ ). Therefore, the groundwater protection RAGs have been attained.

\subsubsection{Nonradionuclides}

Table 4 compares the shallow and deep zone nonradionuclide cleanup verification statistical values presented in Table 2 to the soil RAG for groundwater protection presented in Table 1. Residual shallow zone concentrations of hexavalent chromium, the sole nonradionuclide COC for the 116-K-2 waste site, are less than the soil RAG for groundwater protection. However, the statistical residual concentration of hexavalent chromium in the eastern deep zone exceed this RAG. Because the soil RAG was not met, a detailed assessment of the potential impact of residual hexavalent chromium to groundwater was made using the 100-D, 100-H, and 100-F Area hexavalent chromium leach studies (BHI 1999, 2000b, and 2001b). 
Table 4. Attainment of Nonradionuclide Groundwater and River Protection Standards.

\begin{tabular}{|c|c|c|c|c|}
\hline $\operatorname{coc}$ & $\begin{array}{c}\text { Cleanup } \\
\text { Verification Data } \\
\text { Set } \\
(\mathrm{mg} / \mathrm{kg})\end{array}$ & $\begin{array}{l}\text { Soil RAG for } \\
\text { Groundwater } \\
\text { Protection } \\
\text { (mg/kg) }\end{array}$ & $\begin{array}{l}\text { Soil RAG for } \\
\text { River } \\
\text { Protection } \\
\text { (mg/kg) }\end{array}$ & $\begin{array}{l}\text { Cleanup Criteria } \\
\text { Attained? }\end{array}$ \\
\hline \multicolumn{5}{|c|}{ 116-K-2 (East) Shallow Zone } \\
\hline Hexavalent chromium & 0.39 & $4.8^{\mathrm{a}}$ & $2^{b}$ & Yes \\
\hline \multicolumn{5}{|c|}{ 116-K-2 (East) Deep Zone } \\
\hline Hexavalent chromium & 5.3 & $4.8^{\mathrm{a}}$ & $2^{b}$ & $\mathrm{Yes}^{\mathrm{c}}$ \\
\hline \multicolumn{5}{|c|}{ 116-K-2 (West) Shallow Zone } \\
\hline Hexavalent chromium & 0.23 & $4.8^{\mathrm{a}}$ & $2^{\mathrm{b}}$ & Yes \\
\hline \multicolumn{5}{|c|}{ 116-K-2 (West) Deep Zone } \\
\hline Hexavalent chromium & 2.4 & $4.8^{\mathrm{a}}$ & $2^{b}$ & Yes $^{c}$ \\
\hline \multicolumn{5}{|c|}{ Overburden } \\
\hline Hexavalent chromium & 0.27 & $4.8^{\mathrm{a}}$ & $2^{b}$ & Yes \\
\hline
\end{tabular}

${ }^{a}$ Criterion is comparison to soil RAG for groundwater protection.

${ }^{b}$ Soil RAG based on "100 times groundwater cleanup" rule.

Soil RAG based on 100 times dilution attenuation factor times surface water quality standard as presented in the Remedial Design Report/Remedial Action Work Plan for the 100 Area (DOE-RL 2005b).

${ }^{\circ}$ Based on the 100-D, 100-F, and 100-H Area hexavalent chromium leach studies, groundwater and surface water quality criteria will not be exceeded where hexavalent chromium concentrations in soil are less than $6.8 \mathrm{mg} / \mathrm{kg}$.

$\mathrm{COC}=$ contaminant of concern

RAG $=$ remedial action goal

The groundwater protection soil RAG is based on the Model Toxics Control Act Cleanup Regulation (MTCA) "100 times groundwater quality criteria" rule. The MTCA Method B hexavalent chromium cleanup level for groundwater is $48 \mu \mathrm{g} / \mathrm{L}$ (WAC 173-340-720(3)) based on the 1998 update to the Integrated Risk Information System data for hexavalent chromium. The near-shore river water hexavalent chromium cleanup level of $20 \mu \mathrm{g} / \mathrm{L}$ (surface water quality criteria of $10 \mu \mathrm{g} / \mathrm{L}$ [WAC 173-201A] times the dilution attenuation factor of 2 ) is more restrictive than the groundwater quality criteria. Based on the 100-D, 100-F, and 100-H Area hexavalent chromium leach studies, the hexavalent chromium soil concentration at which the leachate did not exceed the more restrictive surface water quality criteria was $6.8 \mathrm{mg} / \mathrm{kg}$. Therefore, the statistical residual eastern 116-K-2 deep zone concentration of hexavalent chromium $(5.3 \mathrm{mg} / \mathrm{kg})$ will not leach to groundwater at a concentration exceeding the groundwater quality criteria and is, therefore, protective of groundwater. 
CVP-2006-00001

Rev. 0

\subsection{COLUMBIA RIVER REMEDIAL ACTION GOALS ATTAINED}

\subsubsection{Radionuclides}

Radionuclide COCs associated with the 116-K-2 site are not predicted to reach groundwater within 1,000 years, based on the model described in Section 4.3 and the RESRAD calculation briefs (Appendix $C$ ). The only pathway for contaminant migration to the Columbia River is via groundwater; therefore, the river protection RAGs have been attained.

\subsubsection{Nonradionuclides}

Residual shallow zone concentrations of hexavalent chromium, the sole nonradionuclide $\mathrm{COC}$ for the 116-K-2 waste site, are less than the applicable soil RAG for protection of the Columbia River (Table 4). However, the deep zone residual hexavalent chromium concentrations exceed this RAG. Because the soil RAG was not met, a detailed assessment of the potential impact of residual hexavalent chromium to groundwater was made using the $100-\mathrm{D}, 100-\mathrm{H}$, and 100-F Area hexavalent chromium leach studies (BHI 1999, 2000b, and 2001b).

The river protection soil RAG is based on the MTCA "100 times dilution attenuation factor times surface water quality criteria" rule. The near-shore river water hexavalent chromium cleanup level is $20 \mu \mathrm{g} / \mathrm{L}$ (surface water quality criteria of $10 \mu \mathrm{g} / \mathrm{L}$ [WAC $173-201 \mathrm{~A}]$ times the dilution attenuation factor of 2). Based on the 100-D, 100-F, and 100-H Area hexavalent chromium leach studies, the hexavalent chromium soil concentration at which the leachate did not exceed the surface water quality criteria was $6.8 \mathrm{mg} / \mathrm{kg}$. Therefore, statistical residual 116-K-2 deep zone concentrations of hexavalent chromium $(5.3 \mathrm{mg} / \mathrm{kg}$ and $2.4 \mathrm{mg} / \mathrm{kg}$ ) will not leach to the Columbia River at concentrations exceeding the surface water quality criteria and are, therefore, protective of the river.

\subsection{WAC 173-340 THREE-PART TEST FOR NONRADIONUCLIDES}

The WAC 173-340-740(7)(e) three-part test is required for nonradionuclide statistical verification data sets. The three-part test consists of the following criteria: (1) the cleanup verification statistical value must be less than the most restrictive cleanup level, (2) no single detection within the data set can exceed two times the most restrictive cleanup criteria, and (3) the percentage of samples in the data set exceeding the most restrictive cleanup criteria must be less than $10 \%$.

Table 5 summarizes the results of the WAC 173-340-740[7][e] three-part test for the 116-K-2 cleanup verification nonradionuclide data sets in comparison to the most restrictive applicable RAG. The table lists the most restrictive RAG (from Table 1), the maximum detected value, the total number of samples collected, and the percentage of samples exceeding the RAG. The final column of the table describes the result of applying the three criteria using the values listed in the preceding columns. 
Table 5. Application of the WAC 173-340 Three-Part Test.

\begin{tabular}{|c|c|c|c|c|c|c|}
\hline coc & $\begin{array}{c}\text { Most } \\
\text { Restrictive } \\
\text { Applicable } \\
\text { RAG }^{\mathrm{a}}\end{array}$ & $\begin{array}{c}\text { Statistical } \\
\text { Cleanup } \\
\text { Verification } \\
\text { Value } \\
(\mathrm{mg} / \mathrm{kg})^{\mathrm{b}}\end{array}$ & $\begin{array}{l}\text { Maximum } \\
\text { Detected } \\
\text { Cleanup } \\
\text { Verification } \\
\text { Value } \\
(\mathbf{m g} / \mathbf{k g})^{c}\end{array}$ & $\begin{array}{c}\text { Total } \\
\text { Number } \\
\text { of } \\
\text { Samples }^{d}\end{array}$ & $\begin{array}{l}\text { Percentage of } \\
\text { Cleanup } \\
\text { Verification Data } \\
\text { Set Exceeding } \\
\text { RAG }^{\mathrm{e}}\end{array}$ & $\begin{array}{c}\text { Cleanup } \\
\text { Criteria } \\
\text { Attained? }\end{array}$ \\
\hline \multicolumn{7}{|c|}{ 116-K-2 (East) Shallow Zone } \\
\hline $\begin{array}{l}\text { Hexavalent } \\
\text { chromium }\end{array}$ & $2^{f}$ & 0.39 & 0.85 & 9 & 0 & Yes \\
\hline \multicolumn{7}{|c|}{ 116-K-2 (East) Deep Zone } \\
\hline $\begin{array}{l}\text { Hexavalent } \\
\text { chromium }\end{array}$ & $2^{f}$ & 5.3 & 8.7 & 7 & $100 \%$ & Yes $^{g}$ \\
\hline \multicolumn{7}{|c|}{ 116-K-2 (West) Shallow Zone } \\
\hline $\begin{array}{l}\text { Hexavalent } \\
\text { chromium }\end{array}$ & $2^{f}$ & 0.23 & 0.31 & 17 & 0 & Yes \\
\hline \multicolumn{7}{|c|}{ 116-K-2 (West) Deep Zone } \\
\hline $\begin{array}{l}\text { Hexavalent } \\
\text { chromium }\end{array}$ & $2^{f}$ & 2.4 & 4.6 & 19 & $47 \%$ & Yes $^{g}$ \\
\hline \multicolumn{7}{|c|}{ Overburden } \\
\hline $\begin{array}{l}\text { Hexavalent } \\
\text { chromium }\end{array}$ & $2^{f}$ & 0.27 & 0.34 & 9 & 0 & Yes \\
\hline
\end{tabular}

${ }^{\text {a }}$ From Table 1, the most restrictive RAG is the soil RAG for protection of the Columbia River.

${ }^{\mathrm{b}}$ Criterion is statistical value cannot exceed most restrictive applicable RAG.

${ }^{c}$ Criterion is no single detection can exceed two times the most restrictive applicable RAG.

${ }^{d}$ Total number of samples in the decision unit includes field duplicate samples, which are included in the evaluation as separate samples.

${ }^{\mathrm{e}}$ Criterion is percentage of data set exceeding the most restrictive applicable RAG cannot exceed $10 \%$.

${ }^{\dagger}$ Soil RAG based on 100 times dilution attenuation factor times surface water quality standard as presented in the Remedial Design Report/Remedial Action Work Plan for the 100 Area (DOE-RL 2005b).

${ }^{9}$ Based on the 100-D, 100-F, and 100-H Area hexavalent chromium leach studies, groundwater and surface water quality criteria will not be exceeded where hexavalent chromium concentrations in soil are less than $6.8 \mathrm{mg} / \mathrm{kg}$. The 116-K-2 deep zone passes the three-part test in comparison against this level.

$\mathrm{COC}=$ contaminant of concern

RAG = remedial action goal

WAC $=$ Washington Administrative Code

As demonstrated in Table 5, residual shallow zone concentrations of hexavalent chromium (the sole nonradionuclide $\mathrm{COC}$ ) at the 116-K-2 site pass the three-part test in comparison to the most restrictive applicable RAG. Residual concentrations of hexavalent chromium in the deep zone of the 116-K-2 site fail the three-part test in comparison to the most restrictive applicable RAG. However, as described in Sections 5.2.2 and 5.3.2, hexavalent chromium will not leach to groundwater or the Columbia River at concentrations exceeding applicable water quality criteria where the concentration in soil is less than $6.8 \mathrm{mg} / \mathrm{kg}$. The 116-K-2 deep zone passes the threepart test in comparison against this threshold; therefore, the RAOs for protection of groundwater and the river have been attained. 


\subsection{RADIONUCLIDE RISK INFORMATION}

The radionuclide RAG for direct exposure is derived from the ROD (EPA 1995) and is expressed in terms of an allowable radiation dose rate above background (i.e., $15 \mathrm{mrem} / \mathrm{yr}$ ). The RAG evaluation (Section 5.0) involved using the RESRAD model to estimate total annual radiation dose rates for 1,000 years for comparison to the RAG. Radiation presents a carcinogenic risk, and the RESRAD model also calculates the excess lifetime cancer risk associated with the estimated radiation dose rates using the EPA's Health Effects Assessment Summary Tables (updated April 16, 2001, "Update of Radionuclide Carcinogenicity Slope Factors," available on the Internet at www.epa.gov/radiation/heast). The "National Oil and Hazardous Substances Pollution Contingency Plan" (40 Code of Federal Regulations 300) presents a target range for residual risk of $10^{-4}$ to $10^{-6}$.

Figures 9, 10, and 11 illustrate excess lifetime cancer risk for the eastern and western portions and overburden material at the 116-K-2 site, respectively. Because of radioactive decay, the risk decreases over time. The estimated risks for the eastern and western portions of the 116-K-2 site are largest at present (year 2006), with estimates of $4.39 \times 10^{-5}$ and $9.70 \times 10^{-5}$, respectively. These values decrease to $2.72 \times 10^{-5}$ and $6.13 \times 10^{-5}$, respectively, in 2018 , and to $8.19 \times 10^{-9}$ and $6.03 \times 10^{-9}$, respectively, in 1,000 years. The estimated risk associated with the 116-K-2 overburden material is largest $\left(8.72 \times 10^{-6}\right)$ at present (year 2006), decreasing to $4.43 \times 10^{-6}$ in 2018 , and to $1.78 \times 10^{-21}$ in 1,000 years. The 2018 date corresponds to the original 30-year site cleanup schedule of the Hanford Federal Facility Agreement and Consent Order (Ecology et al. 1989).

Figure 9. Combined Radionuclide Excess Lifetime Cancer Risk for the Eastern 116-K-2 Shallow and Deep Zone Decision Units.

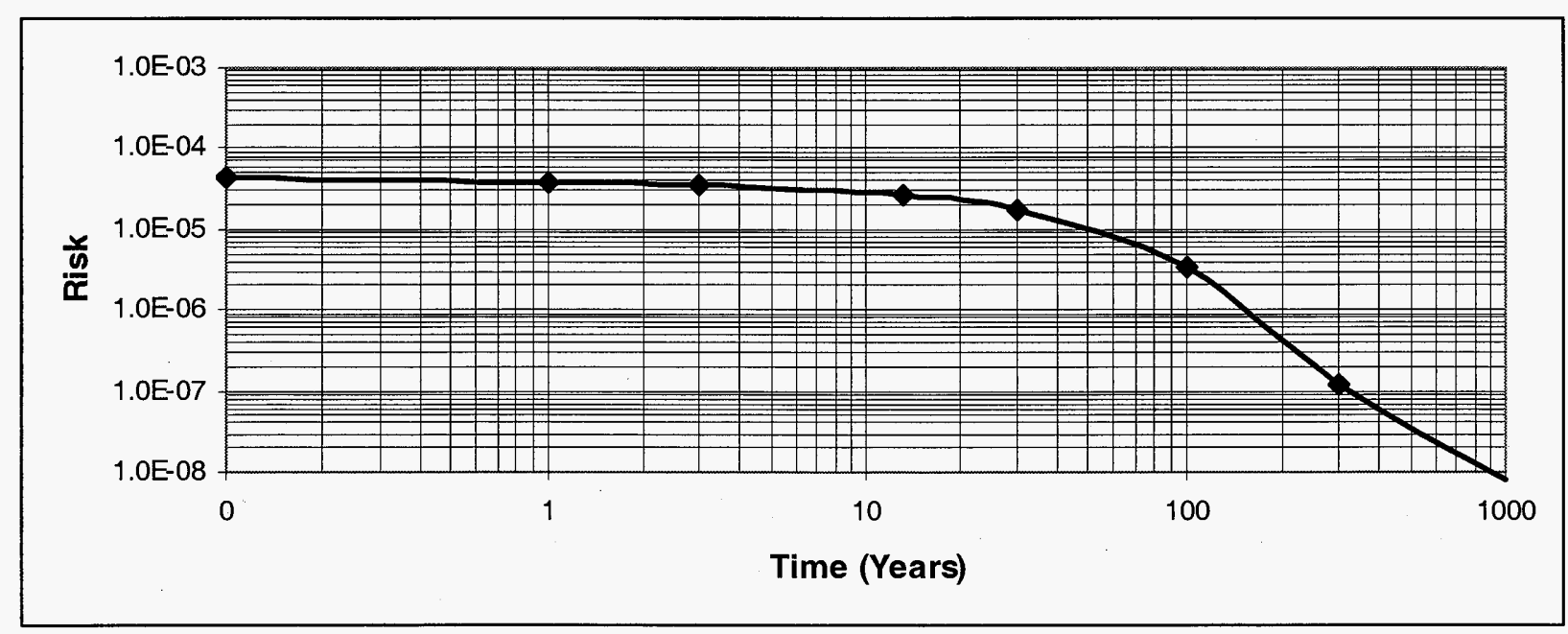


Figure 10. Combined Radionuclide Excess Lifetime Cancer Risk for the Western 116-K-2 Shallow and Deep Zone Decision Units.

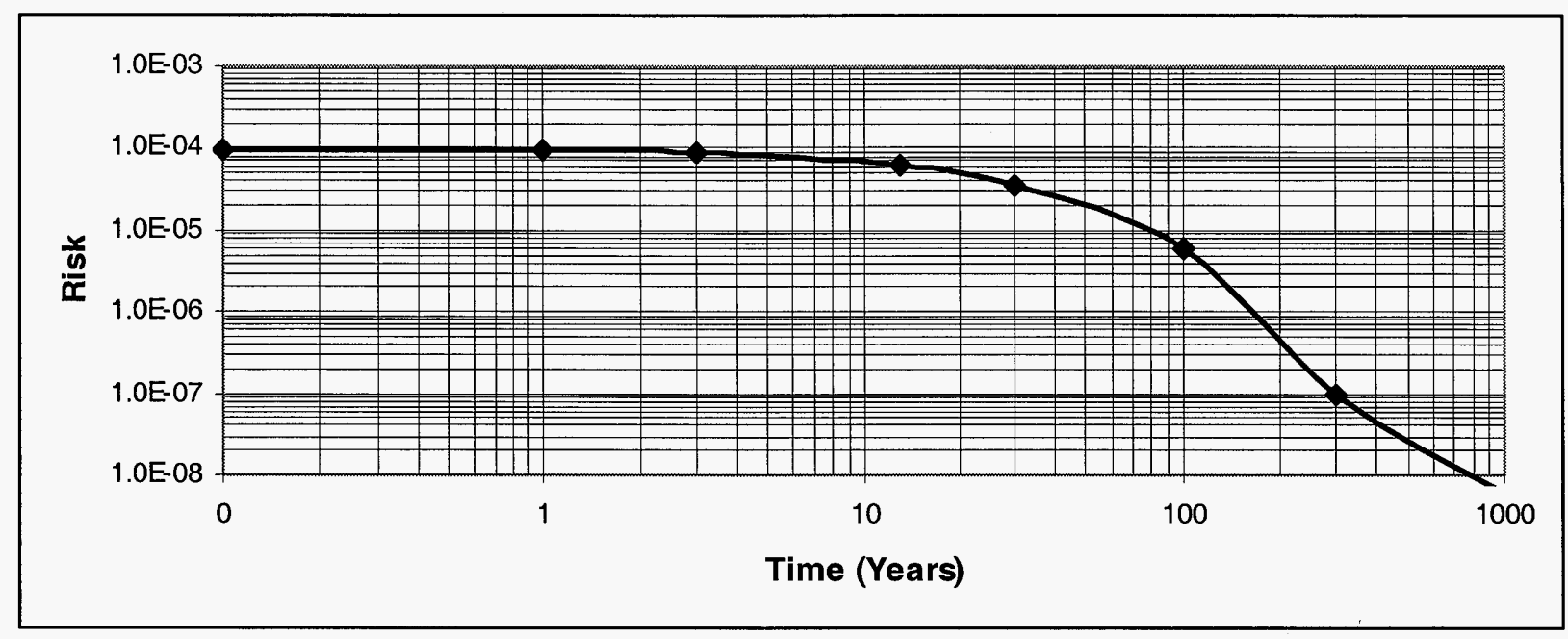

Figure 11. Radionuclide Excess Lifetime Cancer Risk for the 116-K-2 Overburden Material.

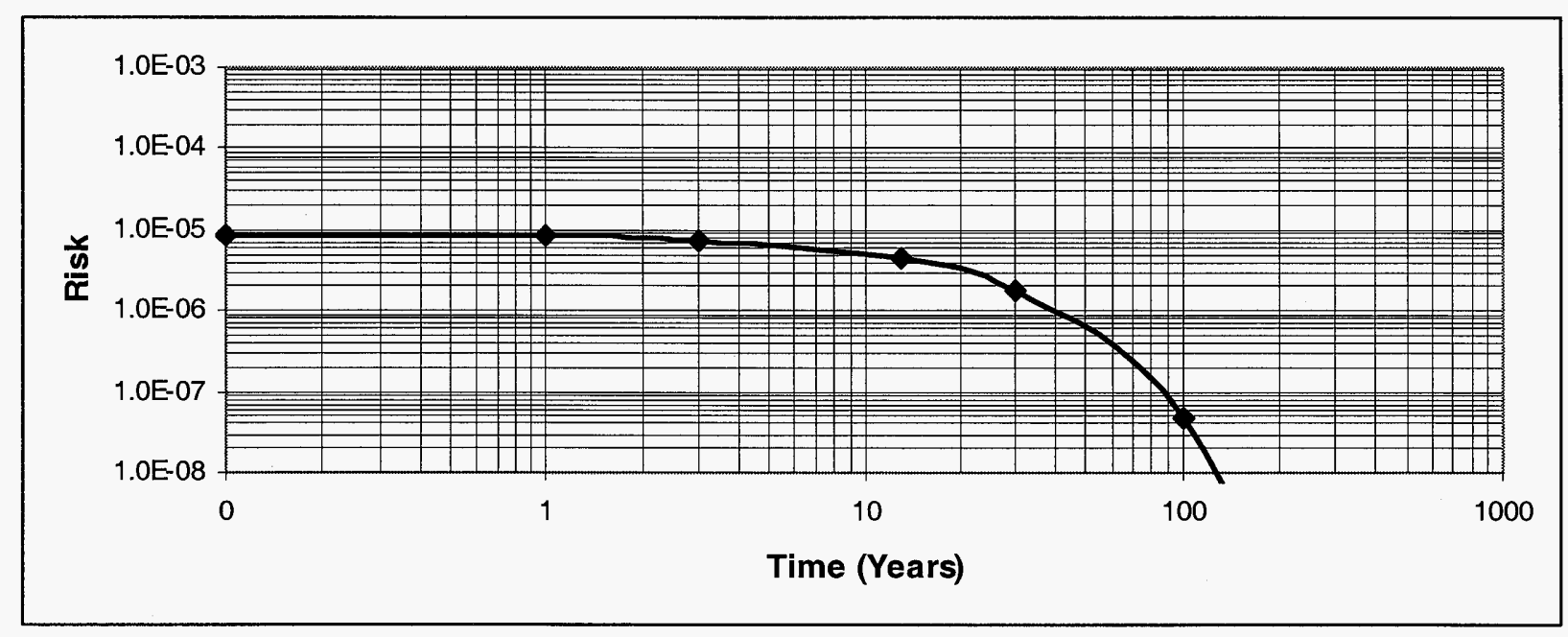




\subsection{STATEMENT OF PROTECTIVENESS}

This CVP demonstrates that remedial action at the 116-K-2 waste site has achieved the RAOs and corresponding RAGs established in the ROD (EPA 1995) and the RDR/RAWP (DOE-RL 2005b). The contaminated materials from this site have been excavated and disposed at the ERDF. The remaining soils at the site have been sampled, analyzed, and modeled, and the results do not preclude any future uses (as bounded by the rural-residential scenario), allow unrestricted use of shallow zone soils, and pose no threat to groundwater or the Columbia River. Institutional controls are required for the site to prevent drilling or excavation into deep zone soils.

\subsection{REFERENCES}

40 CFR 141, "National Primary Drinking Water Regulations," Code of Federal Regulations, as amended.

40 CFR 300, "National Oil and Hazardous Substances Pollution Contingency Plan," Code of Federal Regulations, as amended.

65 FR 76708, "National Primary Drinking Water Regulations; Radionuclides; Final Rule," Federal Register, Vol. 65, No. 236, p. 76708, December 7, 2000.

ANL, 2005, RESRAD for Windows, Version 6.3, Argonne National Laboratory, Environmental Assessment Division, Argonne, Illinois.

BHI, 1999, Cleanup Verification Package for the 116-D-7 Retention Basin, CVP-99-00007, Rev. 0, Bechtel Hanford, Inc., Richland, Washington.

BHI, 2000a, Calculation of Hexavalent Chromium Carcinogenic Risk, 0100X-CA-V0031, Rev. 0, Bechtel Hanford, Inc., Richland, Washington.

BHI, 2000b, Cleanup Verification Package for the 116- $\mathrm{H}-7$ Retention Basin, CVP-2000-00027, Rev. 0, Bechtel Hanford, Inc., Richland, Washington.

BHI, 2001a, Calculation of Total Uranium Activity Corresponding to a Maximum Contaminant Level for Total Uranium of 30 Micrograms per Liter in Groundwater, 0100X-CA-V0038, Rev. 0, Bechtel Hanford, Inc., Richland, Washington.

BHI, 2001b, Cleanup Verification Package for the 100-F-19:1 North Pipelines, 100-F-19:3 West Pipelines, 100-F-34 Biology Facility French Drain, and 116-F-12 French Drain, CVP-2001-00002, Rev. 0, Bechtel Hanford, Inc., Richland, Washington. 
BHI, 2004, October 27, 2004, 100-K Remedial Action, Department of Energy, and U. S. Environmental Protection Agency Meeting, Meeting Minutes, CCN 117424, Bechtel Hanford, Inc., Richland, Washington.

BHI, 2005, 100K Remedial Sampling, Logbook EL-1572-3, Bechtel Hanford, Inc., Richland, Washington.

Carpenter, R. W. and S. L. Cote, 1994, 100-K Area Technical Baseline Report, WHC-SD-EN-TI-239, Rev. 0, Westinghouse Hanford Company, Richland, Washington.

DOE Order 5400.5, Radiation Protection of the Public and the Environment, U.S. Department of Energy, Washington, D.C.

DOE-RL, 1994, Limited Field Investigation Report for the 100-KR-1 Operable Unit, DOE/RL-93-78, Rev. 0, U.S. Department of Energy, Richland Operations Office, Richland, Washington.

DOE-RL, 1996, Hanford Site Background: Part 2, Soil Background for Radionuclides, DOE/RL-96-12, Rev. 0, U.S. Department of Energy, Richland Operations Office, Richland, Washington.

DOE-RL, 1998, Tri-Party Agreement Handbook Management Procedures, RL-TPA-90-0001, Guideline Number TPA-MP-14, "Maintenance of the Waste Information Data System (WIDS)," U.S. Department of Energy, Richland Operations Office, Richland, Washington.

DOE-RL, 2001, 100 Area Remedial Action Sampling and Analysis Plan, DOE/RL-96-22, Rev. 3, U.S. Department of Energy, Richland Operations Office, Richland, Washington.

DOE-RL, 2005a, 100 Area Remedial Action Sampling and Analysis Plan, DOE/RL-96-22, Rev. 4, U.S. Department of Energy, Richland Operations Office, Richland, Washington.

DOE-RL, 2005b, Remedial Design Report/Remedial Action Work Plan for the 100 Area, DOE/RL-96-17, Rev. 5, U.S. Department of Energy, Richland Operations Office, Richland, Washington.

Ecology, EPA, and DOE, 1989, Hanford Federal Facility Agreement and Consent Order, 2 vols., as amended, Washington State Department of Ecology, U.S. Environmental Protection Agency, and U.S. Department of Energy, Olympia, Washington. 
EPA, 1995, Interim Action Record of Decision for the 100-BC-1, 100-DR-1, and 100-HR-1 Operable Units, Hanford Site, Benton County, Washington, U.S. Environmental Protection Agency, Region 10, Seattle, Washington.

EPA, 1997, Amendment to the Interim Action Record of Decision for the 100-BC-1, 100-DR-1, and 100-HR-1 Operable Units, Hanford Site, Benton County, Washington, EPA/AMD/R10-97/044, U.S. Environmental Protection Agency, Region 10, Seattle, Washington.

WAC 173-201A, "Water Quality Standards for Surface Waters of the State of Washington," Washington Administrative Code, as amended.

WAC 173-340, 1996, "Model Toxics Control Act - Cleanup," Washington Administrative Code. 


\section{APPENDIX A}

\section{SUMMARY OF VERIFICATION SOIL SAMPLING} AND ANALYTICAL RESULTS 
CVP-2006-00001

Rev. 0 
Table A-1. 116-K-2 (East End) Shallow Zone Cleanup Verification Data.

\begin{tabular}{|c|c|c|c|c|c|c|c|c|c|c|c|c|c|c|c|c|c|}
\hline \multirow{2}{*}{$\begin{array}{l}\text { Sampling } \\
\text { Area }\end{array}$} & \multirow{2}{*}{$\begin{array}{c}\text { HEIS } \\
\text { Number }\end{array}$} & \multirow{2}{*}{$\begin{array}{c}\text { Sample } \\
\text { Date }\end{array}$} & \multicolumn{3}{|c|}{ Hexavalent Chromium } & \multicolumn{3}{|c|}{ Carbon-14 } & \multicolumn{3}{|c|}{ Cesium-137 } & \multicolumn{3}{|c|}{ Cobalt-60 } & \multicolumn{3}{|c|}{ Europium-152 } \\
\hline & & & $\mathrm{mg} / \mathrm{kg}$ & $\mathbf{Q}$ & PQL & $\mathrm{pCi} / \mathrm{g}$ & $\mathbf{Q}$ & MDA & $\mathrm{pCi} / \mathrm{g}$ & $\mathbf{Q}$ & MDA & $\mathrm{pCi} / \mathrm{g}$ & $\mathbf{Q}$ & MDA & $\mathrm{pCi} / \mathrm{g}$ & $\mathbf{Q}$ & MDA \\
\hline B7 & J03JW8 & $8 / 18 / 2005$ & $2.0 \mathrm{E}-01$ & $U$ & $2.0 \mathrm{E}-01$ & $-1.04 \mathrm{E}+00$ & $u$ & $2.6 \mathrm{E}+00$ & $1.67 \mathrm{E}-01$ & & $3.2 \mathrm{E}-02$ & $3.0 \mathrm{E}-02$ & $u$ & $3.0 \mathrm{E}-02$ & 1.1E-01 & $\mathrm{u}$ & 1.1E-01 \\
\hline $\begin{array}{c}\text { Duplicate of } \\
\text { J03JW8 }\end{array}$ & J03JW9 & 8/18/2005 & $2.0 \mathrm{E}-01$ & $u$ & $2.0 \mathrm{E}-01$ & 4.5E-02 & u & $2.8 \mathrm{E}+00$ & $1.48 \mathrm{E}-01$ & & $3.0 \mathrm{E}-02$ & 3.0E-02 & $u \mid$ & $3.0 \mathrm{E}-02$ & $1.1 \mathrm{E}-01$ & u & $1.1 \mathrm{E}-01$ \\
\hline $\begin{array}{l}\text { Split of } \\
\text { J03JW8 }\end{array}$ & J03JX1 & $8 / 18 / 2005$ & $3.50 \mathrm{E}+00$ & UR & $3.50 \mathrm{E}+00$ & $-1.51 E-01$ & $u$ & $8.02 \mathrm{E}-01$ & 2.04E-01 & & $1.50 \mathrm{E}-02$ & 3.89E-06 & $u$ & 1.54E-02 & 1.09E-01 & $u$ & $4.86 \mathrm{E}-02$ \\
\hline A1 & J103C0 & 9/6/2005 & $2.0 \mathrm{E}-01$ & $U$ & $2.0 \mathrm{E}-01$ & $-2.87 E+00$ & $\mathrm{U}$ & $4.4 \mathrm{E}+00$ & $3.46 \mathrm{E}-01$ & & $2.8 \mathrm{E}-02$ & $4.1 \mathrm{E}-02$ & $u$ & $4.1 \mathrm{E}-02$ & 3.46E-01 & & $7.5 \mathrm{E}-02$ \\
\hline $\mathrm{A} 2$ & J03JW3 & $8 / 18 / 2005$ & 2.5E-01 & & $2.0 \mathrm{E}-01$ & $2.98 \mathrm{E}+00$ & & $2.9 \mathrm{E}+00$ & $5.1 \mathrm{E}-02$ & $U$ & $5.1 \mathrm{E}-02$ & $3.2 \mathrm{E}-02$ & $U$ & $3.2 \mathrm{E}-02$ & $7.5 \mathrm{E}-02$ & U & $7.5 \mathrm{E}-02$ \\
\hline A3 & J03W34 & 8/18/2005 & $2.2 \mathrm{E}-01$ & & $2.0 \mathrm{E}-01$ & $-5.18 E-01$ & $U$ & $1.8 \mathrm{E}+00$ & 3.4E-02 & $U$ & 3.4E-02 & $3.1 \mathrm{E}-02$ & $u$ & 3.1E-02 & $1.1 \mathrm{E}-01$ & $U$ & $1.1 \mathrm{E}-01$ \\
\hline $\mathrm{A} 4$ & J03JW5 & $8 / 18 / 2005$ & $2.0 \mathrm{E}-01$ & U & $2.0 \mathrm{E}-01$ & 5.14E-01 & $\mathrm{U}$ & $2.6 \mathrm{E}+00$ & $1.46 \mathrm{E}-01$ & & $3.4 \mathrm{E}-02$ & $3.2 \mathrm{E}-02$ & $U$ & $3.2 \mathrm{E}-02$ & $1.2 \mathrm{E}-01$ & $U$ & $1.2 \mathrm{E}-01$ \\
\hline B5 & J03JW6 & $7 / 6 / 2005$ & 2.3E-01 & & 2.0E-01 & -8.08E-01 & $\mathrm{U}$ & $2.2 E+00$ & $1.45 \mathrm{E}+00$ & & $1.0 \mathrm{E}-01$ & $3.5 \mathrm{E}-02$ & $U$ & 3.5E-02 & $1.7 \mathrm{E}-01$ & $U$ & $1.7 \mathrm{E}-01$ \\
\hline B6 & J03JW7 & $8 / 19 / 2005$ & $2.0 \mathrm{E}-01$ & u & $2.0 \mathrm{E}-01$ & $-5.96 \mathrm{E}-01$ & $U$ & $2.6 \mathrm{E}+00$ & 3.8E-02 & $\mathrm{u}$ & $3.8 \mathrm{E}-02$ & $3.5 \mathrm{E}-02$ & $U$ & $3.5 \mathrm{E}-02$ & $1.2 \mathrm{E}-01$ & $\bar{U}$ & $1.2 \mathrm{E}-01$ \\
\hline B8 & J03JX0 & $7 / 7 / 2005$ & $8.5 \mathrm{E}-01$ & & $2.0 \mathrm{E}-01$ & $-1.92 \mathrm{E}-01$ & $U$ & $1.6 \mathrm{E}+00$ & 3.2E-02 & $\mathrm{u}$ & $3.2 \mathrm{E}-02$ & $2.8 \mathrm{E}-02$ & $u$ & $2.8 \mathrm{E}-02$ & $1.0 \mathrm{E}-01$ & $U$ & $1.0 \mathrm{E}-01$ \\
\hline
\end{tabular}

\begin{tabular}{|c|c|c|c|c|c|c|c|c|c|c|c|c|c|c|}
\hline \multirow{2}{*}{$\begin{array}{c}\text { Sampling } \\
\text { Area }\end{array}$} & \multirow{2}{*}{$\begin{array}{c}\text { NEIS } \\
\text { Number }\end{array}$} & $\begin{array}{c}\text { Sample } \\
\text { Date }\end{array}$ & \multicolumn{3}{|c|}{ Europium-154 } & \multicolumn{3}{c|}{ Nickel-63 } & \multicolumn{2}{c|}{ Plutonium-239/240 } & \multicolumn{3}{c|}{ Strontium-90 } \\
\hline & & pCi/g & $\mathbf{Q}$ & MDA & pCi/g & $\mathbf{Q}$ & MDA & pCi/g & Q & MDA & pCi/g & Q & MDA \\
\hline B7 & J03JW8 & $8 / 18 / 2005$ & $1.0 \mathrm{E}-01$ & $\mathrm{U}$ & $1.0 \mathrm{E}-01$ & $1.09 \mathrm{E}+00$ & $\mathrm{U}$ & $3.3 \mathrm{E}+00$ & 0 & $\mathrm{U}$ & $3.1 \mathrm{E}-01$ & $-6.3 \mathrm{E}-02$ & $\mathrm{U}$ & $2.6 \mathrm{E}-01$ \\
\hline $\begin{array}{c}\text { Duplicate of } \\
\text { J03JW8 }\end{array}$ & J03JW9 & $8 / 18 / 2005$ & $9.8 \mathrm{E}-02$ & $\mathrm{U}$ & $9.8 \mathrm{E}-02$ & $3.41 \mathrm{E}-01$ & $\mathrm{U}$ & $3.2 \mathrm{E}+00$ & $5.3 \mathrm{E}-02$ & $\mathrm{U}$ & $4.1 \mathrm{E}-01$ & $6.0 \mathrm{E}-02$ & $\mathrm{U}$ & $2.8 \mathrm{E}-01$ \\
\hline $\begin{array}{c}\text { Split of } \\
\text { J03JW8 }\end{array}$ & J03JX1 & $8 / 18 / 2005$ & $-7.73 \mathrm{E}-03$ & $\mathrm{U}$ & $4.90 \mathrm{E}-02$ & $4.55 \mathrm{E}+00$ & $\mathrm{U}$ & $1.06 \mathrm{E}+01$ & $2.82 \mathrm{E}-02$ & $\mathrm{U}$ & $7.64 \mathrm{E}-02$ & $8.63 \mathrm{E}-02$ & $\mathrm{U}$ & $1.24 \mathrm{E}-01$ \\
\hline A1 & J103C0 & $9 / 6 / 2005$ & $1.2 \mathrm{E}-01$ & $\mathrm{U}$ & $1.2 \mathrm{E}-01$ & $1.97 \mathrm{E}+00$ & $\mathrm{U}$ & $4.3 \mathrm{E}+00$ & 0 & $\mathrm{U}$ & $2.9 \mathrm{E}-01$ & $1.94 \mathrm{E}-01$ & $1.8 \mathrm{E}-01$ \\
\hline A2 & J03JW3 & $8 / 18 / 2005$ & $1.1 \mathrm{E}-01$ & $\mathrm{U}$ & $1.1 \mathrm{E}-01$ & $3.56 \mathrm{E}-01$ & $\mathrm{U}$ & $3.4 \mathrm{E}+00$ & 0 & $\mathrm{U}$ & $2.7 \mathrm{E}-01$ & $-5.1 \mathrm{E}-02$ & $\mathrm{U}$ & $2.3 \mathrm{E}-01$ \\
\hline A3 & J03W34 & $8 / 18 / 2005$ & $1.0 \mathrm{E}-01$ & $\mathrm{U}$ & $1.0 \mathrm{E}-01$ & $4.99 \mathrm{E}-01$ & $\mathrm{U}$ & $3.3 \mathrm{E}+00$ & $3.7 \mathrm{E}-02$ & $\mathrm{U}$ & $2.8 \mathrm{E}-01$ & $1.80 \mathrm{E}-01$ & $1.8 \mathrm{E}-01$ \\
\hline A4 & J03JW5 & $8 / 18 / 2005$ & $1.1 \mathrm{E}-01$ & $\mathrm{U}$ & $1.1 \mathrm{E}-01$ & $2.24 \mathrm{E}+00$ & $\mathrm{U}$ & $3.3 \mathrm{E}+00$ & $3.6 \mathrm{E}-02$ & $\mathrm{U}$ & $2.7 \mathrm{E}-01$ & $1.2 \mathrm{E}-02$ & $\mathrm{U}$ & $2.4 \mathrm{E}-01$ \\
\hline B5 & J03JW6 & $7 / 6 / 2005$ & $1.1 \mathrm{E}-01$ & $\mathrm{U}$ & $1.1 \mathrm{E}-01$ & $7.83 \mathrm{E}+00$ & $3.7 \mathrm{E}+00$ & 0 & $\mathrm{U}$ & $2.5 \mathrm{E}-01$ & $1.02 \mathrm{E}-01$ & $\mathrm{U}$ & $3.0 \mathrm{E}-01$ \\
\hline B6 & J03JW7 & $8 / 19 / 2005$ & $1.1 \mathrm{E}-01$ & $\mathrm{U}$ & $1.1 \mathrm{E}-01$ & $8.75 \mathrm{E}-01$ & $\mathrm{U}$ & $3.2 \mathrm{E}+00$ & 0 & $\mathrm{U}$ & $3.0 \mathrm{E}-01$ & $1.7 \mathrm{E}-02$ & $\mathrm{U}$ & $2.3 \mathrm{E}-01$ \\
\hline B8 & J03JX0 & $7 / 7 / 2005$ & $9.7 \mathrm{E}-02$ & $\mathrm{U}$ & $9.7 \mathrm{E}-02$ & $-1.25 \mathrm{E}+01$ & $\mathrm{U}$ & $7.3 \mathrm{E}+00$ & 0 & $\mathrm{U}$ & $3.5 \mathrm{E}-01$ & $2.13 \mathrm{E}-01$ & $\mathrm{U}$ & $2.3 \mathrm{E}-01$ \\
\hline
\end{tabular}

NOTE: The following acronyms and abbreviations apply to all tables in this appendix.

$\mathrm{HEIS}=$ Hanford Environmental Information system $\quad \mathrm{PQL}=$ practical quantitation limi

$\mathrm{MDA}=$ minimum detectable activity

$Q \quad=$ qualifier

$R=$ rejected

$\mathrm{U}=$ undetected 
Table A-2. 116-K-2 (East End) Deep Zone Cleanup Verification Data.

\begin{tabular}{|c|c|c|c|c|c|c|c|c|c|c|c|c|c|c|c|c|c|}
\hline \multirow{2}{*}{$\underset{\text { Area }}{\text { Sampling }}$} & \multirow{2}{*}{$\begin{array}{c}\text { HEIS } \\
\text { Number }\end{array}$} & \multirow{2}{*}{$\begin{array}{l}\text { Sample } \\
\text { Date }\end{array}$} & \multicolumn{3}{|c|}{ Hexavalent Chromium } & \multicolumn{3}{|c|}{ Carbon-14 } & \multicolumn{3}{|c|}{ Cesium-137 } & \multicolumn{3}{|c|}{ Cobalt-60 } & \multicolumn{3}{|c|}{ Europium-152 } \\
\hline & & & $\mathbf{m g} / \mathbf{k g}$ & $\mathbf{Q}$ & PQL & $\mathrm{pCi} / \mathrm{g}$ & $\mathbf{Q}$ & MDA & $\mathrm{pCi} / \mathrm{g}$ & $\mathbf{Q}$ & MDA & $\mathrm{pCi} / \mathrm{g}$ & $\mathbf{Q}$ & MDA & $\mathrm{pCi} / \mathrm{g}$ & $\mathbf{Q}$ & MDA \\
\hline B6 & J03D02 & $7 / 26 / 2005$ & $8.7 E+00$ & & 4.0E-01 & $2.00 \mathrm{E}+00$ & & $1.9 E+00$ & $1.83 \mathrm{E}+02$ & & 3.0E-01 & $4.12 \mathrm{E}+00$ & & $1.2 \mathrm{E}-01$ & $8.92 E+01$ & & $9.0 \mathrm{E}-01$ \\
\hline $\begin{array}{c}\text { Duplicate of } \\
\text { J03D02 }\end{array}$ & J03D03 & $7 / 26 / 2005$ & $7.3 E+00$ & & 4.0E-01 & 8.54E-01 & $\mathrm{U}$ & $2.1 E+00$ & 1.64E+02 & & 3.3E-01 & $3.56 \mathrm{E}+00 \mid$ & & $1.5 \mathrm{E}-01$ & $8.18 \mathrm{E}+01$ & & 8.9E-01 \\
\hline $\begin{array}{l}\text { Split of } \\
\text { J03D02 } \\
\end{array}$ & J03D04 & $7 / 26 / 2005$ & $1.33 E+01$ & & $3.50 \mathrm{E}+00$ & 1.50E-01 & $\mathrm{U}$ & $8.06 \mathrm{E}-01$ & 2.27E+02 & & $1.10 \mathrm{E}-01$ & $6.16 \mathrm{E}+00$ & & 4.29E-02 & $1.32 E+02$ & & 3.66E-01 \\
\hline $\mathrm{A} 1$ & J03CY7 & $7 / 7 / 2005$ & $2.7 E+00$ & & 2.0E-01 & $1.69 \mathrm{E}-01$ & $\mathrm{U}$ & $1.5 \mathrm{E}+00$ & $1.03 E+02$ & & $2.8 E-01$ & $1.52 E+00$ & & 9.5E-02 & $2.53 E+01$ & & $7.9 \mathrm{E}-01$ \\
\hline $\mathrm{A} 2$ & J03CY8 & $7 / 25 / 2005$ & $2.5 E+00$ & & $4.1 E-01$ & 4.23E-01 & $\mathrm{U}$ & $1.7 E+00$ & $1.04 \mathrm{E}+02$ & & $2.6 \mathrm{E}-01$ & 7.35E-01 & & $7.8 \mathrm{E}-02$ & $2.02 E+01$ & & 1.0E-01 \\
\hline A3 & J03CY9 & $7 / 7 / 2005$ & $3.3 E+00$ & & $2.0 \mathrm{E}-01$ & $1.32 E+00$ & $\mathrm{U}$ & $1.4 \mathrm{E}+00$ & $1.10 \mathrm{E}+02$ & & 3.3E-01 & $1.66 \mathrm{E}+00$ & & 1.1E-01 & $6.29 E+01$ & & $1.1 \mathrm{E}+00$ \\
\hline B4 & J03D00 & $7 / 25 / 200$ & $3.6 \mathrm{E}+00$ & & 4.0E-01 & $7.58 \mathrm{E}-01$ & $U$ & $1.7 \mathrm{E}+00$ & $6.06 \mathrm{E}+01$ & & 1.7E-01 & 8.33E-01 & & $5.5 \mathrm{E}-02$ & $1.06 \mathrm{E}+01$ & & 5.9E-01 \\
\hline B5 & J03D01 & $7 / 7 / 2005$ & $3.3 E+00$ & & 2.0E-01 & $1.33 E+00$ & $\mathrm{U}$ & $1.6 \mathrm{E}+00$ & $6.18 E+01$ & & 2.3E-01 & $1.79 \mathrm{E}+00$ & & 8.9E-02 & $5.46 \mathrm{E}+01$ & & $6.2 E-01$ \\
\hline
\end{tabular}

\begin{tabular}{|c|c|c|c|c|c|c|c|c|c|c|c|c|c|c|}
\hline \multirow{2}{*}{$\begin{array}{c}\text { Sampling } \\
\text { Area }\end{array}$} & \multirow{2}{*}{$\begin{array}{c}\text { HEIS } \\
\text { Number }\end{array}$} & \multirow{2}{*}{$\begin{array}{l}\text { Sample } \\
\text { Date }\end{array}$} & \multicolumn{3}{|c|}{ Europium-154 } & \multicolumn{3}{|c|}{ Nickel-63 } & \multicolumn{3}{|c|}{ Plutonium-239/240 } & \multicolumn{3}{|c|}{ Strontium-90 } \\
\hline & & & $\mathrm{pCi} / \mathrm{g}$ & $\mathbf{Q}$ & MDA & $\mathrm{pCi} / \mathrm{g}$ & $\mathbf{Q}$ & MDA & $\mathrm{pCi} / \mathrm{g}$ & $\mathbf{Q}$ & MDA & $\mathrm{pCi} / \mathrm{g}$ & $\mathbf{Q}$ & MDA \\
\hline B6 & J03D02 & $7 / 26 / 2005$ & $8.14 \mathrm{E}+00$ & & 4.5E-01 & $1.22 \mathrm{E}+03$ & & $9.5 E+00$ & $7.22 \mathrm{E}+00$ & & 2.3E-01 & $9.83 E+00$ & & $3.2 E-01$ \\
\hline $\begin{array}{c}\text { Duplicate of } \\
\text { J03D02 }\end{array}$ & J03D03 & $7 / 26 / 2005$ & $5.90 \mathrm{E}+00$ & & 5.5E-01 & $1.45 E+03$ & & $1.0 \mathrm{E}+01$ & $1.02 \mathrm{E}+01$ & & $2.8 \mathrm{E}-01$ & $8.80 E+00$ & & 2.9E-01 \\
\hline $\begin{array}{c}\text { Split of } \\
\text { J03D02 } \\
\end{array}$ & J03D04 & $7 / 26 / 2005$ & $1.15 \mathrm{E}+01$ & & $1.60 \mathrm{E}-01$ & $2.18 E+03$ & & $7.15 E+00$ & $1.31 \mathrm{E}+01$ & & 5.56E-02 & $1.30 \mathrm{E}+01$ & & 1.65E-01 \\
\hline $\mathrm{A} 1$ & $\mathrm{~J} 03 \mathrm{CY} 7$ & $7 / 7 / 2005$ & $2.75 \mathrm{E}+00$ & & 3.1E-01 & $2.48 \mathrm{E}+02$ & & $7.9 E+00$ & $1.58 \mathrm{E}+00$ & & 2.6E-01 & $2.96 \mathrm{E}+00$ & & 2.2E-01 \\
\hline $\mathrm{A} 2$ & J03CY8 & $7 / 25 / 2005$ & $2.04 \mathrm{E}+00$ & & 3.1E-01 & $2.27 E+02$ & & $4.1 \mathrm{E}+00$ & $2.56 \mathrm{E}+00$ & & 2.1E-01 & $2.60 \mathrm{E}+00$ & & 2.9E-01 \\
\hline A3 & J03CY9 & $7 / 7 / 2005$ & $5.28 \mathrm{E}+00$ & & 4.4E-01 & $8.22 E+02$ & & $7.2 \mathrm{E}+00$ & $7.38 \mathrm{E}+00$ & & $2.9 \mathrm{E}-01$ & $6.65 \mathrm{E}+00$ & & 2.1E-01 \\
\hline B4 & $\mathrm{J} 03 \mathrm{D} 00$ & $7 / 25 / 200$ & 7.70E-01 & & 2.1E-01 & $3.38 E+02$ & & $4.9 \mathrm{E}+00$ & $2.30 \mathrm{E}+00$ & & 2.6E-01 & $4.84 \mathrm{E}+00$ & & 3.1E-01 \\
\hline B5 & J03D01 & $7 / 7 / 2005$ & $5.20 \mathrm{E}+00$ & & 3.6E-01 & $5.64 \mathrm{E}+02$ & & $7.4 \mathrm{E}+00$ & $6.40 \mathrm{E}+00$ & & 3.3E-01 & $6.21 \mathrm{E}+00$ & & $2.9 \mathrm{E}-01$ \\
\hline
\end{tabular}


Table A-3. 116-K-2 (West End) Shallow Zone Cleanup Verification Data. (2 Pages)

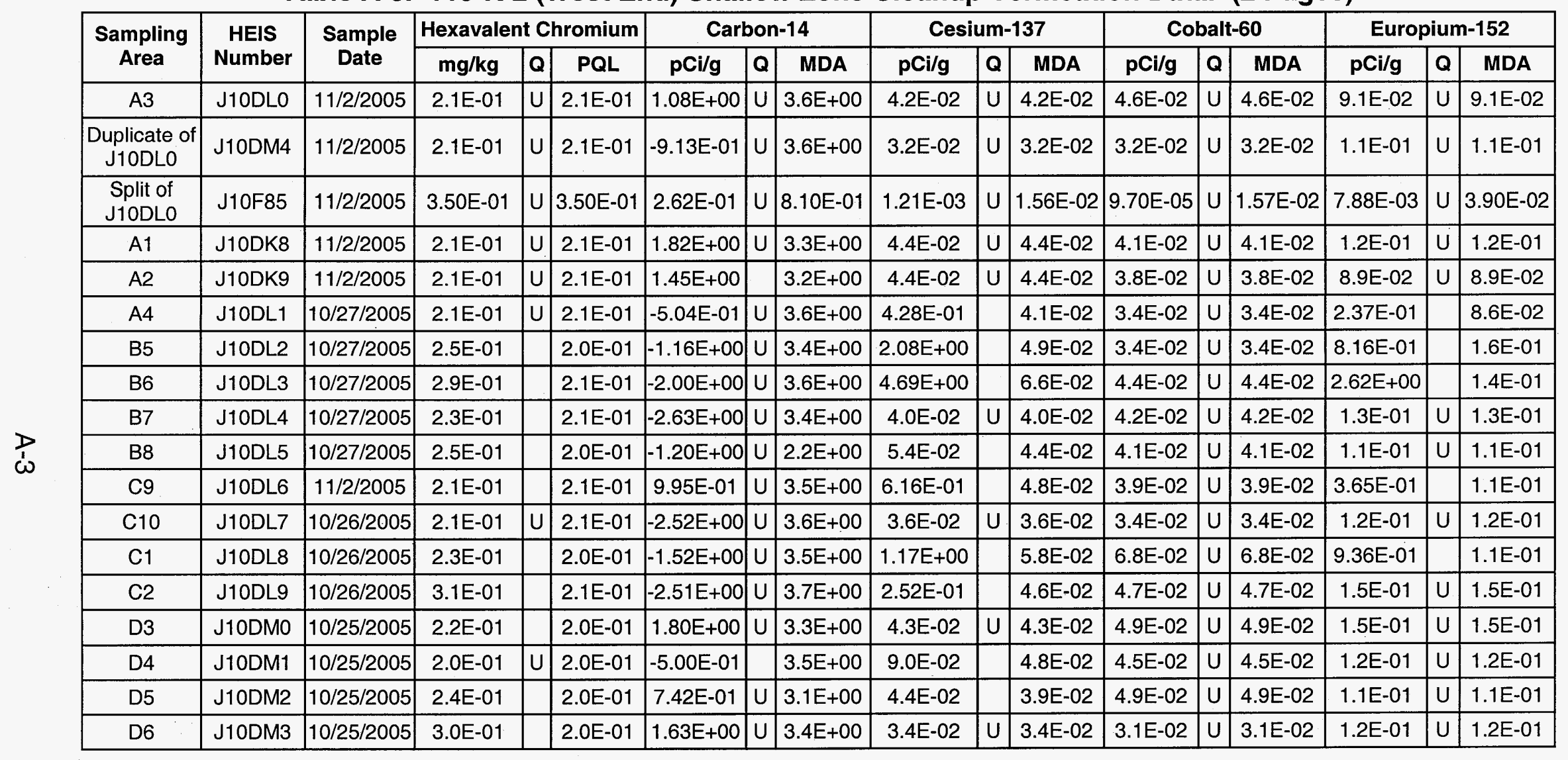


Table A-3. 116-K-2 (West End) Shallow Zone Cleanup Verification Data. (2 Pages)

\begin{tabular}{|c|c|c|c|c|c|c|c|c|c|c|c|c|c|c|}
\hline \multirow{2}{*}{$\begin{array}{l}\text { Sampling } \\
\text { Area }\end{array}$} & \multirow{2}{*}{$\begin{array}{c}\text { HEIS } \\
\text { Number }\end{array}$} & \multirow{2}{*}{$\begin{array}{l}\text { Sample } \\
\text { Date }\end{array}$} & \multicolumn{3}{|c|}{ Europium-154 } & \multicolumn{3}{|c|}{ Nickel-63 } & \multicolumn{3}{|c|}{ Plutonium-239/240 } & \multicolumn{3}{|c|}{ Strontium-90 } \\
\hline & & & $\mathrm{pCi} / \mathrm{g}$ & $\mathbf{Q}$ & MDA & $\mathrm{pCi} / \mathrm{g}$ & $Q$ & MDA & $\mathrm{pCi} / \mathrm{g}$ & $\mathbf{Q}$ & MDA & $\mathrm{pCi} / \mathrm{g}$ & $\mathbf{Q}$ & MDA \\
\hline A3 & DLO & $11 / 2 / 2005$ & $1.5 \mathrm{E}-01$ & $\mathrm{U}$ & 1.5E-01 & $-5.04 \mathrm{E}-01$ & $u \mid$ & $3.8 E+00$ & 0 & $U$ & $3.2 \mathrm{E}-01$ & 9.0E-02 & $u$ & $1.5 \mathrm{E}-01$ \\
\hline & ODM4 & /2/2005 & $1.1 \mathrm{E}-01$ & $\mathrm{U}$ & $1.1 \mathrm{E}-01$ & $-1.83 E+00$ & $u \mid$ & $3.9 \mathrm{E}+00$ & 0 & $u$ & $2.8 \mathrm{E}-01$ & $1.36 \mathrm{E}-01$ & $u$ & $1.1 \mathrm{E}-01$ \\
\hline & OF85 & 1/2/2005 & $6.80 \mathrm{E}-03$ & $\mathrm{U}$ & $5.04 \mathrm{E}-02$ & $6.59 \mathrm{E}+00$ & $\mathrm{U}$ & $9.34 \mathrm{E}+00$ & $0.00 \mathrm{E}+00$ & $U$ & $5.18 \mathrm{E}-02$ & $6.11 \mathrm{E}-02$ & $u$ & 1.29E-01 \\
\hline $\mathrm{A} 1$ & & 1/2/2005 & $1.4 \mathrm{E}-01$ & $\mathrm{U}$ & 1.4E-01 & $-1.04 E+00$ & $u$ & $3.7 \mathrm{E}+00$ & 0 & U & 3.1E-01 & $1.54 \mathrm{E}-01$ & & $1.4 \mathrm{E}-01$ \\
\hline$A$ & DK9 & 005 & $1.4 \mathrm{E}-01$ & $U$ & $1.4 \mathrm{E}-01$ & $-1.11 \mathrm{E}+00$ & $U$ & $3.7 \mathrm{E}+00$ & 3.5E-02 & $U$ & $2.7 \mathrm{E}-01$ & $1.24 \mathrm{E}-01$ & $\mathrm{U}$ & $1.4 \mathrm{E}-01$ \\
\hline $\mathrm{A4}$ & $\overline{O D L 1}$ & $/ 27 / 2005$ & $1.1 \mathrm{E}-01$ & $U$ & $1.1 \mathrm{E}-01$ & $-3.94 \mathrm{E}-01$ & $\mathrm{U}$ & $3.6 \mathrm{E}+00$ & 0 & $\mathrm{U}$ & $1.3 \mathrm{E}-01$ & $3.74 \mathrm{E}-01$ & & $1.1 \mathrm{E}-01$ \\
\hline & & 005 & $1.3 \mathrm{E}-01$ & $U$ & $\mathrm{E}-01$ & $2.07 \mathrm{E}+00$ & $U$ & $6 \mathrm{E}+00$ & & U & $.5 \mathrm{E}-01$ & 2.85E-01 & & $1.3 \mathrm{E}-01$ \\
\hline $\mathrm{B} 6$ & ODL3 & //27/2005 & 2.95E-01 & & $1.5 \mathrm{E}-01$ & $4.22 \mathrm{E}+00$ & & $3.4 \mathrm{E}+00$ & $3.6 \mathrm{E}-02$ & $U$ & $2.7 \mathrm{E}-01$ & $2.71 \mathrm{E}-01$ & & $2.95 \mathrm{E}-01$ \\
\hline B7 & ODL4 & $10 / 27 / 2005$ & $1.5 \mathrm{E}-01$ & $\mathrm{u}$ & $1.5 \mathrm{E}-01$ & $2.10 \mathrm{E}+00$ & $\mathrm{U}$ & $3.8 \mathrm{E}+00$ & 0 & $\mathrm{U}$ & $2.6 \mathrm{E}-01$ & $9.9 \mathrm{E}-02$ & $U$ & $1.5 \mathrm{E}-01$ \\
\hline B8 & & 005 & $3 \mathrm{E}-01$ & $U$ & $\mathrm{E}-01$ & $1.27 \mathrm{E}+00$ & $\mathrm{U}$ & $3.5 \mathrm{E}+00$ & 0 & 0 & 3.6E-01 & 1.57E-01 & $\mathrm{U}$ & $1.3 \mathrm{E}-01$ \\
\hline $\mathrm{C9}$ & & $11 / 2 / 2005$ & $1.3 \mathrm{E}-01$ & $\mathrm{U}$ & $1.3 \mathrm{E}-01$ & 2.25E-01 & $U$ & $3.7 \mathrm{E}+00$ & 0 & $U$ & $2.3 \mathrm{E}-01$ & $2.40 \mathrm{E}-01$ & & 1.3E-01 \\
\hline C10 & J10DL7 & $10 / 26 / 2005$ & 1.3E-01 & $\mathrm{U}$ & $1.3 \mathrm{E}-01$ & $7.6 \mathrm{E}-02$ & $\mathrm{U}$ & $3.2 \mathrm{E}+00$ & c & $\mathrm{U}$ & $1.8 \mathrm{E}-01$ & $-3.5 \mathrm{E}-02$ & $u$ & $1.3 \mathrm{E}-01$ \\
\hline $\mathrm{C} 1$ & J10DL8 & 10/26/2005 & $2.99 \mathrm{E}-01$ & & $1.6 \mathrm{E}-01$ & $5.03 \mathrm{E}+00$ & & $3.5 \mathrm{E}+00$ & 0 & 0 & $2.4 \mathrm{E}-01$ & $3.6 \mathrm{E}-02$ & $\mathrm{U}$ & $2.99 \mathrm{E}-01$ \\
\hline $\mathrm{C} 2$ & ODL9 & $10 / 26 / 2005$ & $1.5 \mathrm{E}-01$ & $\mathrm{U}$ & 1.5E-01 & $1.87 \mathrm{E}+00$ & $\mathrm{U}$ & $3.2 \mathrm{E}+00$ & 0 & $U$ & $2.4 \mathrm{E}-01$ & 2.E-03 & U & $1.5 \mathrm{E}-01$ \\
\hline D3 & ODMO & $10 / 25 / 2005$ & 1.5E-01 & $\mathrm{U}$ & $1.5 \mathrm{E}-01$ & $9.08 \mathrm{E}-01$ & $\mathrm{U}$ & $3.6 \mathrm{E}+00$ & 0 & $\mathrm{U}$ & $2.9 \mathrm{E}-01$ & $3.20 \mathrm{E}-01$ & & $1.5 \mathrm{E}-01$ \\
\hline D4 & ODM1 & 10/25/2005 & $1.4 \mathrm{E}-01$ & $U$ & $1.4 \mathrm{E}-01$ & $3.50 \mathrm{E}-01$ & $U$ & $4.3 \mathrm{E}+00$ & 1.13E-01 & $U$ & 2.2E-01 & $1.09 \mathrm{E}-01$ & $U$ & $1.4 \mathrm{E}-01$ \\
\hline D5 & J10DM2 & $10 / 25 / 2005$ & 1.7E-01 & $U$ & 1.7E-01 & $1.14 \mathrm{E}+00$ & $u$ & $4.3 \mathrm{E}+00$ & 0 & U & $2.6 \mathrm{E}-01$ & 1.16E-01 & $\mathrm{U}$ & 1.7E-01 \\
\hline D6 & J10DM3 & $0 / 25 / 2005$ & $1.1 \mathrm{E}-01$ & $U$ & 1.1E-01 & $1.18 \mathrm{E}+00$ & $U$ & $4.1 \mathrm{E}+00$ & 0 & $U$ & $3.4 \mathrm{E}-01$ & $1.00 \mathrm{E}-01$ & $U$ & $1.1 \mathrm{E}-01$ \\
\hline
\end{tabular}


Table A-4. 116-K-2 (West End) Deep Zone Cleanup Verification Data. (2 Pages)

\begin{tabular}{|c|c|c|c|c|c|c|c|c|c|c|c|c|c|c|c|c|c|}
\hline \multirow{2}{*}{$\begin{array}{c}\text { Sampling } \\
\text { Area }\end{array}$} & \multirow{2}{*}{$\begin{array}{c}\text { HEIS } \\
\text { Number }\end{array}$} & \multirow{2}{*}{$\begin{array}{c}\text { Sample } \\
\text { Date }\end{array}$} & \multicolumn{3}{|c|}{ Hexavalent Chromium } & \multicolumn{3}{|c|}{ Carbon-14 } & \multicolumn{3}{|c|}{ Cesium-137 } & \multicolumn{3}{|c|}{ Cobalt-60 } & \multicolumn{3}{|c|}{ Europium-152 } \\
\hline & & & $\mathbf{m g} / \mathbf{k g}$ & $\mathbf{Q}$ & PQL & $\mathrm{pCi} / \mathrm{g}$ & $\mathbf{Q}$ & MDA & $\mathrm{pCi} / \mathrm{g}$ & $\mathbf{Q}$ & MDA & $\mathrm{pCi} / \mathrm{g}$ & $\mathbf{Q}$ & MDA & $\mathrm{pCi} / \mathrm{g}$ & $\mathbf{Q}$ & MDA \\
\hline D10 & $\mathrm{J} 10 \mathrm{CW} 7$ & $10 / 18 / 2005$ & 9.7E-01 & & $2.1 \mathrm{E}-01$ & $1.08 \mathrm{E}+00$ & $\mathrm{U}$ & $2.4 \mathrm{E}+00$ & $2.18 \mathrm{E}+02$ & & $4.0 \mathrm{E}-01$ & $6.68 \mathrm{E}+00$ & & $1.5 \mathrm{E}-01$ & $1.94 \mathrm{E}+02$ & & $1.1 \mathrm{E}+00$ \\
\hline $\begin{array}{c}\text { Duplicate of } \\
\text { J10CW7 }\end{array}$ & J10CX6 & $10 / 18 / 2005$ & $2.0 \mathrm{E}+00$ & & 2.0E-01 & $1.71 \mathrm{E}+00$ & $\mathrm{U}$ & $2.4 \mathrm{E}+00$ & $1.09 \mathrm{E}+02$ & & $3.0 \mathrm{E}-01$ & $3.45 \mathrm{E}+00$ & & 1.3E-01 & $1.02 E+02$ & & $8.6 \mathrm{E}-01$ \\
\hline $\begin{array}{c}\text { Split of } \\
\text { J10CW7 }\end{array}$ & J10CX7 & $10 / 18 / 2005$ & 7.50E-01 & & 3.50E-01 & $5.59 \mathrm{E}-01$ & $u$ & $8.01 \mathrm{E}-01$ & $1.22 \mathrm{E}+02$ & & $1.13 \mathrm{E}-01$ & $4.30 E+00$ & & $5.10 \mathrm{E}-02$ & $1.13 E+02$ & & 3.11E-01 \\
\hline $\mathrm{A} 1$ & J10CV8 & $10 / 11 / 2005$ & $2.1 \mathrm{E}+00$ & & 2.0E-01 & 4.87E-01 & $\mathrm{U}$ & $4.2 \mathrm{E}+00$ & 3.77E+01 & & $2.4 \mathrm{E}-01$ & $4.71 \mathrm{E}+00$ & & $1.1 \mathrm{E}-01$ & $4.65 E+01$ & & 6.4E-01 \\
\hline $\mathrm{A} 3$ & J10CW0 & $10 / 11 / 2005$ & $2.0 \mathrm{E}+00$ & & $2.1 \mathrm{E}-01$ & $-2.19 \mathrm{E}+00$ & & $4.0 \mathrm{E}+00$ & $1.13 \mathrm{E}+02$ & & $3.9 \mathrm{E}-01$ & $4.85 \mathrm{E}+00$ & & $1.5 \mathrm{E}-01$ & $9.00 \mathrm{E}+01$ & & $1.0 \mathrm{E}+00$ \\
\hline B4 & J10CW1 & $10 / 12 / 2005$ & $9.3 \mathrm{E}-01$ & & $2.0 \mathrm{E}-01$ & $-7.82 E-01$ & & $2.5 \mathrm{E}+00$ & $9.48 \mathrm{E}-01$ & & $4.4 \mathrm{E}-02$ & 7.7E-02 & $U$ & 7.7E-02 & $1.42 \mathrm{E}+00$ & & 1.3E-01 \\
\hline B5 & J10CW2 & $10 / 12 / 2005$ & $1.2 \mathrm{E}+00$ & & $2.0 \mathrm{E}-01$ & 5.52E-01 & & $2.2 \mathrm{E}+00$ & $2.71 \mathrm{E}+01$ & & $1.9 \mathrm{E}-01$ & $1.54 \mathrm{E}+00$ & & $9.3 \mathrm{E}-02$ & $1.93 \mathrm{E}+01$ & & 3.3E-01 \\
\hline$B 6$ & J10CW3 & $10 / 12 / 2005$ & $2.2 \mathrm{E}+00$ & & 2.0E-01 & $1.56 \mathrm{E}+00$ & & $2.2 \mathrm{E}+00$ & $8.87 \mathrm{E}+01$ & & $2.1 \mathrm{E}-01$ & $7.53 \mathrm{E}+00$ & & $9.6 \mathrm{E}-02$ & $3.70 \mathrm{E}+01$ & & $6.5 \mathrm{E}-01$ \\
\hline $\mathrm{C7}$ & J10CW4 & $10 / 17 / 2005$ & $3.6 \mathrm{E}+00$ & & $2.0 \mathrm{E}-01$ & $4.62 \mathrm{E}+00$ & & $2.3 \mathrm{E}+00$ & $1.11 \mathrm{E}+02$ & & $3.4 \mathrm{E}-01$ & $9.62 \mathrm{E}+00$ & & $1.8 \mathrm{E}-01$ & $1.24 \mathrm{E}+02$ & & $8.8 \mathrm{E}-01$ \\
\hline $\mathrm{C} 8$ & J10CW5 & $10 / 17 / 2005$ & $2.0 \mathrm{E}-01$ & $\mathrm{U}$ & $2.0 \mathrm{E}-01$ & $-5.13 \mathrm{E}-01$ & $\mathrm{U}$ & $2.4 \mathrm{E}+00$ & $1.21 \mathrm{E}+00$ & & 4.3E-02 & 4.40E-02 & & $3.1 \mathrm{E}-02$ & $1.15 \mathrm{E}+00$ & & $9.9 \mathrm{E}-02$ \\
\hline D1 & J10CW8 & $10 / 18 / 2005$ & $1.1 \mathrm{E}+00$ & & $2.1 \mathrm{E}-01$ & $8.21 \mathrm{E}-01$ & $\mathrm{U}$ & $2.4 \mathrm{E}+00$ & $4.64 \mathrm{E}+01$ & & $2.4 \mathrm{E}-01$ & $2.01 \mathrm{E}+00$ & & $1.2 \mathrm{E}-01$ & $6.93 \mathrm{E}+01$ & & $5.0 \mathrm{E}-01$ \\
\hline $\mathrm{D} 2$ & J10CW9 & $10 / 18 / 2005$ & $4.6 \mathrm{E}+00$ & & $2.0 \mathrm{E}-01$ & $1.48 \mathrm{E}+00$ & $\mathrm{U}$ & $2.3 \mathrm{E}+00$ & $2.61 \mathrm{E}+02$ & & $2.7 \mathrm{E}-01$ & $6.80 \mathrm{E}+00$ & & $1.5 \mathrm{E}-01$ & $1.51 \mathrm{E}+02$ & & $1.2 \mathrm{E}+00$ \\
\hline E3 & $\mathrm{J} 10 \mathrm{CX} 0$ & $10 / 18 / 2005$ & $2.2 \mathrm{E}+00$ & & $2.0 \mathrm{E}-01$ & 2.34E-01 & $\mathrm{U}$ & $2.3 \mathrm{E}+00$ & $1.73 \mathrm{E}+02$ & & $4.1 \mathrm{E}-01$ & $4.38 \mathrm{E}+00$ & & $1.5 \mathrm{E}-01$ & $5.56 \mathrm{E}+01$ & & $1.1 \mathrm{E}+00$ \\
\hline E4 & $\mathrm{J} 10 \mathrm{CX} 1$ & $10 / 18 / 2005$ & $1.0 \mathrm{E}+00$ & & $2.0 \mathrm{E}-01$ & \begin{tabular}{|l|} 
4.7E-02 \\
\end{tabular} & $\mathrm{U}$ & $2.3 \mathrm{E}+00$ & $1.12 \mathrm{E}+00$ & & $4.0 \mathrm{E}-02$ & $3.6 \mathrm{E}-02$ & $U$ & $3.6 \mathrm{E}-02$ & $9.15 \mathrm{E}-01$ & & $1.4 \mathrm{E}-01$ \\
\hline E5 & J10CX2 & $10 / 24 / 2005$ & $1.7 \mathrm{E}+00$ & & $2.0 \mathrm{E}-01$ & $1.02 \mathrm{E}+00$ & $\mathrm{U}$ & $3.5 \mathrm{E}+00$ & $4.63 \mathrm{E}+01$ & & $1.4 \mathrm{E}-01$ & $6.15 \mathrm{E}-01$ & & 7.6E-02 & $1.79 \mathrm{E}+01$ & & $5.7 \mathrm{E}-01$ \\
\hline F6 & J10CX3 & $10 / 24 / 2005$ & $4.3 \mathrm{E}+00$ & & $2.0 \mathrm{E}-01$ & $3.66 \mathrm{E}+00$ & $\mathrm{U}$ & $5.0 \mathrm{E}+00$ & $2.32 \mathrm{E}+02$ & & $5.7 \mathrm{E}-01$ & $5.09 \mathrm{E}+00$ & & $2.1 \mathrm{E}-01$ & $1.61 \mathrm{E}+02$ & & $1.5 \mathrm{E}+00$ \\
\hline $\mathrm{F} 7$ & J10CX4 & $10 / 24 / 2005$ & $1.7 \mathrm{E}+00$ & & $2.0 \mathrm{E}-01$ & $1.48 \mathrm{E}+00$ & $\mathrm{U}$ & $3.1 \mathrm{E}+00$ & $4.12 \mathrm{E}+01$ & & $1.6 \mathrm{E}-01$ & $6.18 \mathrm{E}-01$ & & $7.1 \mathrm{E}-02$ & $2.41 E+01$ & & $3.5 \mathrm{E}-01$ \\
\hline F8 & J10CX5 & $10 / 24 / 2005$ & $2.3 \mathrm{E}+00$ & & $2.0 \mathrm{E}-01$ & $8.19 \mathrm{E}-01$ & & $3.2 \mathrm{E}+00$ & $1.63 \mathrm{E}+02$ & & 1.7E-01 & 3.87E-01 & & $5.3 \mathrm{E}-02$ & $1.52 \mathrm{E}+01$ & & $4.7 \mathrm{E}-01$ \\
\hline
\end{tabular}


Table A-4. 116-K-2 (West End) Deep Zone Cleanup Verification Data. (2 Pages)

\begin{tabular}{|c|c|c|c|c|c|c|c|c|c|c|c|c|}
\hline \multirow{2}{*}{$\begin{array}{c}\text { Sampling } \\
\text { Area }\end{array}$} & \multirow{2}{*}{$\begin{array}{c}\text { HEIS } \\
\text { Number }\end{array}$} & \multirow{2}{*}{$\begin{array}{c}\text { Sample } \\
\text { Date }\end{array}$} & \multicolumn{3}{|c|}{ Europium-154 } & \multicolumn{2}{|c|}{ Nickel-63 } & \multicolumn{3}{|c|}{ Plutonium-239/240 } & \multicolumn{2}{|c|}{ Strontium-90 } \\
\hline & & & $\mathrm{pCi} / \mathrm{g}$ & $\mathbf{Q}$ & MDA & $\mathrm{pCi} / \mathrm{g}$ & MDA & $\mathrm{pCi} / \mathrm{g}$ & $\mathbf{Q}$ & MDA & $\mathrm{pCi} / \mathrm{g}$ & MDA \\
\hline D10 & J10CW7 & 10/18/2005 & $1.80 \mathrm{E}+01$ & & $5.8 \mathrm{E}-01$ & $4.80 E+02$ & $4.0 \mathrm{E}+00$ & $4.33 E+00$ & & $2.8 \mathrm{E}-01$ & $3.27 E+00$ & 2.6E-01 \\
\hline & $10 C \times 6$ & 0/18/2005 & $1.00 \mathrm{E}+01$ & & $5.2 \mathrm{E}-01$ & $5.30 \mathrm{E}+02$ & $3.9 \mathrm{E}+00$ & $5.47 \mathrm{E}+00$ & & $1.8 \mathrm{E}-01$ & $5.38 \mathrm{E}+00$ & 2.3E-01 \\
\hline & 10CX7 & o/18/2005 & $.09 \mathrm{E}+01$ & & 1.91E-01 & $5.28 \mathrm{E}+02$ & $5.73 \mathrm{E}+00$ & $7.33 E+00$ & & $1.25 \mathrm{E}-01$ & $4.30 \mathrm{E}+00$ & $1.39 \mathrm{E}-0$ \\
\hline A1 & & 2005 & $4.75 E+00$ & & E-01 & $3.93 E+02$ & $5.6 \mathrm{E}+00$ & $2.67 \mathrm{E}+00$ & & 3.2E-01 & $2.48 \mathrm{E}+00$ & $2.2 \mathrm{E}-01$ \\
\hline A2 & & & $59 \mathrm{E}+00$ & & & $1.92 E+02$ & $1 \mathrm{E}+00$ & 4.62E-01 & & $E-01$ & $4.27 \mathrm{E}+00$ & $1 \mathrm{E}-01$ \\
\hline A3 & J10CW0 & $10 / 11 / 2005$ & $9.45 \mathrm{E}+00$ & & $5.5 \mathrm{E}-01$ & $5.88 \mathrm{E}+02$ & $6.7 \mathrm{E}+00$ & $3.81 E+00$ & & $2.9 \mathrm{E}-01$ & $3.93 E+00$ & $2.2 \mathrm{E}-01$ \\
\hline B4 & OCW1 & $10 / 12 / 2005$ & $1.2 \mathrm{E}-01$ & $U$ & $1.2 \mathrm{E}-01$ & $2.16 \mathrm{E}+01$ & $3.5 \mathrm{E}+00$ & 0 & $\mathrm{u}$ & 3.7E-01 & 8.24E-01 & $2.9 \mathrm{E}-01$ \\
\hline $\mathrm{B} 5$ & & $2 / 2005$ & $2.21 \mathrm{E}+00$ & & $2 \mathrm{E}-01$ & $2.43 \mathrm{E}+02$ & $4.4 \mathrm{E}+00$ & $1.43 \mathrm{E}+00$ & & $2.8 \mathrm{E}-01$ & $2.19 \mathrm{E}+00$ & $2.8 \mathrm{E}-01$ \\
\hline $\mathrm{B} 6$ & ocW3 & $10 / 12 / 2005$ & $3.45 \mathrm{E}+00$ & & $3.0 \mathrm{E}-01$ & $7.39 E+02$ & $7.5 \mathrm{E}+00$ & $1.71 E+00$ & & $4.2 \mathrm{E}-01$ & $1.88 \mathrm{E}+00$ & $2.8 \mathrm{E}-01$ \\
\hline $\mathrm{C} 7$ & J10CW4 & $10 / 17 / 2005$ & $1.03 E+01$ & & $.2 \mathrm{E}-01$ & $1.57 \mathrm{E}+03$ & $4.4 \mathrm{E}+00$ & $1.34 \mathrm{E}+01$ & & $2.6 \mathrm{E}-01$ & $2.01 E+01$ & 3.1E-01 \\
\hline $\mathrm{C} 8$ & OCW5 & $0 / 17 / 2005$ & $1.4 \mathrm{E}-01$ & $U$ & 1.4E-01 & $9.10 E+00$ & $4.2 \mathrm{E}+00$ & $8.8 \mathrm{E}-02$ & $\mathrm{U}$ & $3.4 \mathrm{E}-01$ & $1.44 \mathrm{E}+00$ & $2.8 \mathrm{E}-01$ \\
\hline C9 & J10CW6 & $10 / 17 / 2005$ & 7.3E-02 & $u$ & $7.3 \mathrm{E}-02$ & $4.49 E+02$ & $3.9 \mathrm{E}+00$ & $3.82 E+00$ & & 3.3E-01 & $2.79 E+00$ & 2.5E-01 \\
\hline D1 & J10CW8 & $10 / 18 / 2005$ & $5.67 \mathrm{E}+00$ & & $4.6 \mathrm{E}-01$ & $5.15 \mathrm{E}+02$ & $3.6 \mathrm{E}+00$ & $2.56 \mathrm{E}+00$ & & $3.8 \mathrm{E}-01$ & $1.14 \mathrm{E}+00$ & $2.5 \mathrm{E}-01$ \\
\hline $\mathrm{D} 2$ & J10CW9 & $10 / 18 / 2005$ & $1.48 \mathrm{E}+01$ & & $5.6 \mathrm{E}-01$ & $1.11 E+03$ & $3.8 \mathrm{E}+00$ & $7.28 \mathrm{E}+00$ & & 3.3E-01 & $6.62 E+00$ & 2.7E-01 \\
\hline E3 & J10CX0 & $10 / 18 / 2005$ & $5.07 \mathrm{E}+00$ & & $5.3 \mathrm{E}-01$ & $6.69 E+02$ & $3.4 \mathrm{E}+00$ & $2.60 \mathrm{E}+00$ & & $1.9 \mathrm{E}-01$ & $5.68 \mathrm{E}+00$ & $2.4 \mathrm{E}-01$ \\
\hline E4 & J10CX1 & $10 / 18 / 2005$ & \begin{tabular}{|l|}
$1.2 \mathrm{E}-01$ \\
\end{tabular} & $U$ & $1.2 \mathrm{E}-01$ & $6.88 \mathrm{E}+00$ & $3.7 \mathrm{E}+00$ & 1.71E-01 & & 3.3E-01 & $4.57 \mathrm{E}+00$ & $2.5 \mathrm{E}-01$ \\
\hline E5 & $10 \mathrm{CX} 2$ & $10 / 24 / 2005$ & $1.66 \mathrm{E}+00$ & & 3.0E-01 & $1.56 \mathrm{E}+02$ & $5.1 \mathrm{E}+00$ & $1.10 \mathrm{E}+00$ & & 3.5E-01 & $4.24 \mathrm{E}+00$ & $2.0 \mathrm{E}-01$ \\
\hline F6 & J10CX3 & $10 / 24 / 2005$ & $1.57 E+01$ & & 8.2E-01 & $1.17 \mathrm{E}+03$ & $9.8 \mathrm{E}+00$ & $1.11 \mathrm{E}+01$ & & 3.3E-01 & $8.41 \mathrm{E}+00$ & $2.0 \mathrm{E}-01$ \\
\hline F7 & J10CX4 & $10 / 24 / 2005$ & $2.38 \mathrm{E}+00$ & & \begin{tabular}{|l|}
$2.7 \mathrm{E}-01$ \\
\end{tabular} & $1.36 \mathrm{E}+02$ & $3.7 \mathrm{E}+00$ & $1.11 \mathrm{E}+00$ & & $3.7 \mathrm{E}-01$ & $2.81 \mathrm{E}+00$ & 2.3E-01 \\
\hline F8 & J10CX5 & $10 / 24 / 2005$ & $1.30 \mathrm{E}+00$ & & $2.1 \mathrm{E}-01$ & $8.89 E+01$ & $4.0 \mathrm{E}+00$ & $6.50 \mathrm{E}-01$ & & $4.1 \mathrm{E}-01$ & $4.82 \mathrm{E}+00$ & $2.7 \mathrm{E}-01$ \\
\hline
\end{tabular}


Table A-1. 116-K-2 Overburden Cleanup Verification Data.

\begin{tabular}{|c|c|c|c|c|c|c|c|c|c|c|c|c|c|c|}
\hline \multirow{2}{*}{$\begin{array}{l}\text { Sampling } \\
\text { Area }\end{array}$} & \multirow{2}{*}{$\begin{array}{c}\text { HEIS } \\
\text { Number }\end{array}$} & \multirow{2}{*}{$\begin{array}{l}\text { Sample } \\
\text { Date }\end{array}$} & \multicolumn{3}{|c|}{ Hexavalent Chromium } & \multicolumn{3}{|c|}{ Carbon-14 } & \multicolumn{3}{|c|}{ Cesium-137 } & \multicolumn{3}{|c|}{ Cobalt-60 } \\
\hline & & & $\mathrm{mg} / \mathrm{kg}$ & $\mathbf{Q}$ & PQL & pCi/g & $\mathbf{Q}$ & MDA & $\mathrm{pCi} / \mathrm{g}$ & $\mathbf{Q}$ & MDA & $\mathrm{pCi} / \mathrm{g}$ & $\mathbf{Q}$ & MDA \\
\hline B8 & J10M07 & 11/15/2005 & $3.0 \mathrm{E}-01$ & & $2.2 \mathrm{E}-01$ & $-1.37 E+00$ & $\mathrm{U}$ & $2.4 \mathrm{E}+00$ & 4.3E-02 & $u$ & $4.3 \mathrm{E}-02$ & 4.7E-02 & $u$ & 4.7E-02 \\
\hline $\begin{array}{c}\text { Duplicate of } \\
\text { J10M07 }\end{array}$ & J10M08 & $11 / 15 / 2005$ & $2.6 \mathrm{E}-01$ & & 2.2E-01 & $-6.04 \mathrm{E}-01$ & $\mathrm{u}$ & $2.4 \mathrm{E}+00$ & $3.2 \mathrm{E}-02$ & $\mathrm{U}$ & 3.2E-02 & $2.9 \mathrm{E}-02$ & $u$ & $2.9 \mathrm{E}-02$ \\
\hline $\begin{array}{l}\text { Split of } \\
\text { J10M07 }\end{array}$ & J10M09 & $|11 / 15 / 2005|$ & $3.50 \mathrm{E}-01$ & $\mathrm{u}$ & $3.50 \mathrm{E}-01$ & $-7.93 \mathrm{E}-02$ & $u$ & 7.92E-01 & 6.76E-03 & $u$ & 1.99E-02 & $-3.73 E-04$ & $u$ & 1.97E-02 \\
\hline $\mathrm{A} 1$ & J10M00 & $11 / 14 / 2005$ & 2.1E-01 & $\mathrm{U}$ & 2.1E-01 & $-1.06 \mathrm{E}+00$ & U & $2.4 \mathrm{E}+00$ & $1.01 \mathrm{E}-01$ & & $3.9 \mathrm{E}-02$ & $3.8 \mathrm{E}-02$ & U & $3.8 \mathrm{E}-02$ \\
\hline A2 & J10M01 & $11 / 14 / 2005$ & 2.6E-01 & & $2.2 \mathrm{E}-01$ & $-3.78 \mathrm{E}-01$ & $\mathrm{U}$ & $2.2 \mathrm{E}+00$ & $2.6 \mathrm{E}-02$ & $\mathrm{U}$ & $2.6 \mathrm{E}-02$ & $2.6 \mathrm{E}-02$ & U & $2.6 \mathrm{E}-02$ \\
\hline A3 & J10M02 & $11 / 14 / 2005$ & $2.1 \mathrm{E}-01$ & $\mathrm{U}$ & $2.1 \mathrm{E}-01$ & $-1.08 \mathrm{E}+00$ & $U$ & $2.4 \mathrm{E}+00$ & $1.30 \mathrm{E}-01$ & & $4.9 \mathrm{E}-02$ & $3.9 \mathrm{E}-02$ & $U$ & $3.9 \mathrm{E}-02$ \\
\hline A4 & J10M03 & $11 / 14 / 2005$ & $2.2 \mathrm{E}-01$ & $\mathrm{U}$ & 2.2E-01 & $-2.58 \mathrm{E}-01$ & $\mathrm{U}$ & $2.3 E+00$ & $4.1 \mathrm{E}-02$ & $\mathrm{u}$ & $4.1 \mathrm{E}-02$ & $3.9 \mathrm{E}-02$ & $\bar{U}$ & $3.9 \mathrm{E}-02$ \\
\hline B5 & J10M04 & $11 / 14 / 2005$ & $2.7 \mathrm{E}-01$ & & $2.2 \mathrm{E}-01$ & $2.13 \mathrm{E}+00$ & $U$ & $2.2 \mathrm{E}+00$ & $1.08 \mathrm{E}-01$ & & $3.8 \mathrm{E}-02$ & $3.8 \mathrm{E}-02$ & U & $3.8 \mathrm{E}-02$ \\
\hline $\mathrm{B} 6$ & J10M05 & $11 / 14 / 2005$ & $3.4 \mathrm{E}-01$ & & $2.1 \mathrm{E}-01$ & $1.74 \mathrm{E}+00$ & $\mathrm{U}$ & $2.3 E+00$ & $3.0 \mathrm{E}-02$ & $\mathrm{U}$ & $3.0 \mathrm{E}-02$ & $3.2 \mathrm{E}-02$ & $U$ & $3.2 \mathrm{E}-02$ \\
\hline B7 & J10M06 & 11/14/2005 & $2.7 \mathrm{E}-01$ & & $2.2 \mathrm{E}-01$ & $-5.45 \mathrm{E}-01$ & $\mathrm{U}$ & $2.4 \mathrm{E}+00$ & $4.4 \mathrm{E}-02$ & $\mathrm{U}$ & $4.4 \mathrm{E}-02$ & 4.7E-02 & $U$ & 4.7E-02 \\
\hline \multirow{2}{*}{$\begin{array}{l}\text { Sampling } \\
\text { Area }\end{array}$} & \multirow{2}{*}{$\begin{array}{c}\text { HEIS } \\
\text { Number }\end{array}$} & \multirow{2}{*}{$\begin{array}{l}\text { Sample } \\
\text { Date }\end{array}$} & \multicolumn{3}{|c|}{ Europium-154 } & \multicolumn{3}{|c|}{ Nickel-63 } & \multicolumn{3}{|c|}{ Plutonium-239/240 } & \multicolumn{3}{|c|}{ Strontium-90 } \\
\hline & & & $\mathrm{pCi} / \mathrm{g}$ & $\mathbf{Q}$ & MDA & $\mathrm{pCi} / \mathrm{g}$ & $\mathbf{Q}$ & MDA & $\mathrm{pCi} / \mathrm{g}$ & $\mathbf{Q}$ & MDA & $\mathrm{pCi} / \mathrm{g}$ & $\mathbf{Q}$ & MDA \\
\hline B8 & J10M07 & $11 / 15 / 2005$ & $1.6 \mathrm{E}-01$ & $\mathrm{U}$ & 1.6E-01 & 1.15E-01 & $\mathrm{U}$ & $3.2 E+00$ & 0 & $\mathrm{U}$ & $2.9 \mathrm{E}-01$ & $-3.1 \mathrm{E}-02$ & $\mathrm{U}$ & 2.6E-01 \\
\hline $\begin{array}{c}\text { Duplicate of } \\
\text { J10M07 }\end{array}$ & J10M08 & $11 / 15 / 2005$ & 1.1E-01 & $u$ & 1.1E-01 & 8.42E-01 & $u$ & $3.4 \mathrm{E}+00$ & $1.20 \mathrm{E}-01$ & $u$ & $4.6 \mathrm{E}-01$ & $-6.8 \mathrm{E}-02 \mid$ & u & 2.2E-01 \\
\hline $\begin{array}{l}\text { Split of } \\
\text { J10M07 }\end{array}$ & J10M09 & $\mid 11 / 15 / 2005$ & $-1.90 \mathrm{E}-02$ & $u \mid$ & 6.46E-02 & $-1.46 E+00$ & $\mathrm{u}$ & $6.89 E+00$ & $0.00 \mathrm{E}+00$ & $\mathrm{u}$ & 4.17E-02 & $-3.37 E-02$ & $u$ & $9.76 \mathrm{E}-02$ \\
\hline $\mathrm{A} 1$ & J10M00 & $11 / 14 / 2005$ & $1.2 \mathrm{E}-01$ & $\mathrm{U}$ & 1.2E-01 & 9.24E-01 & $\mathrm{U}$ & $4.5 \mathrm{E}+00$ & 0 & $\mathrm{U}$ & 3.4E-01 & $-3.2 E-02$ & $\mathrm{U}$ & 2.3E-01 \\
\hline $\mathrm{A} 2$ & J10M01 & $11 / 14 / 2005$ & $8.3 \mathrm{E}-02$ & $\mathrm{U}$ & $8.3 E-02$ & 4.49E-01 & $\mathrm{U}$ & $2.9 \mathrm{E}+00$ & 0 & $\mathrm{U}$ & $2.6 \mathrm{E}-01$ & $8.3 \mathrm{E}-02$ & U & $2.6 \mathrm{E}-01$ \\
\hline A3 & J10M02 & $11 / 14 / 2005$ & $1.3 \mathrm{E}-01$ & $\mathrm{U}$ & $1.3 \mathrm{E}-01$ & 7.06E-01 & $\mathrm{U}$ & $4.5 \mathrm{E}+00$ & $3.1 \mathrm{E}-02$ & $\mathrm{U}$ & 2.4E-01 & $1.70 \mathrm{E}-01$ & $U$ & $1.8 \mathrm{E}-01$ \\
\hline A4 & J10M03 & $11 / 14 / 2005$ & $1.4 \mathrm{E}-01$ & $U$ & $1.4 \mathrm{E}-01$ & -5.94E-01 & $\mathrm{U}$ & $2.9 \mathrm{E}+00$ & 0 & $\mathrm{U}$ & $3.1 \mathrm{E}-01$ & \begin{tabular}{|l|}
$-3.1 \mathrm{E}-02$ \\
\end{tabular} & U & 2.7E-01 \\
\hline B5 & J10M04 & $11 / 14 / 2005$ & $1.2 \mathrm{E}-01$ & $\mathrm{U}$ & 1.2E-01 & 9.75E-01 & $\mathrm{U}$ & $2.9 \mathrm{E}+00$ & 0 & $U$ & 2.1E-01 & $-7 . E-03$ & $U$ & $2.4 \mathrm{E}-01$ \\
\hline $\mathrm{B} 6$ & J10M05 & $11 / 14 / 2005$ & $1.1 \mathrm{E}-01$ & $U$ & $1.1 \mathrm{E}-01$ & $-5.54 \mathrm{E}-01$ & $\mathrm{U}$ & $3.3 \mathrm{E}+00$ & 0 & $U$ & $2.1 \mathrm{E}-01$ & $-3 . E-03$ & $U$ & $2.0 \mathrm{E}-01$ \\
\hline B7 & J10M06 & $11 / 14 / 2005$ & $1.5 \mathrm{E}-01$ & $\mathrm{U}$ & 1.5E-01 & 2.99E-01 & $\mathrm{U}$ & $3.6 E+00$ & $4.4 \mathrm{E}-02$ & $\mathrm{U}$ & $3.4 \mathrm{E}-01$ & $2.5 \mathrm{E}-02$ & $U$ & $2.7 \mathrm{E}-01$ \\
\hline
\end{tabular}


CVP-2006-00001

Rev. 0 
CVP-2006-00001

Rev. 0

APPENDIX B

DATA QUALITY ASSESSMENT

B-i 
CVP-2006-00001

Rev. 0 


\section{B1.0 DATA QUALITY ASSESSMENT FOR THE 116-K-2 WASTE SITE}

\section{B1.1 OVERVIEW}

The data quality assessment (DQA) completes the data life cycle (i.e., planning, implementation, and assessment) that was initiated by the data quality objectives process. The DQA includes a review of the field logbook information (BHI 2005a, 2005b, 2005c) to verify sample location, date, and time. It also involves the scientific and statistical evaluation of the data to determine if they are of the right type, quality, and quantity to support their intended use for closeout decisions (EPA 2000).

This DQA was performed in accordance with WCH-EE-01, Environmental Investigations Procedures. Specific data quality objectives for the site are found in the 100 Area Remedial Action Sampling and Analysis Plan (SAP) (DOE-RL 2005a). The DQA is based on the guidelines presented in Guidance for Data Quality Assessment (EPA 2000). Statistical tests used in this DQA were performed as specified in the SAP and the Remedial Design Report/Remedial Action Work Plan for the 100 Area (RDR/RAWP) (DOE-RL 2005b).

Prior to performing statistical tests, the field logbooks (BHI 2005a, 2005b, 2005c), sample designs (Appendix C), and sample analytical data are evaluated. A portion of the cleanup verification sample analytical data are validated for compliance requirements (DOE-RL 2005a). Data evaluation is performed to determine if the laboratory carried out all steps required by the SAP and the laboratory contract governing the conduct of analysis and reporting of the data. This evaluation also examines the available laboratory data to determine if an analyte is present or absent in a sample and the degree of overall uncertainty associated with that determination. Data validation is done in accordance with validation procedures (BHI 2000a, 2000b) as part of data evaluation. After data evaluation and validation, the appropriate statistical analyses are performed on the adjusted raw analytical data (Appendix $\mathrm{C}$ ) to determine statistical values for each contaminant. The cleanup verification sample analytical data are stored in the Hanford Environmental Information System and are summarized in Appendix A.

For the 116-K-2 site, 66 samples in 18 sample delivery groups (SDGs) have been evaluated in the following sections.

\section{B1.2 LABORATORY QUALITY MEASURES}

All verification samples are subject to laboratory-specific quality assurance (QA) requirements, including instrument procurement, maintenance, calibration, and operation. Additional laboratory quality control $(\mathrm{QC})$ checks are performed, as 
appropriate, for the analytical method at a rate of 1 per SDG, or 1 in 20 , whichever is more frequent. Laboratory internal QC checks include the following:

- Laboratory Contamination. Each analytical batch contains a laboratory (method) blank (material of similar composition as the samples with known/minimal contamination of the analytes of interest) carried through the complete analytical process. The method blank is used to evaluate false-positive results in samples due to contamination during handling at the laboratory.

- Analytical Accuracy. For most analyses, a known quantity of representative analytes of interest (matrix spike/matrix spike duplicate [MS/MSD]) is added to a separate aliquot of a sample from the analytical batch. The recovery percentage of the added MS is used to evaluate analytical accuracy. For analyses not amenable to MS techniques (e.g., gamma energy analysis) or where analytical recovery is corrected via internal standards (e.g., alpha spectral analyses), accuracy is evaluated from recovery of the QC reference sample (e.g., laboratory control spike or blank spike sample).

- Analytical Precision. Separate aliquots removed from the same sample container (replicate samples) are analyzed for each analytical batch. The replicate sample results (evaluated as relative percent differences [RPDs]) are used to assess analytical precision.

- QC Reference Samples. A QC reference sample is prepared from an independent standard at a concentration other than that used for calibration, but within the calibration range. Reference samples provide an independent check on analytical technique and methodology.

Laboratories are also subject to periodic and random assessments of the laboratory performance, systems, and overall program. These assessments are performed by the Washington Closure Hanford QA group to ensure that the laboratories are performing within laboratory contract requirements.

\section{B1.3 DATA VALIDATION}

After sampling was completed, all of the fixed-base laboratory data from SDG H3328 were validated by a third-party validator to Level $\mathrm{C}$ per WCH-EE-01, Procedure 2.5, "Data Package Validation Process." Level C validation procedures are specified in Data Validation Procedure for Radiochemical Analysis (BHI 2000b) and Data Validation Procedure for Chemical Analysis (BHI 2000a).

Use of Level C validation procedures was included in the review of the following items, as appropriate, for each analytical method:

- Sample holding times

- Method blanks 
- MS/MSD recovery

- Surrogate recovery

- Sample replicates

- Associated batch laboratory control sample results

- Data package completeness

- Achievement of required (or contractual) detection limits (RDLs).

Data flagged by the validator as estimated (i.e., "J") indicate that the associated concentration is an estimate, but that the data may be used for decision-making purposes. Data flagged as below detection limits (i.e., "U") indicate the contaminant was analyzed for but not detected, and the concentration is below the minimum detectable activity (MDA) for radionuclides or the practical quantitation limit (PQL) (i.e., reporting limit) for nonradionuclides. For nonradionuclides, nondetects are reported at the PQL. For radionuclides, nondetects report the actual value obtained from analysis (positive or negative but less than the MDA) except for limited analyses where no value can be calculated. In these cases, the MDA is reported. This situation is applicable for sample results that are below detection limits. All other validated results are considered to be accurate within the standard errors associated with the methods.

The adequacy of laboratory QA/QC was evaluated for precision, accuracy, completeness, and RDLs pursuant to the SAP (DOE-RL 2005a). The organization performing the data validation reported that, of the data given formal validation, the laboratory met the standards for performance for precision $( \pm 30 \%)$, accuracy $( \pm 30 \%)$, and completeness $(>90 \%)$. Comparison of the RDL with the respective MDA or PQL is discussed in Section B1.4.

The validated SDG H3328 contains six samples (J03JW3, J03W34, J03JW5, J03JW7, J03JW8, and J03JW9). A summary of deficiencies noted during validation follows.

- Radionuclides. The validation DQA noted no major deficiencies.

Sample number J03W34, in SDG 3328, had errors in the quantitation of carbon-14 and nickel-63. The sample was rerun for those two results only. The validator qualified the carbon-14 and nickel-63 results with a "J" as estimated values. Data qualified with a "J" are useable for decision-making purposes.

- Nonradionuclides. The validation DQA noted no major or minor deficiencies.

\section{B1.4 LABORATORY DATA EVALUATION}

The following paragraphs include the results of the data evaluation of 18 verification sample SDGs. 
The context for assessing the data includes evaluating the sample data using the statistical methodology of the SAP (DOE-RL 2005a) (included in the calculation briefs in Appendix C) and a comparison of analytical results to the parameters as specified in the SAP. This section summarizes the results of the comparison and presents an evaluation of the affected data.

\section{MAJOR DEFICIENCIES}

Any data anomaly that causes final data to be qualified as rejected is considered a major deficiency. The project has identified one major deficiency in the 116-K-2 data set. The hexavalent chromium result for sample J03JX1 has been qualified, by the project, with an "R" as rejected data. Sample J03JX1 is the only sample in SDG W04740. The sample was non-detect for hexavalent chromium, the MDLs were not met, and the associated MS(s) had zero recovery. J03JX1 was a split sample, sent to a separate laboratory from the rest of the data. The primary sample and its duplicate are not affected by the J03JX1 result. No other major deficiencies were found in the 116-K2 data set.

\section{MINOR DEFICIENCIES}

Sample Holding Times. All of the method-specific holding times were met for all samples in the 116-K-2 data set.

Method Blanks. The method blank is used to evaluate false-positive results in samples due to contamination during handling at the laboratory.

Radionuclides. In the radionuclide analyses, low-level positive results were observed in several SDGs. Most radiological analytical techniques are counting methods. Due to the nature of this type of analysis, positive, but insignificant, results are not uncommon in the method blank and are not considered contamination.

Nonradionuclides. No analytes were reported in the nonradiological method blanks associated with $116-\mathrm{K}-2$.

MS/MSDs Recoveries. Recovery of spiked analytes in the MS/MSD pair is used to evaluate method efficiency and the effect of the matrix on an environmental sample.

Radionuclides. All MS/MSD recoveries for radionuclide analytes were within acceptance criteria.

Nonradionuclides. In SDG J00004, the MS/MSD pair for hexavalent chromium had low recoveries ( $49 \%$ and $60 \%$, respectively). The laboratory control sample had good recovery, indicating that the analytical instrumentation was in good operating condition. It is not uncommon to see reactive species, such as hexavalent chromium, react with the sample media and give a low response in the MS/MSD pair. This conclusion is 
supported by the similarly low recovery in both the MS and the MSD. Low recoveries in the MS/MSD pair may be cause to consider the data estimated. However, the data remain usable for decision-making purposes.

RDL Comparison. Reported analytical detection levels for nondetected analytes were compared to the RDLs specified in the SAP (DOE-RL 2005a). When detected results were obtained, evaluation of detection limits was not performed. The data validation and supplemental data evaluation noted any analyses in which the detection limit (MDA or PQL) was above the SAP RDLs for nondetected analytes.

Radionuclides. All of the reported MDAs are sufficiently low for decision-making purposes. All values meet the site cleanup criteria as demonstrated in the calculation briefs (Appendix $\mathrm{C}$ ) and discussed in this cleanup verification package.

Nonradionuclides. All of the reported method detection limits are less than applicable remedial action goals (RAGs), and the data are of sufficient quality for decision-making purposes.

Precision and Accuracy Evaluation. Analytical accuracy and precision were evaluated by examination of the RPD of the main and duplicate samples. Only the contaminants of concern (COCs) detected at five times the detection limit (or greater) are used for data analysis with respect to accuracy and precision.

Radionuclides. In SDG H3256, the RPD for the duplicate analysis of sample J03D01 for uranium-233/234 is slightly above acceptance criteria at $35.0 \%$. In SDG K0010, the RPD for the duplicate analysis of sample J103C0 for nickel-63 is 93\%. In SDG J00004, the RPD for the duplicate analysis of plutonium-238 is slightly above acceptance criteria at $35.5 \%$.

Nonradionuclides. The RPD for the duplicate analysis of sample J03JW2 for hexavalent chromium in SDG 3250 is above acceptance criteria at $44.3 \%$. The RPD for the duplicate analysis of sample J03JX0 for hexavalent chromium in SDG 3256 is above criteria at $39.5 \%$.

Duplicates are produced using field-collected materials. The natural heterogeneity of these materials adds to elevated RPDs, such as those listed above. This variability is expected and does not indicate a problem with the analytical system. RPDs of analytes detected at low concentrations (less than five times the detection limit) are also not considered to be indicative of the analytical system performance. The data are useable for decision-making purposes.

\section{B1.5 FIELD QUALITY ASSURANCE/QUALITY CONTROL}

Field QA/QC measures were used to assess potential sources of error and cross contamination of soil samples that could bias results. Field QA/QC samples listed in the 
field logbook (BHI 2005b, 2005c) are summarized in Table B-1. All main and QA/QC sample results are presented in Appendix A.

Table B-1. Summary of Field Quality Control Samples.

\begin{tabular}{|c|c|c|c|}
\hline Sample Area & Main Sample & Duplicate & Split \\
\hline $\begin{array}{c}\text { B7 (East Shallow } \\
\text { Zone) }\end{array}$ & J03JW8 & J03JW9 & J03JX1 \\
\hline B6 (East Deep Zone) & J03D02 & J03D03 & J03D04 \\
\hline $\begin{array}{c}\text { A3 (West Shallow } \\
\text { Zone) }\end{array}$ & J10DL0 & J10DM4 & J10F85 \\
\hline $\begin{array}{c}\text { D10 (West Deep } \\
\text { Zone) }\end{array}$ & J10CW7 & J10CX6 & J10CX7 \\
\hline B8 (Overburden) & J10M07 & J10M08 & J10M09 \\
\hline
\end{tabular}

Field duplicate samples were collected to provide a relative measure of the degree of local heterogeneity in the sampling medium, unlike laboratory duplicates that are used to evaluate precision in the analytical process. The field duplicates are evaluated by computing the RPD of the duplicate samples for each COC. Only analytes with values above five times the detection limits for both the main and duplicate samples are compared. The 95\% upper confidence limit (UCL) calculation briefs in Appendix C provide details on duplicate pair evaluation and RPD calculation. The data are suitable for the intended purpose of cleanup verification.

Split samples were collected to provide a relative measure of the degree of variability in the sampling, sample handling, and analytical techniques used by commercial laboratories. The field main and split samples are evaluated by computing the RPD of the split samples for each COC to determine the usability of the verification data. The U.S. Environmental Protection Agency Contract Laboratory Program duplicate sample comparison methodology, USEPA Contract Laboratory Program National Functional Guidelines for Inorganic Data Review (EPA 1994), is used as an initial test of the data from the splits. Only analytes that had values above five times the contractual RDL for both the main and split sample were compared. The $95 \%$ UCL calculation briefs in Appendix $C$ provide details on split pair RPD calculation. These results are typical of the heterogeneity found in the sample matrices and do not indicate a problem with either of the laboratory's analytical systems.

\section{B1.6 SUITABILITY OF DATA}

The DQA for the 116-K-2 site determined that the data are of the right type, quality, and quantity to support site cleanup verification decisions within specified error tolerances. The evaluation verified that the sample design was sufficient for the purpose of clean 
site verification. All analytical data, except the hexavalent chromium result in sample J03JX1, were found to be acceptable for decision-making purposes.

\section{B2.0 REFERENCES}

BHI, 2000a, Data Validation Procedure for Chemical Analysis, BHI-01435, Rev. 0 , Bechtel Hanford, Inc., Richland, Washington.

BHI, 2000b, Data Validation Procedure for Radiochemical Analysis, BHI-01433, Rev. 0, Bechtel Hanford, Inc., Richland, Washington.

BHI, 2005a, 100K Remedial Sampling, Logbook EL-1572-2, Bechtel Hanford, Inc., Richland, Washington.

BHI, 2005b, 100K Remedial Sampling, Logbook EL-1572-3, Bechtel Hanford, Inc., Richland, Washington.

BHI, 2005c, 100K Remedial Sampling, Logbook EL-1572-4, Bechtel Hanford, Inc., Richland, Washington.

DOE-RL, 2005a, 100 Area Remedial Action Sampling and Analysis Plan, DOE/RL-96-22, Rev. 4, U.S. Department of Energy, Richland Operations Office, Richland, Washington.

DOE-RL, 2005b, Remedial Design Report/Remedial Action Work Plan for the 100 Area, DOE/RL-96-17, Rev. 5, U.S. Department of Energy, Richland Operations Office, Richland, Washington.

EPA, 1994, USEPA Contract Laboratory Program National Functional Guidelines for Inorganic Data Review, EPA 540/R-94/013, U.S. Environmental Protection Agency, Washington, D.C.

EPA, 2000, Guidance for Data Quality Assessment, EPA QA/G-9, QA00 Update, U.S. Environmental Protection Agency, Office of Environmental Information, Washington, D.C.

WCH-EE-01, Environmental Investigations Procedures, Washington Closure Hanford, Richland, Washington. 
CVP-2006-00001

Rev. 0

B-8 


\section{APPENDIX C}

RESRAD INPUT PARAMETERS

\section{AND CALCULATION BRIEF EXCERPTS}

C-i 
CVP-2006-00001

Rev. 0 
RESRAD INPUT PARAMETERS FOR THE 116-K-2 (EAST END) SHALLOW ZONE 
CVP-2006-00001

Rev. 0

C-2 
CVP-2006-00001

Rev. 0

IRESRAD, Version 6.3 T Limit $=180$ days $\quad 10 / 10 / 2005 \quad 09: 35$ Page

Summary : 116-K-2 Trench (East End) Cleanup Verification RESRAD Calculation

File : 116-K-2-SZ-SWC.RAD

\section{Table of Content}

$\ddot{A} \ddot{A} \ddot{A} \ddot{A} \ddot{A} \ddot{A} \ddot{A} \ddot{A} \ddot{A} \ddot{A} \ddot{A} \ddot{A} \ddot{A} \ddot{A} \ddot{A} \ddot{A} \ddot{A}$

Part I: Mixture Sums and Single Radionuclide Guidelines

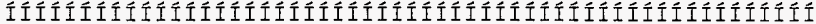

Dose Conversion Factor (and Related) Parameter Summary ...

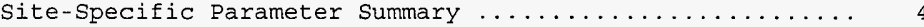

Summary of Pathway selections . . . . . . . . . . . . . 8

Contaminated zone and Total Dose Summary ............

Total Dose Components

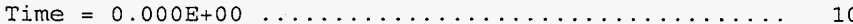

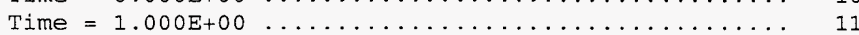

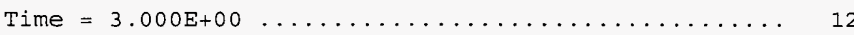

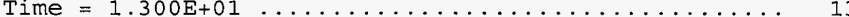

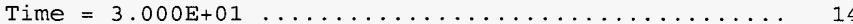

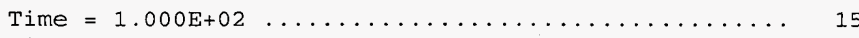

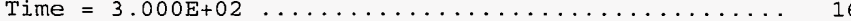

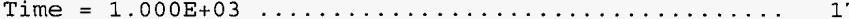

Dose/Source Ratios Summed over All Pathways ........... 18

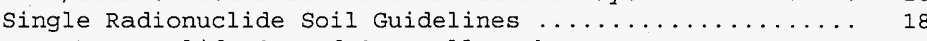

Dose Pex Nuclide Summed Over All Pathways ............... 19

Soil Concentration Per Nuclide .................. 19 


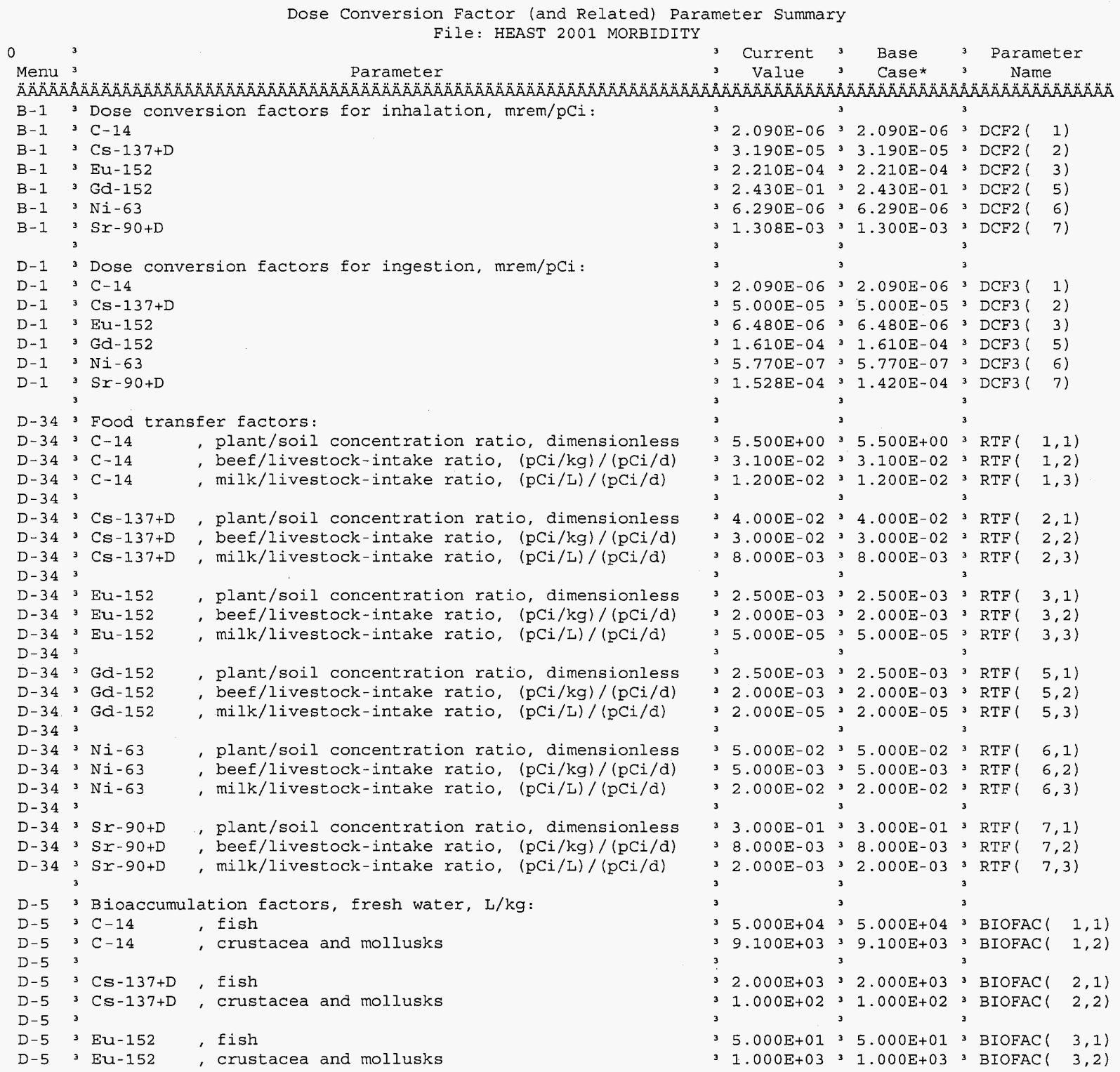


CVP-2006-00001

Rev. 0

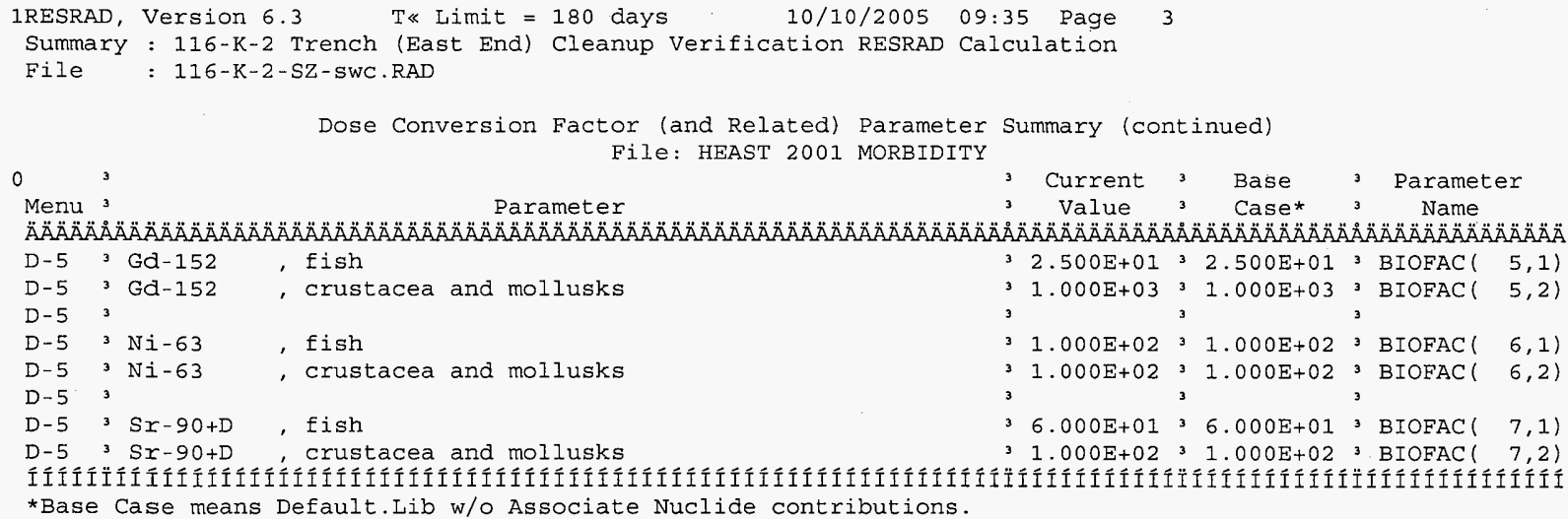


Summary : $116-\mathrm{K}-2$ Trench (East End) Cleanup Verification RESRAD Calculation

File : $116-\mathrm{K}-2-\mathrm{SZ}-\mathrm{SWC} \cdot \mathrm{RAD}$

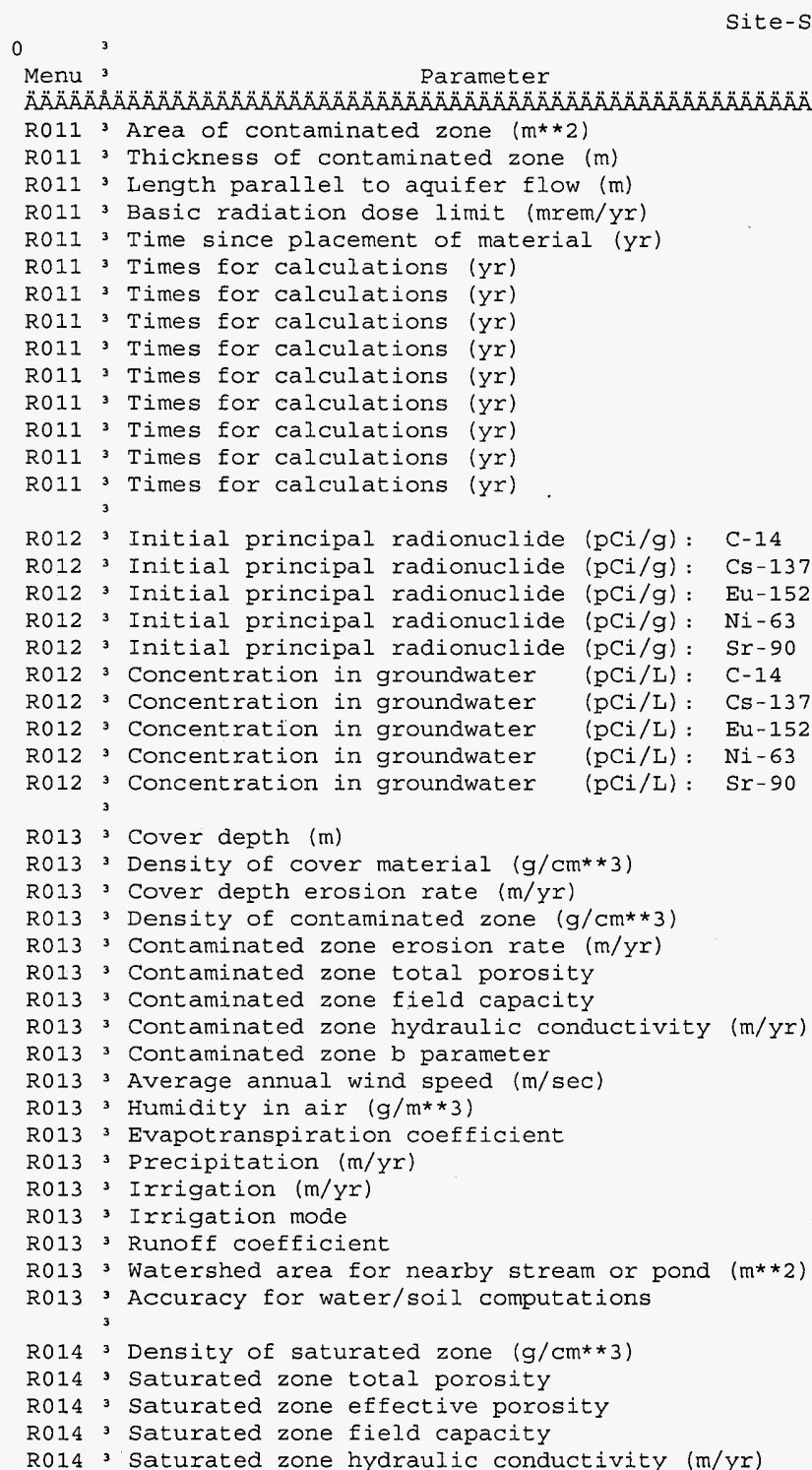
ite-Specific Parameter Summary 31 Tnput 3 - Default 30 Used by RESRAD 3 Parameter

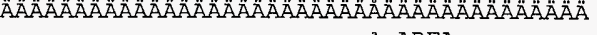
$32.920 \mathrm{E}+02 \quad 3 \quad 1.000 \mathrm{E}+02 \quad 3$ ${ }^{3} 1.500 \mathrm{E}+01 \mathrm{~B}^{3} 3.000 \mathrm{E}+01^{3}$ $30.000 \mathrm{E}+003 \quad 0.000 \mathrm{E}+00$ $3 \quad 1.000 \mathrm{E}+00^{3} \quad 1.000 \mathrm{E}+00^{3}$ $3.000 \mathrm{E}+00^{3} 3.000 \mathrm{E}+00^{3}$ $31.300 \mathrm{E}+01^{3} 1.000 \mathrm{E}+01$

$33.000 E+0133.000 E+013$ $1.000 \mathrm{E}+0231.000 \mathrm{E}+02$ $3.000 \mathrm{E}+0233.000 \mathrm{E}+02$ $31.000 E+0331.000 E+03$ not used $30.000 \mathrm{E}+00$ not used $30.000 \mathrm{E}+00$

$7.000 \mathrm{E}-0130.000 \mathrm{E}+00$ $35.600 \mathrm{E}-0130.000 \mathrm{E}+00$

$3 \quad 1.530 \mathrm{E}-01^{3} 0.000 \mathrm{E}+00^{3}$ $3.560 \mathrm{E}+00^{3} 0.000 \mathrm{E}+00$ 1.430E-01 $30.000 E+00$

3 not used $30.000 E+00$

not used $30.000 \mathrm{E}+00^{3}$

3 not used $30.000 E+00$

not used $30.000 \mathrm{E}+00$ not used $30.000 \mathrm{E}+00$

$0.000 \mathrm{E}+00^{3} \quad 0.000 \mathrm{E}+00$

not used $3 \quad 1.500 E+00^{3}$

not used $31.000 \mathrm{E}-03^{3}$

$31.600 \mathrm{E}+00^{3} 1.500 \mathrm{E}+00$

$1.000 E-0331.000 E-03$

4.000E-01 3 4.000E-01

$32.000 \mathrm{E}-0132.000 \mathrm{E}-013$

$32.500 E+0231.000 E+01$

$4.050 E+003 \quad 5.300 E+00$

$3.400 E+00 \quad 3 \quad 2.000 E+00$

not used $38.000 E+00^{3}$

$9.100 E-013 \quad 5.000 E-01$

$1.600 \mathrm{E}-0131.000 \mathrm{E}+00$

$7.600 \mathrm{E}-01$ 3 $2.000 \mathrm{E}-01$

3 overhead ${ }^{3}$ overhead 3

$2.000 E-0132.000 E-01$

$1.000 E+06 \quad 3 \quad 1.000 E+06 \quad 3$ ${ }^{3} 1.000$ E-03 3 1.000E-03.

$1.600 E+003 \quad 1.500 E+00$

$4.000 E-0134.000 E-01$

$2.500 E-0132.000 E-01$

$1.500 \mathrm{E}-01{ }^{3} 2.000 \mathrm{E}-01$

$35.530 E+03 \quad 3 \quad 1.000 E+02^{3}$
3 AREA

$\begin{array}{ll}-.- & 3 \text { AREA } \\ -- & 3 \text { THICKO }\end{array}$

3 BRD

3 TT

-. $3 \mathrm{~T}(2)$

-. $3 \mathrm{~T}(3)$

-.. $3 \mathrm{~T}(5)$

- 3 T $(6)$

-. 3 T 7 ( 7 )

$3 \mathrm{~T}(8)$

-. $3 \mathrm{~T}(9)$

- $3 \mathrm{~T}(10)$

-. $351(1)$

-.- 3 S1 $(2)$

-. $\quad 31(3)$

$3 \operatorname{Si}(6)$

-. 3 S1 $(7)$

3 W1 $(1)$

3 WI ( 2 )

3 W1 ( 3 )

3 WI $(6)$

-.. 3 W1 $(7)$

-. 3 COVERO

-.- 3 DENSCV

... 3 VCV

-.. 3 DENSCZ

$3 \mathrm{VCZ}$

- - 3 TPCZ

3 FCCZ

$3 \mathrm{HCCZ}$

- - $3 \mathrm{BCZ}$

-.- 3 WIND

-.. 3 HUMID

- 3 EVAPTR

- 3 PRECIP

-. 3 RI

-.- 3 IDITCH

3 RUNOFF

3 WAREA

3 EPS

--- 3 DENSAQ

- - 3 TPSZ

-

3 FCSZ

- - $3 \mathrm{HCSZ}$
3 LCZPAQ 
IRESRAD, Version $6.3 \quad T$ « Limit $=180$ days $\quad 10 / 10 / 2005$ 09:35 Page 5 Summary : 116-K-2 Trench (East End) Cleanup Verification RESRAD Calculation File : 116-K-2-SZ-SWC.RAD

\begin{tabular}{|c|c|c|c|c|c|c|c|}
\hline \multirow{2}{*}{0} & \multicolumn{7}{|c|}{ Site-Specific Parameter Summary (continued) } \\
\hline & $=0$ & $3 \quad$ User & 3 & 3 & Used by RESRAD & 3 & Parameter \\
\hline \multirow{2}{*}{\multicolumn{8}{|c|}{ 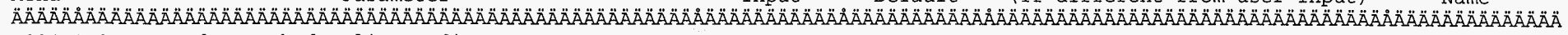 }} \\
\hline & & & & & & & \\
\hline R014 & 3 Saturated zone hydraulic gradient & $31.250 \mathrm{E}-03$ & $32.000 E-02$ & 3 & -- & 3 & HGWT \\
\hline R014 & 3 Saturated zone b parameter & $34.050 E+00$ & $35.300 E+00$ & 3 & --- & 3 & BSZ \\
\hline R014 & 3 Water table drop rate $(\mathrm{m} / \mathrm{yr})$ & $31.000 \mathrm{E}-03$ & $31.000 E-03$ & 3 & $-\ldots$ & 3 & VWT \\
\hline R014 & 3ell pump intake depth (m below water table) & $4.600 \mathrm{E}+00$ & $31.000 E+01$ & 3 & $-\cdots$ & 3 & DWIBWT \\
\hline R014 & 3 Model: Nondispersion (ND) or Mass-Balance (MB) & $\mathrm{ND}$ & ${ }^{3} \mathrm{ND}$ & 3 & --- & 3 & MODEL \\
\hline \multirow[t]{2}{*}{$\mathrm{R} 014$} & ${ }_{3}$ Well pumping rate $(m * * 3 / y r)$ & $32.500 \mathrm{E}+02$ & $32.500 \mathrm{E}+02$ & 3 & $-\cdots$ & 3 & UW \\
\hline & з & 3 & 3 & 3 & & 3 & \\
\hline R015 & 3 Number of unsaturated zone strata & 31 & 31 & 3 & --- & 3 & NS \\
\hline R015 & 3 Unsat. zone 1 , thickness $(\mathrm{m})$ & $31.040 \mathrm{E}+01$ & $34.000 E+00$ & 3 & --- & 3 & $\mathrm{H}(1)$ \\
\hline R015 & 3 Unsat. zone 1 , soil density $\left(\mathrm{g} / \mathrm{cm}^{* *} 3\right)$ & $31.600 \mathrm{E}+00$ & $31.500 \mathrm{E}+00$ & 3 & --- & 3 & DENSUZ (1) \\
\hline R015 & 3 Unsat. zone 1 , total porosity & $34.000 \mathrm{E}-01$ & $34.000 \mathrm{E}-01$ & 3 & --- & 3 & TPUZ (1) \\
\hline R015 & 3 Unsat. zone 1 , effective porosity & $32.500 \mathrm{E}-01$ & $32.000 \mathrm{E}-01$ & 3 & --- & 3 & EPUZ (1) \\
\hline R015 & 3 Unsat. zone 1 , field capacity & $31.500 \mathrm{E}-01$ & $32.000 E-01$ & 3 & --- & 3 & FCUZ (1) \\
\hline $\mathrm{R} 015$ & 3 Unsat. zone 1 , soil-specific b parameter & $34.050 \mathrm{E}+00$ & $35.300 E+00$ & 3 & --- & 3 & $\mathrm{BUZ}(1)$ \\
\hline \multirow[t]{2}{*}{ R015 } & 3. Unsat. zone 1, hydraulic conductivity (m/yr) & $32.500 \mathrm{E}+02$ & $31.000 E+01$ & 3 & $-\cdots$ & 3 & HCUZ (1) \\
\hline & 3 & 3 & 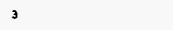 & 3 & & 3 & \\
\hline R016 & ${ }^{3}$ Distribution coefficients for $\mathrm{C}-14$ & 3 & 3 & 3 & & 3 & \\
\hline R016 & 3 Contaminated zone $(\mathrm{cm} * \star 3 / \mathrm{g})$ & $32.000 E+02$ & $30.000 E+00$ & 3 & $-\ldots$ & 3 & DCNUCC $(1)$ \\
\hline R016 & Unsaturated zone $1(\mathrm{~cm} * * 3 / \mathrm{g})$ & $32.000 \mathrm{E}+02$ & $30.000 E+00$ & 3 & --- & 3 & DCNUCU $(1,1)$ \\
\hline R016 & Saturated zone $(\mathrm{cm} * * 3 / \mathrm{g})$ & $32.000 E+02$ & $30.000 E+00$ & 3 & $-\ldots$ & 3 & DCNUCS $(1)$ \\
\hline R016 & Leach rate $(/ y r)$ & $30.000 \mathrm{E}+00$ & $30.000 E+00$ & 3 & $5.426 E-05$ & 3 & ALEACH ( 1) \\
\hline \multirow[t]{2}{*}{ R016 } & Solubility constant & $30.000 E+00$ & $30.000 E+00$ & 3 & not used & 3 & SOLUBK ( 1) \\
\hline & 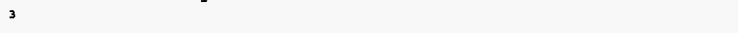 & 3 & 3 & 3 & & 3 & \\
\hline R016 & 3 Distribution coefficients for $\mathrm{Cs}-137$ & 3 & 3 & 3 & & 3 & \\
\hline $\mathrm{R} 016$ & 3 Contaminated zone $(\mathrm{cm} * * 3 / \mathrm{g})$ & $35.000 \mathrm{E}+01$ & $34.600 E+03$ & 3 & --- & 3 & DCNUCC $(2)$ \\
\hline $\mathrm{R} 016$ & Unsaturated zone $1\left(\mathrm{~cm}^{\star *} 3 / \mathrm{g}\right)$ & $35.000 \mathrm{E}+01$ & $34.600 \mathrm{E}+03$ & 3 & --- & 3 & DCNUCU $(2,1)$ \\
\hline R016 & Saturated zone $(\mathrm{cm} * * 3 / \mathrm{g})$ & $35.000 \mathrm{E}+01$ & $34.600 \mathrm{E}+03$ & 3 & $\ldots$ & 3 & DCNUCS $(2)$ \\
\hline R016 & Leach rate (/yr) & $30.000 \mathrm{E}+00$ & $30.000 \mathrm{E}+00$ & 3 & $2.166 E-04$ & 3 & ALEACH ( 2) \\
\hline \multirow[t]{2}{*}{ R016 } & Solubility constant & $30.000 E+00$ & $30.000 E+00$ & 3 & not used & 3 & SOLUBK( 2) \\
\hline & (0) & 3 & 3 & 3 & & 3 & \\
\hline R016 & 3 Distribution coefficients for $\mathrm{Eu}-152$ & 3 & 3 & 3 & & 3 & \\
\hline $\mathrm{R} 016$ & 3 Contaminated zone $\left(\mathrm{cm}^{*} * 3 / \mathrm{g}\right)$ & $32.000 \mathrm{E}+02$ & $3-1.000 E+00$ & 3 & --- & 3 & DCNUCC ( 3$)$ \\
\hline R016 & Unsaturated zone $1\left(\mathrm{~cm}^{\star} \star 3 / \mathrm{g}\right)$ & $32.000 \mathrm{E}+02$ & $3-1.000 E+00$ & 3 & --- & 3 & DCNUCU $(3,1)$ \\
\hline $\mathrm{R} 016$ & Saturated zone $(\mathrm{cm} * * 3 / \mathrm{g})$ & $32.000 \mathrm{E}+02$ & $3-1.000 E+00$ & 3 & --- & 3 & DCNUCS $(3)$ \\
\hline $\mathrm{R} 016$ & Leach rate (/yr) & $0.000 \mathrm{E}+00$ & $30.000 \mathrm{E}+00$ & 3 & $5.426 \mathrm{E}-05$ & 3 & ALEACH ( 3) \\
\hline \multirow[t]{2}{*}{ R016 } & Solubility constant & $30.000 \mathrm{E}+00$ & $30.000 \mathrm{E}+00$ & 3 & not used & 3 & SOLUBK $(3)$ \\
\hline & 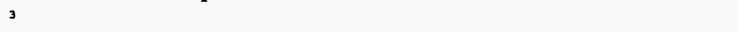 & 3 & 3 & 3 & & 3 & \\
\hline R016 & 3 Distribution coefficients for $\mathrm{Ni}-63$ & 3 & 3 & 3 & & 3 & \\
\hline $\mathrm{R} 016$ & 3 Contaminated zone $\left(\mathrm{cm}^{* * 3} / \mathrm{g}\right)$ & $33.000 E+01$ & $31.000 E+03$ & 3 & --- & 3 & $\operatorname{DCNUCC}(6)$ \\
\hline $\mathrm{R} 016$ & Unsaturated zone $1(\mathrm{~cm} * * 3 / \mathrm{g})$ & $33.000 \mathrm{E}+01$ & $31.000 \mathrm{E}+03$ & 3 & --- & 3 & $\operatorname{DCNUCU}(6,1)$ \\
\hline R016 & Saturated zone $(\mathrm{cm} * * 3 / \mathrm{g})$ & $3.000 E+01$ & $31.000 E+03$ & 3 & --- & 3 & DCNUCS $(6)$ \\
\hline $\mathrm{R} 016$ & Leach rate $(/ y r)$ & 3. $0.000 \mathrm{E}+00$ & $30.000 E+00$ & 3 & $3.605 \mathrm{E}-04$ & 3 & ALEACH ( 6) \\
\hline \multirow[t]{2}{*}{$\mathrm{R} 016$} & Solubility constant & $30.000 \mathrm{E}+00$ & $30.000 E+00$ & 3 & not used & 3 & $\operatorname{SOLUBK}(6)$ \\
\hline & 3 & 3 & 3 & 3 & & 3 & \\
\hline R016 & ${ }^{3}$ Distribution coefficients for $\mathrm{Sr}-90$ & 3 & 3 & 3 & & 3 & \\
\hline $\mathrm{R} 016$ & 3 Contaminated zone $(\mathrm{cm} * * 3 / \mathrm{g})$ & $32.500 \mathrm{E}+01$ & $33.000 \mathrm{E}+01$ & 3 & --- & 3. & DCNUCC $(7)$ \\
\hline R016 & Unsaturated zone $1(\mathrm{~cm} * * 3 / \mathrm{g})$ & $32.500 \mathrm{E}+01$ & $33.000 E+01$ & 3 & -- & 3. & DCNUCU $(7,1)$ \\
\hline $\mathrm{R} 016$ & Saturated zone $(\mathrm{cm} * * 3 / \mathrm{g})$ & $32.500 \mathrm{E}+01$ & $33.000 E+01$ & 3 & -- & 3 & DCNUCS $(7)$ \\
\hline R016 & Leach rate $(/ y r)$ & $30.000 \mathrm{E}+00$ & $30.000 E+00$ & 3 & $4.322 E-04$ & 3 & ALEACH ( 7) \\
\hline R016 & Solubility constant & $30.000 \mathrm{E}+00$ & $30.000 \mathrm{E}+00$ & 3 & not used & 3 & SOLUBK $(7)$ \\
\hline
\end{tabular}


$\begin{array}{llccc}\text { 1RESRAD, Version } 6.3 & T \ll \text { Limit }=180 \text { days } & 10 / 10 / 2005 & 09: 35 \text { Page } \\ \text { Summary : } 116-\mathrm{K}-2 & \text { Trench (East End) Cleanup Verification RESRAD Calculation }\end{array}$

File : 116-K-2-SZ-SWC.RAD

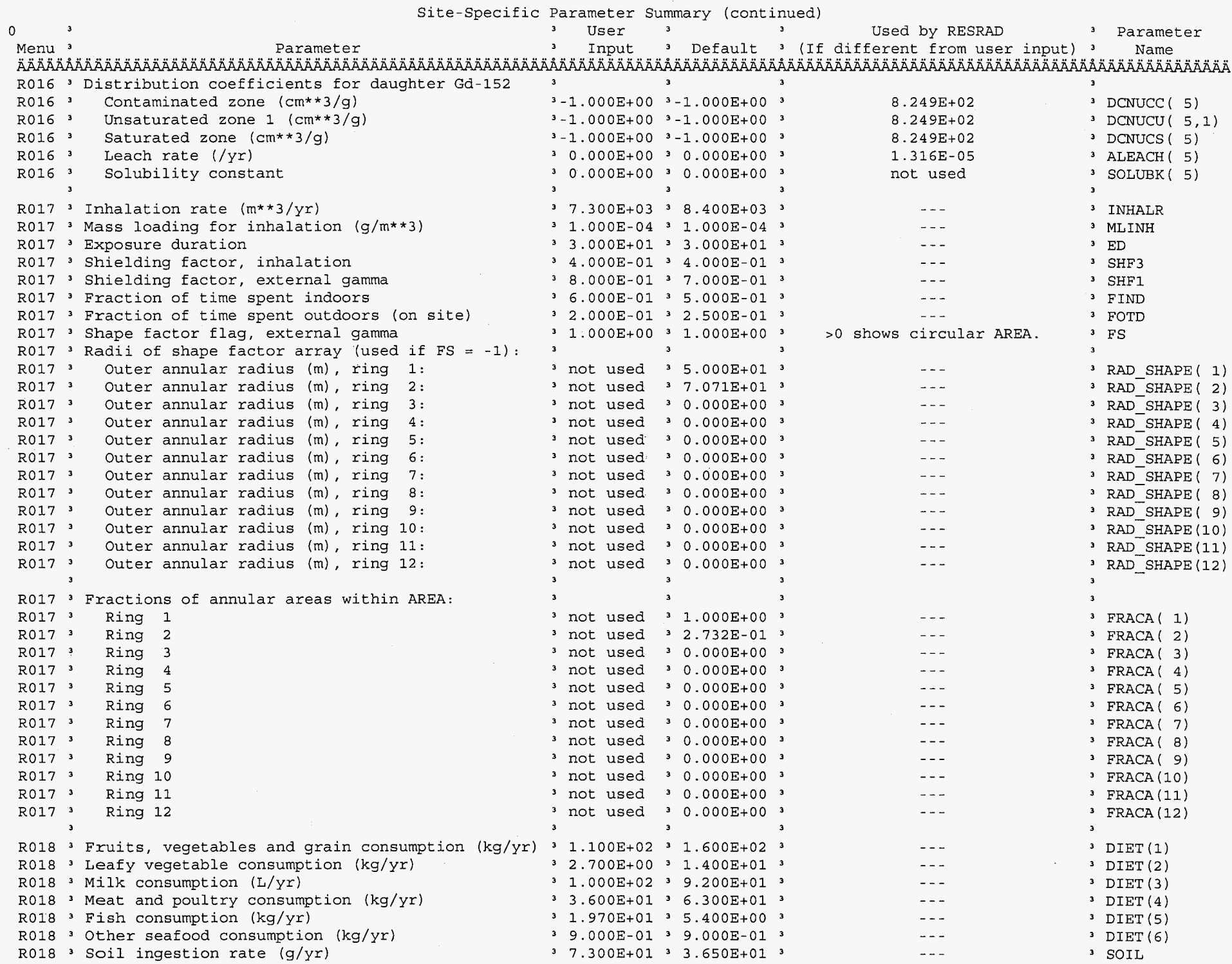


1RESRAD, Version 6.3 T Limit $=180$ days $\quad 10 / 10 / 2005 \quad 09: 35$ Page 7 Summary : 116-K-2 Trench (East End) Cleanup Verification RESRAD Calculation File : 116-K-2-SZ-SWC.RAD

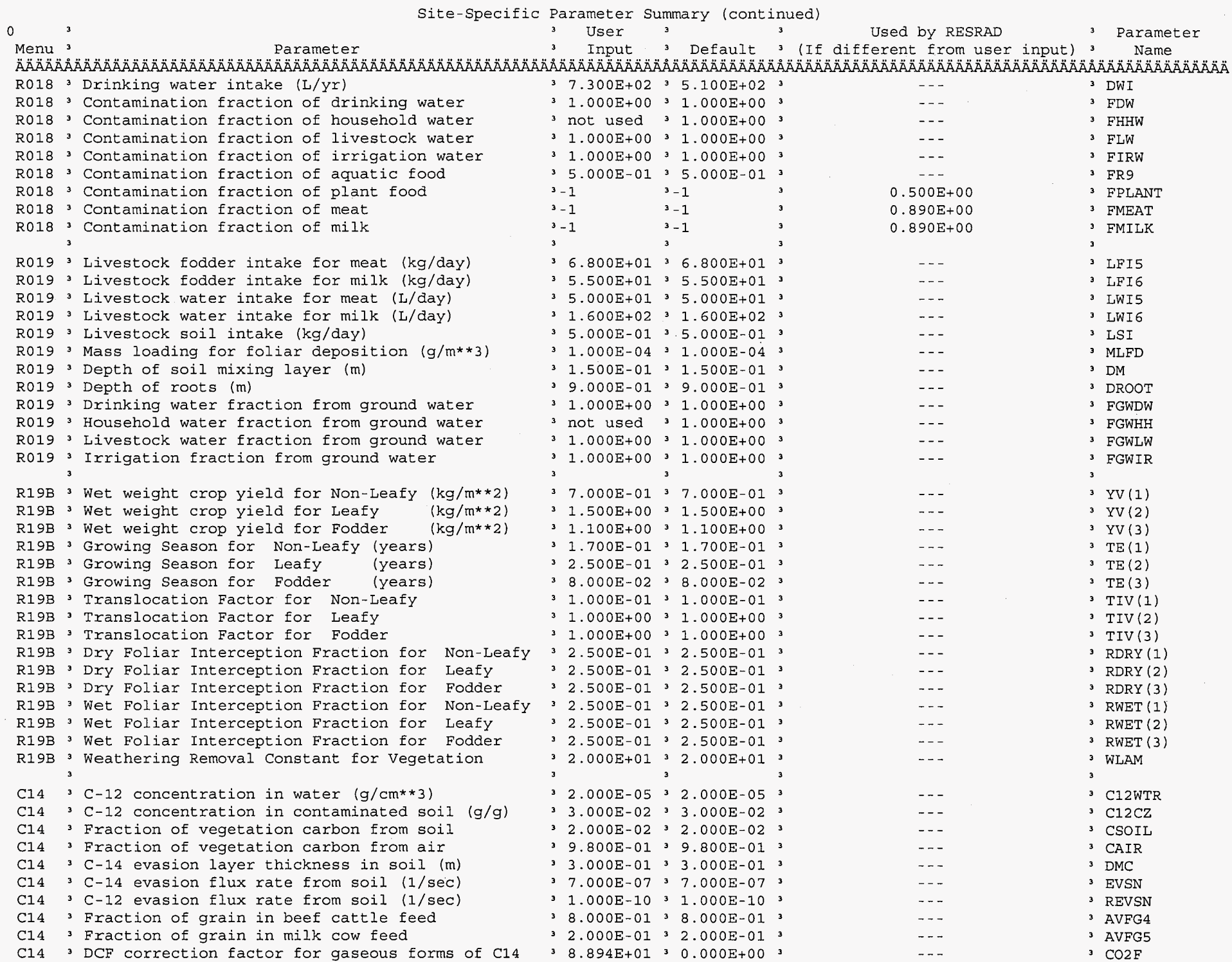


1RESRAD, Version 6.3

$T \ll$ Limit $=180$ days

10/10/2005 09:35 Page

Summary : 116-K-2 Trench (East End) Cleanup Verification RESRAD Calculation

File : 116-K-2-SZ-SWC.RAD

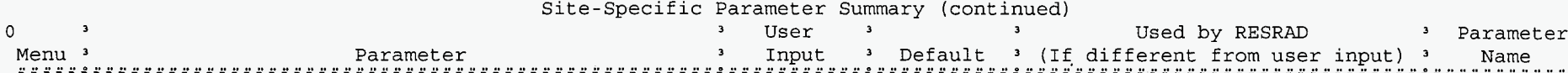

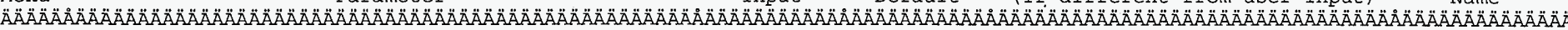

STOR 3 Storage times of contaminated foodstuffs (days) :

STOR ${ }^{3}$ Fruits, non-leafy vegetables, and grain

STOR ${ }^{3}$ Leafy vegetables

STOR ${ }^{3} \quad$ Milk

STOR ${ }^{3}$ Meat and poultry

STOR ${ }^{3}$ Fish

STOR ${ }^{3}$ Crustacea and mollusks

STOR ${ }^{3}$ Well water

$\mathrm{STOR}^{3}$ Surface water

STOR ${ }^{3}$ Livestock fodder

R021 3 Thickness of building foundation (m)

R021 3 Bulk density of building foundation $(\mathrm{g} / \mathrm{cm} * * 3)$

R021 3 Total porosity of the cover material

R021 3 Total porosity of the building foundation

R021 ${ }^{3}$ Volumetric water content of the cover material

R021 3 Volumetric water content of the foundation

R021 3 Diffusion coefficient for radon gas $(\mathrm{m} / \mathrm{sec})$ :

R02 3 in cover material

R021 ${ }^{3}$ in foundation material

R021 3 in contaminated zone soil

R021 3 Radon vertical dimension of mixing (m)

R021 3 Average building air exchange rate ( $1 / \mathrm{hr}$ )

R021 3 Height of the building (room) (m)

R021 3 Building interior area factor

R021 3 Building depth below ground surface (m)

R021 3 Emanating power of Rn-222 gas

R021 3 Emanating power of Rn-220 gas 3

TITL 3 Number of graphical time points

TITL 3 Maximum number of integration points for dose

TITL 3 Maximum number of integration points for risk
$31.400 E+01 \quad 3 \quad 1.400 E+013$

$31.000 \mathrm{E}+0031.000 \mathrm{E}+00^{3}$

$31.000 E+00^{3} 1.000 E+00^{3}$

$32.000 E+0132.000 E+013$

$37.000 E+00$ 3 $7.000 E+00^{3}$

$37.000 E+00 \quad 3 \quad 7.000 E+00^{3}$

$31.000 \mathrm{E}+00^{3} \quad 1.000 \mathrm{E}+00^{3}$

$31.000 E+00^{3} 1.000 E+00^{3}$

$34.500 \mathrm{E}+011^{3} 4.500 \mathrm{E}+01^{3}$

3 not used $3 \quad 1.500 E^{3}-01^{3}$

3 not used $32.400 E+00^{3}$

3 not used $34.000 \mathrm{E}-01^{3}$

3 not used 31.000 E-01,

3 not used 3 5.000E-02 3

3 not used 3.000 E-02

3

3 not used $32.000 \mathrm{E}-063$

3 not used 3 3.000E-07 3

3 not used $32.000 \mathrm{E}^{3} 06^{3}$

3 not used $32.000 \mathrm{E}+00$

3 not used $35.000 \mathrm{E}-013$

${ }^{3}$ not used ${ }^{3} 2.500 \mathrm{E}+00^{3}$

3 not used $30.000 \mathrm{E}+00^{3}$

3 not used ${ }^{3}-1.000 \mathrm{E}+00^{3}$

3 not used $32.500 E^{3} 01^{3}$

3 not used ${ }^{3} 1.500 \mathrm{E}^{3} 01^{3}$

3

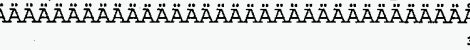

STOR_T (1)

3 STORT 12

... 3 STORT T (3)

3 STOR T (4)

-. 3 STOR_T (5)

-.. $3 \operatorname{STOR}^{-T}(6)$

3 STORTT(7)

-. 3 STOR_T (8)

-. 3 STOR_T (9)

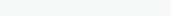

3 FLOORI

3 TPCV

3 TPFI

${ }^{3} \mathrm{PH} 2 \mathrm{OCV}$

3 PH2OFI

3 DIFCV

3 DIFFL

- 3 DIFCZ

-. 3 HMIX

-. 3 REXG

$\begin{array}{lll}-- & & \text { REXG } \\ -- & 3 & \text { HRM }\end{array}$

-.. 3 FAI

-.- 3 DMFI

3 EMANA (1)

3 EMANA (2)

NPTS

LYMAX

3 KYMAX

Summary of Pathway Selections

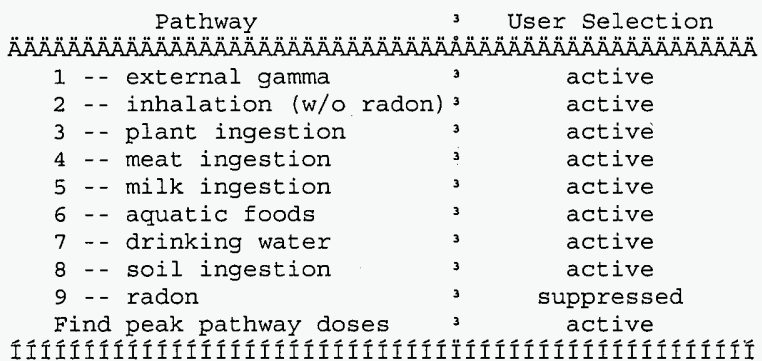




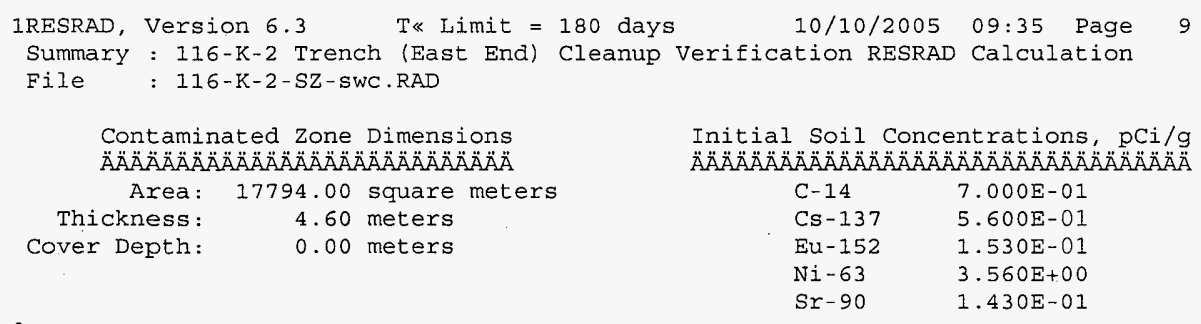

0

Total Dose TDOSE(t), mrem/yr

Basic Radiation Dose Limit $=1.500 \mathrm{E}+01 \mathrm{mrem} / \mathrm{yr}$

Total Mixture Sum $M(t)=$ Fraction of Basic Dose Limit Received at Time ( $t$ )

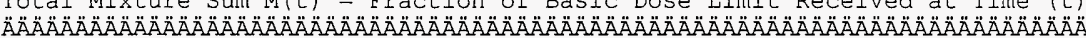

(years): $\quad \begin{array}{lllllllll}0.000 \mathrm{E}+00 & 1.000 \mathrm{E}+00 & 3.000 \mathrm{E}+00 & 1.300 \mathrm{E}+01 & 3.000 \mathrm{E}+01 & 1.000 \mathrm{E}+02 & 3.000 \mathrm{E}+02 & 1.000 \mathrm{E}+03\end{array}$

TDOSE $(t): \begin{array}{lllllllll}4.624 \mathrm{E}+00 & 3.130 \mathrm{E}+00 & 2.489 \mathrm{E}+00 & 1.830 \mathrm{E}+00 & 1.139 \mathrm{E}+00 & 2.008 \mathrm{E}-01 & 3.657 \mathrm{E}-03 & 9.730 \mathrm{E}-06\end{array}$ $\mathrm{M}(\mathrm{t}): \begin{array}{llllllll}3.082 \mathrm{E}-01 & 2.087 \mathrm{E}-01 & 1.659 \mathrm{E}-01 & 1.220 \mathrm{E}-01 & 7.595 \mathrm{E}-02 & 1.339 \mathrm{E}-02 & 2.438 \mathrm{E}-04 & 6.487 \mathrm{E}-07\end{array}$

OMaximum TDOSE $(t): 4.624 \mathrm{E}+00 \mathrm{mrem} / \mathrm{yr}$ at $t=0.000 \mathrm{E}+00$ years 


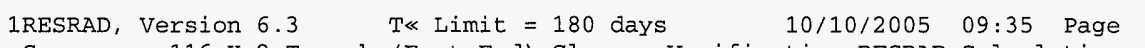

Milk $\ddot{A} \ddot{A} \ddot{A} \ddot{A} \ddot{A} \ddot{A} \ddot{A} \ddot{A} \ddot{A} \ddot{A} \ddot{A} \ddot{A} \ddot{A}$

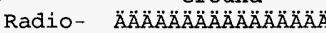
Nuclide mrem/Yx fract. Ӓ̈̈̈̈̈̈̈̈

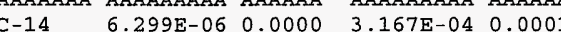
$\begin{array}{llllll}\mathrm{Cs}-137 & 1.225 \mathrm{E}+00 & 0.2648 & 7.655 \mathrm{E}-07 & 0.0000\end{array}$ $\begin{array}{lllll}\text { Eu-152 } & 6.911 E-01 & 0.1495 & 1.449 \mathrm{E}-06 & 0.0000\end{array}$ $\begin{array}{llllll}\mathrm{Ni}-63 & 0.000 \mathrm{E}+00 & 0.0000 & 9.596 \mathrm{E}-07 & 0.0000\end{array}$ $\begin{array}{lllll}\mathrm{Sr}-90 & 2.264 \mathrm{E}-03 & 0.0005 & 8.018 \mathrm{E}-06 & 0.0000\end{array}$ Total $1.918 \mathrm{E}+00 \quad 0.4148 \quad 3.278 \mathrm{E}-04 \quad 0.0001$

\section{AAAAAAAAAAAAÄA} $\mathrm{mrem} / \mathrm{yr}$ fract. $0.000 \mathrm{E}+00 \quad 0.0000$ $0.000 \mathrm{E}+00 \quad 0.0000$ $0.000 \mathrm{E}+00 \quad 0.0000$ $0.000 \mathrm{E}+00 \quad 0.0000$ $0.000 E+00 \quad 0.0000$ Ifítítít tíííí $0.000 \mathrm{E}+00 \quad 0.0000$

\section{MAAAAAAAAAAAAAA}

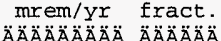
$\begin{array}{ll}1.183 \mathrm{E}+00 & 0.2559\end{array}$ $6.311 \mathrm{E}-02 \quad 0.0137$ $1.397 \mathrm{E}-04 \quad 0.0000$ $5.788 \mathrm{E}-03 \quad 0.0013$ $\begin{array}{lll}3.694 \mathrm{E}-01 & 0.0799\end{array}$ fítifítí ífítí $1.621 \mathrm{E}+00 \quad 0.3507$ $\mathrm{mrem} / \mathrm{yr}$ fract. 4.110E-01 0.0889 $8.664 \mathrm{E}-02 \quad 0.0187$ $4.257 \mathrm{E}-05 \quad 0.0000$ $1.283 \mathrm{E}-03 \quad 0.0003$ $1.170 \mathrm{E}-01 \quad 0.0253$ itítítít fit́tít $6.160 \mathrm{E}-01 \quad 0.1332$

\section{AAAAAAAAAAAAAAA}

$\mathrm{mrem} / \mathrm{yr}$ fract. $3.3295-010.0720$ 5. $381 \mathrm{E}-02 \quad 0.0116$ $2.813 \mathrm{E}-06 \quad 0.0000$ 1. $188 \mathrm{E}-02 \quad 0.0026$ $6.610 \mathrm{E}-02 \quad 0.014$ ííítíííi ííítí

$4.647 \mathrm{E}-01 \quad 0.1005$

Total Dose Contributions TDOSE $(i, p, t)$ for Individual Radionuclides (i) and Pathways (p) As mrem/yr and Fraction of Total Dose At $t=0.000 \mathrm{E}+00$ years Water Dependent Pathways

Fish

\section{Radio- Water} Nuclide mrem/yr fract.

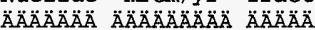
C-14 $0.000 E+00 \quad 0.0000$ Cs-137 $0.000 \mathrm{E}+00 \quad 0.0000$

$\begin{array}{lll}\text { Eu-152 } & 0.000 E+00 \quad 0.0000\end{array}$

$\begin{array}{lll}\mathrm{Ni}-63 & 0.000 \mathrm{E}+00 \quad 0.0000\end{array}$

$\begin{array}{lll}\mathrm{Sr}-90 & 0.000 \mathrm{E}+00 \quad 0.0000\end{array}$

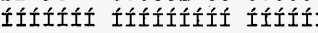

Total $0.000 \mathrm{E}+00 \quad 0.0000$ Radon

АÄAAAAAAÄ̈̈̈̈̈̈̈̈̈̈̈̈

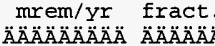

$0.000 \mathrm{E}+00 \quad 0.0000$

$0.000 \mathrm{E}+00 \quad 0.0000$

$0.000 \mathrm{E}+00 \quad 0.0000$

$0.000 E+00 \quad 0.0000$

$0.000 E+00 \quad 0.0000$

tífítitit ifítít

$0.000 \mathrm{E}+000.0000$

\section{A}

Plant

\section{AAAAAAAAAÄÄ̈̈̈̈̈̈}

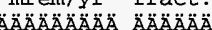

$0.000 \mathrm{E}+00 \quad 0.0000$

$0.000 \mathrm{E}+00 \quad 0.0000$

$0.000 \mathrm{E}+00 \quad 0.0000$

$0.000 \mathrm{E}+00 \quad 0.0000$

$0.000 \mathrm{E}+00 \quad 0.0000$

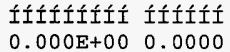

$0.000 E+00 \quad 0.0000$

$0.000 E+00 \quad 0.0000$

$0.000 E+00 \quad 0.0000$

$0.000 \mathrm{E}+00 \quad 0.0000$

$0.000 \mathrm{E}+00 \quad 0.0000$

Íííííííi ííííí

$0.000 \mathrm{E}+00 \quad 0.0000$
Meat

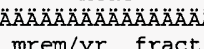

$\mathrm{mrem} / \mathrm{yr}$ fract.

$\begin{array}{lllll}0.000 \mathrm{E}+00 & 0.0000 & 0.000 \mathrm{E}+00 & 0.0000\end{array}$

$\begin{array}{lllll}0.000 \mathrm{E}+00 & 0.0000 & 0.000 \mathrm{E}+00 & 0.0000\end{array}$

$\begin{array}{lllll}0.000 \mathrm{E}+00 & 0.0000 & 0.000 \mathrm{E}+00 & 0.0000\end{array}$

$\begin{array}{llll}0.000 E+00 & 0.0000 & 0.000 E+00 & 0.0000\end{array}$

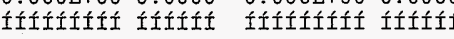

$0.000 \mathrm{E}+00 \quad 0.0000 \quad 0.000 \mathrm{E}+00 \quad 0.0000$
Soi1 $\triangle \ddot{A} \ddot{A} \ddot{A} \ddot{A} \ddot{A} \ddot{A} \ddot{A} \ddot{A} \ddot{A} \ddot{A} \ddot{A} \ddot{A}$ mrem/yr fract. $8.544 \mathrm{E}-05 \quad 0.0000$ $1.635 \mathrm{E}-03 \quad 0.0004$ $5.790 \mathrm{E}-05 \quad 0.0000$ $1.200 \mathrm{E}-04 \quad 0.0000$ $1.276 \mathrm{E}-03 \quad 0.0003$ ífítítít fítítít $3.175 \mathrm{E}-03 \quad 0.0007$ 


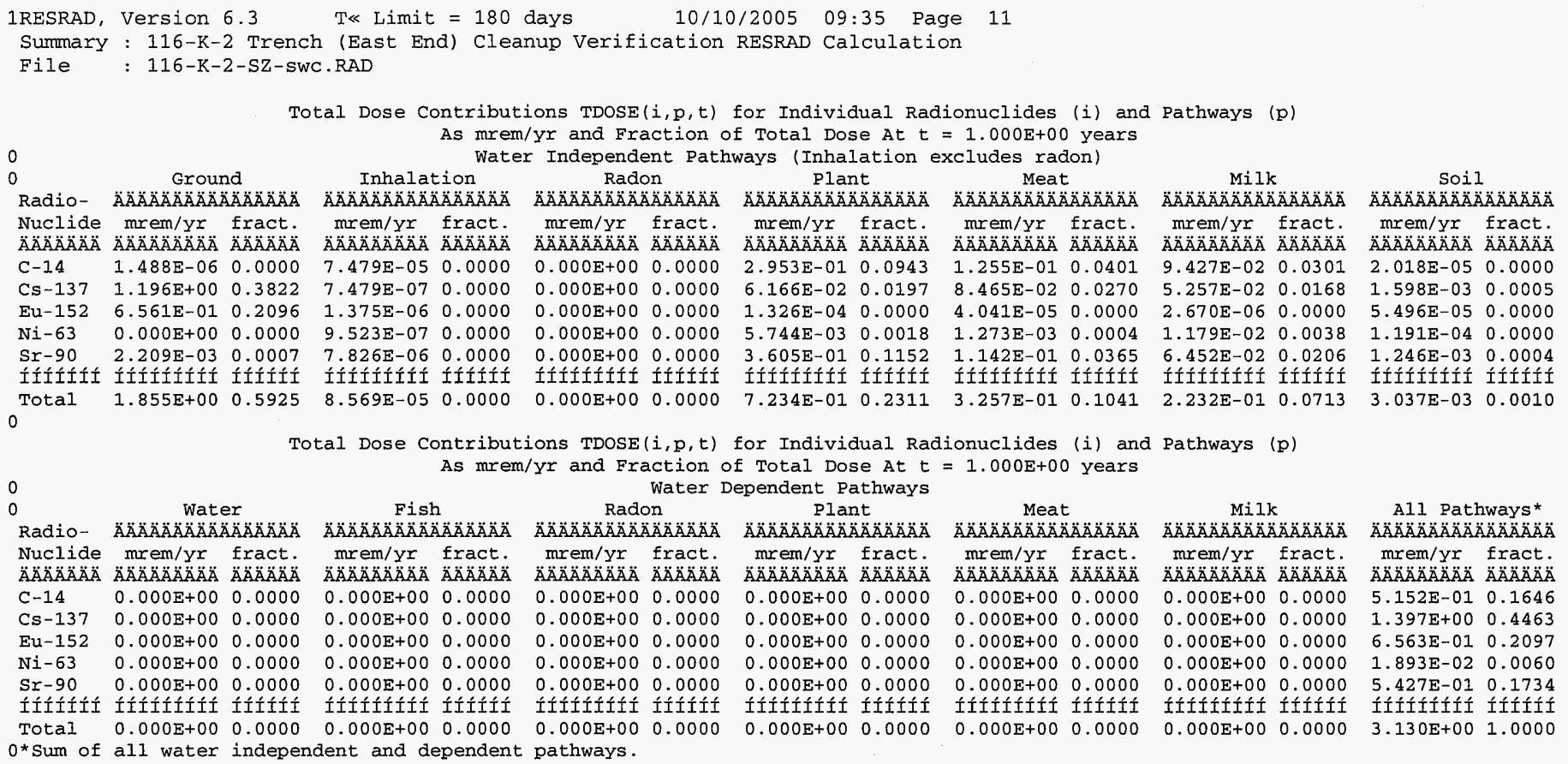


Rev. 0

1RESRAD, Version $6.3 \quad$ T« Limit $=180$ days $10 / 10 / 2005 \quad 09: 35$ Page 12

Summary : 116-K-2 Trench (East End) Cleanup Verification RESRAD Calculation

File : 116-K-2-SZ-SWC.RAD

Total Dose Contributions TDOSE $(i, p, t)$ for Individual Radionuclides ( $i$ ) and Pathways (p)

As mrem/yr and Fraction of Total Dose At $t=3.000 \mathrm{E}+00$ years

0

Dation excludes radon)

Thalation

$$
\text { Radon }
$$

Plant

Meat

Milk

Soil

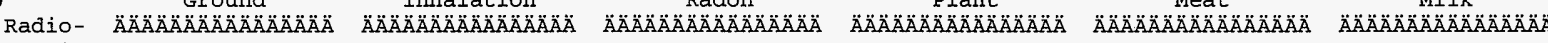
Nuclide $\mathrm{mrem} / \mathrm{yr}$ fract. mrem/yr fract. mrem/yr fract. mrem/yr fract. mrem/yr fract. $\mathrm{mrem} / \mathrm{yr}$ fract.

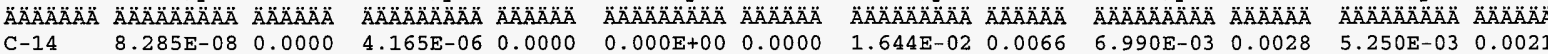

$\begin{array}{lllll}\mathrm{Cs}-137 & 1.142 \mathrm{E}+00 & 0.4587 & 7.138 \mathrm{E}-07 & 0.0000 \\ \mathrm{Eu}-152 & 5.912 \mathrm{E}-01 & 0.2375 & 1.239 \mathrm{E}-06 & 0.0000\end{array}$

$0.000 \mathrm{E}+00 \quad 0.0000$

$5.885 \mathrm{E}-020.0236$

$8.079 \mathrm{E}-02 \quad 0.0325$

$0.000 \mathrm{E}+00 \quad 0.0000$

$1.195 \mathrm{E}-04 \quad 0.0000$

$\mathrm{Ni}-63 \quad 0.000 \mathrm{E}+00 \quad 0.0000 \quad 9.380 \mathrm{E}-07 \quad 0.0000$

$\begin{array}{lll}-03 & 0.0021 \\ 0 & 0 & 0 \\ 0 & 0 & 0\end{array}$

5.658E-03 $0.0023 \quad 1.254 \mathrm{E}-030.00052 .1 .16$

$\begin{array}{llllllll}0.000 \mathrm{E}+00 & 0.0000 & 3.435 \mathrm{E}-01 & 0.1380 & 1.088 \mathrm{E}-01 & 0.0437 & 6.147 \mathrm{E}-02 & 0.0247\end{array}$

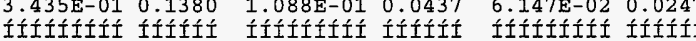

$735 \mathrm{E}+000.6971 \quad 1.451 \mathrm{E}-050.0000$

$0.000 \mathrm{E}+00$ 0.0000

$4.246 \mathrm{E}-01 \quad 0.1706$

$1.9790-01$ 0.0795

$1.285 E-010.0516$

0

Total Dose Contributions TDOSE $(i, p, t)$ for Individual Radionuclides ( $i$ ) and Pathways (p)

As mrem/yr and Fraction of Total Dose At $t=3.000 \mathrm{E}+00$ years

0 Water Dependent Pathways Fish Radon Plant

Meat

$\mathrm{Milk}$

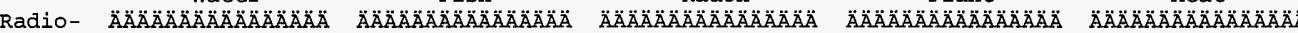
Nuclide mrem/yr fract.

\section{mäm/Yr fract.}

mrem/yr fract.

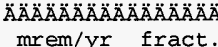

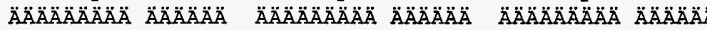

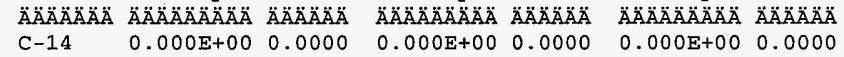

$0.000 \mathrm{E}+00 \quad 0.0000$

$\begin{array}{lllll}0.000 \mathrm{E}+00 & 0.0000 & 0.000 \mathrm{E}+00 & 0.0000\end{array}$

$0.000 \mathrm{E}+00 \quad 0.0000 \quad 0.000 \mathrm{E}+00 \quad 0.0000$

$0.000 \mathrm{E}+000.0000$

$1520.000 \mathrm{E}$

$\mathrm{Ni}-63$

$0.000 \mathrm{E}+00 \quad 0.0000$

$0.000 \mathrm{E}+000.0000$

$0.000 \mathrm{E}+00 \quad 0.0000$

$0.000 \mathrm{E}+00 \quad 0.0000$

$0.000 \mathrm{E}+00 \quad 0.0000$

$\begin{array}{llll}0.000 \mathrm{E}+00 & 0.0000 & 0.000 \mathrm{E}+00 & 0.0000\end{array}$

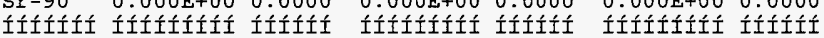

Total $0.000 \mathrm{E}+00 \quad 0.0000 \quad 0.000 \mathrm{E}+00 \quad 0.0000$

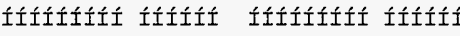

Ítíííítíi ÍÍtítí

0 *Sum of all water independent and dependent pathways.

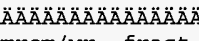
mrem/yr fract. $\ddot{A} \ddot{A} \ddot{A} \ddot{A} \ddot{A} \ddot{A} \ddot{A} \ddot{A} \ddot{A}$ Ä $\ddot{A} \ddot{A} \ddot{A} \ddot{A}$ $0.000 E+00 \quad 0.0000$ $0.000 \mathrm{E}+00 \quad 0.0000$

$0.000 \mathrm{E}+00 \quad 0.0000$

$0.000 E+00 \quad 0.0000$

$0.000 \mathrm{E}+00 \quad 0.0000$

Íííííít ifíít

$0.000 \mathrm{E}+00 \quad 0.0000$

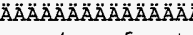

$\mathrm{mrem} / \mathrm{yr}$ fract.

$1.124 \mathrm{E}-06 \quad 0.0000$

$1.525 \mathrm{E}-03 \quad 0.0006$

$4.953 \mathrm{E}-05 \quad 0.0000$

$1.173 \mathrm{E}-04 \quad 0.0000$

$1.187 \mathrm{E}-03 \quad 0.0005$

íífííííi íííít

2.879E-03 0.0012
All Pathways* A mrem/yr fract. $\ddot{A} \ddot{A} \ddot{A} \ddot{A} \ddot{A} \ddot{A} \ddot{A} \ddot{A} \ddot{A}$ 苂获苂苂 2.869E-02 0.0115 $1.333 \mathrm{E}+00 \quad 0.5356$ $5.914 \mathrm{E}-01 \quad 0.2376$ $1.864 \mathrm{E}-02 \quad 0.0075$ $\begin{array}{lll}5.171 E-01 & 0.2077\end{array}$ iffífítít ífííí $2.489 \mathrm{E}+001.0000$ 
CVP-2006-00001

Rev. 0

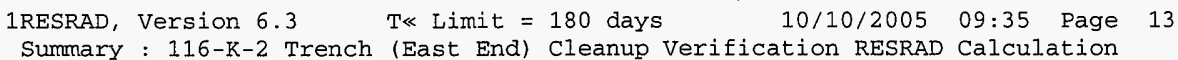

File : 116-K-2-SZ-SWC.RAD

Total Dose Contributions TDOSE $(i, p, t)$ for Individual Radionuclides ( $i$ ) and Pathways (p)

As mrem/yr and Fraction of Total Dose At $t=1.300 \mathrm{E}+01$ years

0 water Independent Pathways (Inhalation excludes radon) Ground Inhalation

$$
\text { Radon Plant Meat }
$$

Meat Milk

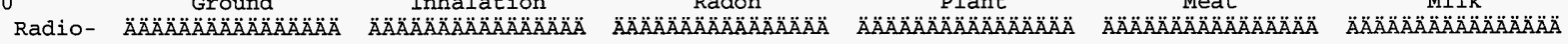
Nuclide mrem/yr fract. mrem/yr fract. mrem/yr fract. mrem/yr fract. mrem/yr fract. mrem/yr fract.

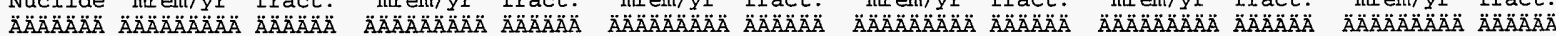
$\begin{array}{llllllllllllll}\mathrm{C}-14 & 4.271 \mathrm{E}-14 & 0.0000 & 2.147 \mathrm{E}-12 & 0.0000 & 0.000 \mathrm{E}+00 & 0.0000 & 8.479 \mathrm{E}-09 & 0.0000 & 3.607 \mathrm{E}-09 & 0.0000 & 2.708 \mathrm{E}-09 & 0.0000\end{array}$ Cs-137 9.043E-01 $0.4941 \quad 5.653 E-07 \quad 0.0000$

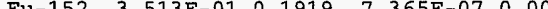
Eu-152 3.51 $\begin{array}{lllll}\mathrm{Ni}-63 & 0.000 \mathrm{E}+00 & 0.0000 & 8.695 \mathrm{E}-07 & 0.0000\end{array}$

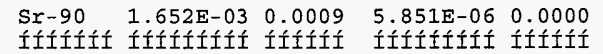
$\begin{array}{llllllll}0.000 \mathrm{E}+00 & 0.0000 & 4.661 \mathrm{E}-02 & 0.0255 & 6.398 \mathrm{E}-02 & 0.0350 & 3.974 \mathrm{E}-02 & 0.0217\end{array}$ $\begin{array}{llllllll}0.000 \mathrm{E}+00 & 0.0000 & 7.102 \mathrm{E}-05 & 0.0000 & 2.164 \mathrm{E}-05 & 0.0000 & 1.430 \mathrm{E}-06 & 0.0000\end{array}$ $\begin{array}{llllllll}0.000 \mathrm{E}+00 & 0.0000 & 5.244 \mathrm{E}-03 & 0.0029 & 1.163 \mathrm{E}-03 & 0.0006 & 1.077 \mathrm{E}-02 & 0.0059\end{array}$ $\begin{array}{llllllll}0.000 \mathrm{E}+00 & 0.0000 & 2.696 \mathrm{E}-01 & 0.1473 & 8.540 \mathrm{E}-02 & 0.0467 & 4.824 \mathrm{E}-02 & 0.0264\end{array}$ Total $1.257 \mathrm{E}+00 \quad 0.6869$

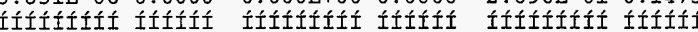

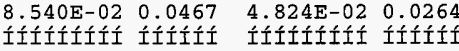

Total Dose Contributions TDOSE $(i, p, t)$ for Individual Radionuclides (i) and Pathways ( $p$ ) As mrem/yr and Fraction of Total Dose At $t=1.300 \mathrm{E}+01$ years

0 Water Dependent Pathways Water Fish Radon Plant

Meat

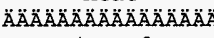
mrem/yr fract.

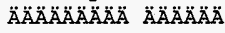
$0.000 \mathrm{E}+00 \quad 0.0000$ $0.000 E+00 \quad 0.0000$ $0.000 \mathrm{E}+00 \quad 0.0000$ $0.000 \mathrm{E}+00 \quad 0.0000$ $0.000 E+00 \quad 0.0000$ íítítííi tíííí $0.000 \mathrm{E}+000.0000$

\section{mrem/yr fract.} $\ddot{A} \ddot{A} \ddot{A} \ddot{A} \ddot{A} \ddot{A} \ddot{A} \ddot{A} \ddot{A}$ Ä $\ddot{A} \ddot{A} \ddot{A} \ddot{A}$ $0.000 \mathrm{E}+0$ $0.000 \mathrm{E}+00$ $0.000 \mathrm{E}+00$ $0.000 E+000.0000$

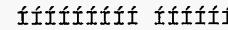
$0.000 \mathrm{E}+00 \quad 0.0000$
$0.000 \mathrm{E}+00 \quad 0.0$ íííítííi ííííi $0.000 \mathrm{E}+00 \quad 0.0000$

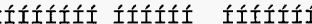
$0 *$ Sum of all water independent and dependent pathways.

\begin{tabular}{|c|c|c|c|}
\hline \multirow{2}{*}{\multicolumn{2}{|c|}{$\frac{M i l k}{M i}$}} & \multirow{2}{*}{\multicolumn{2}{|c|}{$\begin{array}{l}\text { All Pathways* } \\
\ddot{A} \ddot{A} \ddot{A} \ddot{A} \ddot{A} \ddot{A} \ddot{A} \ddot{A} \ddot{A} \ddot{A} \ddot{A} \ddot{A} \ddot{A} \ddot{A}\end{array}$}} \\
\hline & & & \\
\hline 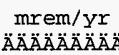 & $\begin{array}{l}\text { fract. } \\
\ddot{A} \ddot{A} \ddot{A} \ddot{A} \ddot{A} \ddot{A}\end{array}$ & $\begin{array}{c}m r e m / y r \\
\ddot{A} \ddot{A} \ddot{A} \ddot{A} \ddot{A} \ddot{A} \ddot{A} \ddot{A} \ddot{A}\end{array}$ & $\begin{array}{l}\text { fract. } \\
\ddot{A} \ddot{A} \ddot{A} \ddot{A} \ddot{A} \ddot{A}\end{array}$ \\
\hline $0.000 \mathrm{E}+00$ & 0.0000 & $1.480 \mathrm{E}-08$ & 0.0000 \\
\hline $0.000 \mathrm{E}+00$ & 0.0000 & $1.056 \mathrm{E}+00$ & 0.5769 \\
\hline $0.000 \mathrm{E}+00$ & 0.0000 & $3.514 \mathrm{E}-01$ & 0.1920 \\
\hline $0.000 \mathrm{E}+00$ & 0.0000 & $1.728 \mathrm{E}-02$ & 0.0094 \\
\hline $\begin{array}{l}0.000 \mathrm{E}+00 \\
\text { ÍÍÍtít }\end{array}$ & $\begin{array}{l}0.0000 \\
\text { Iftítít }\end{array}$ & $\begin{array}{l}\text { 4.058E-01 } \\
\text { ÍÍÍtítíí }\end{array}$ & $\begin{array}{l}0.2217 \\
\text { Ítííí }\end{array}$ \\
\hline $0.000 \mathrm{E}+00$ & 0.0000 & $1.830 \mathrm{E}+00$ & 1.0000 \\
\hline
\end{tabular}


CVP-2006-00001

Rev. 0

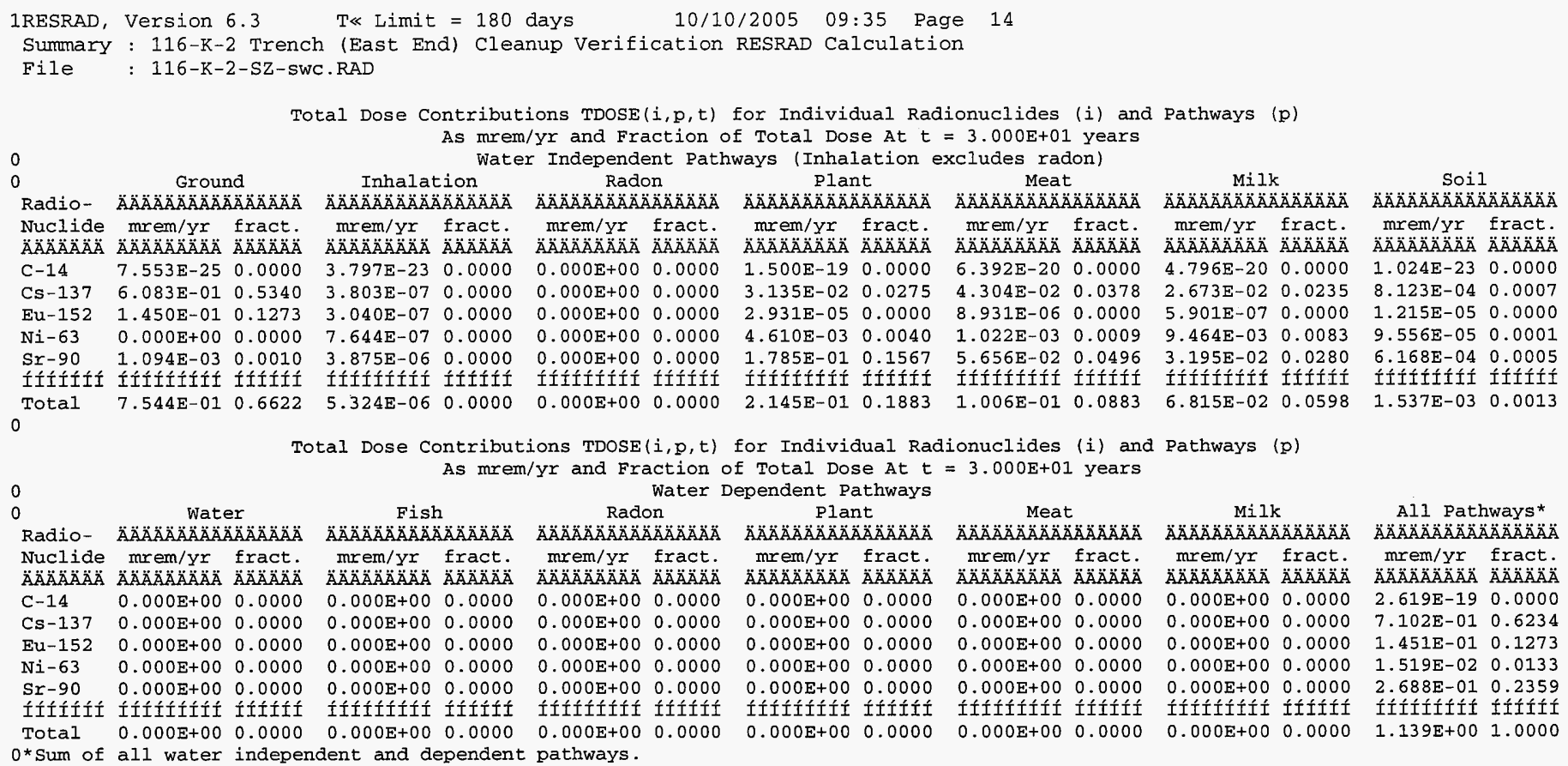




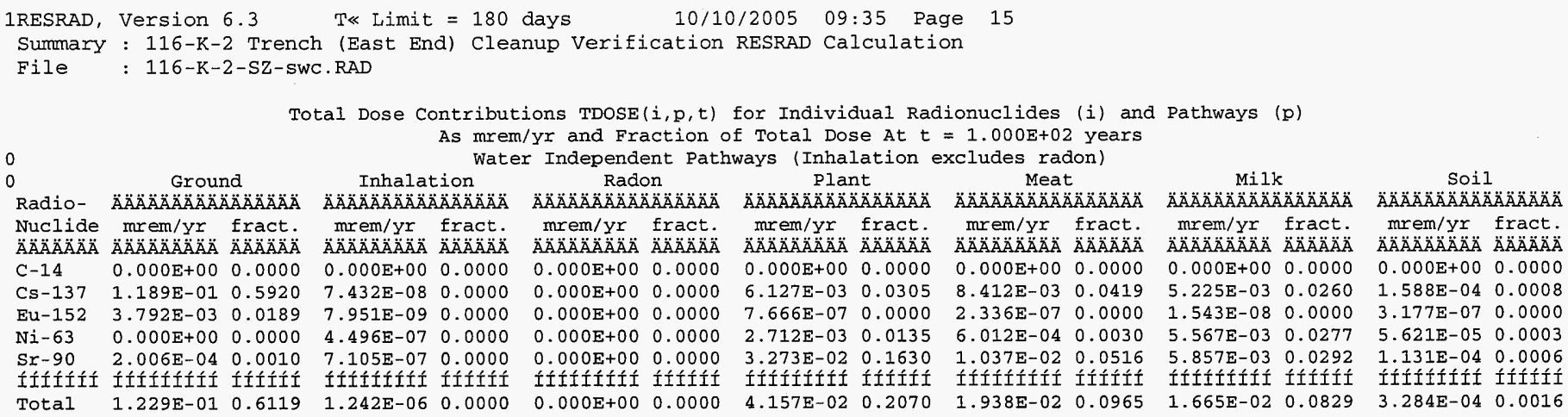

Total Dose Contributions TDOSE $(i, p, t)$ for Individual Radionuclides (i) and Pathways (p) As mrem/yr and Fraction of Total Dose At $t=1.000 \mathrm{E}+02$ years Water Dependent Pathways

0 Fish Radon

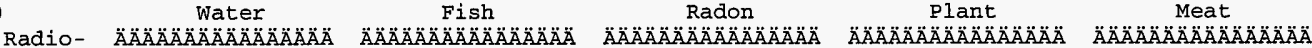
Nuclide mrem/yr fract. mrem/yr fract. mrem/yr fract. mrem/yr fract. mrem/yr fract.

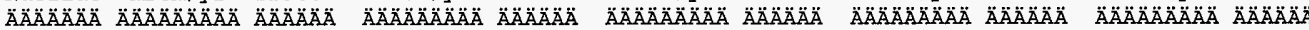
$\begin{array}{llllllllllll}C-14 & 0.000 \mathrm{E}+00 & 0.0000 & 0.000 \mathrm{E}+00 & 0.0000 & 0.000 \mathrm{E}+00 & 0.0000 & 0.000 \mathrm{E}+00 & 0.0000 & 0.000 \mathrm{E}+00 & 0.0000\end{array}$ $\begin{array}{llllllllllll}\mathrm{Cs}-137 & 0.000 \mathrm{E}+00 & 0.0000 & 0.000 \mathrm{E}+00 & 0.0000 & 0.000 \mathrm{E}+00 & 0.0000 & 0.000 \mathrm{E}+00 & 0.0000 & 0.000 \mathrm{E}+00 & 0.0000\end{array}$ $\begin{array}{lllllllllllll}\mathrm{Eu}-152 & 0.000 \mathrm{E}+00 & 0.0000 & 0.000 \mathrm{E}+00 & 0.0000 & 0.000 \mathrm{E}+00 & 0.0000 & 0.000 \mathrm{E}+00 & 0.0000 & 0.000 \mathrm{E}+00 & 0.0000\end{array}$ $\begin{array}{llllllllllll}\mathrm{Ni}-63 & 0.000 \mathrm{E}+00 & 0.0000 & 0.000 \mathrm{E}+00 & 0.0000 & 0.000 \mathrm{E}+00 & 0.0000 & 0.000 \mathrm{E}+00 & 0.0000 & 0.000 \mathrm{E}+00 & 0.0000\end{array}$ $\begin{array}{llllllllllll}S r-90 & 0.000 E+00 & 0.0000 & 0.000 \mathrm{E}+00 & 0.0000 & 0.000 \mathrm{E}+00 & 0.0000 & 0.000 \mathrm{E}+00 & 0.0000 & 0.000 \mathrm{E}+00 & 0.0000\end{array}$

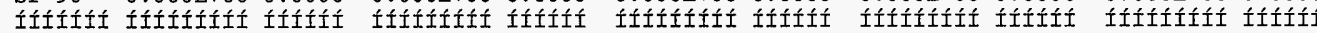
$\begin{array}{lllllllllllll}\text { Total } & 0.000 \mathrm{E}+00 & 0.0000 & 0.000 \mathrm{E}+00 & 0.0000 & 0.000 \mathrm{E}+00 & 0.0000 & 0.000 \mathrm{E}+00 & 0.0000 & 0.000 \mathrm{E}+00 & 0.0000\end{array}$ 0 * Sum of all water independent and dependent pathways. 
Rev. 0

$\begin{array}{rllll}\text { 1RESRAD, Version } 6.3 & \text { T } \ll \text { Limit }=180 \text { days } & 10 / 10 / 2005 & 09: 35 \text { Page } & 16 \\ \text { Summary : 116-K-2 Trench } & \text { (East End) Cleanup Verification RESRAD Calculation }\end{array}$

File : 116-K-2-SZ-SWC.RAD

Total Dose Contributions TDOSE $(i, p, t)$ for Individual Radionuclides (i) and Pathways (p)

As mrem/yr and Fraction of Total Dose At $t=3.000 \mathrm{E}+02$ years Water Independent Pathways (Inhalation excludes radon)

Inhalation

$$
\text { Radon Plant }
$$

Meat

Milk

Soil

Radio- $\ddot{A} \ddot{A} \ddot{A} \ddot{A} \ddot{A} \ddot{A} \ddot{A} \ddot{A} \ddot{A} \ddot{A} \ddot{A} \ddot{A} \ddot{A} \ddot{A}$

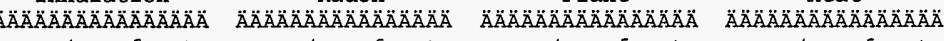
$\mathrm{mrem} / \mathrm{yr}$ fract. Nuclide mrem/yr fract.

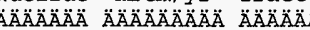
$\begin{array}{llllll}C-14 & 0.000 E+00 & 0.0000 & 0.000 E+00 & 0.0000\end{array}$ Cs-137 $1.121 \mathrm{E}-03 \quad 0.3064 \quad 7.005 \mathrm{E}-10 \quad 0.0000$ mrem/yr fract. mrem/Yx fract. mrem/yr fract. $\begin{array}{llllll}\text { AAAAAAAAA AAAAAA } & \text { AAAAAAAAA AAAAAA } & \text { AAAAAAAAA AAAAAA } \\ 0.000 \mathrm{E}+00 & 0.0000 & 0.000 \mathrm{E}+00 & 0.0000 & 0.000 \mathrm{E}+00 & 0.0000\end{array}$ $\begin{array}{lll}\mathrm{Eu}-152 & 1.142 \mathrm{E}-07 & 0.0000\end{array}$ $0.000 \mathrm{P}+000.0000$ $\begin{array}{lllll}\mathrm{Ni}-63 & 0.000 \mathrm{E}+00 & 0.0000 & 9.872 \mathrm{E}-08 & 0.0000\end{array}$ $\begin{array}{llllllll}\mathrm{Sr}-90 & 1.575 \mathrm{E}-06 & 0.0004 & 5.578 \mathrm{E}-09 & 0.0000 & 0.000 \mathrm{E}+00 & 0.0000\end{array}$

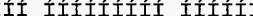
íf́títít fítít $2.308 \mathrm{E}-110.0000$ $7.033 \mathrm{E}-12 \quad 0.0000$ $\begin{array}{llll}5.954 \mathrm{E}-04 & 0.1628 & 1.320 \mathrm{E}-04 & 0.0361 \\ 2.570 \mathrm{E}-04 & 0.0703 & 8.141 \mathrm{E}-05 & 0.0223\end{array}$

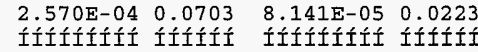
tífífítíf tífíti Total $1.122 \mathrm{E}-03 \quad 0.3069$

Total Dose Contributions TDOSE $(i, p, t)$ for Individual Radionuclides (i) and Pathways (p) As mrem/yr and Fraction of Total Dose At $t=3.000 \mathrm{E}+02$ years

0 Water Dependent Pathways Fish Radon P1ant. Meat Milk

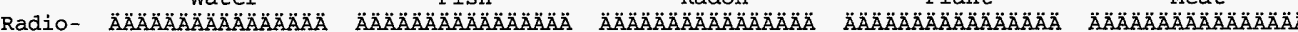
Nuclide mrem/yr fract. mrem/yr fract. mrem/yr fract. mrem/yr fract. mrem/yr fract.

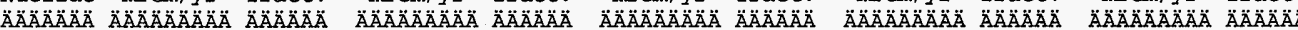
$\begin{array}{lllllllllll}C-14 & 0.000 \mathrm{E}+00 & 0.0000 & 0.000 \mathrm{E}+00 & 0.0000 & 0.000 \mathrm{E}+00 & 0.0000 & 0.000 \mathrm{E}+00 & 0.0000 & 0.000 \mathrm{E}+00 & 0.0000\end{array}$

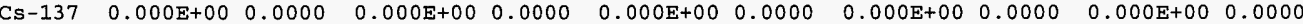
$\begin{array}{lllllll}\mathrm{Eu}-152 & 0.000 \mathrm{E}+00 & 0.0000 & 0.000 \mathrm{E}+00 & 0.0000 & 0.000 \mathrm{E}+00 & 0.0000\end{array}$ $0.000 \mathrm{E}+00 \quad 0.0000 \quad 0.000 \mathrm{E}+00 \quad 0.0000$ $0.000 \mathrm{E}+00 \quad 0.0000 \quad 0.000 \mathrm{E}+00 \quad 0.0000$ $\begin{array}{lllllllllll}\mathrm{Sr}-90 & 0.000 \mathrm{E}+00 & 0.0000 & 0.000 \mathrm{E}+00 & 0.0000 & 0.000 \mathrm{E}+00 & 0.0000 & 0.000 \mathrm{E}+00 & 0.0000 & 0.000 \mathrm{E}+00 & 0.0000\end{array}$

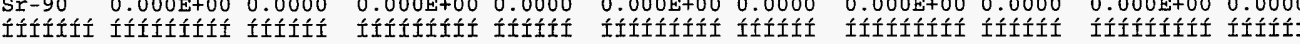
Total $0.000 \mathrm{E}+00 \quad 0.0000 \quad 0.000 \mathrm{E}+00 \quad 0.0000 \quad 0.000 \mathrm{E}+00 \quad 0.0000$ 0 *Sum of all water independent and dependent pathways.

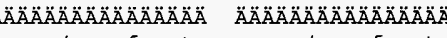
$\mathrm{mrem} / \mathrm{Yr}$ fract. $0.000 \mathrm{E}+00 \quad 0.0000$ $4.925 \mathrm{E}-05 \quad 0.0135$ $\begin{array}{lll}647 \mathrm{E}-13 & 0.0000\end{array}$ $\begin{array}{ll}1.222 \mathrm{E}-03 & 0.3342\end{array}$ $4.599 \mathrm{E}-05 \quad 0.012$ tífífítít fitít

$1.317 \mathrm{E}-03 \quad 0.3602$ mrem/yr fract. $0.000 \mathrm{E}+00 \quad 0.0000$ $1.496 \mathrm{E}-06 \quad 0.0004$ $9.566 \mathrm{E}-12 \quad 0.0000$ $1.234 \mathrm{E}-05 \quad 0.0034$ $\begin{array}{lll}8.878 \mathrm{E}-07 \quad 0.0002 & 0.003\end{array}$ fítítííí fíííí $1.473 \mathrm{E}-05 \quad 0.0040$ 
Total Dose Contributions $\operatorname{TDOSE}(i, p, t)$ for Individual Radionuclides ( $i$ ) and Pathways ( $p$ ) As mrem/yx and Fraction of Total Dose At $t=1.000 \mathrm{E}+03$ years

0 Radio- Ground Nuclide mrem/yr fract.

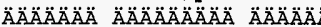
$\begin{array}{lll}C-14 & 0.000 \mathrm{E}+00 & 0.0000\end{array}$ Cs-137 $9.111 \mathrm{E}-11 \quad 0.0000$ Eu-152 $1.710 \mathrm{E}-23 \quad 0.0000$ $\mathrm{Ni}-63 \quad 0.000 \mathrm{E}+00 \quad 0.0000$ Sr-90 6.753E-14 0.0000 fítífít ífífítít títít Total $9.118 \mathrm{E}-110.0000$ Radio- $\ddot{A} \ddot{A} \ddot{A} \ddot{A} \ddot{A} \ddot{A} \ddot{A} \ddot{A} \ddot{A} \ddot{A} \ddot{A} \ddot{A} \ddot{A} \ddot{A}$ Nuclide mrem/yr fract. Ä $\begin{array}{lll}C-14 & 0.000 E+00 & 0.0000\end{array}$ Cs-137 $0.000 \mathrm{E}+00 \quad 0.0000$ $\mathrm{Eu}-152 \quad 0.000 \mathrm{E}+00 \quad 0.0000$ $\begin{array}{lllllll}\mathrm{Ni}-63 & 0.000 \mathrm{E}+00 & 0.0000 & 0.000 \mathrm{E}+00 & 0.0000 & 0.000 \mathrm{E}+00 & 0.0000\end{array}$

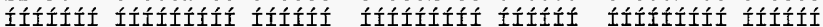
$\begin{array}{lllllll}\text { Total } & 0.000 \mathrm{E}+00 & 0.0000 & 0.000 \mathrm{E}+00 & 0.0000 & 0.000 \mathrm{E}+00 & 0.0000\end{array}$ $0 *$ Sum of all water independent and dependent pathways. Inhalation mrem/yr fract. $0.000 \mathrm{E}+00 \quad 0.0000$ $5.696 \mathrm{E}-17 \quad 0.0000$ $4.896 \mathrm{E}-100.0001$ $2.392 \mathrm{E}-16 \quad 0.0000$ Ííítííi íítítí

$0.000 \mathrm{E}+000.0000$ Radon
Total Dose Contributions TDOSE $(i, p, t)$ for Individual Radionuclides (i) and Pathways (p) As mrem/yr and Fraction of Total Dose At $t=1.000 \mathrm{E}+03$ years Plant

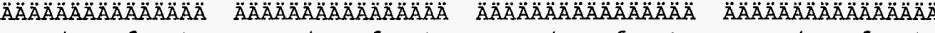

\section{mrem/yr fract.}

$0.000 \mathrm{E}+00 \quad 0.0000$

$0.000 \mathrm{E}+00 \quad 0.0000$

tífítífít íííít

$0.000 \mathrm{E}+00 \quad 0.0000$ $4.696 \mathrm{E}-12 \quad 0.0000$

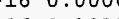
 $1.102 \mathrm{E}-110.0000$ Ífítítíi ífítít $2.953 \mathrm{E}-06 \quad 0.3035$

\section{mrem/yr fract.} $\ddot{A} \ddot{A} \ddot{A} \ddot{A} \ddot{A} \ddot{A} \ddot{A} \ddot{A} \ddot{A}$ Ä $\ddot{A} \ddot{A} \ddot{A} \ddot{A} \ddot{A}$ $0.000 \mathrm{E}+00 \quad 0.0000$ $6.446 \mathrm{E}-12 \quad 0.0000$ E-17 0.0000 $6.546 \mathrm{E}-07 \quad 0.0673$ $3.492 \mathrm{E}-12 \quad 0.0000$ Íítifítí íítít $6.546 \mathrm{E}-070.0673$ Milk Soil

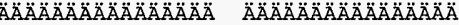
mrem/yr fract. ÄÄÄÄÄÄÄ̈ $0.000 \mathrm{E}+00 \quad 0.0000$ $4.004 \mathrm{E}-12 \quad 0.0000$ $\begin{array}{ll}.499 \mathrm{E}-19 & 0.0000\end{array}$ $6.061 \mathrm{E}-06 \quad 0.6229$ $1.972 \mathrm{E}-12 \quad 0.0000$ ííítííti ííítí $6.061 E-06 \quad 0.6229$ Fish Plant

Meat mrem/yr fract. mrem/yr fract. mrem/yr fract. $\ddot{A} \ddot{A} \ddot{A} \ddot{A} \ddot{A} \ddot{A} \ddot{A} \ddot{A} \ddot{A} \ddot{A} \ddot{A}_{A} \ddot{A} \ddot{A}$ $0.000 \mathrm{E}+00 \quad 0.0000$ $0.000 \mathrm{E}+00 \quad 0.0000$ $0.000 \mathrm{E}+00 \quad 0.0000$

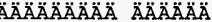
$0.000 \mathrm{E}+00 \quad 0.0000$ $0.000 \mathrm{E}+00 \quad 0.0000$ $0.000 \mathrm{E}+00 \quad 0.0000$ $0.000 E+00 \quad 0.00000 .000 E+00 \quad 0.0000$ $\begin{array}{lllll}0.000 \mathrm{E}+00 & 0.0000 & 0.000 \mathrm{E}+00 & 0.0000\end{array}$ $\begin{array}{lllll}0.000 \mathrm{E}+00 & 0.0000 & 0.000 \mathrm{E}+00 & 0.0000\end{array}$ $\begin{array}{lllll}0.000 E+00 & 0.0000 & 0.000 E+00 & 0.0000\end{array}$ $0.000 \mathrm{E}+00 \quad 0.0000 \quad 0.000 \mathrm{E}+00 \quad 0.0000$

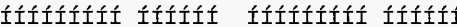
$0.000 \mathrm{E}+000.00000 .000 \mathrm{E}+000.0000$
Milk

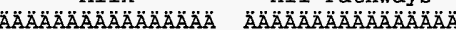
mrem/yr fract mrem/yr fract.

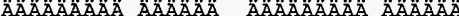
$0.000 \mathrm{E}+00 \quad 0.0000 \quad 0.000 \mathrm{E}+00 \quad 0.0000$ $\begin{array}{llll}0.000 \mathrm{E}+00 & 0.0000 & 1.064 \mathrm{E}-10 & 0.0000\end{array}$

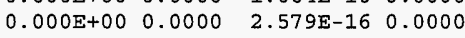
$0.000 \mathrm{E}+00 \quad 0.0000 \quad 9.730 \mathrm{E}-06 \quad 1.0000$ $0.000 \mathrm{E}+00 \quad 0.0000 \quad 1.659 \mathrm{E}-11 \quad 0.0000$

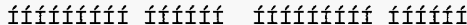
$0.000 \mathrm{E}+00 \quad 0.0000 \quad 9.730 \mathrm{E}-06 \quad 1.0000$ 
Dose/Source Ratios Summed Over All Pathways

Parent Product Thread $\operatorname{DSR}(j, t)$ At Time in Years (mrem/yr)/(pCi/g)

(i) (j) Fraction $0.000 \mathrm{E}+00 \quad 1.000 \mathrm{E}+00 \quad 3.000 \mathrm{E}+00 \quad 1.300 \mathrm{E}+01 \quad 3.000 \mathrm{E}+01 \quad 1.000 \mathrm{E}+023.000 \mathrm{E}+02 \quad 1.000 \mathrm{E}+03$

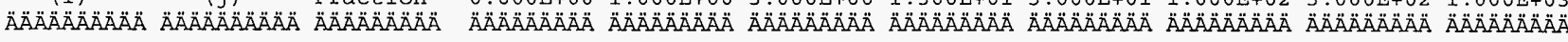

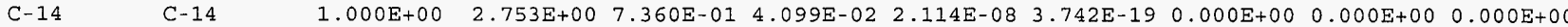
$0 \mathrm{Cs}-137+\mathrm{D} \quad \mathrm{Cs}-137+\mathrm{D} \quad 1.000 \mathrm{E}+00 \quad 2.553 \mathrm{E}+00 \quad 2.494 \mathrm{E}+00 \quad 2.381 \mathrm{E}+00 \quad 1.885 \mathrm{E}+00 \quad 1.268 \mathrm{E}+00 \quad 2.479 \mathrm{E}-01 \quad 2.336 \mathrm{E}-03 \quad 1.900 \mathrm{E}-10$ $\begin{array}{lllllllllll}0 \mathrm{Eu}-152 & \mathrm{EU}-152 & 7.208 \mathrm{E}-01 & 3.257 \mathrm{E}+00 & 3.092 \mathrm{E}+00 & 2.786 \mathrm{E}+00 & 1.656 \mathrm{E}+00 & 6.834 \mathrm{E}-01 & 1.787 \mathrm{E}-02 & 5.382 \mathrm{E}-07 & 8.061 \mathrm{E}-23\end{array}$

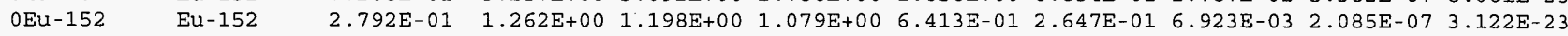
$\begin{array}{lllllllllll}E & \text { Gd-152 } & 2.792 \mathrm{E}-01 & 0.000 \mathrm{E}+00 & 8.660 \mathrm{E}-17 & 2.469 \mathrm{E}-16 & 8.395 \mathrm{E}-16 & 1.349 \mathrm{E}-15 & 1.696 \mathrm{E}-15 & 1.701 \mathrm{E}-15 & 1.686 \mathrm{E}-15\end{array}$ Eu-152 äDSR (j) $0 \mathrm{Ni}-63$ $0 \mathrm{Sr}-90+\mathrm{D} \quad \mathrm{S} r-90+\mathrm{D}$

$1.262 \mathrm{E}+00 \quad 1.198 \mathrm{E}+00 \quad 1.079 \mathrm{E}+00 \quad 6.413 \mathrm{E}-01 \quad 2.647 \mathrm{E}-01 \quad 6.923 \mathrm{E}-03 \quad 2.085 \mathrm{E}-07 \quad 1.686 \mathrm{E}-15$ $\begin{array}{lllllllll}1.000 \mathrm{E}+00 & 5.357 \mathrm{E}-03 & 5.317 \mathrm{E}-03 & 5.237 \mathrm{E}-03 & 4.855 \mathrm{E}-03 & 4.268 \mathrm{E}-03 & 2.510 \mathrm{E}-03 & 5.511 \mathrm{E}-04 & 2.733 \mathrm{E}-06\end{array}$ $\begin{array}{lllllllllll}1.000 \mathrm{E}+00 & 3.888 \mathrm{E}+00 & 3.795 \mathrm{E}+00 & 3.616 \mathrm{E}+00 & 2.838 \mathrm{E}+00 & 1.879 \mathrm{E}+00 & 3.446 \mathrm{E}-01 & 2.705 \mathrm{E}-03 & 1.160 \mathrm{E}-10\end{array}$

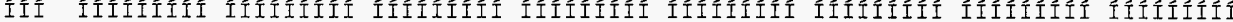
0

Single Radionuclide Soil Guidelines $G(i, t)$ in $\mathrm{pCi} / \mathrm{g}$ Basic Radiation Dose Limit $=1.500 \mathrm{E}+01 \mathrm{mrem} / \mathrm{yr}$

\begin{tabular}{|c|c|c|c|c|c|c|c|c|c|}
\hline $\begin{array}{c}(i) \\
\ddot{A} \ddot{A} \ddot{A} \ddot{A} \ddot{A} \ddot{A} \ddot{A}\end{array}$ & $t=$ & $\begin{array}{l}0.000 E+00 \\
\ddot{A} \ddot{A} \ddot{A} \ddot{A} \ddot{A} \ddot{A} \ddot{A} \ddot{A} \ddot{A}\end{array}$ & $\begin{array}{l}1.000 E+00 \\
\text { ÄÄÄÄÄÄÄÄÄ }\end{array}$ & $\begin{array}{l}3.000 E+00 \\
\ddot{A} \ddot{A} \ddot{A} \ddot{A} \ddot{A} \ddot{A} \ddot{A} A \ddot{A}\end{array}$ & $\begin{array}{l}1.300 E+01 \\
\ddot{A} \ddot{A} \ddot{A} \ddot{A} \ddot{A} \ddot{A} \ddot{A} \ddot{A} \ddot{A}\end{array}$ & $\begin{array}{l}3.000 E+01 \\
\ddot{A} \ddot{A} \ddot{A} \ddot{A} \ddot{A} \ddot{A} \ddot{A} \ddot{A} \ddot{A}\end{array}$ & $\begin{array}{l}1.000 E+02 \\
\ddot{A} \ddot{A} \ddot{A} \ddot{A} \ddot{A} \ddot{A} \ddot{A} \ddot{A} \ddot{A}\end{array}$ & $\begin{array}{l}3.000 E+02 \\
\ddot{A} \ddot{A} \ddot{A} \ddot{A} \ddot{A} \ddot{A} \ddot{A} \ddot{A} \ddot{A}\end{array}$ & $\begin{array}{l}1.000 E+03 \\
\ddot{A} \ddot{A} \ddot{A} \ddot{A} \ddot{A} \ddot{A} \ddot{A} \ddot{A} \ddot{A}\end{array}$ \\
\hline$C-14$ & & $5.448 E+00$ & $2.038 E+01$ & $3.660 \mathrm{E}+02$ & $7.096 \mathrm{E}+08$ & $* 4.455 E+12$ & *4. $455 E+12$ & $* 4.455 E+12$ & $* 4.455 E+12$ \\
\hline $\mathrm{Cs}-137$ & & $5.875 \mathrm{E}+00$ & $6.014 \mathrm{E}+00$ & $6.301 E+00$ & $7.956 \mathrm{E}+00$ & $1.183 E+01$ & $6.052 E+01$ & $6.420 \mathrm{E}+03$ & $7.897 \mathrm{E}+10$ \\
\hline$E u-152$ & & $3.319 \mathrm{E}+00$ & $3.497 E+00$ & $3.880 \mathrm{E}+00$ & $6.530 \mathrm{E}+00$ & $1.582 E+01$ & $6.049 \mathrm{E}+02$ & $2.009 E+07$ & $\star 1.765 \mathrm{E}+14$ \\
\hline $\mathrm{Ni}-63$ & & $2.800 E+03$ & $2.821 \mathrm{E}+03$ & $2.864 E+.03$ & $3.090 \mathrm{E}+03$ & $3.515 E+03$ & $5.975 E+03$ & $2.722 E+04$ & $5.488 E+06$ \\
\hline $\begin{array}{l}\text { Sr }-90 \\
\text { IIIIITí }\end{array}$ & & $\begin{array}{l}3.858 \mathrm{E}+00 \\
\text { IIIIIIIII }\end{array}$ & $\begin{array}{l}\text { 3.952E+00 } \\
\text { IIIIIIIIIII }\end{array}$ & 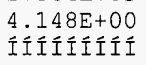 & $\begin{array}{l}5.286 \mathrm{E}+00 \\
\text { IIIIIIIII }\end{array}$ & $\begin{array}{l}7.981 E+00 \\
\text { IIIIIIIII }\end{array}$ & $\begin{array}{l}4.353 E+01 \\
\text { IIIIIIIII }\end{array}$ & $\begin{array}{l}5.545 E+03 \\
\text { IIIIIIIIII }\end{array}$ & $\begin{array}{l}\text { 1.293E+11 } \\
\text { İIIIIIÍI }\end{array}$ \\
\hline
\end{tabular}

Summed Dose/Source Ratios DSR ( $i, t)$ in (mrem/yr)/(pCi/g) and Single Radionuclide Soil Guidelines $\mathrm{G}(i, t)$ in $\mathrm{pCi} / \mathrm{g}$ at $\operatorname{tmin}=$ time of minimum single radionuclide soil guideline and at $t \max =t i m e$ of maximum total dose $=0.000 \mathrm{E}+00$ years

\begin{tabular}{|c|c|c|c|c|c|c|}
\hline $\begin{array}{l}\text { ONuclide } \\
\quad \text { (i) } \\
\ddot{A} \ddot{A} \ddot{A} \ddot{A} \ddot{A} \ddot{A} A ̈\end{array}$ & $\begin{array}{c}\text { Initial } \\
\text { (pCi/g) } \\
\ddot{A} \ddot{A} \ddot{A} \ddot{A} \ddot{A} \ddot{A} \ddot{A} \ddot{A} \ddot{A}\end{array}$ & $\begin{array}{c}\text { tmin } \\
(\text { years) } \\
\ddot{A} \ddot{A} \ddot{A} \ddot{A} \ddot{A} \ddot{A} \ddot{A} \ddot{A} \ddot{A} \ddot{A} \ddot{A} \ddot{A} \ddot{A} \ddot{A} A\end{array}$ & $\operatorname{DSR}(i, t \min )$ & $\begin{array}{l}G(i, t \min ) \\
(p C i / g) \\
\ddot{A} \ddot{A} \ddot{A} \ddot{A} \ddot{A} \ddot{A} \ddot{A} \ddot{A}\end{array}$ & $\operatorname{DSR}(i, t \max )$ & $\begin{array}{l}G(i, t \max ) \\
(p C i / g) \\
\ddot{A} \ddot{A} \ddot{A} \ddot{A} \ddot{A} \ddot{A} \ddot{A} \ddot{A} \ddot{A}\end{array}$ \\
\hline$C-14$ & $7.000 E-01$ & $0.000 \mathrm{E}+00$ & $2.753 E+00$ & $5.448 E+00$ & $2.753 E+00$ & $5.448 E+00$ \\
\hline Cs -137 & $5.600 E-01$ & $0.000 \mathrm{E}+00$ & $2.553 \mathrm{E}+00$ & $5.875 E+00$ & $2.553 E+00$ & $5.875 E+00$ \\
\hline Eu-152 & $1.530 \mathrm{E}-01$ & $0.000 \mathrm{E}+00$ & $4.519 E+00$ & $3.319 E+00$ & $4.519 E+00$ & $3.319 E+00$ \\
\hline $\mathrm{Ni}-63$ & $3.560 \mathrm{E}+00$ & $0.000 \mathrm{E}+00$ & $5.357 \mathrm{E}-03$ & $2.800 E+03$ & $5.357 E-03$ & $2.800 E+03$ \\
\hline $\begin{array}{l}\text { Sr }-90 \\
\text { İIIIIIII }\end{array}$ & $\begin{array}{l}\text { I. } 430 E-01 \\
\text { İIIIIIIIIÍI }\end{array}$ & $\begin{array}{l}0.000 \mathrm{E}+00 \\
\text { IIIIIIIIIIIIIIIIII }\end{array}$ & $\begin{array}{l}3.888 E+00 \\
\text { İIIIIIIII }\end{array}$ & $\begin{array}{l}3.858 E+00 \\
\text { İIIIIIIII }\end{array}$ & $\begin{array}{l}3.888 E+00 \\
\text { İIIIIIIII }\end{array}$ & $\begin{array}{l}3.858 E+00 \\
\text { IIIITIIII }\end{array}$ \\
\hline
\end{tabular}




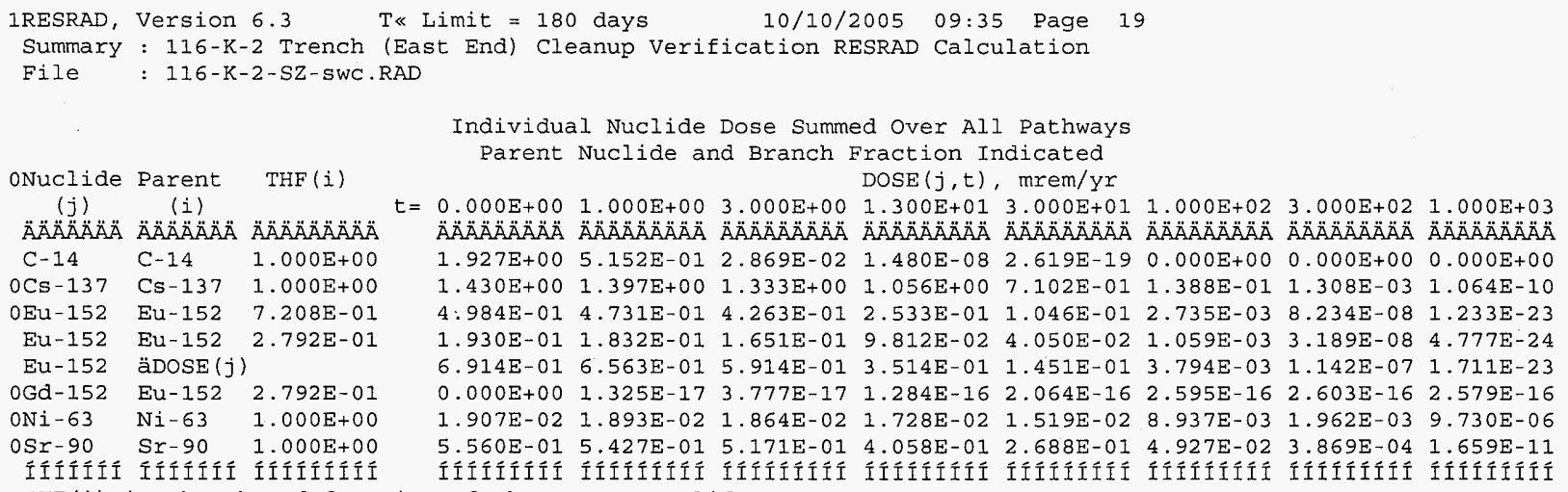
THF(i) is the thread fraction of the parent nuclide.

Individual Nuclide Soil Concentration Parent Nuclide and Branch Fraction Indicated $\mathrm{S}(j, \mathrm{t}), \mathrm{pCi} / \mathrm{g}$

\begin{tabular}{|c|c|c|c|c|c|c|c|c|c|c|}
\hline $\begin{array}{c}\text { onuclide } \\
\qquad(j) \\
\ddot{A} \ddot{A} \ddot{A} \ddot{A} \ddot{A} \ddot{A} \ddot{A}\end{array}$ & $\begin{array}{l}\text { Parent } \\
\text { (i) } \\
\ddot{A} \ddot{A} \ddot{A} \ddot{A} \ddot{A} \ddot{A} \ddot{A}\end{array}$ & $\ddot{A} \ddot{A} \ddot{A} \ddot{A} \ddot{A} \ddot{A} \ddot{A} \ddot{A}$ & $t=\begin{array}{l}0.000 E+00 \\
\ddot{A} \ddot{A} \ddot{A} \ddot{A} \vec{A} \ddot{A} \ddot{A} \ddot{A} \ddot{A}\end{array}$ & $\begin{array}{l}1.000 E+00 \\
\ddot{A} \ddot{A} \ddot{A} \ddot{A} \ddot{A} \ddot{A} \ddot{A} \ddot{A} \ddot{A}\end{array}$ & $\begin{array}{l}3.000 E+00 \\
\ddot{A} \ddot{A} \ddot{A} \ddot{A} \ddot{A} \ddot{A} \ddot{A} \ddot{A} \ddot{A}\end{array}$ & $\begin{array}{r}S(j, t), \\
1.300 E+01 \\
\ddot{A} \ddot{A} \ddot{A} \ddot{A} \ddot{A} \ddot{A} \ddot{A} \ddot{A} \ddot{A}\end{array}$ & $\begin{array}{l}\mathrm{pCi} / \mathrm{g} \\
3.000 \mathrm{E}+01 \\
\ddot{\mathrm{A}} \ddot{\mathrm{A}} \ddot{\mathrm{A}} \mathrm{A} \ddot{A} \mathrm{~A} \ddot{A} \mathrm{~A} \ddot{A} \mathrm{~A}\end{array}$ & $\begin{array}{l}1.000 E+02 \\
\ddot{A} \ddot{A} \ddot{A} \ddot{A} \ddot{A} \ddot{A} \ddot{A} \ddot{A} \ddot{A}\end{array}$ & $\begin{array}{l}3.000 E+02 \\
\ddot{A} \ddot{A} \ddot{A} \ddot{A} \ddot{A} \ddot{A} \ddot{A} \ddot{A} \ddot{A}\end{array}$ & $\begin{array}{l}1.000 E+03 \\
\ddot{A} \ddot{A} \ddot{A} \ddot{A} \ddot{A} \ddot{A} \ddot{A} \ddot{A} A\end{array}$ \\
\hline C- 14 & C- 14 & $1.000 \mathrm{E}+00$ & $7.000 \mathrm{E}-01$ & $1.653 E-01$ & $9.206 \mathrm{E}-03$ & $4.746 E-09$ & $8.393 E-20$ & $0.000 E+00$ & $0.000 E+00$ & $0.000 E+00$ \\
\hline OCs -137 & $\mathrm{Cs}-137$ & $1.000 \mathrm{E}+00$ & $5.600 \mathrm{E}-01$ & $5.471 \mathrm{E}-01$ & $5.222 E-01$ & $4.135 E-01$ & $2.782 \mathrm{E}-01$ & $5.437 E-02$ & $5.125 E-04$ & 4. $167 \mathrm{E}-11$ \\
\hline OEu-152 & Eu-152 & $7.208 \mathrm{E}-01$ & $1.103 \mathrm{E}-01$ & $1.047 \mathrm{E}-01$ & $9.434 \mathrm{E}-02$ & $5.606 E-02$ & $2.314 E-02$ & $6.051 E-04$ & $1.822 E-08$ & $2.729 E-24$ \\
\hline$E u-152$ & $\mathrm{Eu}-152$ & $2.792 \mathrm{E}-01$ & $4.272 \mathrm{E}-02$ & $4.055 \mathrm{E}-02$ & $3.654 \mathrm{E}-02$ & $2.171 E-02$ & $8.962 \mathrm{E}-03$ & $2.344 \mathrm{E}-04$ & $7.058 \mathrm{E}-09$ & $1.057 E-24$ \\
\hline$E u-152$ & äs $(j):$ & & $1.530 \mathrm{E}-01$ & $1.452 \mathrm{E}-01$ & $1.309 \mathrm{E}-01$ & $7.777 \mathrm{E}-02$ & 3.210E-02 & $8.395 E-04$ & $2.528 E-08$ & $3.786 E-24$ \\
\hline OGd-152 & $E u-152$ & $2.792 \mathrm{E}-01$ & $0.000 E+00$ & $2.671 E-16$ & $7.615 E-16$ & $2.590 \mathrm{E}-15$ & $4.161 E-15$ & $5.232 \mathrm{E}-15$ & $5.248 E-15$ & $5.199 E-15$ \\
\hline ONi-63 & $\mathrm{Ni}-63$ & $1.000 \mathrm{E}+00$ & $3.560 E+00$ & $3.533 E+00$ & $3.480 E+00$ & $3.226 \mathrm{E}+00$ & $2.836 E+00$ & $1.668 \mathrm{E}+00$ & $3.662 \mathrm{E}-01$ & $1.816 \mathrm{E}-03$ \\
\hline $\begin{array}{l}\text { OSx }-90 \\
\text { IIIIIII }\end{array}$ & $\begin{array}{l}\text { Sr-90 } \\
\text { İIIIIII }\end{array}$ & $\begin{array}{l}1.000 E+00 \\
\text { IIÍIIIIIII }\end{array}$ & $\begin{array}{l}1.430 E-01 \\
\text { IIIIIIIIIII }\end{array}$ & $\begin{array}{l}1.396 \mathrm{E}-01 \\
\text { İIIIIIIIII }\end{array}$ & $\begin{array}{l}1.330 E-01 \\
\text { İIIIIIÍII }\end{array}$ & $\begin{array}{l}1.044 \mathrm{E}-01 \\
\text { İIÍIIIIIII }\end{array}$ & $\begin{array}{l}6.912 \mathrm{E}-02 \\
\text { İIIIIIIIII }\end{array}$ & $\begin{array}{l}1.267 E-02 \\
\text { İİIIIIIIII }\end{array}$ & $\begin{array}{l}9.948 E-05 \\
\text { İİIIIIIII }\end{array}$ & $\begin{array}{l}4.267 E-12 \\
\text { IIIIIIIIIII }\end{array}$ \\
\hline
\end{tabular}
ORESCALC.EXE execution time $=0.35$ seconds 
CVP-2006-00001

Rev. 0

C-22 
CVP-2006-00001

Rev. 0

RESRAD INPUT PARAMETERS FOR THE 116-K-2 (EAST END) DEEP ZONE 
CVP-2006-00001

Rev. 0

C-24 
CVP-2006-00001

Rev. 0

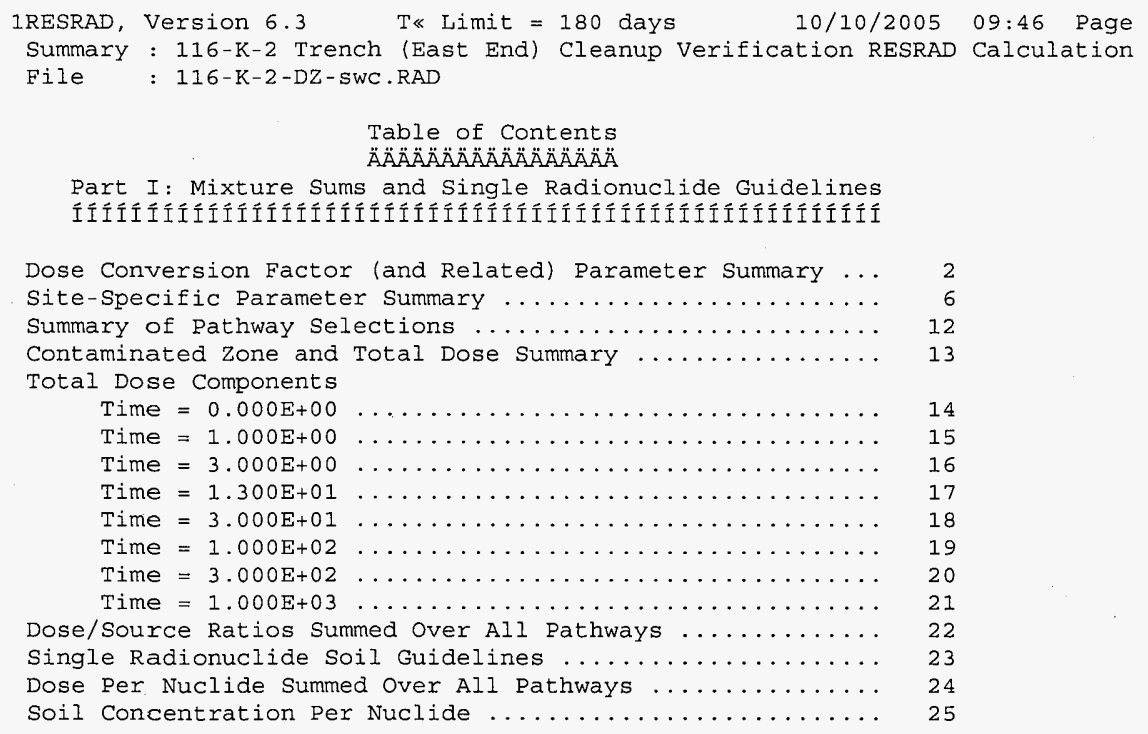


IRESRAD, Version 6.3

$\mathrm{T} \ll$ Limit $=180$ days

10/10/2005 09:46 Page

Summary : $116-\mathrm{K}-2$ Trench (East End) Cleanup Verification RESRAD Calculation

File : 116-K-2-DZ-SwC.RAD

Dose Conversion Factor (and Related) Parameter Summary File: HEAST 2001 MORBIDIEY

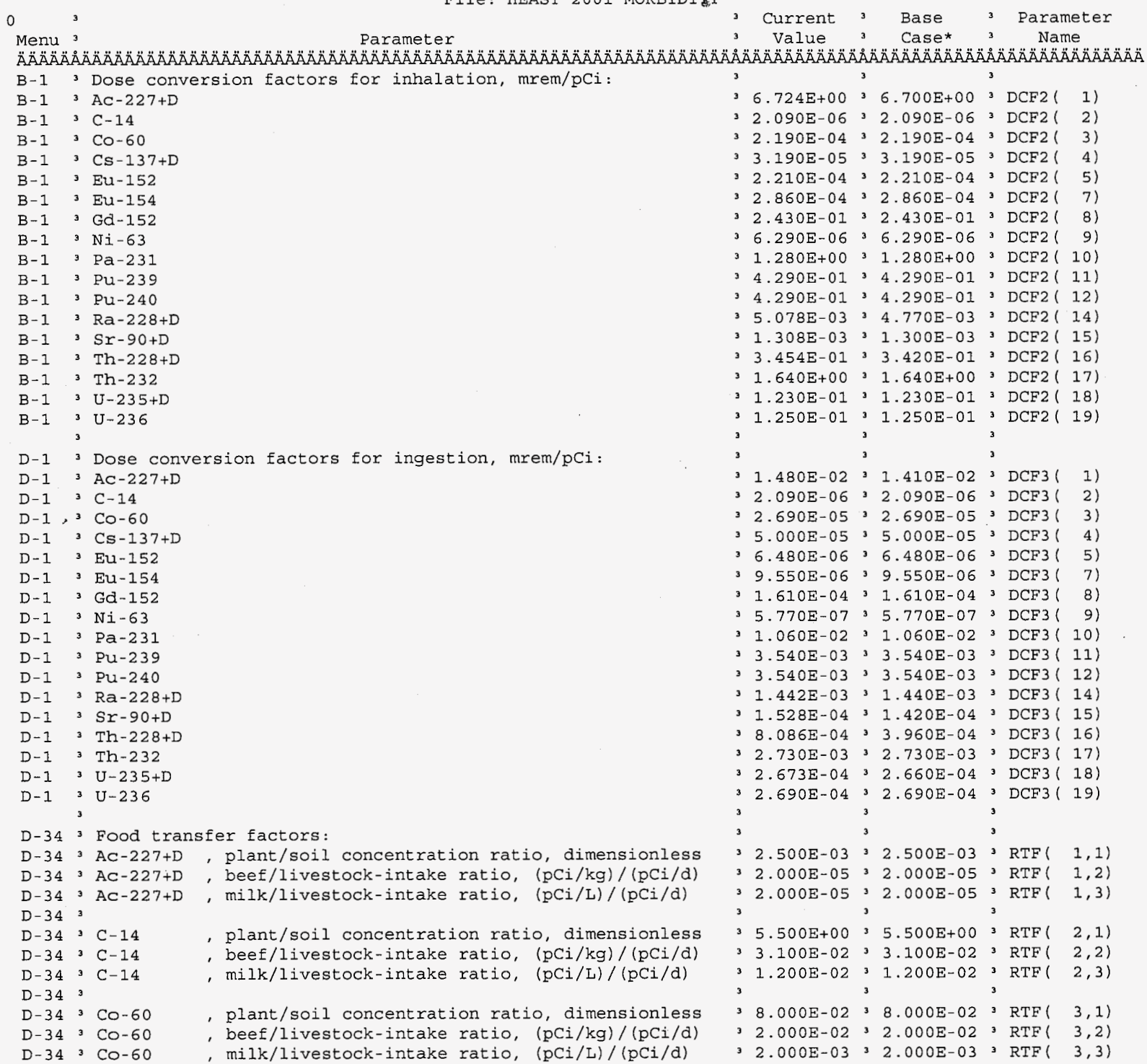




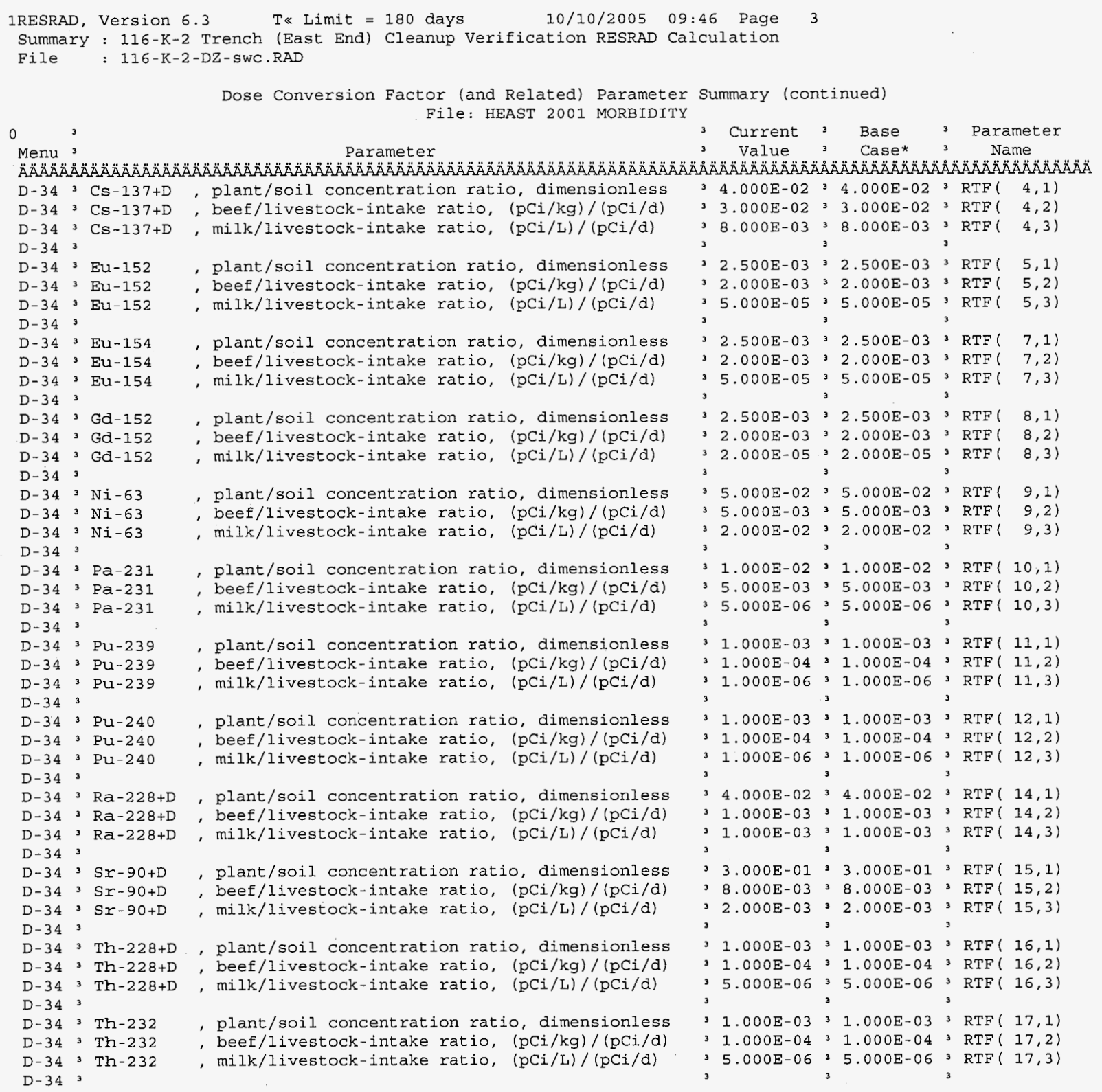




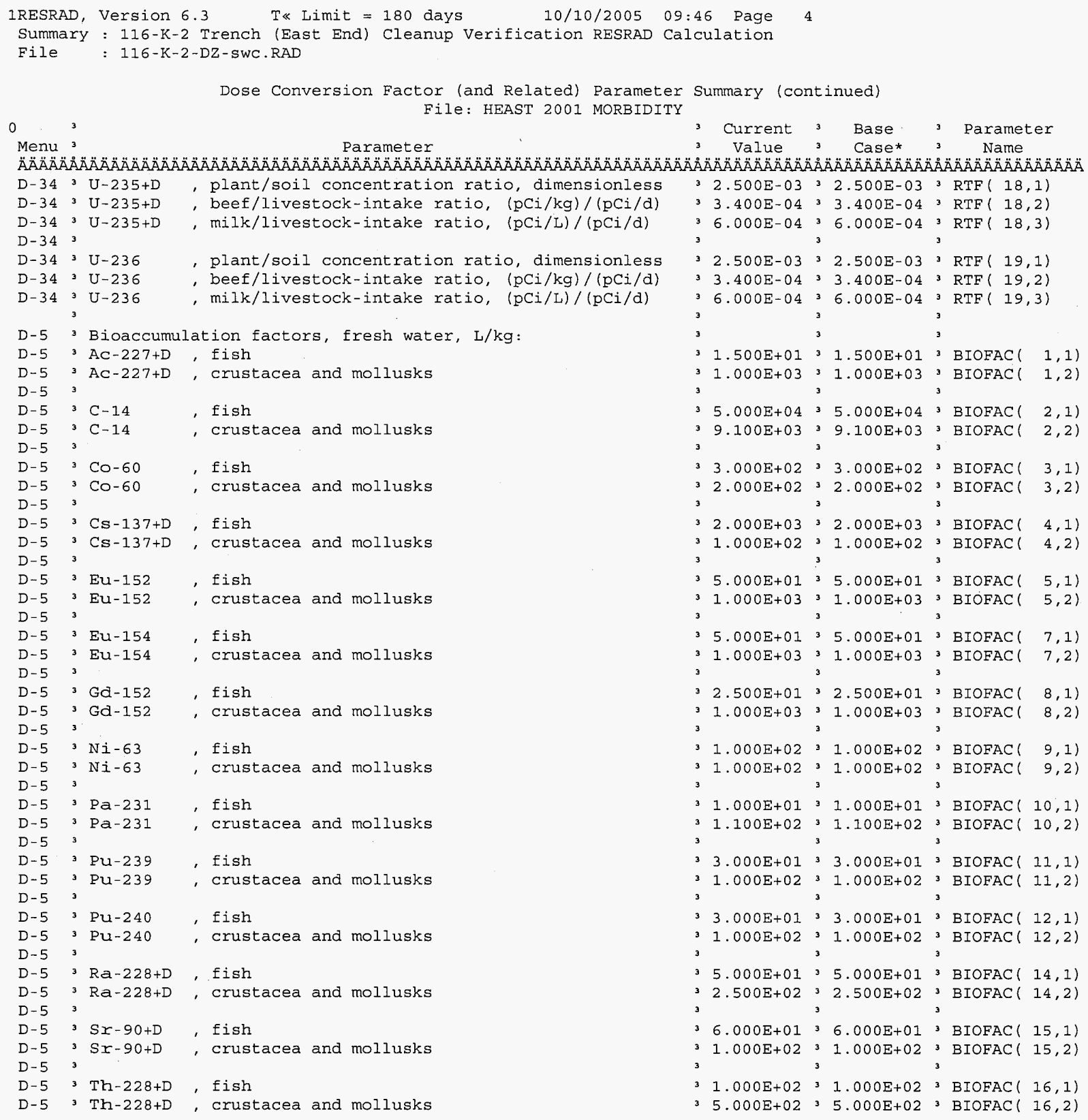


CVP-2006-00001

Rev. 0

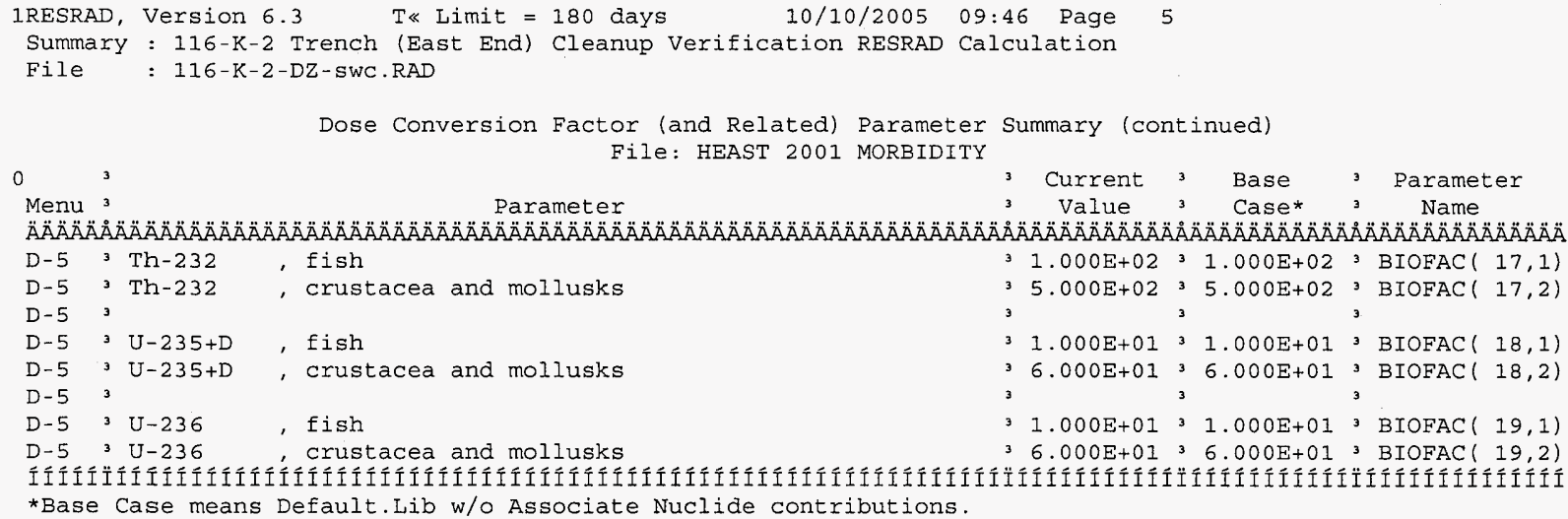


CVP-2006-00001

Rev. 0

$\begin{array}{rlrr}\text { IRESRAD, Version } 6.3 & \text { T } \text { Limit }=180 \text { days } & 10 / 10 / 2005 & 09: 46 \text { Page } \\ \text { Summary : } 116-\mathrm{K}-2 \text { Trench } & \text { (East End) Cleanup Verification RESRAD Calculation }\end{array}$ $\begin{array}{lll}\text { Summary } & : 116-\mathrm{K}-2 \text { Trench } \\ \text { File } & : 116-\mathrm{K}-2-\mathrm{DZ}-\mathrm{SwC} \cdot \mathrm{RAD}\end{array}$

\begin{tabular}{|c|c|c|c|c|c|c|c|c|c|c|c|}
\hline \multirow[b]{2}{*}{0} & \multicolumn{11}{|c|}{ Site-Specific Parameter Summary } \\
\hline & 3 & & & 3 & User & 3 & & Used & by RESRAD & 3 & Parameter \\
\hline $\begin{array}{l}\text { Menu } \\
\ddot{A} \ddot{A} \ddot{A} \ddot{A} \ddot{A}\end{array}$ & 3 & 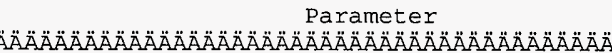 & 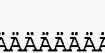 & 3 & 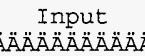 & 3 & $\begin{array}{l}\text { Default } \\
\ddot{A} \ddot{A} \ddot{A} \ddot{A} \ddot{A} \ddot{A} \ddot{A} \ddot{A} \ddot{z}\end{array}$ & 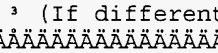 & 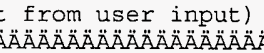 & 3 & $\begin{array}{c}\text { Name } \\
\ddot{A} \ddot{A} \ddot{A} \ddot{A} \ddot{A} \ddot{A} \ddot{A} \ddot{A} \ddot{A} \ddot{A} \ddot{A} \ddot{A}\end{array}$ \\
\hline R011 & 3 & Area of contaminated zone $(m * * 2)$ & & 3 & $1.779 \mathrm{E}+04$ & 3 & $1.000 E+04$ & 3 & -1 & 3 & AREA \\
\hline R011 & & Thickness of contaminated zone (m) & & 3 & $7.300 \mathrm{E}+00$ & 3 & $2.000 \mathrm{E}+00$ & 3 & -- & 3 & THICKO \\
\hline R011 & & Length parallel to aquifer flow $(\mathrm{m})$ & & 3 & $2.920 \mathrm{E}+02$ & 3 & 1. $000 E+02$ & 3 & --- & 3 & LCZPAQ \\
\hline R011 & & Basic radiation dose limit (mrem/yr) & & 3 & $1,500 \mathrm{E}+01$ & 3 & $3.000 E+01$ & 3 & -- & 3 & BRDL \\
\hline R011 & & Time since placement of material (yr) & & 3 & $0.000 E+00$ & 3 & $0.000 \mathrm{E}+00$ & 3 & $\ldots$ & 3 & $\mathrm{TI}$ \\
\hline R011 & & Times for calculations ( $y r$ ) & & 3 & $1.000 E+00$ & 3 & $1.000 E+00$ & 3 & --- & 3 & $T(2)$ \\
\hline R011 & & Times for calculations (yr) & & 3 & $3.000 \mathrm{E}+00$ & 3 & $3.000 \mathrm{E}+00$ & 3 & $-\cdots$ & 3 & $T(3)$ \\
\hline R011 & 3 & Times for calculations (yr) & & 3 & $1.300 E+01$ & 3 & $1.000 E+01$ & 3 & -- & 3 & $T(4)$ \\
\hline R011 & & Times for calculations (yr) & & 3 & $3.000 E+01$ & 3 & $3.000 E+01$ & 3 & --- & 3 & $T(5)$ \\
\hline R011 & & Times for calculations (yr) & & 3 & $1.000 \mathrm{E}+02$ & 3 & $1.000 E+02$ & 3 & $-\ldots$ & 3 & $T(6)$ \\
\hline R011 & & Times for calculations (yr) & & 3 & $3.000 \mathrm{E}+02$ & 3 & $3.000 E+02$ & 3 & --- & 3 & $\mathrm{~T}(7)$ \\
\hline R011 & 3 & Times for calculations (yr) & & 3 & $1.000 E+03$ & 3 & $1.000 E+03$ & 3 & -- & 3 & $T(8)$ \\
\hline R011 & 3 & Times for calculations (yr) & & 3 & not used & 3 & $0.000 E+00$ & 3 & $\ldots$ & 3 & $T(9)$ \\
\hline R011 & $\begin{array}{l}3 \\
3\end{array}$ & Times for calculations (yr) & & 3 & not used & 3 & $0.000 E+00$ & $\begin{array}{l}3 \\
3\end{array}$ & -- & 3 & $T(10)$ \\
\hline R012 & 3 & Initial principal radionuclide (pCi/g): & $\mathrm{C}-14$ & 3 & $1.260 E+00$ & 3 & $0.000 E+00$ & 3 & --- & 3 & $S 1(2)$ \\
\hline R012 & 3 & Initial principal radionuclide $(\mathrm{pCi} / \mathrm{g})$ : & $\mathrm{CO}-60$ & 3 & $2.480 E+00$ & 3 & $0.000 E+00$ & 3 & --- & 3 & S1 ( 3) \\
\hline $\mathrm{R} 012$ & 3 & Initial principal radionuclide $(\mathrm{pCi} / \mathrm{g})$ : & $\mathrm{Cs}-137$ & 3 & $1.300 E+02$ & 3 & $0.000 \mathrm{E}+00$ & 3 & -- & 3 & $\mathrm{S1}(4)$ \\
\hline $\mathrm{R} 012$ & & Initial principal radionuclide & Eu- -152 & 3 & $6.270 E+01$ & 3 & $0.000 \mathrm{E}+00$ & 3 & -- & 3 & S1 ( 5) \\
\hline $\mathrm{R} 012$ & & Initial principal radionuclide & $\mathrm{Eu}-154$ & 3 & $5.400 E+00$ & 3 & $0.000 \mathrm{E}+00$ & 3 & --- & 3 & $\mathrm{~S} 1(7)$ \\
\hline R012 & & Initial principal radionuclide & $\mathrm{Ni}-63$ & 3 & $8.800 E+02$ & 3 & $0.000 \mathrm{E}+00$ & 3 & -- & 3 & $\mathrm{SI}(9)$ \\
\hline $\mathrm{R} 012$ & & Initial principal radionuclide & $\mathrm{Pu}-239$ & 3 & $5.570 E+00$ & 3 & $0.000 \mathrm{E}+00$ & 3 & $-\cdots$ & 3 & $S 1(11)$ \\
\hline $\mathrm{RO} 22$ & & Initial principal radionuclide & $\mathrm{Pu}-240$ & 3 & $1.330 E+00$ & 3 & $0.000 \mathrm{E}+00$ & 3 & -- & 3 & $\mathrm{~S} 1(12)$ \\
\hline $\mathrm{RO} 12$ & & Initial principal radionuclide & $\operatorname{Sr}-90$ & 3 & $7.120 E+00$ & 3 & $0.000 \mathrm{E}+00$ & 3 & --- & 3 & S1 (15) \\
\hline $\mathrm{R} 012$ & & Concentration in groundwater & $C-14$ & 3 & not used & 3 & $0.000 \mathrm{E}+00$ & 3 & -- & 3 & W1 ( 2) \\
\hline $\mathrm{R} 012$ & & Concentration in groundwater & $\mathrm{CO}-6 \mathrm{O}$ & 3 & not used & 3 & $0.000 \mathrm{E}+00$ & 3 & -- & 3 & W1 ( 3) \\
\hline R012 & & Concentration in groundwater & $\mathrm{Cs}-137$ & 3 & not used & 3 & $0.000 \mathrm{E}+00$ & 3 & -- & 3 & W1 (4) \\
\hline $\mathrm{R} 012$ & & Concentration in groundwater & $\mathrm{Eu}-152$ & 3 & not used & 3 & $0.000 \mathrm{E}+00$ & 3 & -- & 3 & W1( 5) \\
\hline $\mathrm{R} 012$ & & Concentration in groundwater & $\mathrm{Eu}-154$ & 3 & not used & 3 & $0.000 \mathrm{E}+00$ & 3 & --- & 3 & W1 $(7)$ \\
\hline $\mathrm{R} 012$ & & Concentration in groundwater & $\mathrm{Ni}-63$ & 3 & not used & 3 & $0.000 \mathrm{E}+00$ & 3 & -- & 3 & W1(9) \\
\hline $\mathrm{R} 012$ & & Concentration in groundwater & Pu -239 & 3 & not used & 3 & $0.000 E+00$ & 3 & -- & 3 & W1 (11) \\
\hline $\mathrm{R} 012$ & & Concentration in groundwater & $\mathrm{Pu}-240$ & 3 & not used & 3 & $0.000 \mathrm{E}+00$ & 3 & $-\cdots$ & 3 & W1 (12) \\
\hline $\mathrm{R} 012$ & $\begin{array}{l}3 \\
3\end{array}$ & Concentration in groundwater & $\mathrm{Sr}-90$ & ${ }_{3}^{3}$ & not used & 3 & $0.000 \mathrm{E}+00$ & $\begin{array}{l}3 \\
3\end{array}$ & --- & $\begin{array}{l}3 \\
3\end{array}$ & W1 (15) \\
\hline R013 & 3 & Cover depth $(\mathrm{m})$ & & 3 & $4.600 \mathrm{E}+00$ & 3 & $0.000 \mathrm{E}+00$ & 3 & --- & 3 & COVERO \\
\hline $\mathrm{R} 013$ & & Density of cover material $(\mathrm{g} / \mathrm{cm} * * 3)$ & & 3 & $1.500 E+00$ & 3 & $1.500 \mathrm{E}+00$ & 3 & --- & 3 & DENSCV \\
\hline $\mathrm{R} 013$ & & Cover depth erosion rate (m/yr) & & 3 & $1.000 E-03$ & 3 & $1.000 \mathrm{E}-03$ & 3 & -- & 3 & VCV \\
\hline $\mathrm{R} 013$ & & Density of contaminated zone $\left(\mathrm{g} / \mathrm{cm}^{*} * 3\right)$ & & 3 & $1.600 \mathrm{E}+00$ & 3 & $1.500 \mathrm{E}+00$ & 3 & --- & 3 & DENSCZ \\
\hline $\mathrm{R} 013$ & 3 & Contaminated zone erosion rate $(\mathrm{m} / \mathrm{yr})$ & & 3 & $1.000 E-03$ & 3 & $1.000 E-03$ & 3 & --- & 3 & $\mathrm{VCZ}$ \\
\hline $\mathrm{R} 013$ & 3 & Contaminated zone total porosity & & 3 & $4.000 E-01$ & 3 & $4.000 E-01$ & 3 & --- & 3 & TPCZ \\
\hline R013 & 3 & Contaminated zone field capacity & & 3 & $2.000 E-01$ & 3 & $2.000 \mathrm{E}-01$ & 3 & -- & 3 & $\mathrm{FCCZ}$ \\
\hline $\mathrm{R} 013$ & 3 & Contaminated zone hydraulic conductivity & $(\mathrm{m} / \mathrm{yr})$ & 3 & $2.500 \mathrm{E}+02$ & 3 & $1.000 \mathrm{E}+01$ & 3 & -- & 3 & $\mathrm{HCCZ}$ \\
\hline R013 & 3 & Contaminated zone $b$ parameter & & 3 & $4.050 E+00$ & 3 & $5.300 E+00$ & 3 & --- & 3 & $\mathrm{BCZ}$ \\
\hline $\mathrm{R} 013$ & & Average annual wind speed ( $\mathrm{m} / \mathrm{sec})$ & & 3 & $3.400 \mathrm{E}+00$ & 3 & $2.000 E+00$ & 3 & --- & 3 & WIND \\
\hline $\mathrm{R} 013$ & & Humidity in air $(\mathrm{g} / \mathrm{m} * * 3)$ & & 3 & not used & 3 & $8.000 E+00$ & 3 & --- & 3 & HUMID \\
\hline R013 & & Evapotranspiration coefficient & & 3 & $9.100 \mathrm{E}-01$ & 3 & $5.000 E-01$ & 3 & $\ldots$ & 3 & EVAPTR \\
\hline $\mathrm{R} 013$ & & Precipitation $(\mathrm{m} / \mathrm{y} r)$ & & 3 & 1. $600 \mathrm{E}-01$ & 3 & $1.000 \mathrm{E}+00$ & 3 & -- & 3 & PRECIP \\
\hline $\mathrm{R} 013$ & 3 & Irrigation $(\mathrm{m} / \mathrm{yr})$ & & 3 & $7.600 E-01$ & 3 & $2.000 E-01$ & 3 & --- & 3 & RI \\
\hline $\mathrm{R} 013$ & 3 & Irrigation mode & & 3 & overhead & 3 & overhead & 3 & -- & 3 & IDITCH \\
\hline R013 & & Runoff coefficient & & 3 & $2.000 E-01$ & 3 & $2.000 E-01$ & 3 & --- & 3 & RUNOFF \\
\hline
\end{tabular}




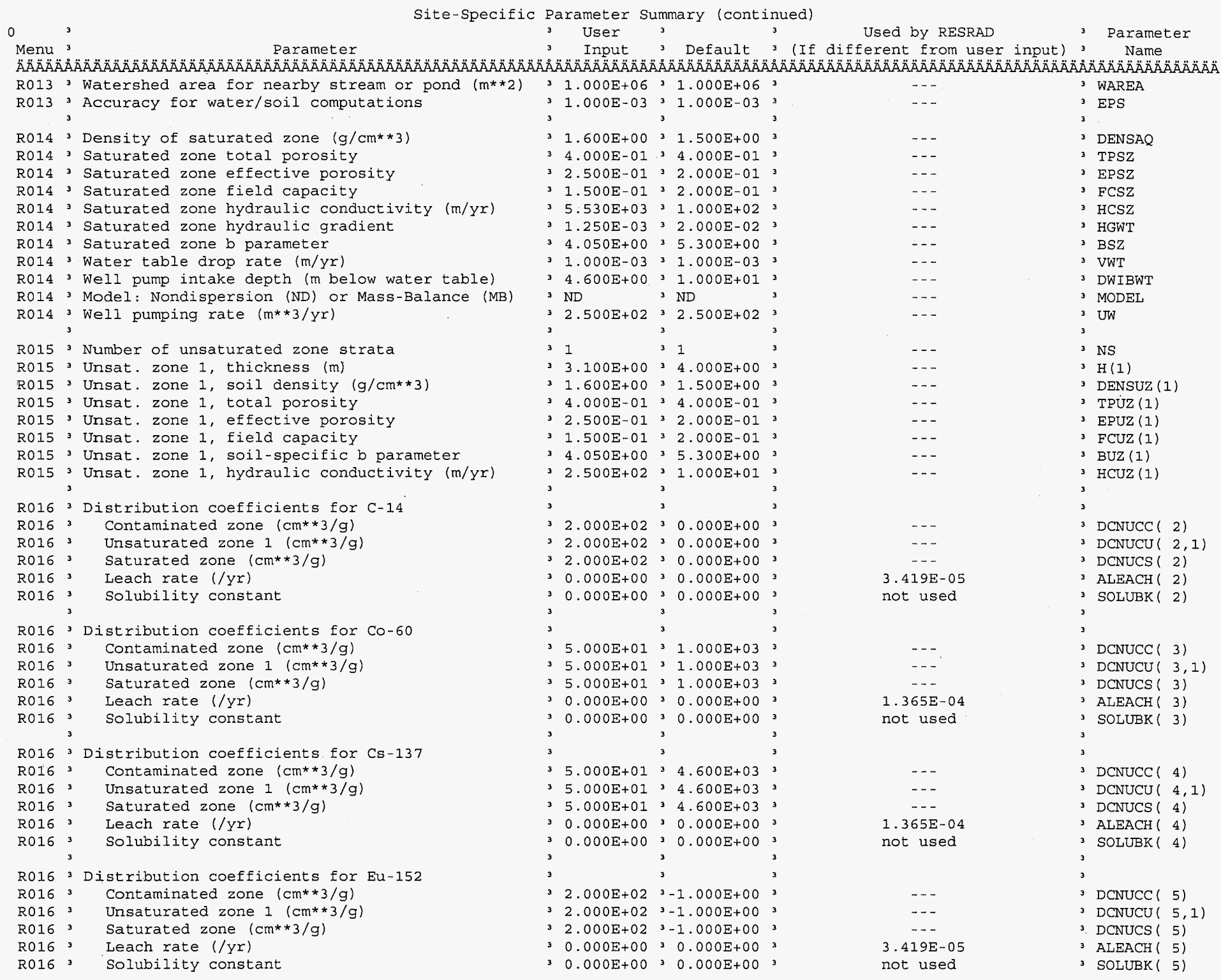


$\begin{array}{rlrr}\text { 1RESRAD, Version } 6.3 & \text { T Limit }=180 \text { days } & 10 / 10 / 2005 & 09: 46 \text { Page } \\ \text { Summary : } 116-\mathrm{K}-2 \text { Trench } & \text { (East End) Cleanup Verification RESRAD Calculation }\end{array}$ File : $116-\mathrm{K}-2-\mathrm{DZ}-\mathrm{SWC} \cdot \mathrm{RAD}$

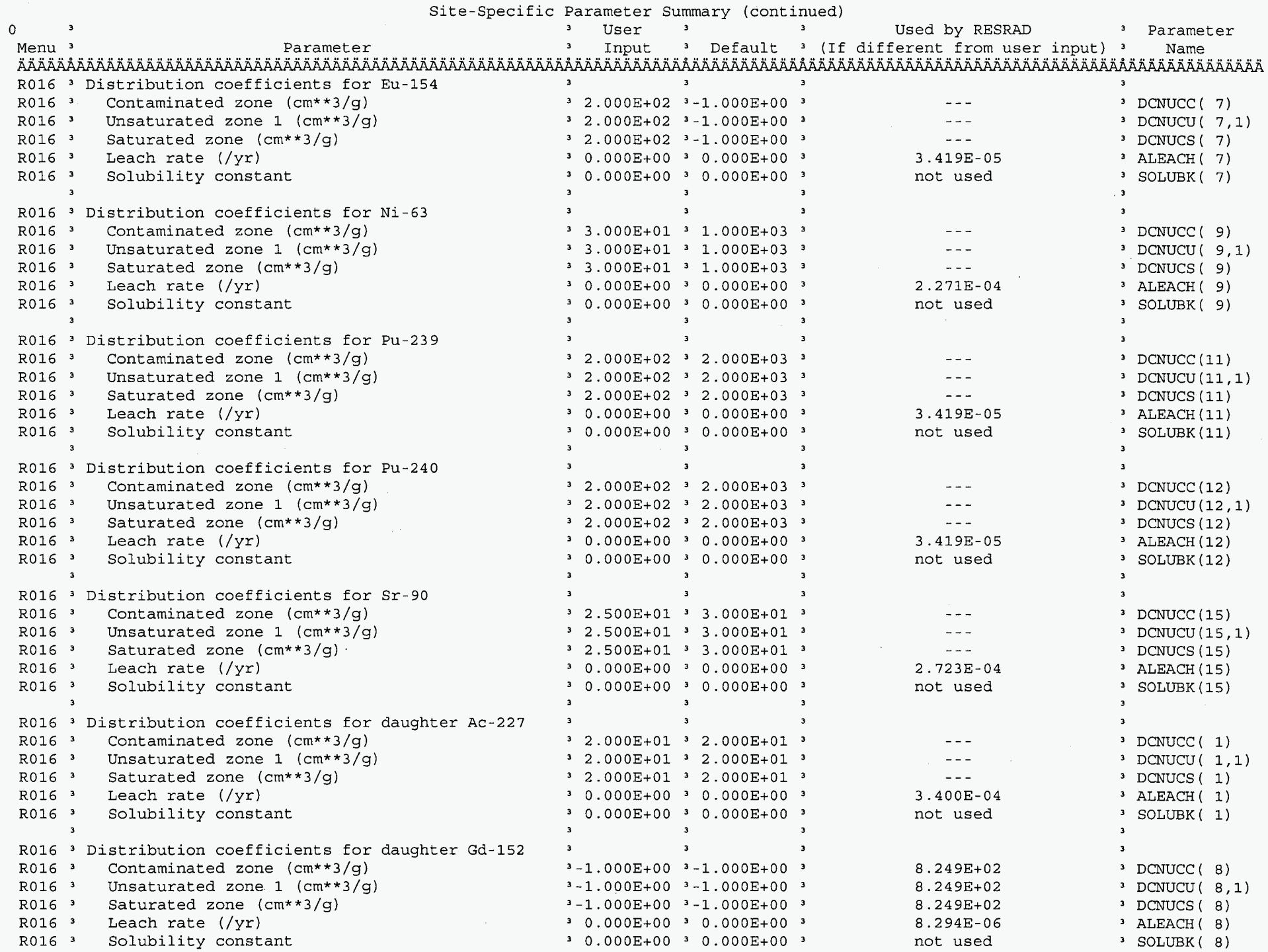




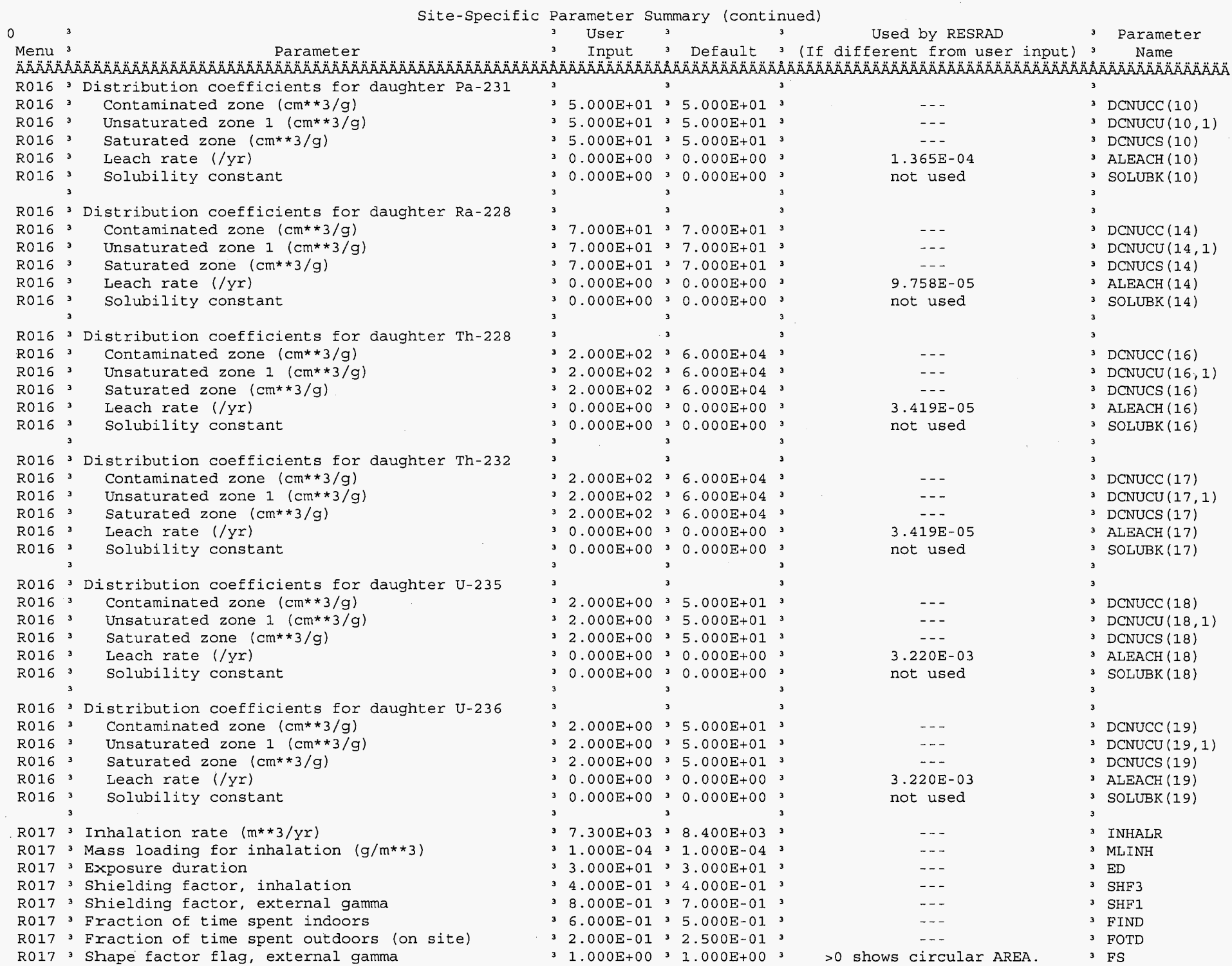




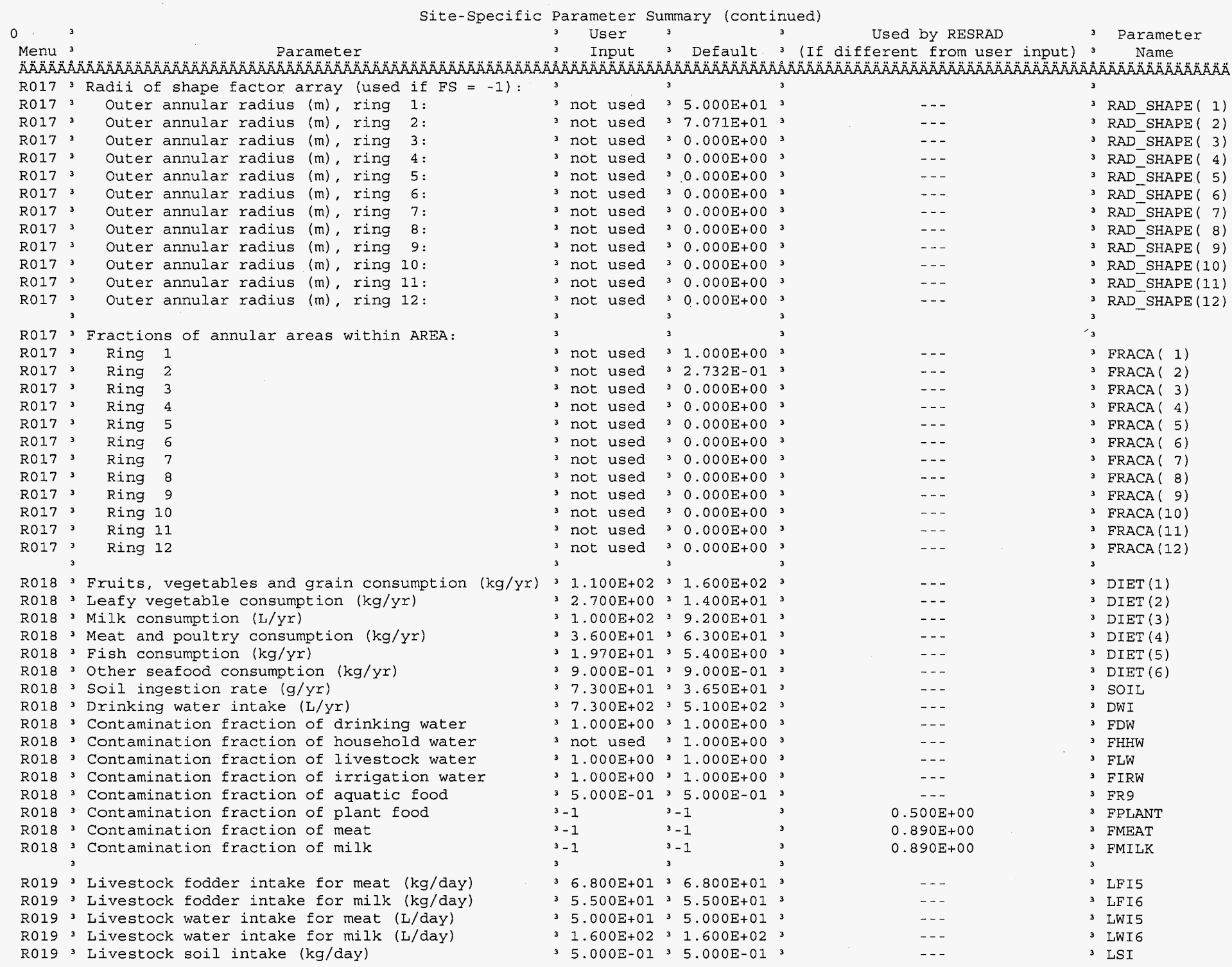




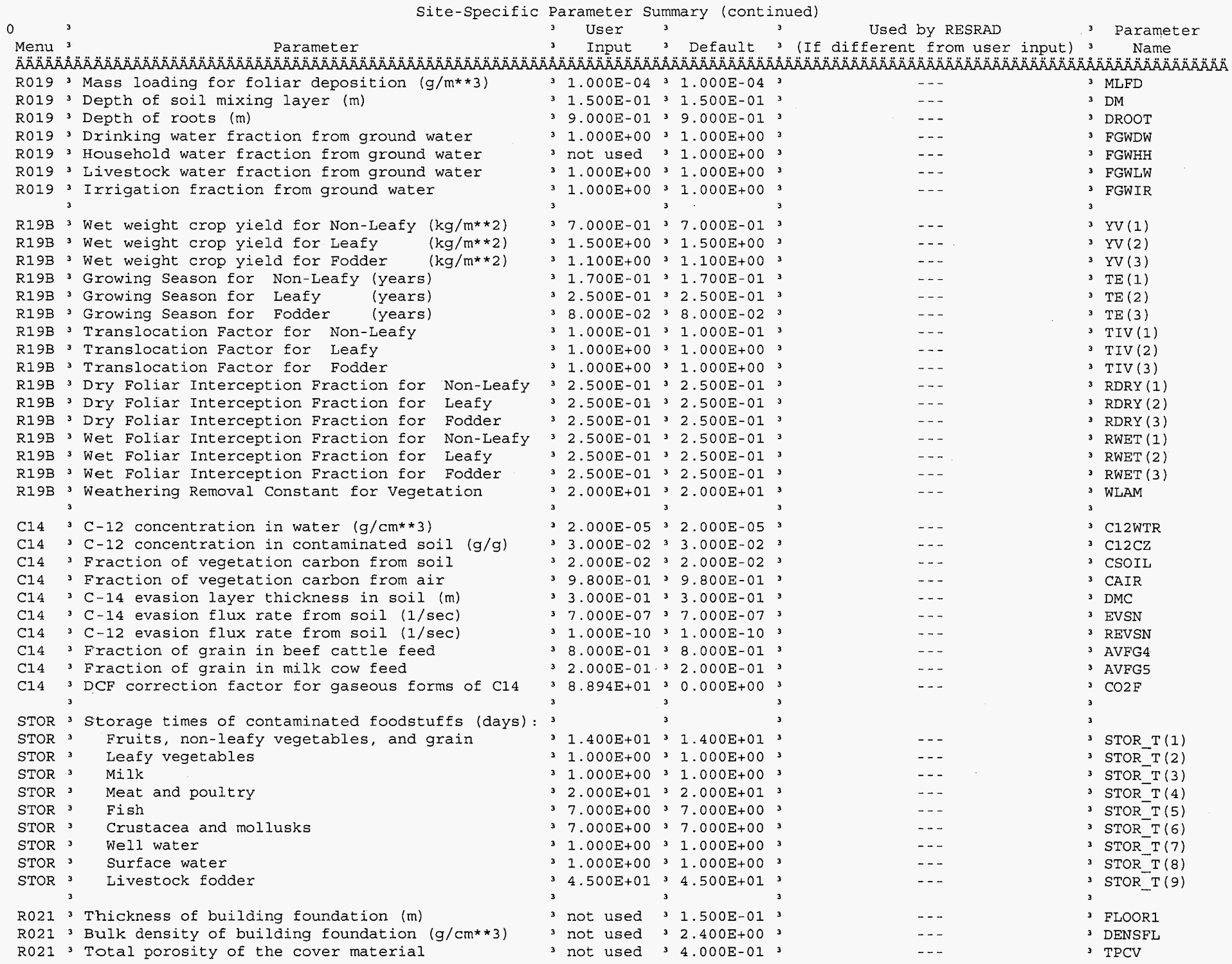


CVP-2006-00001

Rev. 0

1RESRAD, Version 6.3 T« Limit $=180$ days $10 / 10 / 2005$ 09:46 Page 12

Summary : 116-K-2 Trench (East End) Cleanup Verification RESRAD Calculation

File : $116-\mathrm{K}-2-\mathrm{DZ}-\mathrm{SWC} \cdot \mathrm{RAD}$

\begin{tabular}{|c|c|c|c|c|c|c|c|c|}
\hline \multicolumn{9}{|c|}{ Site-Specific Parameter Summary (continued) } \\
\hline 0 & 3 & $3 \quad \mathrm{Us}$ & ser & 3 & & 3 & Used by RESRAD & Parameter \\
\hline $\begin{array}{l}\text { Menu } \\
\ddot{A} \ddot{A} \ddot{A} \ddot{A} \ddot{A}\end{array}$ & 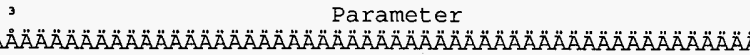 & $\stackrel{\text { In }}{3} \stackrel{2}{A} \ddot{A} \ddot{A} \ddot{A} \ddot{A} \ddot{A}$ & 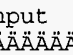 & 3 & $\begin{array}{l}\text { Default } \\
\ddot{A} \ddot{A} \ddot{A} \ddot{A} \ddot{A} \ddot{A} \ddot{A} \ddot{A} \ddot{A}\end{array}$ & ${ }^{3}$ (If & 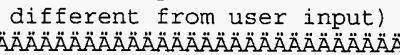 & 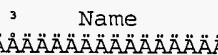 \\
\hline R021 & 3 Total porosity of the building foundation & 3 not & used & 3 & $1.000 \mathrm{E}-01$ & 3 & $-\ldots$ & 3 TPFL \\
\hline R021 & 3 Volumetric water content of the cover material & 3 not & used & 3 & $5.000 \mathrm{E}-02$ & 3 & --- & $3 \mathrm{PH} 2 \mathrm{OCV}$ \\
\hline $\mathrm{R} 021$ & 3 Volumetric water content of the foundation & 3 not & used & 3 & $3.000 \mathrm{E}-02$ & 3 & -- & 3 PH2OFL \\
\hline $\mathrm{R} 021$ & 3 Diffusion coefficient for radon gas (m/sec): & 3 & & 3 & & 3 & & 3 \\
\hline R021 & 3 in cover material & 3 not & used & 3 & $2.000 \mathrm{E}-06$ & 3 & --- & 3 DIFCV \\
\hline $\mathrm{R} 021$ & in foundation material & 3 not & used & 3 & $3.000 \mathrm{E}-07$ & 3 & --- & 3 DIFFL \\
\hline R021 & 3 in contaminated zone soil & 3 not & used & 3 & $2.000 \mathrm{E}-06$ & 3 & --- & 3 DIFCZ \\
\hline R021 & 3 Radon vertical dimension of mixing $(\mathrm{m})$ & 3 not & used & 3 & $2.000 E+00$ & 3 & -- & 3 HMIX \\
\hline R021 & 3 Average building air exchange rate $(1 / \mathrm{hr})$ & 3 not & used & 3 & $5.000 E-01$ & 3 & -- & 3 REXG \\
\hline R021 & 3 Height of the building (room) (m) & 3 not & used & 3 & $2.500 E+00$ & 3 & --- & 3 HRM \\
\hline R021 & 3 Building interior area factor & 3 not & used & 3 & $0.000 E+00$ & 3 & $-\ldots$ & 3 FAI \\
\hline R021 & 3 Building depth below ground surface $(\mathrm{m})$ & 3 not & used & & $-1.000 \mathrm{E}+00$ & 3 & --- & 3 DMFL \\
\hline R02I & 3 Emanating power of $\mathrm{Rn}-222$ gas & 3 not & used & 3 & $2.500 \mathrm{E}-01$ & 3 & -- & 3 EMANA (1) \\
\hline R021 & 3 Emanating power of $\mathrm{Rn}-220$ gas & 3 not & used & 3 & $1.500 \mathrm{E}-01$ & 3 & --- & 3 EMANA (2) \\
\hline TITL & 3 Number of graphical time points & 3 & 32 & 3 & - - - & 3 & --- & 3 NPTS \\
\hline TITL & 3 Maximum number of integration points for dose & 3 & 1 & 3 & --- & 3 & --- & 3 LYMAX \\
\hline TITL & 3 Maximum number of integration points for risk & 3 & & 3 & --- & & $\cdots-$ & 3 KYMAX \\
\hline
\end{tabular}

Summary of Pathway Selections

Pathway 3 User Selection

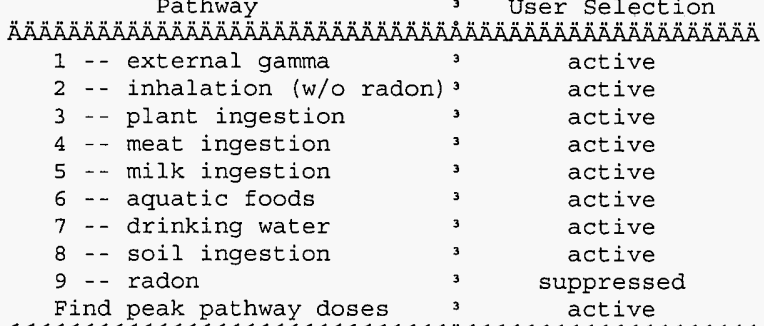

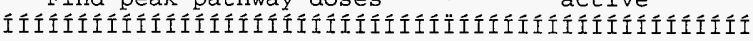




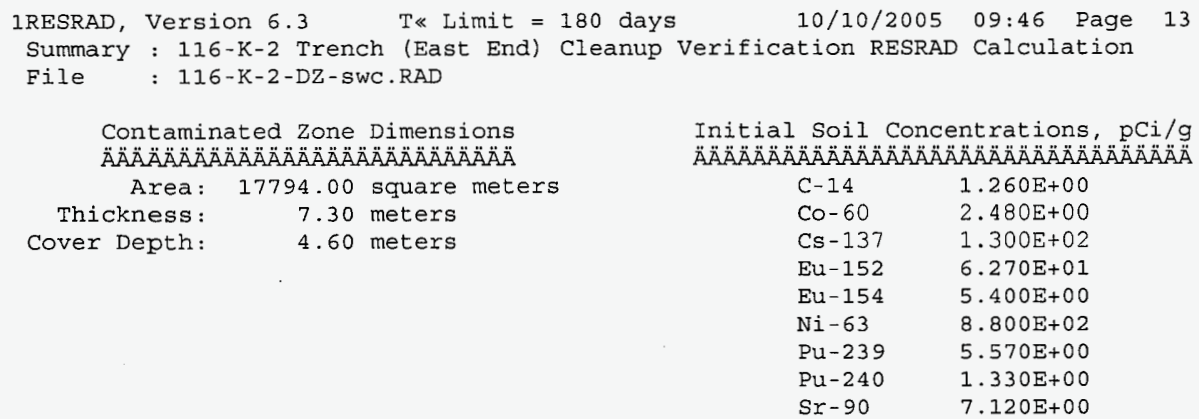

0

Total Dose TDOSE(t), mrem/yr Basic Radiation Dose Iimit $=1.500 \mathrm{E}+01 \mathrm{mrem} / \mathrm{yr}$

Total Mixture Sum $M(t)=$ Fraction of Basic Dose Limit Received at Time (t)

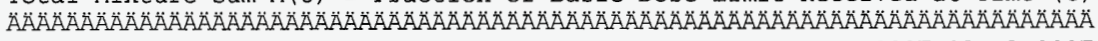

$t$ (years) : $\begin{array}{lllllllll}0.000 \mathrm{E}+00 & 1.000 \mathrm{E}+00 & 3.000 \mathrm{E}+00 & 1.300 \mathrm{E}+01 & 3.000 \mathrm{E}+01 & 1.000 \mathrm{E}+02 & 3.000 \mathrm{E}+02 & 1.000 \mathrm{E}+03\end{array}$

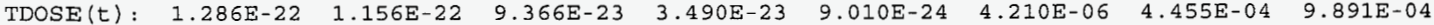

$M(t): \begin{array}{llllllll}8.574 E-24 & 7.707 E-24 & 6.244 \mathrm{E}-24 & 2.326 \mathrm{E}-24 & 6.007 \mathrm{E}-25 & 2.806 \mathrm{E}-07 & 2.970 \mathrm{E}-05 & 6.594 \mathrm{E}-05\end{array}$ OMaximum TDOSE(t): $9.891 \mathrm{E}-04 \mathrm{mrem} / \mathrm{yr}$ at $t=1.000 \mathrm{E}+03$ years 
1RESRAD, Version 6.3 T« Limit $=180$ days $\quad 10 / 10 / 2005 \quad 09: 46$ Page 14 Summary : 116-K-2 Trench (East End) Cleanup Verification RESRAD Calculation File : 116-K-2-DZ-SWC.RAD

Total Dose Contributions TDOSE $(i, p, t)$ for Individual Radionuclides (i) and Pathways (p)

As mrem/yr and Fraction of Total Dose At $t=0.000 \mathrm{E}+00$ years

0 water Independent Pathways (Inhalation excludes radon) Ground Nuclide mrem/Yr

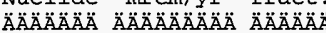
$\begin{array}{lll}\mathrm{C}-14 & 0.000 \mathrm{E}+00 & 0.0000\end{array}$ $\begin{array}{lll}\text { Co- } 60 & 1.069 \mathrm{E}-22 & 0.8308\end{array}$ Cs-137 $9.524 \mathrm{E}-27 \quad 0.0001$ $\mathrm{Eu}-152 \quad 1.830 \mathrm{E}-23 \quad 0.1423$ $\begin{array}{lll}\mathrm{Eu}-154 & 3.444 \mathrm{E}-24 & 0.0268\end{array}$ Ni-63 $0.000 \mathrm{E}+00 \quad 0.0000$ $\begin{array}{lll}\mathrm{Pu}-239 & 0.000 \mathrm{E}+00 \quad 0.0000\end{array}$ $\mathrm{Pu}-240 \quad 0.000 \mathrm{E}+00 \quad 0.0000$ Sr-90 $0.000 \mathrm{E}+00 \quad 0.0000$

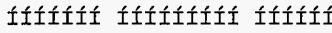
Total $1.286 \mathrm{E}-221.0000$ Inhalation Radon Plant Meat

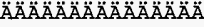

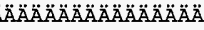

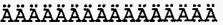
$\mathrm{mrem} / \mathrm{yr}$ fract. Ä $0.000 \mathrm{E}+00 \quad 0.0000$ mrem/yr fract. mrem/yr fract. mrem/yr fract. $\ddot{A} \ddot{A} \ddot{A} \ddot{A} \ddot{A} \ddot{A} \ddot{A} \ddot{A}$ Ä $\ddot{A} \ddot{A} \ddot{A} \ddot{A} \ddot{A}$ $0.000 \mathrm{E}+00 \quad 0.0000$ $0.000 \mathrm{E}+00 \quad 0.0000$ $0.000 \mathrm{E}+00 \quad 0.0000$ $0.000 \mathrm{E}+00 \quad 0.0000$ $0.000 \mathrm{E}+00 \quad 0.0000$ $\begin{array}{llllll}0.000 \mathrm{E}+00 & 0.0000 & 0.000 \mathrm{E}+00 & 0.0000\end{array}$ $\begin{array}{lllll}0.000 \mathrm{E}+00 & 0.0000 & 0.000 \mathrm{E}+00 & 0.0000\end{array}$ $\begin{array}{lllll}0.000 \mathrm{E}+00 & 0.0000 & 0.000 \mathrm{E}+00 & 0.0000\end{array}$ $0.000 \mathrm{E}+00 \quad 0.0000$ $0.000 \mathrm{E}+00 \quad 0.0000$ $0.000 \mathrm{E}+00 \quad 0.0000$

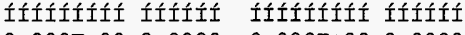
$0.000 \mathrm{E}+000.0000$ 0.000 0.000 .000 $0.000 \mathrm{E}+00 \quad 0.0000 \quad 0.000 \mathrm{E}+00 \quad 0.0000$ $0.000 \mathrm{E}+00 \quad 0.0000$ $0.000 \mathrm{E}+00 \quad 0.0000$ $0.000 \mathrm{E}+00 \quad 0.0000$ $0.000 \mathrm{E}+00 \quad 0.0000$ $0.000 \mathrm{E}+00 \quad 0.0000$ $0.000 \mathrm{E}+00 \quad 0.0000$ $0.000 \mathrm{E}+00 \quad 0.0000$ $0.000 \mathrm{E}+00 \quad 0.0000$

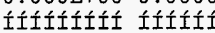
$0.000 \mathrm{E}+00 \quad 0.0000$

Total Dose Contributions TDOSE( $(i, p, t)$ for Individual Radionuclides ( $i$ ) and Pathways (p) As mrem/yr and Fraction of Total Dose At $t=0.000 \mathrm{E}+00$ years Water Dependent Pathways

$$
\text { Radon }
$$
Plant

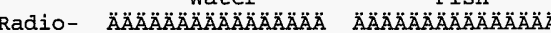
Nuclide mrem/yr fract. $\ddot{A} \ddot{A} \ddot{A} \ddot{A} \ddot{A} \ddot{A} \ddot{A} \ddot{A} \ddot{A} \ddot{A} \ddot{A} \ddot{A} \ddot{A} \ddot{A} \ddot{A}$ Ä $\ddot{A} \ddot{A} \ddot{A} \ddot{A}$ C-14 $0.000 \mathrm{E}+00 \quad 0.0000$ Co-60 $0.000 \mathrm{E}+00 \quad 0.0000$ $\begin{array}{lll}\mathrm{Cs}-137 & 0.000 \mathrm{E}+00 \quad 0.0000\end{array}$ Eu-152 $0.000 \mathrm{E}+00 \quad 0.0000$ $\begin{array}{lll}\mathrm{Eu}-1.54 & 0.000 \mathrm{E}+00 \quad 0.000\end{array}$ $\mathrm{Ni}-63 \quad 0.000 \mathrm{E}+00 \quad 0.0000$ Pu-239 $0.000 \mathrm{E}+00 \quad 0.0000$ $\begin{array}{lll}\mathrm{Pu}-240 & 0.000 \mathrm{E}+00 & 0.000\end{array}$

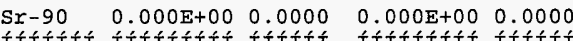

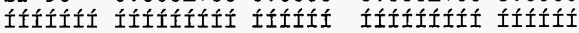
0 * Sum of all water independent and dependent pathwars. $\ddot{A} \ddot{A} \ddot{A} \ddot{A} \ddot{A} \ddot{A} \ddot{A} \ddot{A} \ddot{A} \ddot{A} \ddot{A} \ddot{A} \ddot{A} \ddot{A} \ddot{A} \ddot{A}$ mrem/yx fract. mrem/yr fract. A $0.000 \mathrm{E}+00 \quad 0.0000$ $00.0000 \quad 0.000 \mathrm{E}+000.0000$ $0.000 \mathrm{E}+00 \quad 0.0000$ $0.000 \mathrm{E}+00 \quad 0.0000$

$0.000 E+00 \quad 0.0000$ $0.000 \mathrm{E}+00 \quad 0.0000$ $0.000 \mathrm{E}+00 \quad 0.0000$ $0.000 \mathrm{E}+00 \quad 0.0000$ $0.000 \mathrm{E}+00 \quad 0.0000$ $0.000 \mathrm{E}+00 \quad 0.0000$ $0.000 \mathrm{E}+00 \quad 0.0000$ $0.000 \mathrm{E}+00 \quad 0.0000$ $0.000 \mathrm{E}+000.0000$ Ítífítít ífííí

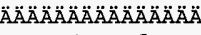
mrem/yr fract. A $\triangle$ AAAAÄ̈̈̈ ÄÄ̈̈̈̈̈̈̈ $E+00 \quad 0.0000$ $\begin{array}{ll}0.000 \mathrm{E}+00 & 0.0000 \\ 0.000 \mathrm{E}+00 & 0.0000\end{array}$ $\begin{array}{ll}0.000 \mathrm{E}+00 & 0.0000 \\ 0.000 \mathrm{E}+00 & 0.0000\end{array}$ $\begin{array}{ll}0.000 \mathrm{E}+00 & 0.0000 \\ 0.000 \mathrm{E}+00 & 0.0000\end{array}$ $0.000 \mathrm{E}+00 \quad 0.0000$ $0.000 \mathrm{E}+00 \quad 0.0000$ $0.000 \mathrm{E}+00 \quad 0.0000$ $0.000 \mathrm{E}+00 \quad 0.0000$ ítítítítí ítítít $0.000 \mathrm{E}+00 \quad 0.0000$ $0.000 \mathrm{E}+00 \quad 0.0000$
Meat

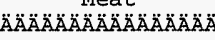
mrem/yr fract. AAAÄÄÄÄ ÄÄÄÄ $0.000 \mathrm{E}+00 \quad 0.0000$ $0.000 \mathrm{E}+00 \quad 0.0000$ $0.000 \mathrm{E}+00 \quad 0.0000$ $0.000 \mathrm{E}+00 \quad 0.0000$ $0.000 \mathrm{E}+00 \quad 0.0000$ $0.000 \mathrm{E}+00 \quad 0.0000$ $0.000 \mathrm{E}+00 \quad 0.0000$ $0.000 \mathrm{E}+00 \quad 0.0000$ ííífítí fitít $0.000 \mathrm{E}+000.0000$ Milk

Ӓ $\ddot{A} \ddot{A} \ddot{A} \ddot{A} \ddot{A} \ddot{A} \ddot{A} \ddot{A} \ddot{A} \ddot{A} \ddot{A} \ddot{A} \ddot{A} \ddot{A}$ mrem/yr fract. $\ddot{\mathrm{A}} \ddot{\mathrm{A}} \ddot{\mathrm{A}} \ddot{\mathrm{A}} \ddot{\mathrm{A}} \ddot{\mathrm{A}} \ddot{\mathrm{A}} \ddot{\mathrm{A}} \ddot{\mathrm{A}}$ Ä $\ddot{\mathrm{A}} \ddot{\mathrm{A}} \ddot{\mathrm{A}} \ddot{\mathrm{A}} \ddot{\mathrm{A}}$ $0.000 E+00 \quad 0.0000$ $0.000 E+00 \quad 0.0000$ $0.000 \mathrm{E}+00 \quad 0.0000$ $0.000 \mathrm{E}+00 \quad 0.0000$ $0.000 \mathrm{E}+00 \quad 0.0000$ $0.000 E+00 \quad 0.0000$ $0.000 \mathrm{E}+00 \quad 0.0000$ $0.000 \mathrm{E}+00 \quad 0.0000$ $0.000 \mathrm{E}+000.0000$ íífífítí íífíí $0.000 \mathrm{E}+000.0000$

\section{Soil}

Ä $\ddot{A} \ddot{A} \ddot{A} \ddot{A} \ddot{A} \ddot{A} \ddot{A} \ddot{A} \ddot{A} \ddot{A} \ddot{A} \ddot{A} \ddot{A}$ mrem/yr fract. A $\ddot{A} \ddot{A} \ddot{A} \ddot{A} \ddot{A} \ddot{A} \ddot{A} \ddot{A} \ddot{A} \ddot{A} \ddot{A} \ddot{A} \ddot{A}$ $0.000 \mathrm{E}+00 \quad 0.0000$ $0.000 \mathrm{E}+00 \quad 0.0000$ $0.000 \mathrm{E}+00 \quad 0.0000$ $0.000 E+00 \quad 0.0000$ $0.000 \mathrm{E}+00 \quad 0.0000$ $0.000 \mathrm{E}+00 \quad 0.0000$ $0.000 \mathrm{E}+00 \quad 0.0000$ $0.000 E+00 \quad 0.0000$ $0.000 \mathrm{E}+00 \quad 0.0000$

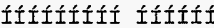
$0.000 \mathrm{E}+000.0000$
Milk

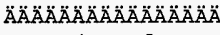
mrem/yr fract. . $0.000 \mathrm{E}+000.0000$ $0.000 \mathrm{E}+00 \quad 0.0000$ $0.000 E+00 \quad 0.0000$ $0.000 E+00 \quad 0.0000$ $0.000 \mathrm{E}+00 \quad 0.0000$ $0.000 E+00 \quad 0.0000$ $0.000 \mathrm{E}+00 \quad 0.0000$ $0.000 \mathrm{E}+00 \quad 0.0000$ ítítítíi ítítí $0.000 \mathrm{E}+00 \quad 0.0000$
A11 Pathways* $\ddot{A} \ddot{A} \ddot{A} \ddot{A} \ddot{A} \ddot{A} \ddot{A} \ddot{A} \ddot{A} \ddot{A} \ddot{A} \ddot{A} \ddot{A} \ddot{A} \ddot{A} \ddot{A} \ddot{A}$ mrem/yr fract. AAAAA AAAAAX $.069 \mathrm{E}-22 \quad 0.8308$ $9.524 \mathrm{E}-27 \quad 0.0001$
$0.1830 \mathrm{E}$ $1.830 \mathrm{E}-23 \quad 0.1423$ $3.444 \mathrm{E}-24 \quad 0.0268$ $0.000 \mathrm{E}+00 \quad 0.0000$ $0.000 \mathrm{E}+00 \quad 0.0000$ $\begin{array}{lll}0.000 E+00 & 0.0000\end{array}$ $0.000 \mathrm{E}+00 \quad 0.0000$ ítííítíi itííti $1.286 \mathrm{E}-221.0000$ 
Total Dose Contributions TDOSE(i,p,t) for Individual Radionuclides (i) and Pathways (p) As mrem/yr and Fraction of Total Dose At $t=1.000 \mathrm{E}+00$ years

0

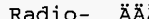
Nuclide mrem/Yr fract.

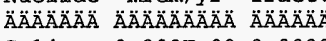
C-14 $0.000 \mathrm{E}+00 \quad 0.0000$ $\begin{array}{lll}\mathrm{C}-60 & 9.478 \mathrm{E}-23 & 0.8198\end{array}$ Cs-137 $9.439 \mathrm{E}-27 \quad 0.0001$ $\mathrm{Eu}-152 \quad 1.760 \mathrm{E}-23 \quad 0.1522$ $\begin{array}{lll}\mathrm{Eu}-154 & 3.223 \mathrm{E}-24 & 0.0279\end{array}$ Ni-63 $0.000 \mathrm{E}+00 \quad 0.0000$ $\mathrm{Pu}-239 \quad 0.000 \mathrm{E}+00 \quad 0.0000$ $\begin{array}{lll}\mathrm{Pu}-240 & 0.000 \mathrm{E}+00 & 0.0000\end{array}$ Sr-90 $0.000 \mathrm{E}+00 \quad 0.0000$ Íííítí íííítítí ítítít

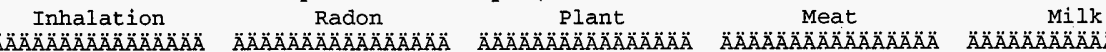
lation excludes radon) AAAAAAAÄÄÄÄÄÄÄ $\operatorname{mrem} / y r$ fract. $0.000 \mathrm{E}+00 \quad 0.0000$ $0.000 \mathrm{E}+00 \quad 0.0000$ $0.000 \mathrm{E}+00 \quad 0.0000$ $0.000 \mathrm{E}+00 \quad 0.0000$ $0.000 \mathrm{E}+00 \quad 0.0000$ $0.000 E+00 \quad 0.0000$ $0.000 \mathrm{E}+00 \quad 0.0000$ $0.000 \mathrm{E}+00 \quad 0.0000$ $0.000 \mathrm{E}+00 \quad 0.0000$ ítítítít ítítí

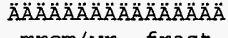

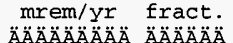
$\begin{array}{lllllll}0.000 \mathrm{E}+00 & 0.0000 & 0.000 \mathrm{E}+00 & 0.0000 & 0.000 \mathrm{E}+00 & 0.0000\end{array}$ $0.000 \mathrm{E}+000.0000$ $0.000 \mathrm{E}+00 \quad 0.0000$ $0.000 \mathrm{E}+00 \quad 0.0000$ $0.000 \mathrm{E}+00 \quad 0.0000$ $0.000 \mathrm{E}+00 \quad 0.0000$ $0.000 \mathrm{E}+00 \quad 0.0000$ $0.000 \mathrm{E}+0.0 \quad 0.0000$ $0.000 \mathrm{E}+00 \quad 0.0000$ íííííít ítítít $0.000 \mathrm{E}+00 \quad 0.0000$ $0.000 \mathrm{E}+00 \quad 0.0000$ $0.000 \mathrm{E}+00 \quad 0.0000$ $0.000 \mathrm{E}+00 \quad 0.0000$ $0.000 \mathrm{E}+00 \quad 0.0000$ $0.000 \mathrm{E}+00 \quad 0.0000$ $0.000 \mathrm{E}+00 \quad 0.0000$ íííííííi ííííí $0.000 \mathrm{E}+00 \quad 0.0000$ $0.000 \mathrm{E}+00 \quad 0.0000$ $0.000 \mathrm{E}+00 \quad 0.0000$ $0.000 E+00 \quad 0.0000$ $0.000 \mathrm{E}+00 \quad 0.0000$ $0.000 E+00 \quad 0.0000$ $0.000 \mathrm{E}+00 \quad 0.0000$ $0.000 \mathrm{E}+00 \quad 0.0000$ ífítítít ítííí $\begin{array}{lllllll}0.000 \mathrm{E}+00 & 0.0000 & 0.000 \mathrm{E}+00 & 0.0000 & 0.000 \mathrm{E}+00 & 0.0000\end{array}$
$\ddot{A} \ddot{A} \ddot{A} \ddot{A} \ddot{A} \ddot{A} \ddot{A} \ddot{A} \ddot{A} \ddot{A} \ddot{A} \ddot{A} \ddot{A} \ddot{A}$ mrem/yr fract. $0.000 \mathrm{E}+00 \quad 0.0000$ $0.000 \mathrm{E}+00 \quad 0.0000$ $0.000 \mathrm{E}+00 \quad 0.0000$ $0.000 \mathrm{E}+00 \quad 0.0000$ $0.000 \mathrm{E}+00 \quad 0.0000$ $0.000 \mathrm{E}+00 \quad 0.0000$ $0.000 \mathrm{E}+00 \quad 0.0000$ $0.000 \mathrm{E}+00 \quad 0.0000$ $0.000 \mathrm{E}+00 \quad 0.0000$ ííííííí fítítí $0.000 \mathrm{E}+00 \quad 0.0000$

Total Dose Contributions TDOSE $(i, p, t)$ for Individual Radionuclides (i) and Pathways (p) As mrem/yr and Fraction of Total Dose At $t=1.000 \mathrm{E}+00$ years Water Dependent Pathways

0 Fish Radon Plant

Meat Milk АВ̈ӒА

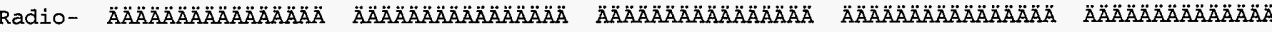
Nuclide mrem/yr fract. mrem/yr fract.

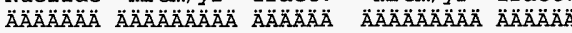

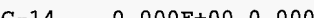
$\begin{array}{lllll}\mathrm{CO}-60 & 0.000 \mathrm{E}+00 & 0.0000 & 0.000 \mathrm{E}+00 & 0.0000\end{array}$ Cs-137 $0.000 \mathrm{E}+00 \quad 0.0000 \quad 0.000 \mathrm{E}+00 \quad 0.0000$

Eu-152 $0.000 E+00 \quad 0.0000 \quad 0.000 E+00 \quad 0.0000$

$\begin{array}{llllll}\mathrm{Eu}-154 & 0.000 \mathrm{E}+00 & 0.0000 & 0.000 \mathrm{E}+00 & 0.0000\end{array}$

$\mathrm{Ni}-63 \quad 0.000 \mathrm{E}+00 \quad 0.0000 \quad 0.000 \mathrm{E}+00 \quad 0.0000$

$\begin{array}{lllll}\mathrm{Pu}-239 & 0.000 \mathrm{E}+00 & 0.0000 & 0.000 \mathrm{E}+00 & 0.0000\end{array}$

$\begin{array}{llllll}\mathrm{Pu}-240 & 0.000 \mathrm{E}+00 & 0.0000 & 0.000 \mathrm{E}+00 & 0.0000\end{array}$

$\begin{array}{llllll}\mathrm{Sr}-90 & 0.000 \mathrm{E}+00 & 0.0000 & 0.000 \mathrm{E}+00 & 0.0000\end{array}$

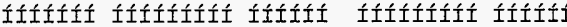

\section{$\mathrm{mrem} / \mathrm{yr}$ fract.}

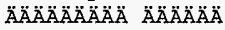
$\begin{array}{lllllll}0.000 \mathrm{E}+00 & 0.0000 & 0.000 \mathrm{E}+00 & 0.0000 & 0.000 \mathrm{E}+00 & 0.0000\end{array}$ $\begin{array}{lllllll}0.000 \mathrm{E}+00 & 0.0000 & 0.000 \mathrm{E}+00 & 0.0000 & 0.000 \mathrm{E}+00 & 0.0000\end{array}$ $\begin{array}{lllllll}0.000 \mathrm{E}+00 & 0.0000 & 0.000 \mathrm{E}+00 & 0.0000 & 0.000 \mathrm{E}+00 & 0.0000\end{array}$ $\begin{array}{lllllll}0.000 \mathrm{E}+00 & 0.0000 & 0.000 \mathrm{E}+00 & 0.0000 & 0.000 \mathrm{E}+00 & 0.0000\end{array}$ $\begin{array}{lllllll}0.000 \mathrm{E}+00 & 0.0000 & 0.000 \mathrm{E}+00 & 0.0000 & 0.000 \mathrm{E}+00 & 0.0000\end{array}$ $\begin{array}{lllllll}0.000 \mathrm{E}+00 & 0.0000 & 0.000 \mathrm{E}+00 & 0.0000 & 0.000 \mathrm{E}+00 & 0.0000\end{array}$ $\begin{array}{lllllll}0.000 \mathrm{E}+00 & 0.0000 & 0.000 \mathrm{E}+00 & 0.0000 & 0.000 \mathrm{E}+00 & 0.0000\end{array}$

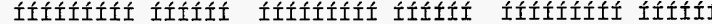
$\begin{array}{lllllllll}0.000 & 0.000 \mathrm{E}+00 & 0.0000\end{array}$ 0 *Sum of all water independent and dependent pathways. mrem/yr fract. $\ddot{A} \ddot{A} \ddot{A} \ddot{A} \ddot{A} \ddot{A} \ddot{A} \ddot{A} \ddot{A} \ddot{A} \ddot{A} \ddot{A} \ddot{A} \ddot{A}$ $0.000 \mathrm{E}+00 \quad 0.0000$ $0.000 \mathrm{E}+00 \quad 0.0000$ $0.000 \mathrm{E}+00 \quad 0.0000$ $0.000 \mathrm{E}+00 \quad 0.0000$ $0.000 \mathrm{E}+00 \quad 0.0000$ $0.000 E+00 \quad 0.0000$ $0.000 \mathrm{E}+00 \quad 0.0000$ $0.000 \mathrm{E}+00 \quad 0.0000$ $0.000 \mathrm{E}+00 \quad 0.0000$ İííííííi ííííí $0.000 \mathrm{E}+00 \quad 0.0000$
mem/Yr fract. mrem/Yr $9.478 \mathrm{E}-23 \quad 0.8198$ $9.439 \mathrm{E}-27 \quad 0.0001$ $1.760 \mathrm{E}-23 \quad 0.1522$ $0.000 \mathrm{E}+00 \quad 0.0000$ $0.000 \mathrm{E}+00 \quad 0.0000$ $0.000 E+00 \quad 0.0000$ $0.000 \mathrm{E}+00 \quad 0.0000$

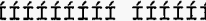
$1.156 \mathrm{E}-22 \quad 1.0000$
Soil

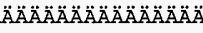

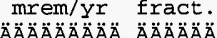
$0.000 \mathrm{E}+00 \quad 0.0000$ $0.000 E+00 \quad 0.0000$ $0.000 \mathrm{E}+00 \quad 0.0000$ $0.000 \mathrm{E}+00 \quad 0.0000$ $0.000 \mathrm{E}+00 \quad 0.0000$ $0.000 \mathrm{E}+00 \quad 0.0000$ $0.000 \mathrm{E}+00 \quad 0.0000$ $0.000 \mathrm{E}+00 \quad 0.0000$ $0.000 \mathrm{E}+00 \quad 0.0000$ ítííítí ítííi $0.000 \mathrm{E}+000.0000$ 
Total Dose Contributions TDOSE $(i, p, t)$ for Individual Radionuclides ( $i$ ) and pathways ( $p$ )

As mrem/yr and Fraction of Total Dose At $t=3.000 \mathrm{E}+00$ years water Independent Pathways (Inhalation excludes radon)

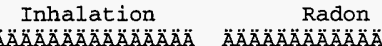
Radon Plant Meat

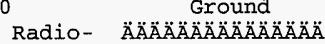
Nuclide mrem/Y⿱ fract. Plant Milk

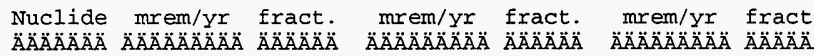
$\begin{array}{lllllll}C-14 & 0.000 E+00 & 0.0000 & 0.000 E+00 & 0.0000 & 0.000 E+00 & 0.0000\end{array}$ $\begin{array}{llllll}C 0-60 & 7.456 \mathrm{E}-23 & 0.7961 & 0.000 \mathrm{E}+00 & 0.0000\end{array}$ $E+00 \quad 0.0000$ $\begin{array}{lllll}\mathrm{Eu}-152 & 1.626 \mathrm{E}-23 & 0.1736 & 0.000 \mathrm{E}+00 & 0.0000\end{array}$ $\begin{array}{llllll}\mathrm{Eu}-154 & 2.823 \mathrm{E}-24 & 0.0301 & 0.000 \mathrm{E}+00 & 0.0000\end{array}$ $\begin{array}{lllll}\mathrm{Ni}-63 & 0.000 \mathrm{E}+00 & 0.0000 & 0.000 \mathrm{E}+00 & 0.0000\end{array}$ $\begin{array}{llllll}\mathrm{Pu}-239 & 0.000 \mathrm{E}+00 & 0.0000 & 0.000 \mathrm{E}+00 & 0.0000\end{array}$ $\begin{array}{lllll}\mathrm{Pu}-240 & 0.000 \mathrm{E}+00 & 0.0000 & 0.000 \mathrm{E}+00 & 0.0000\end{array}$ $0.000 \mathrm{E}+00 \quad 0.0000$ $\begin{array}{llllll}5 r-90 & 0.000 E+00 & 0.0000 & 0.000 E+00 & 0.0000\end{array}$ $0.000 E+00 \quad 0.0000$ $0.000 E+00 \quad 0.0000$ $0.000 \mathrm{E}+00 \quad 0.0000$ $0.000 \mathrm{E}+00 \quad 0.0000$ $0.000 \mathrm{E}+00 \quad 0.0000$ $0.000 \mathrm{E}+00 \quad 0.0000$ $0.000 \mathrm{E}+000.000$ Íííítíí ííííi

\section{AAAAAAAAAAAAAAAA} mrem/yr fract.

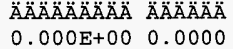
$0.000 \mathrm{E}+00 \quad 0.0000$ $0.000 E+00 \quad 0.0000$ $0.000 E+00 \quad 0.0000$ $0.000 \mathrm{E}+00 \quad 0.0000$ $0.000 E+00 \quad 0.0000$ $0.000 \mathrm{E}+00 \quad 0.0000$ $0.000 \mathrm{E}+00 \quad 0.0000$ $0.000 \mathrm{E}+00 \quad 0.0000$ ííítítít ítítí Ítíítíí tífítí $0.000 \mathrm{E}+00 \quad 0.0000$

\section{Meat} mrem/Yr fract.
$\ddot{A} \ddot{A} \ddot{A} \ddot{A} \ddot{A} \ddot{A} \ddot{A} \ddot{A} \ddot{A}$ Ä̈̈̈̈̈̈̈̈̈̈ $0.000 \mathrm{E}+00 \quad 0.0000$ $0.000 \mathrm{E}+00 \quad 0.0000$ $0.000 \mathrm{E}+00 \quad 0.0000$ $\begin{array}{lll}0.000 \mathrm{E}+00 & 0.0000\end{array}$ $0.000 \mathrm{E}+000.0000$ $0.000 \mathrm{E}+00 \quad 0.0000$ $0.000 \mathrm{E}+00 \quad 0.0000$ $0.000 \mathrm{E}+00 \quad 0.0000$ $0.000 \mathrm{E}+00 \quad 0.0000$ Íítítítí ítífí $0.000 \mathrm{E}+000.0000$

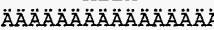
mrem/yr fract. $\ddot{\mathrm{A}} \ddot{\mathrm{A}} \ddot{\mathrm{A}} \ddot{\mathrm{A}} \ddot{\mathrm{A}} \ddot{\mathrm{A}} \ddot{\mathrm{A}} \ddot{\mathrm{A}} \ddot{\mathrm{A}}$ 苂̈̈$\ddot{\mathrm{A}} \ddot{\mathrm{A}} \ddot{\mathrm{A}}$ $0.000 \mathrm{E}+00 \quad 0.0000$ $0.000 \mathrm{E}+00 \quad 0.0000$ $0.000 \mathrm{E}+00 \quad 0.0000$ $0.000 \mathrm{E}+00 \quad 0.0000$ $0.000 \mathrm{E}+00 \quad 0.0000$ $0.000 \mathrm{E}+00 \quad 0.0000$ $0.000 \mathrm{E}+00 \quad 0.0000$ $0.000 \mathrm{E}+00 \quad 0.0000$ $0.000 \mathrm{E}+00 \quad 0.0000$ fítítítí 任任

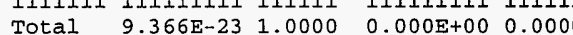

Total Dose Contributions $\operatorname{TDOSE}(i, p, t)$ for Individual Radionuclides (i) and Pathways (p) As mrem/yr and Fraction of Total Dose At $t=3.000 \mathrm{E}+00$ years As mrem/yr and Fraction of Total Dose At $t$
Water Dependent Pathways

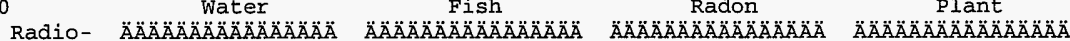
Nuclide mrem/yr fract. mrem/yr fract mrem/yr fract. mrem/yr fract.

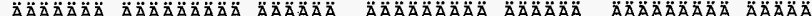
$0.0008+000.00000 .000 E+000.0000-0.000 E+000.0000$ C-14 $\begin{array}{llllll}C 0-60 & 0.000 \mathrm{E}+00 & 0.0000 & 0.000 \mathrm{E}+00 & 0.0000\end{array}$ $\begin{array}{llllll}C S-137 & 0.000 E+00 & 0.0000 & 0.000 E+00 & 0.0000\end{array}$ $\begin{array}{lllll}\mathrm{Eu}-152 & 0.000 \mathrm{E}+00 & 0.0000 & 0.000 \mathrm{E}+00 & 0.0000\end{array}$ $\begin{array}{llllll}\mathrm{Eu}-154 & 0.000 \mathrm{E}+00 & 0.0000 & 0.000 \mathrm{E}+00 & 0.0000\end{array}$ $\begin{array}{lllll}\mathrm{Ni}-63 & 0.000 \mathrm{E}+00 & 0.0000 & 0.000 \mathrm{E}+00 & 0.0000\end{array}$ $\begin{array}{llllll}\mathrm{Pu}-239 & 0.000 \mathrm{E}+00 & 0.0000 & 0.000 \mathrm{E}+00 & 0.0000\end{array}$ $\begin{array}{lllll}\mathrm{Pu}-240 & 0.000 \mathrm{E}+00 & 0.0000 & 0.000 \mathrm{E}+00 & 0.000\end{array}$

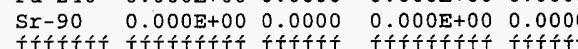
$\begin{array}{lllll}\text { Total } & 0.000 \mathrm{E}+00 & 0.0000 & 0.000 \mathrm{E}+00 & 0.0000\end{array}$ $0 * \mathrm{Sum}$ of all water independent and dependent pathways.

\section{Meat}

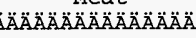
$\mathrm{mrem} / \mathrm{yr}$ fract. $\ddot{A} \ddot{A} \ddot{A} \ddot{A} \ddot{A} \ddot{A} \ddot{A} \ddot{A} \ddot{A} \ddot{A} \ddot{A} \ddot{A} \ddot{A} \ddot{A} \ddot{A}$ $0.000 \mathrm{E}+00 \quad 0.0000$ $\begin{array}{ll}0.000 \mathrm{E}+00 & 0.0000 \\ 0.000 \mathrm{E}+00 & 0.0000\end{array}$ $0.000 \mathrm{E}+00 \quad 0.0000$ $0.000 \mathrm{E}+00 \quad 0.0000$ $0.000 \mathrm{E}+00 \quad 0.0000$ $0.000 \mathrm{E}+00 \quad 0.0000$ $0.000 \mathrm{E}+00 \quad 0.0000$ $0.000 \mathrm{E}+00 \quad 0.0000$ $0.000 E+00 \quad 0.0000$ $0.000 \mathrm{E}+00 \quad 0.0000$ Ííííníí íííí

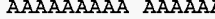
$0.000 \mathrm{E}+00 \quad 0.0000$ $0.000 \mathrm{E}+00 \quad 0.0000$ $0.000 \mathrm{E}+00 \quad 0.0000$ $0.000 \mathrm{E}+00 \quad 0.0000$ $0.000 \mathrm{E}+00 \quad 0.0000$ $0.000 E+00 \quad 0.0000$ $0.000 \mathrm{E}+00 \quad 0.0000$ $0.000 \mathrm{E}+00 \quad 0.0000$ $0.000 \mathrm{E}+00 \quad 0.0000$ ífífítít fífíf $0.000 \mathrm{E}+00 \quad 0.0000$ $0.000 E+00 \quad 0.0000$ $0.000 E+00 \quad 0.0000$ $0.000 \mathrm{E}+00 \quad 0.0000$ $0.000 \mathrm{E}+00 \quad 0.0000$ $0.000 \mathrm{E}+00 \quad 0.0000$ $0.000 \mathrm{E}+00 \quad 0.0000$ $0.000 \mathrm{E}+00 \quad 0.0000$ Íítítítíi íííníi íftítútí ítítít

Milk mrem/yr fract.

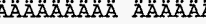
$0.000 \mathrm{E}+00 \quad 0.0000$ $0.000 \mathrm{E}+00 \quad 0.0000$ $0.000 E+00 \quad 0.0000$ $0.000 \mathrm{E}+00 \quad 0.0000$ $0.000 \mathrm{E}+00 \quad 0.0000$ $0.000 \mathrm{E}+00 \quad 0.0000$ $0.000 \mathrm{E}+00 \quad 0.0000$ $0.000 \mathrm{E}+00 \quad 0.0000$ $0.000 \mathrm{E}+00 \quad 0.000$ $0.000 \mathrm{E}+00 \quad 0.0000$

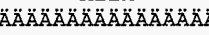

Soil

Ä

mrem/yr fract. $\ddot{A} \ddot{A} \ddot{A} \ddot{A} \ddot{A} \ddot{A} \ddot{A} \ddot{A} \ddot{A} \ddot{A} \ddot{A} \ddot{A} \ddot{A} \ddot{A}$ $0.000 E+00 \quad 0.0000$ $0.000 \mathrm{E}+00 \quad 0.0000$ $0.000 \mathrm{E}+00 \quad 0.0000$ $0.000 \mathrm{E}+00 \quad 0.0000$ $0.000 \mathrm{E}+00 \quad 0.0000$ $0.000 \mathrm{E}+00 \quad 0.0000$ $0.000 E+00 \quad 0.0000$ $0.000 E+00 \quad 0.0000$ $0.000 \mathrm{E}+00 \quad 0.0000$ ííítííí íííí $0.000 \mathrm{E}+00 \quad 0: 0000$
All Pathways* mrem/yx fract. $0.000 \mathrm{E}+00 \quad 0.0000$ $\begin{array}{lll}7.456 \mathrm{E}-23 & 0.7961\end{array}$ $9.271 \mathrm{E}-27 \quad 0.0001$ $1.626 \mathrm{E}-23 \quad 0.1736$ $2.823 E-24 \quad 0.0301$ $0.000 \mathrm{E}+00 \quad 0.0000$ $0.000 \mathrm{E}+00 \quad 0.0000$ $0.000 \mathrm{E}+00 \quad 0.0000$ $0.000 E+00 \quad 0.0000$ ífítítíti fítítí $9.366 \mathrm{E}-231.0000$ 
1RESRAD, Version 6.3 T Limit $=180$ days $\quad 10 / 10 / 2005 \quad 09: 46$ Page 17

Summary : 116-K-2 Trench (East End) Cleanup Verification RESRAD Calculation

File : 116-K-2-DZ-swC.RAD

Total Dose Contributions TDOSE $(i, p, t)$ for Individual Radionuclides (i) and Pathways (p)

As mrem/yr and Fraction of Total Dose At $t=1.300 \mathrm{E}+01$ years

0 Water Independent Pathways (Inhalation excludes radon) Inhalation Radon Plant Meat Milk

RadioNucli Inhalation

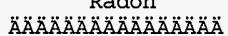
$\ddot{A} \ddot{A} \ddot{A} \ddot{A} \ddot{A} \ddot{A} \ddot{A} \ddot{A} \ddot{A} \ddot{A} \ddot{A} \ddot{A} \ddot{A} \ddot{A} \ddot{A}$ $\begin{array}{lllll}C-14 & 0.000 \mathrm{E}+00 & 0.0000 & 0.000 \mathrm{E}+00 & 0.0000\end{array}$ $\begin{array}{llllll}\mathrm{Co}-60 & 2.247 \mathrm{E}-23 & 0.6440 & 0.000 \mathrm{E}+00 & 0.0000\end{array}$ C $-137 \quad 8.473=-27 \quad 0.00020 .000 E+000.0000$ $\begin{array}{lllll}\mathrm{Eu}-152 & 1.096 \mathrm{E}-23 & 0.3141 & 0.000 \mathrm{E}+00 & 0.0000\end{array}$ $\begin{array}{lllll}\mathrm{Eu}-154 & 1.454 \mathrm{E}-24 & 0.0417 & 0.000 \mathrm{E}+00 & 0.0000\end{array}$ $\begin{array}{lllll}\mathrm{Ni}-63 & 0.000 \mathrm{E}+00 & 0.0000 & 0.000 \mathrm{E}+00 & 0.0000\end{array}$ $\begin{array}{llllll}\mathrm{Pu}-239 & 0.000 \mathrm{E}+00 & 0.0000 & 0.000 \mathrm{E}+00 & 0.0000\end{array}$ $\begin{array}{lllll}\mathrm{Pu}-240 & 0.000 \mathrm{E}+00 & 0.0000 & 0.000 \mathrm{E}+00 & 0.0000\end{array}$ $\begin{array}{llllll}S r-90 & 0.000 E+00 & 0.0000 & 0.000 E+00 & 0.0000\end{array}$

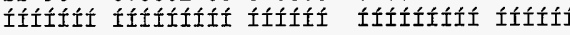
Total $3.490 \mathrm{E}-231.0000$ iffítítíi ífííti

\section{mrem/yr fract.} $\ddot{A} \ddot{A} \ddot{A} \ddot{A} \ddot{A} \ddot{A} \ddot{A} \ddot{A} \ddot{A} \ddot{A} \ddot{A} \ddot{A} \ddot{A} \ddot{A} \ddot{A}$ $0.000 \mathrm{E}+00 \quad 0.0000$ $0.000 \mathrm{E}+00 \quad 0.0000$

$0.000 E+00 \quad 0.0000$

$0.000 \mathrm{E}+00 \quad 0.0000$

$0.000 \mathrm{E}+00 \quad 0.0000$

$0.000 \mathrm{E}+00 \quad 0.0000$

$0.000 \mathrm{E}+00 \quad 0.0000$

$0.000 \mathrm{E}+00 \quad 0.0000$

$0.000 E+00 \quad 0.0000$

Íííítííí ííííi

$0.000 \mathrm{E}+000.0000$

mrem/yr fract.

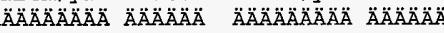
$0.000 \mathrm{E}+00 \quad 0.0000$ $0.000 \mathrm{E}+00 \quad 0.0000$ $0.000 \mathrm{E}+00 \quad 0.0000$ $0.000 \mathrm{E}+00 \quad 0.0000$

$0.000 \mathrm{E}+00 \quad 0.0000$ $0.000 \mathrm{E}+00 \quad 0.0000$ $0.000 \mathrm{E}+00 \quad 0.0000$ $0.000 \mathrm{E}+00 \quad 0.0000$ $0.000 \mathrm{E}+00 \quad 0.0000$ ííííííí ííííí $0.000 \mathrm{E}+000.0000$ $0.000 E+00 \quad 0.0000$ $0.000 \mathrm{E}+00 \quad 0.0000$ $0.000 \mathrm{E}+00 \quad 0.0000$ $0.000 \mathrm{E}+00 \quad 0.0000$ $0.000 \mathrm{E}+00 \quad 0.0000$ $0.000 \mathrm{E}+00 \quad 0.0000$ $0.000 \mathrm{E}+00 \quad 0.0000$ $0.000 E+00 \quad 0.0000$ $0.000 \mathrm{E}+00 \quad 0.0000$ ítítítít ítítít Íítífítít ífííí
$0.000 \mathrm{E}+000.0000$ Total Dose Contributions TDOSE $(i, p, t)$ for Individual Radionuclides ( $i$ ) and Pathways (p) As mrem/yr and Fraction of Total Dose At $t=1.300 \mathrm{E}+01$ years

0 Water Dependent Pathways Fish Plant

Meat

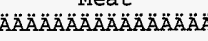

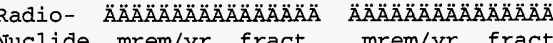

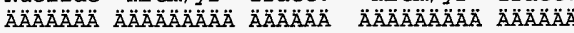
$\begin{array}{lllll}C-14 & 0.000 \mathrm{E}+00 & 0.0000 & 0.000 \mathrm{E}+00 & 0.0000\end{array}$ $\begin{array}{llllll}C 0-60 & 0.000 \mathrm{E}+00 & 0.0000 & 0.000 \mathrm{E}+00 & 0.0000\end{array}$ $\begin{array}{llllll}\mathrm{Cs}-137 & 0.000 \mathrm{E}+00 & 0.0000 & 0.000 \mathrm{E}+00 & 0.0000\end{array}$ $\begin{array}{lllll}\mathrm{Eu}-152 & 0.000 \mathrm{E}+00 & 0.0000 & 0.000 \mathrm{E}+00 & 0.0000\end{array}$ $\mathrm{Eu}-154 \quad 0.000 \mathrm{E}+00 \quad 0.0000 \quad 0.000 \mathrm{E}+00 \quad 0.0000$ Ni-63 $0.000 \mathrm{E}+00 \quad 0.0000 \quad 0.000 \mathrm{E}+00 \quad 0.0000$ $\begin{array}{lllll}\mathrm{Pu}-239 & 0.000 \mathrm{E}+00 & 0.0000 & 0.000 \mathrm{E}+00 & 0.0000\end{array}$ $\begin{array}{lllll}\mathrm{Pu}-240 & 0.000 \mathrm{E}+00 & 0.0000 & 0.000 \mathrm{E}+00 & 0.0000\end{array}$ $\begin{array}{lllll}\mathrm{S} x-90 & 0.000 \mathrm{E}+00 & 0.0000 & 0.000 \mathrm{E}+00 & 0.0000\end{array}$

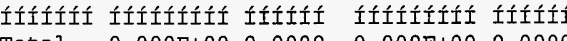
Total $0.000 \mathrm{E}+000.0000 \quad 0.000 \mathrm{E}+000.0000 \quad 0.000 \mathrm{E}+000.0000$ $0 *$ Sum of all water independent and dependent pathways. mrem/yr fract. mrem/yr fract. mrem/yr fract. Ä $0.000 \mathrm{E}+000.0000$ $0.000 \mathrm{E}+00 \quad 0.0000$ $0.000 \mathrm{E}+00 \quad 0.0000$ $0.000 \mathrm{E}+00 \quad 0.0000$ $0.000 \mathrm{E}+00 \quad 0.0000$ $0.000 \mathrm{E}+00 \quad 0.0000$ $0.000 \mathrm{E}+00 \quad 0.0000$ $0.000 \mathrm{E}+00 \quad 0.0000$ $0.000 \mathrm{E}+00 \quad 0.0000$

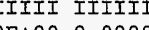
$0.000 \mathrm{E}+000.0000 \quad 0.000 \mathrm{E}+000.0000$ $\begin{array}{llll}0.000 \mathrm{E}+00 & 0.0000 & 0.000 \mathrm{E}+00 & 0.0000\end{array}$ $0.000 \mathrm{E}+00 \quad 0.0000 \quad 0.000 \mathrm{E}+00 \quad 0.0000$ $0.000 \mathrm{E}+00 \quad 0.0000 \quad 0.000 \mathrm{E}+00 \quad 0.0000$ $0.000 \mathrm{E}+00 \quad 0.0000 \quad 0.000 \mathrm{E}+00 \quad 0.0000$ $0.000 \mathrm{E}+000.0000 \quad 0.000 \mathrm{E}+00 \quad 0.0000$ $\begin{array}{lllll}0.000 \mathrm{E}+00 & 0.0000 & 0.000 \mathrm{E}+00 & 0.0000\end{array}$ $0.000 \mathrm{E}+00 \quad 0.0000 \quad 0.000 \mathrm{E}+00 \quad 0.0000$ $\begin{array}{llll}0.000 \mathrm{E}+00 & 0.0000 & 0.000 \mathrm{E}+00 \quad 0.0000\end{array}$

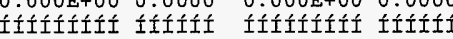
$0.000 \mathrm{E}+00 \quad 0.0000 \quad 0.000 \mathrm{E}+00 \quad 0.0000$

$\ddot{A} \ddot{A} \ddot{A} \ddot{A} \ddot{A} \ddot{A} \ddot{A} \ddot{A} \ddot{A} \ddot{A} \ddot{A} \ddot{A} \ddot{A}$ mrem/Yr fract.

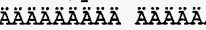
$0.000 \mathrm{E}+00 \quad 0.0000$ $0.000 \mathrm{E}+00 \quad 0.0000$ $0.000 \mathrm{E}+00 \quad 0.0000$ $0.000 \mathrm{E}+00 \quad 0.000$ $0.000 \mathrm{E}+00 \quad 0.0000$ $0.000 \mathrm{E}+00 \quad 0.0000$ $0.000 \mathrm{E}+00 \quad 0.0000$ $0.000 E+00 \quad 0.0000$ $0.000 \mathrm{E}+00 \quad 0.0000$ Ítítítít ftítít $0.000 \mathrm{E}+00 \quad 0.0000$

Milk $\stackrel{A}{ } \ddot{A} \ddot{A} \ddot{A} \ddot{A} \ddot{A} \ddot{A} \ddot{A} \ddot{A} \ddot{A} \ddot{A} \ddot{A} \ddot{A} \ddot{A}$ mrem/yr fract $0.000 \mathrm{E}+00 \quad 0.0000$ $0.000 \mathrm{E}+00 \quad 0.0000$ $0.000 \mathrm{E}+00 \quad 0.0000$ $0.000 \mathrm{E}+00 \quad 0.0000$ $0.000 \mathrm{E}+00 \quad 0.0000$ $0.000 \mathrm{E}+00 \quad 0.0000$ $0.000 E+00 \quad 0.0000$ $0.000 \mathrm{E}+00 \quad 0.0000$ $0.000 \mathrm{E}+00 \quad 0.000$ Ííííííí ífítít $0.000 \mathrm{E}+00 \quad 0.0000$
Soil

满 mrem/yr fract. Ä $0.000 E+00 \quad 0.0000$ $0.000 E+00 \quad 0.0000$ $0.000 E+00 \quad 0.0000$ $0.000 \mathrm{E}+00 \quad 0.0000$ $0.000 \mathrm{E}+00 \quad 0.0000$ $0.000 \mathrm{E}+00 \quad 0.0000$ $0.000 E+00 \quad 0.0000$ $0.000 \mathrm{E}+00 \quad 0.0000$ $0.000 \mathrm{E}+00 \quad 0.0000$ ítífítit fitifí $0.000 \mathrm{E}+000.0000$ 
Total Dose Contributions $\operatorname{TDOSE}(i, p, t)$ for Individual Radionuclides ( $i$ ) and Pathways (p)

As mrem/yr and Fraction of Total Dose At $t=3.000 E+01$ years water Independent Pathways (Inhalation excludes radon)

Ground

Inhalation

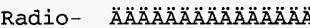
Nuclide mrem/yr fract. $\ddot{A} \ddot{A} \ddot{A} \ddot{A} \ddot{A} \ddot{A} \ddot{A}$ 苂 $\ddot{A} \ddot{A} \ddot{A} \ddot{A} \ddot{A} \ddot{A} \ddot{A} \ddot{A} \quad \ddot{A} \ddot{A} \ddot{A} \ddot{A}$ $\begin{array}{lll}C-14 & 0.000 \mathrm{E}+00 \quad 0.0000\end{array}$ Co-60 2.925E-24 0.3246 $\begin{array}{llll}\mathrm{Cs}-137 & 7.272 \mathrm{E}-27 & 0.0008\end{array}$ $\begin{array}{lll}\mathrm{Eu}-152 & 5.607 \mathrm{E}-24 & 0.6223\end{array}$ $\begin{array}{lll}\mathrm{Eu}-154 & 4.706 \mathrm{E}-25 & 0.0522\end{array}$ $\mathrm{Ni}-63 \quad 0.000 \mathrm{E}+00 \quad 0.0000$ $\begin{array}{lll}\mathrm{Pu}-239 & 0.000 \mathrm{E}+00 \quad 0.0000\end{array}$ $\begin{array}{lll}\text { Pu-240 } & 0.000 E+00 & 0.0000\end{array}$ $\begin{array}{llll}\mathrm{Sr}-90 & 0.000 \mathrm{E}+00 & 0.0000\end{array}$

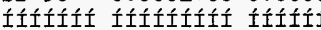
Total $9.010 \mathrm{E}-241.0000$

$$
\text { Radon }
$$$$
\text { Plant }
$$

$$
\text { Meat }
$$

\section{AAAAAAAAAAAAÄ} $m r e m / y r$ fract.

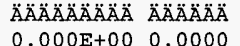
$0.000 \mathrm{E}+00 \quad 0.0000$ $0.000 \mathrm{E}+00 \quad 0.0000$ $0.000 \mathrm{E}+00 \quad 0.0000$ $0.000 E+00 \quad 0.0000$ $0.000 \mathrm{E}+00 \quad 0.0000$ $0.000 \mathrm{E}+00 \quad 0.0000$ $0.000 \mathrm{E}+00 \quad 0.0000$ $0.000 E+00 \quad 0.0000$ ítítítíí ítíít Ä mrem/yr fract.

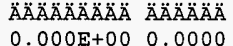
$0.000 \mathrm{E}+00 \quad 0.0000$ $0.000 \mathrm{E}+00 \quad 0.0000$ $0.000 \mathrm{E}+00 \quad 0.0000$ $0.000 E+00 \quad 0.0000$ $0.000 \mathrm{E}+00 \quad 0.0000$ $0.000 E+00 \quad 0.0000$ $0.000 E+00 \quad 0.0000$ $0.000 \mathrm{E}+00 \quad 0.0000$ fítitíít ííít $0.000 \mathrm{E}+00 \quad 0.0000$ mrem/yr fract. $\mathrm{mrem} / \mathrm{yr}$ fract.

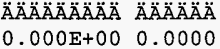
$0.000 \mathrm{E}+00 \quad 0.0000$ $0.000 \mathrm{E}+00 \quad 0.0000$ $0.000 \mathrm{E}+00 \quad 0.0000$ $0.000 \mathrm{E}+00 \quad 0.0000$ $0.000 \mathrm{E}+00 \quad 0.0000$ $0.000 \mathrm{E}+00 \quad 0.0000$ $0.000 \mathrm{E}+00 \quad 0.0000$ $0.000 \mathrm{E}+00 \quad 0.0000$ íífítíít ítíít $0.000 \mathrm{E}+00 \quad 0.0000$ mrem/yr fract. mrem/yr fract.

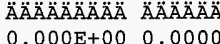
$0.000 \mathrm{E}+00 \quad 0.0000$ $0.000 \mathrm{E}+00 \quad 0.0000$ $0.000 \mathrm{E}+00 \quad 0.0000$ $0.000 \mathrm{E}+00 \quad 0.0000$ $0.000 \mathrm{E}+00 \quad 0.0000$ $0.000 \mathrm{E}+00 \quad 0.0000$ $0.000 \mathrm{E}+00 \quad 0.0000$ $0.000 \mathrm{E}+00 \quad 0.0000$

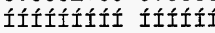
$0.000 \mathrm{E}+00 \quad 0.0000$

$$
\text { Milk }
$$

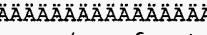
mrem/yr fract. $\ddot{A} \ddot{A} \ddot{A} \ddot{A} \ddot{A} \ddot{A} \ddot{A} \ddot{A} \ddot{A}$ A $\ddot{A} \ddot{A} \ddot{A} \ddot{A} \ddot{A}$ $0.000 \mathrm{E}+00 \quad 0.0000$ $0.000 \mathrm{E}+00 \quad 0.0000$ $0.000 \mathrm{E}+00 \quad 0.0000$ $0.000 \mathrm{E}+00 \quad 0.0000$ $0.000 \mathrm{E}+00 \quad 0.0000$ $0.000 \mathrm{E}+00 \quad 0.0000$ $0.000 \mathrm{E}+00 \quad 0.0000$ $0.000 \mathrm{E}+00 \quad 0.0000$ $0.000 \mathrm{E}+00 \quad 0.0000$ Itífítíti ítítí $0.000 \mathrm{E}+00 \quad 0.0000$

Total Dose Contributions TDOSE(i,p,t) for Individual Radionuclides (i) and Pathways (p) As mrem/yr and Fraction of Total Dose At $t=3.000 \mathrm{E}+01$ years

0

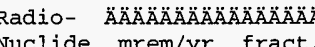
Nuclide $\mathrm{mrem} / \mathrm{yr}$ fract.

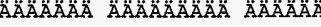
$\begin{array}{lll}\mathrm{C}-14 & 0.000 \mathrm{E}+00 & 0.0000\end{array}$ Co-60 $0.000 \mathrm{E}+00 \quad 0.0000$ Cs-137 $0.000 \mathrm{E}+00 \quad 0.000$ $\begin{array}{lll}\text { Eu-152 } & 0.000 \mathrm{E}+00 \quad 0.0000\end{array}$ $\mathrm{Eu}-154 \quad 0.000 \mathrm{E}+00 \quad 0.0000$ $\mathrm{Ni}-63 \quad 0.000 \mathrm{E}+00 \quad 0.0000$ $\begin{array}{lll}\mathrm{Pu}-239 & 0.000 \mathrm{E}+00 \quad 0.0000\end{array}$ $\mathrm{Pu}-240 \quad 0.000 \mathrm{E}+00 \quad 0.000$ $\begin{array}{lll}\mathrm{Sr}-90 & 0.000 \mathrm{E}+00 & 0.0000\end{array}$

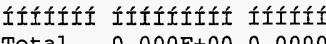
$0.000 \mathrm{E}+00 \quad 0.0000$

\section{Fish}

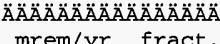

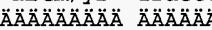

$0.000 \mathrm{E}+00 \quad 0.0000$ $0.000 E+00 \quad 0.0000$ $0.000 \mathrm{E}+00 \quad 0.0000$ $0.000 \mathrm{E}+00 \quad 0.0000$ $0.000 \mathrm{E}+00 \quad 0.0000$ $0.000 \mathrm{E}+00 \quad 0.0000$ $0.000 \mathrm{E}+00 \quad 0.0000$ $0.000 \mathrm{E}+00 \quad 0.0000$ $0.000 \mathrm{E}+00 \quad 0.0000$ Ííííííí íííít

$$
\text { Radon }
$$

$$
\text { water Dependent Pathways }
$$
mrem/yr fract.

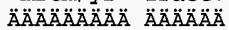
$0.000 \mathrm{E}+00 \quad 0.0000$ $0.000 E+00 \quad 0.0000$ $0.000 E+00 \quad 0.0000$ $0.000 \mathrm{E}+00 \quad 0.0000$ $0.000 \mathrm{E}+00 \quad 0.0000$ $0.000 E+00 \quad 0.0000$ $0.000 \mathrm{E}+00 \quad 0.0000$ $0.000 \mathrm{E}+00 \quad 0.0000$ $0.000 \mathrm{E}+00 \quad 0.0000$ fifííííí ítíííi $0.000 \mathrm{E}+00 \quad 0.0000$ pathways.

\section{Plant}

AAAAAAAAAAÄÄÄÄ

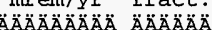
$0.000 \mathrm{E}+00 \quad 0.0000$ $0.000 \mathrm{E}+00 \quad 0.0000$ $0.000 \mathrm{E}+00 \quad 0.0000$ $0.000 \mathrm{E}+00 \quad 0.0000$ $0.000 E+00 \quad 0.0000$ $0.000 E+00 \quad 0.0000$ $0.000 \mathrm{E}+00 \quad 0.0000$ $0.000 \mathrm{E}+00 \quad 0.0000$ $0.000 \mathrm{E}+00 \quad 0.0000$ Íííííííi ííííí $0.000 \mathrm{E}+00 \quad 0.0000$
Meat

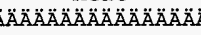
mrem/yr fract. A $0.000 \mathrm{E}+00 \quad 0.0000$ $0.000 \mathrm{E}+00 \quad 0.0000$ $0.000 \mathrm{E}+00 \quad 0.0000$ $0.000 \mathrm{E}+00 \quad 0.0000$ $0.000 \mathrm{E}+00 \quad 0.0000$ $0.000 \mathrm{E}+00 \quad 0.0000$ $0.000 \mathrm{E}+00 \quad 0.0000$ $0.000 \mathrm{E}+00 \quad 0.0000$ $0.000 \mathrm{E}+00 \quad 0.0000$ Íííííííí ííííis $0.000 \mathrm{E}+00 \quad 0.0000$
Milk ÄA mrem/yr fract.

AAAAAAAAB AAAAAX $0.000 \mathrm{E}+00 \quad 0.0000$ $0.000 \mathrm{E}+00 \quad 0.0000$ $0.000 E+00 \quad 0.0000$ $0.000 \mathrm{E}+00 \quad 0.0000$ $0.000 E+00 \quad 0.0000$ $0.000 \mathrm{E}+00 \quad 0.0000$ $0.000 \mathrm{E}+00 \quad 0.0000$ $0.000 \mathrm{E}+00 \quad 0.0000$ ífíififít íííít $0.000 \mathrm{E}+00 \quad 0.0000$
Soil

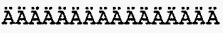
mrem/yr fract. $\ddot{A} \ddot{A} \ddot{A} \ddot{A} \ddot{A} \ddot{A} \ddot{A} \ddot{A} \ddot{A} \ddot{A}$ Ä $\ddot{A} \ddot{A} \ddot{A} \ddot{A} \ddot{A}$ $0.000 \mathrm{E}+00 \quad 0.0000$ $0.000 \mathrm{E}+00 \quad 0.0000$ $0.000 \mathrm{E}+00 \quad 0.0000$ $0.000 \mathrm{E}+00 \quad 0.0000$ $0.000 \mathrm{E}+00 \quad 0.0000$ $0.000 \mathrm{E}+00 \quad 0.0000$ $0.000 \mathrm{E}+00 \quad 0.0000$ $0.000 E+00 \quad 0.0000$ $0.000 \mathrm{E}+00 \quad 0.0000$ ítítitití ítitín $0.000 \mathrm{E}+00 \quad 0.0000$ 


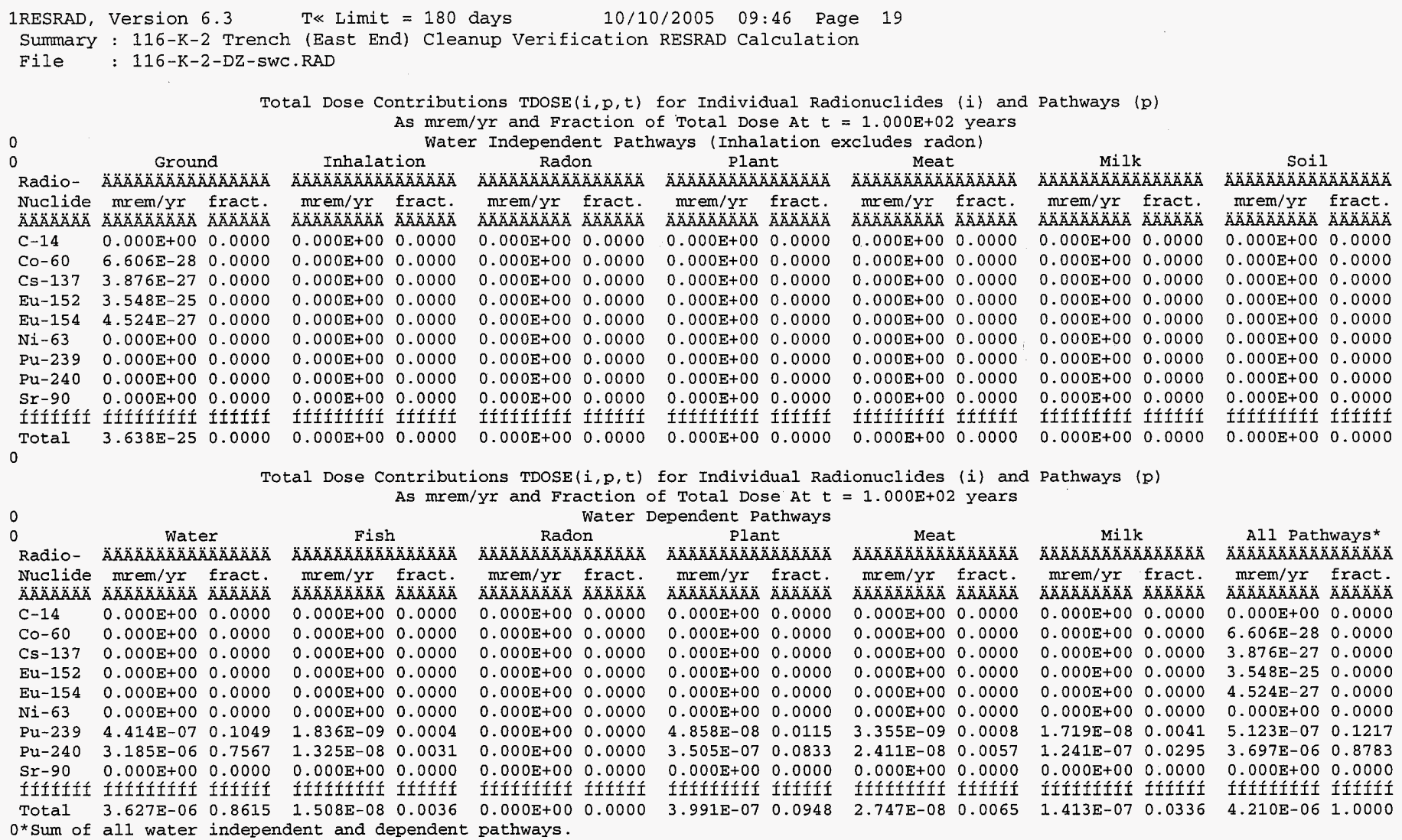




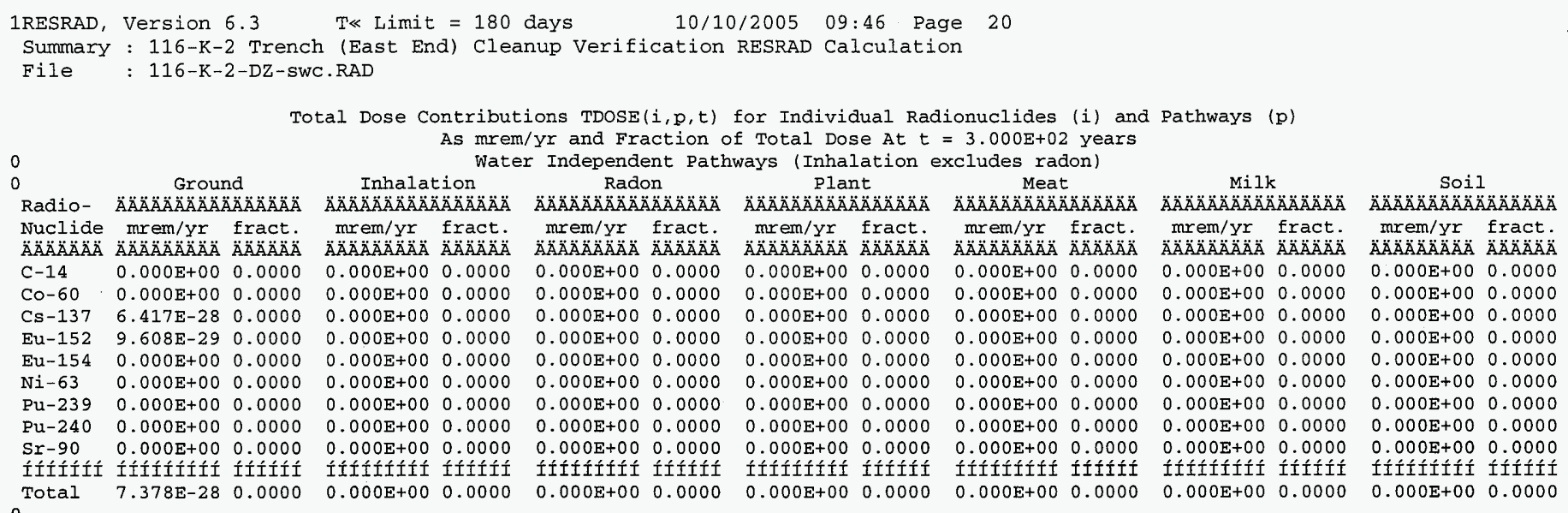

$$
0
$$

Total Dose Contributions TDOSE $(i, p, t)$ for Individual Radionuclides ( $i$ ) and Pathways (p) As mrem/yr and Fraction of Total Dose At $t=3.000 \mathrm{E}+02$ years

0 As mrem/yr and Fraction of Total Dose At
Water Dependent Pathways Fish Plant

Meat Ӓ̈Ӓ̈̈̈̈̈̈̈̈̈̈̈̈̈̈̈̈̈̈̈̈̈̈̈

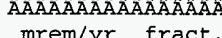
mrem/Yr fract. $0.000 \mathrm{E}+00 \quad 0.0000$ $0.000 E+00 \quad 0.0000$ $0.000 \mathrm{E}+00 \quad 0.0000$ $0.000 \mathrm{E}+00 \quad 0.0000$ $0.000 \mathrm{E}+00 \quad 0.0000$ $0.000 \mathrm{E}+00 \quad 0.0000$ $0.000 \mathrm{E}+00 \quad 0.0000$ $0.000 \mathrm{E}+00 \quad 0.0000$ $0.000 \mathrm{E}+00 \quad 0.0000$ íítítíti ítítí $0.000 \mathrm{E}+00 \quad 0.0000$ mrem/yr fract. mrem/yr fract. A $\begin{array}{lllll}0.000 E+00 & 0.0000 & 0.000 E+00 & 0.0000\end{array}$ $\begin{array}{lllll}0.000 \mathrm{E}+00 & 0.0000 & 0.000 \mathrm{E}+00 & 0.0000\end{array}$ $\begin{array}{lllll}0.000 \mathrm{E}+00 & 0.0000 & 0.000 \mathrm{E}+00 & 0.0000\end{array}$ $\begin{array}{lllll}0.000 \mathrm{E}+00 & 0.0000 & 0.000 \mathrm{E}+00 & 0.0000\end{array}$ $\begin{array}{lllll}0.000 \mathrm{E}+00 & 0.0000 & 0.000 \mathrm{E}+00 & 0.0000\end{array}$ $\begin{array}{llll}0.000 \mathrm{E}+00 & 0.0000 & 0.000 \mathrm{E}+00 & 0.0000\end{array}$ $\begin{array}{llll}5.243 \mathrm{E}-06 & 0.0118 & 3.815 \mathrm{E}-07 & 0.0009\end{array}$ $\begin{array}{lllll}3.714 \mathrm{E}-05 & 0.0834 & 2.598 \mathrm{E}-06 & 0.0058\end{array}$ $\begin{array}{lllll}0.000 E+00 & 0.0000 & 0.000 E+00 & 0.0000\end{array}$

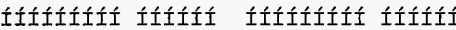
$\begin{array}{llll}4.239 \mathrm{E}-05 & 0.0951 & 2.980 \mathrm{E}-06 & 0.0067\end{array}$ mrem/yr fract.

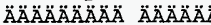
$0.000 \mathrm{E}+00 \quad 0.0000$ $0.000 \mathrm{E}+00 \quad 0.0000$ $0.000 \mathrm{E}+00 \quad 0.0000$ $0.000 \mathrm{E}+00 \quad 0.0000$ $0.000 \mathrm{E}+00 \quad 0.0000$ $0.000 \mathrm{E}+00 \quad 0.0000$ $1.847 \mathrm{E}-06 \quad 0.0041$ $1.324 \mathrm{E}-05 \quad 0.0297$ $0.000 \mathrm{E}+00 \quad 0.0000$ $1.509 \mathrm{E}-05 \quad 0.0339$
Al1 Pathways * AAAAAAAAA Mract. $0.000=+00$ 0.0000 $0.000 \mathrm{E}+00 \quad 0.0000$ $6.417 \mathrm{E}-28 \quad 0.0000$ $9.608 \mathrm{E}-290.0000$ $0.000 \mathrm{E}+00 \quad 0.0000$ $0.000 \mathrm{E}+00 \quad 0.0000$ $5.511 \mathrm{E}-05 \quad 0.1237$ $3.904 \mathrm{E}-04 \quad 0.8763$ $0.000 \mathrm{E}+00 \quad 0.0000$ Íííííííí ííííí $4.455 \mathrm{E}-041.0000$ Total
$0 *$ Sum of all water independent and dependent pathways 
CVP-2006-00001

Rev. 0

1RESRAD, Version 6.3 T« Limit $=180$ days $\quad 10 / 10 / 2005 \quad 09: 46$ Page 21 Summary : 116-K-2 Trench (East End) Cleanup Verification RESRAD Calculation File : 116-K-2-DZ-SWC.RAD

Total Dose Contributions TDOSE $(i, p, t)$ for Individual Radionuclides (i) and Pathways (p) As mrem/yr and Fraction of Total Dose At $t=1.000 \mathrm{E}+03$ years water Independent Pathways (Inhalation excludes radon) halation Plant Meat Radio- Ground

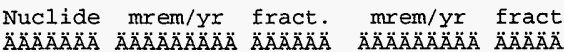

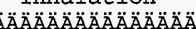
Radon

$1 k$ Soil $\begin{array}{lllll}\text { AAAAAAA } & \text { AAAAAAAA AAAAA } & \text { AAAAAAAAA } & \text { AAAAAA } \\ \mathrm{C}-14 & 0.000 \mathrm{E}+00 & 0.0000 & 0.000 \mathrm{E}+00 & 0.0000\end{array}$ $\begin{array}{llllll}\mathrm{CO}-60 & 0.000 \mathrm{E}+00 & 0.0000 & 0.000 \mathrm{E}+00 & 0.0000\end{array}$

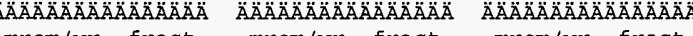

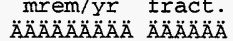
$\begin{array}{lllllll}0.000 \mathrm{E}+00 & 0.0000 & 0.000 \mathrm{E}+00 & 0.0000 & 0.000 \mathrm{E}+00 & 0.0000\end{array}$ $\begin{array}{lllllll}0.000 \mathrm{E}+00 & 0.0000 & 0.000 \mathrm{E}+00 & 0.0000 & 0.000 \mathrm{E}+00 & 0.0000\end{array}$ $\begin{array}{llllllll}0.000 E+00 & 0.0000 & 0.000 E+00 & 0.0000 & 0.000 E+00 & 0.0000\end{array}$ $0.000 \mathrm{E}+00 \quad 0.0000$ Eu-152 $0.000 E+00 \quad 0.0000$ $\mathrm{Eu}-154 \quad 0.000 \mathrm{E}+00 \quad 0.0000$ $\mathrm{Ni}-63 \quad 0.000 \mathrm{E}+00 \quad 0.0000$ $\mathrm{Pu}-239 \quad 0.000 \mathrm{E}+00 \quad 0.0000$ $0.000 \mathrm{E}+00 \quad 0.0$ $0.000 \mathrm{E}+00 \quad 0.0000$ $0.000 \mathrm{E}+00 \quad 0.000$ $0.000 \mathrm{E}+00 \quad 0.0000$ $3.316 \mathrm{E}-27 \quad 0.0000 \quad 0.000 \mathrm{E}+00 \quad 0.0000$ $\begin{array}{llllll}\mathrm{Sr}-90 & 0.000 \mathrm{E}+00 & 0.0000 & 0.000 \mathrm{E}+00 & 0.0000\end{array}$ $\begin{array}{llllll}0.000 \mathrm{E}+00 & 0.0000 & 0.000 \mathrm{E}+00 & 0.0000 & 0.000 \mathrm{E}+00 & 0.0000\end{array}$ $\begin{array}{llllll}0.000 \mathrm{E}+00 & 0.0000 & 0.000 \mathrm{E}+00 & 0.0000 & 0.000 \mathrm{E}+00 & 0.0000\end{array}$ $\begin{array}{lllllll}0.000 \mathrm{E}+00 & 0.0000 & 0.000 \mathrm{E}+00 & 0.0000 & 0.000 \mathrm{E}+00 & 0.0000\end{array}$ $0.000 \mathrm{E}+00 \quad 0.0000$ Ífítítít if́ítí $0.000 \mathrm{E}+000.0000 \quad 0.000 \mathrm{E}+000.0000$

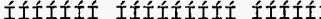
Total $3.316 \mathrm{E}-270.0000$ íífíítíí íííít

Total Dose Contributions TDOSE(i,p,t) for Individual Radionuclides ( $i$ ) and Pathways (p) As mrem/yr and Fraction of Total Dose At $t=1.000 \mathrm{E}+03$ years 0 Water Fish Radon $\begin{aligned} & \text { Water Dependent Pathw } \\ & 0 \text { Plant }\end{aligned}$ Radon Plant Milk

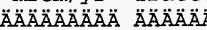
$0.000 \mathrm{E}+00 \quad 0.0000$ $0.000 \mathrm{E}+00 \quad 0.0000$ $0.000 \mathrm{E}+00 \quad 0.0000$ $0.000 \mathrm{E}+00 \quad 0.0000$ $0.000 \mathrm{E}+00 \quad 0.0000$ $0.000 E+00 \quad 0.0000$ $0.000 \mathrm{E}+00 \quad 0.0000$ $0.000 E+00 \quad 0.0000$ $0.000 \mathrm{E}+00 \quad 0.0000$ ífíítííí ííítít Ififitifi fifiti mrem/yr fract.

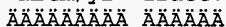
$0.000 \mathrm{E}+00 \quad 0.0000$ $0.000 \mathrm{E}+00 \quad 0.0000$ $0.000 \mathrm{E}+00 \quad 0.0000$ $0.000 \mathrm{E}+00 \quad 0.0000$ $0.000 E+00 \quad 0.0000$ $0.000 \mathrm{E}+00 \quad 0.0000$ $0.000 E+00 \quad 0.0000$ $0.000 \mathrm{E}+00 \quad 0.0000$ $0.000 E+00 \quad 0.0000$ $0.000 \mathrm{0}+000.0000$ Radio- Water

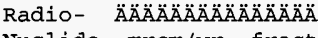
Nuclide mrem/Yr fract.

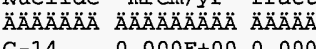
$\begin{array}{lll}C-14 & 0.000 E+00 & 0.000 \\ C 0-60 & 0.000 E+00 & 0.000\end{array}$ $\begin{array}{lll}\mathrm{Co}-60 & 0.000 \mathrm{E}+00 & 0.0000 \\ \mathrm{Cs}-137 & 0.000 \mathrm{E}+00 & 0.0000\end{array}$ $\begin{array}{lll}\mathrm{Cs}-137 & 0.000 \mathrm{E}+00 & 0.0000 \\ \mathrm{Eu}-152 & 0.000 \mathrm{E}+00 & 0.0000\end{array}$ Eu-154 $0.000 \mathrm{E}+00 \quad 0.0000$

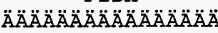
$\begin{array}{lllllll}0.000 \mathrm{E}+00 & 0.0000 & 0.000 \mathrm{E}+00 & 0.0000 & 0.000 \mathrm{E}+00 & 0.0000\end{array}$ $\begin{array}{llllllll}\text { Pu-239 } & 1.168 \mathrm{E}-04 & 0.1181 & 6.132 \mathrm{E}-07 & 0.0006 & 0.000 \mathrm{E}+00 & 0.0000\end{array}$ $\begin{array}{llllllll}\mathrm{Pu}-240 & 7.345 \mathrm{E}-04 & 0.7426 & 3.061 \mathrm{E}-06 & 0.0031 & 0.000 \mathrm{E}+00 & 0.0000\end{array}$ $\begin{array}{lllllll}\mathrm{S} x-90 & 0.000 \mathrm{E}+00 & 0.0000 & 0.000 \mathrm{E}+00 & 0.0000 & 0.000 \mathrm{E}+00 & 0.0000\end{array}$

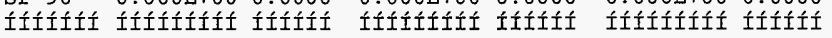
Total $\quad 8.513 \mathrm{E}-04 \quad 0.8607 \quad 3.675 \mathrm{E}-06 \quad 0.0037$

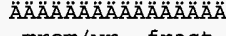
$\mathrm{mrem} / \mathrm{yr}$ fract. $\ddot{A} \ddot{A} \ddot{A} \ddot{A} \ddot{A} \ddot{A} \ddot{A} \ddot{A} \ddot{A} \ddot{A} \ddot{A} \ddot{A} \ddot{A} \ddot{A}$ mrem/yr fract. Ä̈̈̈̈̈̈̈̈̈̈̈̈

$0.000 \mathrm{E}+00 \quad 0.0000$ $0.000 \mathrm{E}+00 \quad 0.0000$ $0.000 E+00 \quad 0.0000$ $0.000 \mathrm{E}+000.0000$ $0.000 \mathrm{E}+00 \quad 0.0000$ $0.000 \mathrm{E}+000.0000$

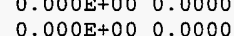
$0.000 E+00 \quad 0.0000$ $0.000 \mathrm{E}+00 \quad 0.0000$ $0.000 \mathrm{E}+00 \quad 0.0000$ $0.000 \mathrm{E}+00 \quad 0.0000$ $0.000 \mathrm{E}+00 \quad 0.0000$ $0.000 \mathrm{E}+00 \quad 0.0000$ $0.000 \mathrm{E}+00 \quad 0.0000$ $1.292 \mathrm{E}-05 \quad 0.0131$ $8.120 \mathrm{E}-05 \quad 0.0821$ $0.000 \mathrm{E}+00 \quad 0.0000$ Ííííííííi ííííí

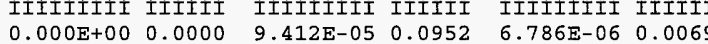
0 *Sum of all water independent and dependent pathways.

\begin{abstract}
Meat
\end{abstract}

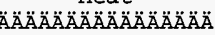
$0.000 \mathrm{E}+000.0000$ $0.000 \mathrm{E}+00 \quad 0.0000$ $0.000 \mathrm{E}+00 \quad 0.0000$ $0.000 \mathrm{E}+00 \quad 0.0000$ $0.000 \mathrm{E}+00 \quad 0.0000$ $1.102 \mathrm{E}-06 \quad 0.0011$ 0.0057 $\mathrm{mrem} / \mathrm{yr}$ fract. $0.000 \mathrm{E}+00 \quad 0.0000$

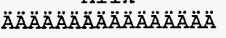
mrem/Yr fract. AAAAAAAAA AAAAA $0.000 \mathrm{E}+00 \quad 0.0000$ $0.000 E+00 \quad 0.0000$ $0.000 \mathrm{E}+00 \quad 0.0000$ $0.000 \mathrm{E}+00 \quad 0.0000$ $0.000 \mathrm{E}+00 \quad 0.0000$ $4.210 \mathrm{E}-06 \quad 0.0043$ $2.896 \mathrm{E}-05 \quad 0.0293$ $0.000 \mathrm{E}+00 \quad 0.0000$ íítíííít íííí $3.317 \mathrm{E}-05 \quad 0.0335 \quad 9.891 \mathrm{E}-04 \quad 1.0000$

A11 Pathways* $\ddot{A} \ddot{A} \ddot{A} \ddot{A} \ddot{A} \ddot{A} \ddot{A} \ddot{A} \ddot{A} \ddot{A} \ddot{A} \ddot{A} \ddot{A} \ddot{A} \ddot{A} \ddot{A}$ $\mathrm{mrem} / \mathrm{yr}$ fract. $0.000 E+000.0000$ $0.000 E+00 \quad 0.0000$ $0.000 E+00 \quad 0.0000$ $0.000 \mathrm{E}+00 \quad 0.0000$ $0.000 \mathrm{E}+00 \quad 0.0000$ $1.357 \mathrm{E}-04 \quad 0.1372$ $8.534 \pm-$ 


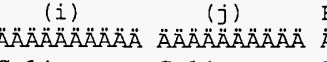

C -14

$0 \mathrm{CO}-60$ OCS $-137+\mathrm{D}$

OEu -152

oEu-152

C-14

1.000E+00

$0.000 \mathrm{E}+00 \quad 1.000 \mathrm{E}+00 \quad 3.000 \mathrm{E}+00 \quad 1.300 \mathrm{E}+01 \quad 3.000 \mathrm{E}+01 \quad 1.000 \mathrm{E}+02 \quad 3.000 \mathrm{E}+02 \quad 1.000 \mathrm{E}+03$

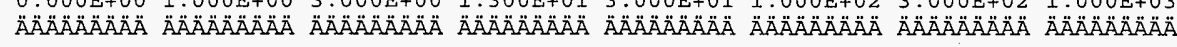
$\begin{array}{llllllllll}0.000 \mathrm{E}+00 & 0.000 \mathrm{E}+00 & 0.000 \mathrm{E}+00 & 0.000 \mathrm{E}+00 & 0.000 \mathrm{E}+00 & 0.000 \mathrm{E}+00 & 0.000 \mathrm{E}+00 & 0.000 \mathrm{E}+00\end{array}$ $\begin{array}{llllllll}4.309 \mathrm{E}-23 & 3.822 \mathrm{E}-23 & 3.007 \mathrm{E}-23 & 9.061 \mathrm{E}-24 & 1.179 \mathrm{E}-24 & 2.664 \mathrm{E}-28 & 1.018 \mathrm{E}-38 & 0.000 \mathrm{E}+00\end{array}$ $\mathrm{Cs}-137+\mathrm{D} \quad 1.000 \mathrm{E}+00$ $\mathrm{Eu}-152$ 7.208E-01

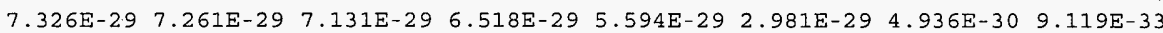

Eul-152

Gd-152 äDSR $(j)$

Eu-152

OEu-154

ONi-63

OPu-239

Pu-239

$\mathrm{Pu}-239$

Pu-239

$\mathrm{Pu}-239$

OPu-240

OPu -240

$\mathrm{Pu}-240$

Pu-240

Pu-240

$\mathrm{Pu}-240$

$\mathrm{Pu}-240$

Eu-154

$\mathrm{Ni}-63$

$\mathrm{Pu}-239$

$\mathrm{U}-235+\mathrm{D}$

Pa-231

$2.792 \mathrm{E}-0$

$2.792 \mathrm{E}-01$

$\begin{array}{llllllll}2.104 E-25 & 2.023 E-25 & 1.869 E-25 & 1.260 E-25 & 6.446 E-26 & 4.079 E-27 & 1.532 E-30 & 1.575 E-42\end{array}$ $\begin{array}{llllllll}8.150 E-26 & 7.835 E-26 & 7.241 E-26 & 4.881 E-26 & 2.497 E-26 & 1.580 E-27 & 5.936 E-31 & 6.110 E-43\end{array}$ $\begin{array}{llllllllll}0.000 E+00 & 0.000 E+00 & 0.000 E+00 & 0.000 E+00 & 0.000 E+00 & 0.000 E+00 & 0.000 E+00 & 0.000 E+00\end{array}$ $\begin{array}{llllllll}8.150 E-26 & 7.835 E-26 & 7.241 E-26 & 4.881 E-26 & 2.497 E-26 & 1.580 E-27 & 5.936 E-31 & 6.110 E-43\end{array}$

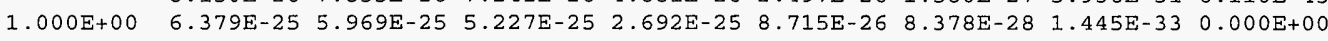
$\begin{array}{llllllllll}1.000 E+00 & 0.000 E+00 & 0.000 E+00 & 0.000 E+00 & 0.000 E+00 & 0.000 E+00 & 0.000 E+00 & 0.000 E+00 & 0.000 E+00\end{array}$

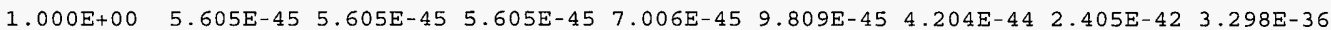
$\begin{array}{lllllllll}1.000 \mathrm{E}+00 & 0.000 \mathrm{E}+00 & 0.000 \mathrm{E}+00 & 0.000 \mathrm{E}+00 & 0.000 \mathrm{E}+00 & 0.000 \mathrm{E}+00 & 9.195 \mathrm{E}-08 & 9.774 \mathrm{E}-06 & 2.222 \mathrm{E}-05\end{array}$ $\begin{array}{llllllllll}1.000 \mathrm{E}+00 & 0.000 \mathrm{E}+00 & 0.000 \mathrm{E}+00 & 0.000 \mathrm{E}+00 & 0.000 \mathrm{E}+00 & 0.000 \mathrm{E}+00 & 2.027 \mathrm{E}-11 & 3.589 \mathrm{E}-08 & 5.286 \mathrm{E}-07\end{array}$ $1.000 \mathrm{E}+00 \quad 0.000 \mathrm{E}+00 \quad 0.000 \mathrm{E}+00 \quad 1.121 \mathrm{E}-44 \quad 9.122 \mathrm{E}-43 \quad 1.247 \mathrm{E}-41 \quad 8.159 \mathrm{E}-12 \quad 8.392 \mathrm{E}-08 \quad 1.613 \mathrm{E}-06$ $A C-227+D$ äDSR $(j)$ $\mathrm{Pu}-240$ $\mathrm{Pu}-240$

$\mathrm{U}-236$

$\mathrm{Th}-232$ $\mathrm{Ra}-228+\mathrm{D}$ $T h-228+D$ äDSR $(j)$ $0 S r-90+D \quad S r-90+D$ $\begin{array}{lllllllll}5.605 \mathrm{E}-45 & 5.605 \mathrm{E}-45 & 1.682 \mathrm{E}-44 & 9.193 \mathrm{E}-43 & 1.248 \mathrm{E}-41 & 9.198 \mathrm{E}-08 & 9.894 \mathrm{E}-06 & 2.436 \mathrm{E}-05\end{array}$ $\begin{array}{lllllllll}4.950 \mathrm{E}-08 & 0.000 \mathrm{E}+00 & 0.000 \mathrm{E}+00 & 0.000 \mathrm{E}+00 & 0.000 \mathrm{E}+00 & 0.000 \mathrm{E}+00 & 0.000 \mathrm{E}+00 & 0.000 \mathrm{E}+00 & 0.000 \mathrm{E}+00\end{array}$ $\begin{array}{llllllllll}1.000 E+00 & 0.000 E+00 & 0.000 E+00 & 0.000 E+00 & 0.000 E+00 & 0.000 E+00 & 0.000 E+00 & 0.000 E+00 & 0.000 E+00\end{array}$ $\begin{array}{llllllllll}1.000 E+00 & 0.000 E+00 & 0.000 E+00 & 0.000 E+00 & 0.000 E+00 & 0.000 E+00 & 2.780 E-06 & 2.935 E-04 & 6.416 E-04\end{array}$ $\begin{array}{lllllllllll}1.000 E+00 & 0.000 E+00 & 0.000 E+00 & 0.000 E+00 & 0.000 E+00 & 0.000 E+00 & 1.778 E-16 & 1.494 E-13 & 2.153 E-12\end{array}$ $\begin{array}{lllllllllll}1.000 \mathrm{E}+00 & 0.000 \mathrm{E}+00 & 1.401 \mathrm{E}-44 & 3.727 \mathrm{E}-43 & 2.622 \mathrm{E}-41 & 2.744 \mathrm{E}-40 & 1.812 \mathrm{E}-16 & 2.165 \mathrm{E}-13 & 3.385 \mathrm{E}-12\end{array}$ $\begin{array}{lllllllll}1.000 \mathrm{E}+00 & 0.000 \mathrm{E}+00 & 1.636 \mathrm{E}-39 & 1.125 \mathrm{E}-37 & 2.042 \mathrm{E}-35 & 2.721 \mathrm{E}-34 & 3.077 \mathrm{E}-17 & 3.923 \mathrm{E}-14 & 6.304 \mathrm{E}-13\end{array}$ $\begin{array}{lllllllll}0.000 E+00 & 1.636 \mathrm{E}-39 & 1.125 \mathrm{E}-37 & 2.042 \mathrm{E}-35 & 2.721 \mathrm{E}-34 & 2.780 \mathrm{E}-06 & 2.935 \mathrm{E}-04 & 6.416 \mathrm{E}-04\end{array}$ $\begin{array}{lllllllll}1.000 E+00 & 6.433 E-38 & 6.393 E-38 & 6.312 E-38 & 5.922 E-38 & 5.314 \mathrm{E}-38 & 3.401 \mathrm{E}-38 & 9.502 \mathrm{E}-39 & 1.096 \mathrm{E}-40\end{array}$

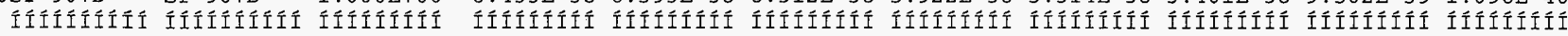
The DSR includes contributions from associated (half-life ó 180 days) daughters. 


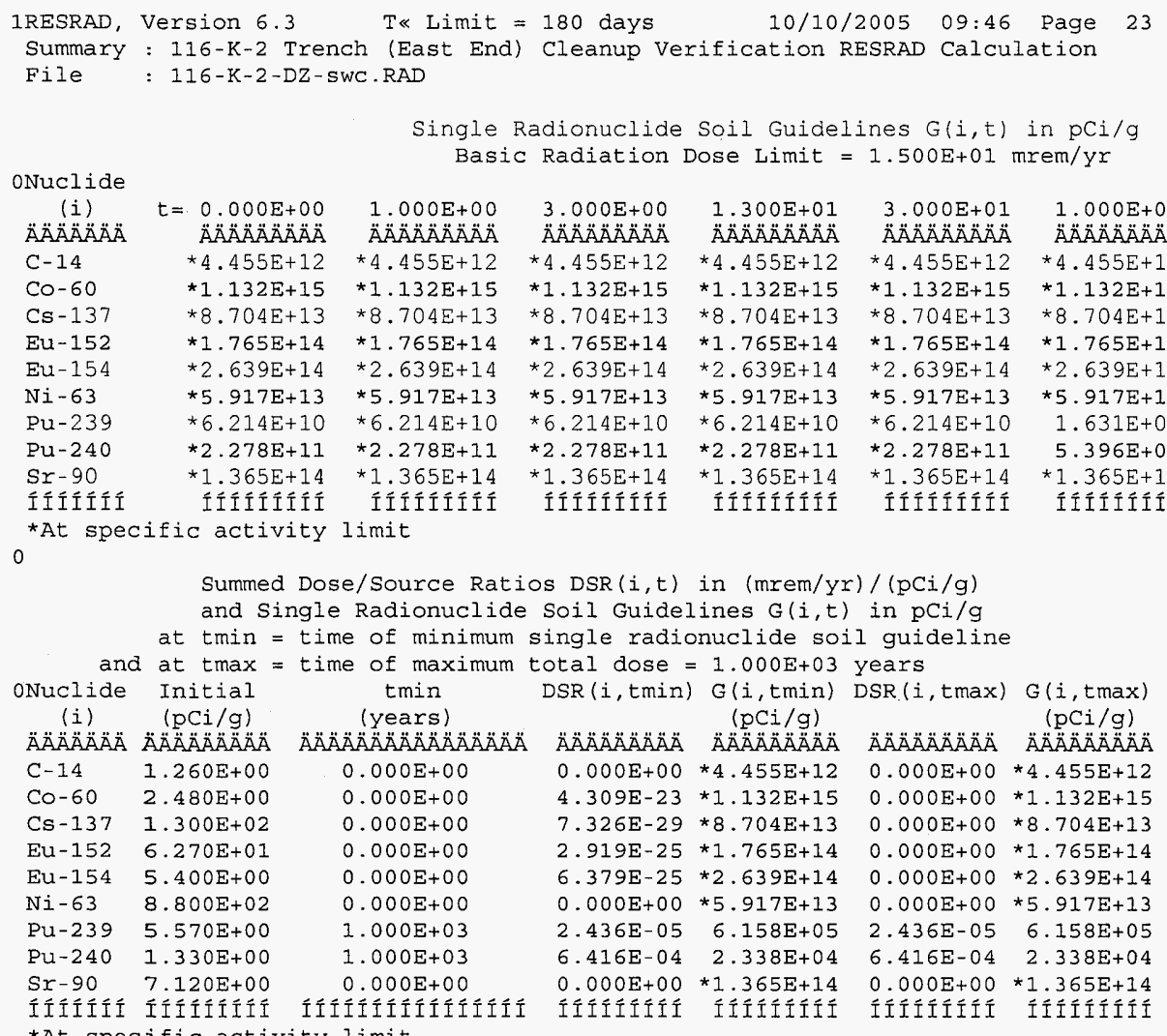

*At specific activity limit 
CVP-2006-00001

Rev. 0

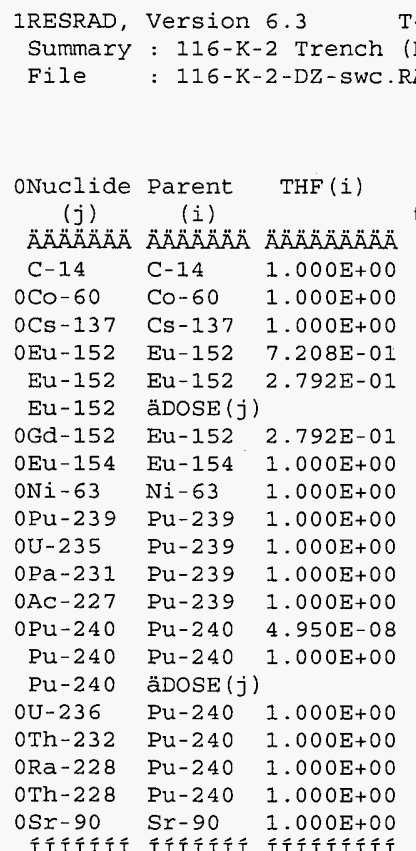

$\mathrm{T} \ll$ Limit $=180$ days

10/10/2005 09:46 Page

24

End) Cleanup Verification RESRAD Calculation

Individual Nuclide Dose Summed Over All Pathways

Parent Nuclide and Branch Fraction Indicated

$\operatorname{DOSE}(j, t), \operatorname{mrem} / \mathrm{yr}$

$=0.000 \mathrm{E}+00 \quad 1.000 \mathrm{E}+00 \quad 3.000 \mathrm{E}+00 \quad 1.300 \mathrm{E}+01 \quad 3.000 \mathrm{E}+01 \quad 1.000 \mathrm{E}+02 \quad 3.000 \mathrm{E}+02 \quad 1.000 \mathrm{E}+03$

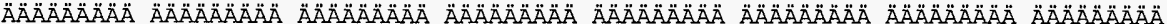
$\begin{array}{lllllllllll}0.000 E+00 & 0.000 E+00 & 0.000 E+00 & 0.000 E+00 & 0.000 E+00 & 0.000 E+00 & 0.000 E+00 & 0.000 E+00\end{array}$ $\begin{array}{llllllll}1.069 \mathrm{E}-22 & 9.478 \mathrm{E}-23 & 7.456 \mathrm{E}-23 & 2.247 \mathrm{E}-23 & 2.925 \mathrm{E}-24 & 6.606 \mathrm{E}-28 & 0.000 \mathrm{E}+00 & 0.000 \mathrm{E}+00\end{array}$ $\begin{array}{llllllll}9.524 \mathrm{E}-27 & 9.439 \mathrm{E}-27 & 9.271 \mathrm{E}-27 & 8.473 \mathrm{E}-27 & 7.272 \mathrm{E}-27 & 3.876 \mathrm{E}-27 & 6.417 \mathrm{E}-28 & 0.000 \mathrm{E}+00\end{array}$ $\begin{array}{llllllll}1.319 E-23 & 1.268 \mathrm{E}-23 & 1.172 \mathrm{E}-23 & 7.901 \mathrm{E}-24 & 4.042 \mathrm{E}-24 & 2.557 \mathrm{E}-25 & 9.608 \mathrm{E}-29 & 0.000 \mathrm{E}+00\end{array}$ $\begin{array}{lllllllll}5.110 \mathrm{E}-24 & 4.913 \mathrm{E}-24 & 4.540 \mathrm{E}-24 & 3.061 \mathrm{E}-24 & 1.566 \mathrm{E}-24 & 9.905 \mathrm{E}-26 & 0.000 \mathrm{E}+00 & 0.000 \mathrm{E}+00\end{array}$ $\begin{array}{llllllll}1.830 E-23 & 1.760 E-23 & 1.626 \mathrm{E}-23 & 1.096 \mathrm{E}-23 & 5.607 \mathrm{E}-24 & 3.548 \mathrm{E}-25 & 9.608 \mathrm{E}-29 & 0.000 \mathrm{E}+00\end{array}$ $\begin{array}{llllllllll}0.000 E+00 & 0.000 E+00 & 0.000 E+00 & 0.000 E+00 & 0.000 E+00 & 0.000 E+00 & 0.000 E+00 & 0.000 E+00\end{array}$ $\begin{array}{llllllll}3.444 \mathrm{E}-24 & 3.223 \mathrm{E}-24 & 2.823 \mathrm{E}-24 & 1.454 \mathrm{E}-24 & 4.706 \mathrm{E}-25 & 4.524 \mathrm{E}-27 & 0.000 \mathrm{E}+00 & 0.000 \mathrm{E}+00\end{array}$ $\begin{array}{lllllllll}0.000 E+00 & 0.000 E+00 & 0.000 E+00 & 0.000 E+00 & 0.000 E+00 & 0.000 E+00 & 0.000 E+00 & 0.000 E+00\end{array}$ $\begin{array}{llllllllll}0.000 \mathrm{E}+00 & 0.000 \mathrm{E}+00 & 0.000 \mathrm{E}+00 & 0.000 \mathrm{E}+00 & 0.000 \mathrm{E}+00 & 0.000 \mathrm{E}+00 & 0.000 \mathrm{E}+00 & 0.000 \mathrm{E}+00\end{array}$ $\begin{array}{lllllllll}0.000 E+00 & 0.000 E+00 & 0.000 E+00 & 0.000 E+00 & 0.000 E+00 & 5.122 E-07 & 5.444 E-05 & 1.237 E-04\end{array}$ $\begin{array}{lllllllll}0.000 \mathrm{E}+00 & 0.000 \mathrm{E}+00 & 0.000 \mathrm{E}+00 & 0.000 \mathrm{E}+00 & 0.000 \mathrm{E}+00 & 1.129 \mathrm{E}-10 & 1.999 \mathrm{E}-07 & 2.945 \mathrm{E}-06\end{array}$ $\begin{array}{llllllllll}0.000 \mathrm{E}+00 & 0.000 \mathrm{E}+00 & 0.000 \mathrm{E}+00 & 0.000 \mathrm{E}+00 & 0.000 \mathrm{E}+00 & 4.545 \mathrm{E}-11 & 4.675 \mathrm{E}-07 & 8.986 \mathrm{E}-06\end{array}$ $\begin{array}{llllllllll}0.000 \mathrm{E}+00 & 0.000 \mathrm{E}+00 & 0.000 \mathrm{E}+00 & 0.000 \mathrm{E}+00 & 0.000 \mathrm{E}+00 & 0.000 \mathrm{E}+00 & 0.000 \mathrm{E}+00 & 0.000 \mathrm{E}+00\end{array}$ $\begin{array}{lllllllll}0.000 \mathrm{E}+00 & 0.000 \mathrm{E}+00 & 0.000 \mathrm{E}+00 & 0.000 \mathrm{E}+00 & 0.000 \mathrm{E}+00 & 0.000 \mathrm{E}+00 & 0.000 \mathrm{E}+00 & 0.000 \mathrm{E}+00\end{array}$ $\begin{array}{llllllllll}0.000 \mathrm{E}+00 & 0.000 \mathrm{E}+00 & 0.000 \mathrm{E}+00 & 0.000 \mathrm{E}+00 & 0.000 \mathrm{E}+00 & 0.000 \mathrm{E}+00 & 0.000 \mathrm{E}+00 & 0.000 \mathrm{E}+00\end{array}$ $\begin{array}{lllllllll}0.000 E+00 & 0.000 E+00 & 0.000 E+00 & 0.000 E+00 & 0.000 E+00 & 3.697 E-06 & 3.904 E-04 & 8.534 E-04\end{array}$ $\begin{array}{llllllllll}0.000 \mathrm{E}+00 & 0.000 \mathrm{E}+00 & 0.000 \mathrm{E}+00 & 0.000 \mathrm{E}+00 & 0.000 \mathrm{E}+00 & 2.364 \mathrm{E}-16 & 1.988 \mathrm{E}-13 & 2.863 \mathrm{E}-12\end{array}$ $\begin{array}{lllllllll}0.000 E+00 & 0.000 E+00 & 0.000 E+00 & 0.000 E+00 & 0.000 E+00 & 2.410 E-16 & 2.879 E-13 & 4.502 E-12\end{array}$ $\begin{array}{lllllllll}0.000 \mathrm{E}+00 & 0.000 \mathrm{E}+00 & 0.000 \mathrm{E}+00 & 0.000 \mathrm{E}+00 & 0.000 \mathrm{E}+00 & 4.093 \mathrm{E}-17 & 5.218 \mathrm{E}-14 & 8.384 \mathrm{E}-13\end{array}$ $\begin{array}{lllllllll}0.000 \mathrm{E}+00 & 0.000 \mathrm{E}+00 & 0.000 \mathrm{E}+00 & 0.000 \mathrm{E}+00 & 0.000 \mathrm{E}+00 & 0.000 \mathrm{E}+00 & 0.000 \mathrm{E}+00 & 0.000 \mathrm{E}+00\end{array}$

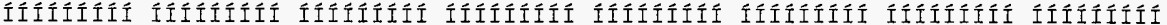
THF (i) is the thread fraction of the parent nuclide. 


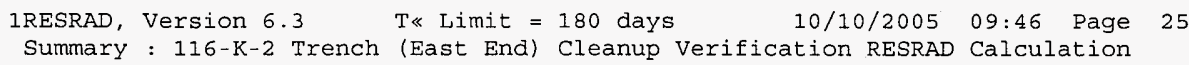

File : $116-\mathrm{K}-2-\mathrm{DZ}-\mathrm{SwC} . \mathrm{RAD}$

Individual Nuclide Soil Concentration

Parent Nuclide and Branch Fraction Indicated

oNuclide Parent THF (i

(j) (i)

$\begin{array}{lll}\ddot{A} \ddot{A} \ddot{A} \ddot{A} \ddot{A} \ddot{A} & \ddot{A} \ddot{A} \ddot{A} \ddot{A} \ddot{A} \ddot{A} & \ddot{A} \ddot{A} \ddot{A} \ddot{A} \ddot{A} \ddot{A} \ddot{A} \ddot{A} \\ C-14 & C-14 & 1.000 E+00\end{array}$

$\begin{array}{cll}\mathrm{C}-14 & \mathrm{C}-14 & 1.000 \mathrm{E}+00 \\ 0 \mathrm{CO}-60 & \mathrm{CO}-60 & 1.000 \mathrm{E}+00\end{array}$

OCs-137 Cs-137 1.000E+00

0Eu-152 Eu-152 7.208E-01

Eu-152 Eu-152 2.792E-01

Eu-152 äs $(j)$ :

OGd-152 Eu-152 2.792E-01

OEu-154 Eu-154 $1.000 \mathrm{E}+00$

ONi-63 Ni-63 $1.000 \mathrm{E}+00$

OPu-239 Pu-239 $1.000 E+00$

OU-235 Pu-239 $1.000 E+00$

$0 P a-231$ Pu-239 1.000E+00

$0 A c-227 \quad \mathrm{Pu}-239 \quad 1.000 \mathrm{E}+00$

OPu-240 $\mathrm{Pu}-240 \quad 4.950 \mathrm{E}-08$

Pu-240 Pu-240 1.000E+00

Pu-240 äs $(j)$ :

OU-236 Pu-240 $1.000 \mathrm{E}+00$

OTh-232 Pu-240 1.000E+00

ORa-228 Pu-240 1.000E+00

$0 \mathrm{Th}-228 \quad \mathrm{Pu}-240 \quad 1.000 \mathrm{E}+00$

$\begin{array}{lll}0 \mathrm{Sr}-90 & \mathrm{Sr}-90 & 1.000 \mathrm{E}+00\end{array}$

IIIIIII IIII

THF (i) is the thread fraction

ORESCALC. EXE execution time =
$S(j, t), \mathrm{pCi} / \mathrm{g}$

$t=0.000 \mathrm{E}+00 \quad 1.000 \mathrm{E}+00 \quad 3.000 \mathrm{E}+00 \quad 1.300 \mathrm{E}+01 \quad 3.000 \mathrm{E}+01 \quad 1.000 \mathrm{E}+02 \quad 3.000 \mathrm{E}+02 \quad 1.000 \mathrm{E}+03$

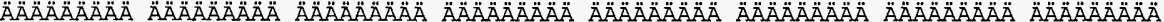
$\begin{array}{llllllll}1.260 \mathrm{E}+00 & 1.260 \mathrm{E}+00 & 1.259 \mathrm{E}+00 & 1.257 \mathrm{E}+00 & 1.254 \mathrm{E}+00 & 1.241 \mathrm{E}+00 & 1.203 \mathrm{E}+00 & 1.079 \mathrm{E}+00\end{array}$ $2.480 E+00 \quad 2.174 E+00 \quad 1.671 E+00 \quad 4.480 E-01 \quad 4.779 E-02 \quad 4.758 E-06 \quad 1.752 E-17 \quad 0.000 E+00$ $\begin{array}{llllllllll}1.300 \mathrm{E}+02 & 1.270 \mathrm{E}+02 & 1.212 \mathrm{E}+02 & 9.610 \mathrm{E}+01 & 6.473 \mathrm{E}+01 & 1.272 \mathrm{E}+01 & 1.219 \mathrm{E}-01 & 1.048 \mathrm{E}-08\end{array}$ $\begin{array}{llllllllll}4.519 \mathrm{E}+01 & 4.290 \mathrm{E}+01 & 3.866 \mathrm{E}+01 & 2.298 \mathrm{E}+01 & 9.487 \mathrm{E}+00 & 2.485 \mathrm{E}-01 & 7.512 \mathrm{E}-06 & 1.141 \mathrm{E}-21\end{array}$

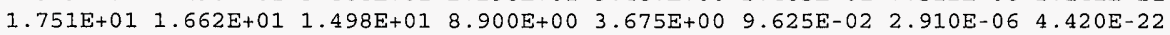
$\begin{array}{lllllllll}6.270 E+01 & 5.952 E+01 & 5.364 E+01 & 3.188 E+01 & 1.316 E+01 & 3.447 E-01 & 1.042 E-05 & 1.583 E-21\end{array}$ $0.000 \mathrm{E}+00 \quad 1.095 \mathrm{E}-13 \quad 3.121 \mathrm{E}-13 \quad 1.061 \mathrm{E}-12 \quad 1.706 \mathrm{E}-12 \quad 2.146 \mathrm{E}-12 \quad 2.154 \mathrm{E}-12 \quad 2.142 \mathrm{E}-12$ $5.400 \mathrm{E}+00 \quad 4.991 \mathrm{E}+00 \quad 4.263 \mathrm{E}+00 \quad 1.939 \mathrm{E}+00 \quad 5.078 \mathrm{E}-01 \quad 2.042 \mathrm{E}-03 \quad 2.921 \mathrm{E}-10 \quad 3.233 \mathrm{E}-34$ $\begin{array}{llllllll}8.800 \mathrm{E}+02 & 8.735 \mathrm{E}+02 & 8.606 \mathrm{E}+02 & 7.988 \mathrm{E}+02 & 7.038 \mathrm{E}+02 & 4.179 \mathrm{E}+02 & 9.423 \mathrm{E}+01 & 5.130 \mathrm{E}-01\end{array}$ $\begin{array}{llllllll}5.570 \mathrm{E}+00 & 5.570 \mathrm{E}+00 & 5.569 \mathrm{E}+00 & 5.565 \mathrm{E}+00 & 5.559 \mathrm{E}+00 & 5.535 \mathrm{E}+00 & 5.466 \mathrm{E}+00 & 5.230 \mathrm{E}+00\end{array}$ $0.000 \mathrm{E}+00 \quad 5.477 \mathrm{E}-09 \quad 1.638 \mathrm{E}-08 \quad 6.981 \mathrm{E}-08 \quad 1.567 \mathrm{E}-07 \quad 4.675 \mathrm{E}-07 \quad 1.044 \mathrm{E}-06 \quad 1.562 \mathrm{E}-06$ $\begin{array}{llllllll}0.000 E+00 & 5.797 E-14 & 5.205 E-13 & 9.663 E-12 & 5.048 E-11 & 5.188 E-10 & 3.789 E-09 & 2.316 E-08\end{array}$ $0.000 E+00 \quad 6.104 E-16 \quad 1.619 E-14 \quad 1.209 E-12 \quad 1.293 E-11 \quad 2.946 E-10 \quad 3.131 E-092.201 E-08$ 6.584E-08 6.583E-08 6.581E-08 $\quad 6.572 \mathrm{E}-08 \quad 6.556 \mathrm{E}-08 \quad 6.492 \mathrm{E}-08 \quad 6.312 \mathrm{E}-08 \quad 5.722 \mathrm{E}-08$ $\begin{array}{llllllll}1.330 E+00 & 1.330 E+00 & 1.329 E+00 & 1.328 E+00 & 1.324 E+00 & 1.311 E+00 & 1.275 E+00 & 1.156 E+00\end{array}$

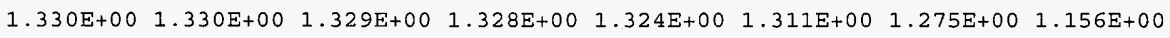
$0.000 \mathrm{E}+00 \quad 3.931 \mathrm{E}-08 \quad 1.175 \mathrm{E}-07 \quad 5.008 \mathrm{E}-07 \quad 1.123 \mathrm{E}-06 \quad 3.341 \mathrm{E}-06 \quad 7.392 \mathrm{E}-06 \quad 1.060 \mathrm{E}-05$ $\begin{array}{llllllll}0.000 E+00 & 9.701 E-19 & 8.711 E-18 & 1.617 E-16 & 8.451 E-16 & 8.696 E-15 & 6.373 E-14 & 3.949 E-13\end{array}$ $0.000 E+00 \quad 3.784 E-20 \quad 9.623 E-19 \quad 5.972 E-17 \quad 5.058 E-16 \quad 7.424 E-15 \quad 6.072 E-14 \quad 3.903 E-13$ $\begin{array}{llllllll}0.000 \mathrm{E}+00 & 3.212 \mathrm{E}-21 & 2.169 \mathrm{E}-19 & 3.579 \mathrm{E}-17 & 4.063 \mathrm{E}-16 & 7.013 \mathrm{E}-15 & 5.974 \mathrm{E}-14 & 3.889 \mathrm{E}-13\end{array}$ $\begin{array}{lllllllll}7.120 \mathrm{E}+00 & 6.951 \mathrm{E}+00 & 6.624 \mathrm{E}+00 & 5.207 \mathrm{E}+00 & 3.458 \mathrm{E}+00 & 6.411 \mathrm{E}-01 & 5.197 \mathrm{E}-03 & 2.493 \mathrm{E}-10\end{array}$

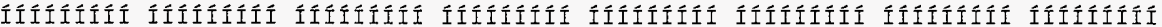
of the parent nuclide. 0.80 seconds 
CVP-2006-00001

Rev. 0 
CVP-2006-00001

Rev. 0

\section{RESRAD INPUT PARAMETERS FOR THE}

116-K-2 (WEST END) SHALLOW ZONE 
CVP-2006-00001

Rev. 0

C-52 
IRESRAD, Version 6.3 T\& Limit $=0.5$ year $12 / 15 / 2005$ 11:50 Page 2

Summary : 116-K-2 Trench (West End) Cleanup Verification RESRAD Calculation

File : 116-K-2_Shallow_Zone_West.RAD

Dose Conversion Factor (and Related) Parameter Summary File: HEAST 2001 Morbidity

\begin{tabular}{|c|c|c|c|c|c|c|c|}
\hline 0 & ${ }^{3}$ & & 3 & Current & 3 & & Parameter \\
\hline Menu & 3 & Parameter & 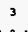 & Value & & Default & $\begin{array}{cc}3 & \text { Name } \\
3 & 3 \\
3\end{array}$ \\
\hline$\ddot{A} \ddot{A} \ddot{A} \ddot{A} \ddot{A}$ & $\therefore \ddot{A} \ddot{A} \ddot{A} \ddot{A} \ddot{A} \ddot{A} \ddot{A} \ddot{A} \ddot{A} \ddot{A}$ & 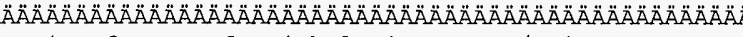 & & $\ddot{A} \ddot{A} \ddot{A} \ddot{A} \ddot{A} \ddot{A} \ddot{A} \ddot{A} \ddot{A}$ & & & \\
\hline$B-1$ & 3 Dose conv & ersion factors for inhalation, mrem/pCi: & 3 & & 3 & & 3 \\
\hline$B-1$ & ${ }^{3} \mathrm{C}-14$ & & 3 & $2.090 \mathrm{E}-06$ & 3 & $2.090 \mathrm{E}-06$ & ${ }^{3}$ DCF2 ( 1) \\
\hline$B-1$ & $\mathrm{Cs}-137+\mathrm{D}$ & & 3 & $3.190 \mathrm{E}-05$ & 3 & $3.190 \mathrm{E}-05$ & ${ }^{3}$ DCF2 $(2)$ \\
\hline$B-1$ & 3 Eu-152 & & 3 & $2.210 \mathrm{E}-04$ & 3 & $2.210 E-04$ & ${ }^{3}$ DCF2 $(3)$ \\
\hline B-1 & ${ }^{3} \mathrm{Eu}-154$ & & 3 & $2.860 \mathrm{E}-04$ & 3 & $2.860 \mathrm{E}-04$ & ${ }^{3} \mathrm{DCF} 2(5)$ \\
\hline B-1 & 3 Gd-152 & & 3 & $2.430 E-01$ & 3 & $2.430 E-01$ & ${ }^{3}$ DCF $2(6)$ \\
\hline B-1 & ${ }^{3} \mathrm{Ni}-63$ & & 3 & $6.290 E-06$ & 3 & $6.290 \mathrm{E}-06$ & ${ }^{3}$ DCF2 $(7)$ \\
\hline$B-1$ & ${ }_{3}^{3} S x-90+D$ & & 3 & $1.310 \mathrm{E}-03$ & 3 & $1.310 E-03$ & ${ }_{3}^{3}$ DCF $2(8)$ \\
\hline$D-1$ & 3 Dose conv & ersion factors for ingestion, $\mathrm{mrem} / \mathrm{pCi}$ : & 3 & & 3 & & 3 \\
\hline$D-1$ & ${ }^{3} \mathrm{C}-14$ & & 3 & $2.090 \mathrm{E}-06$ & 3 & $2.090 \mathrm{E}-06$ & ${ }^{3} \mathrm{DCF} 3(1)$ \\
\hline D-1 & ${ }^{3} \mathrm{Cs}-137+\mathrm{D}$ & & 3 & $5.000 \mathrm{E}-05$ & 3 & $5.000 E-05$ & 3 DCF3 $(2)$ \\
\hline D-1 & ${ }^{3}$ Eu-152 & & 3 & $6.480 \mathrm{E}-06$ & 3 & $6.480 \mathrm{E}-06$ & 3 DCF $3(3)$ \\
\hline$D-1$ & 3 Eu-154 & & 3 & $9.550 \mathrm{E}-06$ & 3 & $9.550 \mathrm{E}-06$ & ${ }^{3} \operatorname{DCF} 3(5)$ \\
\hline$D-1$ & ${ }^{3} \mathrm{Gd}-152$ & & 3 & $1.610 \mathrm{E}-04$ & 3 & 1. $610 \mathrm{E}-04$ & ${ }^{3}$ DCF3 $(6)$ \\
\hline D-1 & $3 \mathrm{Ni}-63$ & & 3 & $5.770 E-07$ & 3 & $5.770 \mathrm{E}-07$ & 3 DCF $3(7)$ \\
\hline D-1 & ${ }_{3} S x-90+D$ & & 3 & $1.530 \mathrm{E}-04$ & 3 & $1.530 \mathrm{E}-04$ & ${ }_{3}^{3} \operatorname{DCF} 3(8)$ \\
\hline$D-34$ & 3 Food tran & isfer factors: & 3 & & 3 & & 3 \\
\hline$D-34$ & $3 \mathrm{C}-14$ & , plant/soil concentration ratio, dimensionless & 3 & $5.500 \mathrm{E}+00$ & 3 & $5.500 \mathrm{E}+00$ & $3 \operatorname{RTF}(1,1)$ \\
\hline$D-34$ & $3 \mathrm{C}-14$ & , beef/Iivestock-intake ratio, (pCi/kg)/(pCi/d) & 3 & $3.100 \mathrm{E}-02$ & 3 & $3.100 \mathrm{E}-02$ & ${ }^{3} \operatorname{RTF}(1,2)$ \\
\hline$D-34$ & $3 \mathrm{C}-14$ & , milk/livestock-intake ratio, $(\mathrm{pCi} / \mathrm{L}) /(\mathrm{pCi} / \mathrm{d})$ & 3 & $1.200 \mathrm{E}-02$ & 3 & $1.200 \mathrm{E}-02$ & $3 \operatorname{RTF}(1,3)$ \\
\hline$D-34$ & 3 & & 3 & & 3 & & 3 \\
\hline$D-34$ & $3 \mathrm{Cs}-137+\mathrm{D}$ & , plant/soil concentration ratio, dimensionless & 3 & $4.000 \mathrm{E}-02$ & 3 & $4.000 \mathrm{E}-02$ & ${ }^{3} \operatorname{RTF}(2,1)$ \\
\hline$D-34$ & $3 \mathrm{Cs}-137+\mathrm{D}$ & , beef/livestock-intake ratio, $(\mathrm{pCi} / \mathrm{kg}) /(\mathrm{pCi} / \mathrm{d})$ & 3 & $3.000 E-02$ & 3 & $3.000 \mathrm{E}-02$ & ${ }^{3} \operatorname{RTF}(2,2)$ \\
\hline$D-34$ & $3 \mathrm{Cs}-137+\mathrm{D}$ & , milk/livestock-intake ratio, $(\mathrm{pCi} / \mathrm{L}) /(\mathrm{pCi} / \mathrm{d})$ & 3 & $8.000 E-03$ & 3 & $8.000 \mathrm{E}-03$ & ${ }^{3} \operatorname{RTF}(2,3)$ \\
\hline$D-34$ & 3 & & 3 & & 3 & & 3 \\
\hline$D-34$ & 3 Eu-152 & , plant/soil concentration ratio, dimensionless & 3 & $2.500 E-03$ & 3 & $2.500 \mathrm{E}-03$ & $3 \operatorname{RTF}(3,1)$ \\
\hline$D-34$ & 3 Eu-152 & , beef/livestock-intake ratio, $(\mathrm{pCi} / \mathrm{kg}) /(\mathrm{pCi} / \mathrm{d})$ & 3 & $2.000 \mathrm{E}-03$ & 3 & $2.000 \mathrm{E}-03$ & $3 \operatorname{RTF}(3,2)$ \\
\hline$D-34$ & 3 Eu-152 & , milk/livestock-intake ratio, $(\mathrm{pCi} / \mathrm{L}) /(\mathrm{pCi} / \mathrm{d})$ & 3 & $2.000 E-05$ & 3 & $2.000 \mathrm{E}-05$ & ${ }^{3} \operatorname{RTF}(3,3)$ \\
\hline$D-34$ & 3 & & 3 & & 3 & & 3 \\
\hline$D-34$ & 3 Eu-154 & , plant/soil concentration ratio, dimensionless & 3 & $2.500 \mathrm{E}-03$ & 3 & $2.500 E-03$ & ${ }^{3} \operatorname{RTF}(5,1)$ \\
\hline$D-34$ & 3 Eu-154 & , beef/livestock-intake ratio, $(\mathrm{pCi} / \mathrm{kg}) /(\mathrm{pCi} / \mathrm{d})$ & 3 & $2.000 \mathrm{E}-03$ & 3 & $2.000 E-03$ & ${ }^{3} \operatorname{RTF}(5,2)$ \\
\hline$D-34$ & 3 Eu-154 & , milk/livestock-intake ratio, $(\mathrm{pCi} / \mathrm{L}) /(\mathrm{pCi} / \mathrm{d})$ & 3 & $2.000 \mathrm{E}-05$ & 3 & $2.000 E-05$ & $3 \operatorname{RTF}(5,3)$ \\
\hline$D-34$ & 3 & & 3 & & 3 & & 3 \\
\hline$D-34$ & ${ }^{3}$ Gd-152 & , plant/soil concentration ratio, dimensionless & 3 & $2.500 \mathrm{E}-03$ & 3 & $2.500 E-03$ & $3 \operatorname{RTF}(6,1)$ \\
\hline$D-34$ & ${ }^{3} \mathrm{Gd}-152$ & , beef/livestock-intake ratio, (pCi/kg)/(pCi/d) & 3 & $2.000 \mathrm{E}-03$ & 3 & $2.000 E-03$ & ${ }^{3} \operatorname{RTF}(6,2)$ \\
\hline$D-34$ & ${ }^{3} \mathrm{Gd}-152$ & , $\operatorname{milk} /$ livestock-intake ratio, $(\mathrm{pCi} / \mathrm{L}) /(\mathrm{pCi} / \mathrm{d})$ & 3 & $2.000 \mathrm{E}-05$ & 3 & $2.000 \mathrm{E}-05$ & $3 \operatorname{RTF}(6,3)$ \\
\hline$D-34$ & 3 & & 3 & & 3 & & 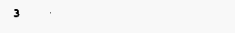 \\
\hline$D-34$ & $3 \mathrm{Ni}-63$ & , plant/soil concentration ratio, dime & 3 & $5.000 \mathrm{E}-02$ & 3 & $5.000 \mathrm{E}-02$ & ${ }^{3} \operatorname{RTF}(7,1)$ \\
\hline$D-34$ & $3 \mathrm{Ni}-63$ & , beef/livestock-intake ratio, $(\mathrm{pCi} / \mathrm{kg}) /(\mathrm{pCi} / \mathrm{d})$ & 3 & $5.000 E-03$ & 3 & $5.000 E-03$ & ${ }^{3} \operatorname{RTF}(7,2)$ \\
\hline$D-34$ & $3 \mathrm{Ni}-63$ & , milk/livestock-intake ratio, $(\mathrm{pCi} / \mathrm{L}) /(\mathrm{pCi} / \mathrm{d})$ & 3 & $2.000 E-02$ & 3 & $2.000 E-02$ & ${ }^{3} \operatorname{RTF}(7,3)$ \\
\hline$D-34$ & 3 & & 3 & & 3 & & 3 \\
\hline$D-34$ & $3 \operatorname{Sr}-90+D$ & plant/soil concentration ratio, dimensionless & 3 & $3.000 \mathrm{E}-01$ & 3 & $3.000 \mathrm{E}-01$ & 3. $\operatorname{RTF}(8,1)$ \\
\hline$D-34$ & 3 Sr $-90+D$ & , beef/livestock-intake ratio, $(\mathrm{pCi} / \mathrm{kg}) /(\mathrm{pCi} / \mathrm{d})$ & 3 & $8.000 \mathrm{E}-03$ & 3 & $8.000 E-03$ & ${ }^{3} \operatorname{RTF}(8,2)$ \\
\hline$D-34$ & ${ }_{3}^{3} \mathrm{Sr}-90+\mathrm{D}$ & , $\operatorname{milk} /$ livestock-intake ratio, $(\mathrm{pCi} / \mathrm{L}) /(\mathrm{pCi} / \mathrm{d})$ & $\begin{array}{l}3 \\
3\end{array}$ & $2.000 E-03$ & 3 & $2.000 E-03$ & $3_{3} \operatorname{RTF}(8,3)$ \\
\hline$D-5$ & 3 Bioaccumu & Iation factors, fresh water, L/kg: & 3 & & 3 & & 3 \\
\hline$D-5$ & ${ }^{3} \mathrm{C}-14$ & fish & 3 & $5.000 \mathrm{E}+04$ & 3 & $5.000 E+04$ & 3 BIOFAC $(1,1$ \\
\hline$D-5$ & $3 \mathrm{C}-14$ & , crustacea and mollusks & 3 & $9.100 E+03$ & 3 & $9.100 \mathrm{E}+03$ & 3 BIOFAC $(1,2)$ \\
\hline
\end{tabular}


CVP-2006-00001

Rev. 0

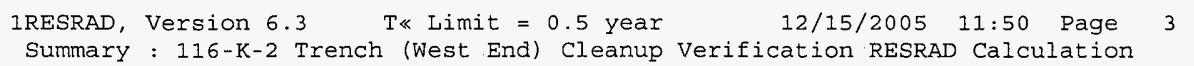

File : 116-K-2_Shallow_Zone_West.RAD

Dose Conversion Factor (and Related) Parameter Summary (continued) File: HEAST 2001 Morbidity

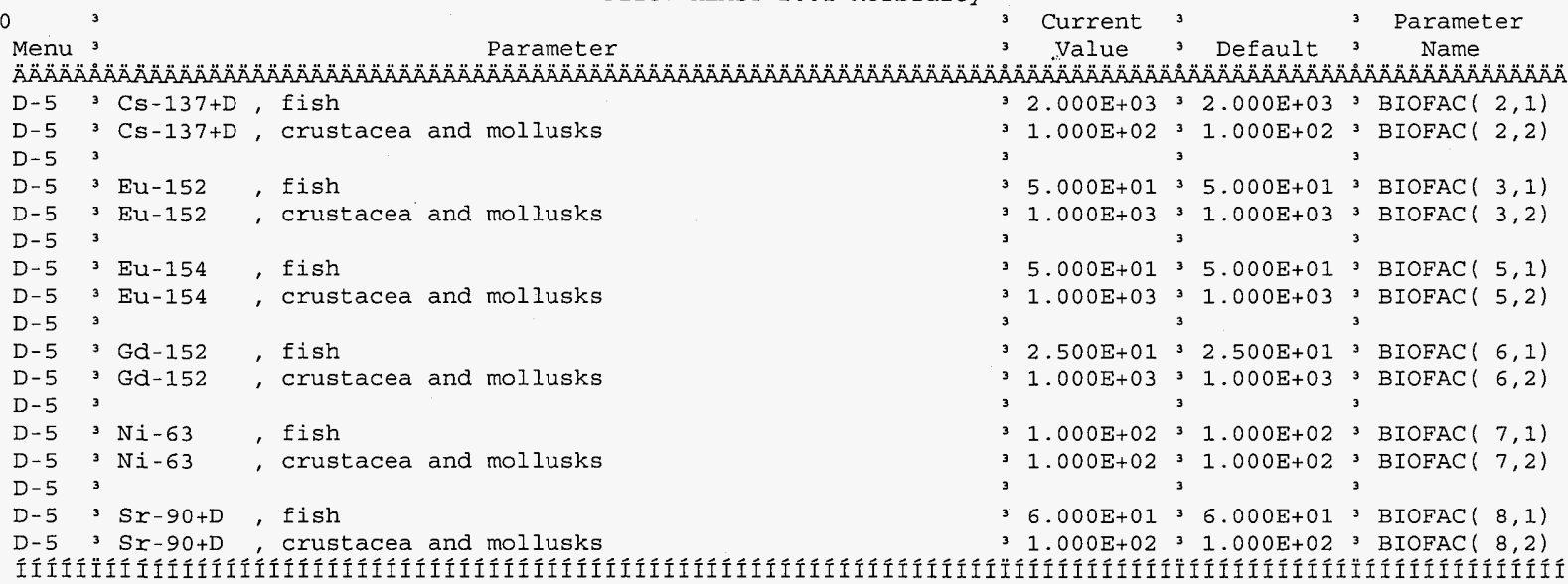




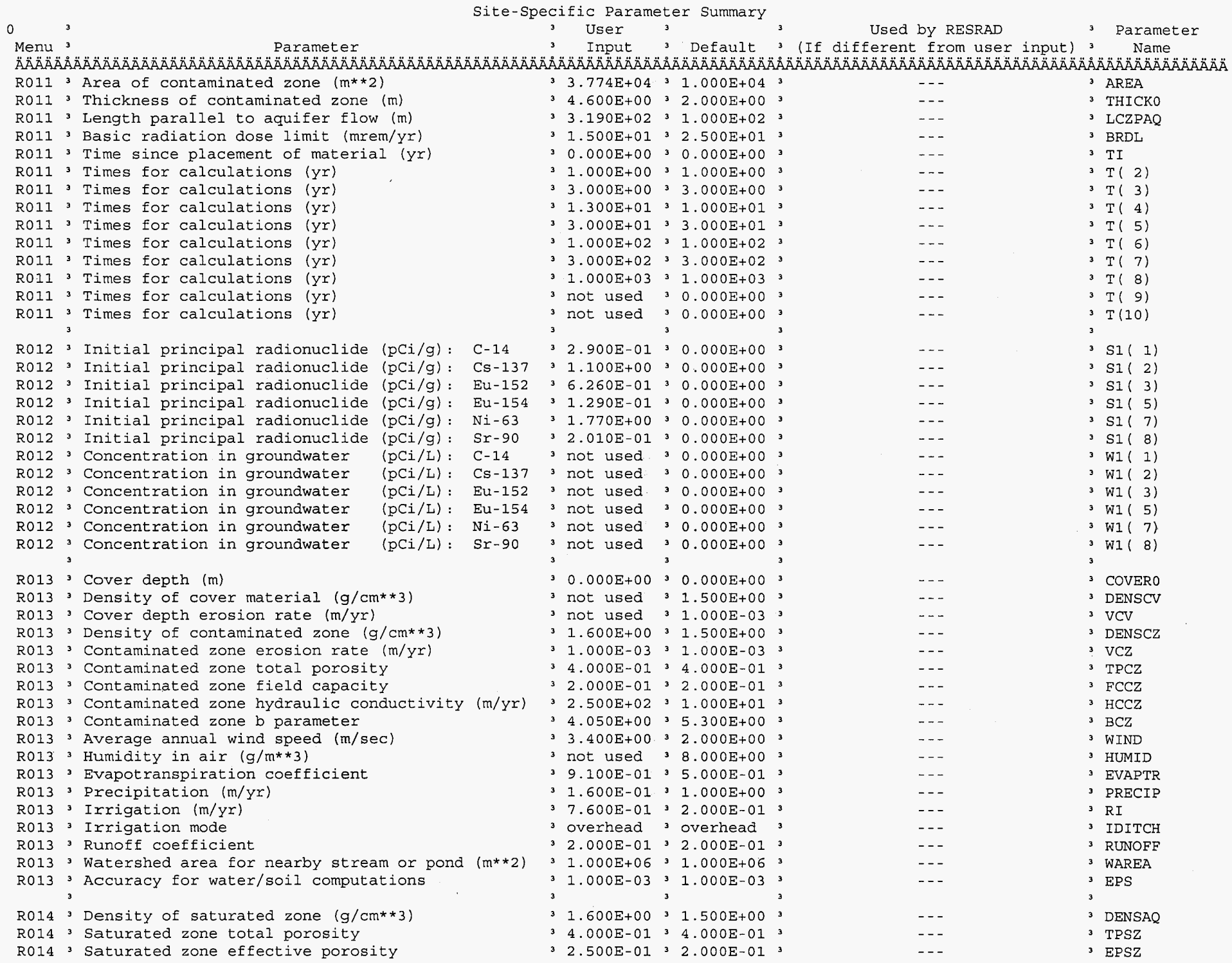


CVP-2006-00001

Rev. 0

\begin{tabular}{|c|c|c|c|c|c|c|c|c|}
\hline \multicolumn{2}{|c|}{$\begin{array}{l}\text { 1RESRAD, } \\
\text { Summary } \\
\text { File }\end{array}$} & \multicolumn{6}{|c|}{ Site-Specific Parameter Summary (continued) } & \\
\hline 0 & ${ }^{3}$ & & 3 User & 3 & 3 & Used by RESRAD & 3 & Parameter \\
\hline $\begin{array}{l}\text { Menu } \\
\ddot{A} \ddot{A} \ddot{A} \ddot{A} \ddot{A}\end{array}$ & & 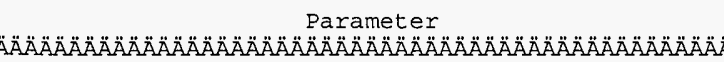 & 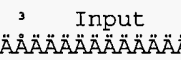 & $\begin{array}{l}3 \quad \text { Default } \\
\dot{A} A \ddot{A} \ddot{A} \ddot{A} \ddot{A} \ddot{A} \ddot{A} \ddot{A} \ddot{A} \ddot{A} \ddot{A} \ddot{A}\end{array}$ & $\stackrel{3}{3}($ If & 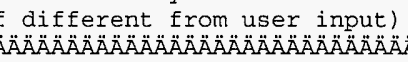 & 3 & $\begin{array}{c}\text { Name } \\
\ddot{A} \ddot{A} \ddot{A} \ddot{A} \ddot{A} \ddot{A} \ddot{A} \ddot{A} \ddot{A} \ddot{A} \ddot{A} \ddot{A} \ddot{A}\end{array}$ \\
\hline $\mathrm{R} 014$ & 3 & Saturated zone field capacity & $31.500 E-01$ & $32.000 E-01$ & 3 & --- & 3 & FCSZ \\
\hline $\mathrm{R} 014$ & 3 & Saturated zone hydraulic conductivity (m/yr) & $35.530 \mathrm{E}+03$ & $31.000 \mathrm{E}+02$ & 3 & -- & 3 & HCSZ \\
\hline $\mathrm{RO} 14$ & 3 & Saturated zone hydraulic gradient & $31.250 E-03$ & $32.000 E-02$ & 3 & $\ldots$ & 3 & HGWT \\
\hline RO14 & 3 & Saturated zone b parameter & $34.050 \mathrm{E}+00$ & $35.300 \mathrm{E}+00$ & 3 & -- & 3 & BSZ \\
\hline $\mathrm{R} 014$ & 3 & Water table drop rate (m/yr) & $31.000 E-03$ & $31.000 E-03$ & 3 & $-\ldots$ & 3 & VWT \\
\hline R014 & & Well pump intake depth (m below water table) & $34.600 E+00$ & ${ }^{3} 1.000 E+01$ & 3 & -- & 3 & DWIBWT \\
\hline R014 & 3 & Model: Nondispersion (ND) or Mass-Balance (MB) & $3 \mathrm{ND}$ & $3 \mathrm{ND}$ & 3 & --- & 3 & MODEL \\
\hline $\mathrm{R} 014$ & 3 & Well pumping rate $(m * * 3 / y r)$ & $3_{3}^{3} 2.500 \mathrm{E}+02$ & $32.500 \mathrm{E}+02$ & ${ }^{3}$ & --- & 3 & UW \\
\hline R015 & 3 & Number of unsaturated zone strata & 31 & 31 & 3 & -- & 3 & NS \\
\hline R015 & 3 & Unsat. zone 1 , thickness (m) & $31.040 E+01$ & $34.000 E+00$ & 3 & --- & 3 & $\mathrm{H}(1)$ \\
\hline $\mathrm{R} 015$ & 3 & Unsat. zone 1 , soil density $\left(\mathrm{g} / \mathrm{cm}^{* *} 3\right)$ & $31.600 \mathrm{E}+00$ & $31.500 E+00$ & 3 & -- & 3 & DENSUZ ( 1 ) \\
\hline R015 & 3 & Unsat. zone 1 , total porosity & $34.000 \mathrm{E}-01$ & $34.000 E-01$ & 3 & --- & 3 & TPUZ (1) \\
\hline $\mathrm{R} 015$ & 3 & Unsat. zone 1 , effective porosity & $32.500 E-01$ & $32.000 E-01$ & 3 & --- & 3 & EPUZ (1) \\
\hline R015 & 3 & Unsat. zone 1 , field capacity & $31.500 \mathrm{E}-01$ & $32.000 \mathrm{E}-01$ & 3 & --- & 3 & FCUZ (1) \\
\hline $\mathrm{R} 015$ & 3 & Unsat. zone 1, soil-specific b parameter & $3.050 E+00$ & $35.300 E+00$ & 3 & --- & 3 & BUZ (1) \\
\hline R015 & 3 & Unsat. zone 1 , hydraulic conductivity ( $\mathrm{m} / \mathrm{y} r)$ & $3.500 \mathrm{E}+02$ & $31.000 E+01$ & 3 & -- & $\begin{array}{l}3 \\
3\end{array}$ & HCUZ (1) \\
\hline R016 & 3 & Distribution coefficients for C-14 & 3 & 3 & 3 & & 3 & \\
\hline R016 & 3 & Contaminated zone $\left(\mathrm{cm}^{* * 3 / \mathrm{g})}\right.$ & $32.000 \mathrm{E}+02$ & $30.000 \mathrm{E}+00$ & 3 & --- & 3 & $\operatorname{DCNUCC}(1)$ \\
\hline R016 & 3 & Unsaturated zone $1\left(\mathrm{~cm}^{*} * 3 / \mathrm{g}\right)$ & $32.000 E+02$ & $30.000 E+00$ & 3 & --- & 3 & DCNUCU $(1,1)$ \\
\hline R016 & 3 & Saturated zone $(\mathrm{cm} * * 3 / \mathrm{g})$ & $32.000 E+02$ & $30.000 E+00$ & 3 & -- & 3 & DCNUCS ( 1) \\
\hline R016 & 3 & Leach rate $(/ y r)$ & $30.000 \mathrm{E}+00$ & $30.000 \mathrm{E}+00$ & 3 & $5.426 \mathrm{E}-05$ & 3 & ALEACH (1) \\
\hline R016 & ${ }^{3}$ & Solubility constant & $0.000 \mathrm{E}+00$ & $30.000 \mathrm{E}+00$ & 3 & not used & ${ }^{3}$ & SOLUBK $(1)$ \\
\hline R016 & 3 & Distribution coefficients for Cs-137 & 3 & 3 & 3 & & 3 & \\
\hline R016 & 3 & Contaminated zone $(\mathrm{cm} * * 3 / \mathrm{g})$ & ${ }^{3} 5.000 E+01$ & $31.000 E+03$ & 3 & $-\cdots$ & 3 & $\operatorname{DCNUCC}(2)$ \\
\hline $\mathrm{R} 016$ & 3 & Unsaturated zone $1(\mathrm{~cm} * * 3 / \mathrm{g})$ & $35.000 E+01$ & $31.000 \mathrm{E}+03$ & 3 & --- & 3 & DCNUCU $(2,1)$ \\
\hline R016 & 3 & Saturated zone $(\mathrm{cm} * * 3 / \mathrm{g})$ & $35.000 E+01$ & $31.000 \mathrm{E}+03$ & 3 & --- & 3 & DCNUCS $(2)$ \\
\hline R016 & 3 & Leach rate (/yr) & $30.000 \mathrm{E}+00$ & $30.000 \mathrm{E}+00$ & 3 & $2.166 \mathrm{E}-04$ & 3 & ALEACH ( 2) \\
\hline R016 & $\begin{array}{l}3 \\
3\end{array}$ & Solubility constant & ${ }_{3}^{3} 0.000 E+00$ & $0.000 E+00$ & 3 & not used & $\begin{array}{l}3 \\
3\end{array}$ & SOLUBK $(2)$ \\
\hline $\mathrm{R} 016$ & 3 & Distribution coefficients for Eu-152 & 3 & 3 & 3 & & 3 & \\
\hline R016 & 3 & Contaminated zone $(\mathrm{cm} * * 3 / \mathrm{g})$ & $32.000 E+02$ & ${ }^{3}-1.000 \mathrm{E}+00$ & 3 & --- & 3 & $\operatorname{DCNUCC}(3)$ \\
\hline R016 & 3 & Unsaturated zone $1(\mathrm{~cm} * * 3 / \mathrm{g})$ & $32.000 E+02$ & ${ }^{3}-1.000 E+00$ & 3 & --- & 3 & $\operatorname{DCNUCU}(3,1)$ \\
\hline $\mathrm{R} 016$ & 3 & Saturated zone $(\mathrm{cm} * * 3 / \mathrm{g}\rangle$ & $32.000 E+02$ & $3-1.000 E+00$ & 3 & --- & 3 & DCNUCS ( 3 ) \\
\hline R016 & 3 & Leach rate $(/ y r)$ & $30.000 E+00$ & ${ }^{3} 0.000 \mathrm{E}+00$ & 3 & $5.426 \mathrm{E}-05$ & 3 & ALEACH ( 3) \\
\hline R016 & $\begin{array}{l}3 \\
3\end{array}$ & Solubility constant & ${ }_{3}^{3} 0.000 \mathrm{E}+00$ & ${ }_{3}^{3} 0.000 \mathrm{E}+00$ & 3 & not used & $\begin{array}{l}3 \\
3\end{array}$ & SOLUBK ( 3$)$ \\
\hline R016 & 3 & Distribution coefficients for Eu-154 & 3 & 3 & 3 & & 3 & \\
\hline $\mathrm{R} 016$ & 3 & Contaminated zone $(\mathrm{cm} * * 3 / \mathrm{g})$ & $32.000 \mathrm{E}+02$ & $3-1.000 E+00$ & 3 & --- & 3 & DCNUCC ( 5) \\
\hline R016 & 3 & Unsaturated zone $1(\mathrm{~cm} * * 3 / \mathrm{g})$ & $32.000 \mathrm{E}+02$ & $3-1.000 E+00$ & 3 & --- & 3 & DCNUCU $(5,1)$ \\
\hline R016 & 3 & Saturated zone $(\mathrm{cm} * * 3 / \mathrm{g})$ & $2.000 \mathrm{E}+02$ & ${ }^{3}-1.000 E+00$ & 3 & --- & 3 & DCNUCS $(5)$ \\
\hline R016 & ${ }^{3}$ & Leach rate (/yr) & $30.000 E+00$ & $30.000 E+00$ & 3 & $5.426 \mathrm{E}-05$ & 3 & ALEACH ( 5) \\
\hline $\mathrm{R} 016$ & 3 & Solubility constant & $30.000 \mathrm{E}+00$ & $30.000 E+00$ & 3 & not used & 3 & SOLUBK( 5) \\
\hline
\end{tabular}


IRESRAD, Version $6.3 \quad$ T\& Limit $=0.5$ year $12 / 15 / 2005 \quad 11: 50$ Page 6

Summary : 116-K-2 Trench (West End) Cleanup Verification RESRAD Calculation

File : 116-K-2_Shallow_zone west.RAD

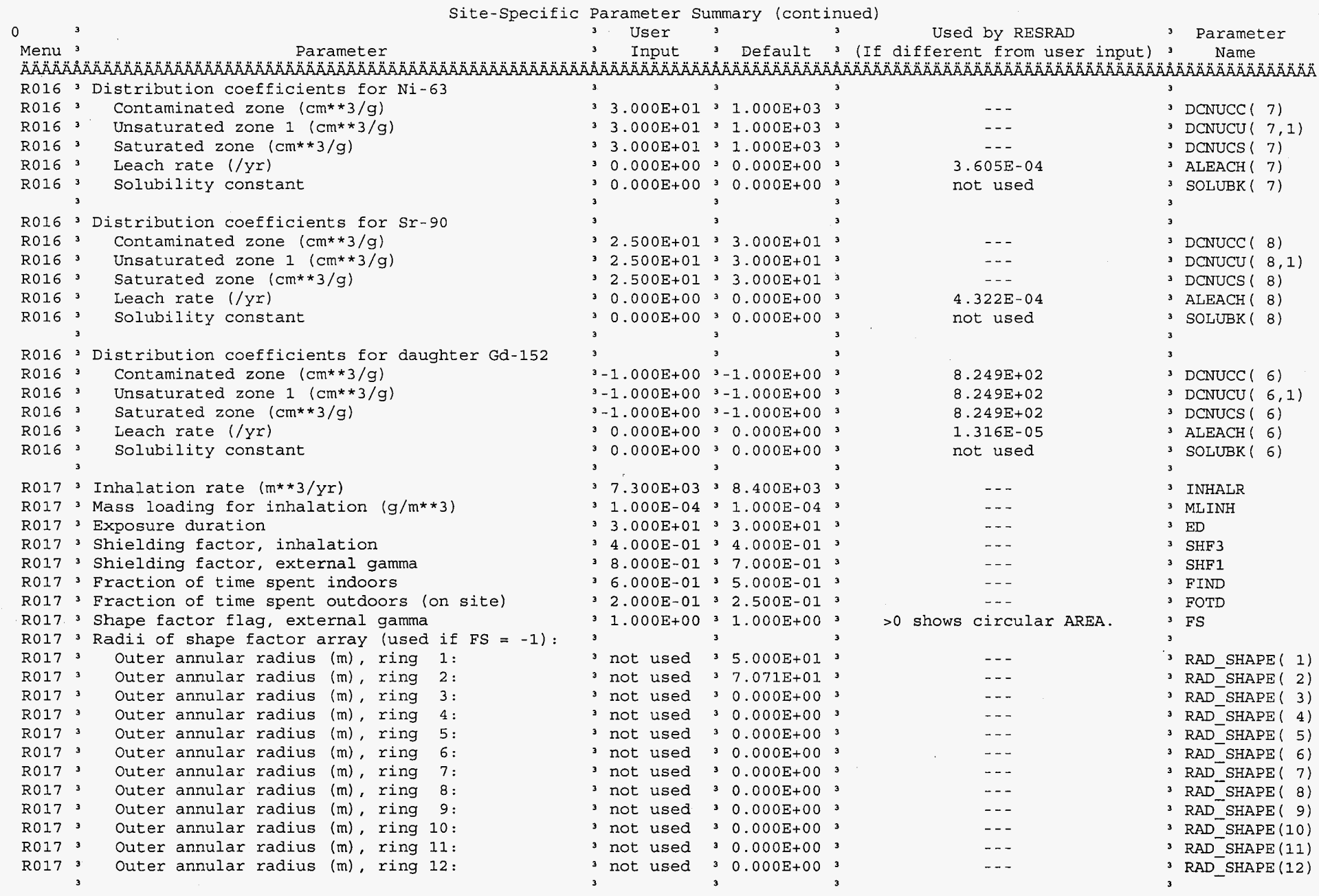


IRESRAD, Version 6.3 T Limit $=0.5$ year $12 / 15 / 2005 \quad 11: 50$ Page 7 Summary : 116-K-2 Trench (West End) Cleanup Verification RESRAD Calculation File : 116-K-2_Shallow_Zone_West.RAD

Site-Specific Parameter Summary (continued)

\begin{tabular}{|c|c|c|}
\hline \multicolumn{3}{|c|}{$0 \quad 3$} \\
\hline $\begin{array}{l}\text { Menu } \\
\ddot{A} \ddot{A} \ddot{A}\end{array}$ & & \\
\hline $\mathrm{R} 017$ & 3 & Fractio \\
\hline R017 & 3 & Ring \\
\hline$R 017$ & 3 & Ring \\
\hline R017 & 3 & Ring \\
\hline R017 & 3 & Ring \\
\hline R017 & 3 & Ring \\
\hline R017 & 3 & Ring \\
\hline R017 & 3 & Ring \\
\hline $\mathrm{R} 017$ & 3 & Ring \\
\hline R017 & 3 & Ring \\
\hline R017 & 3 & Ring \\
\hline R017 & 3 & Ring \\
\hline $\mathrm{R} 017$ & 3 & Ring \\
\hline
\end{tabular}

3 User 3

Used by RESRAD 3 parameter

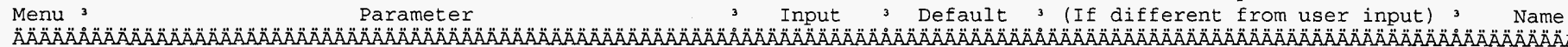

R018 3 Fruits, vegetables and grain consumption $(\mathrm{kg} / \mathrm{yr})$ R018 3 Leafy vegetable consumption $(\mathrm{kg} / \mathrm{yr})$

R018 Milk consumption (L/Yr)

R018 3 Meat and poultry consumption $(\mathrm{kg} / \mathrm{yr}$

R018 3 Fish consumption (kg/yr)

R018 3 Other seafood consumption ( $\mathrm{kg} / \mathrm{yr}$ )

R018 3 Soil ingestion rate ( $\mathrm{g} / \mathrm{yr}$ )

R018 3 Drinking water intake (L/yr)

${ }^{3}$ not used ${ }^{3} 1.000 E+00^{3}$

$3_{3}$ not used ${ }^{3} 1.000 \mathrm{E}+00^{3}$

3 not used $32.732 \mathrm{E}-01{ }^{3}$

3 not used $30.000 \mathrm{E}+00^{3}$

${ }_{3}^{3}$ not used used ${ }_{3}^{3} 0.000 \mathrm{O}+00^{3}$

3 not used $30.000 \mathrm{E}+00^{3}$

3 not used $30.000 E+00^{3}$

3 not used $30.000 E+00^{3}$

3 not used $30.000 \mathrm{E}+00^{3}$

3 not used $30.000 \mathrm{E}+00^{3}$

3 not used $30.000 \mathrm{E}+00^{3}$

${ }^{3}$ not used ${ }^{3} 0.000 \mathrm{E}+00^{3}$ 3

$1.100 \mathrm{E}+02 \quad 3 \quad 1.600 \mathrm{E}+02$ $32.700 E+00^{3} 1.400 E+01^{3}$

$31.000 \mathrm{E}+022^{3} 9.200 \mathrm{E}+013$

$33.600 E+01{ }^{3} 6.300 E+013$

$31.970 E+0135.400 E+00$

$39.000 E-0139.000 E-01$,

${ }^{3} 7.300 \mathrm{E}+011^{3} 3.650 \mathrm{E}+01^{3}$

$37.300 E+02 \quad 35.100 E+02$

$31.000 \mathrm{E}+00^{3} 1.000 \mathrm{E}+00^{3}$

3 not used $31.000 \mathrm{E}+00^{3}$

${ }^{3} 1.000 \mathrm{E}+00^{3} 1.000 \mathrm{E}+00^{3}$

$31.000 \mathrm{E}+0031.000 \mathrm{E}+00$

3 5.000E-01 3 5.000E-01

$3-1 \quad 3-1$

$3-1$

$3-1$ ${ }^{3}-1$

$3-1$

$5.500 \mathrm{E}+01^{3} 5.500 \mathrm{E}+01^{3}$

$35.000 \mathrm{E}+0135.000 \mathrm{E}+01$

$31.600 \mathrm{E}+0231.600 \mathrm{E}+02$

3 , $5.000 \mathrm{E}-0135.000 \mathrm{E}-01$,

31.000 E-04 $31.000 E-04$

$31.500 E-01331.500 E-01$

$39.000 \mathrm{E}-01^{3} 9.000 \mathrm{E}-01^{3}$

${ }_{3} 1.000 \mathrm{E}+00^{3} 1.000 \mathrm{E}+00^{3}$

3 not used $31.000 \mathrm{E}+00$

$31.000 \mathrm{E}+00^{3} 1.000 \mathrm{E}+00^{3}$

$31.000 \mathrm{E}+00^{3} 1.000 \mathrm{E}+00^{3}$

3

$37.000 \mathrm{E}-0137.000 \mathrm{E}-01$

$31.500 \mathrm{E}+00$ 3 $1.500 \mathrm{E}+00$

$31.100 \mathrm{E}+00^{3} 1.100 \mathrm{E}+00^{3}$

$31.700 \mathrm{E}-011^{3} 1.700 \mathrm{E}-01^{3}$

32.500 E-01 $32.500 E-01$

$38.000 \mathrm{E}-022^{3} 8.000 \mathrm{E}-02$,
3 FRACA ( 1$)$

3 FRACA ( 2)

3 FRACA ( 3)

3 FRACA ( 4$)$

3 FRACA ( 5)

3 FRACA ( 6$)$

3 FRACA ( 7)

3 FRACA ( 8)

3 FRACA ( 9)

3 FRACA (10)

3 FRACA (11)

3 FRACA (12)

3 DIET (1)

3 DIET (2)

3 DIET(3)

3 DIET (4)

3 DIET (5)

3 DIET (6)

3 SOIL

3 DWI

3 FDW

3 FHHW

3 FLW

3 FIRW

3 FR9

3 FPLANT

3 FMEAT

3 FMILK

3 LFI5

3 LFI6

3 LWI5

3 LWI 6

3 LSI

3 MLFD

$3 \mathrm{DM}$

3 DROOT

3 FGWDW

3 FGWHH

3 FGWLW

3 FGWIR

3 YV (1)

$3 \mathrm{YV}(2)$

$3 \mathrm{YV}(3)$

3 TE (1)

3 TE (2)

$3 \mathrm{TE}(3)$ 
1RESRAD, Version 6.3 T Limit $=0.5$ year $\quad 12 / 15 / 2005 \quad 11: 50$ Page 8 Summary : $116-\mathrm{K}-2$ Trench (West End) Cleanup Verification RESRAD Calculation File : 116-K-2_Shallow Zone west. RAD

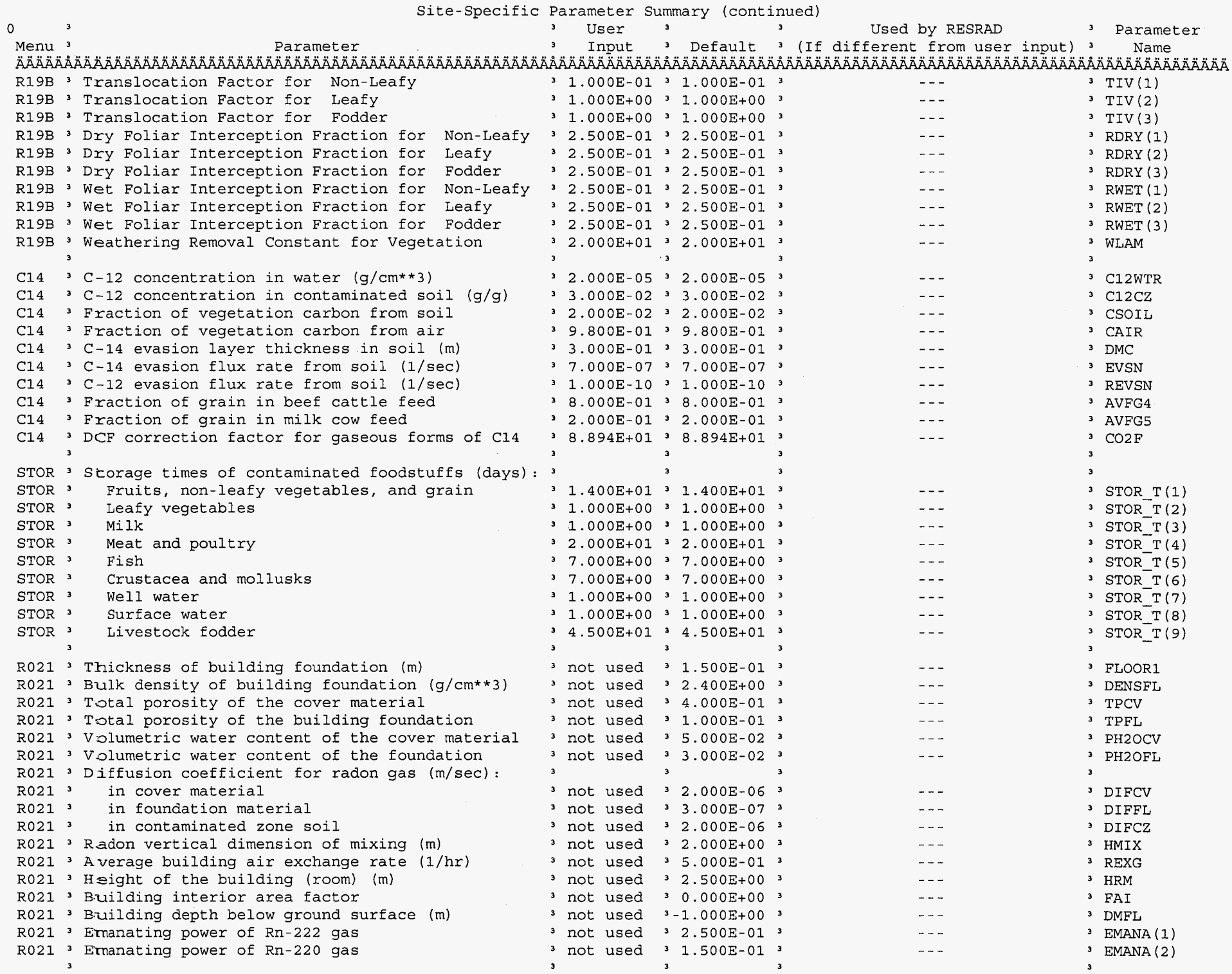


CVP-2006-00001

Rev. 0

1RESRAD, Version $6.3 \quad$ T\& Limit $=0.5$ year
Summary : $116-\mathrm{K}-2$ Trench (West End) Cleanup Verification RESRAD Calculation

$\begin{array}{ll}\text { Summary } & =116-\mathrm{K}-2 \text { Trench (West End) Clean } \\ \text { File } & =116-\mathrm{K}-2 \text { _Shallow_Zone_West.RAD }\end{array}$

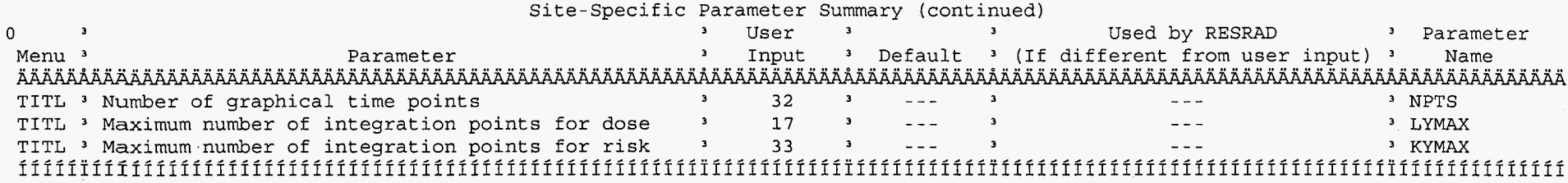

Summary of Pathway Selections

Pathway ${ }^{3}$ User Selection

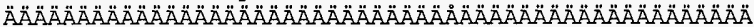

1 - external gamma active

2 - inhalation (w/o radon) ${ }^{3}$ active

3 -- plant ingestion active

4 -- meat ingestion active

5 -. milk ingestion 3 active

6 - aquatic foods ${ }^{3}$ active

7 -- drinking water 3 active

8 - soil ingestion ${ }^{3}$ active

$\begin{array}{llc}9 \text {-- radon } & 3 & \text { suppressed } \\ \text { Find peak pathway doses } & 3 & \text { active }\end{array}$

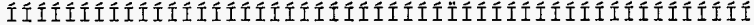


CVP-2006-00001

Rev. 0

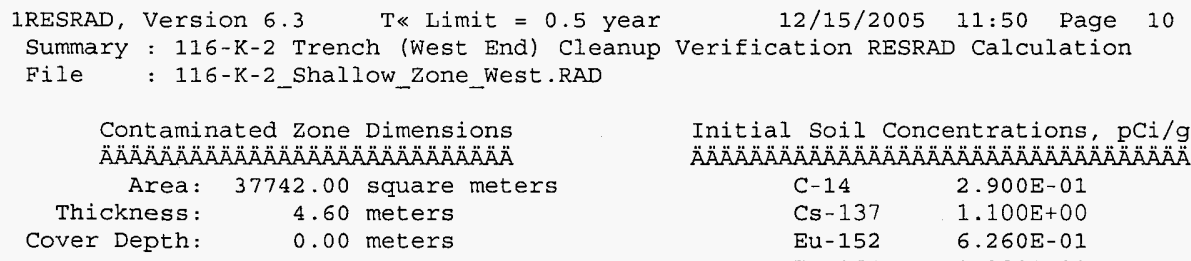

0

Total Dose TDOSE(t), mrem/yr

Basic Radiation Dose Limit $=1.500 \mathrm{E}+01 \mathrm{mrem} / \mathrm{yr}$

Total Mixture Sum $M(t)=$ Fraction of Basic Dose Limit Received at Time ( $t)$

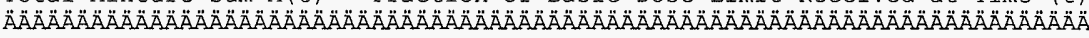

$t$ (years): $\quad \begin{array}{lllllllll}0.000 \mathrm{E}+00 & 1.000 \mathrm{E}+00 & 3.000 \mathrm{E}+00 & 1.300 \mathrm{E}+01 & 3.000 \mathrm{E}+01 & 1.000 \mathrm{E}+02 & 3.000 \mathrm{E}+02 & 1.000 \mathrm{E}+03\end{array}$

$\operatorname{TDOSE}(t): \begin{array}{lllllllll}7.767 E+00 & 6.959 E+00 & 6.287 \mathrm{E}+00 & 4.331 \mathrm{E}+00 & 2.450 \mathrm{E}+00 & 3.673 \mathrm{E}-01 & 4.214 \mathrm{E}-03 & 5.232 \mathrm{E}-06\end{array}$

$M(t):=\begin{array}{lllllllll}5.178 E-01 & 4.639 E-01 & 4.192 \mathrm{E}-01 & 2.888 \mathrm{E}-01 & 1.633 \mathrm{E}-01 & 2.449 \mathrm{E}-02 & 2.809 \mathrm{E}-04 & 3.488 \mathrm{E}-07\end{array}$

OMaximum $\operatorname{TDOSE}(t): \quad 7.767 \mathrm{E}+00 \mathrm{mrem} / \mathrm{yr}$ at $t=0.000 \mathrm{E}+00$ years 


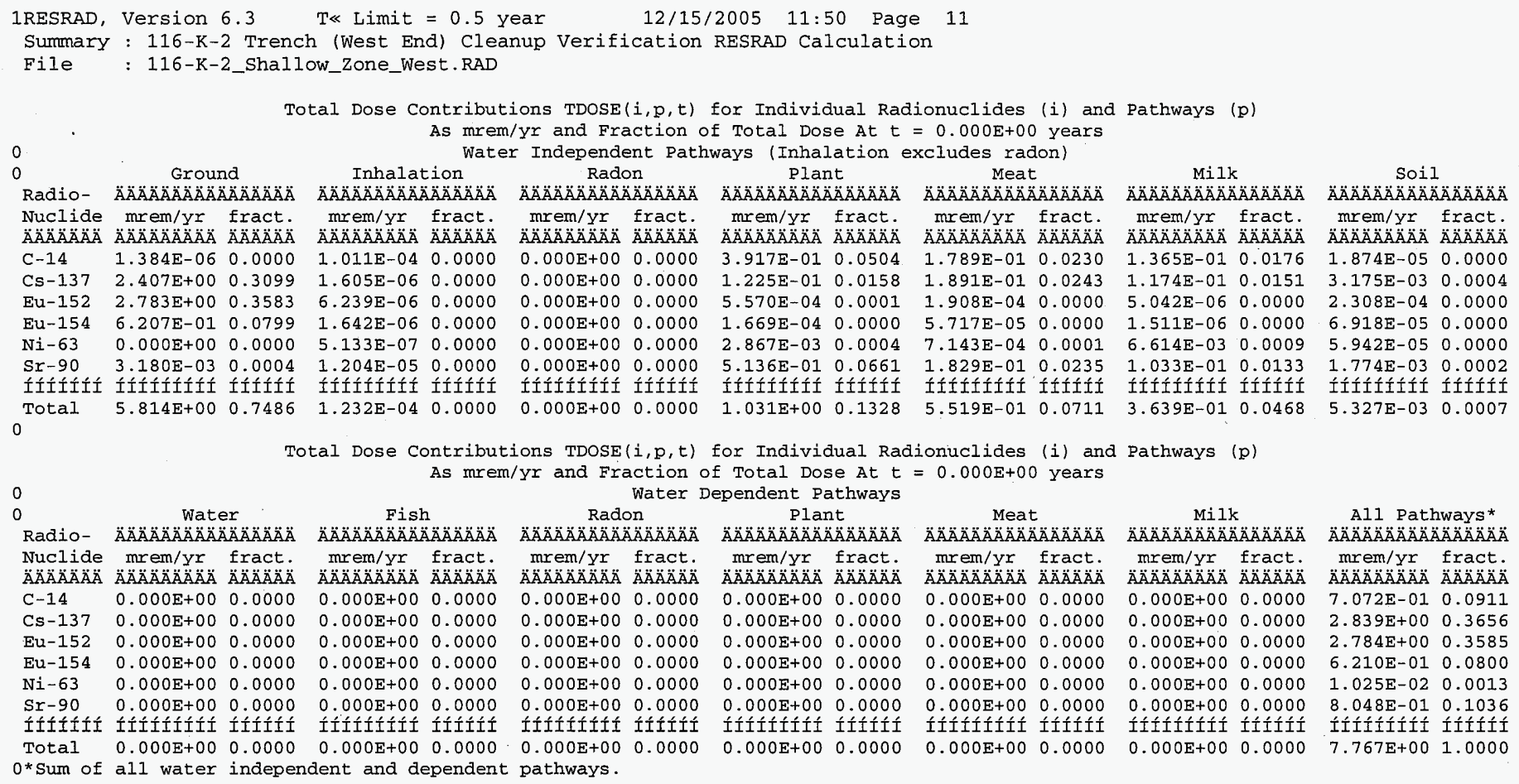




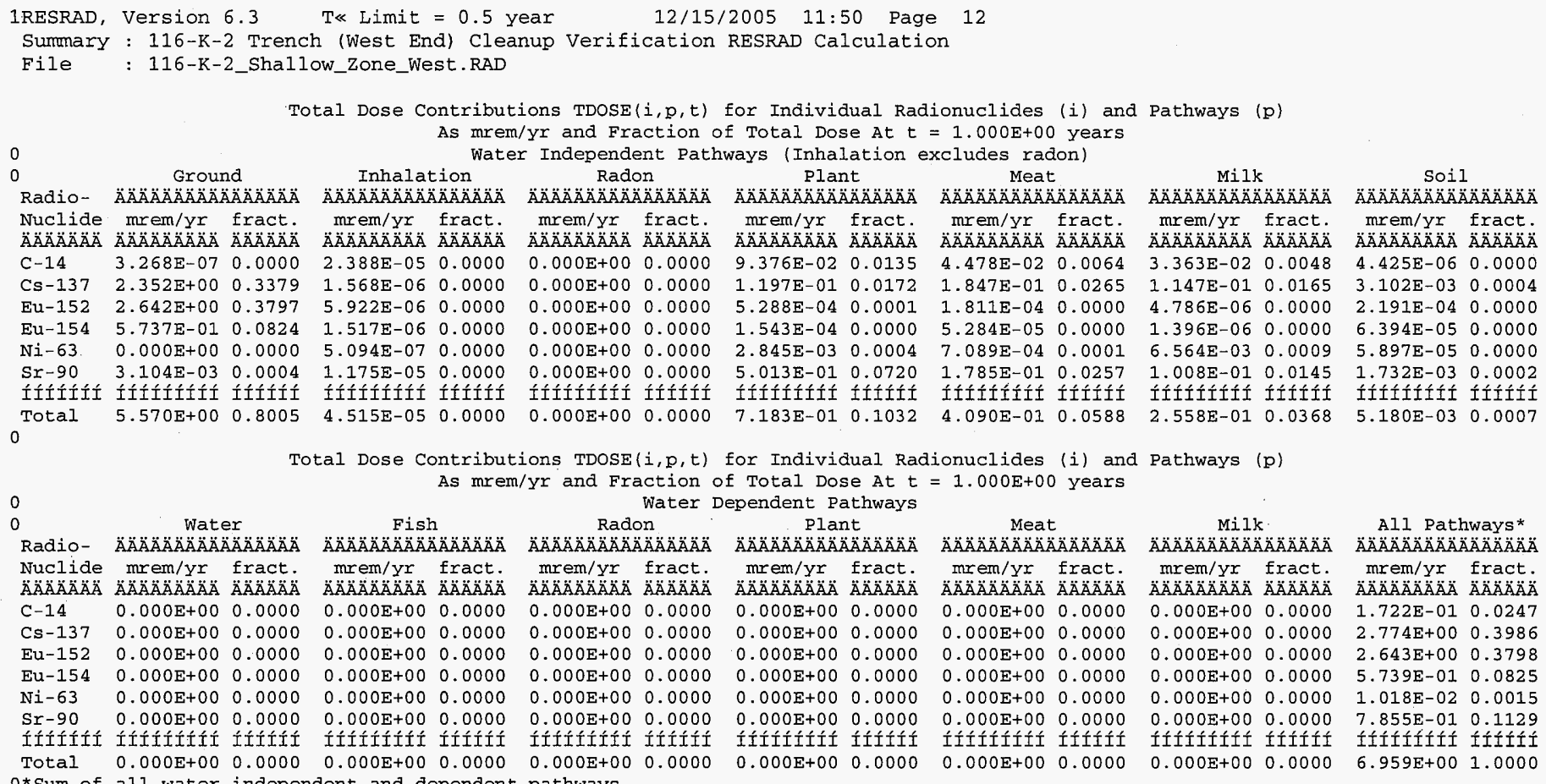
0 * Sum of all watex independent and dependent pathways. 


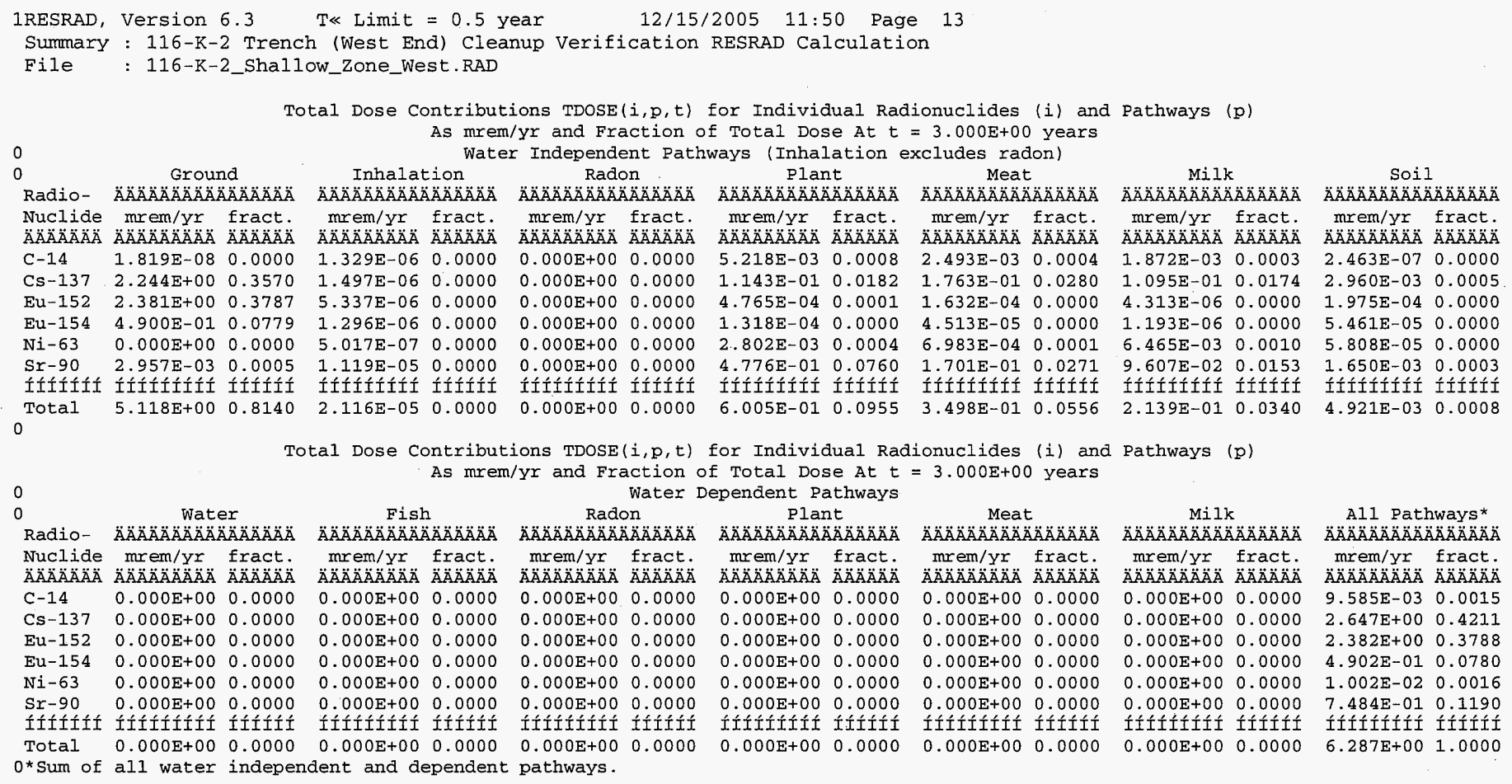


Total Dose Contributions TDOSE( $i, p, t)$ for Individual Radionuclides (i) and Pathways (p)

As mrem/yr and Fraction of Total Dose At $t=1.300 \mathrm{E}+01$ years Water Independent Pathways (Inhalation excludes radon)

Ground

Inhalation Radon Radio- $\ddot{A} \ddot{A} \ddot{A} \ddot{A} \ddot{A} \ddot{A} \ddot{A} \ddot{A} \ddot{A} \ddot{A} \ddot{A} \ddot{A} \ddot{A}$ Nuclide mrem/yr fract. $\ddot{A} \ddot{A} \ddot{A} \ddot{A} \ddot{A} \ddot{A} \ddot{A} \quad \ddot{A} \ddot{A} \ddot{A} \ddot{A} \ddot{A} \ddot{A} \ddot{A} \ddot{A} \ddot{A} \ddot{A} \ddot{A} \ddot{A} \ddot{A} \ddot{A} \ddot{A}$ $\begin{array}{lll}C-14 & 9.352 \mathrm{E}-15 \quad 0.0000\end{array}$ Cs-137 1.778E+00 0.4104 $\mathrm{Eu}-152 \quad 1.415 \mathrm{E}+00 \quad 0.3266$ \begin{tabular}{lll}
$\mathrm{Eu}-154 \quad 2.228 \mathrm{E}-01 \quad 0.0514$ \\
\hline
\end{tabular} $\mathrm{Ni}-63 \quad 0.000 \mathrm{E}+00 \quad 0.0000$ $\begin{array}{lll}\mathrm{Sr}-90 & 2.321 \mathrm{E}-03 & 0.0005\end{array}$ Total $\quad 3.417 \mathrm{E}+00 \quad 0.7890$

0 Radio- $\ddot{A} \ddot{A} \ddot{A} \ddot{A} \ddot{A} \ddot{A} \ddot{A} \ddot{A} \ddot{A} \ddot{A} \ddot{A} \ddot{A} \ddot{A} \ddot{A}$ Nuclide mrem/yr fract.

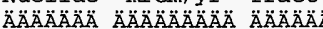
$\begin{array}{lll}C-14 & 0.000 E+00 & 0.0000\end{array}$ $\begin{array}{lll}\text { Cs }-137 & 0.000 E+00 & 0.0000\end{array}$ Eu-152 $0.000 E+00 \quad 0.0000$ (154) $\begin{array}{lll}\mathrm{Ni}-63 & 0.000 \mathrm{E}+00 & 0.0000\end{array}$ $\mathrm{Sr}-90 \quad 0.000 \mathrm{E}+00 \quad 0.000$

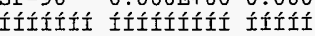

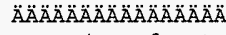
mrem/yr fract. AAAAAAAAAAAAAAA mrem/yr fract.

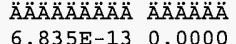
$1.185 \mathrm{E}-06 \quad 0.0000$ $3.171 \mathrm{E}-06 \quad 0.0000$ $5.893 \mathrm{E}-07 \quad 0.0000$ $4.651 \mathrm{E}-07 \quad 0.0000$ $8.785 \mathrm{E}-060.0000$

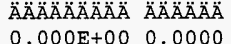
$0.000 E+00 \quad 0.0000$ $0.000 E+00 \quad 0.0000$ $0.000 E+00 \quad 0.0000$ $0.000 E+00 \quad 0.0000$ $0.000 E+00 \quad 0.0000$

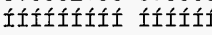
$1.420 \mathrm{E}-050.0000$

$0.000 \mathrm{E}+00 \quad 0.0000$ As mrem/yr and Fraction of Total Dose At $t=1.300 \mathrm{E}+01$ years

\begin{abstract}
Plant Meat

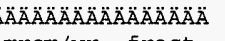
$\mathrm{mrem} / \mathrm{Yr}$ fract. mrem/yr fract.

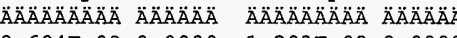
$9.049 \mathrm{E}-020.0209$ $2.831 E-04 \quad 0.0001$ $5.992 \mathrm{E}-05 \quad 0.0000$ $2.598 \mathrm{E}-03 \quad 0.0006$ $3.748 \mathrm{E}-010.0865$ ítítííí ítíít $4.683 \mathrm{E}-010.1083$

0.000
0.052

$6.473 \mathrm{E}-04 \quad 0.0001$

$1.335 \mathrm{E}-010.0308$

ífítífítí ífítít
\end{abstract}

Total Dose Contributions TDOSE ( $i, p, t)$ for Individual Radionuclides ( $i$ ) and Pathways (p) Fish water Dependent Pathways

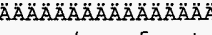
$\mathrm{mrem} / \mathrm{yr}$ fract. $0.000 E+00 \quad 0.0000$ $0.000 \mathrm{E}+00 \quad 0.0000$ $0.000 \mathrm{E}+00 \quad 0.0000$ $0.000 E+00 \quad 0.0000$ $0.000 E+00 \quad 0.0000$ $0.000 \mathrm{E}+00$ 0.0000 Radon Plant

$$
\text { Meat }
$$

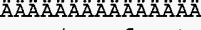

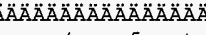
$\begin{array}{cc}m r e m / Y r & \text { fract. } \\ \ddot{A} \ddot{A} \ddot{A} \ddot{A} \ddot{A} \ddot{A} \ddot{A} \ddot{A} \text { Ä } \ddot{A} \ddot{A} \ddot{A} \ddot{A} \ddot{A}\end{array}$ fract $\begin{array}{lllll}0.000 \mathrm{E}+00 & 0.0000 & 0.000 \mathrm{E}+00 & 0.0000\end{array}$ $\begin{array}{lllll}0.000 \mathrm{E}+00 & 0.0000 & 0.000 \mathrm{E}+00 & 0.0000\end{array}$ $0.000 \mathrm{E}+00 \quad 0.0000$ $0.000 \mathrm{E}+00 \quad 0.0000$ $0.000 \mathrm{E}+00 \quad 0.0000$ $0.000 \mathrm{E}+00 \quad 0.0000$ $0.000 \mathrm{E}+00 \quad 0.0000$ $0.000 \mathrm{E}+00 \quad 0.0000$ $0.000 E+00 \quad 0.0000$ $0.000 E+00 \quad 0.0000$ $0.000 \mathrm{E}+00 \quad 0.0000$ $0.000 \mathrm{E}+00 \quad 0.0000$ $0.000 \mathrm{E}+00 \quad 0.0000$ pathways . 


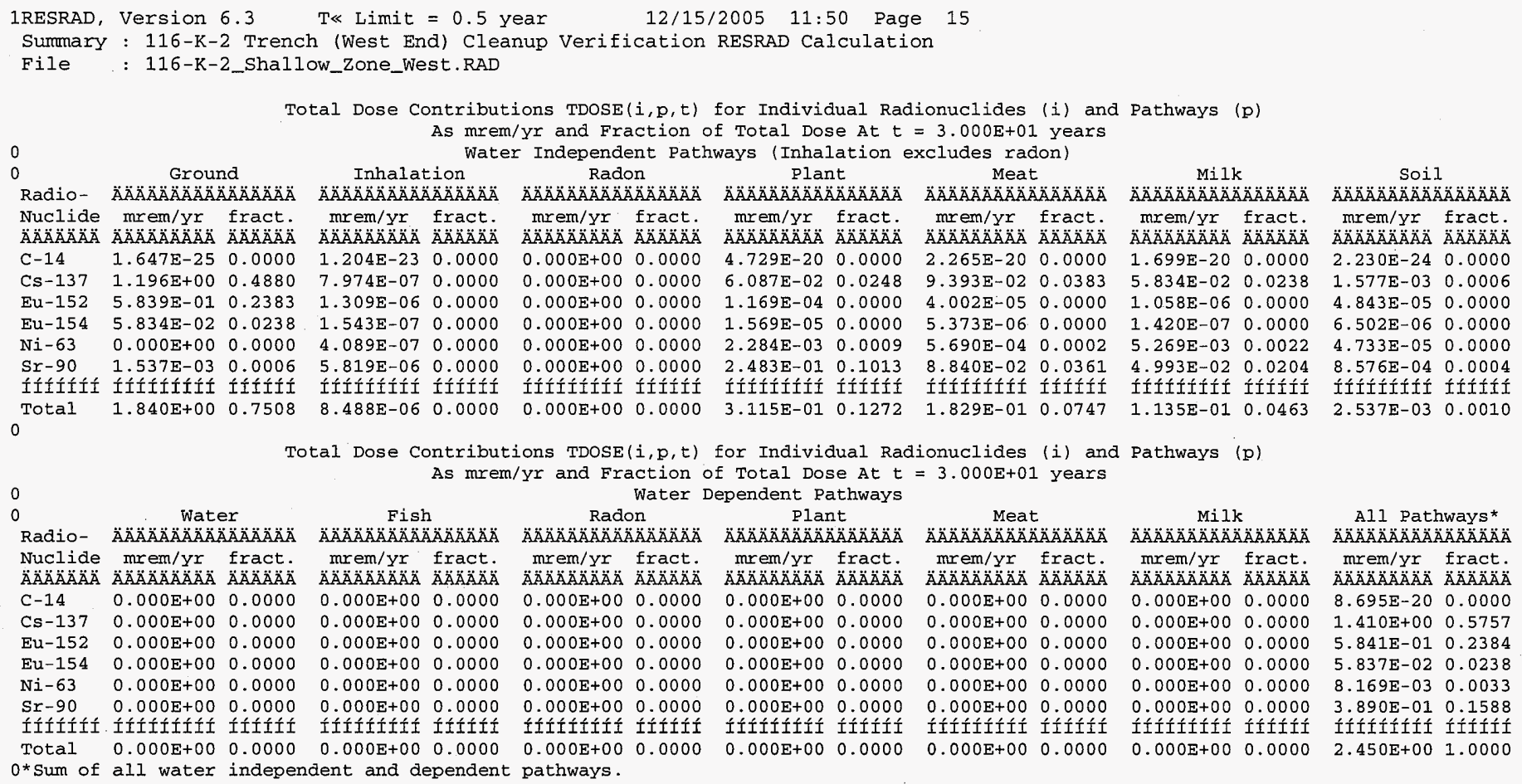




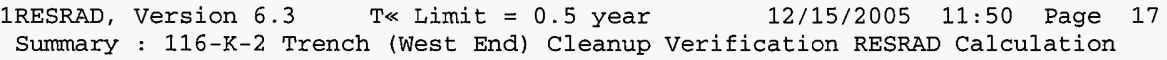

File : 116-K-2_Shallow_zone_West.RAD

Total Dose Contributions TDOSE(i,p,t) for Individual Radionuclides (i) and Pathways ( $p$ ) As mrem/yr and Fraction of Total. Dose At $t=3.000 \mathrm{E}+02$ years

0 Water Independent Pathways (Inhalation excludes radon) Ground Inhalation Radon Plant. Meat

Radio- $\ddot{\mathrm{A}} \ddot{\mathrm{A}} \ddot{\mathrm{A}} \ddot{\mathrm{A}} \ddot{\mathrm{A}} \ddot{\mathrm{A}} \ddot{\mathrm{A}} \ddot{\mathrm{A}} \ddot{\mathrm{A}} \ddot{\mathrm{A}} \ddot{\mathrm{A}} \ddot{\mathrm{A}} \ddot{\mathrm{A}} \ddot{\mathrm{A}} \ddot{\mathrm{A}} \ddot{\mathrm{A}}$

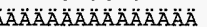

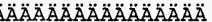

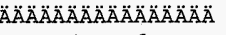

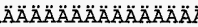
Nuclide mrem/yr fract. mrem/yr fract.

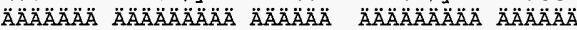
$\begin{array}{llllll}C-14 & 0.000 \mathrm{E}+00 & 0.0000 & 0.000 \mathrm{E}+00 & 0.0000\end{array}$ $\begin{array}{lllll}\mathrm{Cs}-137 & 2.203 \mathrm{E}-03 & 0.5228 & 1.469 \mathrm{E}-09 & 0.0000\end{array}$ mrem/yr fract. mrem/yr fract. mrem/yr fract.

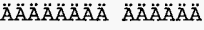
$0.0000+000.0000-0.00$ $\begin{array}{llll}0.000 \mathrm{E}+00 & 0.0000 & 0.000 \mathrm{E}+00 \quad 0.0000\end{array}$ $\begin{array}{lllll}\mathrm{Eu}-152 & 4.598 \mathrm{E}-07 & 0.0001 & 1.031 \mathrm{E}-12 & 0.0000\end{array}$

$\begin{array}{llllll}\mathrm{Eu}-154 & 3.338 \mathrm{E}-11 & 0.0000 & 8.828 \mathrm{E}-17 & 0.0000\end{array}$ $.000 \mathrm{E}+00 \quad 0.0000$ $1.121 \mathrm{E}-04 \quad 0.0266$ $1.730 \mathrm{E}-04 \quad 0.0411$ $0.00 \mathrm{E}+00 \quad 0.0000$ $9.203 \mathrm{E}-110.0000$ $3.152 \mathrm{E}-11 \quad 0.0000$ $8.977 \mathrm{E}-15 \quad 0.0000$ $3.074 \mathrm{E}-15 \quad 0.0000$ $0.000 \mathrm{E}+00 \quad 0.0000$ $2.949 \mathrm{E}-04 \quad 0.0700$

$7.349 \mathrm{E}-05 \quad 0.0174$ $\mathrm{Sr}-90 \quad 2.212 \mathrm{E}-06 \quad 0.0005 \quad 8.376 \mathrm{E}-09 \quad 0.0000$

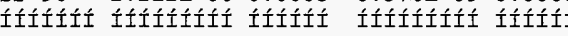
$000 \mathrm{E}+00 \quad 0.0000$ $3.573 \mathrm{E}-04 \quad 0.0848$ $1.272 \mathrm{E}-04 \quad 0.0302$ Total $2.205 \mathrm{E}-03 \quad 0.5234 \quad 6.265 \mathrm{E}-08 \quad 0.0000$

Ííífítít ífítít

ífítíítí ííííi

Total Dose Contributions TDOSE( $i, p, t)$ for Individual Radionuclides ( $i$ ) and Pathways (p) As mrem/yr and Fraction of Total Dose At $t=3.000 \mathrm{E}+02$ years As mrem/yr and Fraction of Total Dose At $t$
water Dependent Pathways Fish Plant

Meat Nuclide mrem/yr fract. mrem/yr fract.

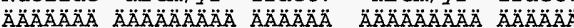
$\begin{array}{lllll}C-14 & 0.000 \mathrm{E}+00 & 0.0000 & 0.000 \mathrm{E}+00 & 0.0000\end{array}$ $\begin{array}{lllll}\mathrm{Cs}-137 & 0.000 \mathrm{E}+00 & 0.0000 & 0.000 \mathrm{E}+00 & 0.000\end{array}$ $\begin{array}{lllll}\mathrm{Eu}-152 & 0.000 \mathrm{E}+00 & 0.0000 & 0.000 \mathrm{E}+00 & 0.0000\end{array}$ Eu-154 $0.000 E+00 \quad 0.0000 \quad 0.000 E+00 \quad 0.0000$ $\begin{array}{llllll}\mathrm{Ni}-63 & 0.000 \mathrm{E}+00 & 0.0000 & 0.000 \mathrm{E}+00 & 0.0000\end{array}$ $\mathrm{Sr}-90 \quad 0.000 \mathrm{E}+00 \quad 0.0000 \quad 0.000 \mathrm{E}+00 \quad 0.0000$

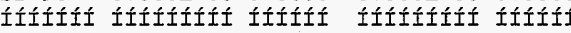
$\begin{array}{lllllllllllll}\text { Total } & 0.000 \mathrm{E}+00 & 0.0000 & 0.000 \mathrm{E}+00 & 0.0000 & 0.000 \mathrm{E}+00 & 0.0000\end{array}$ $0 *$ Sum of all water independent and dependent pathways

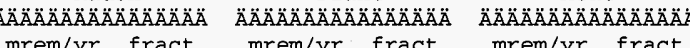
Radon mrems fract. $0.000 \mathrm{E}+00 \quad 0.0000$ $0.000 \mathrm{E}+00 \quad 0.0000$ $0.000 \mathrm{E}+00 \quad 0.0000$ $0.000 \mathrm{E}+00 \quad 0.0000$

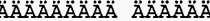
$0.000 \mathrm{E}+00 \quad 0.0000$ $0.000 \mathrm{E}+00 \quad 0.0000$ $0.000 \mathrm{E}+00 \quad 0.0000$ $0.000 E+00 \quad 0.0000$ $0.000 \mathrm{E}+00 \quad 0.0000$ $0.000 \mathrm{E}+00 \quad 0.0000$ ííííítíi ííííi $0.000 \mathrm{E}+00 \quad 0.0000$

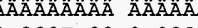

$0.000 \mathrm{E}+00 \quad 0.0000$

$0.000 \mathrm{E}+00 \quad 0.0000$

$0.000 \mathrm{E}+00 \quad 0.0000$

$0.000 \mathrm{E}+00 \quad 0.0000$

$0.000 \mathrm{E}+00 \quad 0.0000$

$0.000 \mathrm{E}+00 \quad 0.0000$

ítíítíti títíít

$0.000 \mathrm{E}+00 \quad 0.0000$
Milk Soil

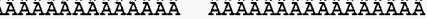
mrem/yr fract. mrem/yr fract. Ä $\begin{array}{lllll}0.000 \mathrm{E}+00 & 0.0000 & 0.000 \mathrm{E}+00 \quad 0.0000\end{array}$ $\begin{array}{llll}1.075 \mathrm{E}-04 & 0.0255 & 2.905 \mathrm{E}-06 & 0.0007\end{array}$ $\begin{array}{llll}.330 \mathrm{E}-13 & 0.0000 & 3.814 \mathrm{E}-11 & 0.0000\end{array}$ 8.125E-17 $0.0000 \quad 3.720 \mathrm{E}-15 \quad 0.0000$ $\begin{array}{cccc}804 \mathrm{E}-04 & 0.1615 & 6.113 \mathrm{E}-06 & 0.0015\end{array}$ $\begin{array}{llll}187 \mathrm{E}-05 & 0.0171 & 1.234 \mathrm{E}-06 & 0.0003\end{array}$

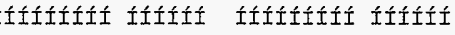
$1.025 \mathrm{E}-05 \quad 0.0024$

\begin{tabular}{|c|c|c|c|}
\hline \multicolumn{2}{|c|}{ 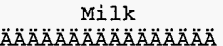 } & \multicolumn{2}{|c|}{ 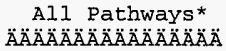 } \\
\hline $\begin{array}{c}m r e m / Y r \\
\ddot{A} \ddot{A} \ddot{A} \ddot{A} \ddot{A} \ddot{A} \ddot{A} \ddot{A} \ddot{A}\end{array}$ & $\begin{array}{l}\text { fract. } \\
\ddot{A} \ddot{A} \ddot{A} \ddot{A} \ddot{A} \ddot{A}\end{array}$ & 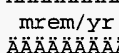 & fract. \\
\hline $0.000 \mathrm{E}+00$ & 0.0000 & $0.000 \mathrm{E}+0$ & 0.0000 \\
\hline $0.000 E+00$ & 0.0000 & $2.598 \mathrm{E}-0$ & 0.6166 \\
\hline $0.000 \mathrm{E}+00$ & 0.0000 & $4.600 \mathrm{E}-0$ & 0.0001 \\
\hline $0.000 \mathrm{E}+00$ & 0.0000 & $3.339 \mathrm{E}-1$ & 0.0000 \\
\hline $0.000 \mathrm{E}+00$ & 0.0000 & $1.055 \mathrm{E}-0$ & 0.2504 \\
\hline $\begin{array}{l}0.000 \mathrm{E}+00 \\
\text { tífítíti }\end{array}$ & $\begin{array}{l}0.0000 \\
\text { ítífít }\end{array}$ & $\begin{array}{l}5.599 \mathrm{E}-0 \\
\text { Iffítíti }\end{array}$ & $\begin{array}{l}0.1329 \\
\text { Itfiff }\end{array}$ \\
\hline $0.000 \mathrm{E}+00$ & 0.0000 & $4.214 \mathrm{E}-0$ & 1.0000 \\
\hline
\end{tabular}




\begin{abstract}
1RESRAD, Version 6.3 T« Limit $=0.5$ year $\quad 12 / 15 / 2005 \quad 11: 50$ Page 18 Summary : 116-K-2 Trench (West End) Cleanup Verification RESRAD Calculation
\end{abstract} File : 116-K-2_Shallow_zone_west.RAD

Total Dose Contributions TDOSE (i,p,t) for Individual Radionuclides (i) and Pathways (p) As mrem/yr and Fraction of Total Dose At $t=1.000 \mathrm{E}+03$ years

0 Water Independent Pathways (Inhalation excludes radon) Inhalation Radon Meat Milk Plant Radio- $\ddot{A} \ddot{A} \ddot{A} \ddot{A} \ddot{A} \ddot{A} \ddot{A} \ddot{A} \ddot{A} \ddot{A} \ddot{A} \ddot{A} \ddot{A} \ddot{A} \ddot{A} \ddot{A}$ Nuclide mrem/Yr fract. yr fract. mrem/yr fract.

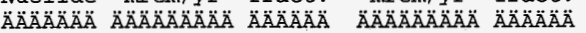
$\begin{array}{llllll}C-14 & 0.000 \mathrm{E}+00 & 0.0000 & 0.000 \mathrm{E}+00 & 0.0000\end{array}$ $\begin{array}{lllll}\mathrm{Cs}-137 & 1.791 \mathrm{E}-10 & 0.0000 & 1.194 \mathrm{E}-16 & 0.0000\end{array}$ $\begin{array}{lllll}\mathrm{Eu}-152 & 6.888 \mathrm{E}-23 & 0.0000 & 2.392 \mathrm{E}-16 & 0.0000\end{array}$ $\begin{array}{llllll}\mathrm{Eu}-154 & 0.000 \mathrm{E}+00 & 0.0000 & 0.000 \mathrm{E}+00 & 0.0000\end{array}$ $\begin{array}{llllll}\mathrm{Ni}-63 & 0.000 \mathrm{E}+00 & 0.0000 & 2.619 \mathrm{E}-10 & 0.0001\end{array}$ Sr-90 $\quad 9.487 \mathrm{E}-14 \quad 0.0000 \quad 3.592 \mathrm{E}-16 \quad 0.0000$

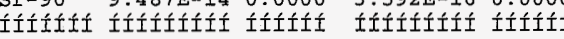
Total $\begin{array}{llll}1.792 \mathrm{E}-10 & 0.0000 & 2.619 \mathrm{E}-10 & 0.0001\end{array}$

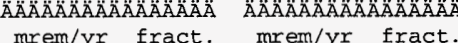
mrem/yr fract. mrem/yr fract. $\ddot{A} \ddot{A} \ddot{A} \ddot{A} \ddot{A} \ddot{A} \ddot{A} \ddot{A}$ Ä $\ddot{A} \ddot{A} \ddot{A} \ddot{A} \ddot{A}$ $0.000 \mathrm{E}+00 \quad 0.0000$ $0.000 \mathrm{E}+00 \quad 0.0000$ $0.000 \mathrm{E}+00 \quad 0.0000$ $0.000 \mathrm{E}+00 \quad 0.0000$ $0.000 \mathrm{E}+00 \quad 0.0000$ $0.000 E+00 \quad 0.0000$ fiffífítí ííítí $0.0000+00$ 0.0000 AAAAAAAÄ ÄÄÄÄ̈̈̈ $\begin{array}{lllll}9.117 \mathrm{E}-12 & 0.0000 & 1.407 \mathrm{E}-11 & 0.0000\end{array}$ $\begin{array}{llll}4.827 \mathrm{E}-16 & 0.0000 & 1.653 \mathrm{E}-16 & 0.0000\end{array}$ $0.000 \mathrm{E}+00.0 .0000$ $1.463 \mathrm{E}-06 \quad 0.2795$ $3.644 \mathrm{E}-070.0697$ $\begin{array}{llll}1.532 \mathrm{E}-11 & 0.0000 & 5.457 \mathrm{E}-12 & 0.0000\end{array}$

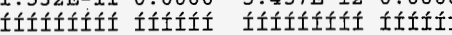
$\begin{array}{llll}1.463 \mathrm{E}-06 & 0.2795 & 3.645 \mathrm{E}-07 & 0.0697\end{array}$ Total Dose Contributions TDOSE $(i, p, t)$ for Individual Radionuclides (i) and Pathways (p) As mrem/yr and Fraction of Total Dose At $t=1.000 \mathrm{E}+03$ years

0 As mrem/yr and Fraction of Total Dose At $t$
Water Dependent Pathways Water Dependent Pathways

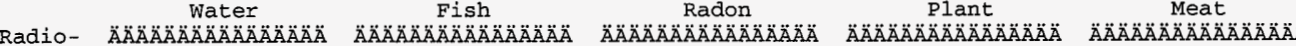
Nuclide mrem/yr fract mrem/yr fract mrem/yr fract. mrem/yr fract. mrem/yr fract.

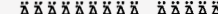
$0.000 E+000.0000$ $0.000 \mathrm{E}+000.0000 \quad 0.000 \mathrm{E}+000.0000$

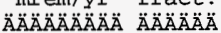
$\ddot{A} \ddot{A} \ddot{A} \ddot{A} \ddot{A} \ddot{A} \ddot{A} \ddot{A} \ddot{A} \ddot{A} \ddot{A} \ddot{A} \ddot{A} \ddot{A} \ddot{A} \ddot{A}$ $0.000 E+000.0000 \quad 0.000 E+000.0000$ $0.000 \mathrm{E}+000.0000$ $\begin{array}{lllllll}\mathrm{E}-154 & 0.000 \mathrm{E}+00 & 0.0000 & 0.000 \mathrm{E}+00 & 0.0000 & 0.000 \mathrm{E}+00 & 0.0000\end{array}$ $\begin{array}{llllllll}\mathrm{Ni}-63 & 0.000 \mathrm{E}+00 & 0.0000 & 0.000 \mathrm{E}+00 & 0.0000 & 0.000 \mathrm{E}+00 & 0.0000\end{array}$ $\begin{array}{llllllll}\mathrm{sr}-90 & 0.000 \mathrm{E}+00 & 0.0000 & 0.000 \mathrm{E}+00 & 0.0000 & 0.000 \mathrm{E}+00 & 0.0000\end{array}$

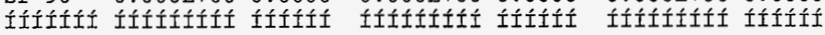
$\begin{array}{lllllllll}\text { Total } & 0.000 E+00 & 0.0000 & 0.000 E+00 & 0.0000 & 0.000 E+00 & 0.0000\end{array}$ $0.000 \mathrm{E}+00 \quad 0.0000$ $\begin{array}{ll}0.000 E+00 & 0.0000 \\ 0.000 E+00 & 0.0000\end{array}$ $0.000 \mathrm{E}+00 \quad 0.0000$ $0.000 \mathrm{E}+000.0000$ Ííííííí íííí́í

$0.000 \mathrm{E}+000.0000$ $0 *$ Sum of all water independent and dependent pathways. $0.000 E+00 \quad 0.0000$ $\ddot{A} \ddot{A} \ddot{A} \ddot{A} \ddot{A} \ddot{A} \ddot{A} \ddot{A} \quad \ddot{A} \ddot{A} \ddot{A} \ddot{A} \ddot{A} \ddot{A}$ $0.000 \mathrm{E}+00 \quad 0.0000$ $0.000 \mathrm{E}+00 \quad 0.0000$ $0.000 \mathrm{E}+00 \quad 0.0000$ $0.000 \mathrm{E}+00 \quad 0.0000$ $0.000 \mathrm{E}+000.0000$ $0.000 \mathrm{E}+000.0000$ mrem/Yr fract.

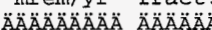
$0.000 \mathrm{E}+00 \quad 0.0000$ $8.738 \mathrm{E}-12 \quad 0.0000$ $4.368 \mathrm{E}-18 \quad 0.0000$ $0.000 \mathrm{E}+00 \quad 0.0000$ $3.374 \mathrm{E}-06 \quad 0.6449$ 3. $082 \mathrm{E}-12 \quad 0.0000$ fíitifiti títiti $3.374 \mathrm{E}-06 \quad 0.6449$ $\operatorname{Mi} 1 k$

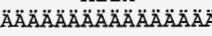
mrem/yr fract.

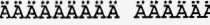
$0.000 \mathrm{E}+00 \quad 0.0000$ $0.000 \mathrm{E}+00 \quad 0.0000$ $0.000 \mathrm{E}+00 \quad 0.0000$ $0.000 \mathrm{E}+00 \quad 0.0000$ $0.000 \mathrm{E}+00 \quad 0.0000$ $0.000 \mathrm{E}+00 \quad 0.0000$ Íííííííi ííítí $0.000 \mathrm{E}+000.0000$
Soil AÄÄAAAAÄÄÄÄÄÄ mrem/yr fract.

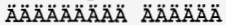
$0.000 \mathrm{E}+00 \quad 0.0000$ $2.362 \mathrm{E}-13 \quad 0.0000$ $2.000 \mathrm{E}-16 \quad 0.0000$ $0.000 E+00 \quad 0.0000$ 3. $031 E-08 \quad 0.0058$ $5.294 \mathrm{E}-14 \quad 0.0000$ íítítítí titútí 3.0315-08 0.0058
All Pathways* mrem/yr fract. 0.000E+00 0.0000 $2.113 E-100.0000$ $1.092 \mathrm{E}-150.0000$ $0.000 E+00 \quad 0.0000$ 5.232E-06 1.0000 $2.401 \mathrm{E}-11 \quad 0.0000$

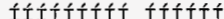
$5.232 \mathrm{E}-061.0000$ 


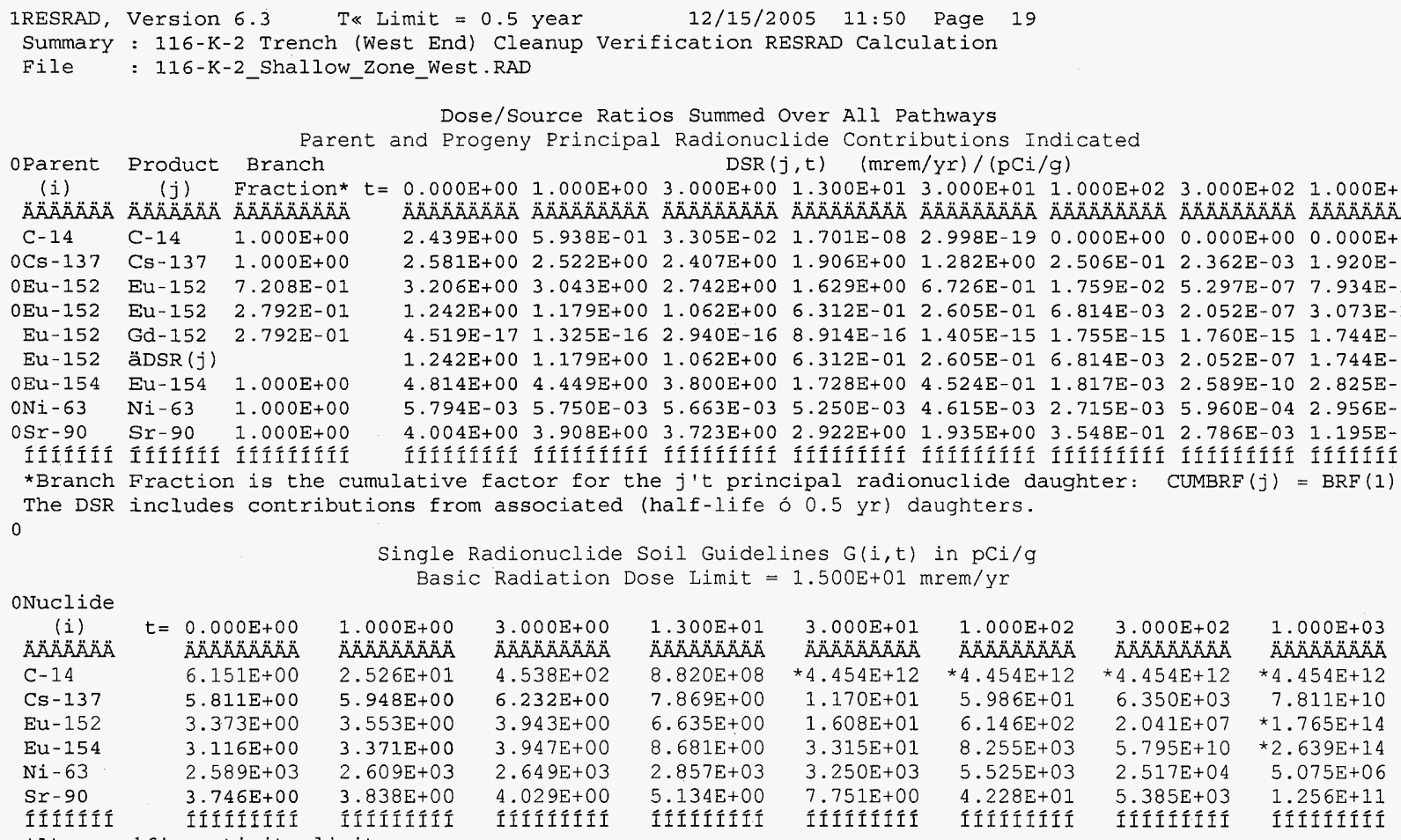


CVP-2006-00001

Rev. 0

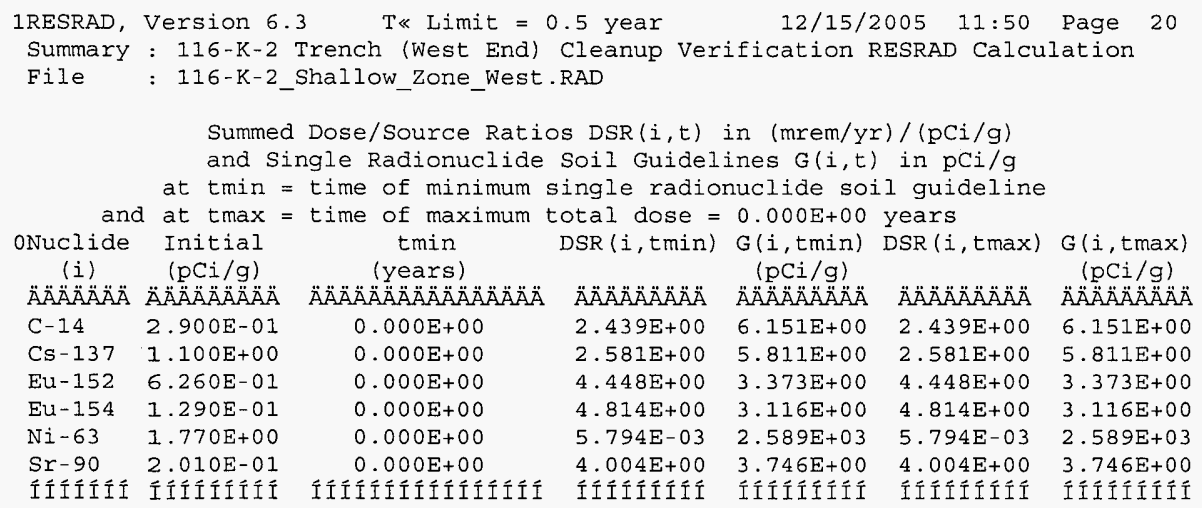


1RESRAD, Version 6.3 T\& Limit $=0.5$ year $12 / 15 / 2005 \quad 11: 50$ Page 21 Summary : 116-K-2 Trench (West End) Cleanup Verification RESRAD Calculation File : 116-K-2_Shallow_Zone_West.RAD

Individual Nuclide Dose Summed Over All Pathways Parent Nuclide and Branch Fraction Indicated

ONuclide Parent BRF(i)

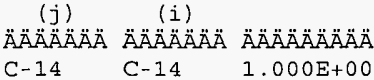

OCs-137 Cs $-137 \quad 1.000 \mathrm{E}+00$

$0 \mathrm{Eu}-152 \quad \mathrm{Eu}-152 \quad 7.208 \mathrm{E}-01$

$\mathrm{Eu}-152$ Eu-152 2.792E-01

$\mathrm{Eu}-152$ äDOSE $(j)$

0Gd-152 Eu-152 2.792E-01

$0 \mathrm{Eu}-154 \quad \mathrm{Eu}-154 \quad 1.000 \mathrm{E}+00$

ONi-63 Ni-63 $1.000 \mathrm{E}+00$

$0 \mathrm{Sr}-90 \quad \mathrm{Sr}-90 \quad 1.000 \mathrm{E}+00$

$\mathrm{BRF}(i)$ is the branch fraction of the parent nuclide.

$t=0.000 E+001.000 E+003.000 E+001300 E+013.000 E+011.000 E+0233.000 E+02 \quad 1.000 E+03$

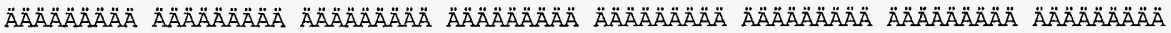
$\begin{array}{lllllllll}7.072 E-01 & 1.722 E-01 & 9.585 E-03 & 4.932 E-09 & 8.695 E-20 & 0.000 E+00 & 0.000 E+00 & 0.000 E+00\end{array}$ $\begin{array}{llllllllll}2.839 E+00 & 2.774 E+00 & 2.647 E+00 & 2.097 E+00 & 1.410 E+00 & 2.757 E-01 & 2.598 E-03 & 2.113 E-10\end{array}$ $2.007 \mathrm{E}+00 \quad 1.905 \mathrm{E}+00 \quad 1.717 \mathrm{E}+00 \quad 1.020 \mathrm{E}+00 \quad 4.210 \mathrm{E}-01 \quad 1.101 \mathrm{E}-02 \quad 3.316 \mathrm{E}-07 \quad 4.967 \mathrm{E}-23$ $\begin{array}{llllllll}7.773 E-01 & 7.379 E-01 & 6.650 E-01 & 3.951 E-01 & 1.631 E-01 & 4.265 E-03 & 1.284 E-07 & 1.924 E-23\end{array}$ $2.784 \mathrm{E}+00.2 .643 \mathrm{E}+00 \quad 2.382 \mathrm{E}+00 \quad 1.415 \mathrm{E}+00 \quad 5.841 \mathrm{E}-01 \quad 1.528 \mathrm{E}-02 \quad 4.600 \mathrm{E}-07 \quad 6.890 \mathrm{E}-23$ $2.829 \mathrm{E}-17 \quad 8.294 \mathrm{E}-17 \quad 1.841 \mathrm{E}-16 \quad 5.580 \mathrm{E}-16 \quad 8.795 \mathrm{E}-16 \quad 1.099 \mathrm{E}-15 \quad 1.102 \mathrm{E}-15 \quad 1.092 \mathrm{E}-15$ $\begin{array}{llllllll}6.210 \mathrm{E}-01 & 5.739 \mathrm{E}-01 & 4.902 \mathrm{E}-01 & 2.229 \mathrm{E}-01 & 5.837 \mathrm{E}-02 & 2.344 \mathrm{E}-04 & 3.339 \mathrm{E}-11 & 0.000 \mathrm{E}+00\end{array}$ $\begin{array}{lllllllll}1.025 \mathrm{E}-02 & 1.018 \mathrm{E}-02 & 1.002 \mathrm{E}-02 & 9.293 \mathrm{E}-03 & 8.169 \mathrm{E}-03 & 4.805 \mathrm{E}-03 & 1.055 \mathrm{E}-03 & 5.232 \mathrm{E}-06\end{array}$ $\begin{array}{lllllllllll}8.048 \mathrm{E}-01 & 7.855 \mathrm{E}-01 & 7.484 \mathrm{E}-01 & 5.873 \mathrm{E}-01 & 3.890 \mathrm{E}-01 & 7.131 \mathrm{E}-02 & 5.599 \mathrm{E}-04 & 2.401 \mathrm{E}-11\end{array}$

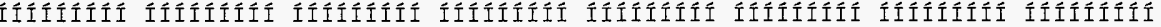

\begin{tabular}{|c|c|c|}
\hline $\begin{array}{c}(j) \\
\ddot{A} \ddot{A} \ddot{A} \ddot{A} \ddot{A} \ddot{A} \ddot{A}\end{array}$ & $\begin{array}{c}\text { (i) } \\
\ddot{A} \ddot{A} \ddot{A} \ddot{A} \ddot{A} \ddot{A} A\end{array}$ & $\ddot{A} \ddot{A} \ddot{A} \ddot{A} \ddot{A} \ddot{A} \ddot{A} \ddot{A} \ddot{A}$ \\
\hline C- 14 & $C-14$ & $1.000 \mathrm{E}+00$ \\
\hline $0 \mathrm{Cs}-137$ & $\mathrm{Cs}-137$ & $1.000 E+00$ \\
\hline OEU-152 & $E u-152$ & $7.208 E-01$ \\
\hline Eu-152 & Eu-152 & $2.792 \mathrm{E}-01$ \\
\hline Eu-152 & äs $(j):$ & \\
\hline OGd-152 & Eu-152 & $2.792 \mathrm{E}-01$ \\
\hline OEu-154 & $\mathrm{Eu}-154$ & $1.000 \mathrm{E}+00$ \\
\hline ONi-63 & $\mathrm{Ni}-63$ & $1.000 \mathrm{E}+00$ \\
\hline $0 \mathrm{Sr}-90$ & Sr -90 & $1.000 E+00$ \\
\hline
\end{tabular}

Individual Nuclide Soil Concentration

Parent Nuclide and Branch Fraction Indicated

$S(j, t), \mathrm{pCi} / \mathrm{g}$

$t=0.000 \mathrm{E}+00 \quad 1.000 \mathrm{E}+00 \quad 3.000 \mathrm{E}+00 \quad 1.300 \mathrm{E}+01 \quad 3.000 \mathrm{E}+01 \quad 1.000 \mathrm{E}+02 \quad 3.000 \mathrm{E}+02 \quad 1.000 \mathrm{E}+03$

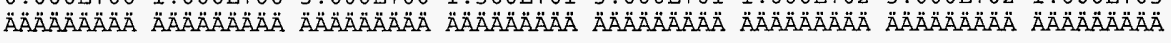
$2.900 \mathrm{E}-01 \quad 6.850 \mathrm{E}-02 \quad 3.814 \mathrm{E}-03 \quad 1.966 \mathrm{E}-09 \quad 3.477 \mathrm{E}-20 \quad 0.000 \mathrm{E}+00 \quad 0.000 \mathrm{E}+00 \quad 0.000 \mathrm{E}+00$ $1.100 \mathrm{E}+00 \quad 1.075 \mathrm{E}+00 \quad 1.026 \mathrm{E}+00 \quad 8.123 \mathrm{E}-01 \quad 5.464 \mathrm{E}-01 \quad 1.068 \mathrm{E}-01 \quad 1.007 \mathrm{E}-03 \quad 8.184 \mathrm{E}-11$

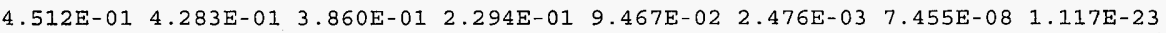
$\begin{array}{llllllll}1.748 \mathrm{E}-01 & 1.659 \mathrm{E}-01 & 1.495 \mathrm{E}-01 & 8.884 \mathrm{E}-02 & 3.667 \mathrm{E}-02 & 9.591 \mathrm{E}-04 & 2.888 \mathrm{E}-08 & 4.325 \mathrm{E}-24\end{array}$ $\begin{array}{lllllllll}6.260 \mathrm{E}-01 & 5.942 \mathrm{E}-01 & 5.355 \mathrm{E}-01 & 3.182 \mathrm{E}-01 & 1.313 \mathrm{E}-01 & 3.435 \mathrm{E}-03 & 1.034 \mathrm{E}-07 & 1.549 \mathrm{E}-23\end{array}$ $0.000 \mathrm{E}+00 \quad 1.093 \mathrm{E}-15 \quad 3.116 \mathrm{E}-15 \quad 1.060 \mathrm{E}-14 \quad 1.702 \mathrm{E}-14 \quad 2.141 \mathrm{E}-14 \quad 2.147 \mathrm{E}-14 \quad 2.127 \mathrm{E}-14$

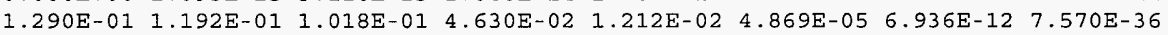
$\begin{array}{llllllll}1.770 E+00 & 1.757 E+00 & 1.730 E+00 & 1.604 E+00 & 1.410 E+00 & 8.294 E-01 & 1.821 E-01 & 9.030 E-04\end{array}$ $\begin{array}{lllllllll}2.010 E-01 & 1.962 \mathrm{E}-01 & 1.869 \mathrm{E}-01 & 1.467 \mathrm{E}-01 & 9.715 \mathrm{E}-02 & 1.781 \mathrm{E}-02 & 1.398 \mathrm{E}-04 & 5.997 \mathrm{E}-12\end{array}$

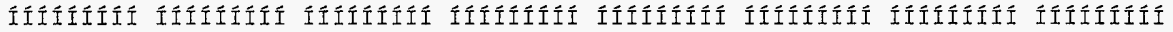
BRF(i) is the branch fraction of the parent nuclide.

ORESCALC. EXE execution time $=0.60$ seconds 
CVP-2006-00001

Rev. 0

C-74 
CVP-2006-00001

Rev. 0

RESRAD INPUT PARAMETERS FOR THE 116-K-2 (WEST END) DEEP ZONE 
CVP-2006-00001

Rev. 0

C-76 
Dose Conversion Factor (and Related) Parameter Summary File: HEAST 2001 Morbidity

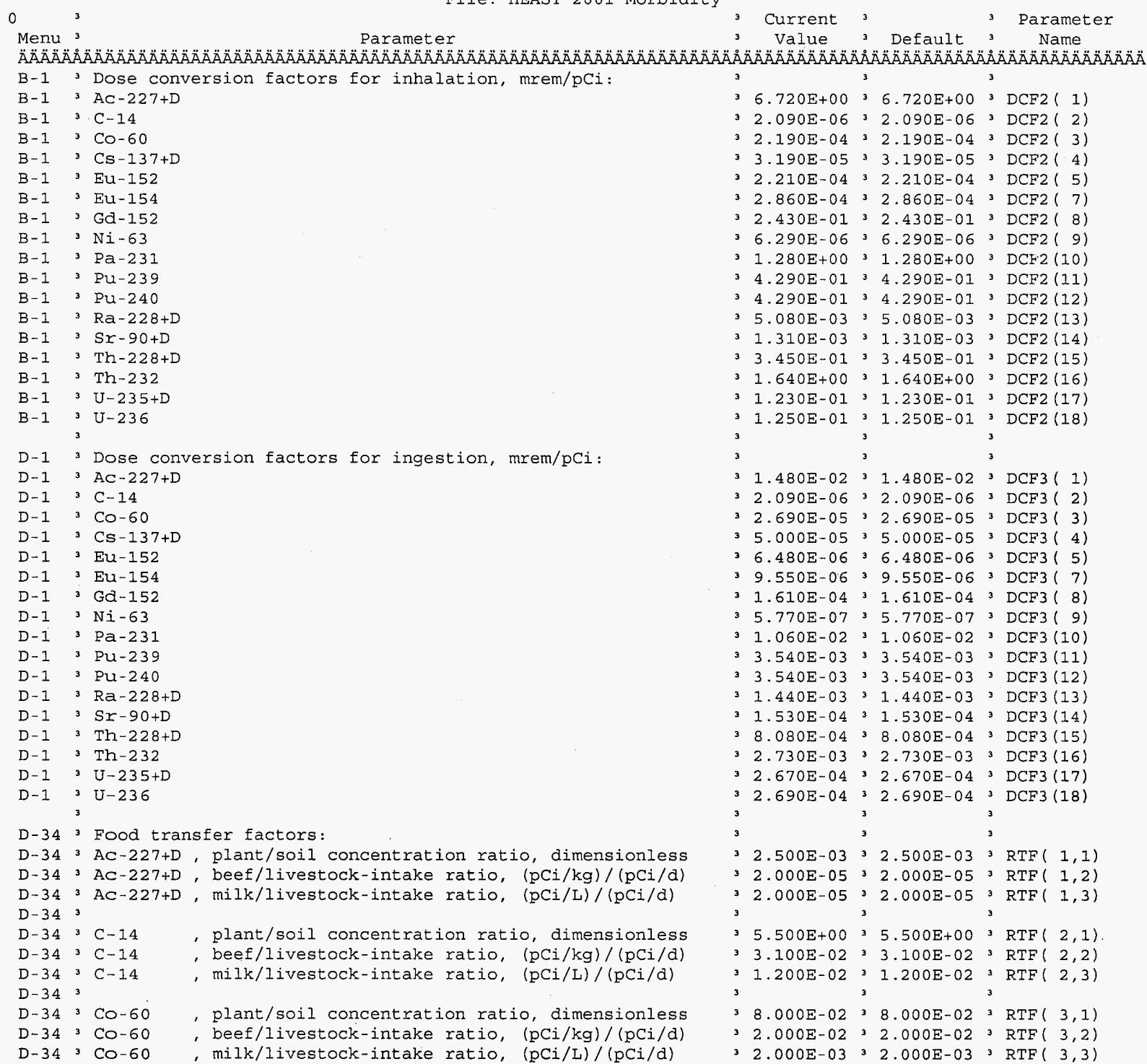




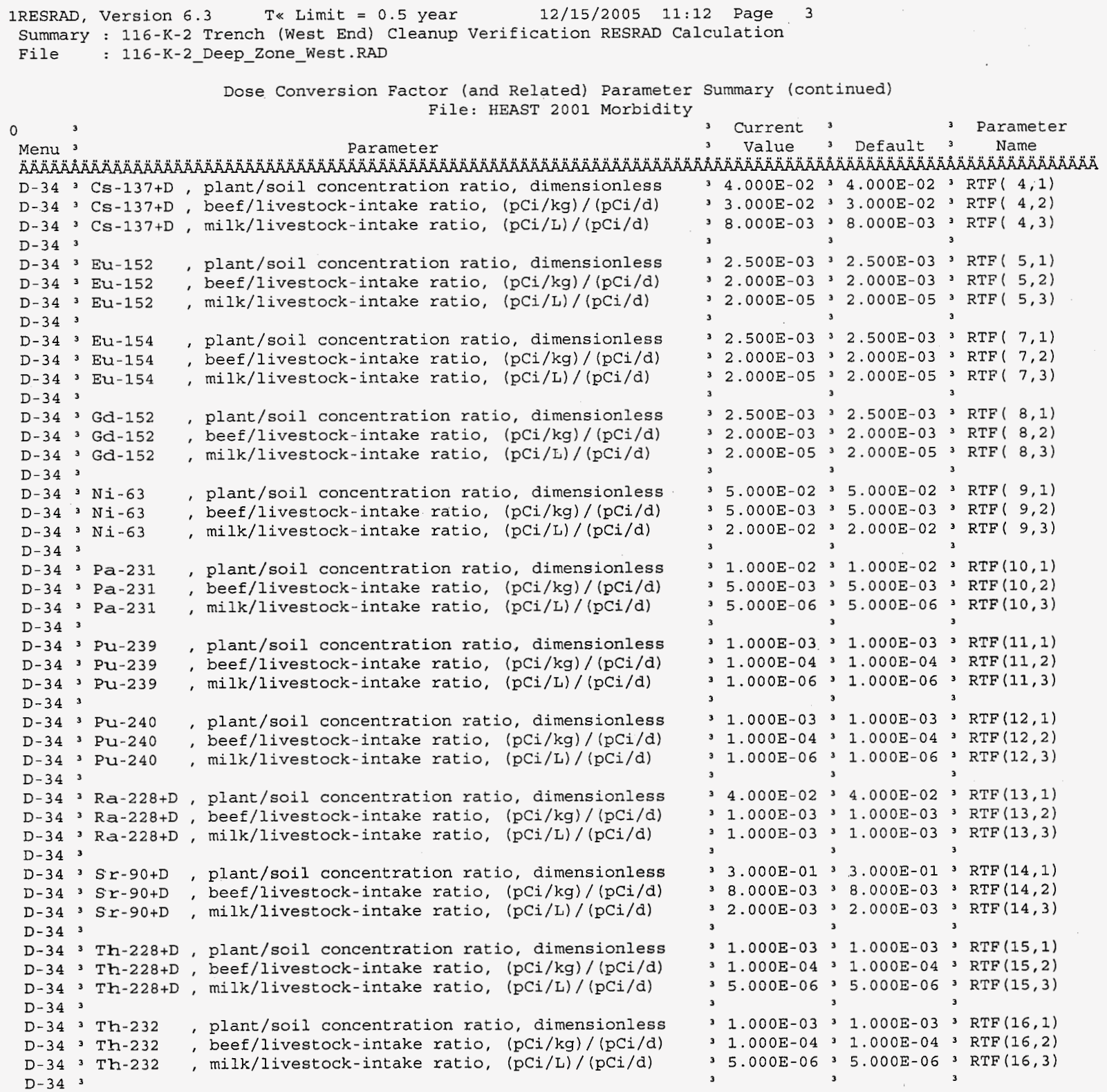


1RESRAD, Version $6.3 \quad T \ll$ Limit $=0.5$ year $12 / 15 / 2005 \quad 11: 12$ Page 4

Summary : 116-K-2 Trench (West End) Cleanup verification RESRAD Calculation

File : 116-K-2_Deep_Zone_West.RAD

Dose Conversion Factor (and Related) Parameter Summary (continued) File: HEAST 2001 Morbidity

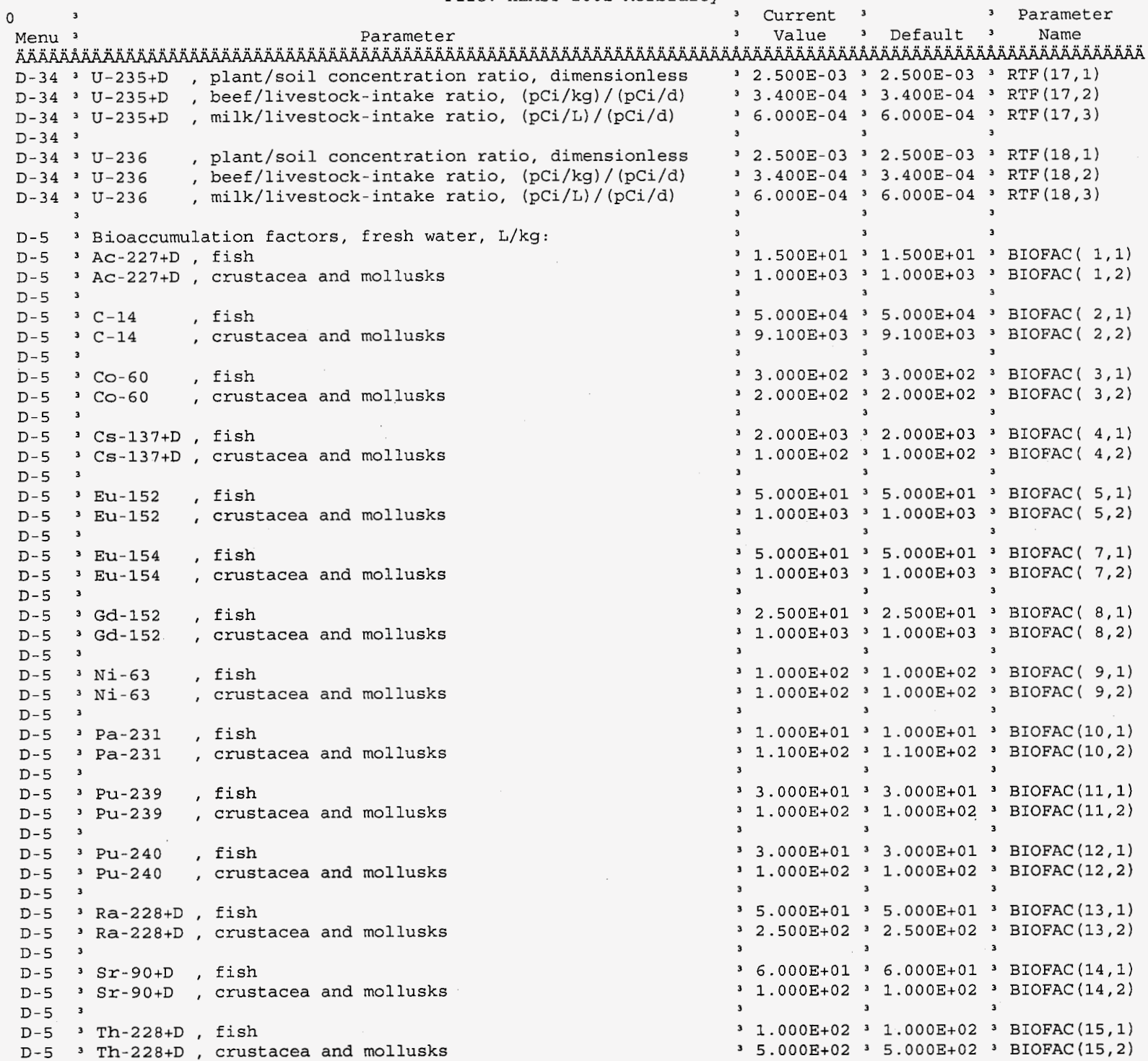


CVP-2006-00001

Rev. 0

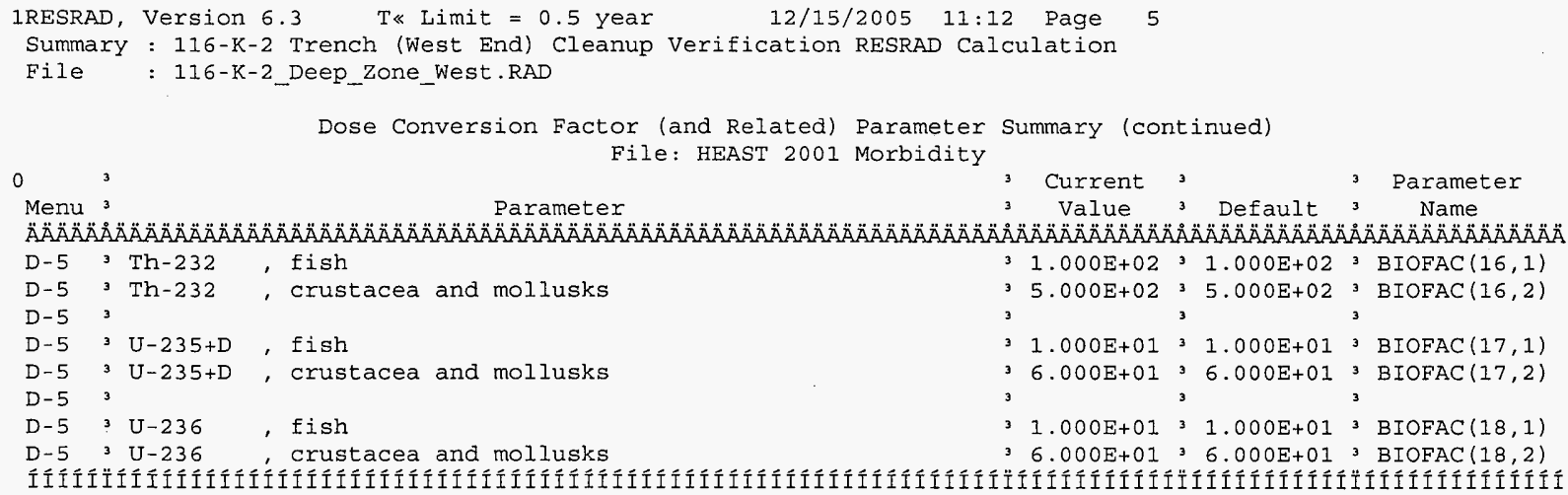


IRESRAD, Version $6.3 \quad T \ll$ Limit $=0.5$ year $\quad 12 / 15 / 2005 \quad 11: 12$ Page 6

Summary : 116-K-2 Trench (West End) Cleanup Verification RESRAD Calculation

File : 116-K-2_Deep_Zone_West.RAD

Site-Specific Parameter Summary

0

Parameter

3 User 3 Used by RESRAD

Used by RESRAD ${ }^{3}$ Parameter

R011 3 Area of contaminated zone ( $m * * 2)$

R011 3 Thickness of contaminated zone (m)

ROI1 Length parallel to aquifer flow (m)

R011 3 Basic radiation dose limit (mrem/yr)

R011 3 Time since placement of material (yr)

R011 Times for calculations (yr)

R011 3 Times for calculations ( $y r$

R011 3 Times for calculations ( $y r$

R011 3 Times for calculations $(y r)$

R011 ${ }^{3}$ Times for calculations ( $y r$ )

R011 3 Times for calculations ( $y r$ )

R011 3 Times for calculations $(y r)$

R011 3 Times for calculations $(\mathrm{yr})$

R011 3 Times for calculations $(y r)$ 3

R012 ${ }^{3}$ Initial principal radionuclide (pCi/g):

$\mathrm{R} 012^{3}$ Initial principal radionuclide ( $\mathrm{pCi} / \mathrm{g}$ ):

$\mathrm{R} 0123$ Initial principal radionuclide (pCi/g):

R012 3 Initial principal radionuclide (pCi/g):

R012 Initial principal radionuclide ( $\mathrm{pCi} / \mathrm{g}$ ):

$\mathrm{R} 012^{3}$ Initial principal radionuclide (pCi/g):

R012 Initial principal radionuclide (pCi/g):

R012 Initial principal radionuclide (pCi/g):

R012 Initial principal radionuclide (pCi/g):

R012 3 Concentration in groundwater ( $\mathrm{pCi} / \mathrm{L}$ ):

R012 Concentration in groundwater (pCi/L):

R012 3 Concentration in groundwater

R012 Concentration in groundwater

R012 3 Concentration in groundwater

R012 3 Concentration in groundwater

R012 3 Concentration in groundwater

$(\mathrm{pCi} / \mathrm{L}):$

(pCi/L):

$(\mathrm{pCi} / \mathrm{L}):$

$(\mathrm{pCi} / \mathrm{L}):$

$(\mathrm{pCi} / \mathrm{L}):$

$(\mathrm{pCi} / \mathrm{L}):$

Pu -240
Sr -90

R012 Concentration in groundwater 3

R013 3 Cover depth $(\mathrm{m})$

R013 3 Density of cover material $(\mathrm{g} / \mathrm{cm} * * 3)$

R013 3 Cover depth erosion rate (m/yr)

R013 3 Density of contaminated zone $(\mathrm{g} / \mathrm{cm} * * 3)$

R013 ${ }^{3}$ Contaminated zone erosion rate (m/yr)

R013 3 Contaminated zone total porosity

R013 3 Contaminated zone field capacity

R013 3 Contaminated zone hydraulic conductivity (m/yr)

R013 3 Contaminated zone b parameter

R013 A Average annual wind speed (m/sec)

R013 3 Humidity in air $(\mathrm{g} / \mathrm{m} * * 3)$

R013 3 Evapotranspiration coefficient

R013 3 Precipitation ( $\mathrm{m} / \mathrm{yr}$ )

R013 3 Irrigation ( $\mathrm{m} / \mathrm{yr}$ )

R013 3 Irrigation mode

R013 3 Runoff coefficient
Input ${ }^{3}$ Default ${ }^{3}$ (If different from usex input) ${ }^{3}$ Name

$3 \quad 3.774 \mathrm{E}+04331.000 \mathrm{E}+043$

$37.300 \mathrm{E}+00 \quad 3 \quad 2.000 \mathrm{E}+00$

$3.190 \mathrm{E}+02,3,1.000 \mathrm{E}+02^{3}$

${ }^{3} 1.500 \mathrm{E}+01^{3} 2.500 \mathrm{E}+01^{3}$

$30.000 \mathrm{E}+00 \rightarrow 0.000 \mathrm{E}+00$

$3 \quad 1.000 \mathrm{E}+00^{3} \quad 1.000 \mathrm{E}+00^{3}$

$3.000 \mathrm{E}+00^{3} 3.000 \mathrm{E}+00^{3}$

$31.300 \mathrm{E}+01^{3} 1.000 \mathrm{E}+01$

$33.000 \mathrm{E}+01^{3} 3.000 \mathrm{E}+01^{3}$

$31.000 \mathrm{E}+02^{3} 1.000 \mathrm{E}+02^{3}$

$33.000 \mathrm{E}+0233.000 \mathrm{E}+02^{3}$

$31.000 \mathrm{E}+03 \quad 3 \quad 1.000 \mathrm{E}+03_{3}$

3 not used $30.000 \mathrm{E}+00^{3}$

3 not used $30.000 \mathrm{E}+00$

3

- - 3 AREA

-.- 3 THICKO

-.- 3 LCZPAQ

3 BRDI

3 TI

${ }^{3} \mathrm{~T}(2)$

${ }^{3} \mathrm{~T}(3)$

-.- $3 \mathrm{~T}(4)$

$3 \mathrm{~T}(5)$

-.. 3 T ( 6)

$3 \mathrm{~T}(7)$

-. $3 \mathrm{~T}(8)$

-

-.- $3 \mathrm{~T}(10)$

-.. $\quad 3$ S1 ( 2)

- - $\quad 3$ SI ( 3$)$

-. $\quad 3$ SI ( 4$)$

3 SI ( 5)

-.- 3 SI $(7)$

- $\quad{ }^{3} \mathrm{SI}(9)$

3 S1 (12)

3 S1 (14)

3 WI ( 2$)$

3 .

3 WI 5 )

-. 3 WI ( 7$)$

- - 3 W1 (11)

3 WI (12)

3 W1 (14)

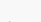

$-\cdots$
$---$

-.

$--$

-. -

$--$

$---$

-.-

$--$

$---$

-..

$-\cdots$

$-\cdots$

$---$

3 COVERo

3 DENSCV

3 VCV

3 DENSCZ

$3 \mathrm{VCZ}$

3 TPCZ

$3 \mathrm{FCCZ}$

$3 \mathrm{HCCZ}$

$3 \mathrm{BCZ}$

3 WIND

3 HUMID

3 EVAPTR

3 PRECIP

3 RI

3 IDITCH

overhead ${ }^{3}$ overhead
3 RUNOFF 
IRESRAD, Version $6.3 \quad T \ll$ Limit $=0.5$ year $12 / 15 / 2005 \quad 11: 12$ Page 7

Summary : 116-K-2 Trench (West End) Cleanup Verification RESRAD Calculation

File : 116-K-2_Deep_zone_west.RAD

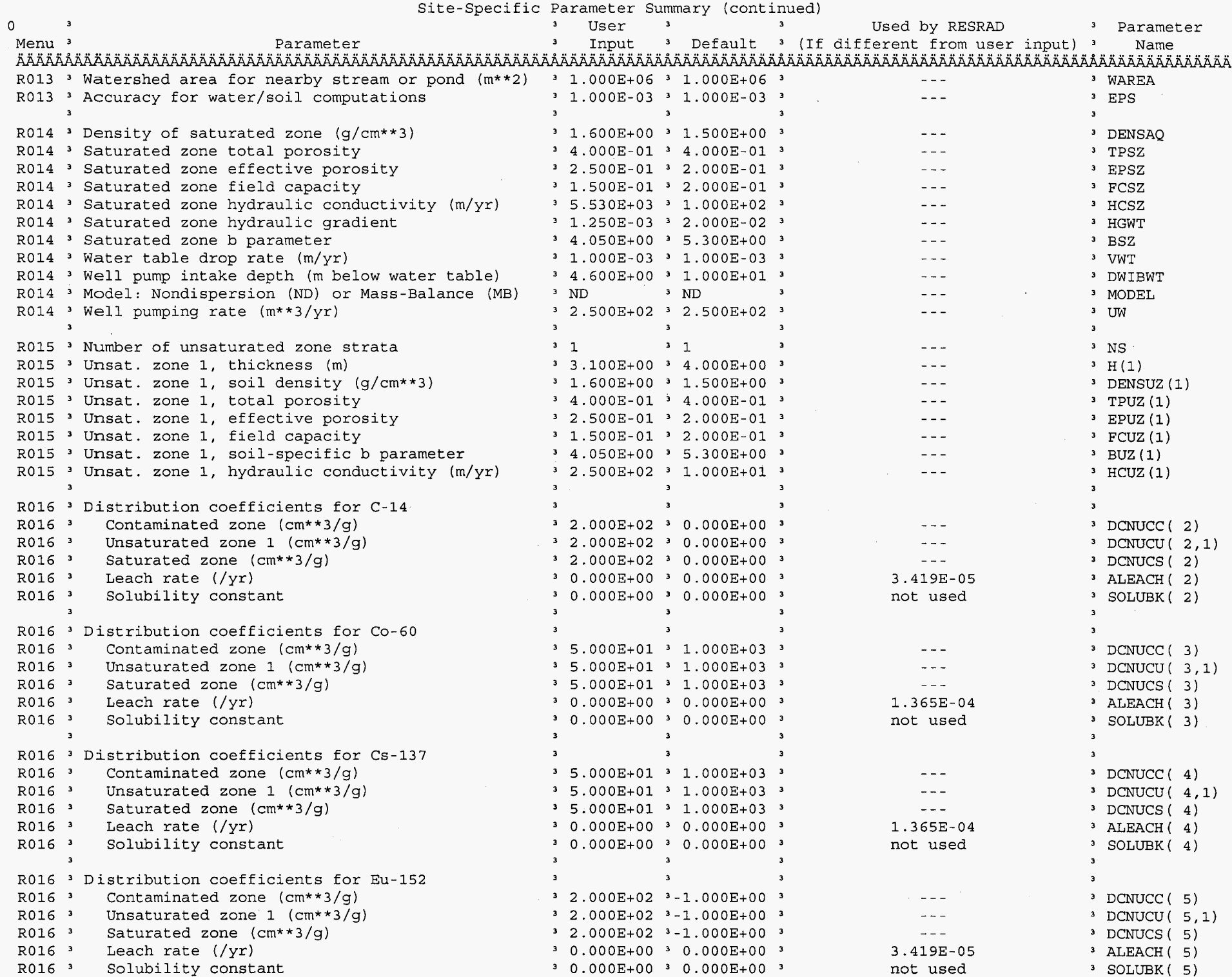


IRESRAD, Version 6.3 T« Limit $=0.5$ year $12 / 15 / 2005$ 11:12 Page 8 Summary : 116-K-2 Trench (West End) Cleanup Verification RESRAD Calculation File : 116-K-2_Deep_Zone_West.RAD

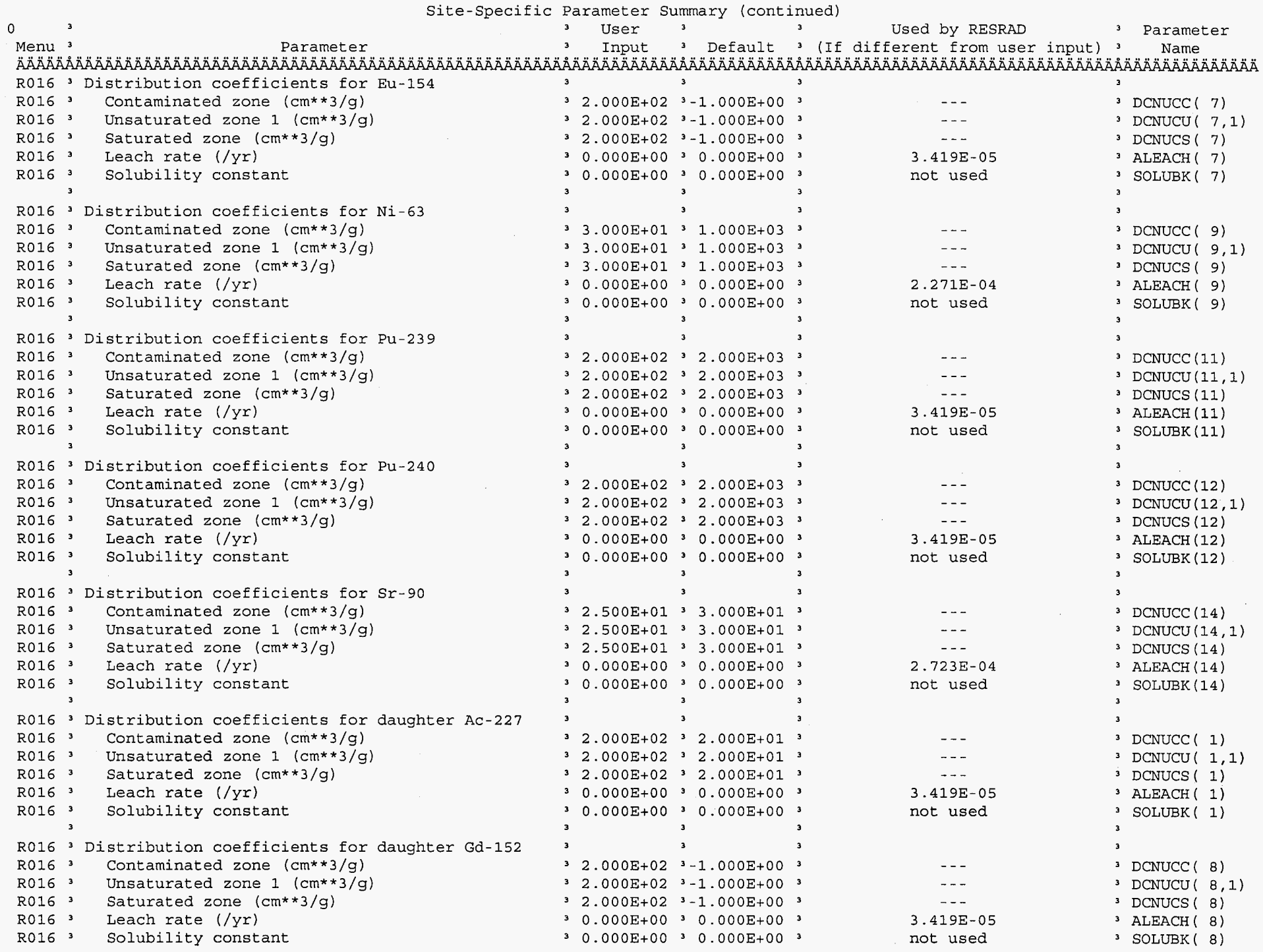




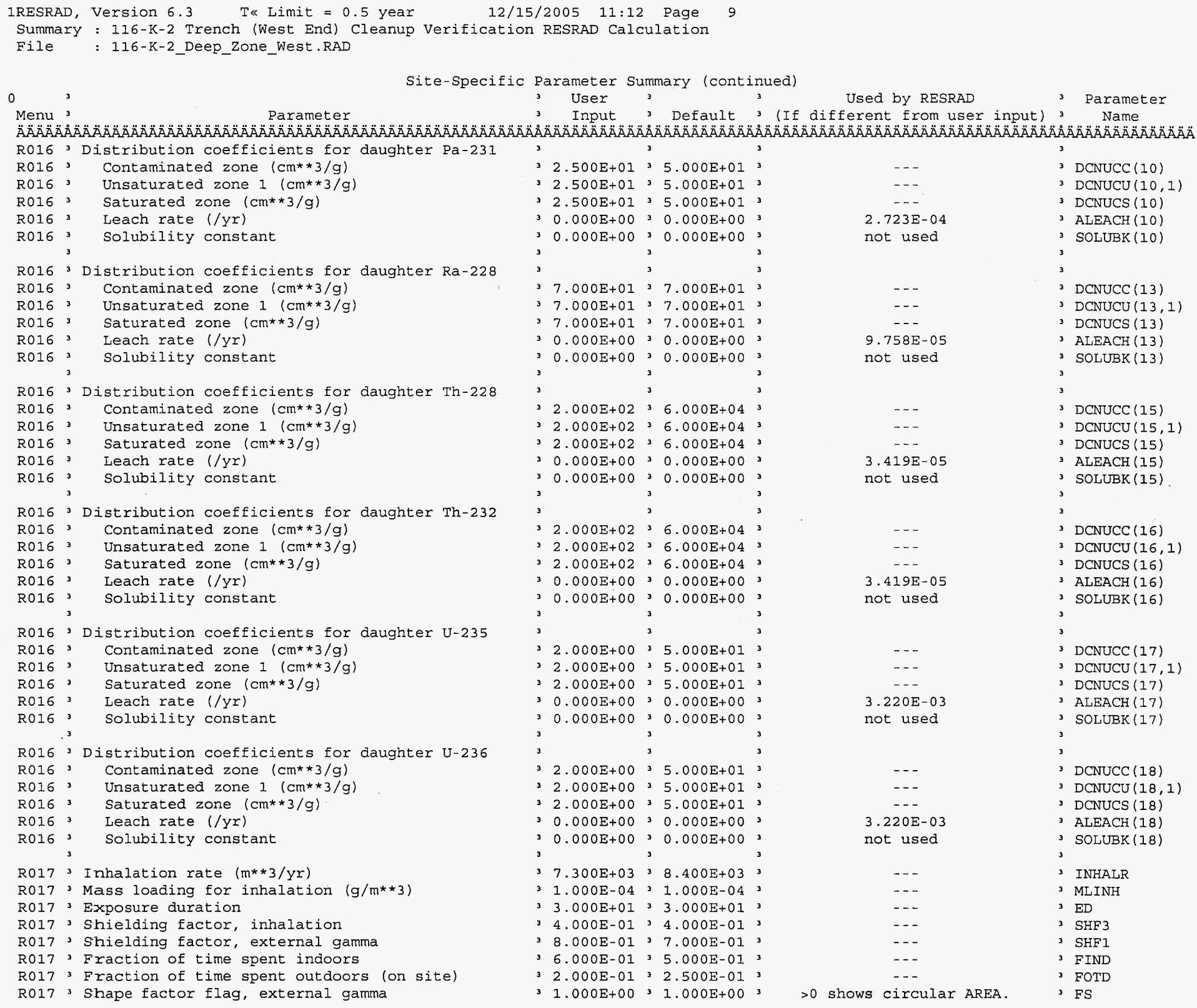


1RESRAD, Version $6.3 \quad T$ Limit $=0.5$ year $12 / 15 / 2005 \quad 11: 12$ Page 10 Summary : 116-K-2 Trench (West End) Cleanup Verification RESRAD Calculation File : 116-K-2_Deep_Zone_West.RAD

Site-Specific Parameter Summary (continued)

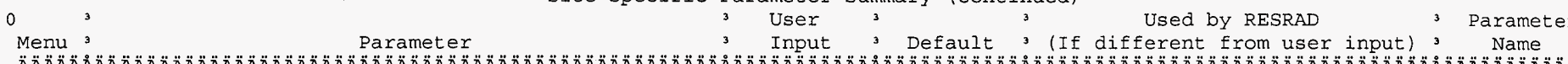

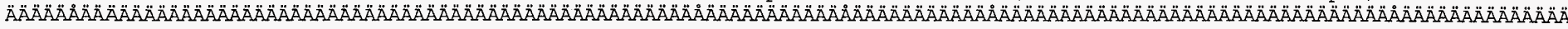

R017 3 Radii of shape factor array (used if FS $=-1$ ) R017 3 Outer annular radius $(\mathrm{m})$, ring 1 $\mathrm{R}_{017} 3$ Outer annular radius $(\mathrm{m})$, ring 2 : R017 3 Outer annular radius (m), ring 3 : $\mathrm{R}_{017^{3}}$ Outer annular radius (m), ring 4 . $\mathrm{R}_{017}{ }^{3}$ Outer annular radius (m), ring 5: R017 3 Outer annular radius (m), ring 6 R017 3 Outer annular radius (m), ring 7 R017 3 Outer annular radius (m), ring 8 : R017 3 Outer annular radius $(m)$, ring 9: R017 3 Outer annular radius $(\mathrm{m})$, ring 10 R017 3 Outer annular radius (m), ring 11 : R017 3 Outer annular radius (m), ring 12

R017 3 Fractions of annular areas within AREA:

$\mathrm{R}_{017^{3} \text { Ring } 1}$

R017 3 Ring 2

R017 3 Ring 3

R017 3 Ring 4

R017 3 Ring 5

R017 3 Ring 6

R017 3 Ring 7

R017 3 Ring 8

R017 3 Ring 9

R017 3 Ring 10

R017 3 Ring 11

R017 3 Ring 12

R018 3 Fruits, vegetables and grain consumption (kg/yr)

R018 3 Leafy vegetable consumption ( $\mathrm{kg} / \mathrm{yr}$ )

R018 3 Milk consumption ( $\mathrm{L} / \mathrm{Yr}$ )

R018 3 Meat and poultry consumption $(\mathrm{kg} / \mathrm{yr})$

R018 3 Fish consumption $(\mathrm{kg} / \mathrm{yr})$

R018 3 Other seafood consumption ( $\mathrm{kg} / \mathrm{yr}$ )

R018 3 Soil ingestion rate ( $/ \mathrm{yr}$ )

R018 3 Drinking water intake (L/yr)

R018 3 Contamination fraction of drinking water

R018 Contamination fraction of household water

R018 3 Contamination fraction of livestock water

R018 ${ }^{3}$ Contamination fraction of irrigation water

R018 3 Contamination fraction of aquatic food

R018 3 Contamination fraction of plant food

R018 3 Contamination fraction of meat

R018 3 Contamination fraction of milk 3

R019 ${ }^{3}$ Livestock fodder intake for meat ( $\mathrm{kg} /$ day)

R019 3 Livestock fodder intake for milk ( $\mathrm{kg} /$ day)

R019 3 Livestock water intake for meat ( $\mathrm{L} /$ day)

R019 3 Livestock water intake for milk (L/day)

R019 3 Livestock soil intake ( $\mathrm{kg} /$ day)
${ }_{3}^{3}$ not used ${ }^{3} 5.000 E+01_{3}^{3}$

3 not used, $7.071 \mathrm{E}+01$,

3 not used $30.000 E+00^{3}$

3 not used $30.000 E+00^{3}$

3 not used $30.000 \mathrm{E}+00^{3}$

3 not used $30.000 \mathrm{E}+00$

3 not used $30.000 \mathrm{E}+00^{3}$

3 not used $30.000 E+00^{3}$

3 not used $30.000 E+00^{3}$

3 not used $30.000 E+00^{3}$

3 not used $30.000 E+00^{3}$

3 not used $30.000 \mathrm{E}+00^{3}$

3

3 not used $31.000 E+00$

3 not used $32.732 \mathrm{E}-01$,

3 not used $30.000 E+00$

3 not used $30.000 E+003$

3 not used $30.000 E+00^{3}$

3 not used, $0.000 E+00^{3}$

3 not used $30.000 E+00=$

3 not used $30.000 \mathrm{E}+00^{3}$

3 not used $30.000 \mathrm{E}+00^{3}$

3 not used $30.000 E+00^{3}$

3 not used $30.000 \mathrm{E}+00$

3 not used $30.000 E+00^{3}$ 3 3

$1.100 \mathrm{E}+02 \quad 3 \quad 1.600 \mathrm{E}+02$

$32.700 E+00 \quad 3 \quad 1.400 E+01$

${ }^{3} 1.000 E+022^{3} 9.200 E+01^{3}$

${ }^{3} 3.600 \mathrm{E}+01^{3} 6.300 \mathrm{E}+01^{3}$

$31.970 \mathrm{E}+01$ 3 $5.400 \mathrm{E}+00$

$39.000 E-0133.000 E-013$

$37.300 \mathrm{E}+01 \quad 3 \quad 3.650 \mathrm{E}+01 \quad 3$

${ }_{3} 7.300 E+022^{3} 5.100 E+02 \quad 3$

$31.000 E+0031.000 E+00$

3 not used $31.000 \mathrm{E}+00^{3}$

${ }_{3} 1.000 \mathrm{E}+00^{3} 1.000 \mathrm{E}+00^{3}$

$31.000 \mathrm{E}+00^{3} 1.000 \mathrm{E}+00^{3}$

3 5.000E-01 3 5.000E-01 3

$3-1$

$3-1$

$3-1$

$3-1$

(1)

$36.800 E+01,6.800 E+01$

$35.500 \mathrm{E}+0135.500 \mathrm{E}+013$

$35.000 \mathrm{E}+0135.000 \mathrm{E}+01$

$31.600 \mathrm{E}+022^{3} 1.600 \mathrm{E}+02$

3 $5.000 \mathrm{E}-01^{3} 5.000 \mathrm{E}-01^{3}$
3 RAD_SHAPE ( 1)

3 RAD_SHAPE ( 2)

3 RAD SHAPE ( 3 )

3 RAD_SHAPE ( 4)

3 RAD_SHAPE ( 5)

3 RAD SHAPE ( 6)

3 RAD SHAPE ( 7 )

3 RAD_SHAPE ( 8)

3 RAD SHAPE ( 9)

RAD SHAPE (10)

RAD SHAPE (11)

3 RAD_SHAPE (12)

FRACA ( 1$)$

3 FRACA ( 2)

3 FRACA ( 3)

3 FRACA ( 4)

FRACA ( 5$)$

3 FRACA ( 6)

3 FRACA ( 7)

3 FRACA ( 8)

FRACA ( 9)

3 FRACA (10)

FRACA (11)

3 FRACA (12)

DIET (2)

DIET (3)

3 DIET (4)

DIET (5)

3 DIET (6)

3 SOIL

DWI

FDW

3 FHHW

3 FLW

3 FIRW

3 FR9

3 FPLANT

3 FMEAT

3 FMILK

3 LFI5

3 LFI6

3 LWI5

3 LWI6

3 LSI 
IRESRAD, Version $6.3 \quad T \ll$ Limit $=0.5$ year $\quad 12 / 15 / 2005 \quad 11: 12$ Page 11

Summary : 116-K-2 Trench (West End) Cleanup Verification RESRAD Calculation

File : 116-K-2_Deep_zone_West.RAD

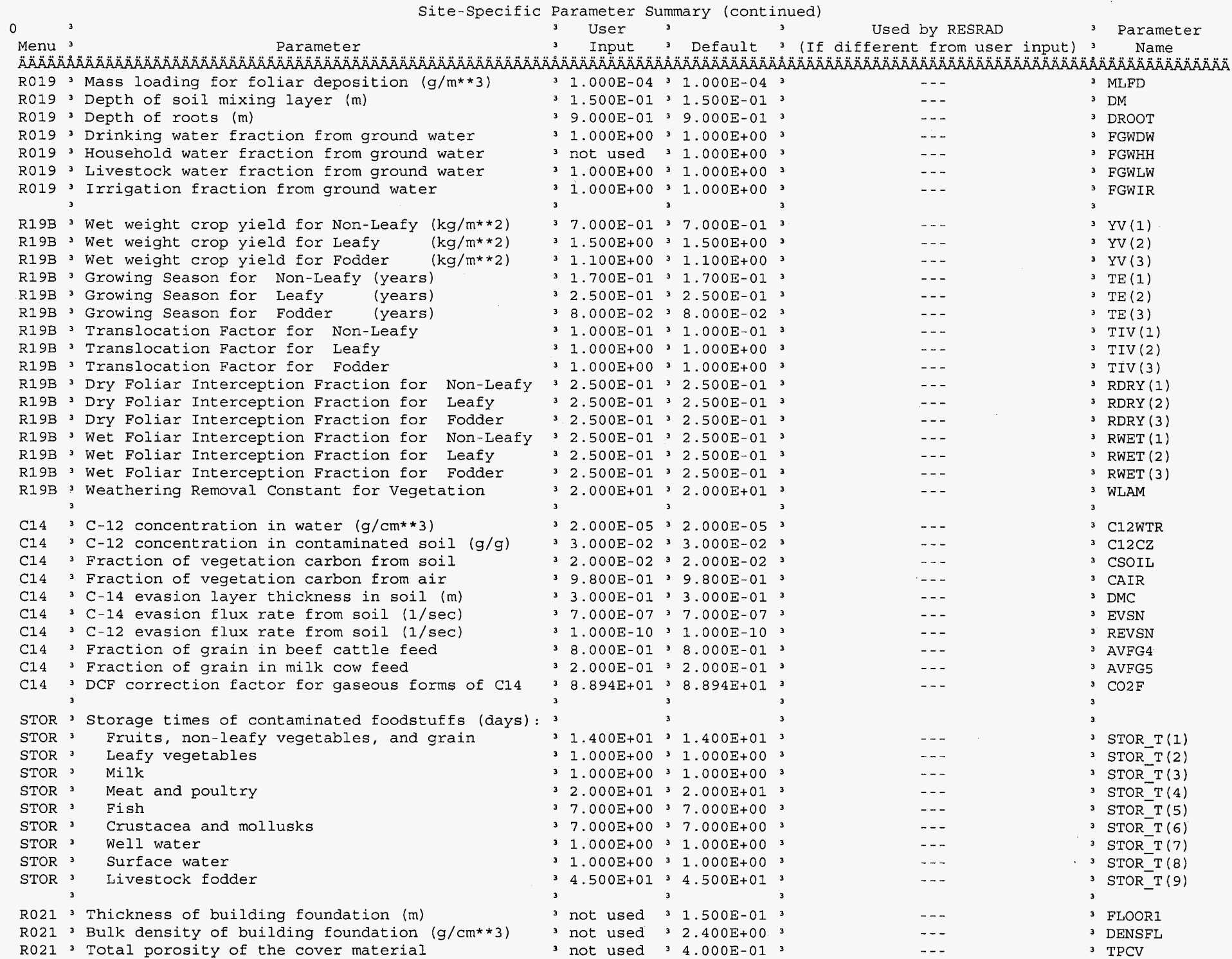


R021 3 Emanating power of Rn-222 gas 3 not used $3^{3.500 E-013}$

TITL 3 Number of graphical time points

TITL 3 Maximum number of integration points for dose

3

3 not used $32.000 \mathrm{E}^{3} 06^{3}$

3 not used 3 3.000E-07,

3 not used $32.000 \mathrm{E}-06^{3}$

3 not used $32.000 \mathrm{E}+00^{3}$

${ }_{3}^{3}$ not used used ${ }^{3} 5.000 \mathrm{E}+0 \mathrm{E}^{3}-0 \mathrm{I}^{3}$

3 not used $32.500 E+00^{3}$

3 not used ${ }^{3} 1.500 E-01{ }^{3}$

3

TITL 3 Maximum number of integration points for risk
Summary of Pathway Selections

Pathway 3 User Selection

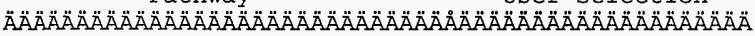

1 - - external gamma active 2 - - inhalation (w/o radon) ${ }^{3}$ active

3 -- plant ingestion 3 active

4 -. meat ingestion active

5 -. milk ingestion active

6 -- aquatic foods ${ }^{3}$ active

7 -- drinking water ${ }^{3}$ active

8 -- soil ingestion 3 active

$\begin{array}{llc}9-- \text { radon } & 3 & \text { suppressed } \\ \text { Find peak pathway doses } & { }_{3} & \text { active }\end{array}$ 


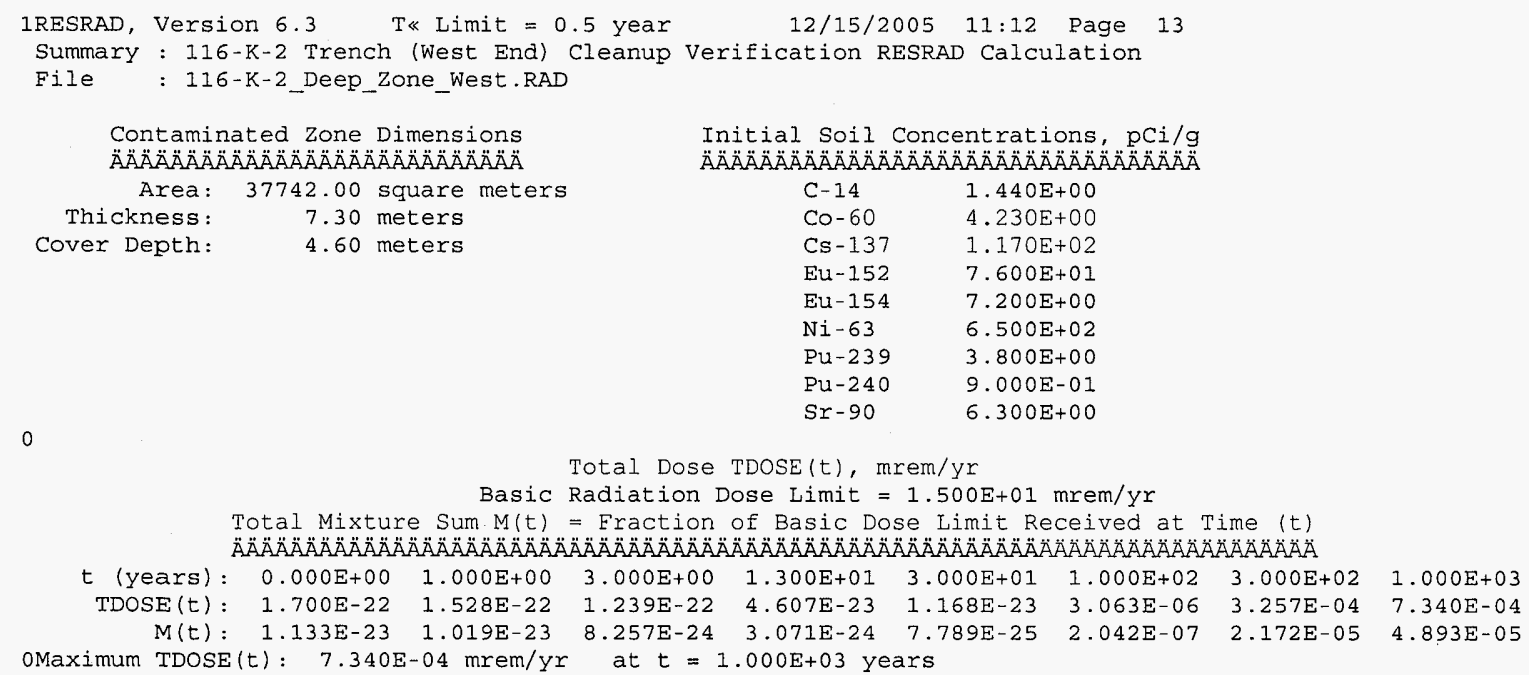

0

\begin{tabular}{|c|c|}
\hline$C-14$ & $1.440 \mathrm{E}+00$ \\
\hline Co- 60 & $4.230 \mathrm{E}+00$ \\
\hline $\mathrm{Cs}-137$ & $1.170 \mathrm{E}+02$ \\
\hline Eu-152 & $7.600 \mathrm{E}+0 \mathrm{I}$ \\
\hline Eu-154 & $7.200 E+00$ \\
\hline $\mathrm{Ni}-63$ & $6.500 \mathrm{E}+02$ \\
\hline$P u-239$ & $3.800 \mathrm{E}+00$ \\
\hline Pu-240 & $9.000 \mathrm{E}-01$ \\
\hline $\mathrm{Sr}-90$ & $6.300 \mathrm{E}+00$ \\
\hline
\end{tabular}

Total Dose TDOSE(t), mrem/yr

Basic Radiation Dose Limit $=1.500 \mathrm{E}+01 \mathrm{mrem} / \mathrm{yr}$

Total Mixture Sum $M(t)=$ Fraction of Basic Dose Limit Received at Time (t)

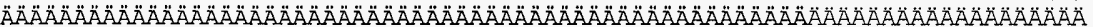

$t$ (years) : $\begin{array}{lllllllll}0.000 \mathrm{E}+00 & 1.000 \mathrm{E}+00 & 3.000 \mathrm{E}+00 & 1.300 \mathrm{E}+01 & 3.000 \mathrm{E}+01 & 1.000 \mathrm{E}+02 & 3.000 \mathrm{E}+02 & 1.000 \mathrm{E}+03\end{array}$

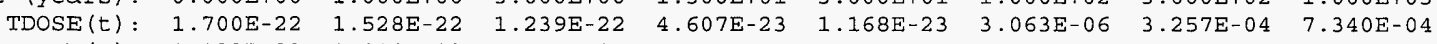
$M(t): \begin{array}{llllllll}1.133 \mathrm{E}-23 & 1.019 \mathrm{E}-23 & 8.257 \mathrm{E}-24 & 3.071 \mathrm{E}-24 & 7.789 \mathrm{E}-25 & 2.042 \mathrm{E}-07 & 2.172 \mathrm{E}-05 & 4.893 \mathrm{E}-05\end{array}$ OMaximum TDOSE(t): $7.340 \mathrm{E}-04 \mathrm{mrem} / \mathrm{yr}$ at $t=1.000 \mathrm{E}+03$ years 


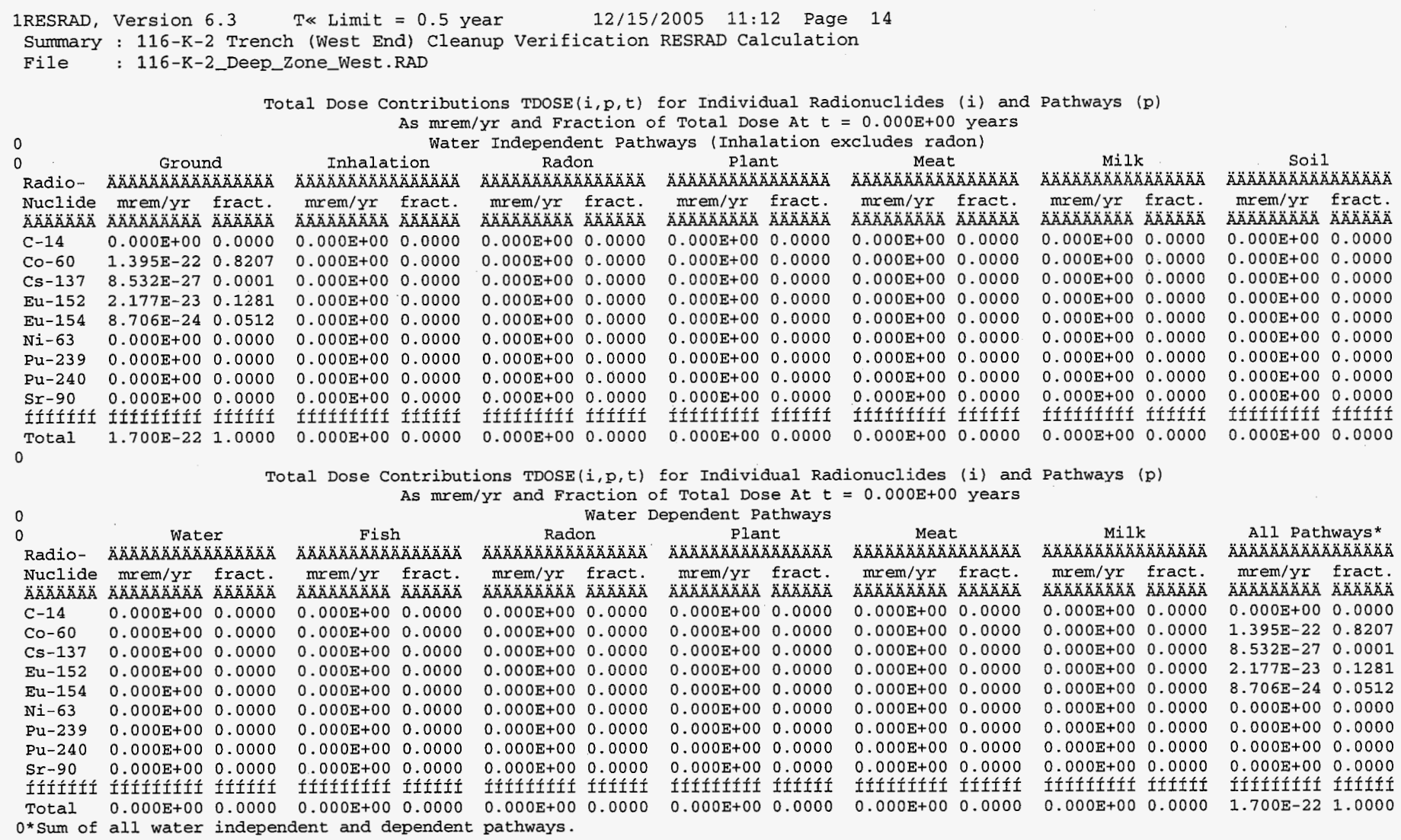




\section{Rev. 0}

1RESRAD, Version $6.3 \quad$ T« Limit $=0.5$ year $12 / 15 / 2005 \quad 11: 12$ Page 15
Summary : 116-K-2 Trench (West End) Cleanup Verification RESRAD Calculation

File : 116-K-2_Deep_Zone_West.RAD

Total Dose Contributions TDOSE $(i, p, t)$ for Individual Radionuclides ( $i$ ) and Pathways (p) As mrem/yr and Fraction of Total Dose At $t=1.000 \mathrm{E}+00$ years

0 Radio- $\ddot{A} \ddot{A} \ddot{A} \ddot{A} \ddot{A} \ddot{A} \ddot{A} \ddot{A} \ddot{A} \ddot{A} \ddot{A} \ddot{A} \ddot{A}$ Nuclide mrem/yr fract.

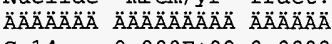
C-14 $0.000 \mathrm{E}+00 \quad 0.0000$ $\begin{array}{lll}\mathrm{C}-60 & 1.238 \mathrm{E}-22 & 0.8097\end{array}$ $\begin{array}{lll}\mathrm{C} 0-60 & 1.238 \mathrm{E}-22 & 0.8097 \\ \mathrm{Cs}-137 & 8.455 \mathrm{E}-27 & 0.0001\end{array}$ $\begin{array}{lll}\mathrm{Cs}-137 & 8.455 \mathrm{E}-27 & 0.0001 \\ \mathrm{Eu}-152 & 2.093 \mathrm{E}-23 & 0.1369\end{array}$ $\begin{array}{lll}\text { Eu-152 } & 2.093 \mathrm{E}-23 & 0.1369 \\ \text { Eu-154 } & 8.145 \mathrm{E}-24 & 0.0533\end{array}$ $\mathrm{Ni}-63 \quad 0.000 \mathrm{E}+00 \quad 0.0000$ $\mathrm{Pu}-239 \quad 0.000 \mathrm{E}+00 \quad 0.0000$ $\mathrm{Pu}-240 \quad 0.000 \mathrm{E}+00 \quad 0.0000$ Sr-90 $0.000 \mathrm{E}+00 \quad 0.0000$

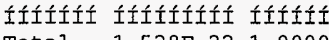
Inhalation Radon Plant Meat $\mathrm{Milk}$ Soil

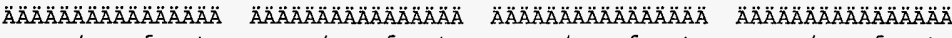
$\mathrm{mrem} / \mathrm{yr}$ fract. $0.000 \mathrm{E}+00 \quad 0.0000 \quad 0.000 \mathrm{E}+00 \quad 0.0000 \quad 0.000 \mathrm{~F}+00 \quad 0.0000 \quad 0.000 \mathrm{E}+000.0000$ $0.000+4+2000$

$0.000 \mathrm{E}+00 \quad 0.0000$

$0.000 \mathrm{E}+00 \quad 0.0000$

$0.000 \mathrm{E}+00 \quad 0.0000$

$0.000 \mathrm{E}+00 \quad 0.0000$

$0.000 \mathrm{E}+00 \quad 0.0000$

$0.000 \mathrm{E}+00 \quad 0.0000$

$0.000 \mathrm{E}+00 \quad 0.0000$

ííííííi tíííti

Total Dose Contributions TDOSE $(i, p, t)$ for Individual Radionuclides (i) and Pathways ( $p$ ) Total Dose Contributions TDOSE $(i, p, t)$ for Individual Radionuclides (i) and
As mrem/yr and Fraction of Total Dose At $t=1.000 \mathrm{E}+00$ years

0 Fish Radon plant

Meat

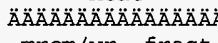
Nuclide

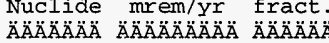
$\begin{array}{lll}C-14 & 0.000 E+00 \quad 0.000\end{array}$ Co-60 $0.000 \mathrm{E}+00 \quad 0.0000$ $\begin{array}{lll}\text { Cs }-137 & 0.000 \mathrm{E}+00 & 0.0000\end{array}$ $\begin{array}{lll}\mathrm{Eu}-152 & 0.000 \mathrm{E}+00 \quad 0.0000\end{array}$ $\mathrm{Eu}-154 \quad 0.000 \mathrm{E}+00 \quad 0.0000$ $\mathrm{Ni}-63 \quad 0.000 \mathrm{E}+00 \quad 0.0000$ Pu-239 $0.000 \mathrm{E}+00 \quad 0.0000$ $\mathrm{Pu}-240 \quad 0.000 \mathrm{E}+00 \quad 0.000$ $\begin{array}{llllllll}\mathrm{Sr}-90 & 0.000 \mathrm{E}+00 & 0.0000 & 0.000 \mathrm{E}+00 & 0.0000 & 0.000 \mathrm{E}+00 & 0.0000\end{array}$

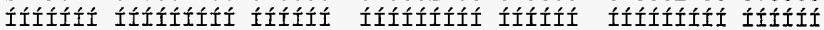
Total $0.000 \mathrm{E}+00 \quad 0.0000 \quad 0.000 \mathrm{E}+00 \quad 0.0000 \quad 0.000 \mathrm{E}+00 \quad 0.0000$ 0 *Sum of all water independent and dependent pathways. $\mathrm{mrem} / \mathrm{yr}$ fract $\ddot{A} \ddot{A} \ddot{A} \ddot{A} \ddot{A} \ddot{A} \ddot{A} \ddot{A}$ Ä $\ddot{A} \ddot{A} \ddot{A} \ddot{A}$

\section{mrem/yr fract. mrem/yr fract.}

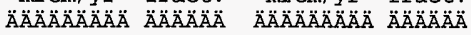
$0.000 \mathrm{E}+00 \quad 0.0000 \quad 0.000 \mathrm{E}+00 \quad 0.0000$ $\begin{array}{llll}0.000 E+00 & 0.0000 & 0.000 E+00 & 0.0000\end{array}$ $\begin{array}{llll}0.000 \mathrm{E}+00 & 0.0000 & 0.000 \mathrm{E}+00 & 0.0000\end{array}$ $\begin{array}{lllll}0.000 \mathrm{E}+00 & 0.0000 & 0.000 \mathrm{E}+00 & 0.0000\end{array}$ $\begin{array}{lllll}0.000 \mathrm{E}+00 & 0.0000 & 0.000 \mathrm{E}+00 & 0.0000\end{array}$ $\begin{array}{llll}0.000 \mathrm{E}+00 & 0.0000 & 0.000 \mathrm{E}+00 & 0.0000\end{array}$ $\begin{array}{llll}0.000 \mathrm{E}+00 & 0.0000 & 0.000 \mathrm{E}+00 & 0.0000 \\ 0.000 \mathrm{E}+00 & 0.0000 & 0.000 \mathrm{E}+00 & 0.0000 \\ 0.000 \mathrm{E}+00 & 0.0000 & 0.000 \mathrm{E}+00 & 0.0000\end{array}$ $\begin{array}{llll}.000 \mathrm{E}+00 & 0.0000 & 0.000 \mathrm{E}+00 & 0.0000\end{array}$

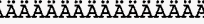
mrem/yr fract. $\ddot{A} \ddot{A} \ddot{A} \ddot{A} \ddot{A} \ddot{A} \ddot{A} \ddot{A} \ddot{A} \quad \ddot{A} \ddot{A} \ddot{A} \ddot{A} \ddot{A} \ddot{A}$ $\begin{array}{lllll}0.000 \mathrm{E}+00 & 0.0000 & 0.000 \mathrm{E}+00 & 0.0000\end{array}$ $\begin{array}{lllll}0.000 \mathrm{E}+00 & 0.0000 & 0.000 \mathrm{E}+00 & 0.0000\end{array}$ $\begin{array}{lllll}0.000 \mathrm{E}+00 & 0.0000 & 0.000 \mathrm{E}+00 & 0.0000\end{array}$ $0.000 E+00 \quad 0.0000 \quad 0.000 E+00 \quad 0.0000$ $\begin{array}{lllll}0.000 E+00 & 0.0000 & 0.000 \mathrm{E}+00 & 0.0000\end{array}$ $\begin{array}{llll}0.000 \mathrm{E}+00 & 0.0000 & 0.000 \mathrm{E}+00 & 0.0000\end{array}$ $0.000 \mathrm{E}+00 \quad 0.0000 \quad 0.000 \mathrm{E}+00 \quad 0.0000$ $\begin{array}{lllll}0.000 \mathrm{E}+00 & 0.0000 & 0.000 \mathrm{E}+00 & 0.0000\end{array}$ $\begin{array}{lllll}0.000 \mathrm{E}+00 & 0.0000 & 0.000 \mathrm{E}+00 & 0.0000\end{array}$

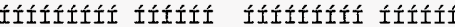
$\begin{array}{lllll}0.000 \mathrm{E}+00 & 0.0000 & 0.000 \mathrm{E}+00 & 0.0000\end{array}$

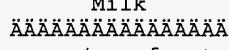
A $\ddot{A} \ddot{A} \ddot{A} \ddot{A} \ddot{A} \ddot{A} \ddot{A} \ddot{A}$ Ä̈̈̈̈̈̈̈̈̈ $0.000 \mathrm{E}+00 \quad 0.0000$ $0.000 \mathrm{E}+00 \quad 0.0000$ $0.000 \mathrm{E}+00 \quad 0.0000$ $0.000 \mathrm{E}+00 \quad 0.0000$ $0.000 \mathrm{E}+00 \quad 0.0000$ $0.000 \mathrm{E}+00 \quad 0.0000$ $0.000 \mathrm{E}+00 \quad 0.0000$ $0.000 \mathrm{E}+00 \quad 0.0000$ $0.000 \mathrm{E}+00 \quad 0.0000$ Íííííííí ííítí $0.000 \mathrm{E}+00 \quad 0.0000$ mrem/yr fract.

\begin{tabular}{|c|c|c|c|}
\hline \multicolumn{2}{|c|}{ 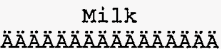 } & \multicolumn{2}{|c|}{ 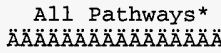 } \\
\hline $\begin{array}{l}m r e m / y x \\
\ddot{A} \ddot{A} \ddot{A} \ddot{A} \ddot{A} \ddot{A} \ddot{A} \ddot{A} \ddot{A}\end{array}$ & $\begin{array}{l}\text { fract. } \\
\ddot{A} \ddot{A} \ddot{A} \ddot{A} \ddot{A} \ddot{A}\end{array}$ & $\begin{array}{l}m r e m / y r \\
\ddot{A} \ddot{A} \ddot{A} \ddot{A} \ddot{A} \ddot{A} \ddot{A} \ddot{A} \ddot{A}\end{array}$ & $\begin{array}{l}\text { fract. } \\
\ddot{A} \ddot{A} \ddot{A} \ddot{A} \ddot{A} \ddot{A}\end{array}$ \\
\hline $0.000 \mathrm{E}+00$ & 0.0000 & $0.000 \mathrm{E}+00$ & 0.0000 \\
\hline $0.000 \mathrm{E}+00$ & 0.0000 & $1.238 \mathrm{E}-22$ & 0.8097 \\
\hline $0.000 \mathrm{E}+00$ & 0.0000 & $8.455 \mathrm{E}-27$ & 0.0001 \\
\hline $0.000 \mathrm{E}+00$ & 0.0000 & $2.093 \mathrm{E}-23$ & 0.1369 \\
\hline $0.000 \mathrm{E}+00$ & 0.0000 & $8.145 \mathrm{E}-24$ & 0.0533 \\
\hline $0.000 \mathrm{E}+00$ & 0.0000 & $0.000 \mathrm{E}+00$ & 0.0000 \\
\hline $0.000 \mathrm{E}+00$ & 0.0000 & $0.000 \mathrm{E}+00$ & 0.0000 \\
\hline 100 & & +00 & 000 \\
\hline $0.000 E+00$ & 0.0000 & $0.000 \mathrm{E}+00$ & 0.0000 \\
\hline İ́íi任任 & & 任任任任 & Íííííi \\
\hline $0.000 \mathrm{E}+00$ & 0.0000 & $1.528 \mathrm{E}-22$ & 1.0000 \\
\hline
\end{tabular}

$\ddot{A} \ddot{A} \ddot{A} \ddot{A} \ddot{A} \ddot{A} \ddot{A} \ddot{A} \ddot{A} \ddot{A} \ddot{A} \ddot{A} \ddot{A} \ddot{A} \ddot{A}$ mrem/yr fract. OOAAA AAAAAA $0.000 E+00 \quad 0.0000$ $0.000 \mathrm{E}+00 \quad 0.0000$ $0.000 \mathrm{E}+00 \quad 0.0000$ $0.000 \mathrm{E}+00 \quad 0.0000$ $0.000 E+00 \quad 0.0000$ $0.000 \mathrm{E}+00 \quad 0.0000$ $0.000 E+00 \quad 0.0000$ $0.000 \mathrm{E}+00 \quad 0.0000$ Itílííi ííííí $0.000 \mathrm{E}+00 \quad 0.0000$ 


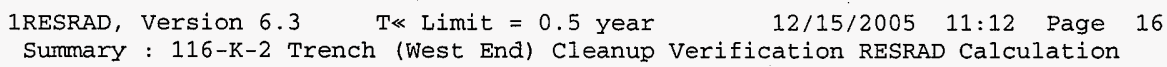

$$
\text { Radon }
$$

$$
\text { Plant }
$$

Meat

Milk

Soil

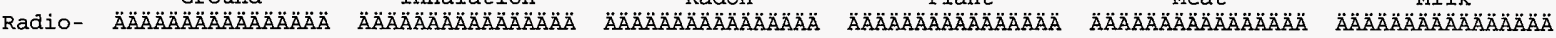
Nuclide mrem/yr fract. mrem/yr fract. mrem/yr fract. mrem/yr fract. mrem/yr fract.

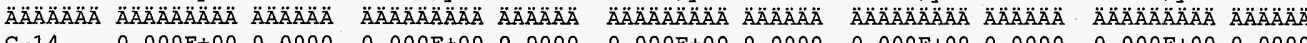
$\begin{array}{llllllllllll}C-14 & 0.000 E+00 & 0.0000 & 0.000 E+00 & 0.0000 & 0.000 E+00 & 0.0000 & 0.000 E+00 & 0.0000 & 0.000 E+00 & 0.0000\end{array}$ $\begin{array}{lllllllllll}\mathrm{C} 0-60 & 9.737 \mathrm{E}-23 & 0.7862 & 0.000 \mathrm{E}+00 & 0.0000 & 0.000 \mathrm{E}+00 & 0.0000 & 0.000 \mathrm{E}+00 & 0.0000 & 0.000 \mathrm{E}+00 & 0.0000\end{array}$ $\begin{array}{llllllllllll}\mathrm{Cs}-137 & 8.305 \mathrm{E}-27 & 0.0001 & 0.000 \mathrm{E}+00 & 0.0000 & 0.000 \mathrm{E}+00 & 0.0000 & 0.000 \mathrm{E}+00 & 0.0000 & 0.000 \mathrm{E}+00 & 0.0000\end{array}$

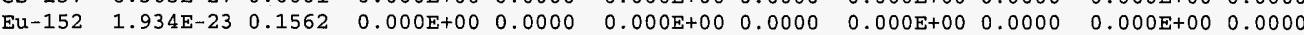
$\begin{array}{llllllllllll}\mathrm{Eu}-154 & 7.131 \mathrm{E}-24 & 0.0576 & 0.000 \mathrm{E}+00 & 0.0000 & 0.000 \mathrm{E}+00 & 0.0000 & 0.000 \mathrm{E}+00 & 0.0000 & 0.000 \mathrm{E}+00 & 0.0000\end{array}$ $\begin{array}{lllllllllllll}\mathrm{Ni}-63 & 0.000 \mathrm{E}+00 & 0.0000 & 0.000 \mathrm{E}+00 & 0.0000 & 0.000 \mathrm{E}+00 & 0.0000 & 0.000 \mathrm{E}+00 & 0.0000 & 0.000 \mathrm{E}+00 & 0.0000\end{array}$ $\begin{array}{llllllllllll}\mathrm{Pu}-239 & 0.000 \mathrm{E}+00 & 0.0000 & 0.000 \mathrm{E}+00 & 0.0000 & 0.000 \mathrm{E}+00 & 0.0000 & 0.000 \mathrm{E}+00 & 0.0000 & 0.000 \mathrm{E}+00 & 0.0000\end{array}$ $\begin{array}{lllllllllll}\mathrm{Pu}-240 & 0.000 \mathrm{E}+00 & 0.0000 & 0.000 \mathrm{E}+00 & 0.0000 & 0.000 \mathrm{E}+00 & 0.0000 & 0.000 \mathrm{E}+00 & 0.0000 & 0.000 \mathrm{E}+00 & 0.0000\end{array}$ $\begin{array}{lllllllllll}\mathrm{Sr}-90 & 0.000 \mathrm{E}+00 & 0.0000 & 0.000 \mathrm{E}+00 & 0.0000 & 0.000 \mathrm{E}+00 & 0.0000 & 0.000 \mathrm{E}+00 & 0.0000 & 0.000 \mathrm{E}+00 & 0.0000\end{array}$

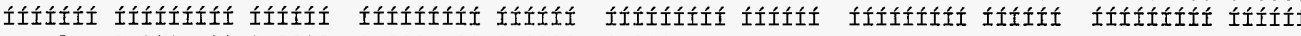
Total $1.239 \mathrm{E}-22 \quad 1.0000$

$0.000 \mathrm{E}+00 \quad 0.0000$

$0.000 E+00 \quad 0.0000$

$0.000 \mathrm{E}+00 \quad 0.0000$

$0.000 \mathrm{E}+00 \quad 0.0000$ $\mathrm{mrem} / \mathrm{yr}$ fract.

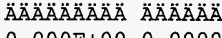
$0.000 \mathrm{E}+000.0000$ $0.000 \mathrm{E}+00 \quad 0.0000$ $0.000 \mathrm{E}+00 \quad 0.0000$ $0.000 \mathrm{E}+00 \quad 0.0000$ $0.000 \mathrm{E}+00 \quad 0.0000$ $0.000 \mathrm{E}+00 \quad 0.0000$ $0.000 E+00 \quad 0.0000$ $0.000 \mathrm{E}+000.0000$ $0.000 \mathrm{E}+00 \quad 0.0000$

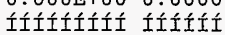
$0.000 \mathrm{E}+00 \quad 0.0000$

Total Dose Contributions TDOSE $(i, p, t)$ for Individual Radionuclides (i) and Pathways ( $p$ ) As mrem/yr and Fraction of Total Dose At $t=3.000 \mathrm{E}+00$ years Water Dependent Pathways

0 Fish Plant

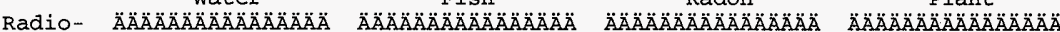
Nuclide mrem/Yr fract. Nuclide mrem/yr. Er $0.0007+00$ 0.0000 0.0000 Co-60 $0.000 \mathrm{E}+00 \quad 0.0000$ Cs-137 $0.000 \mathrm{E}+00 \quad 0.0000$ Eu-152 $0.000 \mathrm{E}+00 \quad 0.0000$ $\begin{array}{lll}\mathrm{Eu}-154 & 0.000 \mathrm{E}+00 & 0.0000\end{array}$ $\begin{array}{lll}\mathrm{Ni}-63 & 0.000 \mathrm{E}+00 \quad 0.0000\end{array}$

$\begin{array}{llllll}\mathrm{Pu}-239 & 0.000 \mathrm{E}+00 & 0.0000 & 0.000 \mathrm{E}+00 & 0.000\end{array}$ $\begin{array}{llllll}\mathrm{Pu}-240 & 0.000 \mathrm{E}+00 & 0.0000 & 0.000 \mathrm{E}+00 & 0.0000\end{array}$ $\begin{array}{llllll}\mathrm{Sr}-90 & 0.000 \mathrm{E}+00 & 0.0000 & 0.000 \mathrm{E}+00 & 0.000\end{array}$ mrem/Yr fract. $0.000 \mathrm{E}+00 \quad 0.0000$ $0.000 \mathrm{E}+00 \quad 0.0000$ $0.000 \mathrm{E}+00 \quad 0.0000$

$0.000 \mathrm{E}+00 \quad 0.0000$

\section{$\mathrm{mrem} / \mathrm{yr}$ fract.} Ä $0.000 \mathrm{E}+00 \quad 0.0000$ $0.000 E+00 \quad 0.0000$ $0.000 \mathrm{E}+00 \quad 0.0000$ $0.000 E+00 \quad 0.0000$ $0.000 \mathrm{E}+00 \quad 0.0000$ $0.000 \mathrm{E}+00 \quad 0.0000$ $0.000 E+00.0 .0000$ $0.000 \mathrm{E}+00 \quad 0.0000$ $0.000 \mathrm{E}+00 \quad 0.0000$ for

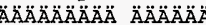
$0.000 \mathrm{E}+000.0000$ $0.000 \mathrm{E}+00 \quad 0.0000$ $0.000 \mathrm{E}+00 \quad 0.0000$ $0.000 \mathrm{E}+00 \quad 0.0000$ $0.000 \mathrm{E}+00 \quad 0.0000$ $0.000 \mathrm{E}+00 \quad 0.0000$ $0.000 \mathrm{E}+00 \quad 0.0000$ $0.000 \mathrm{E}+00 \quad 0.0000$ $0.000 \mathrm{E}+00 \quad 0.0000$ t́títítítít íítíít $0.000 \mathrm{E}+000.0000 \quad 0.000 \mathrm{E}+000.0000 \quad 0.000 \mathrm{E}+000.0000 \quad 0.000 \mathrm{E}+000.0000$ IfIIIII IIIIII IIIfIIIII IIIII

Meat $\mathrm{Milk}$ Ä mrem/yr fract. $\ddot{A} \ddot{A} \ddot{A} \ddot{A} \ddot{A} \ddot{A} \ddot{A} \ddot{A} \ddot{A} \ddot{A} \ddot{A} \ddot{A} \ddot{A} \ddot{A}$ $0.000 \mathrm{E}+00 \quad 0.0000$ $0.000 \mathrm{E}+00 \quad 0.0000$ $0.000 \mathrm{E}+00 \quad 0.0000$ $0.000 \mathrm{E}+00 \quad 0.0000$ $0.000 \mathrm{E}+00 \quad 0.0000$ $0.000 \mathrm{E}+00 \quad 0.0000$ $0.000 \mathrm{E}+00 \quad 0.0000$ $0.000 \mathrm{E}+00 \quad 0.0000$ $0.000 \mathrm{E}+000.00000 .000 \mathrm{E}+000.0000$

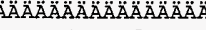
mrem/yr fract. O $0.000 E+00 \quad 0.0000$ $0.000 E+00 \quad 0.0000$ $0.000 \mathrm{E}+00 \quad 0.0000$ $0.000 \mathrm{E}+00 \quad 0.0000$ $0.000 \mathrm{E}+00 \quad 0.0000$ $0.000 \mathrm{E}+00 \quad 0.0000$ $0.000 \mathrm{E}+000.0000$ $0.000 \mathrm{E}+00 \quad 0.0000$ Ííííííít ííííí $0.000 \mathrm{E}+00 \quad 0.0000$ 0 *Sum of all water independent and dependent pathways 
Rev. 0

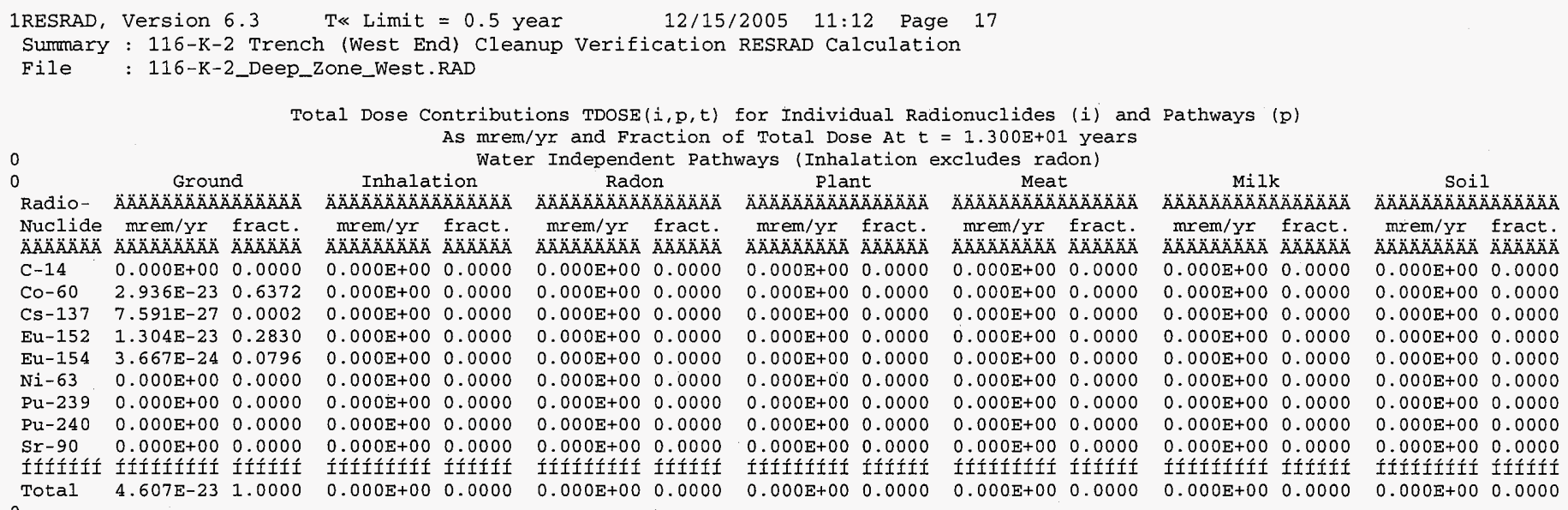

Total Dose Contributions TDOSE $(i, p, t)$ for Individual Radionuclides (i) and Pathways (p) As mrem/yr and Fraction of Total Dose At $t=1.300 \mathrm{E}+01$ years Water Dependent Pathways

0 Water

Radio- $\ddot{A} \ddot{A} \ddot{A} \ddot{A} \ddot{A} \ddot{A} \ddot{A} \ddot{A} \ddot{A} \ddot{A} \ddot{A} \ddot{A} \ddot{A}$

Nuclide mrem/yr fract.

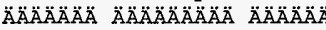

$\begin{array}{lll}C-14 & 0.000 \mathrm{E}+00 & 0.0000\end{array}$ Co-60 $0.000 \mathrm{E}+00 \quad 0.0000$ Cs-137 $0.000 \mathrm{E}+00 \quad 0.0000$

Eu-152 $0.000 \mathrm{E}+00 \quad 0.0000$

$\begin{array}{lll}\mathrm{Eu}-154 & 0.000 \mathrm{E}+00 \quad 0.0000\end{array}$

$\begin{array}{lll}\mathrm{Ni}-63 & 0.000 \mathrm{E}+00 \quad 0.0000\end{array}$

Pu-239 $0.000 \mathrm{E}+00 \quad 0.0000$

$\begin{array}{ll}\mathrm{Pu}-240 & 0.000 \mathrm{E}+00 \quad 0.0000\end{array}$

$\begin{array}{lll}\mathrm{S} x-90 & 0.000 \mathrm{E}+00 \quad 0.0000\end{array}$ Fish Radon Plant

Meat

Milk

mrem/Yr fract. mrem/yr fract

$0.000 \mathrm{E}+00 \quad 0.0000$

$0.000 \mathrm{E}+00 \quad 0.0000$

$0.000 \mathrm{E}+00 \quad 0.0000$

$0.000 \mathrm{E}+00 \quad 0.0000$

$0.000 \mathrm{E}+00 \quad 0.0000$

$0.000 \mathrm{E}+00 \quad 0.0000$

$0.000 \mathrm{E}+00 \quad 0.0000$

$0.000 \mathrm{E}+00 \quad 0.0000$

$0.000 \mathrm{E}+00 \quad 0.0000$

Ä $\ddot{A} \ddot{A} \ddot{A} \ddot{A} \ddot{A} \ddot{A} \ddot{A} \ddot{A} \ddot{A} \ddot{A} \ddot{A} \ddot{A} \ddot{A}$

АÄ $\ddot{A} \ddot{A} \ddot{A} \ddot{A} \ddot{A} \ddot{A} \ddot{A} \ddot{A} \ddot{A} \ddot{A} \ddot{A} \ddot{A}$

$\ddot{\mathrm{A}} \ddot{\mathrm{A}} \ddot{\mathrm{A}} \ddot{\mathrm{A}} \ddot{\mathrm{A}} \mathrm{A} \mathrm{A} \mathrm{A} \ddot{\mathrm{A}} \mathrm{A} \ddot{\mathrm{A}} \mathrm{A} \mathrm{A} \ddot{\mathrm{A}} \mathrm{A}$

mrem/yr fract. mrem/Yr fract. $0.0000+00$ 0.0000 $\begin{array}{llll}0.000 \mathrm{E}+00 & 0.0000 & 0.000 \mathrm{E}+00 & 0.0000 \\ 0.000 \mathrm{E}+00 & 0.0000 & 0.000 \mathrm{E}+00 & 0.0000\end{array}$ $0.000 \mathrm{E}+00 \quad 0.0000$

OE+00 0.0000

$.000 \mathrm{E}+00 \quad 0.0000$

$0.000 \mathrm{E}+000.0000$ $0.000 E+00 \quad 0.0000$ $0.000 \mathrm{E}+00 \quad 0.0000$

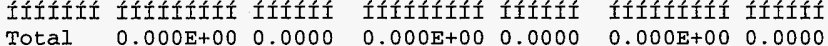
$0 *$ Sum of all water independent and dependent pathways. $0.0000 \quad 0.000 \mathrm{E}+00 \quad 0.0000$ $0.000 \mathrm{E}+00 \quad 0.0000$ $0.000 \mathrm{E}+00 \quad 0.0000$ $0.000 \mathrm{E}+00 \quad 0.0000$ $0.000 \mathrm{E}+00 \quad 0.0000$ $0.000 \mathrm{E}+00 \quad 0.0000$ $0.000 \mathrm{E}+00 \quad 0.0000$ $0.000 \mathrm{E}+00 \quad 0.0000$ ÍIIÍÍÍÍíí ííííí $0.000 \mathrm{E}+00 \quad 0.0000$
$0.000 \mathrm{E}+00 \quad 0.0000$

$0.000 \mathrm{E}+00 \quad 0.0000$

$0.000 \mathrm{E}+00 \quad 0.0000$

$0.000 \mathrm{E}+00 \quad 0.0000$

$0.000 \mathrm{E}+00 \quad 0.0000$

$0.000 \mathrm{E}+00 \quad 0.0000$

$0.000 \mathrm{E}+00 \quad 0.0000$

İííítííí ííííí

$0.000 \mathrm{E}+00 \quad 0.0000$ $0.000 \mathrm{E}+00 \quad 0.0000$

\subsection{0 a $0.0000+00$ 0.0000}

A mrem/yr fract. Ä $\ddot{A} \ddot{A} \ddot{A} \ddot{A} \ddot{A} \ddot{A} \ddot{A} \ddot{A}$ Ä $\ddot{A} \ddot{A} \ddot{A} \ddot{A}$ $0.000 \mathrm{E}+00 \quad 0.0000$ $0.000 \mathrm{E}+00 \quad 0.0000$ $0.000 \mathrm{E}+00 \quad 0.0000$ $0.000 \mathrm{E}+00 \quad 0.0000$ $\begin{array}{lll}0.000 \mathrm{E}+00 & 0.0000\end{array}$ $0.000 \mathrm{E}+00 \quad 0.0000$ $0.000 \mathrm{E}+00 \quad 0.000$ $0.000 \mathrm{E}+00 \quad 0.000$ $0.000 \mathrm{E}+00 \quad 0.000$ IIIIIIÍíí Íxííít

$0.000 \mathrm{E}+00 \quad 0.0000$
All Pathways* AAAAAAAAAAAAAAAA $\mathrm{mrem} / \mathrm{yr}$ fract. $\ddot{A} \ddot{A} \ddot{A} \ddot{A} \ddot{A} \ddot{A} \ddot{A} \ddot{A} \ddot{A} \ddot{A} \quad \ddot{A} \ddot{A} \ddot{A} \ddot{A} \ddot{A} \ddot{A}$ $0.000 \mathrm{E}+00 \quad 0.0000$ $2.936 \mathrm{E}-23 \quad 0.6372$ $7.591 \mathrm{E}-27 \quad 0.0002$ $1.304 \mathrm{E}-23 \quad 0.2830$ $3.667 \mathrm{E}-24 \quad 0.0796$ $0.000 E+00 \quad 0.0000$ $0.000 \mathrm{E}+00 \quad 0.0000$ $0.000 \mathrm{E}+00 \quad 0.0000$ $0.000 \mathrm{E}+00 \quad 0.0000$ Íííííííi ííítí $4.607 \mathrm{E}-231.0000$ 
1RESRAD, Version 6.3 T« Limit $=0.5$ year $12 / 15 / 2005 \quad 11: 12$ Page 18

Summary : 116-K-2 Trench (West End) Cleanup Verification RESRAD Calculation

File : 116-K-2_Deep_zone_West.RAD

\begin{tabular}{|c|c|c|c|c|c|c|c|c|c|c|c|c|c|}
\hline \multicolumn{14}{|l|}{0} \\
\hline 0 & Grour & & Inhalat & tion & Rad & on & Pla & nt & Meat & & MIL & & Soil \\
\hline Radio- & 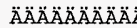 & $\ddot{A} \ddot{A} \ddot{A} \ddot{A} \ddot{A} \ddot{A} \ddot{A}$ & 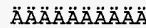 & 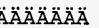 & $\ddot{A} \ddot{A} \ddot{A} \ddot{A} \ddot{A} \ddot{A} \ddot{A} \ddot{A} \ddot{A}$ & $\ddot{A} \ddot{A} \ddot{A} \ddot{A} \ddot{A} \ddot{A} \ddot{A}$ & $\ddot{A} \ddot{A} \ddot{A} \ddot{A} \ddot{A} \ddot{A} \ddot{A} \ddot{A} \ddot{A} \ddot{A}$ & $\ddot{A} \ddot{A} \ddot{A} \ddot{A} \ddot{A} \ddot{A} \ddot{A} \ddot{A}$ & $\ddot{A} \ddot{A} \ddot{A} \ddot{A} \ddot{A} \ddot{A} \ddot{A} \ddot{A} \ddot{A} \dot{A}$ & $\ddot{A} \ddot{A} \ddot{A} \ddot{A} \ddot{A} \ddot{A} \ddot{A}$ & $\ddot{\mathrm{A}} \ddot{\mathrm{A}} \ddot{\mathrm{A}} \ddot{\mathrm{A}} \ddot{\mathrm{A}} \ddot{\mathrm{A}} \ddot{\mathrm{A}} \ddot{\mathrm{A}} \ddot{\mathrm{A}}$ & & 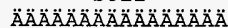 \\
\hline $\begin{array}{l}\text { Nuclide } \\
\ddot{A} \ddot{A} \ddot{A} \ddot{A} \ddot{A} \ddot{A} \ddot{A} \ddot{A}\end{array}$ & $\begin{array}{c}m r e m / y r \\
\ddot{A} \ddot{A} \ddot{A} \ddot{A} \ddot{A} \ddot{A} \ddot{A} \ddot{A} \ddot{A}\end{array}$ & $\begin{array}{l}\text { fract. } \\
\ddot{A} \ddot{A} \ddot{A} \ddot{A} \ddot{A} \ddot{A}\end{array}$ & $\begin{array}{c}\operatorname{mrem} / y r \\
\ddot{A} \ddot{A} \ddot{A} \ddot{A} \ddot{A} \ddot{A} \ddot{A} \ddot{A} \ddot{A}\end{array}$ & $\begin{array}{l}\text { fract. } \\
\ddot{A} \ddot{A} \ddot{A} \ddot{A} \ddot{A} \ddot{A}\end{array}$ & $\begin{array}{c}m r e m / y r \\
\ddot{A} \ddot{A} \ddot{A} \ddot{A} \ddot{A} \ddot{A} \ddot{A} \ddot{A} \ddot{A}\end{array}$ & $\begin{array}{l}\text { fract. } \\
\ddot{A} \ddot{A} \ddot{A} \ddot{A} \ddot{A}\end{array}$ & $\begin{array}{c}m r e m / y x \\
\ddot{A} \ddot{A} \ddot{A} \ddot{A} \ddot{A} \ddot{A} \ddot{A} \ddot{A} \ddot{A}\end{array}$ & $\begin{array}{l}\text { fract. } \\
\ddot{A} \ddot{A} \ddot{A} \ddot{A} \ddot{A} \ddot{A}\end{array}$ & $\begin{array}{c}m r e m / y r \\
\ddot{A} \ddot{A} \ddot{A} \ddot{A} \ddot{A} \ddot{A} \ddot{A} \ddot{A}\end{array}$ & $\begin{array}{l}\text { fract. } \\
\ddot{A} \ddot{A} \ddot{A} \ddot{A} \ddot{A} \ddot{A}\end{array}$ & $\begin{array}{c}m r e m / y r \\
\ddot{A} \ddot{A} \ddot{A} \ddot{A} \ddot{A} \ddot{A} \ddot{A} \ddot{A}\end{array}$ & $\begin{array}{l}\text { fract. } \\
\ddot{A} \ddot{A} \ddot{A} \ddot{A} \ddot{A} \ddot{A}\end{array}$ & $\begin{array}{cc}m r e m / y r & f r \\
\ddot{A} \ddot{A} \ddot{A} \ddot{A} \ddot{A} \ddot{A} \ddot{A} \ddot{A} \quad \ddot{A} \ddot{A}\end{array}$ \\
\hline$C-14$ & $0.000 \mathrm{E}+00$ & 0.0000 & $0.000 \mathrm{E}+00$ & 0.0000 & $0.000 \mathrm{E}+00$ & 0.0000 & $0.000 \mathrm{E}+00$ & & $0.000 \mathrm{E}+00$ & 0.0000 & & & $0 E+000$. \\
\hline Co- 60 & $3.824 \mathrm{E}-24$ & 0.3273 & $0.000 \mathrm{E}+00$ & 0.0000 & $0.000 \mathrm{E}+00$ & 0.0000 & $0.000 \mathrm{E}+00$ & 0.0000 & $0.000 \mathrm{E}+00$ & 0.0000 & $000 E+00$ & & $0 \mathrm{E}+00 \quad 0$. \\
\hline $\mathrm{Cs}-137$ & $6.515 E-27$ & 0.0006 & $0.000 \mathrm{E}+00$ & 0.0000 & $0.000 \mathrm{E}+00$ & 0.0000 & $0.000 \mathrm{E}+00$ & 0.0000 & $0.000 \mathrm{E}+00$ & 0.0000 & $0.000 \mathrm{E}+00$ & 0.0000 & $0.000 \mathrm{E}+00 \quad 0.0000$ \\
\hline $\mathrm{Eu}-152$ & $6.669 \mathrm{E}-24$ & 0.5708 & $0.000 \mathrm{E}+00$ & 0.0000 & $0.000 \mathrm{E}+00$ & 0.0000 & $0.000 \mathrm{E}+00$ & 0.0000 & $0.000 \mathrm{E}+00$ & 0.0000 & $0.000 E+00$ & 0.0000 & $0.000 \mathrm{E}+00 \quad 0.0000$ \\
\hline Eu-154 & $1.184 \mathrm{E}-24$ & 0.1013 & $0.000 E+00$ & 0.0000 & $0 E+00$ & 0.0000 & $0.000 \mathrm{E}+00$ & 0.0000 & $0.000 E+00$ & 0.0000 & $0.000 \mathrm{E}+00$ & 0.0000 & $0.000 \mathrm{E}+00 \quad 0.0000$ \\
\hline $\mathrm{Ni}-63$ & 0.00 & 0.0 & $0.000 \mathrm{E}+00$ & 0.0000 & $0 E+00$ & 0.0000 & $0.000 \mathrm{E}+00$ & 0.0 & +00 & 0.0 & +00 & 0. & $0.000 \mathrm{E}+000$. \\
\hline Pu-239 & 0.00 & 0.0000 & $0.000 \mathrm{E}+00$ & 0.0000 & $\mathrm{E}+00$ & 0.0000 & $0.000 \mathrm{E}$ & 0.0 & +00 & 0.0000 & +00 & & $0 E+000$. \\
\hline $\mathrm{Pu}-240$ & 0.00 & 0.0 & $0.000 \mathrm{E}+00$ & & & & 0.00 & & & 0.0 & & & +000. \\
\hline $\begin{array}{l}\text { Sr-90 } \\
\text { Ítífít }\end{array}$ & $\begin{array}{l}0.000 \mathrm{E}+00 \\
\text { IfÍfítít }\end{array}$ & $\begin{array}{l}0.0000 \\
\text { ffítít }\end{array}$ & $\begin{array}{l}0.000 E+00 \\
\text { ff́́tíf́́tí }\end{array}$ & $\begin{array}{l}0.0000 \\
\text { ÍÍÍÍí }\end{array}$ & $\begin{array}{l}0.000 \mathrm{E}+00 \\
\text { IfÍ1́1́1́1́ }\end{array}$ & $\begin{array}{l}0.0000 \\
\text { If́títi }\end{array}$ & $\begin{array}{l}0.000 \mathrm{E}+00 \\
\text { Iftútíf }\end{array}$ & $\begin{array}{l}0.0000 \\
\text { Íf́ítí }\end{array}$ & 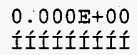 & $\begin{array}{l}0.0000 \\
\text { Í́títí }\end{array}$ & $\begin{array}{l}0.000 \mathrm{E}+00 \\
\text { Íf́́títít }\end{array}$ & $\begin{array}{l}0.0000 \\
\text { If́f́́tí }\end{array}$ & 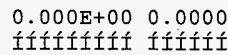 \\
\hline Total & $1.168 \mathrm{E}-23$ & 1.0000 & $0.000 \mathrm{E}+00$ & 0.0000 & $0.000 \mathrm{E}+00$ & 0.0000 & $0.000 \mathrm{E}+00$ & 0.0000 & $0.000 \mathrm{E}+00$ & 0.0000 & $0.000 \mathrm{E}+00$ & 0.0000 & $0.000 \mathrm{E}+00 \quad 0.0000$ \\
\hline
\end{tabular}
$0^{\text {Tot }}$

Total Dose Contributions TDOSE(i,p,t) for Individual Radionuclides (i) and Pathways (p) As mrem/yr and Fraction of Total Dose At $t=3.000 \mathrm{E}+01$ years Water Dependent Pathways

0 Water

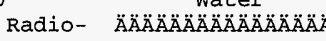
Nuclide mrem/yr fract. Nuclide mrem/Yr fract. $\begin{array}{lll}\triangle & \\ \mathrm{C}-14 \mathrm{~A} & 0.000 \mathrm{E}+00 & 0.0000\end{array}$ $\begin{array}{lll}\mathrm{C}-14 & 0.000 \mathrm{E}+00 & 0.0000 \\ \mathrm{C}-60 & 0.000 \mathrm{E}+00 & 0.0000\end{array}$ $\begin{array}{lll}\mathrm{C}-60 & 0.000 \mathrm{E}+00 & 0.0000 \\ \mathrm{CS}-137 & 0.000 \mathrm{E}+00 & 0.0000\end{array}$ Eu-152 $0.000 \mathrm{E}+00 \quad 0.0000$ $\mathrm{Eu}-154 \quad 0.000 \mathrm{E}+00 \quad 0.0000$ $\begin{array}{lll}\mathrm{Eu}-154 & 0.000 \mathrm{E}+00 & 0.0000 \\ \mathrm{Ni}-63 & 0.000 \mathrm{E}+00 & 0.0000\end{array}$ $\begin{array}{lll}\mathrm{Ni}-63 & 0.000 \mathrm{E}+00 & 0.0000 \\ \mathrm{Pu}-239 & 0.000 \mathrm{E}+00 & 0.0000\end{array}$ $\begin{array}{lll}\mathrm{Pu}-239 & 0.000 \mathrm{E}+00 & 0.0000 \\ \mathrm{Pu}-240 & 0.000 \mathrm{E}+00 & 0.0000\end{array}$ $\begin{array}{lllllll} & 0.000 \mathrm{E}+00 & 0.0000 & 0.000 \mathrm{E}+00 & 0.0000 & 0.000 \mathrm{E}+00 & 0.0000\end{array}$

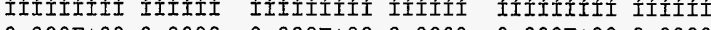
$0 *$ Sum of all water independent and dependent pathways. $0.000 \mathrm{E}+00 \quad 0.0000 \quad 0.000 \mathrm{E}+00 \quad 0.0000$

$$
\text { Radon }
$$
Plant

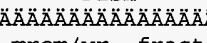

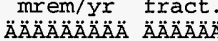
$0.000 \mathrm{E}+00 \quad 0.0000$ $0.000 \mathrm{E}+00 \quad 0.0000$ $0.000 \mathrm{E}+00 \quad 0.0000$ $0.000 E+00 \quad 0.0000$ $0.000 E+00 \quad 0.0000$ $0.000 \mathrm{E}+000.0000$

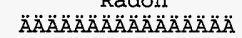
A $\ddot{A} \ddot{A} \ddot{A} \ddot{A} \ddot{A} \ddot{A} \ddot{A} \ddot{A} \ddot{A} \ddot{A} \ddot{A} \ddot{A} \ddot{A} \ddot{A}$

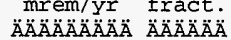
$0.000 \mathrm{E}+00 \quad 0.0000$ $0.000 \mathrm{E}+00 \quad 0.0000$ $0.000 \mathrm{E}+00 \quad 0.0000$ $0.000 \mathrm{E}+00 \quad 0.0000$ $0.000 \mathrm{E}+00 \quad 0.0000$ $0.000 \mathrm{E}+00 \quad 0.0000$ $0.000 \mathrm{E}+00 \quad 0.0000$ mrem/yr fract.

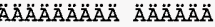
$0.000 E+00 \quad 0.0000$ $0.000 \mathrm{E}+00 \quad 0.0000$ $0.000 \mathrm{E}+00 \quad 0.0000$ $0.000 \mathrm{E}+00 \quad 0.0000$ $0.000 \mathrm{E}+00 \quad 0.0000$ $0.000 \mathrm{E}+00 \quad 0.0000$ $0.000 \mathrm{E}+00 \quad 0.0000$ $0.000 \mathrm{E}+00 \quad 0.0000$ $0.000 \mathrm{E}+00 \quad 0.0000$ Íííííííí ííííi $0.000 \mathrm{E}+00 \quad 0.0000$
Meat $\ddot{A} \ddot{A} \ddot{A} \ddot{A} \ddot{A} \ddot{A} \ddot{A} \ddot{A} \ddot{A} \ddot{A} \ddot{A} \ddot{A} \ddot{A} \ddot{A} \ddot{A} \ddot{A} \ddot{A}$ mrem/yr fract. $\ddot{A} \ddot{A} \ddot{A} \ddot{A} \ddot{A} \ddot{A} \ddot{A} \ddot{A} \ddot{A}$ Ä $\ddot{A} \ddot{A} \ddot{A} \ddot{A}$ $0.000 E+00 \quad 0.0000$ $0.000 \mathrm{E}+00 \quad 0.0000$ $0.000 \mathrm{E}+00 \quad 0.0000$ $0.000 \mathrm{E}+00 \quad 0.0000$ $0.000 \mathrm{E}+00 \quad 0.0000$ $0.000 \mathrm{E}+00 \quad 0.0000$ $0.000 \mathrm{E}+00 \quad 0.0000$ $0.000 \mathrm{E}+00 \quad 0.0000$ $0.000 \mathrm{E}+00 \quad 0.0000$ Ííííítíí ííííti $0.000 \mathrm{E}+000.0000$
Milk

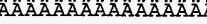
mrem/yr fract. ÄÄ $\ddot{A} \ddot{A} \ddot{A} \ddot{A} \ddot{A} \ddot{A} \ddot{A}$ Ä $\ddot{A} \ddot{A} \ddot{A} \ddot{A}$ $0.000 \mathrm{E}+00 \quad 0.0000$ $0.000 \mathrm{E}+00 \quad 0.0000$ $0.000 \mathrm{E}+00 \quad 0.0000$ $0.000 \mathrm{E}+00 \quad 0.0000$ $0.000 \mathrm{E}+00 \quad 0.0000$ $0.000 \mathrm{E}+00 \quad 0.0000$ $0.000 \mathrm{E}+00 \quad 0.0000$ $0.000 \mathrm{E}+00 \quad 0.0000$ $0.000 \mathrm{E}+00 \quad 0.0000$

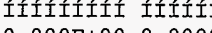
$0.000 \mathrm{E}+00 \quad 0.0000$
All Pathways*

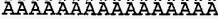
mrem/yr fract. $\ddot{A} \ddot{A} \ddot{A} \ddot{A} \ddot{A} \ddot{A} \ddot{A} \ddot{A} \ddot{A}$ Ä̈̈̈̈̈̈̈̈̈̈ $0.000 \mathrm{E}+00 \quad 0.0000$ $3.824 \mathrm{E}-24 \quad 0.3273$ $6.515 \mathrm{E}-27 \quad 0.0006$ 6. $669 \mathrm{E}-240.5708$ $1.184 \mathrm{E}-24 \quad 0.1013$ $0.000 \mathrm{E}+00 \quad 0.0000$ $0.000 \mathrm{E}+00 \quad 0.0000$ $0.000 \mathrm{E}+00 \quad 0.0000$ $0.000 \mathrm{E}+00 \quad 0.0000$

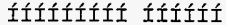
$1.168 \mathrm{E}-231.0000$ 


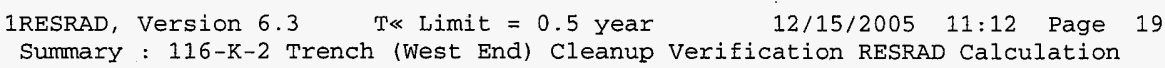


1RESRAD, Version $6.3 \quad$ T« Limit $=0.5$ year $12 / 15 / 2005 \quad 11: 12$ Page 20

Summary : 116-K-2 Trench (West End) Cleanup Verification RESRAD Calculation

File : 116-K-2_Deep_zone_West.RAD

Total Dose Contributions $\operatorname{TDOSE}(i, p, t)$ for Individual Radionuclides (i) and Pathways (p) As mrem/yr and Fraction of Total Dose At $t=3.000 E+02$ years

0 Water Independent Pathways (Inhalation excludes radon) nhalation Radon Plant

Meat

$\operatorname{Mi} 1 \mathrm{k}$ Soi1

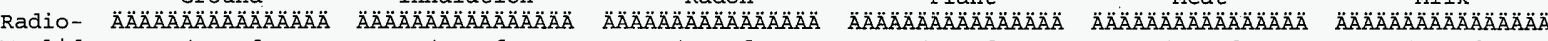
Nuclide mrem/yr fract. mrem/yr fract. mrem/yr fract. mrem/yr fract. mrem/yr fract. mrem/yr fract.

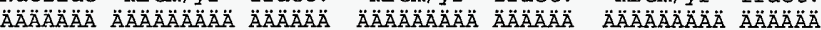
$\begin{array}{llllllll}C-14 & 0.000 E+00 & 0.0000 & 0.000 E+00 & 0.0000 & 0.000 E+00 & 0.0000\end{array}$ $\begin{array}{llllllll}\mathrm{CO}-60 & 0.000 \mathrm{E}+00 & 0.0000 & 0.000 \mathrm{E}+00 & 0.0000 & 0.000 \mathrm{E}+00 & 0.0000\end{array}$

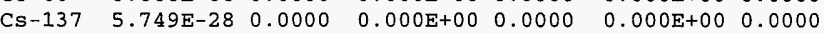
$\mathrm{Eu}-152 \quad 1.143 \mathrm{E}-28 \quad 0.0000 \quad 0.000 \mathrm{E}+00 \quad 0.0000 \quad 0.000 \mathrm{E}+000.0000$ $\begin{array}{llllllll}\mathrm{Eu}-154 & 0.000 \mathrm{E}+00 & 0.0000 & 0.000 \mathrm{E}+00 & 0.0000 & 0.000 \mathrm{E}+00 & 0.0000\end{array}$ $\begin{array}{lllllllll}\mathrm{Ni}-63 & 0.000 \mathrm{E}+00 & 0.0000 & 0.000 \mathrm{E}+00 & 0.0000 & 0.000 \mathrm{E}+00 & 0.0000\end{array}$ $\begin{array}{llllllll}\mathrm{Pu}-239 & 0.000 \mathrm{E}+00 & 0.0000 & 0.000 \mathrm{E}+00 & 0.0000 & 0.000 \mathrm{E}+00 & 0.0000\end{array}$ $\begin{array}{llllllll}\mathrm{Pu}-240 & 0.000 \mathrm{E}+00 & 0.0000 & 0.000 \mathrm{E}+00 & 0.0000 & 0.000 \mathrm{E}+00 & 0.0000\end{array}$ $\begin{array}{lllllllll}\mathrm{S} r-90 & 0.000 \mathrm{E}+00 & 0.0000 & 0.000 \mathrm{E}+00 & 0.0000 & 0.000 \mathrm{E}+00 & 0.0000\end{array}$

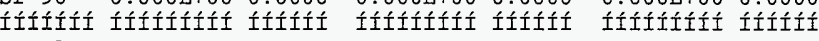

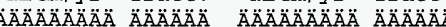
$0.000 \mathrm{E}+00 \quad 0.0000 \quad 0.000 \mathrm{E}+00 \quad 0.0000$ $0.000 \mathrm{E}+000.0000$ $0.000 \mathrm{E}+00 \quad 0.0000$ $0.000 \mathrm{E}+00 \quad 0.0000$ $0.000 \mathrm{E}+00 \quad 0.0000$ $0.000 E+00 \quad 0.0000$ $0.000 \mathrm{E}+00 \quad 0.0000$ $0.000 E+00 \quad 0.0000$ $0.000 E+00 \quad 0.0000$ $0.000 \mathrm{E}+00 \quad 0.0000$ ífífítít ífíítí $0.000 E+00 \quad 0.0000$ $0.000 E+00 \quad 0.0000$ $0.000 \mathrm{E}+00 \quad 0.0000$ $0.000 E+00 \quad 0.0000$ $0.000 E+00 \quad 0.0000$ $0.000 \mathrm{E}+00 \quad 0.0000$ $0.000 \mathrm{E}+00 \quad 0.0000$ Total $\begin{array}{lllll}6.891 \mathrm{E}-28 & 0.0000 & 0.000 \mathrm{E}+00 & 0.0000\end{array}$

$0.000 \mathrm{E}+00 \quad 0.0000$

$0.000 \mathrm{E}+00 \quad 0.0000$

ífítífít ífííí $0.0005+00$ OAAAA $0.000 \mathrm{~s}+000.0000$ $0.000 \mathrm{E}+00 \quad 0.0000$ $0.000 \mathrm{E}+00 \quad 0.0000$ $0.000 E+00 \quad 0.0000$ $0.000 \mathrm{E}+00 \quad 0.0000$ $0.000 E+00 \quad 0.0000$ $0.000 \mathrm{E}+00 \quad 0.0000$ $0.000 \mathrm{E}+00 \quad 0.0000$ ífífítítí fí́ítíi

Total Dose Contributions TDOSE $(i, p, t)$ for Individual Radionuclides (i) and Pathways (p) As mrem/yr and Fraction of Total Dose At $t=3.000 \mathrm{E}+02$ years

0 water Dependent Pathways Fish Radon Qlant

Meat А $\mathrm{mrem} / \mathrm{yr}$ fract $0.000=00$ 0.0000 0.000 A $0.000 \mathrm{E}+00 \quad 0.0000 \quad 0.000 \mathrm{E}+00 \quad 0.0000$ $\begin{array}{llll}0.000 \mathrm{E}+00 & 0.0000 & 0.000 \mathrm{E}+00 & 0.0000\end{array}$ $\begin{array}{llll}0.000 E+00 & 0.0000 & 0.000 E+00 & 0.0000\end{array}$ $0.000 \mathrm{E}+00 \quad 0.0000$ $0.000 \mathrm{E}+00 \quad 0.0000$ $0.000 \mathrm{E}+00 \quad 0.0000 \quad 0.000 \mathrm{E}+00 \quad 0.0000$ $0.000 \mathrm{E}+00 \quad 0.0000 \quad 0.000 \mathrm{E}+00 \quad 0.0000$ $\begin{array}{llll}3.810 \mathrm{E}-06 & 0.0117 & 3.286 \mathrm{E}-07 & 0.0010\end{array}$

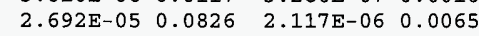
$0.000 \mathrm{E}+00 \quad 0.0000$ ítíítítít ítíti $3.073 \mathrm{E}-050.0943$. 
Ground Inhalation

$$
\text { Radon }
$$

$$
\text { Plant }
$$




\begin{tabular}{|c|c|c|c|c|c|c|}
\hline \multirow{4}{*}{$\begin{array}{l}\text { 1RESRAD, } \\
\text { Summary } \\
\text { File }\end{array}$} & Version 6.3 & \multicolumn{2}{|c|}{$T \ll$ Limit $=0.5$ year } & \multicolumn{2}{|c|}{$12 / 15 / 2005$} & \multirow{2}{*}{$\begin{array}{l}\text { Page } 23 \\
\text { lation }\end{array}$} \\
\hline & \multirow{2}{*}{\multicolumn{6}{|c|}{$\begin{array}{l}\text { : 116-K-2 Trench (West End) Cleanup } \\
: 116-\mathrm{K}-2 \text { _Deep_Zone_West.RAD }\end{array}$}} \\
\hline & & & & & & \\
\hline & \multicolumn{6}{|c|}{$\begin{array}{c}\text { Single Radionuclide soil Guidelines } \mathrm{G}(\mathrm{i}, \mathrm{t}) \text { in } \mathrm{pCi} / \mathrm{g} \\
\text { Basic Radiation Dose Limit }=1.500 \mathrm{E}+01 \mathrm{mrem} / \mathrm{yr}\end{array}$} \\
\hline \multicolumn{7}{|c|}{ (5) } \\
\hline$\ddot{A} \ddot{A} \ddot{A} \ddot{A} \ddot{A} \ddot{A} \ddot{A}$ & $\begin{aligned} t= & 0.000 \mathrm{E}+00 \\
& \ddot{A} \ddot{A} \ddot{A} \ddot{A} \ddot{A} \ddot{A} \ddot{A} \ddot{A} \ddot{A}\end{aligned}$ & $\begin{array}{l}1,000 E+00 \\
\ddot{A} \ddot{A} \ddot{A} \ddot{A} \ddot{A} \ddot{A} \ddot{A} \ddot{A} A \ddot{A}\end{array}$ & $\begin{array}{l}0 E+00 \\
\ddot{A} \ddot{A} \ddot{A} \ddot{A} \ddot{A} \ddot{A}\end{array}$ & $\begin{array}{l}1.300 \mathrm{E} \\
\ddot{A} \ddot{A} \ddot{A} \ddot{A} \ddot{A} \ddot{A}\end{array}$ & $\begin{array}{l}3.000 \mathrm{E} \\
\ddot{A} \ddot{A} \ddot{A} \ddot{A} \ddot{A} \ddot{A}\end{array}$ & $\begin{array}{l}1.000 E+0 \\
\ddot{A} \ddot{A} \ddot{A} \ddot{A} \ddot{A} \ddot{A} \ddot{A} \ddot{A}\end{array}$ \\
\hline$C-14$ & $\star 4.454 \mathrm{E}+12$ & $\star 4.454 \mathrm{E}+12$ & $4 \mathrm{E}+12$ & $\star 4.454 \mathrm{E}+12$ & $\star 4.454$ & \\
\hline $\mathrm{CO}-$ & $\star 1.131 E+15$ & $\star 1.131 \mathrm{E}$ & $* 1$ & $\star 1.1311$ & $\star 1.13$ & \\
\hline $\mathrm{Cs}-$ & $\star 8.701 \mathrm{E}+13$ & $\star 8$. & *8. & $* 8$. & $\star 8.70$ & \\
\hline Eu & $\star 1.765 \mathrm{E}+14$ & $\star 1$. & $\star 1$. & $\star 1$. & $\star 1.76$ & \\
\hline $\mathrm{Eu}-$ & $\star 2.639 \mathrm{E}+14$ & *2. & *2. & & $\star 2.63$ & $* 2$. \\
\hline $\mathrm{Ni}-$ & $\star 5.916 \mathrm{E}+13$ & $\star 5$. & $\star 5$. & $\star 5$. & $* 5.91$ & $\star 5$. \\
\hline Pu-239 & $* 6.212 \mathrm{E}+10$ & *6. & ${ }^{*} 6$. & $* 6$ & $* 6.212$ & \\
\hline Pu-240 & $* 2.277 \mathrm{E}+11$ & $\star 2.2$ & *2. & $\star 2.27$ & $\star 2.27$ & \\
\hline 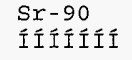 & $\begin{array}{l}\text { *1.365E+14 } \\
\text { IIIIIIIIII }\end{array}$ & $\begin{array}{l}* 1.365 E+14 \\
\text { IIIIIIIII }\end{array}$ & $\begin{array}{l}* 1.365 E+14 \\
\text { IIIIIIIIII }\end{array}$ & $\begin{array}{l}* 1.365 \mathrm{E}+14 \\
\text { IIIIIIIII }\end{array}$ & $\begin{array}{l}* 1 \cdot 365 \mathrm{E}+14 \\
\text { İIIIIIIIII }\end{array}$ & $\begin{array}{l}* 1 \cdot 365 \mathrm{E}+1 \\
\text { IIIIIIIII }\end{array}$ \\
\hline \multicolumn{7}{|c|}{ *At specific activity limit } \\
\hline \\
\hline & \multicolumn{5}{|c|}{$\begin{array}{l}\text { Summed Dose/Source Ratios DSR }(i, t) \text { in }(m r e m / y r) /(p C i / g) \\
\text { and Single Radionuclide Soil Guidelines }(i, t) \text { in pCi/g }\end{array}$} & \\
\hline & at $t \max =t$ & ime of maximum $t$ & total dose $=$ & $=1.000 \mathrm{E}+03$ & years & \\
\hline $\begin{array}{l}\text { ONuclide } \\
\text { (i) }\end{array}$ & $\begin{array}{l}\text { Initial } \\
(\mathrm{pCi} / \mathrm{g})\end{array}$ & $\begin{array}{c}\text { tmin } \\
\text { (years) }\end{array}$ & $\operatorname{DSR}(i, t \min )$ & $\begin{array}{l}G(i, t \min ) \\
(\mathrm{pCi} / \mathrm{g})\end{array}$ & $\operatorname{DSR}(i, t \max )$ & $\begin{array}{c}G(i, t \max ) \\
(p C i / g)\end{array}$ \\
\hline & $\ddot{A} \ddot{A} \ddot{A} \ddot{A} \ddot{A} \ddot{A} \ddot{A} \ddot{A} \ddot{A}$ & $\ddot{A} \ddot{A} \ddot{A} \ddot{A} \ddot{A} \ddot{A} \ddot{A} \ddot{A}$ & $\ddot{A} \ddot{A} \ddot{A} A \ddot{A} \ddot{A} A \ddot{A} \ddot{\partial}$ & & $\ddot{A} \ddot{A} \ddot{A} \ddot{A} \ddot{A} \ddot{P}$ & \\
\hline$C-14$ & $1.440 \mathrm{E}+00$ & $.000 E+00$ & $0 \mathrm{E}+00$ & $\star 4.454 \mathrm{E}+12$ & 00 & $\star 4.454 \mathrm{E}+12$ \\
\hline $\mathrm{Co}-60$ & $4.230 \mathrm{E}+00$ & $0.000 \mathrm{E}+00$ & $8 E-23$ & $\star 1.131 \mathrm{E}+15$ & $0.000 \mathrm{E}+00$ & $* 1.131 E+15$ \\
\hline Cs -137 & 1.170E+02 & $0.000 \mathrm{E}+00$ & $7.292 \mathrm{E}-29$ & $\star 8.701 \mathrm{E}+13$ & $0.000 \mathrm{E}+00$ & $\star 8.701 E+13$ \\
\hline Eu-152 & $7.600 \mathrm{E}+01$ & $0.000 E+00$ & $2.864 E-25$ & $\star 1.765 E+14$ & $0.000 \mathrm{E}+00$ & $\star 1.765 E+14$ \\
\hline Eu-154 & $7.200 \mathrm{E}+00$ & $0.000 E+00$ & $1.209 \mathrm{E}-24$ & $\star 2.639 \mathrm{E}+14$ & $0.000 \mathrm{E}+00$ & $\star 2.639 E+14$ \\
\hline $\mathrm{Ni}-63$ & $6.500 E+02$ & $0.000 \mathrm{E}+00$ & $0.000 \mathrm{E}+00$ & $\star 5.916 E+13$ & $0.000 \mathrm{E}+00$ & $* 5.916 \mathrm{E}+13$ \\
\hline Pu-239 & $3.800 \mathrm{E}+00$ & $1.000 E+03$ & $2.583 \mathrm{E}-05$ & $5.808 E+05$ & $2.583 E-05$ & $5.808 E+05$ \\
\hline Pu -240 & $9.000 \mathrm{E}-01$ & $1.000 \mathrm{E}+03$ & $7.065 \mathrm{E}-04$ & $2.123 E+04$ & $7.065 E-04$ & $2.123 E+04$ \\
\hline $\begin{array}{l}\text { Sr-90 } \\
\text { IIIIIIII }\end{array}$ & $\begin{array}{l}6.300 E+00 \\
\text { İIIIIIIII }\end{array}$ & $\begin{array}{l}0.000 E+00 \\
\text { :IIIIIIIIIIIII }\end{array}$ & $\begin{array}{l}0.000 E+00 \\
\text { IIIIIIIIIII }\end{array}$ & $\begin{array}{c}* 1.365 \mathrm{E}+14 \\
\text { IIIIIIIII }\end{array}$ & $\begin{array}{l}0.000 \mathrm{E}+00 \\
\text { İIIIÍIIIII }\end{array}$ & $\begin{array}{l}\text { *1.365E+14 } \\
\text { IIIIIIIIII }\end{array}$ \\
\hline
\end{tabular}

*At specific activity limit 
CVP-2006-00001

Rev. 0

1RESRAD, Version $6.3 \quad T \ll$ Limit $=0.5$ year $\quad 12 / 15 / 2005 \quad 11: 12$ Page 24

Summary : 116-K-2 Trench (West End) Cleanup Verification RESRAD Calculation

File : 116-K-2_Deep_Zone_West.RAD

Individual Nuclide Dose Summed Over All Pathways

Parent Nuclide and Branch Fraction Indicated

oNuclide Parent BRF(i) (j)

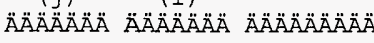

C-14 C-14 1.000E+00

$0 \mathrm{Co}-60$ Co-60 $1.000 \mathrm{E}+00$

0Cs-137 Cs $-137 \quad 1.000 \mathrm{E}+00$

$0 \mathrm{Eu}-152$ Eu-152 7.208E-01

Eu-152 Eu-152 2.792E-01

Eu-152 äDOSE $(j)$

0Gd-152 Eu-152 2.792E-01

$0 \mathrm{Eu}-154 \quad \mathrm{Eu}-154 \quad 1.000 \mathrm{E}+00$

ONi-63 Ni-63 $1.000 \mathrm{E}+00$

OPu-239 Pu-239 $1.000 \mathrm{E}+00$

OU-235 Pu-239 $1.000 \mathrm{E}+00$

OPa-231 $\mathrm{Pu}-239 \quad 1.000 \mathrm{E}+00$

$0 \mathrm{AC}-227$ Pu-239 $1.000 \mathrm{E}+00$

OPu-240 Pu-240 1.000E+00

OU-236 Pu-240 1.000E +00

OTh-232 Pu-240 $1.000 \mathrm{E}+00$

ORa-228 Pu-240 $1.000 \mathrm{E}+00$

OTh-228 Pu-240 1.000E+00

OSr-90 Sr-90 1.000E+00

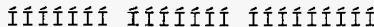

$\operatorname{DOSE}(j, t)$, mrem/yr

$t=0.000 \mathrm{E}+00 \quad 1.000 \mathrm{E}+00 \quad 3.000 \mathrm{E}+00 \quad 1.300 \mathrm{E}+01 \quad 3.000 \mathrm{E}+01 \quad 1.000 \mathrm{E}+02 \quad 3.000 \mathrm{E}+02 \quad 1.000 \mathrm{E}+03$

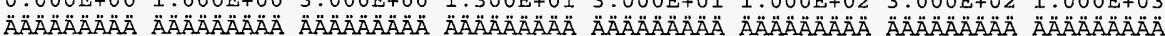
$\begin{array}{llllllllll}0.000 E+00 & 0.000 E+00 & 0.000 E+00 & 0.000 E+00 & 0.000 E+00 & 0.000 E+00 & 0.000 E+00 & 0.000 E+00\end{array}$

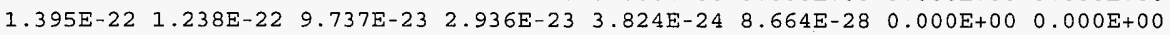
$\begin{array}{llllllll}8.532 E-27 & 8.455 E-27 & 8.305 E-27 & 7.591 E-27 & 6.515 E-27 & 3.472 E-27 & 5.749 E-28 & 0.000 E+00\end{array}$ $\begin{array}{llllllll}1.569 \mathrm{E}-23 & 1.508 \mathrm{E}-23 & 1.394 \mathrm{E}-23 & 9.398 \mathrm{E}-24 & 4.807 \mathrm{E}-24 & 3.042 \mathrm{E}-25 & 1.143 \mathrm{E}-28 & 0.000 \mathrm{E}+00\end{array}$ $\begin{array}{llllllll}6.078 \mathrm{E}-24 & 5.843 \mathrm{E}-24 & 5.400 \mathrm{E}-24 & 3.640 \mathrm{E}-24 & 1.862 \mathrm{E}-24 & 1.178 \mathrm{E}-25 & 0.000 \mathrm{E}+00 & 0.000 \mathrm{E}+00\end{array}$ $\begin{array}{llllllll}2.177 \mathrm{E}-23 & 2.093 \mathrm{E}-23 & 1.934 \mathrm{E}-23 & 1.304 \mathrm{E}-23 & 6.669 \mathrm{E}-24 & 4.220 \mathrm{E}-25 & 1.143 \mathrm{E}-28 & 0.000 \mathrm{E}+00\end{array}$ $\begin{array}{llllllllll}0.000 E+00 & 0.000 E+00 & 0.000 E+00 & 0.000 E+00 & 0.000 E+00 & 0.000 E+00 & 0.000 E+00 & 0.000 E+00\end{array}$ $\begin{array}{lllllllll}8.706 \mathrm{E}-24 & 8.145 \mathrm{E}-24 & 7.131 \mathrm{E}-24 & 3.667 \mathrm{E}-24 & 1.184 \mathrm{E}-24 & 1.126 \mathrm{E}-26 & 0.000 \mathrm{E}+00 & 0.000 \mathrm{E}+00\end{array}$ $\begin{array}{llllllllll}0.000 E+00 & 0.000 E+00 & 0.000 E+00 & 0.000 E+00 & 0.000 E+00 & 0.000 E+00 & 0.000 E+00 & 0.000 E+00\end{array}$ $\begin{array}{lllllllll}0.000 E+00 & 0.000 E+00 & 0.000 E+00 & 0.000 E+00 & 0.000 E+00 & 0.000 E+00 & 0.000 E+00 & 0.000 E+00\end{array}$ $\begin{array}{llllllllll}0.000 \mathrm{E}+00 & 0.000 \mathrm{E}+00 & 0.000 \mathrm{E}+00 & 0.000 \mathrm{E}+00 & 0.000 \mathrm{E}+00 & 3.749 \mathrm{E}-07 & 4.006 \mathrm{E}-05 & 9.281 \mathrm{E}-05\end{array}$ $\begin{array}{llllllll}0.000 \mathrm{E}+00 & 0.000 \mathrm{E}+00 & 0.000 \mathrm{E}+00 & 0.000 \mathrm{E}+00 & 0.000 \mathrm{E}+00 & 1.742 \mathrm{E}-10 & 3.028 \mathrm{E}-07 & 4.626 \mathrm{E}-06\end{array}$ $\begin{array}{llllllll}0.000 E+00 & 0.000 E+00 & 0.000 E+00 & 0.000 E+00 & 0.000 E+00 & 3.605 E-12 & 3.483 E-08 & 7.138 E-07\end{array}$ $\begin{array}{llllllllll}0.000 E+00 & 0.000 E+00 & 0.000 E+00 & 0.000 E+00 & 0.000 E+00 & 0.000 E+00 & 0.000 E+00 & 0.000 E+00\end{array}$ $0.000 \mathrm{E}+00 \quad 0.000 \mathrm{E}+00 \quad 0.000 \mathrm{E}+00 \quad 0.000 \mathrm{E}+00 \quad 0.000 \mathrm{E}+00 \quad 2.687 \mathrm{E}-06 \quad 2.853 \mathrm{E}-04 \quad 6.358 \mathrm{E}-04$ $0.000 \mathrm{E}+00 \quad 0.000 \mathrm{E}+00 \quad 0.000 \mathrm{E}+00 \quad 0.000 \mathrm{E}+00 \quad 0.000 \mathrm{E}+00 \quad 1.359 \mathrm{E}-16 \quad 1.436 \mathrm{E}-13 \quad 2.152 \mathrm{E}-12$ $\begin{array}{lllllllll}0.000 \mathrm{E}+00 & 0.000 \mathrm{E}+00 & 0.000 \mathrm{E}+00 & 0.000 \mathrm{E}+00 & 0.000 \mathrm{E}+00 & 1.175 \mathrm{E}-16 & 2.062 \mathrm{E}-13 & 3.351 \mathrm{E}-12\end{array}$ $\begin{array}{llllllllll}0.000 \mathrm{E}+00 & 0.000 \mathrm{E}+00 & 0.000 \mathrm{E}+00 & 0.000 \mathrm{E}+00 & 0.000 \mathrm{E}+00 & 1.887 \mathrm{E}-17 & 3.766 \mathrm{E}-14 & 6.297 \mathrm{E}-13\end{array}$ $\begin{array}{llllllllll}0.000 E+00 & 0.000 E+00 & 0.000 E+00 & 0.000 E+00 & 0.000 E+00 & 0.000 E+00 & 0.000 E+00 & 0.000 E+00\end{array}$

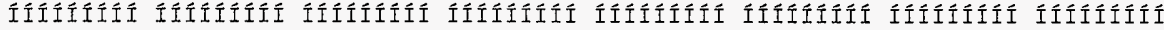
$B R F(i)$ is the branch fraction of the parent nuclide. 


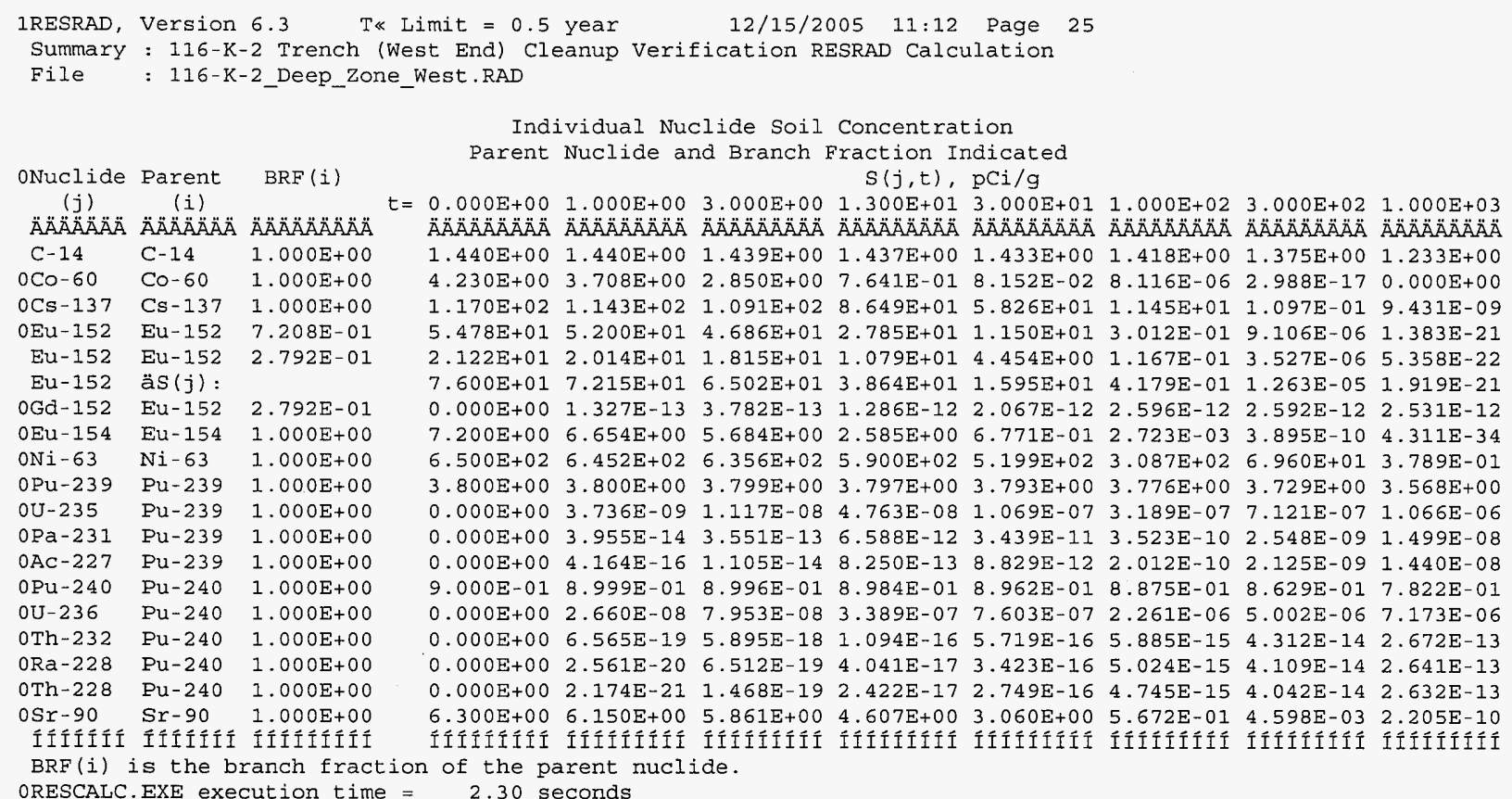

ORESCALC. EXE execution time $=2.30$ seconds 
CVP-2006-00001

Rev. 0

C-102 
CVP-2006-00001

Rev. 0

RESRAD INPUT PARAMETERS FOR THE 116-K-2 OVERBURDEN 
CVP-2006-00001

Rev. 0

C-104 


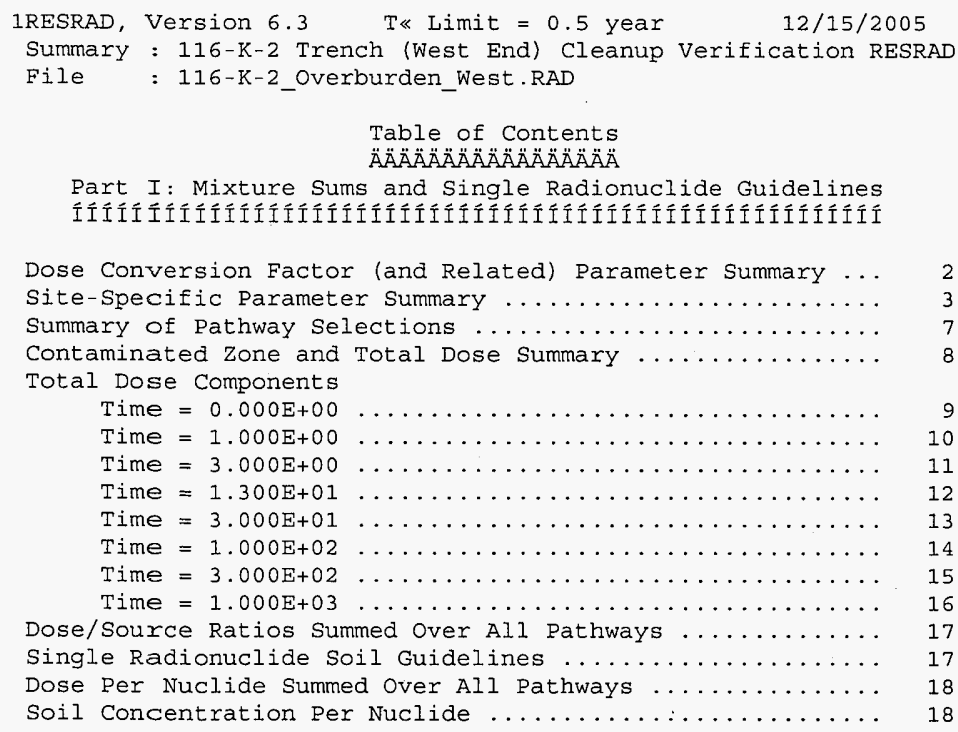




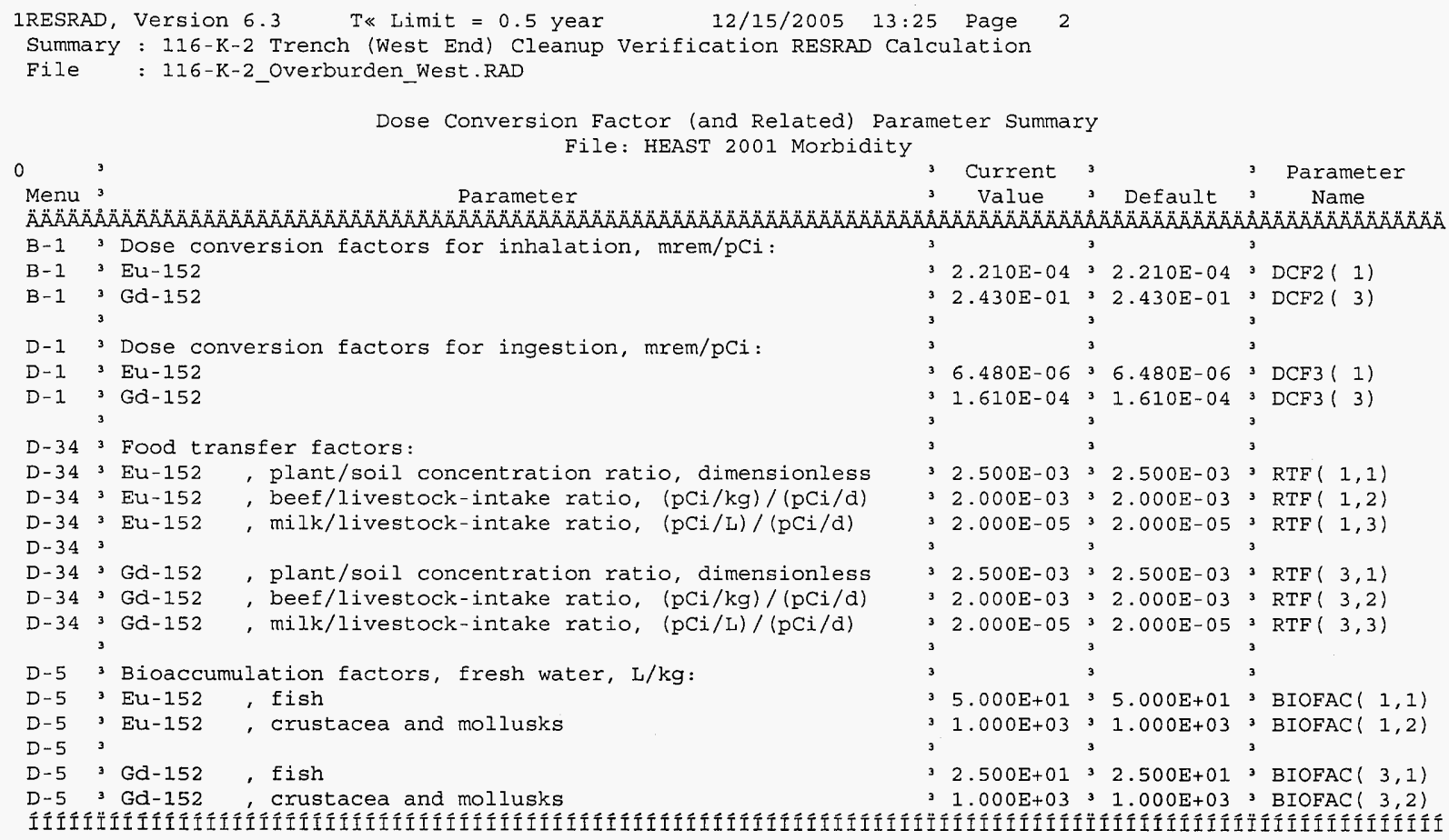


IRESRAD, Version 6.3 T« Limit $=0.5$ year $12 / 15 / 2005 \quad 13: 25$ Page 3

Summary : 116-K-2 Trench (West End) Cleanup Verification RESRAD Calculation

File : 116-K-2_Overburden_West.RAD

Site-Specific Parameter Summary

Menu ${ }^{3}$

Parameter

3 Default

3 Parametex

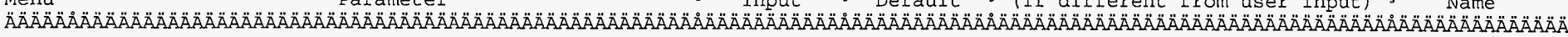

R011 3 Area of contaminated zone ( $m * * 2$ )

$38.676 \mathrm{E}+03 \quad 3 \quad 1.000 \mathrm{E}+04$

R011 3 Thickness of contaminated zone $(\mathrm{m})$

R011 3 Length parallel to aquifer flow $(\mathrm{m})$

R011 3 Basic radiation dose limit (mrem/yr)

R011 3 Time since placement of material ( $y r$ )

$34.600 E+0032.000 E+00$

${ }^{3} 1.220 \mathrm{E}+02^{3} 1.000 \mathrm{E}+02^{3}$

${ }^{3} 1.500 \mathrm{E}+01^{3} 2.500 \mathrm{E}+01^{3}$

$30.000 E+0030.000 E+00$

$31.000 E+00^{3} 1.000 E+00^{3}$

R011 3 Times for calculations (yr)

R011 3 Times for calculations $(y r)$

R011 3 Times for calculations $(y r)$

R011 3 Times for calculations ( $y r$ )

$33.000 E+00^{3} 3.000 E+00^{3}$

$31.300 E+01^{3} 1.000 E+01^{3}$

$33.000 \mathrm{E}+01^{3} 3.000 \mathrm{E}+01^{3}$

$31.000 \mathrm{E}+02 \quad 3 \quad 1.000 \mathrm{E}+02$

$3.000 E+0233.000 E+023$

$31.000 \mathrm{E}+03^{3} 1.000 \mathrm{E}+03^{3}$

3 not used $30.000 E+00^{3}$

RO11 3 Times for calculations (yr)

R011 3 Times for calculations ( $\mathrm{Yr}$ )

3 not used $30.000 \mathrm{E}+00^{3}$

3

3 AREA

3 THICKO

$-1$

R011 3 Times for calculations ( $y r$ )

R012 ${ }^{3}$ Initial principal radionuclide (pCi/g): Eu-152

R012 3 Concentration in groundwater (pCi/L): Eu-152

$31.700 E-0130.000 E+00^{3}$

3 not used $30.000 \mathrm{E}+00^{3}$

R013 3 Cover depth (m)

R013 3 Density of cover material $(\mathrm{g} / \mathrm{cm} * \star 3$ )

R013 3 Cover depth erosion rate (m/yr)

R013 Density of contaminated zone $(\mathrm{g} / \mathrm{cm} * * 3)$

R013 3 Contaminated zone erosion rate $(\mathrm{m} / \mathrm{yr})$

R013 3 Contaminated zone total porosity

R013 3 Contaminated zone field capacity

R013 3 Contaminated zone hydraulic conductivity (m/yr)

R013 3 Contaminated zone b parameter

R013 3 Average annual wind speed ( $\mathrm{m} / \mathrm{sec})$

R013 3 Humidity in $\operatorname{air}(\mathrm{g} / \mathrm{m} * * 3)$

R013 3 Evapotranspiration coefficient

R013 3 Precipitation (m/yr)

R013 3 Irrigation ( $\mathrm{m} / \mathrm{yr}$

R013 3 Irrigation mode

R013 3 Runoff coefficient

$0.000 \mathrm{E}+00^{3} 0.000 \mathrm{E}+00$

3 not used $31.500 E+00^{3}$

3 not used $31.000 \mathrm{E}-03$

$31.600 \mathrm{E}+00^{3} 1.500 \mathrm{E}+00^{3}$

$31.000 \mathrm{E}-03,1.000 \mathrm{E}-03$

$34.000 \mathrm{E}-0134.000 \mathrm{E}-013$

$32.000 E-0132.000 E-013$

$32.500 \mathrm{E}+02^{3} 1.000 \mathrm{E}+01^{3}$

$34.050 E+00 \quad 3 \quad 5.300 E+00$

$33.400 \mathrm{E}+0032.000 \mathrm{E}+00$

3 not used $38.000 E+00^{3}$

3 3.100E-01 3 $5.000 \mathrm{E}-01^{3}$

$31.600 \mathrm{E}-0131.000 \mathrm{E}+00 \quad 3$

$37.600 \mathrm{E}-0132.000 \mathrm{E}-01$

3 overhead 3 overhead 3

$32.000 \mathrm{E}-01,32.000 \mathrm{E}-01,3$

$31.000 E+06 \quad 3 \quad 1.000 E+06$

$31.000 E-03 \quad 3 \quad 1.000 E-03$

3

R013 3 Accuracy for water/soil computation

R014 3 Density of saturated zone $(\mathrm{g} / \mathrm{cm} * * 3)$

R014 3 Saturated zone total porosity

R014 3 Saturated zone effective porosity

R014 ${ }^{3}$ Saturated zone field capacity

R014 3 Saturated zone hydraulic conductivity (m/yr)

R014 3 Saturated zone hydraulic gradient

R014 3 Saturated zone b parameter

Ro14 3 Water table drop rate $(\mathrm{m} / \mathrm{yr}$ )

R014 3 Well pump intake depth (m below water table)

R014 Model: Nondispersion (ND) or Mass-Balance (MB)

R014 3 Well pumping rate $(m * * 3 / \mathrm{yr})$

R015 3 Number of unsaturated zone strata

$1.600 \mathrm{E}+00^{3} 1.500 \mathrm{E}+00$

$34.000 \mathrm{E}-0134.000 \mathrm{E}-01$

$32.500 \mathrm{E}-0132.000 \mathrm{E}-013$

$31.500 \mathrm{E}-01^{3} 2.000 \mathrm{E}-01^{3}$

$35.530 \mathrm{E}+03 \quad 3 \quad 1.000 \mathrm{E}+02$

$31.250 E-03 \quad 3 \quad 2.000 E-02 \quad 3$

$34.050 \mathrm{E}+00^{3} 5.300 \mathrm{E}+00^{3}$

$3^{3} 1.000 \mathrm{E}-03^{3} 1.000 \mathrm{E}-03^{3}$

${ }^{3} 4.600 \mathrm{E}+00^{3} 1.000 \mathrm{E}+01^{3}$

3 ND

$3 \mathrm{ND}$

3 $2.500 \mathrm{E}+02^{3} 2.500 \mathrm{E}+02^{3}$

3

31

3 $\begin{array}{lll}-.- & 3 & \text { LCZPAQ } \\ -.- & 3 & \text { BRDL }\end{array}$

$--$

-..

... 3 T 3 ( 3$)$

$3 \mathrm{~T}(4)$

$3 \mathrm{~T}(5)$

-. 3 T $(6)$

-. 3 T $(7)$

$3 \mathrm{~T}(8)$

-. $3 \mathrm{~T}(9)$

${ }_{3} \mathrm{~T}(10)$

$---$

-.. 3 W1 $(1)$

3 COVERO

3 DENSCV

-. 3 VCV

3 DENSCZ

3 FCCZ

- - $3 \mathrm{HCCZ}$

$3 \mathrm{BCZ}$

3 HUMID

- - 3 EVAPTR

- - 3 RI

-- 3 IDITCF

3 RUNOFF

-. 3 WAREA

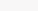

$---$

$-\cdots$

$-\cdots$

$---$

$---$

$---$

-.-

-..

-..

-..

$\cdots$

3 EPS

3 DENSAQ

3 TPSZ

3 EPSZ

3 FCSZ

3 HCSZ

3 HGWT

$3 \mathrm{BSZ}$

3 VWT

3 DWIBWT

3 MODEL

3 UW

3 NS 
1RESRAD, Version $6.3 \quad$ T L Limit $=0.5$ year $12 / 15 / 2005 \quad 13: 25$ Page 4

Summary : 116-K-2 Trench (West End) Cleanup Verification RESRAD Calculation

File : 116-K-2_Overburden_West.RAD

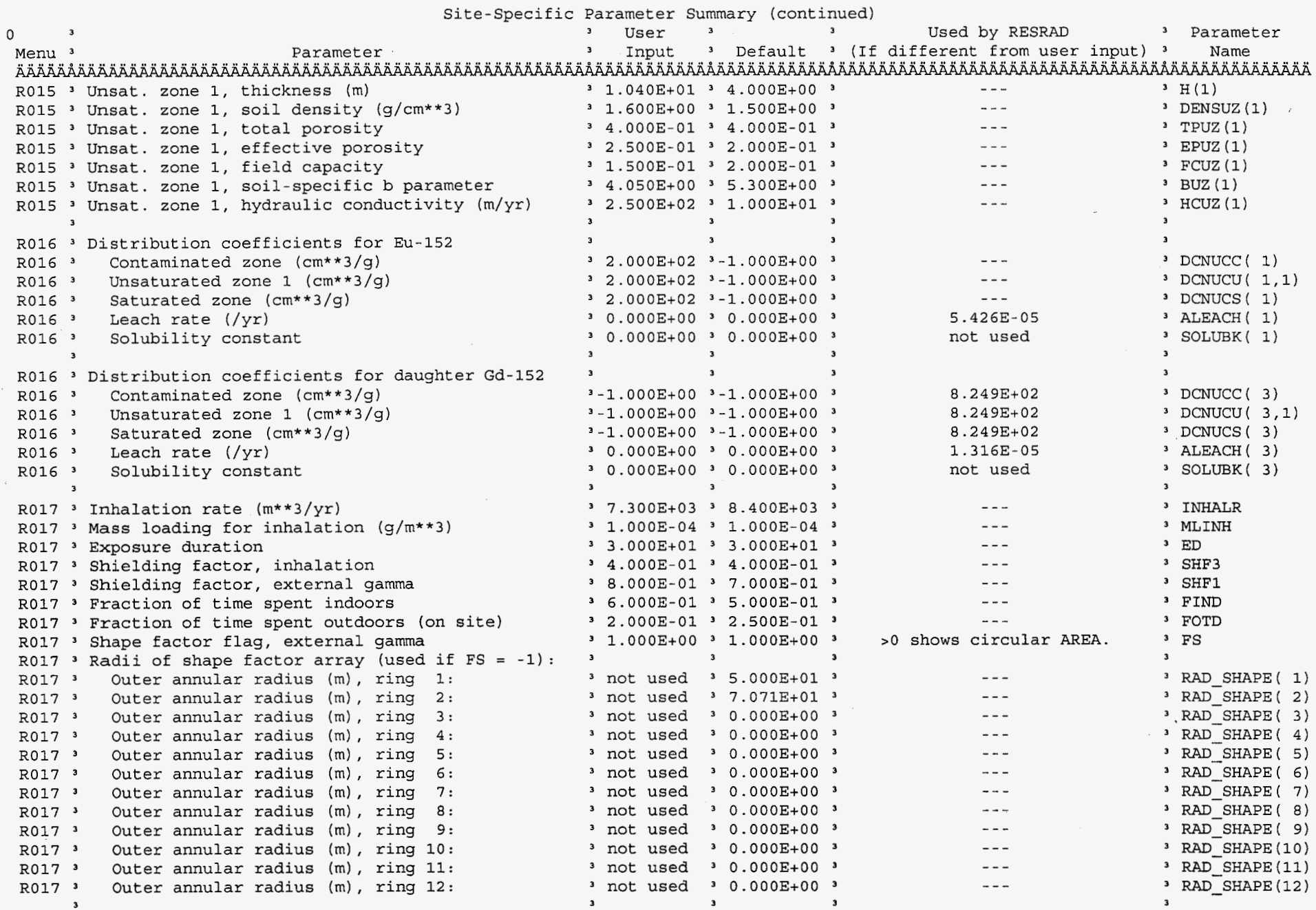


IRESRAD, Version $6.3 \quad$ T« Limit $=0.5$ year $12 / 15 / 2005 \quad 13: 25$ Page

Summary : 116-k-2 Trench (West End) Cleanup Verification RESRAD Calculation

File : 116-K-2_Overburden_West.RAD

$0 \quad{ }_{3} \quad$ Site-Specific Parameter Summary (continued)

Parameter

3 User

3 Default

Used by RESRAD

3 Parameter

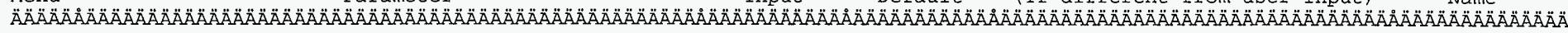

R017 ${ }^{3}$ Fractions of annular areas within AREA:

R017 3 Ring 1

R017 3 Ring 2

R017 3 Ring 3

R017, Ring 4

R017 3 Ring 5

R017 3 Ring 6

R017 3 Ring 7

$\mathrm{R}_{017^{3}}$ Ring 8

$\mathrm{R}^{3} 17^{3}$ Ring 9

$\mathrm{R}^{3} \mathrm{~B}^{3}$ Ring 10

$\mathrm{R}_{017} \mathbf{3}^{3}$ Ring 11

${ }^{3}$ not used ${ }^{3} 1.000 \mathrm{E}+00^{3}$

3 not used $32.732 \mathrm{E}-013$

${ }^{3}$ not used $30.000 \mathrm{E}+00^{3}$

3 not used $30.000 \mathrm{E}+00^{3}$

3 not used $30.000 \mathrm{E}+00^{3}$

3 not used $30.000 \mathrm{E}+00^{3}$

3 not used $30.000 \mathrm{E}+00^{3}$

3 not used $30.000 \mathrm{E}+00^{3}$

3 not used $30.000 \mathrm{E}+00^{3}$

3 not used ${ }^{3} 0.000 \mathrm{E}+00^{3}$

3 not used $30.000 E+00^{3}$

3 not used $30.000 \mathrm{E}+00^{3}$

R018 ${ }^{3}$ Fruits, vegetables and grain consumption (kg/yr) ${ }^{3} 1.100 \mathrm{E}+02^{3} 1.600 \mathrm{E}+02$

R018 3 Leafy vegetable consumption ( $\mathrm{kg} / \mathrm{yr}$ )

R018 3 Milk consumption ( $\mathrm{L} / \mathrm{Yr}$ )

R018 3 Meat and poultry consumption $(\mathrm{kg} / \mathrm{Yr})$

R018 3 Fish consumption (kg/yr)

R018 3 Other seafood consumption $(\mathrm{kg} / \mathrm{Yr})$

R018 3 Soil ingestion rate $(\mathrm{g} / \mathrm{yr})$

R018 3 Drinking water intake (L/yr)

R018 3 Contamination fraction of drinking water

R018 3 Contamination fraction of household water

R018 ${ }^{3}$ Contamination fraction of livestock water

R018 3 Contamination fraction of irrigation water

R018 3 Contamination fraction of aquatic food

R018 Contamination fraction of plant food

R018 ${ }^{3}$ Contamination Eraction of meat

R018 3 Contamination fraction of milk

R019 3 Livestock fodder intake for meat ( $\mathrm{kg} /$ day)

R019 3 Livestock fodder intake for milk ( $\mathrm{kg} /$ day)

R019 3 Livestock water intake for meat ( $\mathrm{L} /$ day)

R019 3 Livestock water intake for milk (L/day)

R019 3 Livestock soil intake ( $\mathrm{kg} /$ day)

R019 Mass loading for foliar deposition $(\mathrm{g} / \mathrm{m} * * 3)$

R019 3 Depth of soil mixing layer (m)

R019 3 Depth of roots (m)

R019 3 Drinking water fraction from ground water

R019 Household water fraction from ground water

R019 3 Iivestock water fraction from ground water

R019 3 Irrigation fraction from ground water

R19B 3 Wet weight crop yield for Non-Leafy $(\mathrm{kg} / \mathrm{m} * * 2)$

R19B 3 Wet weight crop yield for Leafy

R19B ${ }^{3}$ Wet weight crop yield for Fodder

$(\mathrm{kg} / \mathrm{m} * * 2)$

R19B 3 Growing Season for Non-Leafy (years)

$\mathrm{R} 19 \mathrm{~B}{ }^{3}$ Growing Season for Leafy (years)

$\mathrm{R} 19 \mathrm{~B} \cdot$ Growing Season for Fodder (years)
$32.700 \mathrm{E}+00^{3} \quad 1.400 \mathrm{E}+013$

$31.000 \mathrm{E}+02 \quad 3 \quad 9.200 \mathrm{E}+013$

$33.600 E+0136.300 E+01$

$31.970 \mathrm{E}+0135.400 \mathrm{E}+00^{3}$

$39.000 E-0139.000 E-013$

$\begin{array}{llll}3 & 7.300 \mathrm{E}+01 & 3 & 3.650 \mathrm{E}+01\end{array}$

$31.000 \mathrm{E}+00^{3} 1.000 \mathrm{E}+00^{3}$

3 not used $31.000 E+003$

$31.000 E+00 \quad 3 \quad 1.000 E+00$

$31.000 \mathrm{E}+00^{3} 1.000 \mathrm{E}+00^{3}$

$35.000 \mathrm{E}-01^{3} 5.000 \mathrm{E}-01^{3}$

$3-1$

$3-1 \quad 3-1$

$3-1$

$3-1$

$36.800 \mathrm{E}+01$

$5.500 \mathrm{E}+0135.500 \mathrm{E}+01$

$31.600 \mathrm{E}+02,1.600 \mathrm{E}+02$

$35.000 \mathrm{E}-0135.000 \mathrm{E}-013$

${ }^{3} 1.000 \mathrm{E}-04^{3} 1.000 \mathrm{E}-04^{3}$

$31.500 \mathrm{E}-0131.500 \mathrm{E}-01$

$39.000 E-0139.000 E-013$

$31.000 E+00^{3} 1.000 E+00^{3}$

3 not used $31.000 \mathrm{E}+0 \mathrm{O}^{3}$

${ }^{3} 1.000 E+00^{3} 1.000 E+00^{3}$

$31.000 E+00^{3} 1.000 E+00^{3}$

$37.000 \mathrm{E}-01^{3} 7.000 \mathrm{E}-01$

$31.500 \mathrm{E}+00^{3} 1.500 \mathrm{E}+00$

$31.100 \mathrm{E}+00^{3} 1.100 \mathrm{E}+00^{3}$

$31.700 \mathrm{E}-0131.700 \mathrm{E}-01$

$3^{3} 2.500 \mathrm{E}-01,2.500 \mathrm{E}-013$
$37.300 \mathrm{E}+02^{3} 5.100 \mathrm{E}+02^{3}$

$35.000 \mathrm{E}+01,5.000 \mathrm{E}+01$

3 8.000E-02 3 8.000E-02 3
FRACA ( 1)

3 FRACA ( 2)

3 FRACA ( 3)

3 FRACA ( 4)

3 FRACA ( 5)

3 FRACA ( 6)

3 FRACA ( 7)

3 FRACA ( 8)

3 FRACA ( 9$)$

3 FRACA (10)

3 FRACA (11)

3 FRACA (12)

DIET (1)

DIET (2)

3 DIET (3)

3 DIET (4)

3 DIET (5)

3 DIET (6)

3 SOIL

3 DWI

3 FDW

3 FHHW

3 FLW

3 FIRW

3 FR9

3 FPLANT

3 FMEAT

3 FMILK

3 LFI5

3 LFI6

3 LWI5

3 LWI6

3 LSI

3 MLFD

3 DM

3 DROOT

3 FGWDW

3 FGWHH

3 FGWLW

3 FGWIR

$3 \mathrm{YV}(1)$

3 YV (2)

$3 \mathrm{YV}(3)$

$T E(1)$

3 TE (2)

$3 \mathrm{TE}(3)$ 


$$
\text { File : 116-K-2_Overburden_West.RAD }
$$

0

Menu ${ }^{3}$

Site-Specific Parameter Summary (continued)

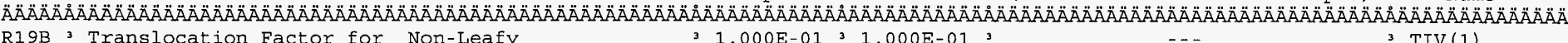

RI9B 3 Tranlor for Non-Leafy $31.000 E-0131.000 E-01$

R19B 3 Translocation Factor for Eodder

$31.000 E+0031.000 E+00$

$31.000 \mathrm{E}+00^{3} 1.000 \mathrm{E}+00$

R19B 3 Dry Foliar Interception Fraction for Non-Leafy ${ }^{3} 2.500 \mathrm{E}-01 \mathbf{3}^{3} 2.500 \mathrm{E}-013$

R19B ${ }^{3}$ Dry Foliar Interception Fraction for Leafy $32.500 E-011^{3} 2.500 \mathrm{E}-01$

R19B 3 Dry Foliar Interception Fraction for Fodder $\quad 3 \quad 2.500 E-0132.500 E-013$

R19B ${ }^{3}$ Wet Foliar Interception Fraction for Non-Leafy ${ }^{3} 2.500 E-013^{3} 2.500 E_{-01}{ }^{3}$

R19B 3 Wet Foliar Interception Fraction for Leafy $32.500 E-013^{3} 2.500 E-013^{3}$

R19B ${ }^{3}$ Wet Foliar Interception Fraction for Fodder ${ }^{3} \quad 2.500 E-013^{3} 2.500 E-013^{3}$

R19B 3 Weathering Removal Constant for vegetation

$3.000 E+0132.000 E+01,3$

C14 $3 \mathrm{C}-12$ concentration in water $(\mathrm{g} / \mathrm{cm} * * 3)$

$3_{3}^{3} 2.000 E+01{ }_{3}^{3} 2.000 E+01$

3 not used $32.000 E-05$

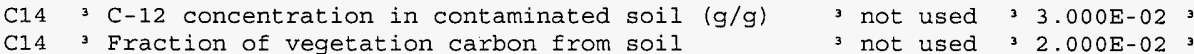

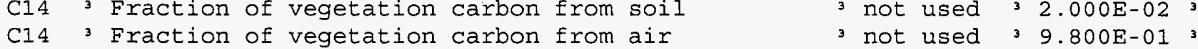

C14 $3 \mathrm{C}-14$ evasion layer thickness in soil (m)

C14 $3 \mathrm{C}-14$ evasion flux rate from soil (1/sec)

C14 3 C-12 evasion flux rate from soil ( $1 / \mathrm{sec})$

C14 3 Fraction of grain in beef cattle feed

C14 3 Fraction of grain in milk cow feed

C14 3 DCF correction factor for gaseous forms of C14

${ }_{3}^{3} \mathrm{DCH}$

STOR ${ }^{3}$ storage times of contaminated foodstuffs (days) :

STOR ${ }^{3}$ Fruits, non-leafy vegetables, and grain

STOR ${ }^{3}$ Leafy vegetables

3 not used $39.800 \mathrm{E}-01$

3 not used 3 7.000E-07 3

3 not used $31.000 \mathrm{E}-10^{3}$

3 not used 3 8.000E-01

3 not used $32.000 E-01$

not used $38.894 \mathrm{E}+01$ 3

$\mathrm{STOR}^{3} \quad$ Milk $^{3}{ }^{3}$ Meat and poult

STOR 3 Fish

STOR $^{3}$ Crustacea and mollusks

$S_{T O R}{ }^{3}$ Well water

STOR 3 Surface water

STOR ${ }^{3}$ Livestock fodder

R021 3 Thickness of building foundation (m)

R021 3 Bulk density of building foundation $(\mathrm{g} / \mathrm{cm} * * 3)$

R021 Total porosity of the cover material

R021 Total porosity of the building foundation

R021 3 Volumetric water content of the cover material

R021 3 Volumetric water content of the foundation

R021 3 Diffusion coefficient for radon gas $(\mathrm{m} / \mathrm{sec})$.

$\mathrm{R}_{0} 1^{3}$ in cover material

$\mathrm{RO}_{2}{ }^{3}$ in foundation material

R021 3 in contaminated zone soil

R021 3 Radon vertical dimension of mixing (m)

R021 3 Average building air exchange rate (1/hr)

R021 3 Height of the building (room) (m)

R021 3 Building interior area factor

R021 3 Building depth below ground surface (m)

R021 3 Emanating power of Rn-222 gas

R021 Emanating power of Rn-220 gas

$1.400 \mathrm{E}+0131^{3} 1.400 \mathrm{E}+01$

$31.000 \mathrm{E}+0031.000 \mathrm{E}+00$

$31.000 \mathrm{E}+00^{3} 1.000 \mathrm{E}+00^{3}$

$32.000 E+0132.000 E+01$

$37.000 \mathrm{E}+00^{3} 7.000 \mathrm{E}+00^{3}$

$37.000 \mathrm{E}+00^{3} \quad 7.000 \mathrm{E}+00^{3}$

$31.000 \mathrm{E}+00^{3} 1.000 \mathrm{E}+00^{3}$

$31.000 \mathrm{E}+00^{3} 1.000 \mathrm{E}+00$

$34.500 \mathrm{E}+0134.500 \mathrm{E}+01$

3 not used 3 1.500E-01

3 not used $32.400 E+00$

3 not used $34.000 \mathrm{E}-013$

3 not used $31.000 \mathrm{E}-013$

${ }^{3}$ not used ${ }^{3} 5.000 \mathrm{E}-02$,

3 not used 33.000 E-02 ${ }^{3}$

3 not used 32.000 E-06

3 not used $3.000 \mathrm{E}-07^{3}$

3 not used $32.000 E^{3} 06^{3}$

3 not used $32.000 E+00^{3}$

3 not used $35.000 \mathrm{E}-0 \mathrm{I}^{3}$

3 not used $32.500 \mathrm{E}+00$

3 not used $30.000 E+00^{3}$

3 not used $3-1.000 E+00^{3}$

3 not used ${ }_{3} 2.500 \mathrm{E}^{-01} 3$

3 not used ${ }_{3} 1.500 E_{-01}{ }^{3}$

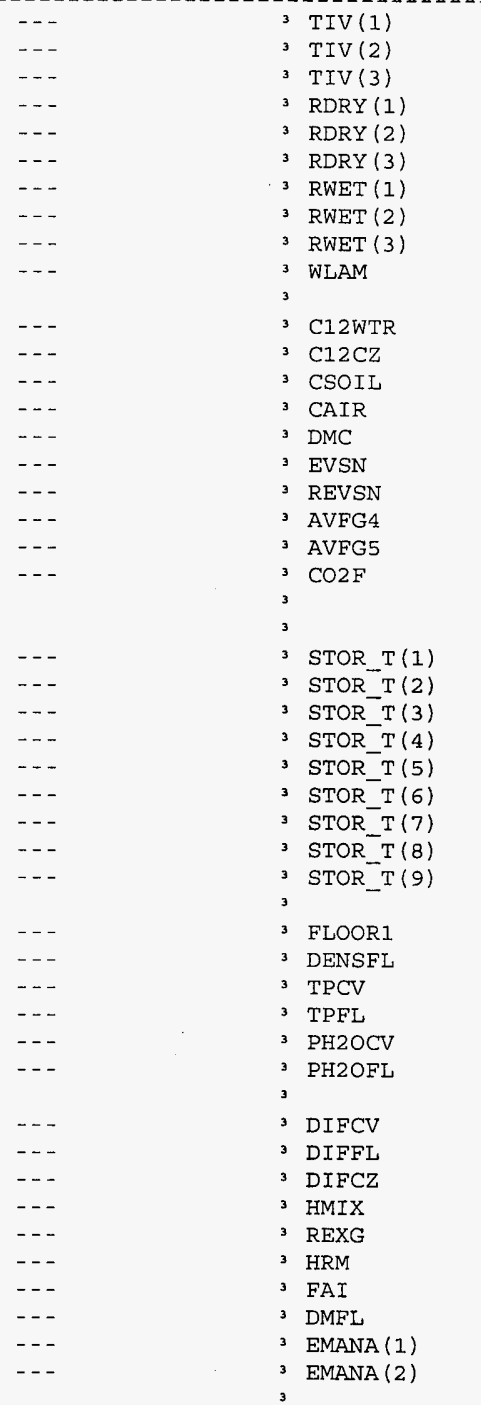


CVP-2006-00001

Rev. 0

IRESRAD, Version $6.3 \quad T \ll$ Limit $=0.5$ year $\quad 12 / 15 / 2005 \quad 13: 25$ Page 7

Summary : 116-K-2 Trench (West End) Cleanup Verification RESRAD Calculation

File

: 116-K-2_Overburden_West.RAD

Site-Specific Parameter Summary (continued)

Menu 3

Parameter

3 User

Default

3 Used by RESRAD

Used by RESRAD 3 Parameter

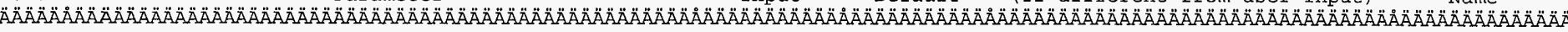

TITL 3 Number of graphical time points

TITL ${ }^{3}$ Maximum number of integration points for dose

3 Max

1730

$---$

$-\cdot-$

3 NPTS

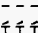

3

3 LYMAX

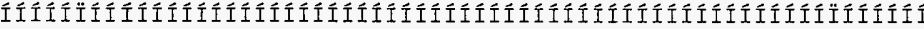

Summary of Pathway Selections

Pathway User Selection

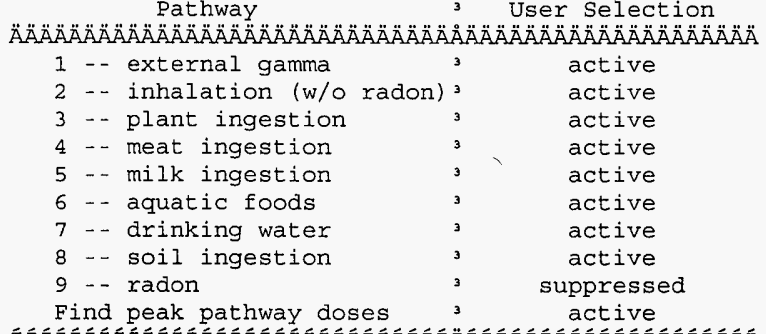

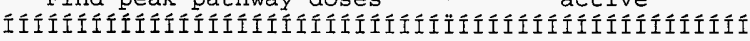


CVP-2006-00001

Rev. 0

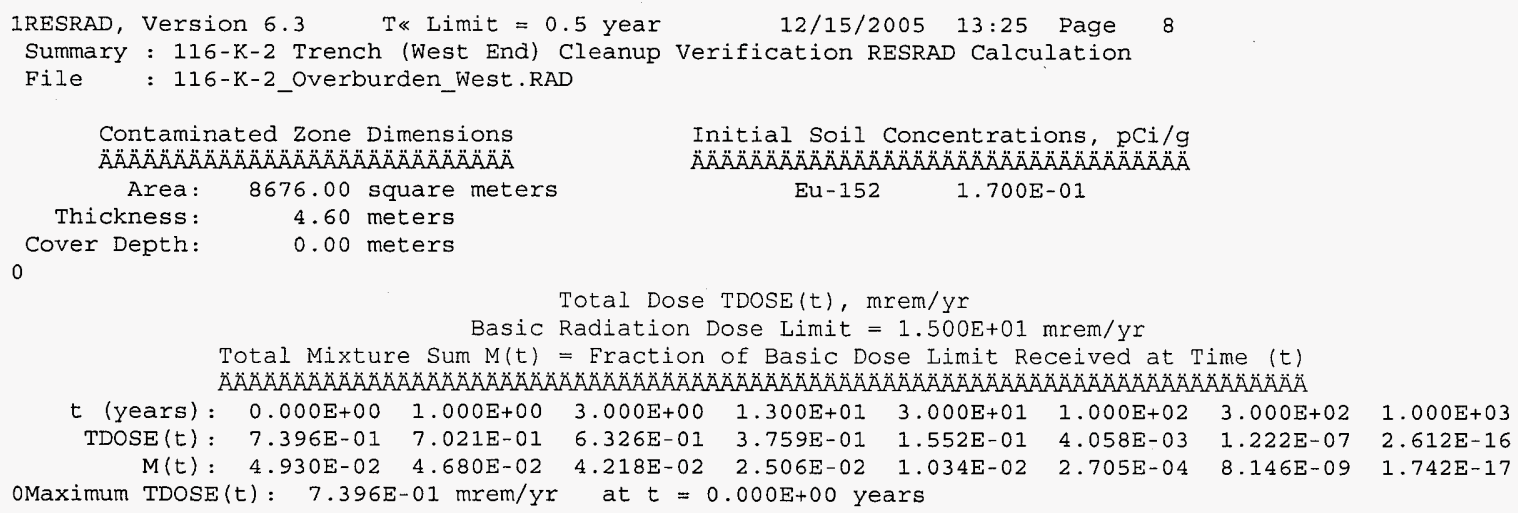




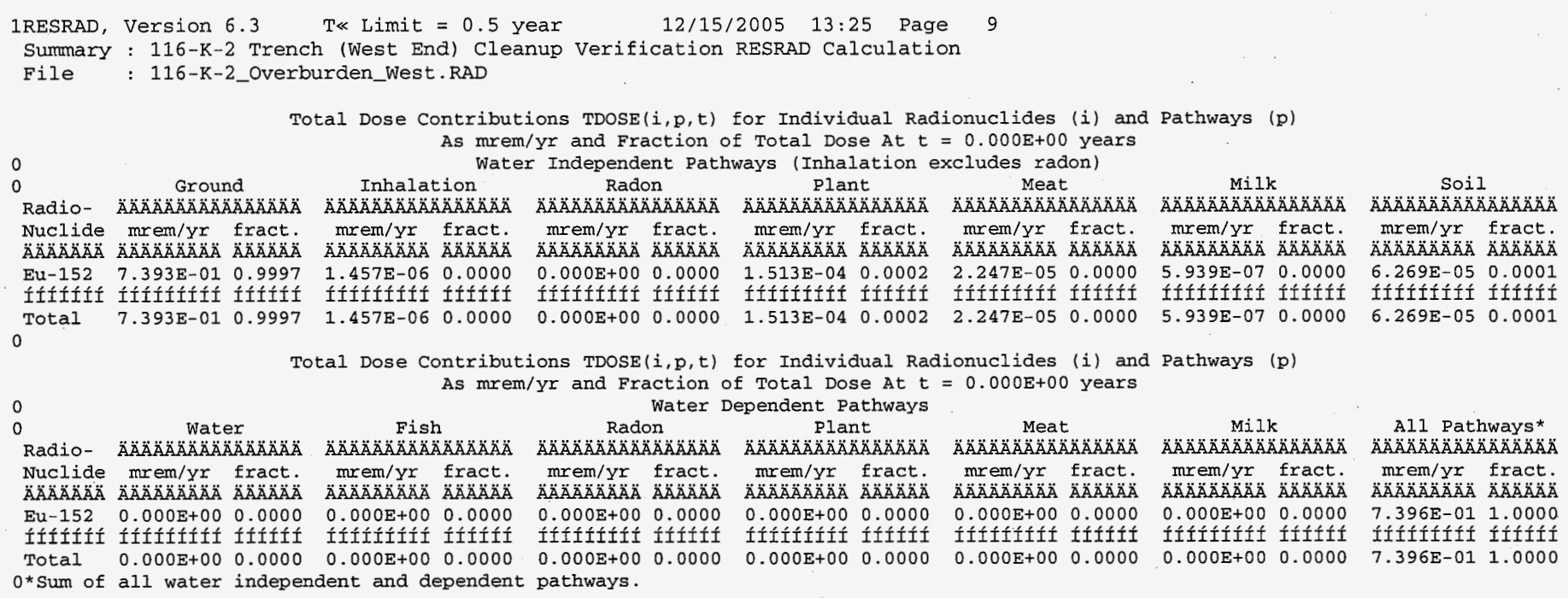


Rev. 0

1RESRAD, Version $6.3 \quad$ T\& Limit $=0.5$ year $12 / 15 / 2005 \quad 13: 25$ Page 10

Summary : 116-K-2 Trench (West End) Cleanup Verification RESRAD Calculation

File : 116-K-2_Overburden_West.RAD

Total Dose Contributions TDOSE $(i, p, t)$ for Individual Radionuclides (i) and Pathways (p) As mrem/yr and Fraction of Total Dose At $t=1.000 \mathrm{E}+00$ years

Fraction of Total Dose At t =

0

Inhalation Radon .... Plant . . . Meat Radio- $\ddot{A} \ddot{A} \ddot{A} \ddot{A} \ddot{A} \ddot{A} \ddot{A} \ddot{A} \ddot{A} \ddot{A} \ddot{A} \ddot{A} \ddot{A} \ddot{A} \ddot{A}$

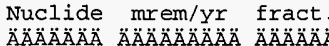
$\ddot{A} \ddot{A} \ddot{A} \ddot{A} \ddot{A} \ddot{A} \ddot{A} \ddot{A} \ddot{A} \ddot{A} \ddot{A} \ddot{A} \ddot{A} \ddot{A} \ddot{A} \ddot{A} \ddot{A} \ddot{A} \ddot{A} \ddot{A}$

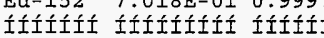
$\begin{array}{lll}\text { Total } & 7.018 \mathrm{E}-01 & 0.9997\end{array}$

0

Total Dose Contributions TDOSE $(i, p, t)$ for Individual Radionuclides (i) and Pathways (p) As mrem/yr and Fraction of Total Dose At $t=1.000 \mathrm{E}+00$ years

0

0 Water Fish Radon Plant

0 Water Fish Radon Plant

0 Water Fish Radon Water Dependent Pathwa Plant Water Dependent Pathways

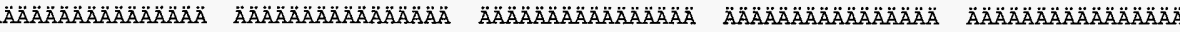

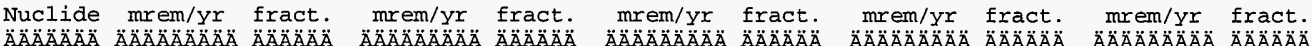

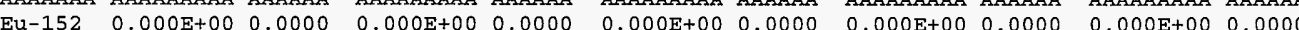

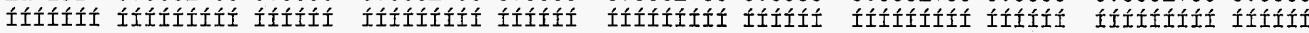

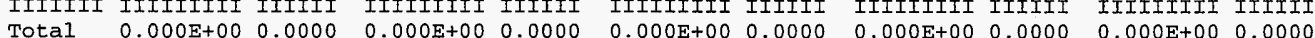

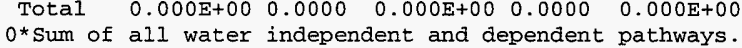

Milk $\ddot{A} \ddot{A} \ddot{A} \ddot{A} \ddot{A} \ddot{A} \ddot{A} \ddot{A} \ddot{A} \ddot{A} \ddot{A} \ddot{A} \ddot{A} \ddot{A}$ mrem/yr fract

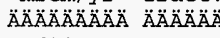
$5.638 \mathrm{E}-07 \quad 0.0000$ íííítítí ífítít $5.638 \mathrm{E}-070.0000$ 
1RESRAD, Version $6.3 \quad$ T« Limit $=0.5$ year $\quad 12 / 15 / 2005 \quad 13: 25$ Page 11
Summary : $116-\mathrm{K}-2$ Trench (West End) Cleanup Verification RESRAD Calculation

File : 116-K-2_Overburden_West.RAD

Total Dose Contributions TDOSE(i,p,t) for Individual Radionuclides (i) and Pathways (p)

As mrem/yr and Fraction of Total Dose At $t=3.000 \mathrm{E}+00$ years

0

Ground Inhalation Radon
Plant

Meat

Milk

Soil

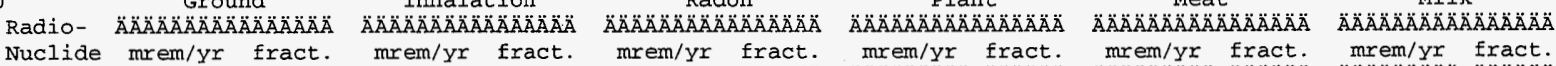
Nuclide mrem/yr fract. mrem/yr fract.

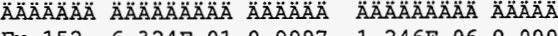

Eu-152 6.324E-01 0.9997

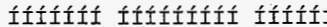

$1.246 \mathrm{E}-06 \quad 0.0000$

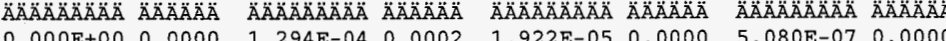

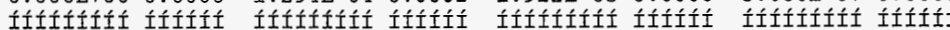

Total $6.324 \mathrm{E}-01 \quad 0.9997$

1.246E-06 0.0000

$0.000 \mathrm{E}+000.0000$

Total Dose Contributions TDOSE $(i, p, t)$ for Individual Radionuclides (i) and Pathways (p) As mrem/yr and Fraction of Total Dose At $t=3.000 \mathrm{E}+00$ years Water Dependent Pathways
Radon

Water

Meat

Milk

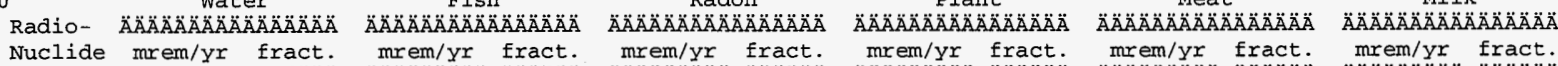

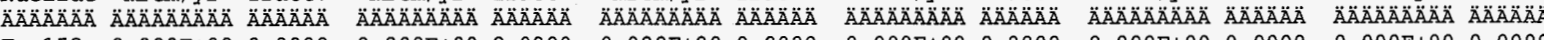
$\begin{array}{lllllllllllll}E & \text { Eu-152 } & 0.000 E+00 & 0.0000 & 0.000 E+00 & 0.0000 & 0.000 E+00 & 0.0000 & 0.000 E+00 & 0.0000\end{array}$

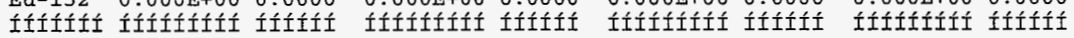
$\begin{array}{llllllllll}\text { Total } & 0.000 \mathrm{E}+00 & 0.0000 & 0.000 \mathrm{E}+00 & 0.0000 & 0.000 \mathrm{E}+00 & 0.0000\end{array}$ $0.000 \mathrm{E}+00 \quad 0.0000$ $0.000 E+00 \quad 0.0000$

$0.0000+00$ 0.0000 $0.0008+00$ 0.0000

ítítítít ítítít 0 * Sum of all water independent and dependent pathways 


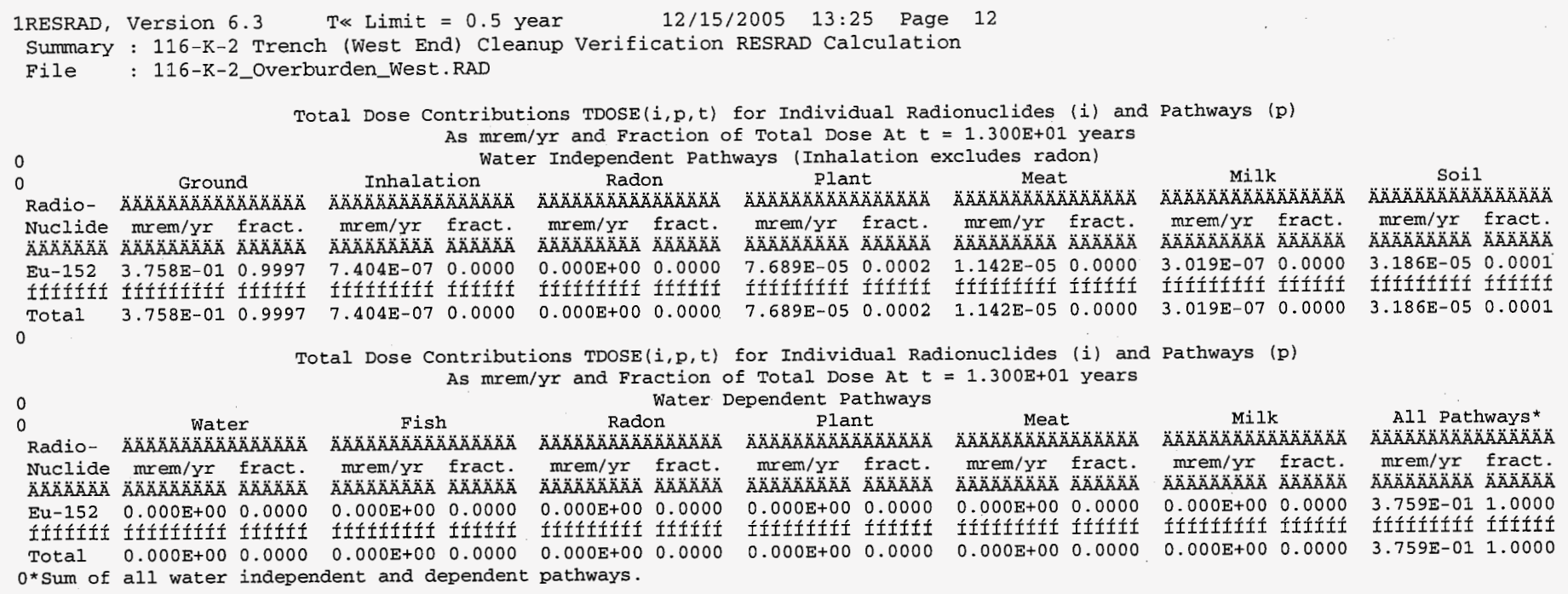


Rev. 0

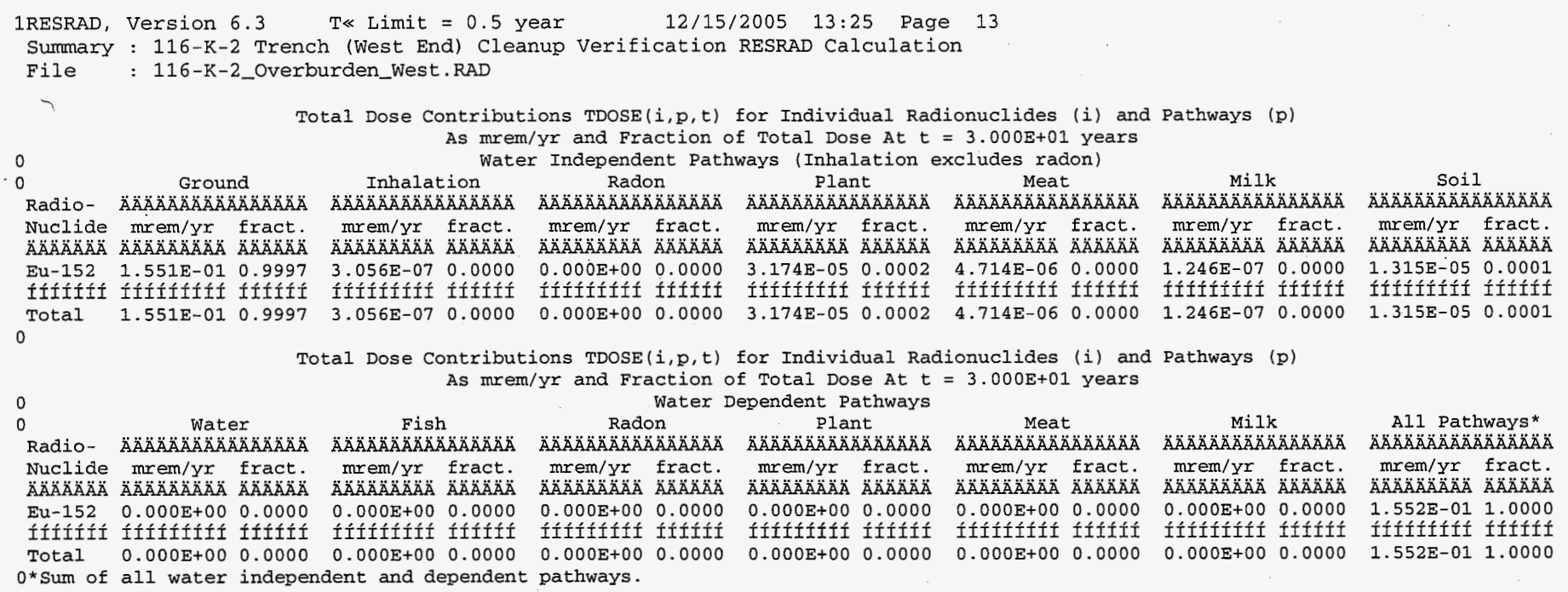


Rev. 0

1RESRAD, Version $6.3 \quad$ T« Limit $=0.5$ year $12 / 15 / 200513: 25$ Page 14
Summary : 116-K-2 Trench (West End) Cleanup Verification RESRAD Calculation

File : 116-K-2_Overburden_West.RAD

Total Dose Contributions TDOSE $(i, p, t)$ for Individual Radionuclides ( $i$ ) and Pathways (p)

As mrem/yr and Fraction of Total Dose At $t=1.000 \mathrm{E}+02$ years

rent Pathways (Inhalation excludes radon)

0

Inhalation

Radon Plant

Meat

Milk

Soi1

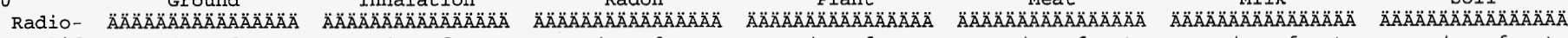
Nuclide mrem/yr fract. mrem/yr fract. mrem/yr fract. mrem/yr fract. mrem/yr fract. mrem/yr fract. mrem/yr fract.

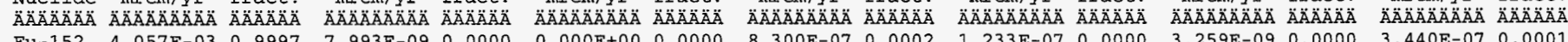

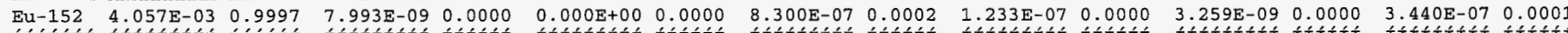

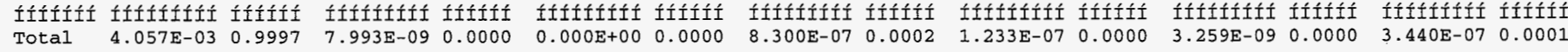

Total Dose Contributions TDOSE $(i, p, t)$ for Individual Radionuclides (i) and Pathways (p) As mrem/yr and Fraction of Total Dose At $t=1.000 \mathrm{E}+02$ years

0

Water Fish Radon Water Dependent Pathways

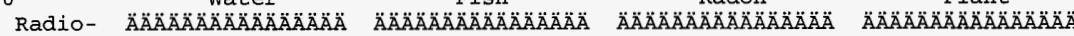
Nuclide mrem $/ y r$ fract. mrem/yr fract. mrem/yr fract. mrem/yr fract.

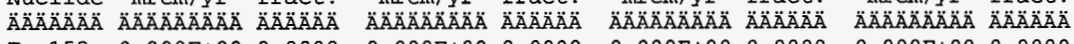
$\begin{array}{llllllllllll}\text { Eu-152 } & 0.000 E+00 & 0.0000 & 0.000 E+00 & 0.0000 & 0.000 E+00 & 0.0000 & 0.000 E+00 & 0.0000\end{array}$

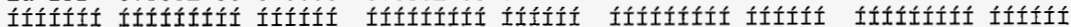
Iotal $0.000 \mathrm{E}+000.00000 .000 \mathrm{E}+000.00000 .000 \mathrm{E}+000.00000 .000 \mathrm{E}+000.0000$

Meat $\mathrm{A} \ddot{A} \ddot{A} \ddot{A} \ddot{A} \ddot{A} \ddot{A} \ddot{A} \ddot{A} \ddot{A} \ddot{A} \ddot{A} \ddot{A} \ddot{A}$
mrem/yr fract mrem/yr fract. Ä 0.000E+00 0.0000 $0.000 \mathrm{E}+00 \quad 0.0000$
Milk Ä mrem/yr fract.

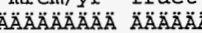
$0.000 \mathrm{E}+00 \quad 0.0000$

Ííútítíí íííti

$0.000 \mathrm{E}+000.0000$ $0^{*}$ Sum of all water independent and dependent pathways. 


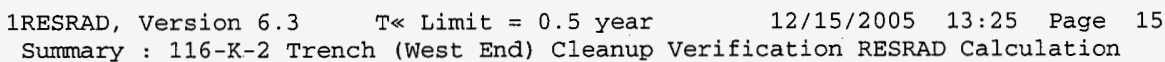

Total Dose Contributions TDOSE( $i, p, t)$ for Individual Radionuclides ( $i$ ) and Pathways (p) As mrem/yr and Fraction of Total Dose At $t=3.000 \mathrm{E}+02$ years Water Independent Pathways (Inhalation excludes radon) Soi1
Meat

Plant

Milk

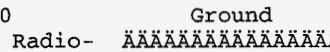

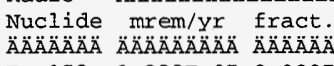
$\begin{array}{lll}\mathrm{Eu}-152 & 1.222 \mathrm{E}-07 & 0.9997\end{array}$ Iotal $1.222 \mathrm{E}-070.9997$ Radon

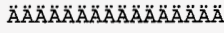

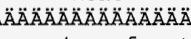
mrem/yr fract.

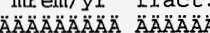
AAAAAAAAAAAAAA $m r e m / y r$ fract. ARAAAAAAA AAAAAA AAAAAAAAA AAAAAA $0.000 \mathrm{E}+00 \quad 0.0000$

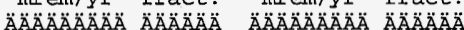
$\begin{array}{llll}2.499 \mathrm{E}-11 & 0.0002 & 3.713 \mathrm{E}-12 & 0.000\end{array}$ $.407 \mathrm{E}-13 \quad 0.0000$

1fititition

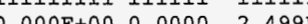
$\begin{array}{ll}.713 E-12 & 0.0000\end{array}$

$3.713 \mathrm{E}-12 \quad 0.0000$ AAAAAAAAAAAAAAA
mrem/yr fract $\mathrm{mrem} / \mathrm{yr}$ fract.

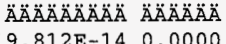
$9.812 \mathrm{E}-140.0000$

Itilití IIIII

Total Dose Contributions TDOSE $(i, p, t)$ for Individual Radionuclides (i) and Pathways (p) As mrem/yr and Fraction of Total Dose At $t=3.000 \mathrm{E}+02$ years

0

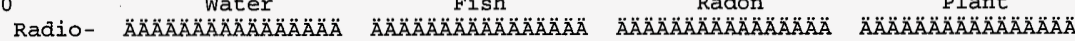
Nuclide mrem/yr fract. mrem/yr fract. mrem/yr fract. mrem/yr fract.

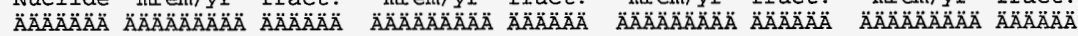
$\begin{array}{llllllllll}\mathrm{Eu}-152 & 0.000 E+00 & 0.0000 & 0.000 \mathrm{E}+00 & 0.0000 & 0.000 \mathrm{E}+00 & 0.0000 & 0.000 \mathrm{E}+00 & 0.0000\end{array}$

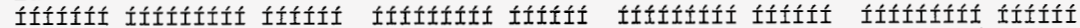
$0.0000+000.0000$ ifitititit titititi $0.000+000000$ $0 *$ Sum of all water independent and dependent pathways. $\mathrm{Mi} 1 \mathrm{k}$ $\begin{array}{ccc}m r e m / Y r & \text { fract. } \\ \ddot{A} \ddot{A} \ddot{A} \ddot{A} \ddot{A} \ddot{A} \ddot{A} \ddot{A} \ddot{A} \ddot{A} \ddot{A} \ddot{A} \ddot{A} \ddot{A}\end{array}$ $\begin{array}{ccc}\ddot{A} \ddot{A} \ddot{A} \ddot{A} \ddot{A} \ddot{A} \ddot{A} \ddot{A} \ddot{A} \ddot{A} \ddot{A} \ddot{A} \\ 0.000 \mathrm{E}+00 & 0.0000\end{array}$ ííítífí fítít $0.000 \mathrm{E}+000.0000$

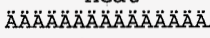

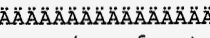
mrem/yr fract.

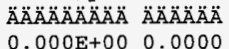
0.000E+00 0.0000 $0.000 \mathrm{E}+000.0000$
A11 Pathways* $\ddot{A} \ddot{A} \ddot{A} \ddot{A} \ddot{A} \ddot{A} \ddot{A} \ddot{A} \ddot{A} \ddot{A} \ddot{A} \ddot{A} \ddot{A} \ddot{A} \ddot{A} \ddot{A}$ mrem/yr fract.

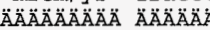
$1.222 \mathrm{E}-07 \quad 1.0000$ $222 \mathrm{E}-07 \quad 1.0000$ 


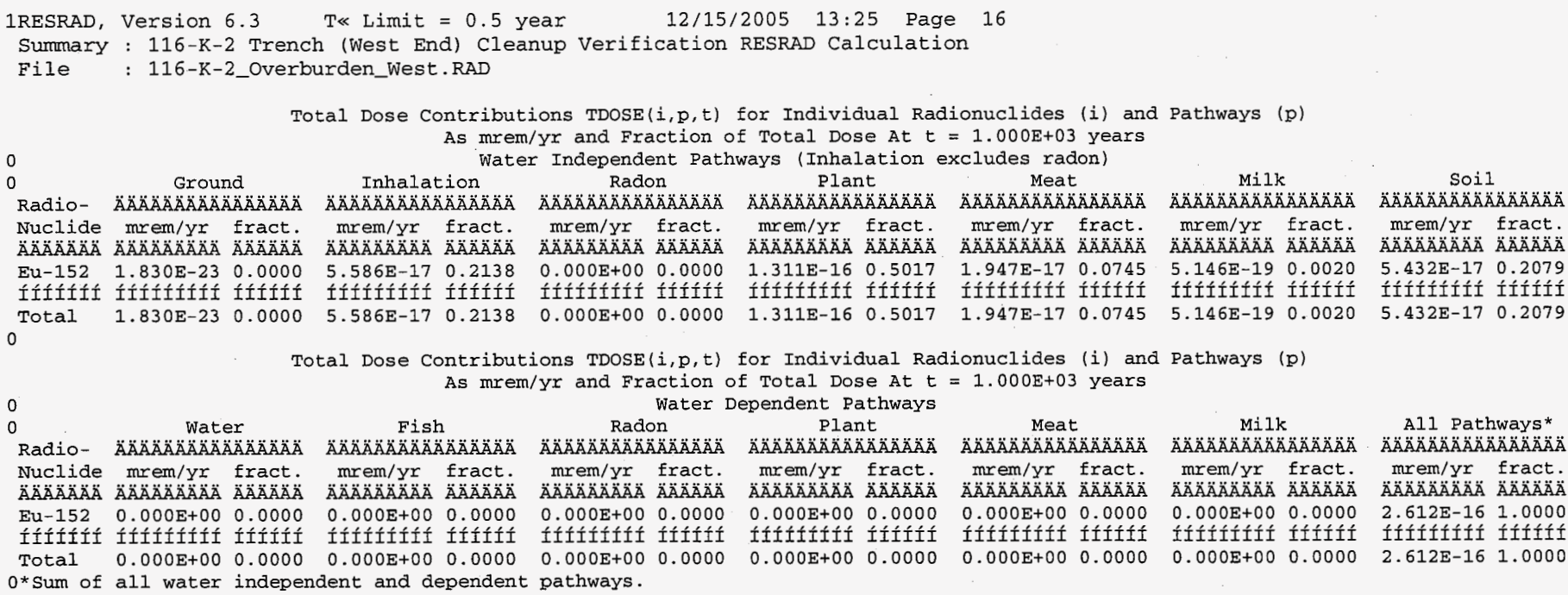$$
\text { Radon Plant }
$$$$
\text { Meat }
$$

Milk

AลАAA mrem/yr fract. mrem/yr fract.

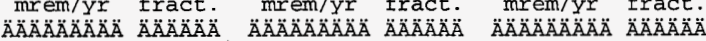
Nuclide mrem/yr fract. mrem/yr fract. mrem/yr $\begin{array}{llllllll}152 & 1.830 \mathrm{E}-23 & 0.0000 & 5.586 \mathrm{E}-17 & 0.2138 & 0.000 \mathrm{E}+00 & 0.0000\end{array}$

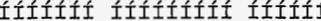
Total $1.830 \mathrm{E}-23 \quad 0.0000$ $1.947 \mathrm{E}-170.0745$ . 0000 fítítítít

$5.586 \mathrm{E}-170.2138$ $0.000 \mathrm{t}+000.0000$

0

Total Dose Contributions TDOSE $(i, p, t)$ for Individual Radionuclides (i) and Pathways (p) As mrem/yx and Fraction of Total Dose At $t=1.000 \mathrm{E}+03$ years

0 Water Fish Radon Plant

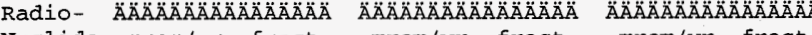

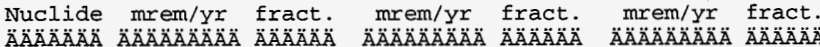
$\begin{array}{lllllll}\mathrm{Eu}-152 & 0.000 \mathrm{E}+00 & 0.0000 & 0.000 \mathrm{E}+00 & 0.0000 & 0.000 \mathrm{E}+00 & 0.0000\end{array}$

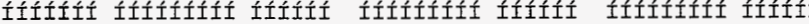
$\begin{array}{lllllllll}\text { Total } & 0.000 \mathrm{E}+00 & 0.0000 & 0.000 \mathrm{E}+00 & 0.0000 & 0.000 \mathrm{E}+00 & 0.0000\end{array}$ mrem/yr fract.

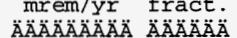
tíítítíi ííííí tifi ifitit $0^{*} \mathrm{Sum}$ of all water independent and dependent pathways.

Meat $\mathrm{Milk}$

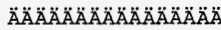
mrem/yr fract

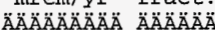
$0.000 E+000.0000$ $0.000 \mathrm{E}+000.0000$

Soil

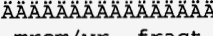
$\mathrm{mrem} / \mathrm{yr}$ fract. $\begin{array}{ll}5.432 \mathrm{E}-17 & 0.2079\end{array}$

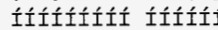

$5.432 \mathrm{E}-17 \quad 0.2079$ 
1RESRAD, Version 6.3 T T Limit $=0.5$ year $12 / 15 / 2005 \quad 13: 25$ Page 17

Summary : 116-K-2 Trench (West End) Cleanup Verification RESRAD Calculation

File : 116-K-2_Overburden_West.RAD

Dose/Source Ratios Summed Over All Pathways

Parent and Progeny Principal Radionuclide Contributions Indicated

oparent Product Branch

$\operatorname{DSR}(j, t) \quad(\mathrm{mrem} / \mathrm{yr}) /(\mathrm{pCi} / \mathrm{g})$

(i) (j) Fraction* $t=0.000 E+00 \quad 1.000 E+00 \quad 3.000 E+00 \quad 1.300 E+013.000 E+01 \quad 1.000 E+02 \quad 3.000 E+02 \quad 1.000 E+03$

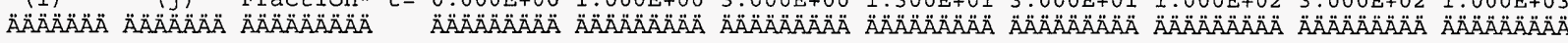

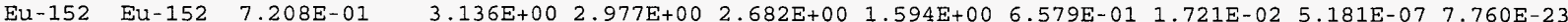

0Eu-152 Eu-152 2.792E-01 $1.215 E+00 \quad 1.153 E+00 \quad 1.039 E+00 \quad 6.174 E-01 \quad 2.548 E-01 \quad 6.665 E-03 \quad 2.007 E-07 \quad 3.006 E-23$

Eu-152 Gd-152 2.792E-01 3.982E-17 $1.168 \mathrm{E}-16 \quad 2.591 \mathrm{E}-16 \quad 7.856 \mathrm{E}-16 \quad 1.238 \mathrm{E}-15 \quad 1.547 \mathrm{E}-15 \quad 1.551 \mathrm{E}-15 \quad 1.537 \mathrm{E}-15$

Eu-152 äDSR(j) $1.215 E+00 \quad 1.153 E+00 \quad 1.039 E+00 \quad 6.174 E-01 \quad 2.548 E-01 \quad 6.665 E-03 \quad 2.007 E-07 \quad 1.537 E-15$

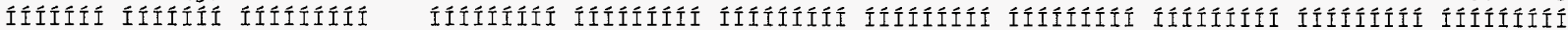

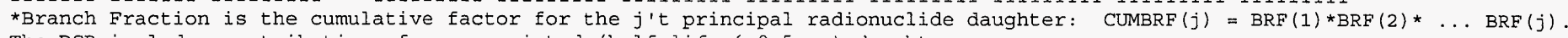
The DSR includes contributions from associated (half-life ó 0.5 yr) daughters.

Single Radionuclide Soil Guidelines $\mathrm{G}(i, t)$ in $\mathrm{pCi} / \mathrm{g}$

Basic Radiation Dose Limit $=1.500 \mathrm{E}+01 \mathrm{mrem} / \mathrm{yr}$

\begin{tabular}{|c|c|c|c|c|c|c|c|c|}
\hline $\begin{array}{c}\text { (i) } \\
\ddot{A} \ddot{A} \ddot{A} \ddot{A} \ddot{A} \ddot{A} \ddot{A}\end{array}$ & $t=\begin{aligned} & 0.000 E+00 \\
& \ddot{A} \ddot{A} \ddot{A} \ddot{A} \ddot{A} \ddot{A} \ddot{A} \ddot{A} \ddot{A} \ddot{A}\end{aligned}$ & $\begin{array}{l}1.000 E+00 \\
\ddot{A} \ddot{A} \ddot{A} \ddot{A} \ddot{A} \ddot{A} \ddot{A} \ddot{A} \ddot{A}\end{array}$ & $\begin{array}{l}3.000 E+00 \\
\ddot{A} \ddot{A} \ddot{A} \ddot{A} \ddot{A} \ddot{A} \ddot{A} \ddot{A} \ddot{A}\end{array}$ & $\begin{array}{l}1.300 E+01 \\
\ddot{A} \ddot{A} \ddot{A} \ddot{A} \ddot{A} \ddot{A} \ddot{A} \ddot{A} \ddot{A}\end{array}$ & $\begin{array}{l}3.000 E+01 \\
\ddot{A} \ddot{A} \ddot{A} \ddot{A} \ddot{A} \ddot{A} \ddot{A} \ddot{A} \ddot{A}\end{array}$ & 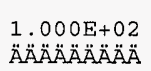 & $\begin{array}{l}3.000 E+02 \\
\ddot{A} \ddot{A} \ddot{A} \ddot{A} \ddot{A} \ddot{A} \ddot{A} \ddot{A} \ddot{A}\end{array}$ & 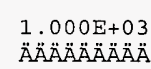 \\
\hline $\begin{array}{l}\text { Eu-152 } \\
\text { ÍIIIIÍÍ }\end{array}$ & $\begin{array}{l}3.448 E+00 \\
\text { IIIIIIIII }\end{array}$ & $\begin{array}{l}3.632 E+00 \\
\text { IIIIIIIII }\end{array}$ & $\begin{array}{l}4.031 E+00 \\
\text { IIIIIIIII }\end{array}$ & $\begin{array}{l}6.783 E+00 \\
\text { IIIIIIIII }\end{array}$ & $\begin{array}{l}1.643 \mathrm{E}+01 \\
\text { IIIIIIIII }\end{array}$ & $\begin{array}{l}6.284 \mathrm{E}+02 \\
\text { IIIIIIIII }\end{array}$ & $\begin{array}{l}2.087 E+07 \\
\text { IIIIIIIII }\end{array}$ & $\begin{array}{l}\text { *1.765E }+14 \\
\text { IIIIIIIII }\end{array}$ \\
\hline
\end{tabular}

*At specific activity limit

0

Summed Dose/Source Ratios DSR $(i, t)$ in (mrem/yr)/(pCi/g)

and Single Radionuclide Soil Guidelines $G(i, t)$ in $\mathrm{pCi} / \mathrm{g}$

at $\operatorname{tmin}=$ time of minimum single radionuclide soil guideline

and at $t \max =t i m e$ of maximum total dose $=0.000 \mathrm{E}+00$ years

\begin{tabular}{|c|c|c|c|c|c|c|}
\hline $\begin{array}{l}\text { ONuclide } \\
\text { (i) } \\
\ddot{A} \ddot{A} A \ddot{A} \ddot{A} \ddot{A} \ddot{A}\end{array}$ & $\begin{array}{c}\text { Initial } \\
(p C i / g) \\
\ddot{A} \ddot{A} \ddot{A} \ddot{A} \ddot{A} \ddot{A} \ddot{A} \ddot{A} \ddot{A}\end{array}$ & $\begin{array}{c}\text { tmin } \\
(y e a r s) \\
\ddot{A} \ddot{A} \ddot{A} \ddot{A} \ddot{A} \ddot{A} \ddot{A} \ddot{A} \ddot{A} \ddot{A} \ddot{A} \ddot{A} \ddot{A} \ddot{A} \mathrm{~A}\end{array}$ & $\operatorname{DSR}(i, \operatorname{tmin})$ & $\begin{array}{l}G(i, t \min ) \\
(p C i / g) \\
\ddot{A} \ddot{A} \ddot{A} \ddot{A} \ddot{A} \ddot{A} \ddot{A} \ddot{A} \ddot{A}\end{array}$ & $\operatorname{DSR}(i, t \max )$ & $\begin{array}{l}\mathrm{G}(i, t \max ) \\
(\mathrm{pC} i / g) \\
\ddot{A} \ddot{A} \ddot{A} \ddot{A} \ddot{A} \ddot{A} \ddot{A} \ddot{A} \ddot{A}\end{array}$ \\
\hline $\begin{array}{l}\mathrm{EU}-152 \\
\text { İIIITII }\end{array}$ & $\begin{array}{l}1.700 E-01 \\
\text { ÍITIOIIIII }\end{array}$ & $\begin{array}{l}0.000 \mathrm{E}+00 \\
\text { IIIIIIIIIIIIIIII }\end{array}$ & $\begin{array}{l}4.350 E+00 \\
\text { IIIIIIIII }\end{array}$ & $\begin{array}{l}3.448 \mathrm{E}+00 \\
\text { IIITIIIII }\end{array}$ & $\begin{array}{l}4.350 E+00 \\
\text { IIIIIIIIII }\end{array}$ & $3.448 \mathrm{E}+00$ \\
\hline
\end{tabular}


IRESRAD, Version 6.3 T\& Limit $=0.5$ year $\quad 12 / 15 / 2005 \quad 13: 25$ Page 18

Summary : 116-K-2 Trench (West End) Cleanup Verification RESRAD Calculation

File : 116-K-2_Overburden_West.RAD

Individual Nuclide Dose Summed Over All Pathways

Parent Nuclide and Branch Fraction Indicated

ONuclide Parent BRF(i) (j) (i)

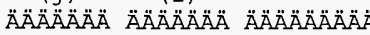
Eu-152 Eu-152 7.208E-01 Eu-152 Eu-152 2.792E-01 $\mathrm{Eu}-152$ äDOSE $(j)$

OGd-152 Eu-152 2.792E-01

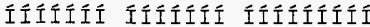
$\mathrm{BRF}(i)$ is the branch fraction of the parent nuclide.

$t=0.000 E+00 \quad 1.000 E+00 \quad 3.000 E+00 \quad 1.300 E+01 \quad 3.000 E+01 \quad 1.000 E+02 \quad 3.000 E+02 \quad 1.000 E+03$

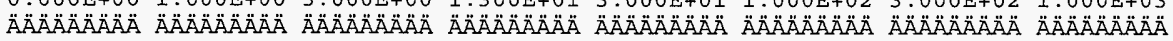

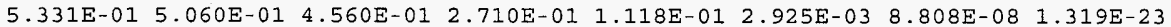
$\begin{array}{llllllll}2.065 E-01 & 1.960 E-01 & 1.766 E-01 & 1.050 E-01 & 4.332 E-02 & 1.133 E-03 & 3.412 E-08 & 5.110 E-24\end{array}$ $\begin{array}{llllllll}7.396 E-01 & 7.021 E-01 & 6.326 E-01 & 3.759 E-01 & 1.552 E-01 & 4.058 E-03 & 1.222 E-07 & 1.830 E-23\end{array}$

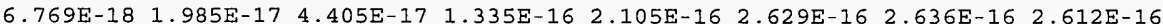

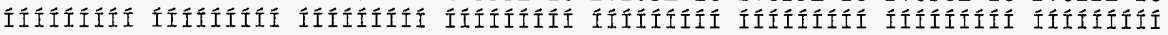

ONuclide Parent BRF(i)

$$
\text { (j) }
$$

$\ddot{A} \ddot{A} \ddot{A} \ddot{A} \ddot{A} \ddot{A} \ddot{A} \quad \ddot{A} \ddot{A} \ddot{A} \ddot{A} \ddot{A} \ddot{A} \ddot{A} \quad \ddot{A} \ddot{A} \ddot{A} \ddot{A} \ddot{A} \ddot{A} \ddot{A} \ddot{A}$

Eu-152 Eu-152 7.208E-01

Eu-152 Eu-152 2.792E-01

Eu-152 äs $(j)$ :

OGd-152 Eu-152 2.792E-01

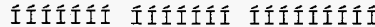

BRF(i) is the branch fraction

ORESCALC.EXE execution time =
Individual Nuclide Soil Concentration

Parent Nuclide and Branch Fraction Indicated

$$
s(j, t), p C i / g
$$

$t=0.000 \mathrm{E}+00 \quad 1.000 \mathrm{E}+00 \quad 3.000 \mathrm{E}+00 \quad 1.300 \mathrm{E}+01 \quad 3.000 \mathrm{E}+01 \quad 1.000 \mathrm{E}+02 \quad 3.000 \mathrm{E}+02 \quad 1.000 \mathrm{E}+03$

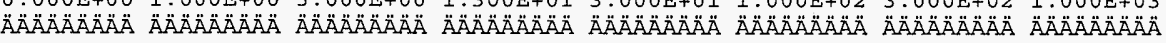
$\begin{array}{lllllllll}1.225 \mathrm{E}-01 & 1.163 \mathrm{E}-01 & 1.048 \mathrm{E}-01 & 6.228 \mathrm{E}-02 & 2.571 \mathrm{E}-02 & 6.724 \mathrm{E}-04 & 2.025 \mathrm{E}-08 & 3.033 \mathrm{E}-24\end{array}$ $\begin{array}{llllllll}4.746 \mathrm{E}-02 & 4.506 \mathrm{E}-02 & 4.060 \mathrm{E}-02 & 2.413 \mathrm{E}-02 & 9.958 \mathrm{E}-03 & 2.604 \mathrm{E}-04 & 7.842 \mathrm{E}-09 & 1.175 \mathrm{E}-24\end{array}$

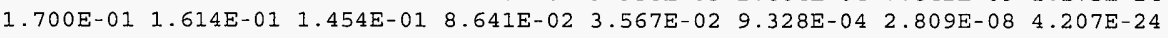
$0.000 \mathrm{E}+00 \quad 2.968 \mathrm{E}-16 \quad 8.461 \mathrm{E}-16 \quad 2.877 \mathrm{E}-15 \quad 4.623 \mathrm{E}-15 \quad 5.814 \mathrm{E}-15 \quad 5.831 \mathrm{E}-15 \quad 5.777 \mathrm{E}-15$ 壬往任任 of the parent nuclide. 0.29 seconds 
CVP-2006-00001

Rev. 0

\section{CALCULATION BRIEF EXCERPTS}


CVP-2006-00001

Rev. 0

\section{DISCLAIMER FOR CALCULATIONS}

The attached calculations have been generated for a specific purpose and task. Use of these calculations by persons who do not have access to all pertinent facts may lead to incorrect conclusions and/or results. Before applying these calculations to your work, the underlying basis, rationale, and other pertinent information relevant to these calculations must be thoroughly reviewed with appropriate Washington Closure Hanford officials or other authorized personnel. Washington Closure Hanford is not responsible for the use of a calculation not under its direct control. 


\section{CALCULATION BRIEFS}

The following calculation briefs have been prepared in accordance with WCH-DE-01, Design Engineering Procedures Manual, EDPI-4.37-01, "Project Calculations," Washington Closure Hanford, Richland, Washington.

116-K-2 (East End) Trench Shallow \& Deep Zone Sampling Plan, Calculation No. 0100K-CA-V0057, Rev. 0, Washington Closure Hanford, Richland, Washington.

116-K-2 Mile Long Trench Variance Calculation, Calculation No. 0100K-CA-V0055, Rev. 0, Washington Closure Hanford, Richland, Washington.

116-K-2 Trench (East End) Cleanup Verification 95\% UCL Calculations, Calculation No. 0100K-CA-V0056, Rev. 1, Washington Closure Hanford, Richland, Washington.

116-K-2 Trench (East End) RESRAD Calculation, Calculation No. 0100K-CA-V0058, Rev. 0, Washington Closure Hanford, Richland, Washington.

116-K-2 (West End) Trench Shallow, Deep Zone and Overburden Sampling Plan, Calculation No. 0100K-CA-V0062, Rev. 0, Washington Closure Hanford, Richland, Washington.

116-K-2 Mile Long Trench Variance Calculation (Phase 2, West), Calculation No. 0100K-CA-V0064, Rev. 0, Washington Closure Hanford, Richland, Washington.

116-K-2 Overburden Variance Calculation, Calculation No. 0100K-CA-V0065, Rev. 0, Washington Closure Hanford, Richland, Washington.

116-K-2 Trench (West End) Cleanup Verification 95\% UCL Calculations, Calculation No. 0100K-CA-V0061, Rev. 0, Washington Closure Hanford, Richland, Washington.

116-K-2 Trench (West End) RESRAD Calculation, Calculation No. 0100K-CA-V0063, Rev. 0, Washington Closure Hanford, Richland, Washington.

NOTE: The calculation briefs referenced in this appendix are kept in the active Washington Closure Hanford project files and are available upon request. When the project is completed, the files will be stored in a U.S. Department of Energy, Richland Operations Office repository. Only excerpts of the calculation briefs are included in this appendix. 
CVP-2006-00001

Rev. 0

C-126 


\section{CALCULATION COVER SHEET}

Project Title:

Area

Discipline

Subject

Computer Program
116-K-2 (East End) Trench Sample Design $100-\mathrm{K}$

\begin{tabular}{lll}
\hline Environmental Engineering & Calc. No. & $0100 \mathrm{~K}-\mathrm{CA}-\mathrm{V} 0057$
\end{tabular}

116-K-2 (East End) Trench Shallow \& Deep Zone Sampling Plan

Excel

Program No. Excel 2003

The attached calculations have been generated to document compliance with established cleanup levels. These documents should be used in conjuction with other relevent documents in the administrative record.

Committed Calculation $\square \quad$ Preliminary $\square \quad$ Superseded $\square \quad$ Voided $\square$

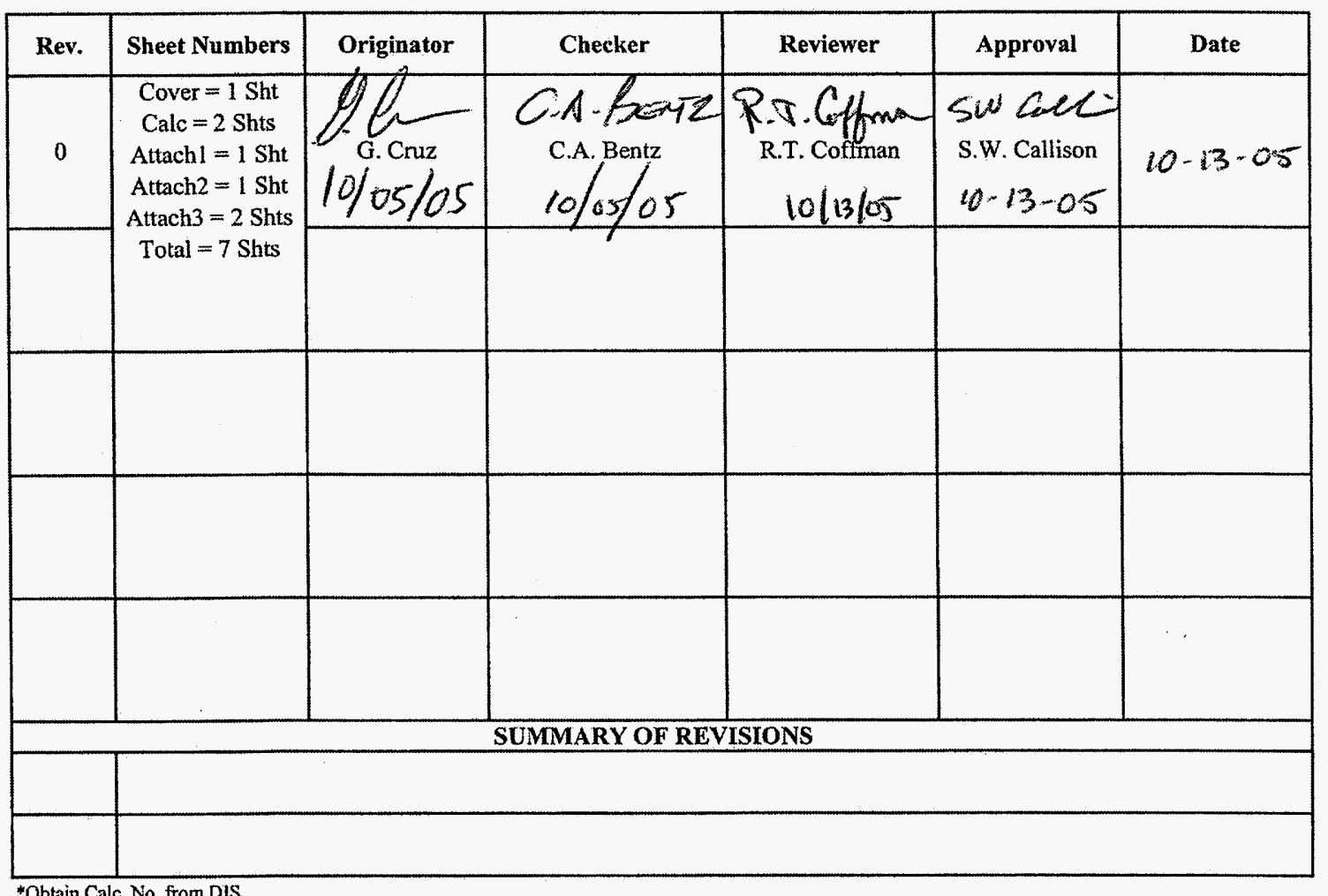

"Obtain Calc. No. from DIS

DE01437.03 (12/09/2004) 


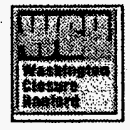

Washington Clusure Hanford

Originator G.Cruz

Date

CALCULATION SHEET
Project 116-K-2 (East End) Trenc

Subject 116-K-2 (East End) Trench Shallow \& Deep Zone Sampling Plan
Calc. No. 0100K-CA-V0057 Rev. No. 0

Job No. 14655 Checked $24 \&$ Date $/ 0 / 5 / 05$ Sheet No. 1 of 2

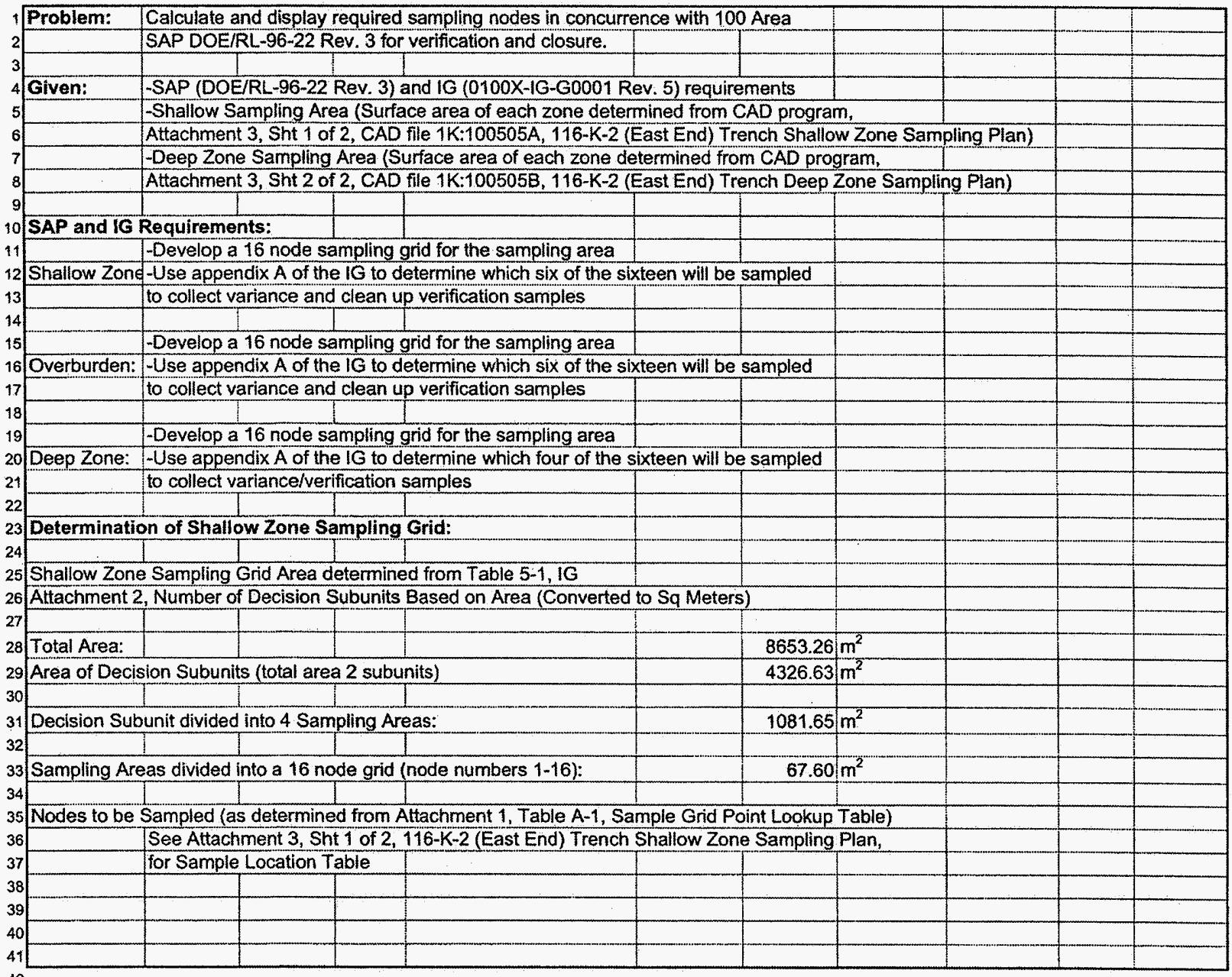


CVP-2006-00001

Rev. 0

Washington Clusure Hanford

CALCULATION SHEET

Originator G. Cruz Date 10/5/2005

Project 116-K-2 (East End) Trench Sample Design Job'No. 14655 Checked $A B$ Date $10 / 5 / 65$ Subject 116-K-2 (East End) Trench Shallow \& Deep Zone Sampling Plan
Sheet No. 2 of 2

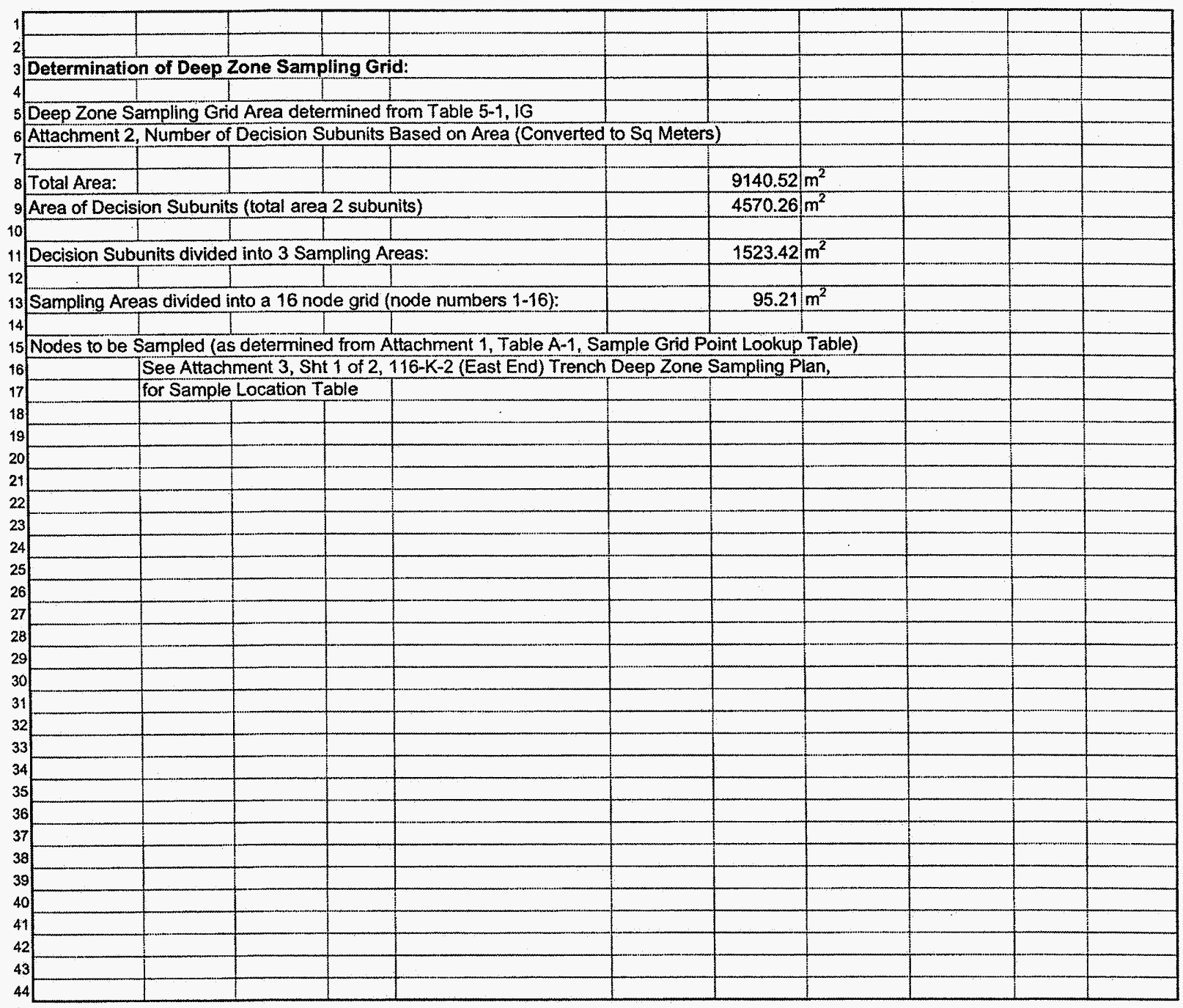


CVP-2006-00001

Rev. 0

Washington Clusure Hanford

1

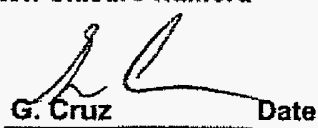

$10 / 5 / 2005$

Calc. No. 0100K-CA-V0057

Rev. No. 0

Project 116-K-2 (East End) Trench Sample Design Job No. 14655

Subject 116-K-2 (East End) Trench Shallow \& Deep Zone Sampling Plan Checked $\frac{01 / 3}{\text { Sheet No } 1 \text { of } 1} 1 / 5 / 05$

1 ATTACHMENT 1

2

${ }_{3}$ Sample Grid Point Lookup Table.

4

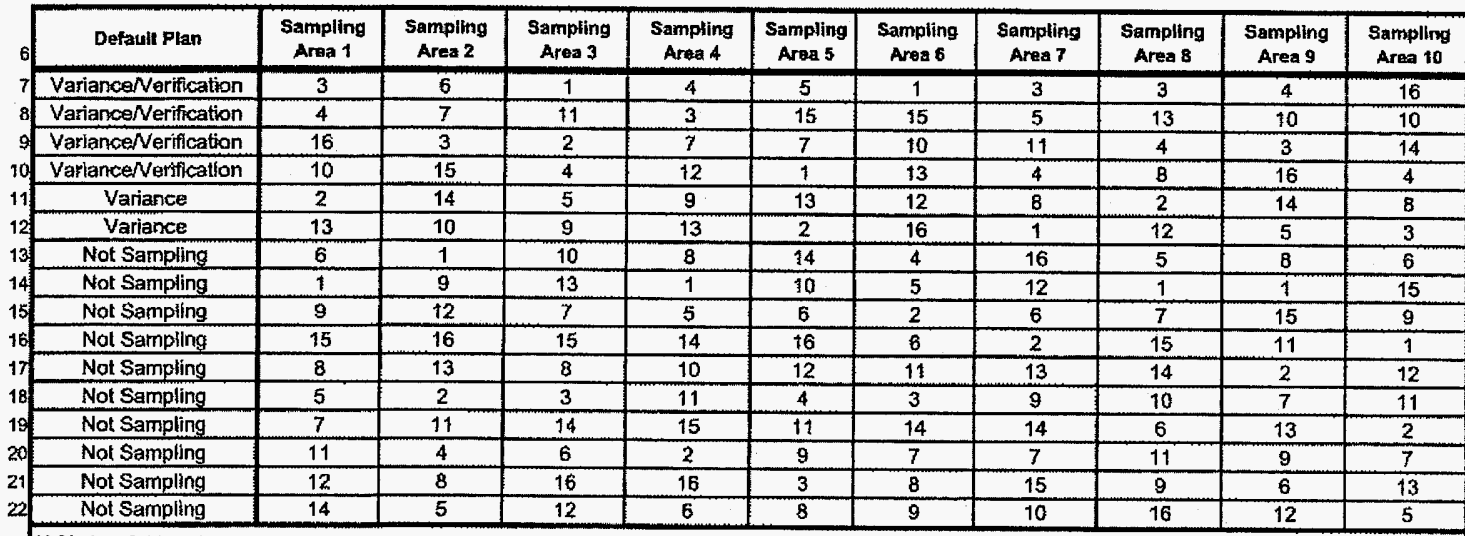

23 "* Note: Grid nodes for each sampling area in each waste site should be numbered consistently, e.g., begin numbering

24 the nodes in the northwestem-most node, then number consecutively left to right. 


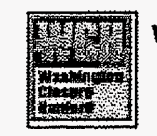

Washington Closure Hanford

Originator G. Cruz

Date $10 / 5 / 2005$

Calc. No. 0100 K-CA-V0057 Rev. No. 0

Project 116-K-2 (East End) Trench Sample Design Job No. 14655 Checked 01/ Date $10 / 5 / 05$

Subject 116-K-2 (East End) Trench Shallow \& Deep Zone Sampling Plan

Sheet No. 1 of 1

1 ATTACHMENT 2

2

${ }_{3}$ Number of Decision Subunits Based on Area.

4

5

\begin{tabular}{|c|c|c|}
\hline & Area of Primary Decision Unit (m2) & Number of Subunits \\
\hline & $<1,394$ & 1 \\
\hline & $>1,394$ to $<2,326$ & 2 \\
\hline & $>2,326$ to $<3,256$ & 3 \\
\hline & $>3,256$ to $<4,186$ & 4 \\
\hline & $>4,186$ to $<9,303$ & 2 \\
\hline & $>9,303$ to $<13,024$ & 3 \\
\hline & $>13,024$ to $<16,745$ & 4 \\
\hline & $>16,745$ to $<20,466$ & 5 \\
\hline & $>20,466$ & ROUNDa (Area/3,720) \\
\hline
\end{tabular}

6 a ROUND is an integer rounding function. 
CVP-2006-00001

Rev. 0

C-132 


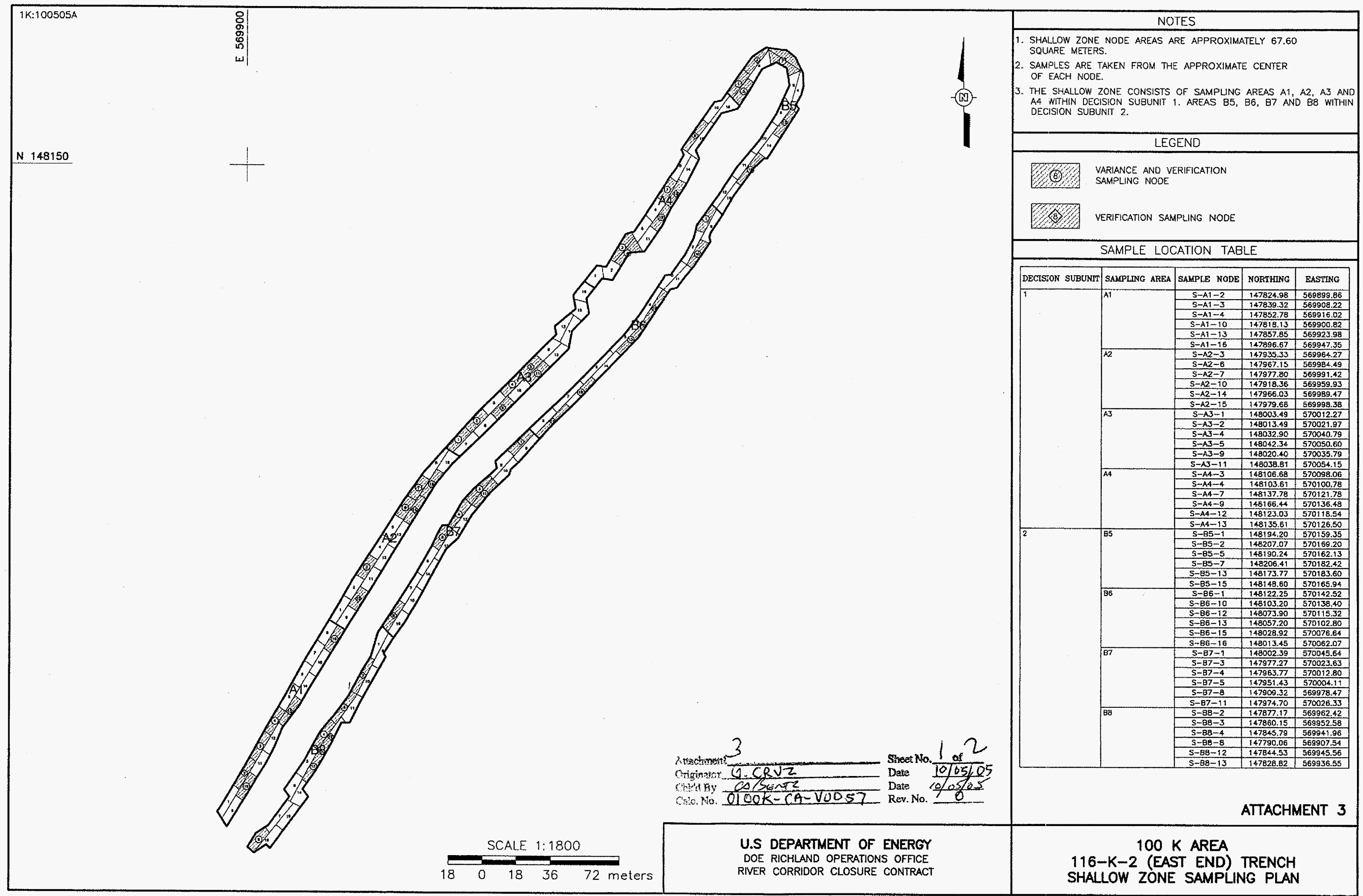




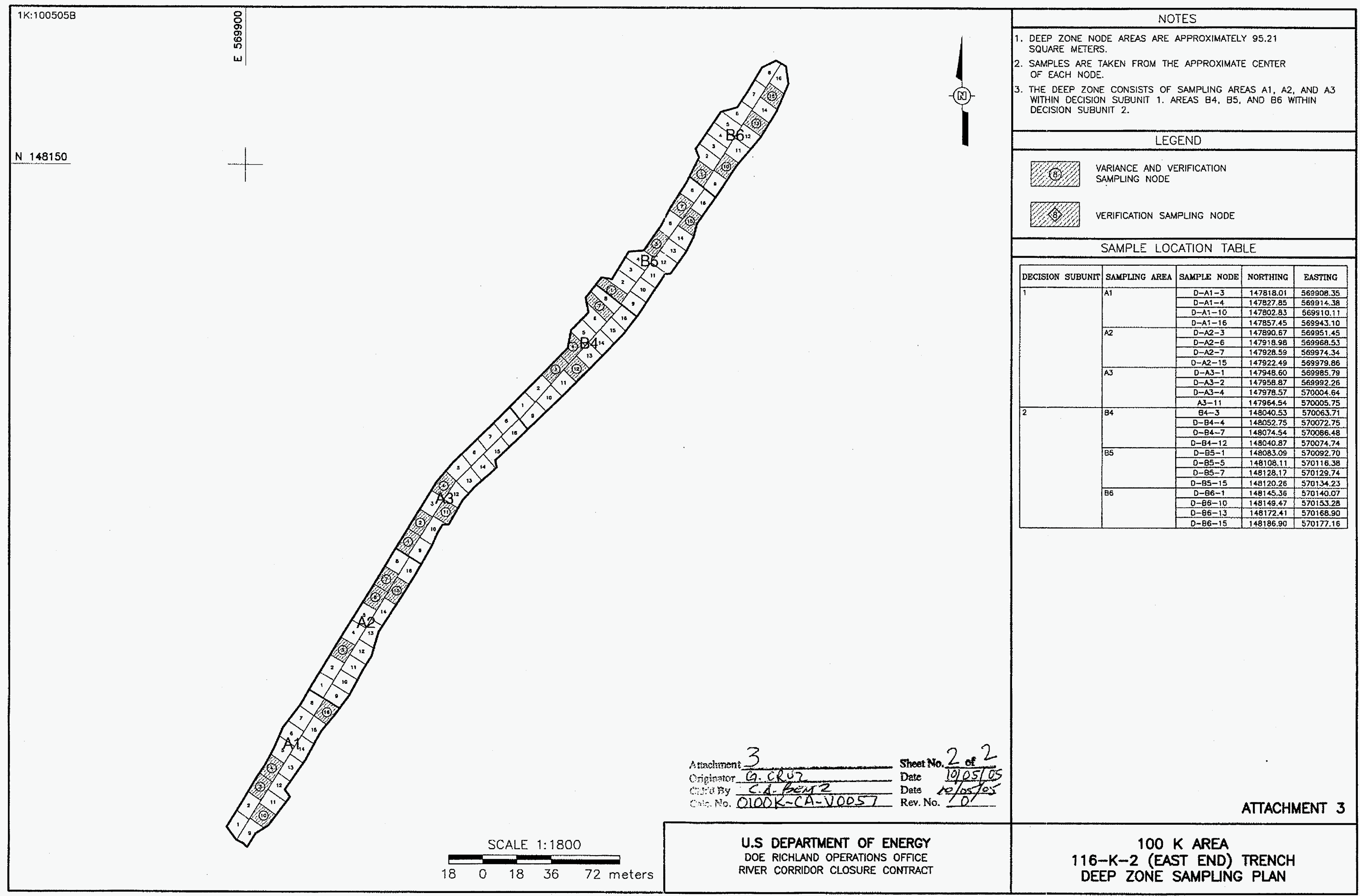




\section{CALCULATION COVER SHEET}

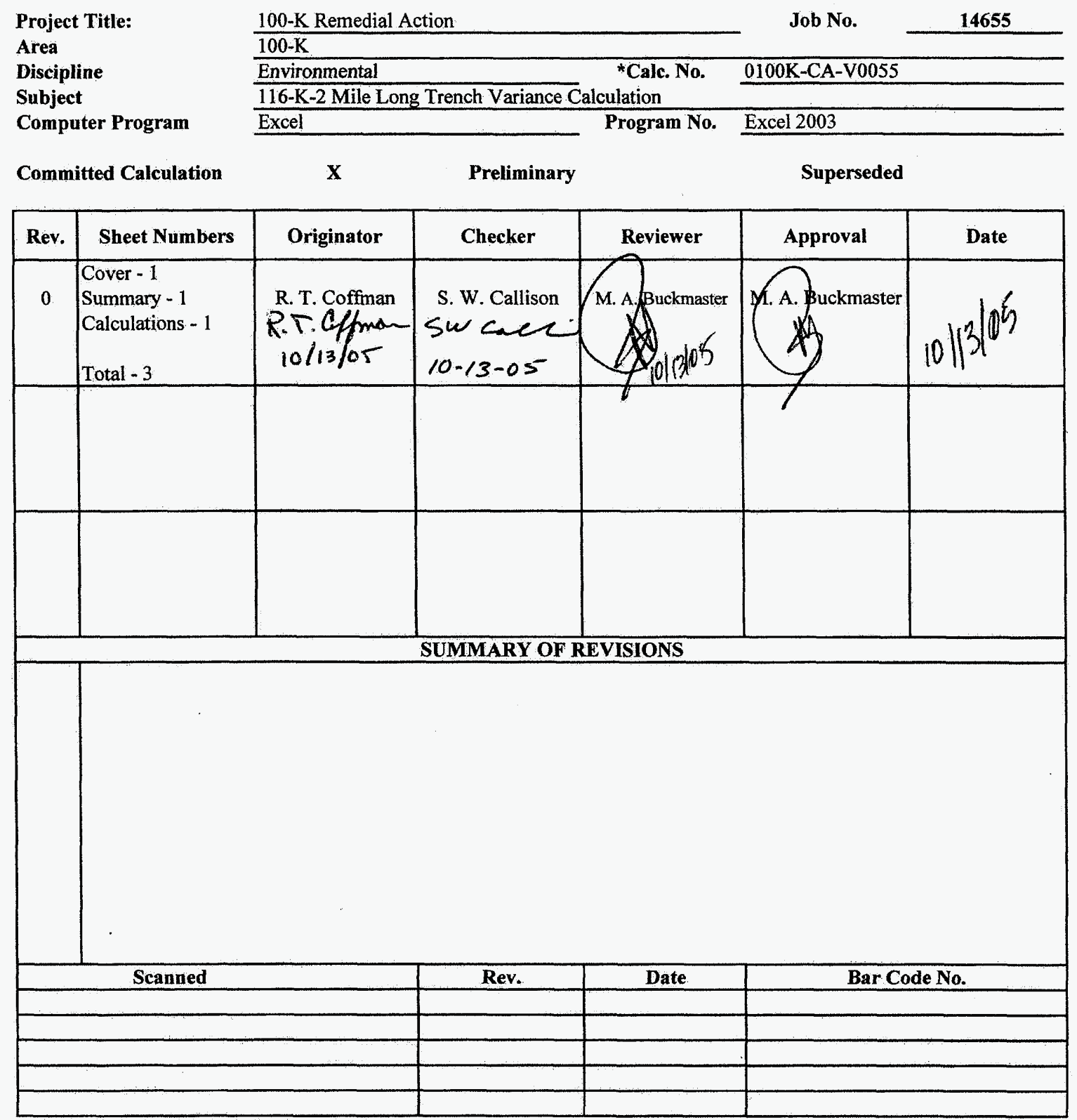

* Obtain Calc. No. from DIS. 


\section{CALCULATION SHEET}

1 Conclusion:

2 The required number of samples calculated $(1,2$, and 3 samples) for each decision sub-unit is less than the 3 default number (4 samples) specified in the DOE/RL-96-22, Rev 4. Therefore, the default number of samples will 4 be collected from each shallow zone decision sub-unit:

5

6 Problem:

7 Calculate the number of close out samples required for 116-K-2 Mile Long Trench Shallow Unit verification 8 sampling as required in "100 Area Remedial Action Sampling and Analysis Plan" (DOE/RL-96-22, Rev 4) and 9 "Instruction Guide for the Remediation of 100 Areas Waste Sites" (0100X-IG-G0001, Rev 5).

10

11. Given:

12 1) Sample locations for the 116-K-2 Mile Long Trench Shallow Decision Unit are identified on the 116-K-2 13 Mile Long Trench Shallow Sample Design, Calculation number 0100K-CA-V0057, Rev. 0.

14 2) Lookup values from DOE/RL-96-22, Rev 4.

15 3) Sample Design requirements from DOE/RL-96-22, Rev 4 and 0100X-IG-G0001, Rev 5.

16 4) Field sampling information from sampling logbook EL-1572-3.

18 Solution:

19 Calculation methodology is described in Appendix A of DOE/RL-96-22, Rev 4. Data from attached worksheets

20 are used to calculate the required number of closeout samples. Variance calculation is based on the same three

21 isotopes used to develop the statistical approach in DOE/RL-96-22, Rev 4. The statistical design is based on the

22 premise that these isotopes are the predominant components of the contamination and are representative of the

23 contamination distribution.

24.

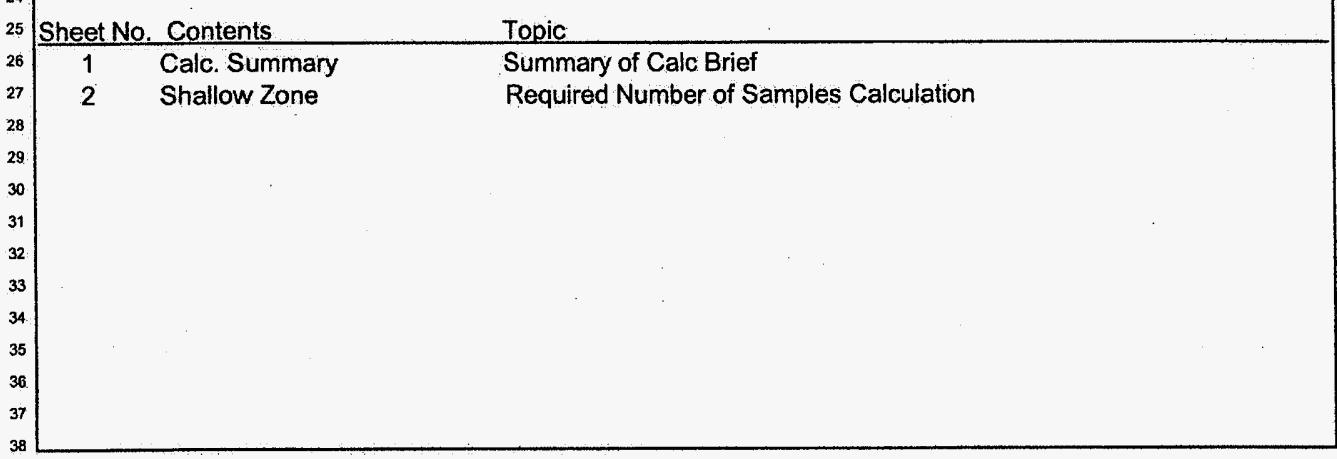




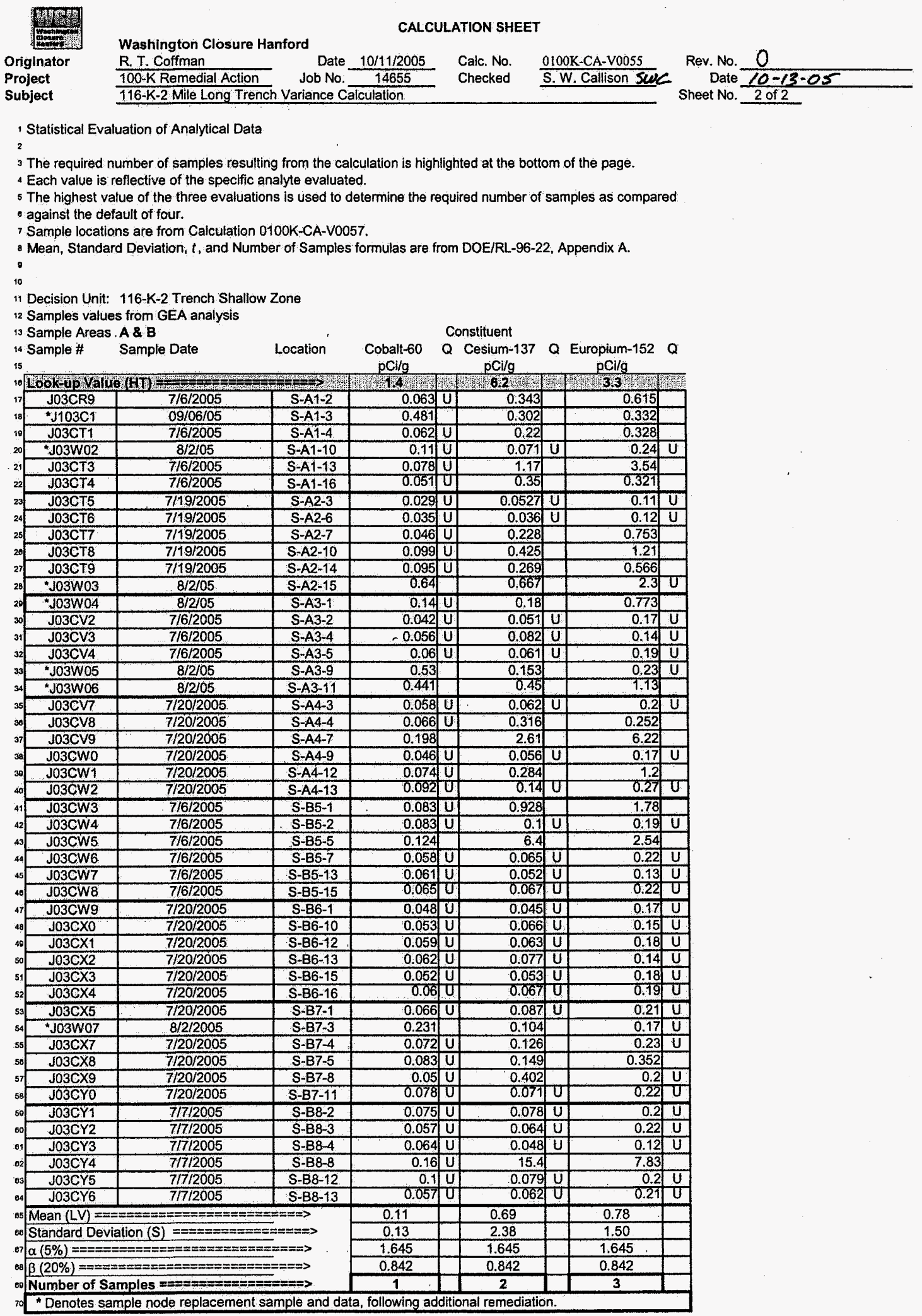


CVP-2006-00001

Rev. 0

C-138 
CVP-2006-00001

Rev. 0

\section{CALCULATION COVER SHEET}

Project Title:

Area

Discipline

Subject

Computer Program
100-K Area Field Remediation

Job No.

14655

Environmental
116-K-2 Trench (East End) Cleanup Verification $95 \%$ UCL Calculations

Excel Program No. Excel 2003

The attached calculations have been generated to document compliance with established cleanup levels. These documents should be used in conjunction with other relevant documents in the administrative record.

$\begin{array}{lllll}\text { Committed Calculation } & \mathbb{X} & \text { Preliminary } & \square & \text { Superseded }\end{array} \quad$ Voided $\square$

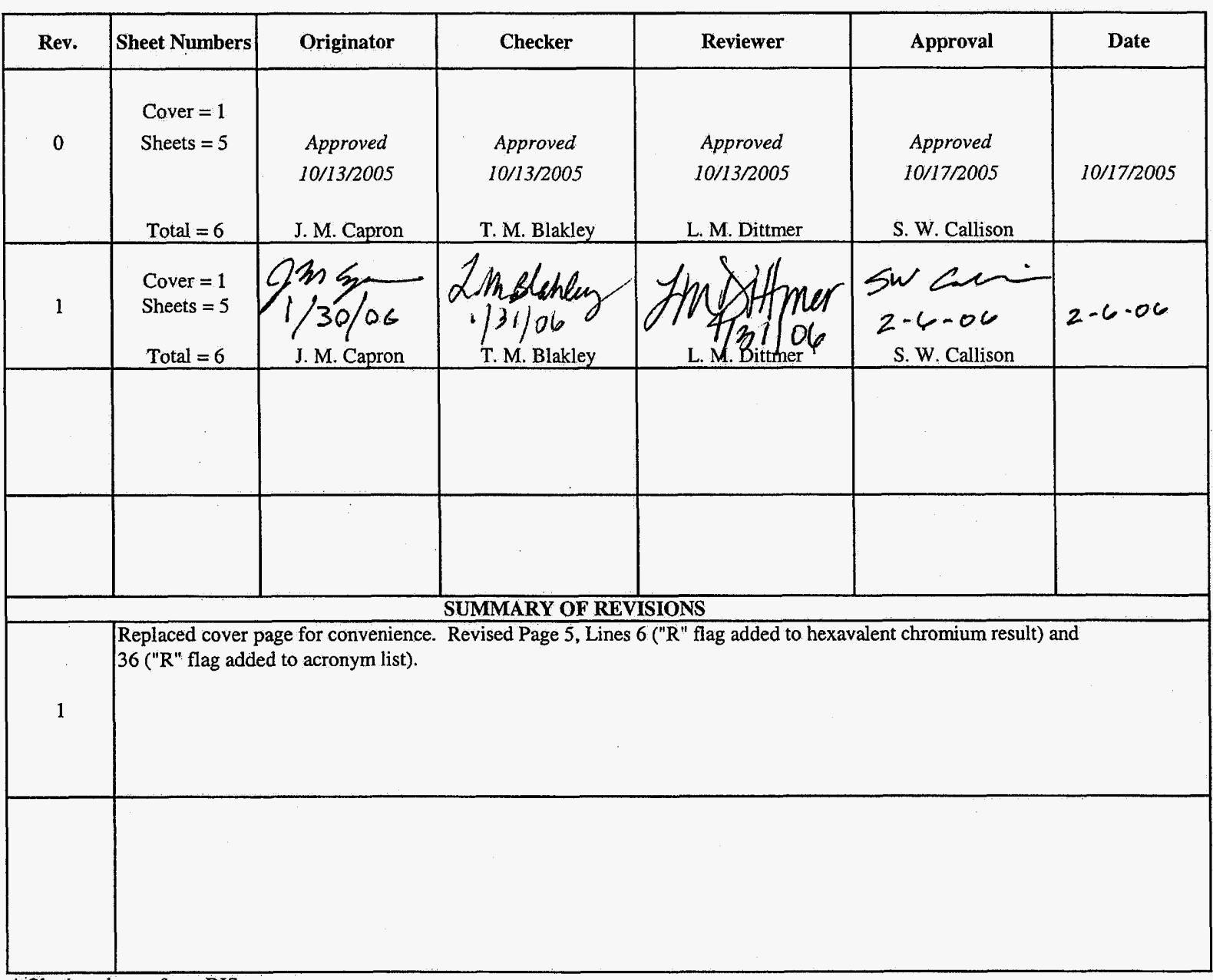

* Obtain calc no. from DIS

DE01437.03 (12/09/2004) 
Washington Closure Hanford

Originator J.M. Capron / J he

Project 100-K Area Reteld Remediation

Subject 116-K-2 Trench (East End) Cle

\section{CALCULATION SHEET}

Date $10 / 13 / 05$

Job No. 14655
Calc. No. 0100K-CA-V0056 Checked T. M. Blakley 2 .

$\begin{aligned} \text { Rev. No. } \frac{0}{\text { Date }} & \text { ro/13/05 } \\ \text { Sheet No. } & \text { of } 5\end{aligned}$

Purpose:

Calculate the $95 \%$ upper confidence limit (UCL) to evaluate compliance with cleanup standards for the subject site. Also, calculate the carcinogenic risk for applicable nonradionuclide analytes, perform the Washington Administrative Code (WAC) 173-340 (Model Toxics Control Act [MTCA]) 3-part test, if required, and calculate the relative percent difference (RPD) for each contaminant of concern (COC)

Table of Contents:

Sheets 1 to 2 - Calculation Sheet Summary

Sheet 3 - Calculation Sheet Shallow Zone Verification

Sheet 4 - Calculation Sheet Deep Zone Veritication

Sheet 5 - Calculation Sheet Split-Duplicate Analysis

Given/References:

1) Sample Results

15 2) All lookup values and remedial action goals (RAGs) are taken from DOE-RL (2005b) and Ecology (1996)

16 3) DOE-RL, 2005a, 100 Area Remedial Action Sampling and Analysis Plan (SAP), DOE/RL-96-22, Rev, 4, U.S. Department of Energy, Richland Operations Office, Richland, Washington.

4) DOE-RL, 2005b, Remedial Design Report/Remedial Action Work Plan for the 100 Area (RDR/RAWP), DOE/RL-96-17, Rev. 5, U.S. Department of Energy, Richland Operations Office, Richland, Washington.

5) Ecology, 1992, Statistical Guidance for Ecology Site Managers, Publication \#92-54, Washington State Department of Ecology, Olympia, Washington.

6) Ecology, 1993, Statistical Guidance for Ecology Site Managers, Supplement S-6, Analyzing Site or Background Data with Below-Detection Limit or Below-PQL

Values (Censored Data Sets), Publication \#92-54, Washington State Department of Ecology, Olympia, Washington.

7) Ecology, 1996, Model Toxics Control Act Cleanup Levels and Risk Calculations (CLARC II), Publication \#94-145, Washington State Department of Ecology, Olympia, Washington.

8) EPA, 1994, USEPA Contract Laboratory Program National Functional Guidelines for Inorganic Data Review, EPA 540/R-94/013, U.S. Environmental Protection Agency, Washington, D.C. Solution:

Calculation methodology is described in Ecology Pub. \#92-54 (Ecology 1992, 1993), below, and in the RDR/RAWP (DOE-RL 2005b). Use data from the attached worksheets to calculate the $95 \%$ UCL, hazard quotients, excess carcinogenic risk, perform the WAC 173-340 3-part test for nonradionuclides, and calculate the RPD for each COC in the primanyduplicate and primary-split sample pairs.

Calculation Description:

The subject calculations were performed on data from soil verification samples from the northeast portion of the 116-K-2 waste site; calculations for the remainder of the waste site will be documented separately. The data were entered into an EXCEL. 2003 spreadsheet and calculations performed by utilizing the built-in spreadsheet functions and/or creating formulae within the cells. The statistical evaluation of data for use in accordance with the RDF/RAWP (DOE-RL 2005b) is documented by this calculation. Split and duplicate RPD results are 1 Methodology:

For nonradioactive analytes with $<50 \%$ of the data below detection limits and all radionuclide analytes, the statistical value calculated to evaluate the effectiveness of cleanup is the $95 \%$. UCL. For nonradioactive analytes with $>50 \%$ of the data below detection limits, the maximum value for the data set is used instead of the $95 \%$ UCL. All nonradionuclide data reported as being below detection limits are set to $1 / 2$ the detection limit value for calculation of the statistics (Ecology 1993). For radionuclide data, calculation of the statistics was don on the reported value. In cases where the laboratory does not report a value below the minimal detectable activity (MDA), half of the MDA is used in the calculation. For the statistical 48

For nonradionuclides, the WAC 173-340 statistical guidance suggests that a test for distributional form be performed on the data and the $95 \%$ UCL calculated on the appropriate distribution using Ecology software. For nonradionuclide small data sets $(n<10)$ and all radionuclide data sets, the calculations are periomed assuming nonparametric distribution, so no test for distribution is performed: For nonradionuclide data sets of ten or greater, distributional testing is done using Ecology's MTCAStat software (Ecology 1993).

The hazard quotient (for shallow zone nonradionuclide COCs) is determined by dividing the statistical value (derived in this caiculation) by the WAC $173-340$ non-carcinogenic cleanup limit. The excess nonradionuclide carcinogenic risk is determined by dividing the statistical value by the WAC $173-340$ carcinogenic cleanup limit and then multiplying by $10^{-6}$

The WAC 173-340 3-part test is performed for nonradionuclide analytes only and determines if:

1) the $95 \%$ UCL value exceeds the most stringent cleanup limit for each non-radionuclide $\mathrm{COC}$

2) greater than $10 \%$ of the raw data exceed the most stringent cleanup limit for each non-radionuclide $\mathrm{COC}$

3) the maximum value of the raw data set exceeds two times the most stringent cleanup limit for each non-radionuclide COC.

The RPD is calculated when both the primary value and either the duplicate or split values are above detection limits and are greater than 5 times the target detection limit (TDL). The TDL is a laboratory detection limit pre-determined for each analytical method, listed in Table II-1 of the SAP (DOE-RL 2005a). The RPD calculations use the following formula: RPD $=[\mid M-S /((M+S) / 2)]^{*} 100$

\section{where, $\quad M=$ Main Sample Value $\quad S=$ Split (or duplicate) Sample Value}

For quality assurance/quality control (QAVQC) split and duplicate RPD calculations, a value less than $+1-30 \%$ indicates the data compare favorably. For regulatory splits, a threshold of $35 \%$ is used (EPA 1994). If the RPD is greater than $30 \%$ (or $35 \%$ for regulatory split data), further investigation regarding the usability of the data is periormed. Additional discussion as necessary is provided in the data quality assessment section of the applicable CVP.

If regulator split comparison is required, an additional parameter is evaluated. A control limit of $+1-2$ times the TDL shall be used if either the main or regulator split value is less than 5 times the TDL and above detection. In the case where only one result is greater than 5 times the TDL and the other is below, the $+/-2$ times the TDL criteria applles. Therefore, the following calculation is performed as part of the evaluation for these two cases involving regulator split data: difference $=$ main - regulator split. If the difference is greater than $+l-2$ times the TDL, then further investigation regarding the usability of the data is performed and presented in the applicable CVP data quality assessment section.

No regulatory split samples were collected for this site.

81 
CVP-2006-00001

Rev. 0

Washington Closure Hanford

Originator J.M. Capron $Q$ me

Project $\frac{100 \text {-Kaprea Field Remediation }}{100}$ Job No. 14655

Job No. 14655

CALCULATION SHEET

Summary (continued)

1 Results:

2 The results presented in the summary tables that follow are for use in RESidual RADioactivity dose/risk analysis and the CVP for this site.

4 Results Summary - Shallow Zone

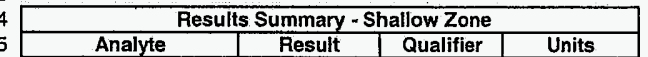

Hexavalent Chromien

7 Carbon-14

8 Cesium-137

Cobalt-60

Europium-152

1 Europium-154

2 Nickel-63

3 Plutonium-239/240

3-Part Test:

$95 \%$ UCL > Cleanup Limit?

$>10 \%$ above Cleanup Limit?

Any sample $>2 \times$ Cleanup Limit?

Risk Estimate:

Nonrad noncarcinogenic index sum: $\quad 1.6 \mathrm{E}-03$

24 Nonrad carcinogenic risk: $\quad 1.9 \mathrm{E}-07$

25

27 Relative.Percent Difference (RPD)

28 Results (Shallow Zone)* QNQC Analysis

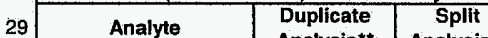

\begin{tabular}{|l|l|l}
30 & Analysis $^{* *}$ & Analysis** \\
\cline { 2 - 3 } & Hexavalent Chromium &
\end{tabular}

31 Carbon-14

32 Cesium-137

33 Cobalt-60

34 Europium-152

35 Europium-154

36 Nickel-63

37 Plutonium-239/240

38 Strontium-90

39 "A blank cell indicates that $\mathrm{RPD}$ evaluation was not required.

40 "'The significance of the reported APD values, inclucing values greater than $30 \%$, is addressed within the Data Quality Assessment for the CVP for this site.

41 QAOC = quality assurance/quality control

42 RESRAD = RESidual RADioactivity (dose model)

43 RPD $=$ relative percent difference

$44 \mathrm{U}=$ undetected

$45 \mathrm{UCL}=$ upper confidence limit

46.
Calc. No. 0100K-CA-V0056

Checked T.M. Blakley 2 mos
Rev. No. $\frac{0}{\text { Date }} \frac{10 /(3 / 05}{2 \text { of } 5}$
Sheet No.

\begin{tabular}{|l|c|c|c|}
\hline \multicolumn{4}{|c|}{ Results Summary - Deep Zone } \\
\hline \multicolumn{1}{|c|}{ Analyte } & Result & Qualifier & Units \\
\hline Hexavalent Chromium & $5.3 \mathrm{E}+00$ & & $\mathrm{mg} / \mathrm{kg}$ \\
\hline Carbon-14 & $1.26 \mathrm{E}+00$ & & $\mathrm{pCi} / \mathrm{g}$ \\
\hline Cesium-137 & $1.30 \mathrm{E}+02$ & & $\mathrm{pCi} / \mathrm{g}$ \\
\hline Cobalt-60 & $2.48 \mathrm{E}+00$ & & $\mathrm{pCi} / \mathrm{g}$ \\
\hline Europium-152 & $6.27 \mathrm{E}+01$ & & $\mathrm{pCi} / \mathrm{g}$ \\
\hline Europium-154 & $5.4 \mathrm{E}+00$ & & $\mathrm{pCi} / \mathrm{g}$ \\
\hline Nickel-63 & $8.8 \mathrm{E}+02$ & & $\mathrm{pCi} / \mathrm{g}$ \\
\hline Plutonium-239/240 & $6.9 \mathrm{E}+00$ & & $\mathrm{pCi} / \mathrm{g}$ \\
\hline Strontium-90 & $7.12 \mathrm{E}+00$ & & $\mathrm{pCi} / \mathrm{g}$ \\
\hline
\end{tabular}

WAC 173-340 Evaluation (Deep Zone)

3-Part Test

$95 \%$ UCL $>$ Cleanup Limit? YES

$\begin{array}{ll}>10 \% \text { above Cleanup LImit? } & \text { YES } \\ \text { Any sample }>2 \times \text { Cleanup Limit? } & \text { YES }\end{array}$

Because of the "yes" answers to the MTCA 3-part test for hexavalent chromium, a detailed assessment will be performed for this contaminant within the CVP.

\begin{tabular}{|l|c|c|}
\hline \multicolumn{3}{|c|}{$\begin{array}{c}\text { Relative Percent Difference (RPD) } \\
\text { Results (Deep Zone) }\end{array}$} \\
\hline \multicolumn{1}{|c|}{ Analyte } & $\begin{array}{c}\text { Duplicate } \\
\text { Analysis** }\end{array}$ & Split Analysis ${ }^{\star *}$ \\
\hline Hexavalent Chromium & $18 \%$ & $42 \%$ \\
\hline Carbon-14 & & \\
\hline Cesium-137 & $11 \%$ & $21 \%$ \\
\hline Cobalt-60 & $15 \%$ & $40 \%$ \\
\hline Europium-152 & $8.7 \%$ & $39 \%$ \\
\hline Europium-154 & $32 \%$ & $34 \%$ \\
\hline Nickel-63 & $17 \%$ & $56 \%$ \\
\hline Plutonium-239/240 & $34 \%$ & $58 \%$ \\
\hline Strontium-90 & $11 \%$ & $28 \%$ \\
\hline
\end{tabular}


CVP-2006-00001

Rev. 0

C-142 


\section{Washington Closure Hanford}

Originator J. M. Capron $\& m_{1} c$

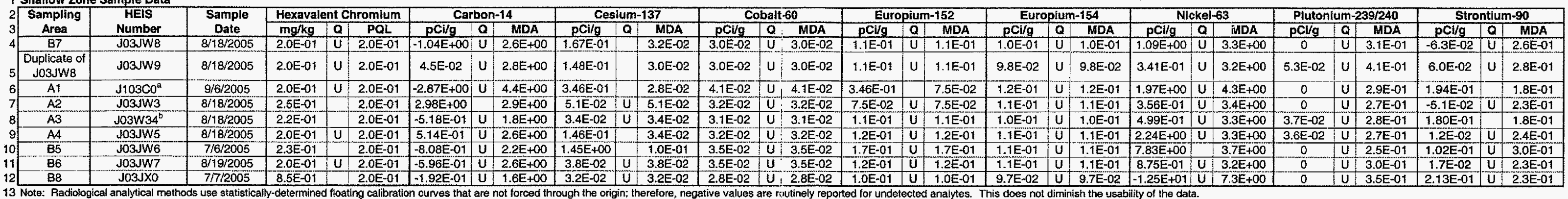

13 Note: Radioiogical analytical methods use
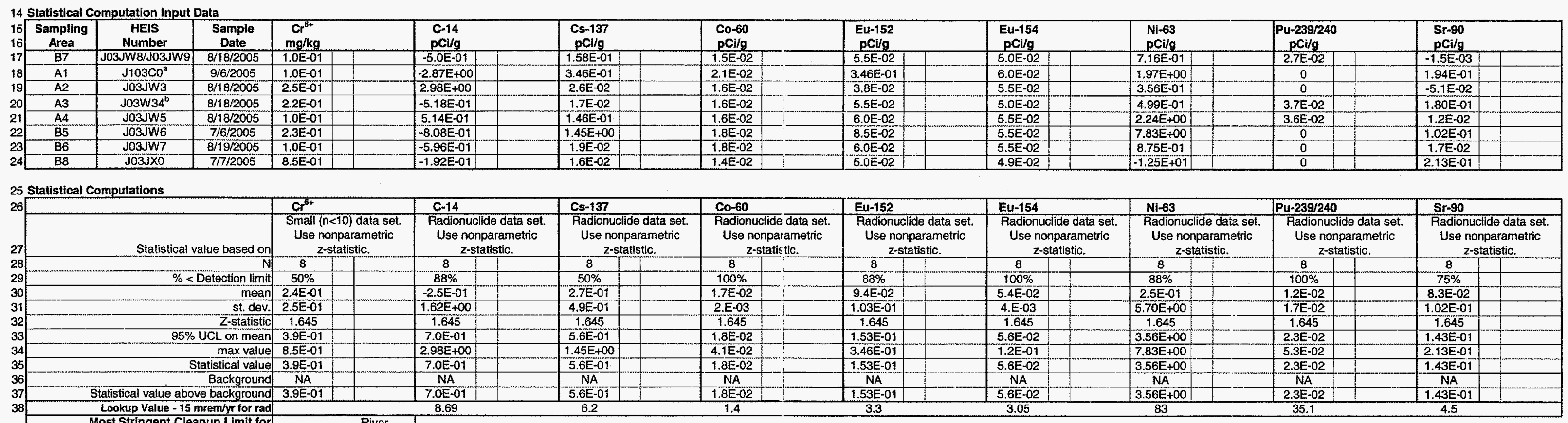

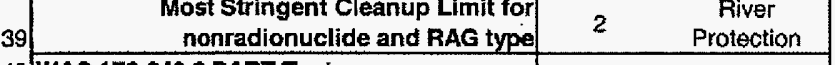

40 WAC 173-340 3-PART Test

$>10 \%$ above Cleanup Limt?

Any sample $>2 \mathrm{X}$
44
44

N Limit?

45 Wa Has-340 Non-Carcinogentic Cleanup: 240

WAC 173-340 Carcinogenic Cleanup: $\frac{1.6 E-03}{21}$

48. Risk for each carcinogenic nonradionuclide: 1 1.9E-07

\begin{tabular}{l|l|l} 
49 & WAC 173-340 3-Part-Test Compliance? & YES \\
50 Nonrad noncarcinogenic index sum: & $1.6 . \mathrm{E}-03$ \\
\hline
\end{tabular}

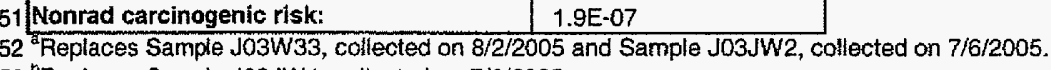

56 NA = not applicable

$Q=$ qualitier
$R A G=$ remedial action goal

$W A C=$ Washington Administrative Code 
Washington Closure Hanford

Originator J.M. Capron $/ 2 \mathrm{C}$

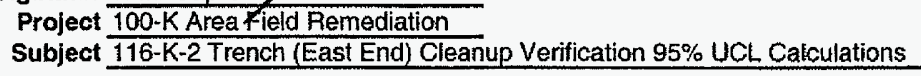

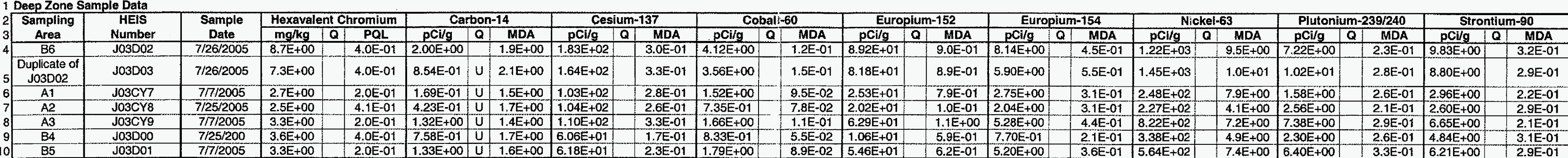

11 Statistical Computation Input Data

\begin{tabular}{|c|c|c|c|c|c|c|c|c|c|c|c|}
\hline \multirow{2}{*}{$\begin{array}{c}\text { Samplings } \\
\text { Aroa } \\
\end{array}$} & $\begin{array}{c}\text { HEIS } \\
\text { Number }\end{array}$ & $\begin{array}{c}\text { Sample } \\
\text { Date }\end{array}$ & $\begin{array}{c}\mathrm{Cr}^{\mathrm{g}^{+}} \\
\mathrm{mg} / \mathrm{kg}\end{array}$ & $\begin{array}{l}\mathrm{C}-14 \\
\mathrm{pCil/g}\end{array}$ & $\begin{array}{l}\mathrm{Cs}-137 \\
\mathrm{pCCl/g}\end{array}$ & 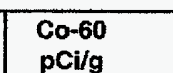 & $\begin{array}{l}\text { Eu-152 } \\
\mathrm{pCl} / \mathrm{g}\end{array}$ & 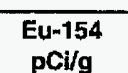 & $\begin{array}{l}\mathrm{N} 1-63 \\
\mathrm{pCCl/g}\end{array}$ & \begin{tabular}{|c|}
$\mathrm{Pu}-239 / 240$ \\
oCC/q
\end{tabular} & $\begin{array}{l}\mathrm{Sr}-90 \\
\mathrm{pCi/g}\end{array}$ \\
\hline & & & & $\frac{1.43 E+00}{1.43 E}$ & $1.74 \mathrm{E}+02$ & & & & & $8.7 E+00$ & $\frac{9.322}{9}+00$ \\
\hline$\frac{\mathrm{A} 1}{\mathrm{~A} 2}$ & $\begin{array}{c}\text { J033Y7 } \\
\text { J03CY8 }\end{array}$ & $\begin{array}{l}77 / 72005 \\
77 / 25 / 2005\end{array}$ & $\frac{2.7 E+00}{2.5 E+00}$ & $\begin{array}{l}\frac{1.69 E-01}{4.23 E-01} \\
4\end{array}$ & $\begin{array}{l}\frac{1.03 E+02}{1.044+02} \\
1.02\end{array}$ & $\mid \begin{array}{l}\mid 1.52 \mathrm{E}+\infty \\
7.35 \mathrm{E}-01\end{array}$ & $\mid \begin{array}{l}2.53 E+01 \\
2.02 E+01\end{array}$ & $\begin{array}{l}2.75 E++00 \\
2.04+00\end{array}$ & $\frac{2.48 E+02}{2.27+02}$ & \begin{tabular}{|c|}
$1.58 \mathrm{E}+00$ \\
$2.56 \mathrm{E}+00$
\end{tabular} & $\begin{array}{l}\frac{2.96 E++00}{2.60+00} \\
2\end{array}$ \\
\hline$\frac{\pi}{A 3}$ & & $-7 \pi / 2005$ & $3.3 E+00$ & $\begin{array}{l}4.520+0 \\
1.32 E+00\end{array}$ & $\begin{array}{l}1,10 \mathrm{E}+02 \\
\end{array}$ & \begin{tabular}{|l|l}
$1.66 E+00$ \\
\end{tabular} & $\frac{6.025+10}{6.29 E+01}$ & & $8.22 E+02$ & $\begin{array}{l}1 \\
7.38 E+00\end{array}$ & 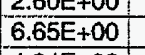 \\
\hline B4 & J03000 & $7 / 25 / 200$ & $\begin{array}{l}3.6 \mathrm{E}+00 \\
3.3 \mathrm{~F}+0\end{array}$ & \begin{tabular}{|l|}
$7.58 E-01$ \\
$133+E$
\end{tabular} & \begin{tabular}{|l|}
$6.06 E+01$ \\
$618 E+01$
\end{tabular} & \begin{tabular}{|l|}
$8.33 E-01$ \\
$19 F+0$
\end{tabular} & $1.06 \mathrm{E}+01$ & $\begin{array}{l}7.70 E-01 \\
5200+01\end{array}$ & $\frac{3.38 E+02}{5.64502}$ & $2.30 \mathrm{E}+00$ & $4.84 E+00$ \\
\hline
\end{tabular}

20 Statistical Computations

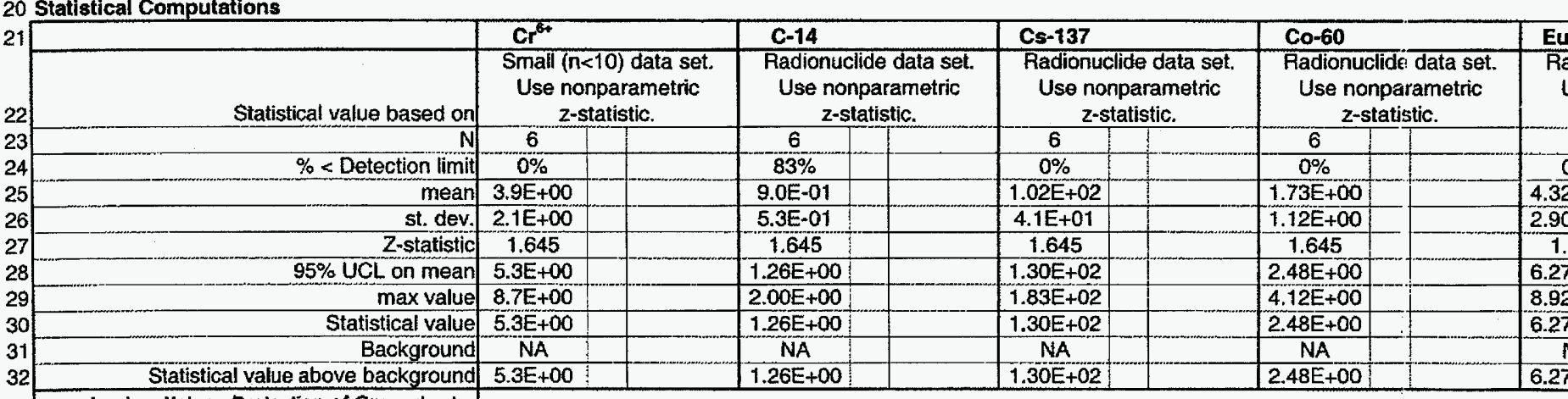

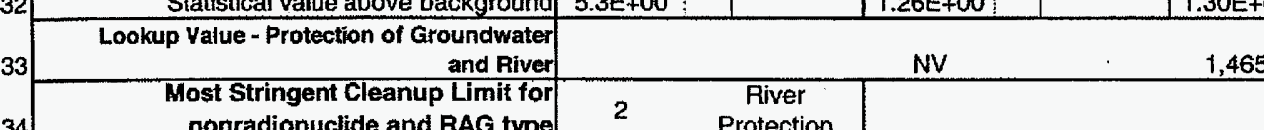

, 465

13,900

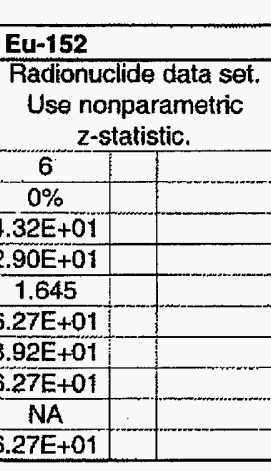

34 nonradionuclide and RAG typ

$95 \%$ UCL $>$ Cleanup Limit? Y YES

$>10 \%$ above Cleanup Limit? YES

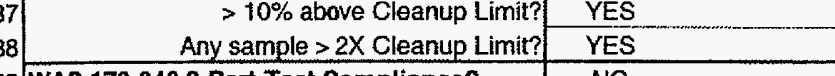

39 WAC 173-340 3-Part-Test Compliance?

$41 \mathrm{MDA}=$ minimum detactable aclivity

$42 \mathrm{NA}=$ not applicable

$Q=$ qualitier
$R A G=$ remedial action goal

WAC $=$ - Watect

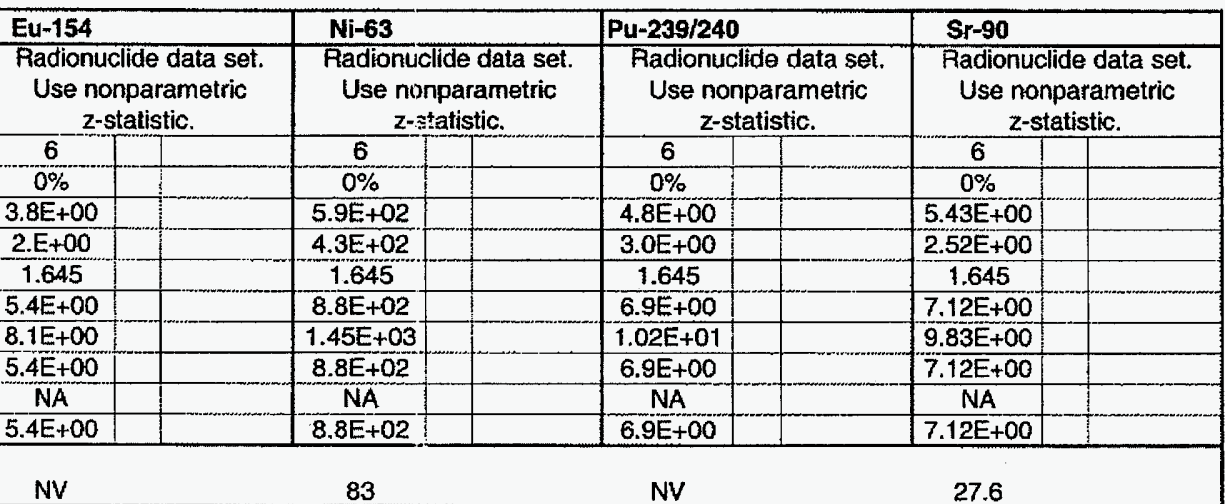
. . 


\section{Washington Closure Hanford}

Originator J.M. Capren

Project
Subject 1100 -K Ar-Kea Fleld Remediation
116rench (East End) Cleanup Veritication 95\% UCL Calculations

Split-Duplicate Analysis

\begin{tabular}{|c|c|c|c|}
\hline $\begin{array}{c}\text { Sampling } \\
\text { Area }\end{array}$ & & Hexavalent Chromium & Carbon-14 \\
\hline $\begin{array}{c}\text { Area } \\
\text { B7 } \\
\end{array}$ & 1031108 & \begin{tabular}{c|c|c|}
$\mathrm{mg} / \mathrm{kg}$ & $\mathrm{Q}$ & $\mathrm{PQL}$ \\
$2.0 \mathrm{E}-01$ & $\mathrm{U}$ & $2.0 \mathrm{E}-01$
\end{tabular} & $\begin{array}{l}\text { Q } \\
2.6 E+00\end{array}$ \\
\hline$\frac{\text { Duplicate of J03JW8 }}{\text { Solit of } 03 \text { W.W8 }}$ & 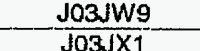 & \begin{tabular}{|c|c|c|c|}
$2.0 \mathrm{E}-01$ & $\mathrm{U}$ & $2.0 \mathrm{E}-01$ \\
$35 \mathrm{O}+00$ & $35 \mathrm{~F}$ \\
\end{tabular} & 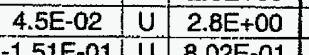 \\
\hline allow Zone Analys & & & \\
\hline & Both > MDA? & No-Stop (acceppable) & No-Stop (acceptable) \\
\hline Duplicate Analysis & $\begin{array}{c}\text { Both }>5 \text { 5TDL? } \\
\text { RPD }\end{array}$ & & \\
\hline Spitt Analysis & $\begin{array}{l}\text { Both > MDA? } \\
\text { Both } 5 \times \text { STDL? } \\
\text { PDD }\end{array}$ & No-Stop (acceptable) & No-Stop (acceptable) \\
\hline
\end{tabular}

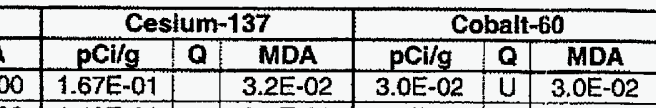

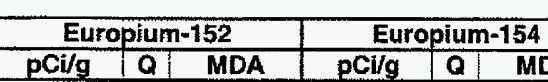

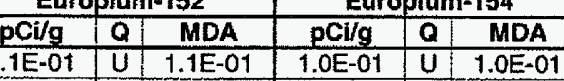

\begin{tabular}{ccccc}
$48 E-01$ & $3.0 E-02$ & $3.0 E-02$ & $\mathrm{U}$ & $3.0 E-02$ \\
$4.04 E-01$ & $1.00 E-02$ & $3.99 E-06$ & $\mathrm{u}$ & $1.54 E-02$ \\
\hline
\end{tabular}

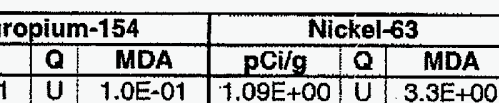

Calc. No. $0100 k$-CA-V0056

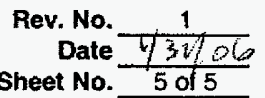

\begin{tabular}{c} 
Yes (continue) \\
\hline No-Stop (acceptable) \\
Yes (continue)
\end{tabular}

No-Stop (acceptable) 15

17 Deep Zone Sample Results:

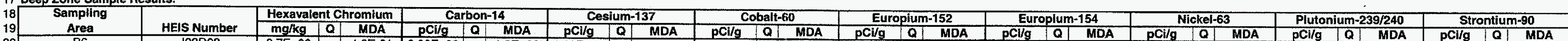

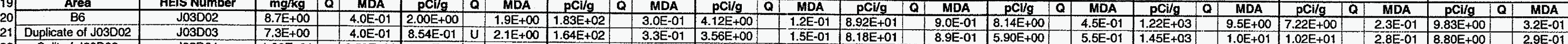

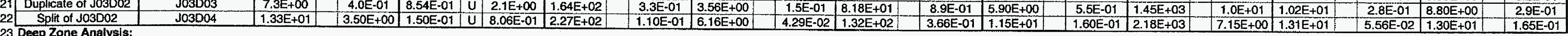

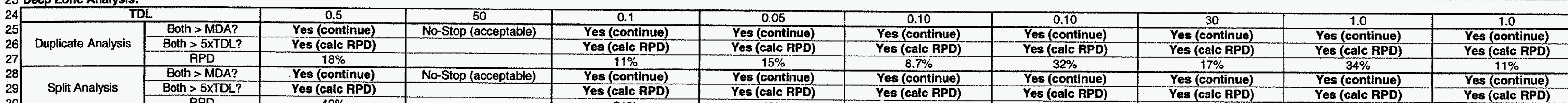

Split Analysis

\begin{tabular}{l|l} 
& Yes (continue \\
\hline Both $>$ 5XTDL? & Yes (calc RPD) \\
\hline
\end{tabular}

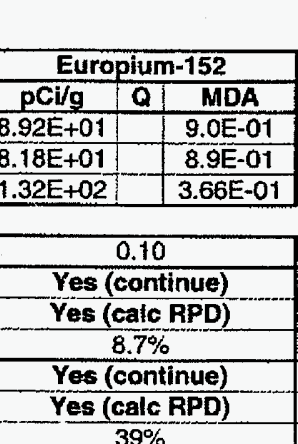

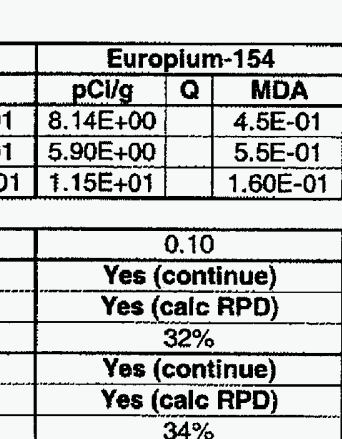

Yes (cantic RPD) Yes $($ calc RPD)
$598 \%$ Yes (continue)
Yes (calc RPD)

31 Note: The significance of the reported APD values, including values greater than $30 \%$, is addressed within the Data Ouality Assessment for the Cleanup Verification Package for this sito 33 MDA = minimum dotectable activity

$35 \mathrm{Q}=$ qualifitior

$37 \mathrm{RPD}=$ relative percent differences

$38 \mathrm{TDL}=$ target detect
$39 \mathrm{U}=$ undetected 


\section{CALCULATION COVER SHEET}

Project Title $100-\mathrm{K}$ Field Remediation Project

Job No. 14655

Area 100-K Area

Discipline Environmental *Calc. No. 0100K-CA-V0058

Subject 116-K-2 Trench (East End) RESRAD Calculation

Computer Program RESRAD Program No.___ Version 6.3

The attached calculations have been generated to document compliance with established cleanup levels.

These documents should be used in conjunction with other relevant documents in the administrative record.

\section{Committed Calculation $\otimes \quad$ Preliminary $\square \quad$ Superseded $\square \quad$ Voided $\square$}

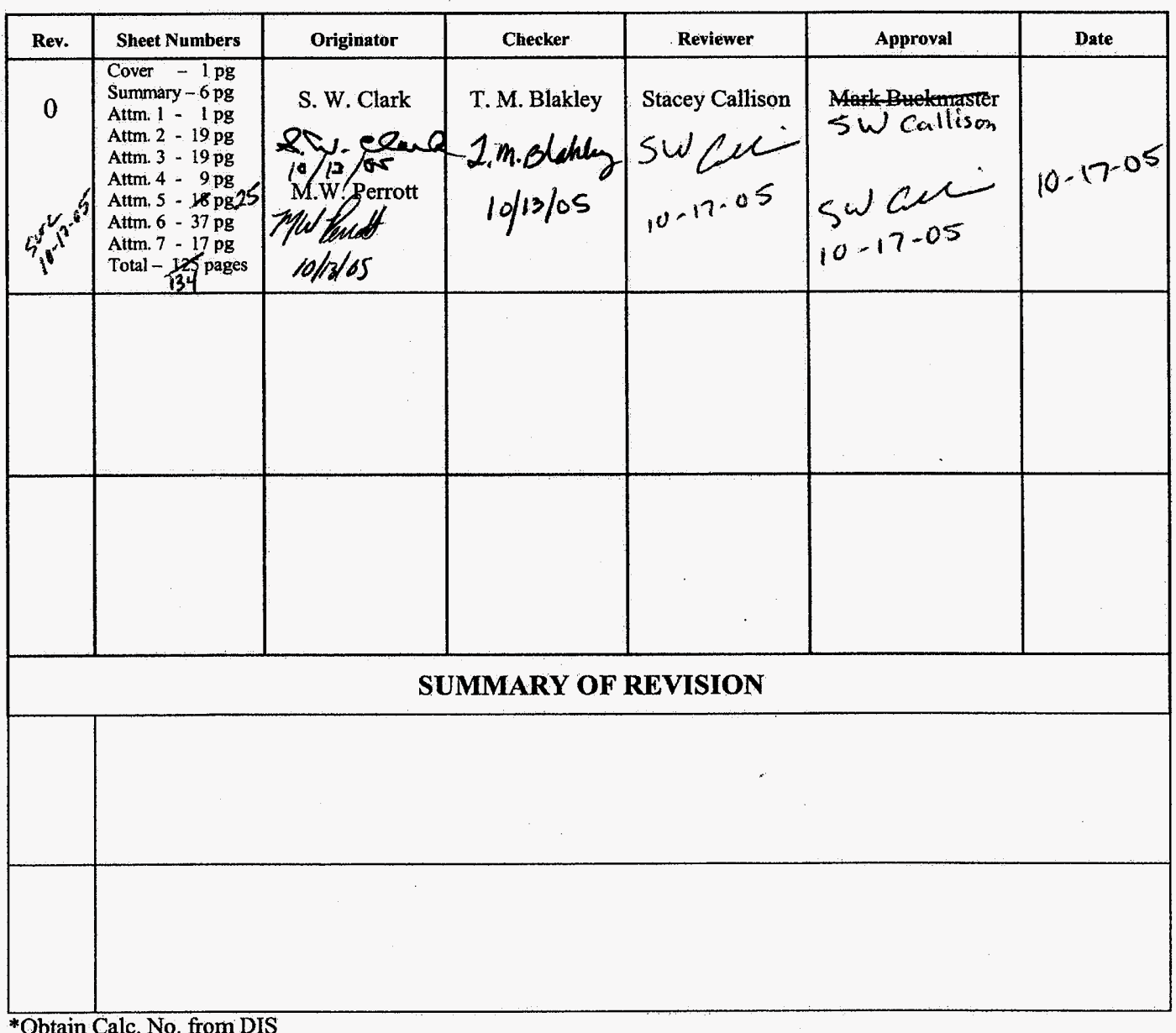

DE01-437.03 (12/09/2004) 
CVP-2006-00001

Rev. 0

\section{MIMIVer Corridor}

Closure Projegt CALCULATION SHEET

\begin{tabular}{|c|c|c|c|c|c|c|}
\hline Originator: & S. W. Clark/M.W.Perrott & Date: $1 / 3 / 05$ & Calc. No.: & $0100 \mathrm{~K}-\mathrm{CA}-\mathrm{V} 0058$ & Rev.: & 0 \\
\hline Project: & 100-K Field Remediation Project & Job No: $/ 74655$ & Checked: & T. M. Blakley $\operatorname{Lin} B$ & Date: & $10 / 13 / 05$ \\
\hline Subject: & \multicolumn{4}{|c|}{ 116-K-2 Trench (East End) RESRAD Calculation } & \multicolumn{2}{|c|}{ Sheet No. 1 of 6} \\
\hline
\end{tabular}

\section{PURPOSE:}

Calculate the soil and groundwater concentrations, dose, and risk contributions from remaining radionuclide contaminants in the vadose zone over a period of 1,000 years.

\section{GIVEN/REFERENCES:}

1) Cleanup verification data from $116-K-2$ Trench (East End) Cleanup Verification $95 \%$ UCL Calculations, Calculation No. 0100K-CA-V0056, Rev. 0, Bechtel Hanford, Inc., Richland, Washington.

2) Remedial Design Report/Remedial Action Work Plan for the 100 Area (RDR/RAWP), DOE/RL-96-17, Rev. 5, U.S. Department of Energy, Richland Operations Office, Richland, Washington.

3) Radioactive and nonradioactive contaminants of concern from the 100 Area Remedial Action Sampling and Analysis Plan (100 Area SAP), DOE/RL-96-22, Rev. 4, U.S. Department of Energy, Richland Operations Office, Richland, Washington. For the purpose of these RESRAD calculations, the radioactive contaminants of concern (COCs) are carbon-14, cesium-137, cobalt-60, europium-152, europium-154, nickel63 , and plutonium $-239 / 240$. Hexavalent chromium is the only nonradionuclide contaminant of concern

4) RESidual RADioactivity (RESRAD) computer code, version 6.3, to calculate compliance with residual radioactivity guidelines, developed for the U.S. Department of Energy by the Environmental Assessment Division of Argonne National Laboratory, Argonne, Illinois.

5) Sample design data from the 116-K-2 Trench (East End) Sampling Plan, Calculation No. 0100K-CA-V0057, Rev. 0, Bechtel Hanford, Inc., Richland, Washington. For the purpose of these RESRAD calculations, the sampling areas identified in the sampling plan calculation brief are $8653.26 \mathrm{~m}^{2}$ for the Shallow Zone and $9140.52 \mathrm{~m}^{2}$ for the Deep Zone. Total sampling area of $17,794 \mathrm{~m}^{2}$ was used for all RESRAD calculations.

6) Plutonium isotope ratios from Ratios of Plutonium Isotopes at 100 Areas Remedial Action Sites, Calculation No. 0100B-CA-V0013, Rev. 0, Bechtel Hanford, Inc., Richland, Washington.

7) Thickness of uncontaminated deep zone determined using analogous site data for the 116-C-1 test pit from Cleanup Verification Package for the 116-C-1 Process Effluent Trench, CVP-98-00006, Rev. 0, Bechtel Hanford, Inc., Richland, Washington.

8) Attainment of the river protection RAGs is based on comparison of results from the 100 F Area Soil Hexavalent Chromium and Carbon-14 Leachability Study Summary Report (Appendix D of the Cleanup Verification Package for the 100-F-19:1 North Pipelines, 100-F-19:3 West Pipelines, 100-F-34 Biology Facility French Drain, and 116-F-12 French Drain, CVP-2001-00002, Rev. 0, Bechtel Hanford, Inc., Richland, Washington.), 100-H Area Soil Hexavalent Chromium Leachability Study Summary Report (Appendix D of the Cleanup Verification Package for the 116-H-7 Retention 


\begin{tabular}{|c|c|c|c|c|c|c|}
\hline Originator: & S.W. Clark / M.W. Perrott MWV & Date: $1 / 2 / 05$ & Calc. No.: & $0100 \mathrm{~K}-\mathrm{CA}-\mathrm{V} 0058$ & Rev.: & 0 \\
\hline Project: & 100-K Field Remediation Project & Job N6: 14655 & Checked: & T. M. Blakley $2 \ln \infty$ & Date: & $10 / 13 / 05$ \\
\hline
\end{tabular}

Basin, CVP-2000-00027, Rev. 0, Bechtel Hanford, Inc., Richland, Washington), and Estimation of Distribution Coefficients and Leachability of Hexavalent Chromium in 100-D Area Hanford Formation Sediments (Appendix D of the Cleanup Verification Package for the 116-D-7 Retention Basin, CVP-99-00007, Rev. 0, Bechtel Hanford, Inc., Richland, Washington).

\section{SOLUTION:}

1) Table 1 shows the waste site parameters used for RESRAD modeling. Based on the conservative assumption (discussed in the 100 Area RDR/RAWP) that residual contamination levels from the deep zone data set extend uniformly to groundwater, RESRAD predicted that the residual soil concentration of nickel-63 would result in groundwater concentrations that exceed the drinking water standard. Because this approach is too conservative, test pit data from the 116-C-1 Process Effluent Trench was used to include an uncontaminated deep zone between the contaminated deep zone and groundwater to more accurately describe the $116-\mathrm{K}-2$ site for modeling using RESRAD.

2) Table 2 shows the radionuclide activities for each COC. Input factors for each RESRAD run are shown in the "Summary" section of the RESRAD "Mixture Sums and Single Radionuclide Guidelines" printouts in Attachments to this Calculation Summary.

3) The year where the peak dose (or concentration) occurs from each individual radionuclide $\mathrm{COC}$ was determined by a preliminary run. This year was then included in all final RESRAD runs. For the direct exposure pathway (i.e. soil ingestion and inhalation and external radiation), the peak year occurred at year zero (year 2005) for all COCs. For the water pathways (i.e., drinking water and food ingestion) the peak year also occurred at year zero (year 2005) for all COCs.

Table 1. Waste Site Dimensions for RESRAD Modeling

\begin{tabular}{|c|c|c|c|}
\hline $\begin{array}{r}\text { Parameter } \\
\end{array}$ & Units & Value & Comments \\
\hline Shallow Zone Cover Depth & $\mathrm{m}$ & 0 & \\
\hline Area of Shallow and Deep Zone & $\mathrm{m}^{2}$ & 17794 & Based on Sample Design ${ }^{2}$ \\
\hline Thickness: Shallow Zone & $\mathrm{m}$ & 4.6 & Appendix B of 100 Area RDR/RAWP \\
\hline Elevation: Ground Surface & $\mathrm{m}$ & 133.5 & NAVD88 \\
\hline Elevation: Groundwater & $\mathrm{m}$ & 118.5 & NAVD88 \\
\hline Thickness: Total Deep Zone & $\mathrm{m}$ & 10.4 & Vadose zone thickness minus shallow zone \\
\hline Thickness: Uncontaminated Deep Zone & $\mathrm{m}$ & 3.1 & Based on deep zone model from 116-C-1 test pit ${ }^{b}$ \\
\hline Thickness: Contaminated Deep Zone & $\mathrm{m}$ & 7.3 & Deep zone thickness minus uncontaminated deep zone \\
\hline Length Parallel to Aquifer Flow & $\mathrm{m}$ & 292 & Based on Sample Design ${ }^{\text {a }}$ \\
\hline
\end{tabular}




\section{River Corridor}

\section{METHODOLOGY:}

1) Runs of RESRAD version 6.3 were completed for the shallow zone and deep zone using the radionuclide concentrations shown in Table 2. RESRAD numerical output reports for dose, risk, and concentration for the shallow zone and deep zone are presented in the Attachments to this calculation summary.

\begin{tabular}{|c|c|c|}
\hline \multicolumn{3}{|c|}{ Table 2. Cleanup Verification Data Set ${ }^{\text {a }}$} \\
\hline COCs & Shallow Zone & Deep Zone \\
\hline \multicolumn{3}{|c|}{ Radionuclide Activity ( $p C i / g$ ) } \\
\hline $\mathrm{C}-14$ & 0.70 & 1.26 \\
\hline Cs-137 & 0.56 & 130 \\
\hline $\mathrm{Co}-60$ & $0.018 \mathrm{U}^{\mathrm{b}}$ & 2.48 \\
\hline Eu-152 & 0.153 & 62.7 \\
\hline Eu-154 & $0.056 \mathrm{U}^{\mathrm{b}}$ & 5.4 \\
\hline $\mathrm{Ni}-63$ & 3.56 & 880 \\
\hline $\mathrm{Pu}-239 / 240$ & $0.023 \mathrm{U}^{\mathrm{b}}$ & 6.9 \\
\hline Sr-90 & 0.143 & 7.12 \\
\hline \multicolumn{3}{|c|}{ Nonradionuclide Concentration $(\mathrm{mg} / \mathrm{Kg}$ ) } \\
\hline $\mathrm{Cr}$ (VI) & 0.39 & 5.3 \\
\hline $\begin{array}{l}\text { a Cleanup verification data } \\
\text { Calculation No. 0100K- } \\
\text { b Value was not imput into }\end{array}$ & $\begin{array}{l}\text { Trench (East End) } C \\
\text { Bechtel Hanford, In } \\
\text { all analyses were bel }\end{array}$ & $\begin{array}{l}5 \% \text { UCL Calculations. } \\
\text { gton. }\end{array}$ \\
\hline
\end{tabular}

2) $\mathrm{Pu}-239 / 240$ Conversion: The relative individual $\mathrm{Pu}-239$ and $\mathrm{Pu}-240$ activities used in the RESRAD model were calculated from the reported combined $\mathrm{Pu}-239 / 249$ analytical results. The calculations were performed in accordance with Calculation No. 0100B-CA-V0013. The relative activities for $\mathrm{Pu}-239$ and $\mathrm{Pu}-240$ were calculated by multiplying the cleanup verification values for $\mathrm{Pu}-239 / 240$ in each decision unit of the site by 0.807 and 0.193 , respectively. Table 3 shows the results of these calculations.

Table 3. Conversion of Pu-239/240 Activity to Relative Pu-239 and Pu-240 Activities

\begin{tabular}{|c|c|c|c|c|}
\hline $\begin{array}{c}\text { RESRAD } \\
\text { Run \# }\end{array}$ & $\begin{array}{c}\text { Vadose Zone } \\
\text { Horizon }\end{array}$ & $\begin{array}{c}\text { Pu-239/240 Activity } \\
\text { (pCi/g) }\end{array}$ & $\begin{array}{c}\text { Pu-239, (pCi/g) } \\
(\mathbf{0 . 8 0 7} \text { multiplier) }\end{array}$ & $\begin{array}{c}\text { Pu-240, (pCi/g) } \\
(\mathbf{0 . 1 9 3} \text { multiplier) }\end{array}$ \\
\hline 1 & Shallow Zone & $0.023 \mathrm{U}$ & $0.0186 \mathrm{U}$ & $.0044 \mathrm{U}$ \\
\hline 2 & Deep Zone & 6.9 & 5.57 & 1.33 \\
\hline
\end{tabular}

3) Protectiveness of residual hexavalent chromium soil concentrations: Shallow zone soil concentrations of hexavalent chromium represented by the $95 \%$ UCL value in 
CVP-2006-00001

Rev. 0

\section{River Corridor}

Closure Project

- CALCULATION SHEET

\begin{tabular}{|c|c|c|c|c|c|}
\hline Originator: & S. W. Clark TM.W. Perrott MWP & Date: $1 / 3 / a r$ & Calc. No.: & $0100 \mathrm{~K}-\mathrm{CA}-\mathrm{V} 0058$ & Rev.: \\
\hline
\end{tabular}

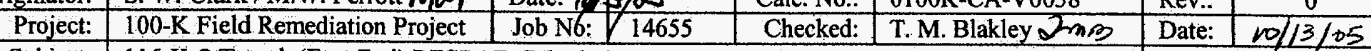

\begin{tabular}{l|l|l} 
Subject: & $116-\mathrm{K}-2$ Trench (East End) RESRAD Calculation & Sheet No. 4 of 6
\end{tabular}

Table 2 are less than the applicable RAGs (i.e., $2.1 \mathrm{mg} / \mathrm{kg}$ for direct exposure or 2.0 $\mathrm{mg} / \mathrm{kg}$ for river protection). However, the deep zone hexavalent chromium soil concentration exceeds the applicable RAG of $2.0 \mathrm{mg} / \mathrm{kg}$ for river protection. Because this RAG was not met for the deep zone soils, a more detailed assessment of the potential impact of residual hexavalent chromium to groundwater was made using the 100-F, 100-H, and 100-D Area-specific hexavalent chromium leach studies (Appendix D of CVP-2001-00002, CVP-2000-00027, and CVP-99-00007). Using the simple and conservative approach of comparing the residual soil concentration of hexavalent chromium to the soil concentrations used in leach testing, and comparing the leachate concentrations from the leach tests to the surface water hexavalent chromium cleanup level, indicates that remaining hexavalent chromium in deep zone soil at the 116-K-2 site is protective of the river. The river protection soil RAG of 2.0 $\mathrm{mg} / \mathrm{kg}$ is based on the MTCA " 100 times dilution attenuation factor (DAF) times surface water quality criteria" rule. The near-shore river water hexavalent chromium cleanup level is $20 \mu \mathrm{g} / \mathrm{L}$ (surface water quality criteria of $10 \mu \mathrm{g} / \mathrm{L}$ times the dilution attenuation factor of 2). Based on the hexavalent chromium leach studies, the hexavalent chromium soil concentration at which the leachate did not exceed the surface water quality criteria was $6.8 \mathrm{mg} / \mathrm{kg}$. Therefore, using the simple and conservative approach of comparing the hexavalent chromium deep zone soil concentration to the leach study hexavalent chromium soil concentrations demonstrates that cleanup of the deep zone hexavalent chromium is protective of the river (i.e., $5.3 \mathrm{mg} / \mathrm{kg}$ is less than $6.8 \mathrm{mg} / \mathrm{kg}$ ).

\section{RESULTS:}

1) Radionuclide "All Pathways" Dose Rate: The "all pathways" (maximum) dose rates are shown in Table 4. The maximum total all-pathways dose rate is $4.62 \mathrm{mrem} / \mathrm{yr}$ which occurs at year zero (2005).

\begin{tabular}{|c|c|c|c|c|c|c|c|c|}
\hline \multicolumn{7}{|c|}{ Table 4. All Pathway Dose Rate (mrem/yr) } \\
\hline $\begin{array}{c}\text { Vadose Zone } \\
\text { Horizon }\end{array}$ & 0 & 1 & 3 & 13 & 30 & 100 & 300 & 1000 \\
\cline { 2 - 9 } & 0 & 1 & All Pathways" Dose Contributions in mrem/yr at each time slice (yr) & \\
\hline Shallow Zone & $4.62 \mathrm{E}+00$ & $3.13 \mathrm{E}+00$ & $2.49 \mathrm{E}+00$ & $1.83 \mathrm{E}+00$ & $1.14 \mathrm{E}+00$ & $2.01 \mathrm{E}-01$ & $3.66 \mathrm{E}-03$ & $9.73 \mathrm{E}-06$ \\
\hline Deep Zone & $1.29 \mathrm{E}-22$ & $1.16 \mathrm{E}-22$ & $9.37 \mathrm{E}-23$ & $3.49 \mathrm{E}-23$ & $9.01 \mathrm{E}-24$ & $4.21 \mathrm{E}-06$ & $4.46 \mathrm{E}-04$ & $9.89 \mathrm{E}-04$ \\
\hline $\begin{array}{c}\text { Total All } \\
\text { Pathways Dose } \\
\text { Rate (mrem/yr) }\end{array}$ & $4.62 \mathrm{E}+00$ & $3.13 \mathrm{E}+00$ & $2.49 \mathrm{E}+00$ & $1.83 \mathrm{E}+00$ & $1.14 \mathrm{E}+00$ & $2.01 \mathrm{E}-01$ & $4.10 \mathrm{E}-03$ & $9.99 \mathrm{E}-04$ \\
\hline
\end{tabular}


CVP-2006-00001

Rev. 0

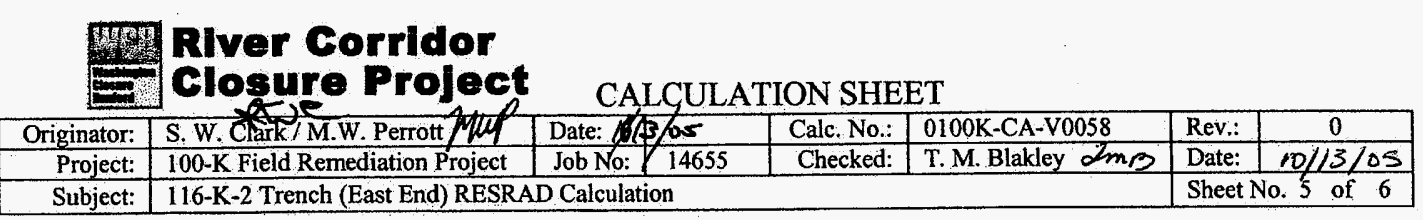

2) Radionuclide Excess Cancer Risk: The radionuclide excess lifetime cancer risk results are shown in Table 5. The maximum total excess lifetime cancer risk ( $4.39 \mathrm{x}$ $10^{-5}$ ) occurs at year zero (2005).

\begin{tabular}{|c|c|c|c|c|c|c|c|c|}
\hline \multicolumn{7}{|c|}{ Table 5. Radionuclide Excess Lifetime Cancer Risk } \\
\hline $\begin{array}{c}\text { Vadose Zone } \\
\text { Horizon }\end{array}$ & 0 & 1 & 3 & 13 & 30 & 100 & 300 & 1000 \\
\cline { 2 - 9 } & 0 & 1 & \multicolumn{7}{c|}{ Excess Cancer Risk at Each Time Slice (y) } \\
\hline Shallow Zone & $4.39 \mathrm{E}-05$ & $3.94 \mathrm{E}-05$ & $3.61 \mathrm{E}-05$ & $2.72 \mathrm{E}-05$ & $1.74 \mathrm{E}-05$ & $3.33 \mathrm{E}-06$ & $1.14 \mathrm{E}-07$ & $4.33 \mathrm{E}-10$ \\
\hline Deep Zone & $9.31 \mathrm{E}-28$ & $8.45 \mathrm{E}-28$ & $6.99 \mathrm{E}-28$ & $2.76 \mathrm{E}-28$ & $6.00 \mathrm{E}-29$ & $1.36 \mathrm{E}-10$ & $3.75 \mathrm{E}-09$ & $7.76 \mathrm{E}-09$ \\
\hline $\begin{array}{c}\text { Total Excess } \\
\text { Cancer Risk }\end{array}$ & $4.39 \mathrm{E}-05$ & $3.94 \mathrm{E}-05$ & $3.61 \mathrm{E}-05$ & $2.72 \mathrm{E}-05$ & $1.74 \mathrm{E}-05$ & $3.33 \mathrm{E}-06$ & $1.17 \mathrm{E}-07$ & $8.19 \mathrm{E}-09$ \\
\hline
\end{tabular}

3) Radionuclide Groundwater Protection: The radionuclide concentrations in groundwater calculated by the RESRAD model are summarized in Table 6 . Because all concentrations presented here are zero no comparison to drinking water standards calculation was necessary.

Table 6. RESRAD Predicted Radionuclide Groundwater (Well Water) Concentrations

\begin{tabular}{|c|c|c|c|c|c|c|c|c|c|c|}
\hline \multirow{2}{*}{$\begin{array}{c}\text { Radio- } \\
\text { nuclides }\end{array}$} & $\begin{array}{c}\text { Vadose Zone } \\
\text { Horizon }\end{array}$ & \multicolumn{6}{|c|}{ Groundwater Concentrations in pCi/L at Each Time Slice (yr) } & \multicolumn{2}{c|}{$\begin{array}{c}\text { RAGs } \\
\text { From RDR }\end{array}$} \\
\cline { 2 - 11 } & & 0 & 1 & 3 & 13 & 30 & 100 & 300 & 1000 & \\
\hline C-14 & Total & 0 & 0 & 0 & 0 & 0 & 0 & 0 & 0 & 2000 \\
\hline Co-60 & Total & 0 & 0 & 0 & 0 & 0 & 0 & 0 & 0 & 100 \\
\hline Cs-137 & Total & 0 & 0 & 0 & 0 & 0 & 0 & 0 & 0 & 60 \\
\hline Eu-152 & Total & 0 & 0 & 0 & 0 & 0 & 0 & 0 & 0 & 200 \\
\hline Eu-154 & Total & 0 & 0 & 0 & 0 & 0 & 0 & 0 & 0 & 60 \\
\hline $\mathrm{Ni}-63$ & Total & 0 & 0 & 0 & 0 & 0 & 0 & 0 & 0 & 50 \\
\hline $\mathrm{Sr}-90$ & Total & 0 & 0 & 0 & 0 & 0 & 0 & 0 & 0 & 8 \\
\hline
\end{tabular}

\section{CONCLUSIONS:}

- The combined maximum all-pathways dose rate for the shallow and deep zones shown in Table 4 is $4.62 \mathrm{mrem} / \mathrm{yr}$ which occurs at year zero (2005).

- The dominant pathway for the dose rate is direct external exposure.

- The primary radionuclide contributing to the direct exposure pathway is cesium-137.

- None of the site COCs are projected to exceed remedial action goals (RAGs). 
CVP-2006-00001

Rev. 0

\begin{tabular}{|c|c|c|c|c|c|c|}
\hline \multicolumn{7}{|c|}{ CALCULATION SHEET } \\
\hline Originator: & S. W. Clark / M.W. Perrott / of & Date: 10 / & Calc. No.: & 0100K-CA-V0058 & Rev.: & 0 \\
\hline Project: & 100-K Field Remediation Project & Job No: 14655 & Checked: & T. M. Blakley $\operatorname{sm} \beta$ & Date: & $3 / 05$ \\
\hline Subject: & 116-K-2 Trench (East End) RESR & Calculation & & & Sheet & \\
\hline
\end{tabular}

- The maximum combined shallow and deep zone excess lifetime cancer risk shown in Table $5\left(4.39 \times 10^{-5}\right)$ occurs at year zero (2005).

- None of the radionuclide contaminants of concern are predicted to reach groundwater in the 1,000 years of the RESRAD model runs.

\section{ATTACHMENTS:}

1. Graphic showing 116-K-2 Cleanup Verification Model (1 page)

2. RESRAD Output: 116-K-2 Shallow Zone Radionuclides, Mixture Sums and Single Radionuclide Guidelines (19 pages)

3. RESRAD Output: 116-K-2 Shallow Zone Radionuclides, Intake Quantities and Health Risk Factors (19 pages)

4. RESRAD Output: 116-K-2 Shallow Zone Radionuclides, Concentration of Radionuclides, ( 9 pages)

5. RESRAD Output: 116-K-2 Deep Zone Radionuclides, Mixture Sums and Single Radionuclide Guidelines (18 pages)

6. RESRAD Output: 116-K-2 Deep Zone Radionuclides, Intake Quantities and Health Risk Factors (37 pages)

7. RESRAD Output: 116-K-2 Deep Zone Radionuclides, Concentration of Radionuclides, (17 pages) 
CVP-2006-00001

Rev. 0

\section{ATTACHMENT 1}

116-K-2 Trench Cleanup Verification Model
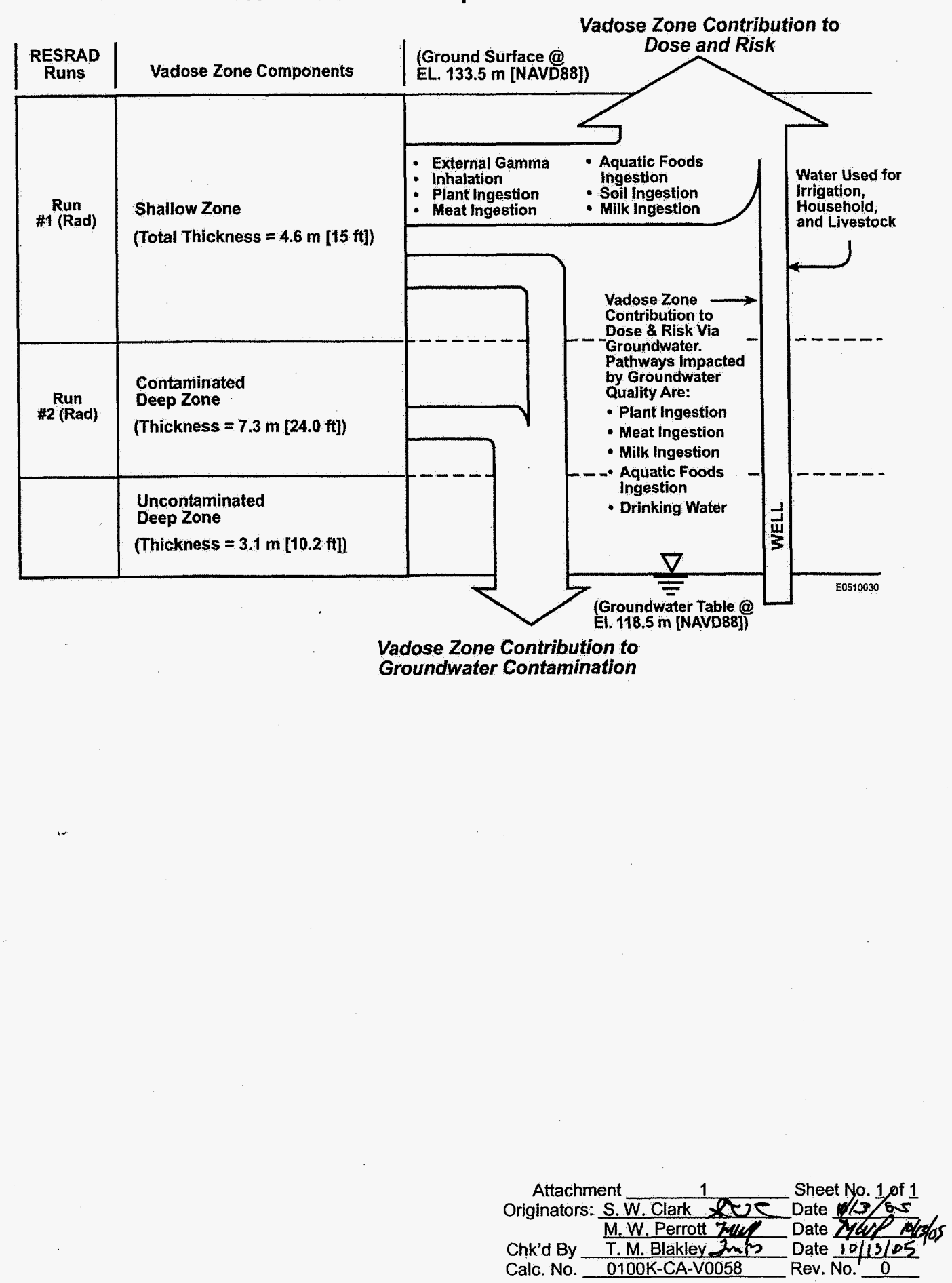


\section{CALCULATION COVER SHEET}

Project Title:

Area

Discipline

Subject

Computer Program
116-K-2 (West End) Trench Sample Design $100-\mathrm{K}$

Environmental Engineering

116-K-2 (West End) Trench Shallow, Deep Zone

and Overburden Sampling Plan

Excel

Program No.
14655

The attached calculations have been generated to document compliance with established cleanup levels. These documents should be used in conjuction with other relevent documents in the administrative record.

$\begin{array}{lllll}\text { Committed Calculation } & \text { Preliminary } & \square \quad \text { Superseded } & \square \quad \text { Voided }\end{array}$

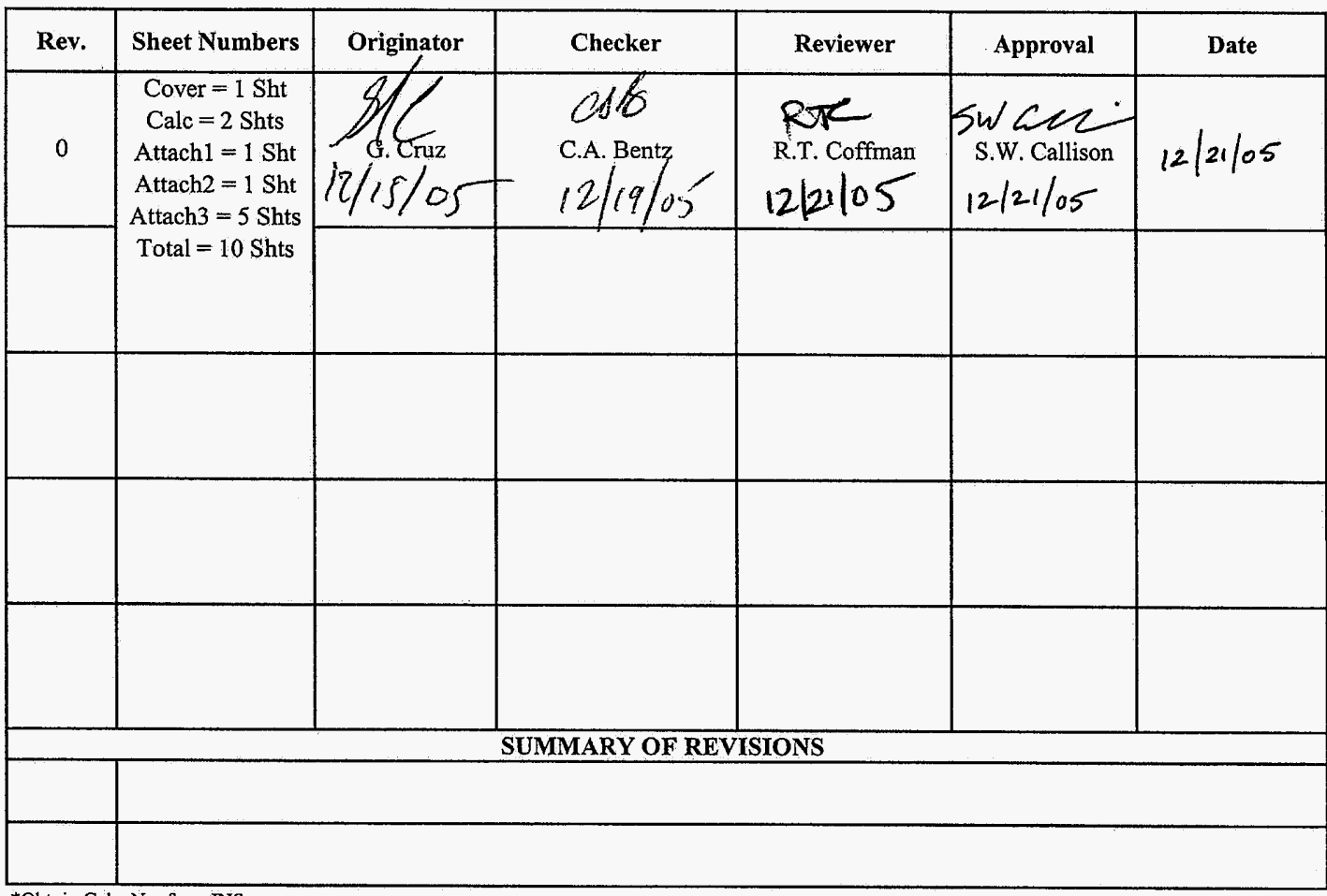

*Obtain Calc, No. from DIS

DE01437.03 (12/09/2004) 
CVP-2006-00001

Rev. 0

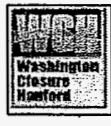

Washingtgh Closure Hanford

Originato

Date 12/15/2005

Project

116-K-2 (West End) Trench Sample Design

116-K-2 (West End) Trench Shallow, Deep Zone

Subject and Overburden Sampling Plan

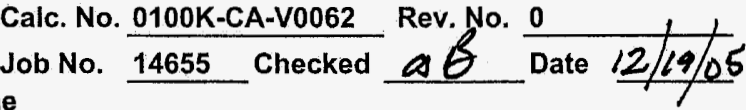

Sheet No. 1 of 2

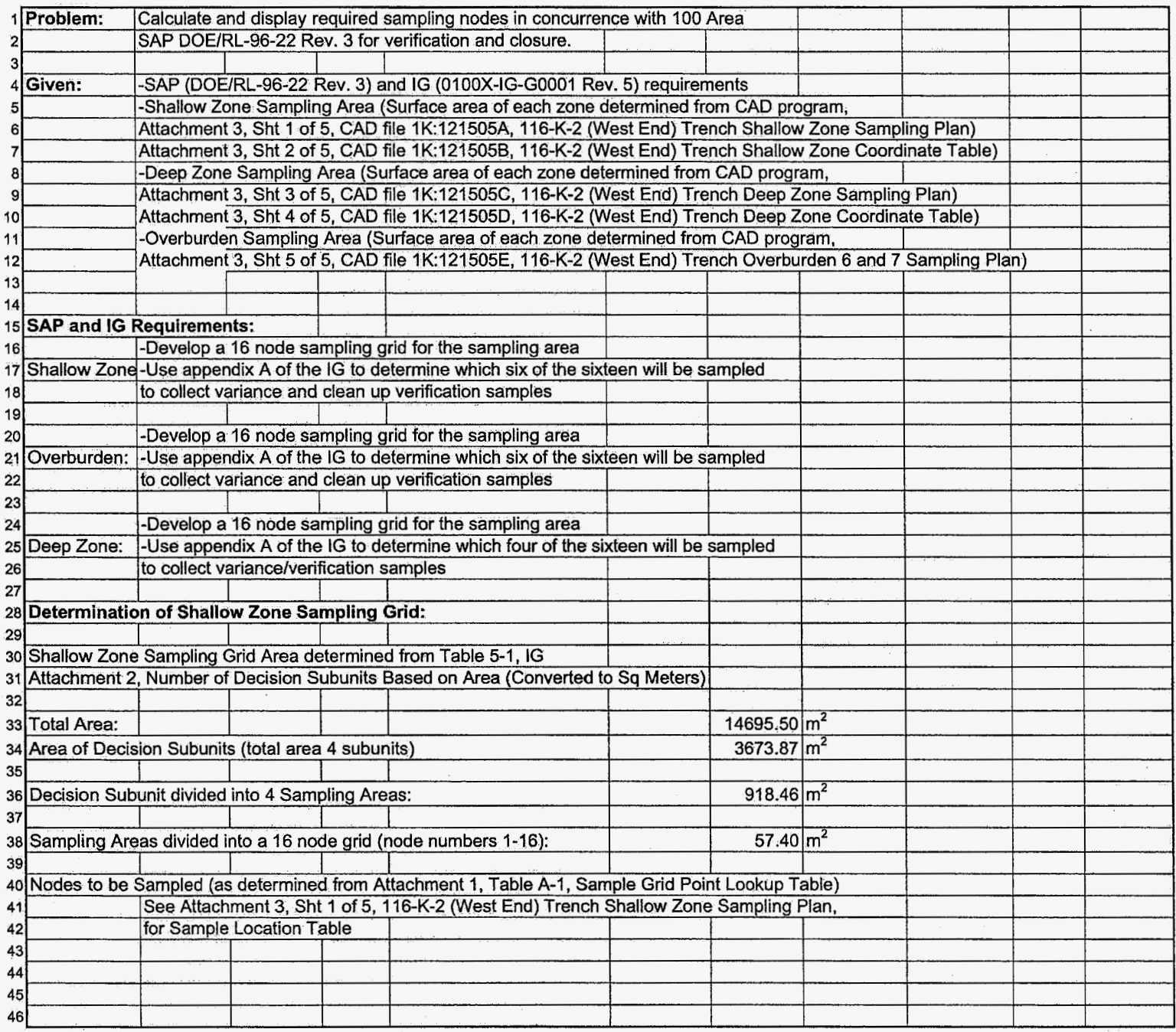


Calc. No. 0100K-CA-V0062 Rev. No.

Project

Date 12/15/2005 Checked $0 \&$ Date 12/19/05 116-K-2 (West End) Trench Shallow, Deep Zone

Subject and Overburden Sampling Plan

Sheet No. 2 of 2

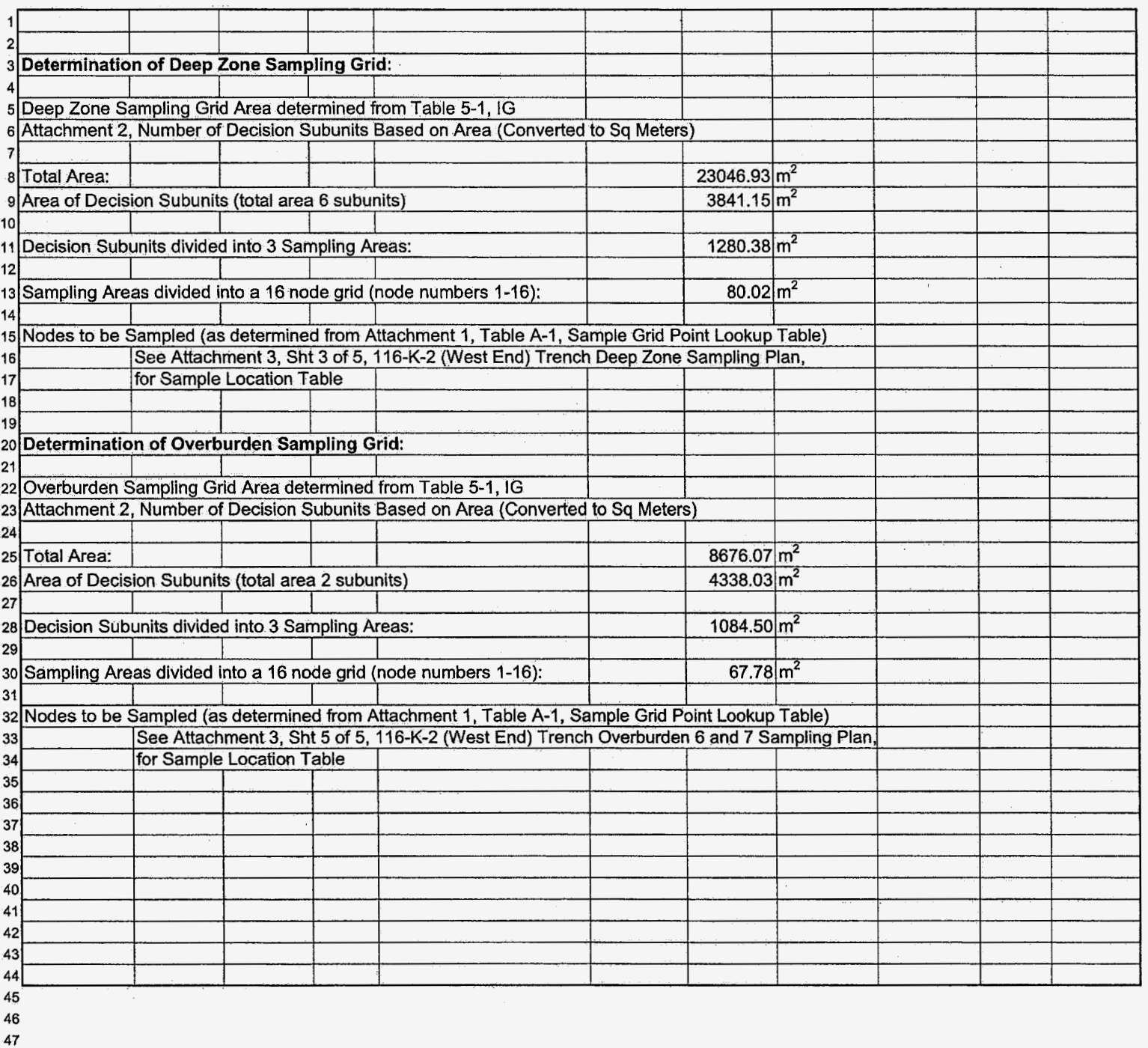


CVP-2006-00001

Rev. 0

C-158 


\section{CALCULATION COVER SHEET}

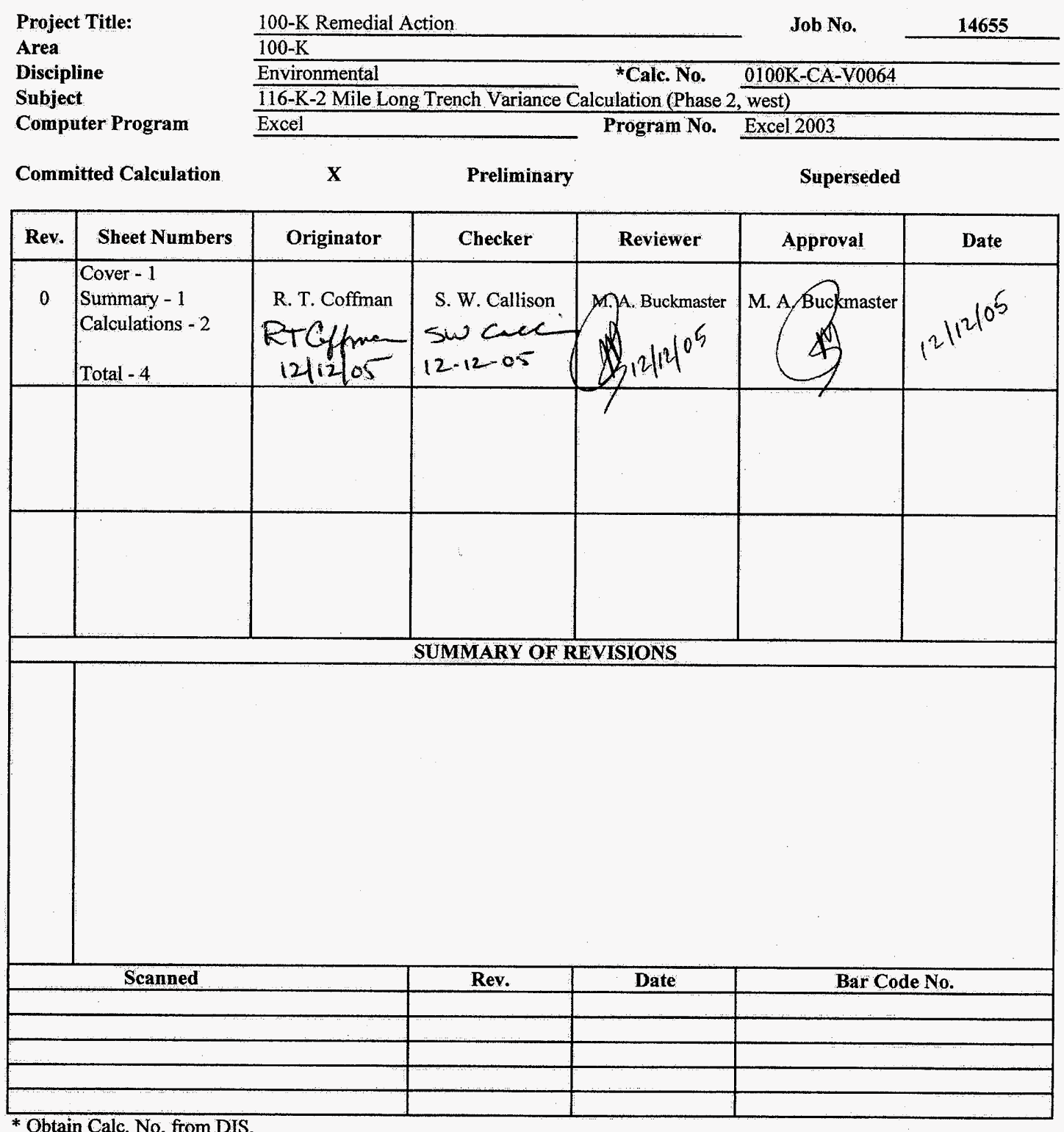




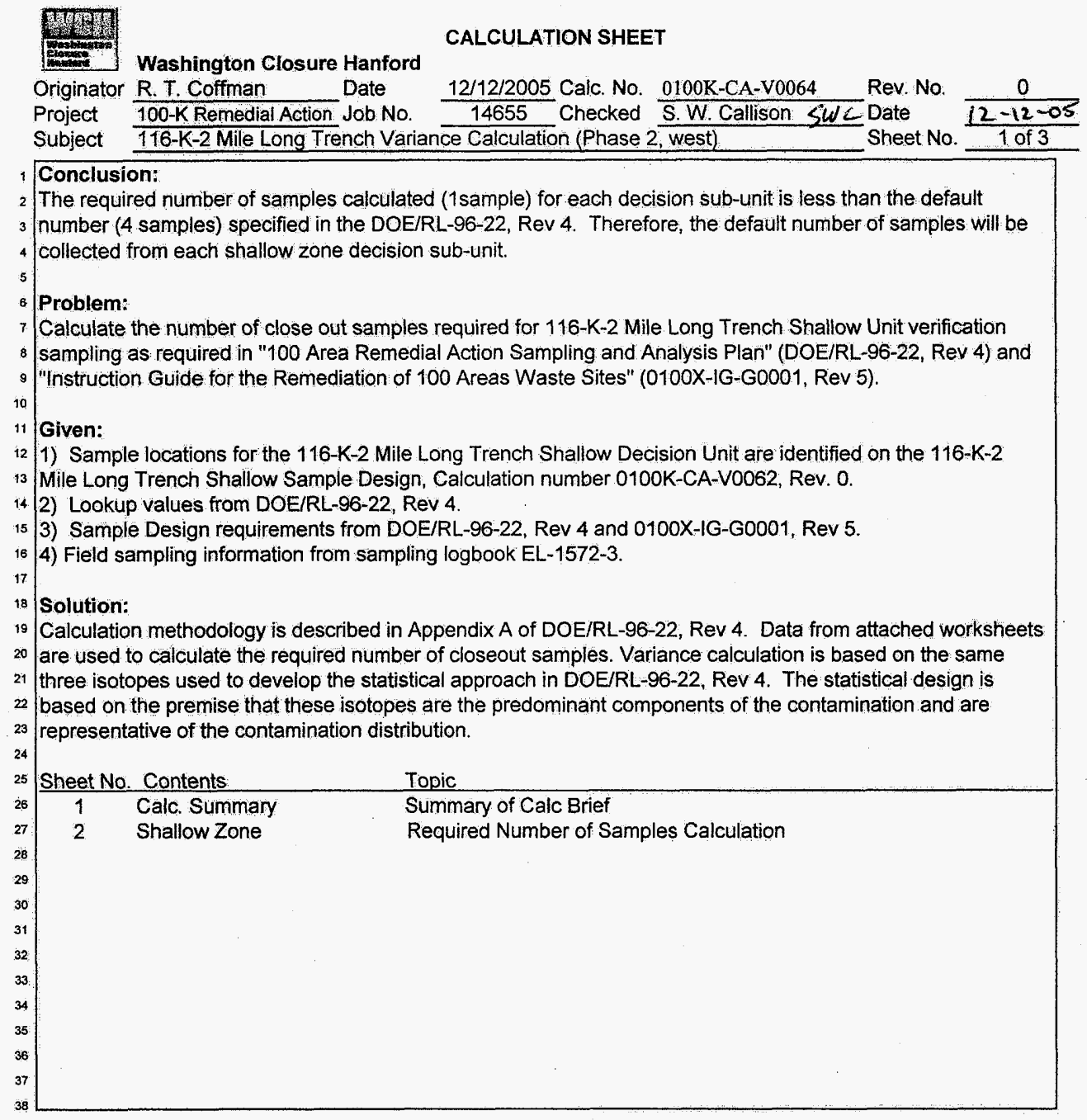




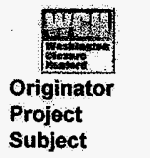

Washington Closure Hanford

CALCULATION SHEET

Subject

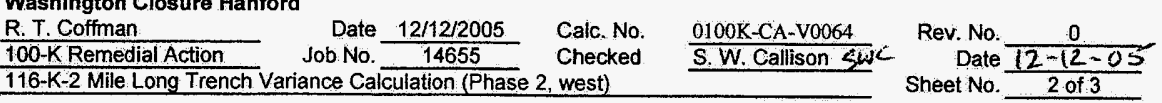

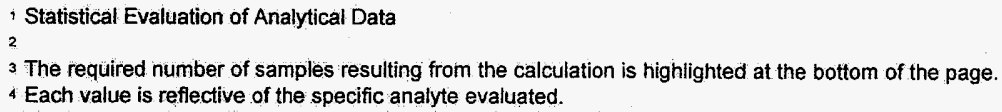

5 The highest value of the three evaluations is used to determine the required number of samples as compared

6 against the default of four.

7 Sample locations are from Calculation 0100K-CA-V0062.

- Mean, Standard Deviation, $t$, and Number of Samples formulas are from DOE/RL-96-22, Appendix A.

11. Decision Unit: 116-K-2 Trench Shallow. Zone

12. Samples values from GEA analysis

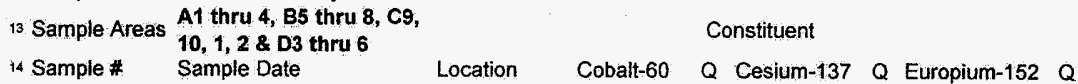

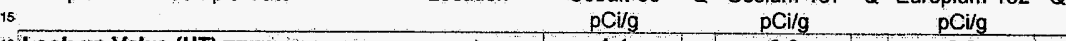

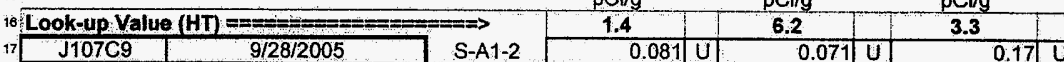

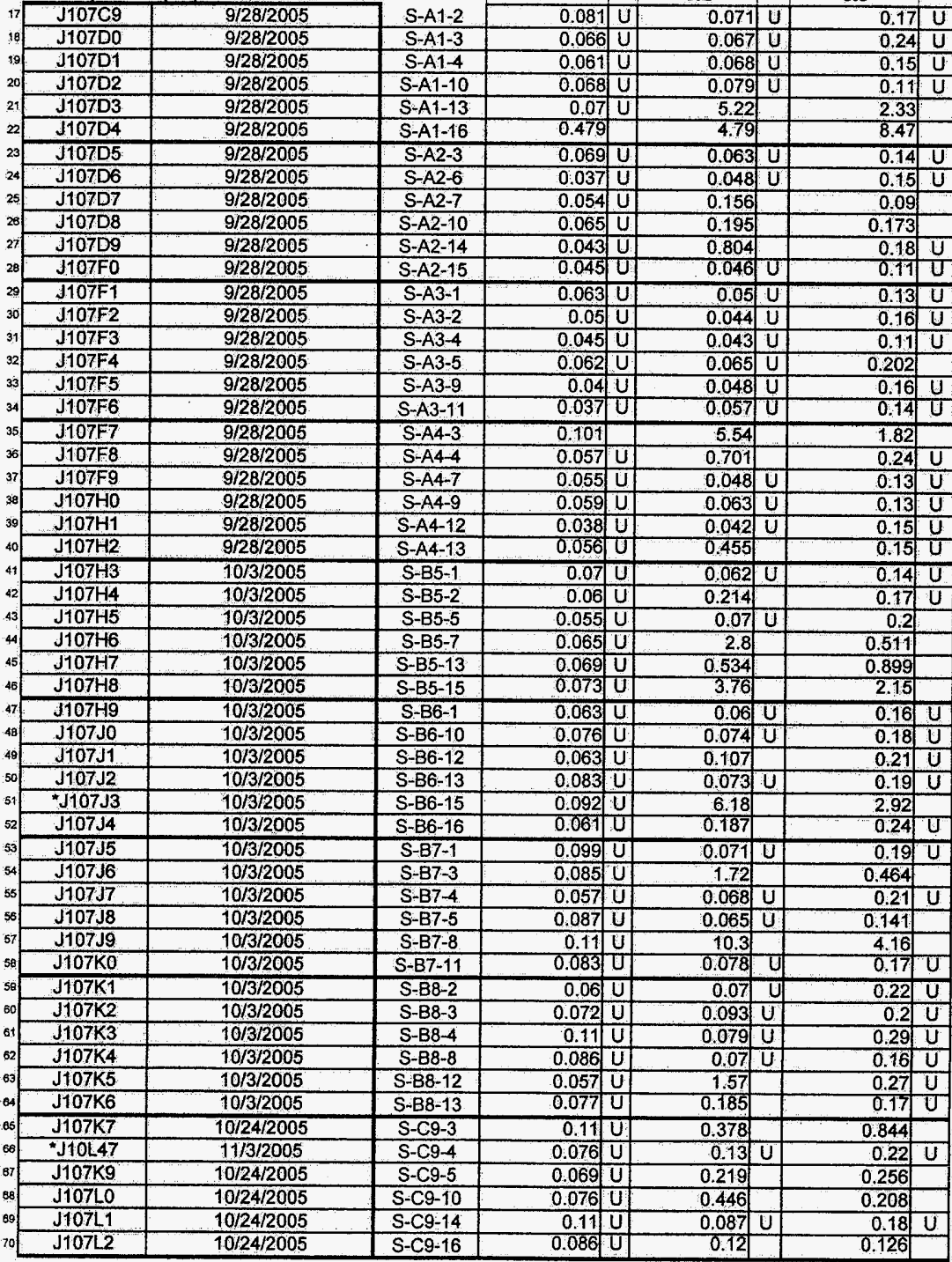




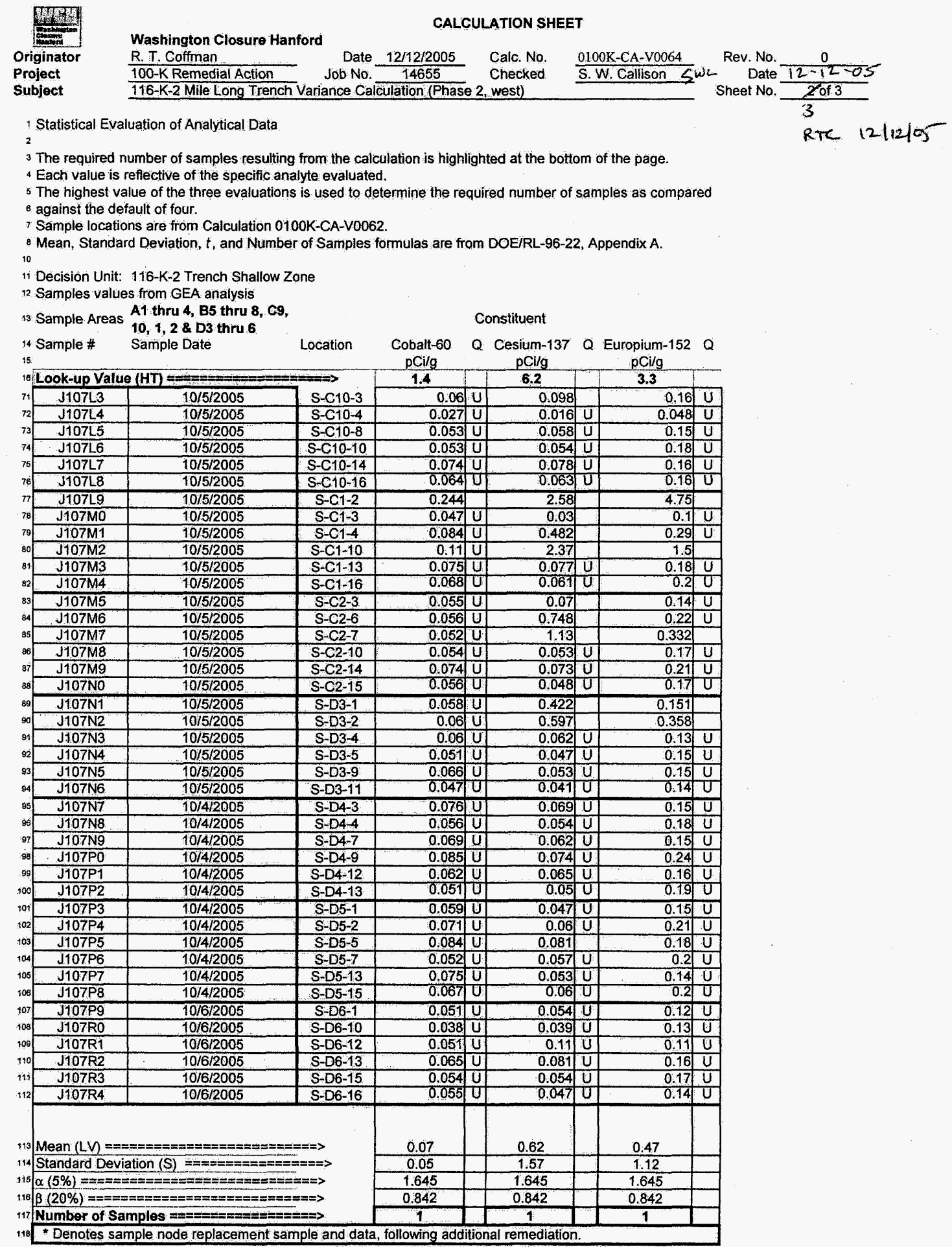


CVP-2006-00001

Rev. 0

\section{CALCULATION COVER SHEET}

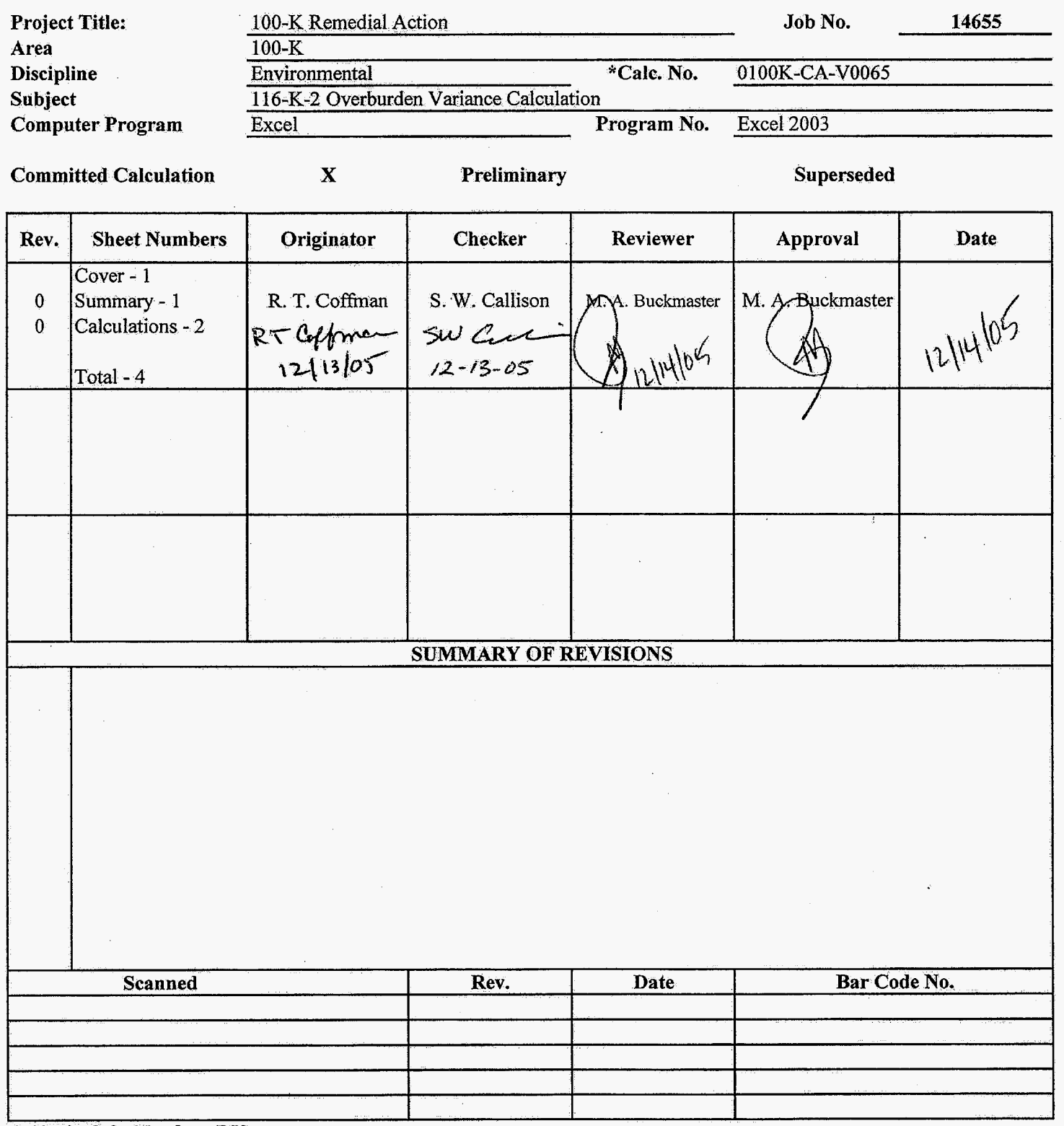

* Obtain Calc. No. from DIS. 
CVP-2006-00001

Rev. 0

\section{CALCULATION SHEET}

Washington Closure Hanford

Originator $R$ T Coffman $P R$.

$\frac{12 / 12 / 2005}{14655}$ Calc. No. $\frac{0100 \mathrm{~K}-\mathrm{CA}-\mathrm{V} 0065}{\text { Checked }}$ Rev. No. Nallison SuL Date

Project 100 -K Remedial Action Job No.

Date

Sheet No.

Subject $110-1$

1 Conclusion:

2 The required number of samples calculated (1sample) for each decision sub-unit is less than the default

3 number (4 samples) specified in the DOE/RL-96-22, Rev 4. Therefore, the default number of samples will be

4 collected from each shallow zone decision sub-unit.

5

6 Problem:

7 Calculate the number of close out samples required for $116-K-2$ Mile Long Trench Shallow Unit verification

8 sampling as required in "100 Area Remedial Action Sampling and Analysis Plan" (DOE/RL-96-22, Rev 4) and

9 "Instruction Guide for the Remediation of 100 Areas Waste Sites" (0100X-IG-G0001, Rev 5).

11 Given:

12 1) Sample locations for the 116-K-2 Mile Long Trench Shallow Decision Unit are identified on the 116-K-2

${ }_{13}$ Mile Long Trench Shallow Sample Design, Calculation number 0100K-CA-V0062, Rev. 0.

14 2) Lookup values from DOE/RL-96-22, Rev 4.

15 3) Sample Design requirements from DOE/RL-96-22, Rev 4 and 0100X-IG-G0001, Rev 5.

16 4) Field sampling information from sampling logbook EL-1572-3.

Solution:

Calculation methodology is described in Appendix A of DOE/RL-96-22, Rev 4. Data from attached worksheets

are used to calculate the required number of closeout samples. Variance calculation is based on the same

21 three isotopes used to develop the statistical approach in DOE/RL-96-22, Rev 4. The statistical design is

22 based on the premise that these isotopes are the predominant components of the contamination and are

23 representative of the contamination distribution,

Sheet No Contents

${ }_{26} \frac{\text { Sheet No. Contents }}{1}$ Calc. Summary $\quad$ Summary of Calc Brief

27 Shallow Zone Required Number of Samples Calculation 


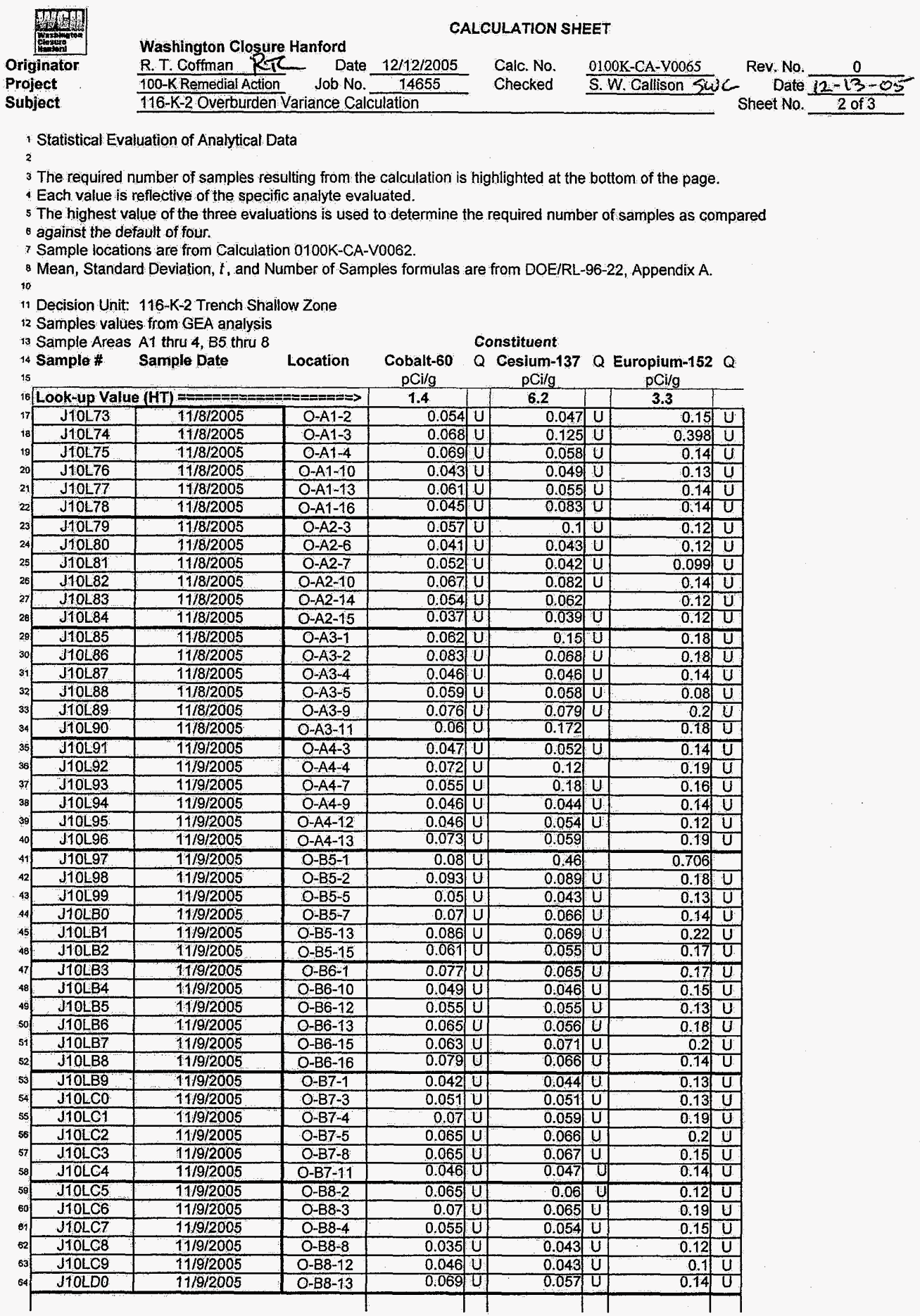


CVP-2006-00001

Rev. 0

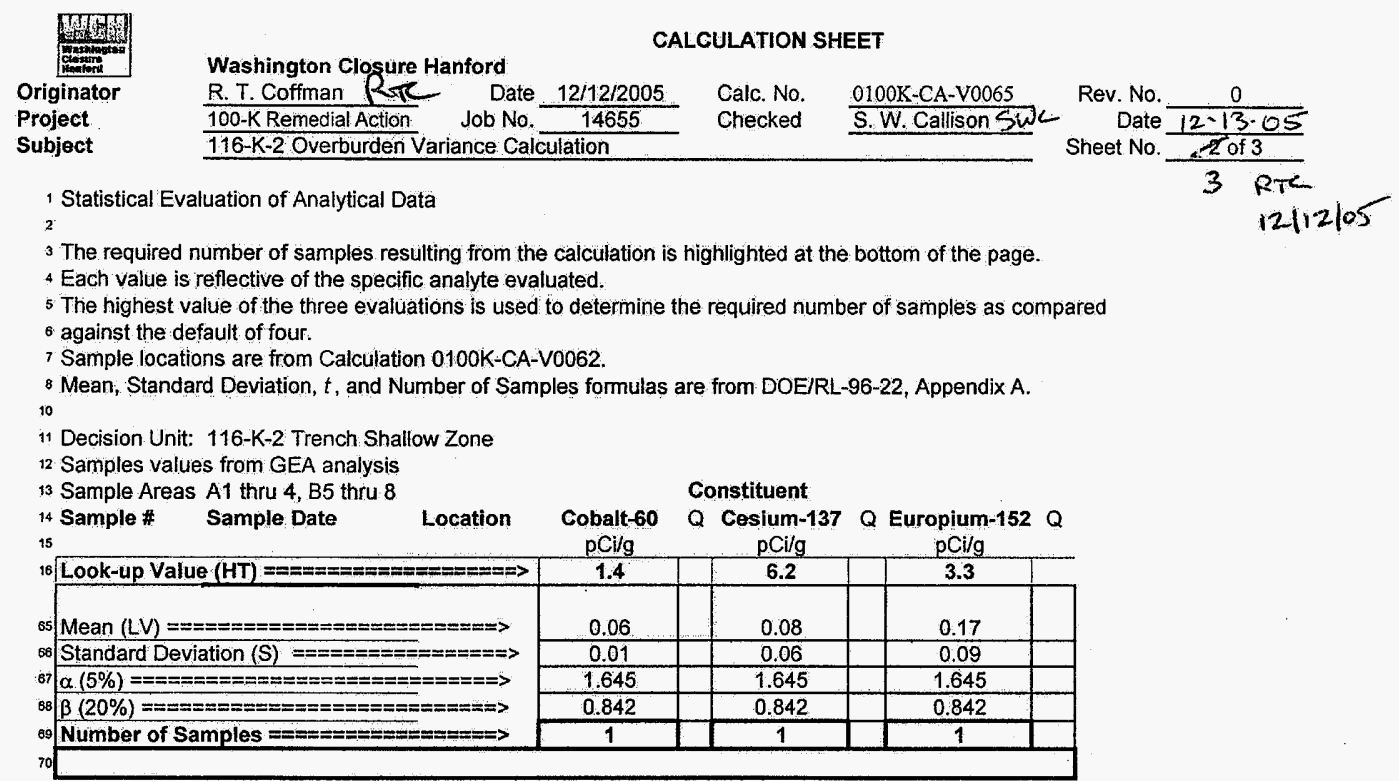


CVP-2006-00001

Rev. 0

\section{CALCULATION COVER SHEET}

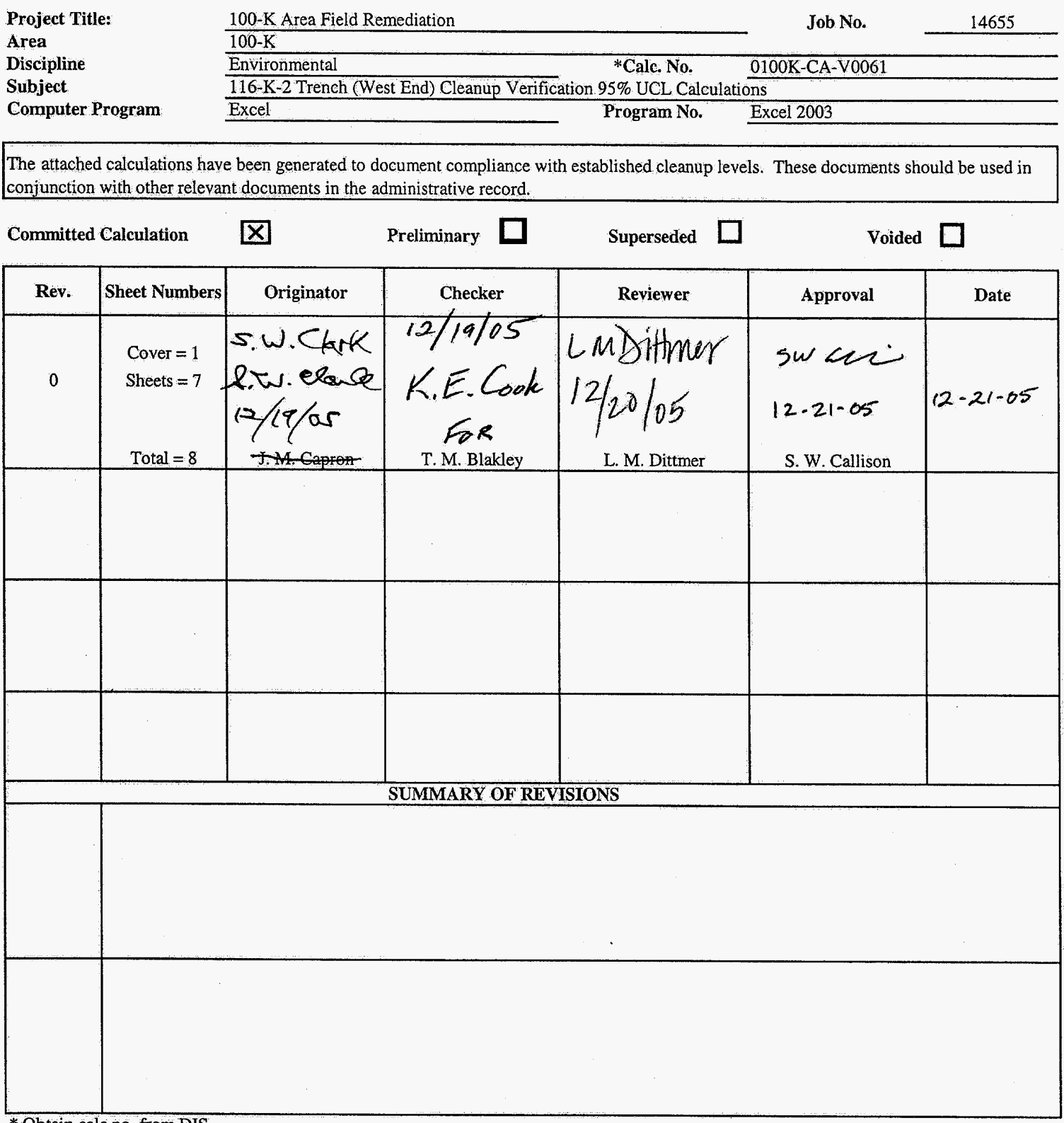

\footnotetext{
* Obtain calc no. from DIS
}

DE01437.03 (12/09/2004) 


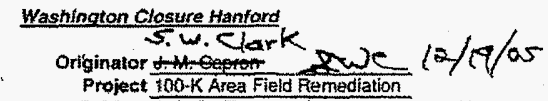

Project $100-K$ Area Field Remediation
CALCULATION SHEET

Date 12/19/05 Job No. 14655 Całc Na O100K-CA-Voust

\section{Purpose:}

Calculate the $95 \%$ upper confidence limit (UCL) to evafuate compliance with cleanup stancards for the subject site. Also, caktutate the carcinogenic risk for applicable nonradioniclide 3 analytes, perform the Washington Adnitristrative Cade (WAC) 173-340 (Model Taxics Control Act [MTCA]) 3-part test, if required, and calculate the relative percent dilference (APD) for each contaminant of concern (COC)

Table of Contents:

Sheets $\$$ to 2 - Calculation Sheet Summary

Sheets $\&$ to 2 - Calculation Sheet Summary
Sheet 3 - Caiculation Sheat Shallow Zone Veritication

Sheet 3 - Calculation Sheat Shallow Zone Verification
Sheet 4 - Caiculation Sheet Deep Zons Verification

Sheet 4 - Calculation Sheet Deep Zons Verification
Sheet 5 - Calculation Sheet Overburcen Verification

Sheet 5 - Calculation Sheet Overburden Verificaticn
Sheet 6 - Calculation Shet Split-Duplicate Astalysis

Sheet 7 . Ecology Software (MTCAStat) Resuts

5 Given/References:

1) Sample Results

2) All lookup values and remedial action goats (FAGs) are taken from DOE-RL (20050) and Ecology (1996)

3) DOE-RL, 2005a, 100 Area Remedial Action Sampling and Anafysis Plan (SAP). DOE/RL-96-22, Fev. 4, U.S. Department of Energy, Rïchland Operations Office, Fichland. Washington.

4) DOE-RL, 20055, Remedial Design Report/Remedial Action Work Flan for the 100 Araa (FOR/RAWP). DOE/RL-96-17, Rev. 5. U.S. Department of Energy, Richland Operations Ottice, Richland, Washington.

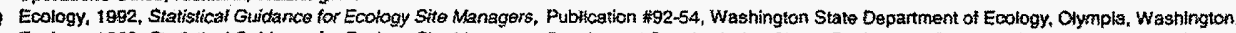

6) Ecology. 1993. Stafistical Guidance tor Ecology Site Managers, Supplement S-6. Anatyzing Site or Background Data with Below-Detection Limit or Betow-PQL

Vatues (Censored Data Sers). Putblication "̈92-54, Washingtori Stale Department of Ecology, Olympia, Washington.

7) Ecology. 1996, Moder Toxics Control Act Clearup Levels and Risk Calculations (CLAFC M), Pubfication \#94-145, Washingtan State Department of Ecology, Oympia.

Washington.

EPA. 1994. USEPA Contract Laboratory Program National Functional Guidelines for Inorganic Data Review, EPA 540/R-94/0:3. U.S. Environmental Ptotection Agency. Washington, D.C.

WAC 173-340, 1996, "Model Toxics Controf Aat-Cleanep," Washington Administrative Code.

10) WCH, 2005, 110 K-2 Trench (East End) Cleanup Verification 95\% VCL Calculations, Calc. No. 0100K-CA-Voo56, Rev. 0, Washington Closure Haniord, Fichland, Washingtion,

Solution:

Calculation methodology is described in Ecology Pub. \#92-54 (Ecology 1992, 1993), below, and in the RDRARAWP (DOE-RL 2005b). Use data from the attached wotksheets to calculate the $95 \%$ UCL, hazard quotents, excess carcinogenic risk, periom the WAC $173-340$ 3-part fest for nonradionustides, and calculate the fPD tor each COC in the primary dowl

The subject calculations were performed on data from soll verification samples trom the southwest portion of the 116-K-2 waste ste; calculations for the remalnder of the waste site are documented in WCH (2005). The data were entered into an EXCEL 2009 spleadsheet and calculations pertomed by utilizing the built-in spreadsheet furctions and/or creating formulae within the cells. The statistical evaluation of data for use in accordance with the RDFiRAWF (DOE.RL 2005b) is documented by this caiculation. Split and dupilicate RPD

For quailty assurfansofquatity control (QACC) sphit and duplicate RPD calculations, a value less than $+1-30 \%$ indicates the data compare lavorably. For regulatory splits, a threshold of $35 \%$ is used (EPA 1994). If the RPD is greater than $30 \%$ (or $35 \%$ for regulatory split data), lerther investigation regarding the usability of the data is performed. Additional discussion as necessary is provided in the data quality assessment section of the applicabie $C V$.

If regulator spllt comparison is required, an adtitional parameter is evaluated. A control limit of $+\%$ - 2 times the TDL shall be used if eimer the main or regulator split value is less than 5 times the TDL and above detection. In the case where only one result is gfeater than 5 times the TDL and the other is below, the $+1,2$ times the TDL. criteria applies. Therefore, the following calculation is pertormed as part of the evaluation for these two cases involving regulator split data: difference = main - regulator spit. If the difference is greater than + l/ 2 times the TDL, then hurther investigation regarding the usabifity of the data is performed and presented in the applicabie CVP data quality assessment section.

No regulatory split samples wert collected for this site. 
Summary (continued)

1 Results:

2. The results presented in the summary tables that follow are for use in RESidual RADioactivity dose/risk analysis and the CVP for this site.

4 Results Summary

\begin{tabular}{|c|c|c|c|c|c|c|c|}
\hline \multirow{2}{*}{ Analyte } & \multicolumn{2}{|c|}{ Shallow Zone } & \multicolumn{2}{|c|}{ Deep Zone } & \multicolumn{2}{|c|}{ Overburden } & \multirow{2}{*}{ Units } \\
\hline & Result & Qualifier & Result & Qualifier & Result & Qualifier & \\
\hline Hexavalent Chromium & $2.3 \mathrm{E}-01$ & & $2.4 E+00$ & & $2.7 \mathrm{E}-01$ & & $\mathrm{mg} / \mathrm{kg}$ \\
\hline Carbon-14 & $2.9 \mathrm{E}-01$ & & $1.44 E+00$ & & $6.8 \mathrm{E}-01$ & $\bar{U}$ & $\mathrm{pCi} / \mathrm{g}$ \\
\hline Cesium-137 & $1.10 \mathrm{E}+00$ & & $1.17 E+02$ & & $0(<B G)$ & & $\mathrm{pCi} / \mathrm{g}$ \\
\hline Cobalt-60 & $2,3 \mathrm{E}-02$ & $\mathrm{U}$ & $4.23 E+00$ & & $1.2 \mathrm{E}-02$ & ú & $\mathrm{pCi} / \mathrm{g}$ \\
\hline Europium-152 & $6.26 \mathrm{E}-01$ & & $7.6 \mathrm{E}+01$ & & $1.7 \mathrm{E}-01$ & & $\mathrm{pCi} / \mathrm{g}$ \\
\hline Europium-154 & $1.29 \mathrm{E}-01$ & & $7.2 E+00$ & & $3.5 \mathrm{E}-02$ & $\mathrm{U}$ & pCi/g \\
\hline Nickel-63 & $1.77 \mathrm{E}+00$ & & $6.5 \mathrm{E}+02$ & & $6.89 \mathrm{E}-01$ & $\bar{U}$ & $\mathrm{pCi} / \mathrm{g}$ \\
\hline Plutonium-239/240 & $2.4 \mathrm{E}-02$ & $\bar{U}$ & $4.7 \mathrm{E}+00$ & & 6.E-03 & $U$ & $\mathrm{pCl} / \mathrm{g}$ \\
\hline Strontium-90 & $2.01 \mathrm{E}-01$ & & $6.3 \mathrm{E}+00$ & & $O(<B G)$ & $U$ & $\mathrm{pCi} / \mathrm{g}$ \\
\hline
\end{tabular}

15 Strontium-90 $\quad 2.01 E-01$

16 WAC 173-340 Evaluation (Shallow Zone)

$95 \%$ UCL $>$ Cleanup Lim

NO Cleanup Limit?

21 Any sample $>2 \times$ Cleanup Limit? NO

3.Part Test

22

23 Risk Estimate:

24 Nonrad noncarcinogenic index sum: $9.6 \mathrm{E}-04$

25 Nonrad carcinogenic risk: $1.1 \mathrm{E}-07$

26

27 WAC 173-340 Evaluation (Overburden)

29 3-Part Test:

$3095 \%$ UCL > Cleanup Limit? NO

$31>10 \%$ above Cleanup Limit? NO

32 Any sample $>2 \times$ Cleanup Limit? NO

34 Risk Estimate:

35 Nonrad noncarcinogenic index sum: $\quad 1.1 \mathrm{E}-03$

36 Nonrad carcinogenic risk: 1.3E-07

39 Relative Percent Difference Results* QAQC Analysis

\begin{tabular}{|c|c|c|c|c|c|c|}
\hline \multirow[t]{2}{*}{40} & \multirow[b]{2}{*}{ Analyte } & \multicolumn{2}{|c|}{ Shallow Zone } & \multicolumn{2}{|c|}{ Deep Zone } & \multirow{2}{*}{$\begin{array}{l}\text { Overburden } \\
\text { Duplicate Analysis }\end{array}$} \\
\hline & & $\begin{array}{l}\text { Duplicate } \\
\text { Analysis }\end{array}$ & $\begin{array}{c}\text { Split } \\
\text { Analysis }\end{array}$ & $\begin{array}{l}\text { Duplicate } \\
\text { Analysis** }\end{array}$ & $\begin{array}{c}\text { Split } \\
\text { Analysis }\end{array}$ & \\
\hline 42 & Hexavalent Chromium & & & & & \\
\hline 43 & Carbon-14 & & & & & \\
\hline 44 & Cesium-137 & & & $67 \%$ & $56 \%$ & \\
\hline 45 & Cobalt-60 & & & $64 \%$ & $43 \%$ & \\
\hline 46 & Europium-152 & & & $62 \%$ & $53 \%$ & \\
\hline 47 & Europium-154 & & & $57 \%$ & $49 \%$ & \\
\hline 48 & Nickel-63 & & & $10 \%$ & $10 \%$ & \\
\hline 49 & Plutonium-239/240 & & & & & \\
\hline 0 & Strontium-90 & & & & & \\
\hline
\end{tabular}

50 Strontium-90

51 *A blank cell indicates that $\mathrm{APD}$ evaluation was not required.

$95 \%$ UCL $>$ Cleanup Limit? YES

$>10 \%$ above Cleanup Limit? YES

Any sample $>2 \times$ Cleanup Limit? YES

$53 \mathrm{QAOC}=$ quality assurance/quality control

54 RESRAD = RESidual RADioactivity (dose model)

$55 \mathrm{RPD}=$ relative percent difference

$56 \mathrm{U}=$ undetected 
CVP-2006-00001

Rev. 0 
Washington Closure Hantord

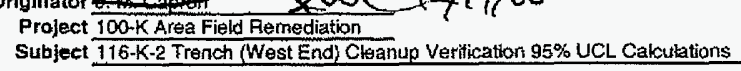

\begin{tabular}{c} 
Date 1212905 \\
Job No. 14655 \\
\hline
\end{tabular}

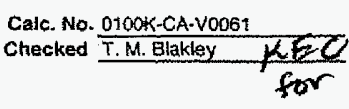

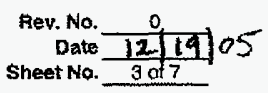
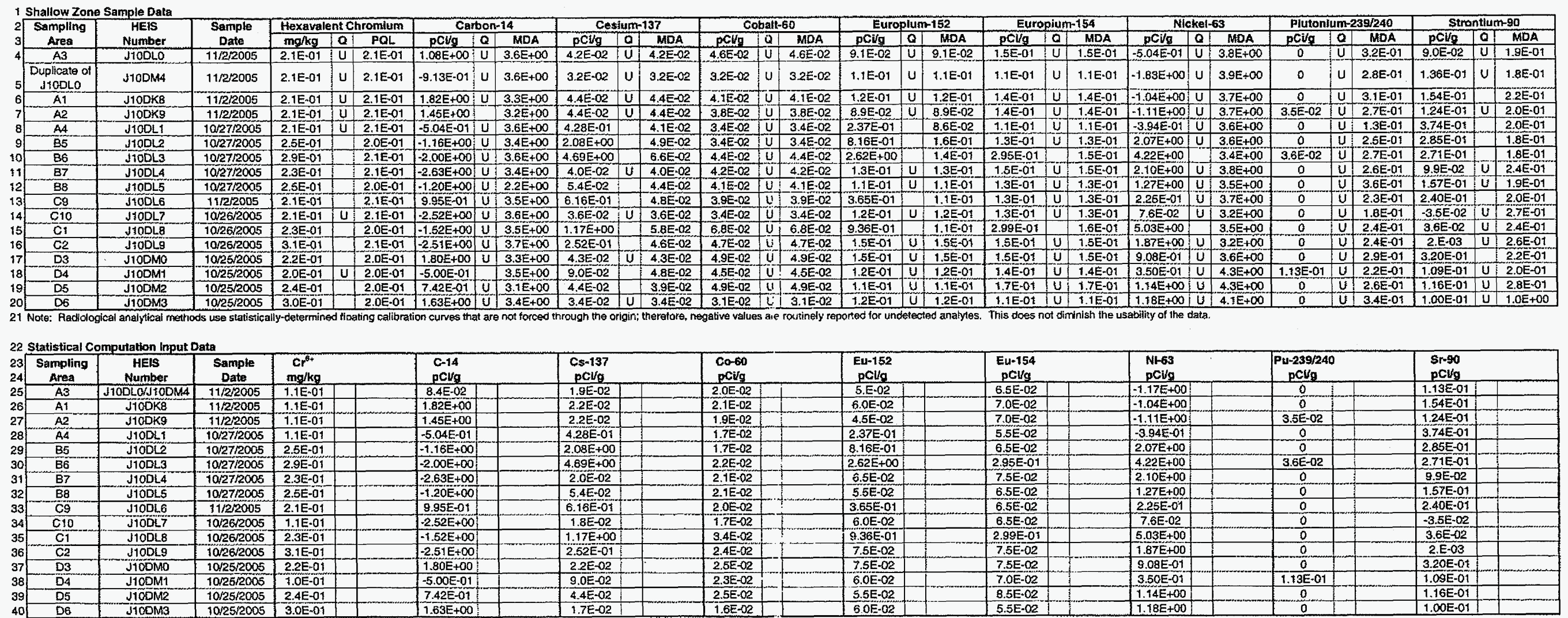

${ }_{41}^{4}$ Statistical Computations

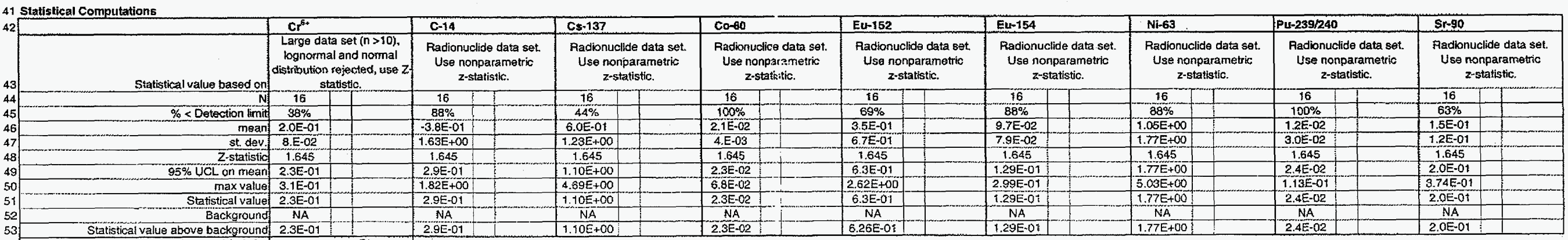

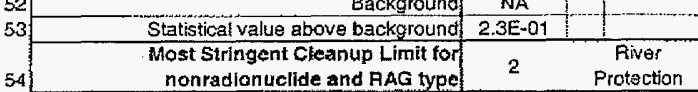

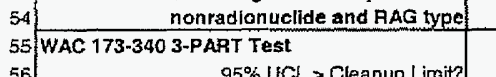

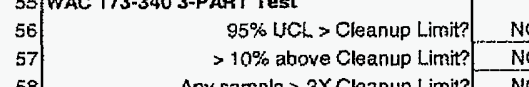

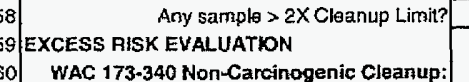

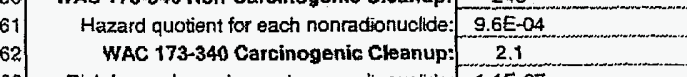

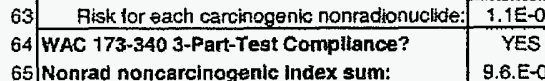

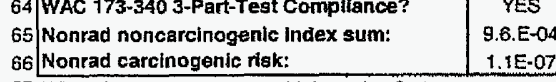

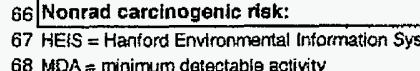

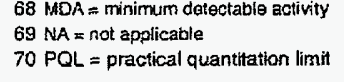

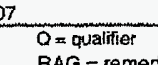

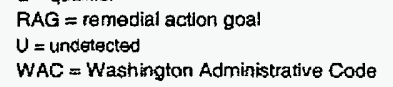


Washington Closure Hantord

Prolect 100-KArea Field Remediation

Date 12191905
Job No. $\frac{14655}{6}$

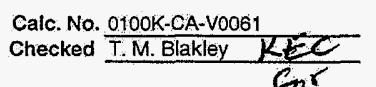

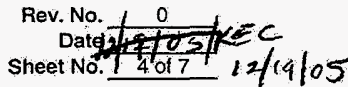

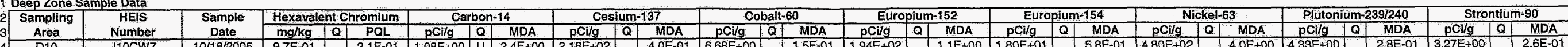

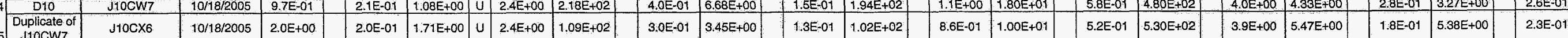

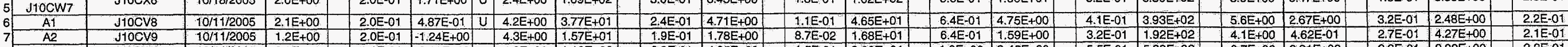

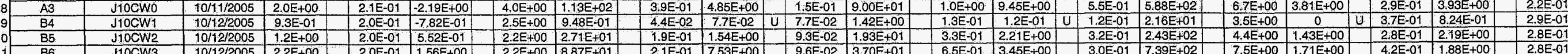

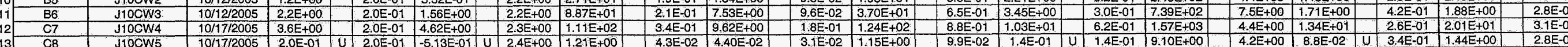

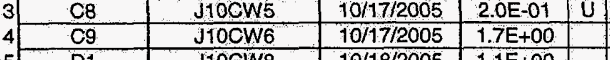

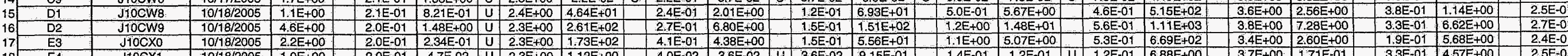

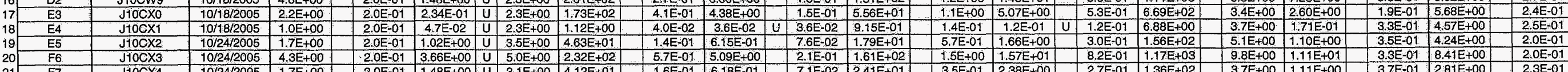

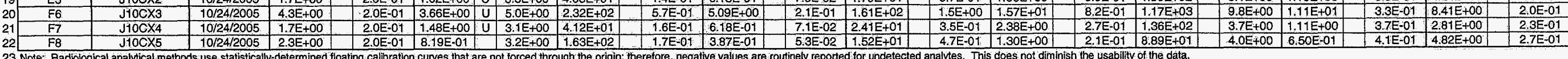
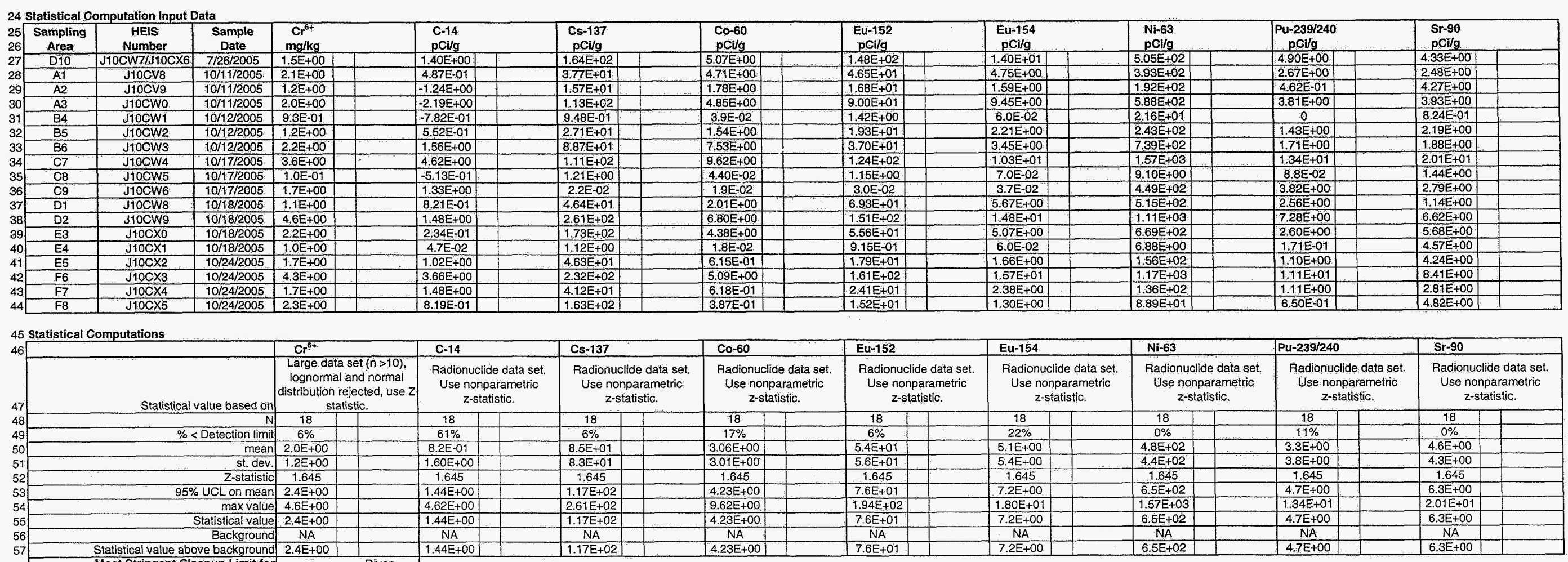

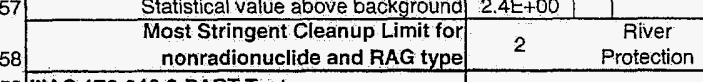

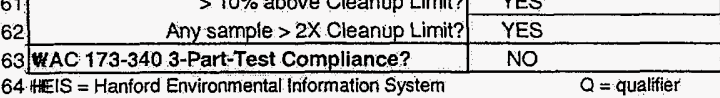

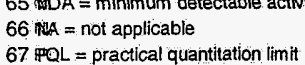

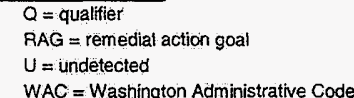


CALCULATION SHEET

$\frac{\text { Washington Closure Hanford }}{5 . \text { W. C(ark }}$

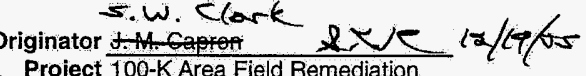

Subject $116-$ K-K-2 Trench (West Endit) Cleanup Verification 95\% UCL Calculations

Date 12119105
Job No. 14655 Calo. No. $0100 \mathrm{0}$-CA-V0061
checked $\frac{\text { T.M. Bakley }}{K E C}$
for

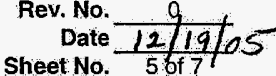

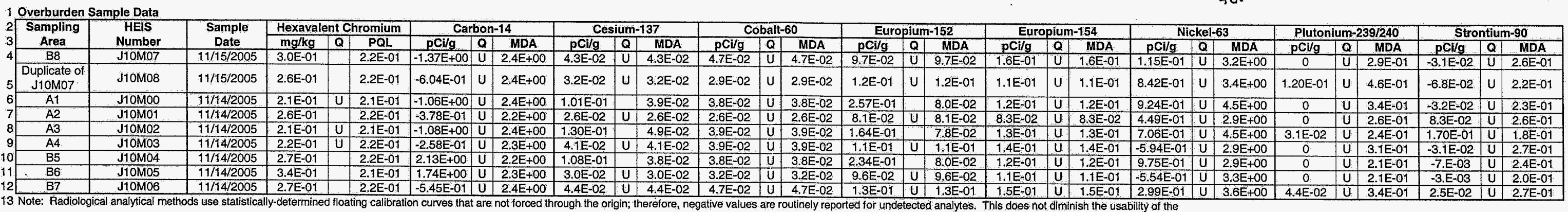

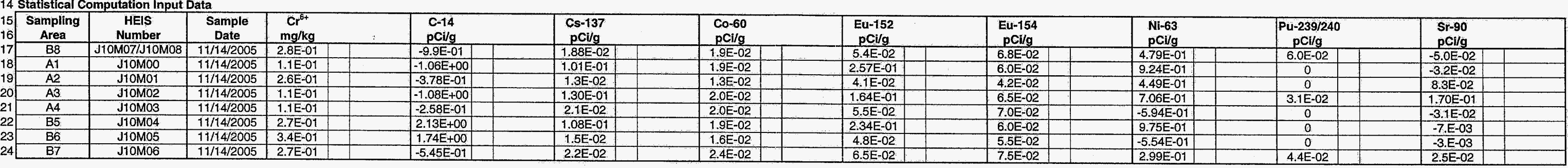
25 Statistical Computations

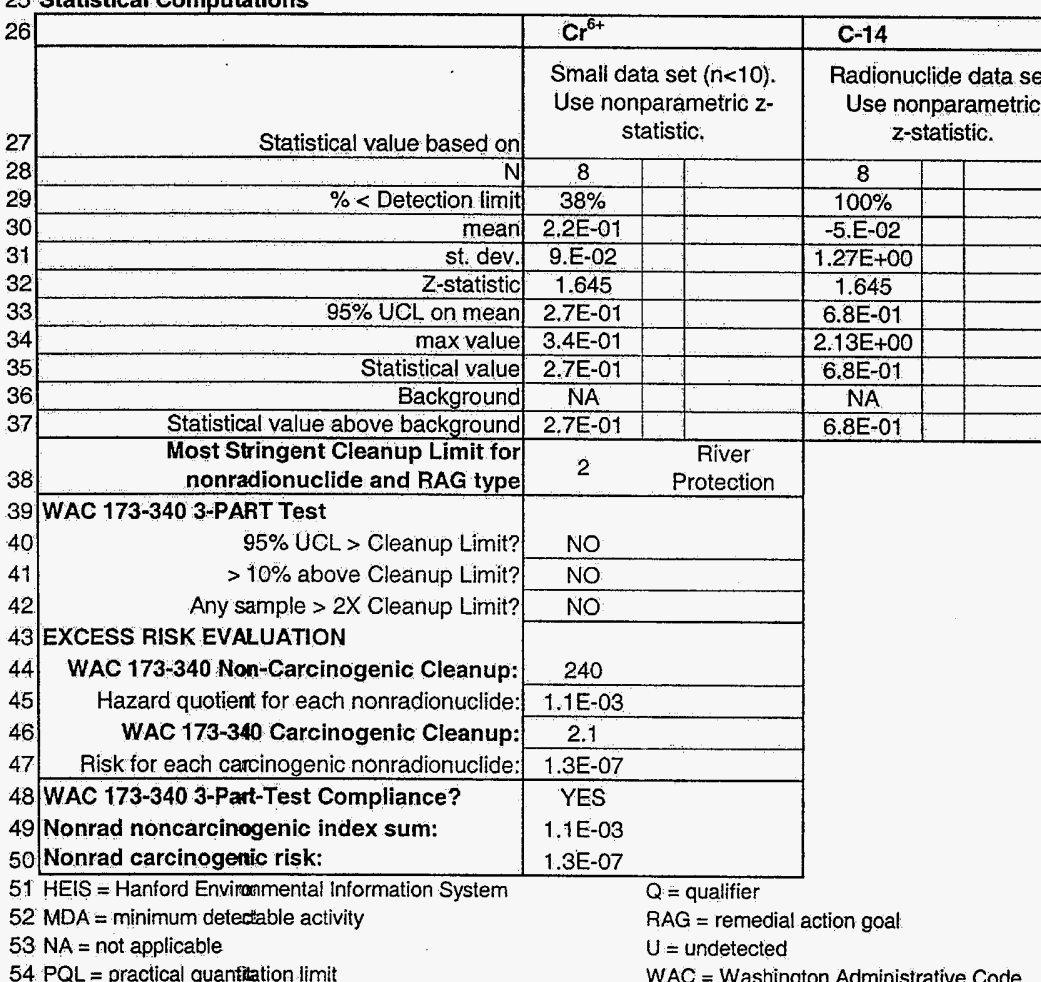


Washington Closure Hanford

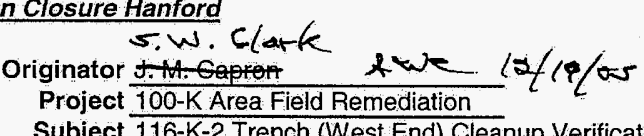

Subject $1166-K-2$ Trench (West End) Cleanup Verification $95 \%$ UCL Calculations
Date $\frac{12 / 19 / 05}{14655}$
Calc. No. $0100 K$-CA-V0061
Checked T.M. Blakley KEe
Rev. No. $\frac{9}{\text { Dote }}$
Sheet No. $\frac{12 / 19 \text { of }}{6 \text { of } 7}$ Split-Duplicate Analysis
Shallow

\begin{tabular}{|c|c|c|c|c|c|c|c|c|c|c|}
\hline Sampling & & Hexavalent Chromium & Carbon-14 & Cesium-137 & Coballt-60 & Europium-152 & Europium-154 & Nickel-63 & Plutonium-239/240 & Strontium-90 \\
\hline Area & HEIS Number & \begin{tabular}{l|l|l|}
$\mathrm{mg} / \mathrm{kg}$ & $\mathrm{PQL}$ \\
\end{tabular} & \begin{tabular}{l|l|l}
$\mathrm{pCi} / \mathrm{g}$ & $\mathrm{Q}$ & $\mathrm{MDA}$ \\
\end{tabular} & \begin{tabular}{l|l|l}
$\mathrm{pCi} / \mathrm{g}$ & $\mathrm{Q}$ & $\mathrm{MDA}$ \\
\end{tabular} & $\begin{array}{l}\text { pCilg a } \\
\text { a }\end{array}$ & \begin{tabular}{l|l|l}
$\mathrm{pCi} / \mathrm{g}$ & $\mathrm{Q}$ & $\mathrm{MDA}$ \\
\end{tabular} & \begin{tabular}{l|l|l}
$\mathrm{PCl} / \mathrm{g}$ & Q & MDA \\
\end{tabular} & \begin{tabular}{ll|l} 
pCilg & a & MDA \\
\end{tabular} & $\begin{array}{lll}\text { pCilg } & \text { Q } & \text { MDA } \\
\end{array}$ & 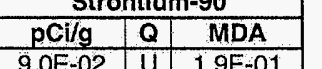 \\
\hline Duplicate of $J 10 D L 0$ & $\begin{array}{l}\mathrm{J1} 10 \mathrm{DLO} \\
\mathrm{J10DM4} \\
\end{array}$ & \begin{tabular}{c|c|c|c|}
$2.1 E-01$ & U.E-1E-1 \\
$2.1 E-01$ & U & $2.1 E-01$
\end{tabular} & $\begin{array}{l}1.08+00 \\
9.13 E-01\end{array}$ & \begin{tabular}{l|l|l}
$4.2 E-02$ & $-4.2 E-02$ \\
$3.2 E-02$ & $U$ & $3.2 E-02$
\end{tabular} & $\begin{array}{l}4.6 E-02 \\
3.2-02\end{array}$ & \begin{tabular}{ll|l|}
$9.1 E-02$ & $9.1 E-02$ \\
$1.1 E-01$ & $U$ & $1.1 E-01$
\end{tabular} & \begin{tabular}{l|l}
$U$ & $1.5 E-01$ \\
& $1.11-0.01$ \\
\end{tabular} & \begin{tabular}{c|c|c}
$-5.04 \mathrm{E}-01$ & $3.8 \mathrm{E}+00$ \\
$-1.83 \mathrm{E}+00$ & $\mathrm{U}$ & $3 \mathrm{~g}+0+00$
\end{tabular} & $\frac{3.2 E-01}{2.8 E-01}$ & $\begin{array}{l}\frac{9.0 E-02}{133 E-01} \\
1.01\end{array}$ \\
\hline & $\sqrt{10 F 85}$ & 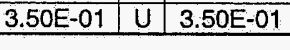 & \begin{tabular}{l|l|l|}
$2.62 E-01$ & 8.10E-01 \\
\end{tabular} & & & & & & & $\begin{array}{l}0.1 .0-2 \\
1.29 E \\
\end{array}$ \\
\hline & & & 50 & & & 0.10 & 0.10 & & & \\
\hline Duplicate Analysis & 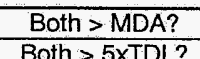 & No-Stop (acceptable) & No-Stop (accepptable) & No-Stop (acceptable) & No-Stop (acceptable) & No-Stop (acceptable) & No-Stop (acceptable) & No-Stop (acceptable) & No-Stop (acceptable) & No-Stop (accepptable) \\
\hline & Both > MDA? & No-Stop (acceptable) & No-Stop (acceptable) & No-Stop (acceptable) & No-Stop (acceptablé) & No-Stop (acceptable) & No-Stop (acceptable) & No-Stop (acceptable) & No-Stop (acceotable) & No-Stop (acceptable) \\
\hline Split Analysis & $\begin{array}{c}\text { Both }>5 \times T D L ? \\
\text { RPD }\end{array}$ & & & & & & & & & \\
\hline
\end{tabular}

\section{Deep Zone Sample Results:}

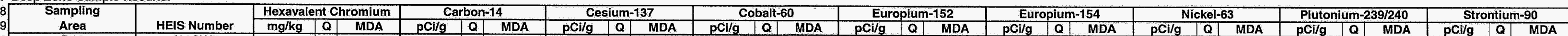

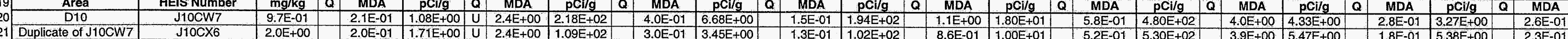

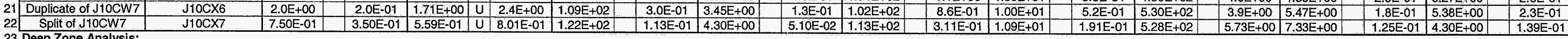
23 Deep Zone Analysis:

\begin{tabular}{|c|c|c|c|c|c|c|c|c|c|c|}
\hline \multirow[b]{3}{*}{ Duplicate Analysis } & & & 50 & & & 0.10 & & & & \\
\hline & Both > MDA? & Yes.(continue) & No-Stop (acceptable) & Yes (continue) & Yes (continue) & Yes (continue) & Yes (continue) & Yes (continue) & Yes (continue) & $\begin{array}{l}1.0 \\
\text { Yes (continue) }\end{array}$ \\
\hline & $\begin{array}{c}\text { Both }>\text { 5xTDL? } \\
\text { RPD }\end{array}$ & No-Stop (acceptable) & & $\begin{array}{l}\text { Yes (calc APD) } \\
67 \%\end{array}$ & $\begin{array}{l}\text { Yes (calc RPD) } \\
64 \%\end{array}$ & $\frac{\text { Yes (calc RPD) }}{66 \%}$ & $\frac{\mid \frac{1 \text { Yes (calc RPD) }}{57 \%}}{5}$ & $\frac{y^{\prime} \text { Yes (calc RPD) }}{10 \%}$ & No-Stop (acceptable) & No-Stop (acceptab \\
\hline \multirow[t]{2}{*}{ Split Analysis } & $\begin{array}{l}\text { Both > MDA? } \\
\text { Both > } 5 \times \mathrm{TDL} ?\end{array}$ & $\begin{array}{c}\text { Yes (continue) } \\
\text { No-Stoo (accetable }\end{array}$ & No-Stop (acceptable) & $\begin{array}{l}\text { Yes (continue) } \\
\text { Yes (calc RPDD }\end{array}$ & $\begin{array}{l}\text { Yes (continue) } \\
\text { Yes (calc RePD) }\end{array}$ & $\begin{array}{l}\text { Yes (continue) } \\
\text { Yes (calt pe) }\end{array}$ & 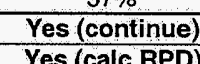 & $\begin{array}{l}\text { Yes (continue) } \\
\text { Yos (call pop) }\end{array}$ & Yes (continue) & Yes (continue) \\
\hline & RPD & & & $\begin{array}{l}\text { res (Calc APD) } \\
56 \%\end{array}$ & $\begin{array}{l}\text { res (calc FiD ) } \\
43 \%\end{array}$ & $\begin{array}{l}\text { Yes (calc HPD) } \\
53 \%\end{array}$ & $\begin{array}{l}\text { res (falc RPD ) } \\
49 \%\end{array}$ & $\begin{array}{l}\text { Yes (calc RPD) } \\
10 \%\end{array}$ & No-Stop (acceppable) & No-Stop (acceptable) \\
\hline
\end{tabular}

33 Overburden Sample Results:

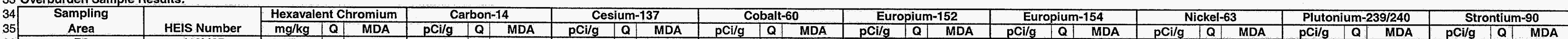

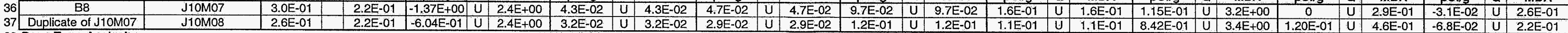

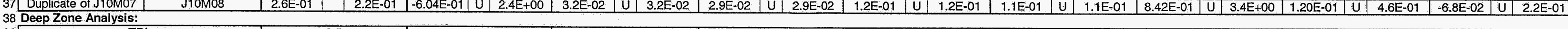

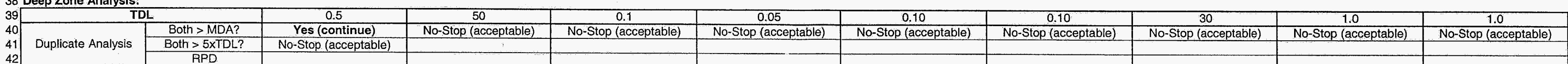
A3 Note: The signiificance of the reported RPD values, including values greater than $30 \%$, is addressed within the Data Quality Assessment for the Cleanup Verification Package for this site

$45 \mathrm{MDA}=$ minimum detectable activin

(8)

$48 \mathrm{RPD}=$ relative percent difierence
$49 \mathrm{TDL}=$ target detection limit

$50 . U=$ undetected 


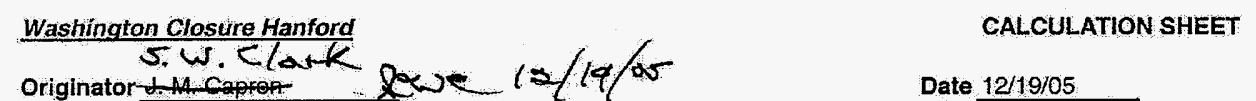

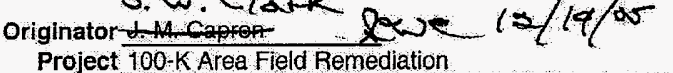

Subject 116-K-2 Trench (West End) Cleanup Verification $95 \%$ UCL Calculations.

\begin{tabular}{|c|c|}
\hline $\begin{array}{r}\text { Date } 12 / 19 / 05 \\
\text { Job No. } 14655\end{array}$ & $\begin{array}{l}\text { Calc. No. } 0100 \mathrm{~K}-\mathrm{CA}-\mathrm{V} 0061 \\
\text { Checked T. M. Blakley KEe }\end{array}$ \\
\hline ns. & for \\
\hline
\end{tabular}

Ecology Software (MTCAStat) Results

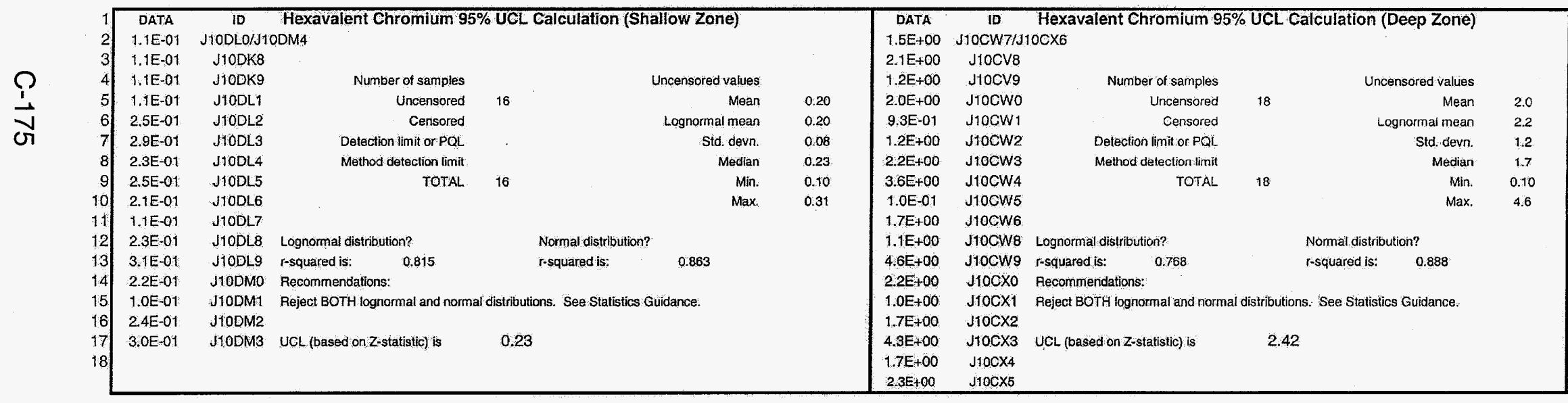


CVP-2006-00001

Rev. 0

C-176 


\section{CALCULATION COVER SHEET}

Project Title $100-\mathrm{K}$ Field Remediation Project

Job No. 14655

Area 100-K Area

Discipline Environmental

*Calc. No.

0100K-CA-V0063

Subject 116-K-2 Trench (West End) RESRAD Calculation

Computer Program RESRAD

Program No.

Version 6.30

The attached calculations have been generated to document compliance with established cleanup levels.

These documents should be used in conjunction with other relevant documents in the administrative record.

\section{Committed Calculation $\bowtie \quad$ Preliminary $\square \quad$ Superseded $\square \quad$ Voided}

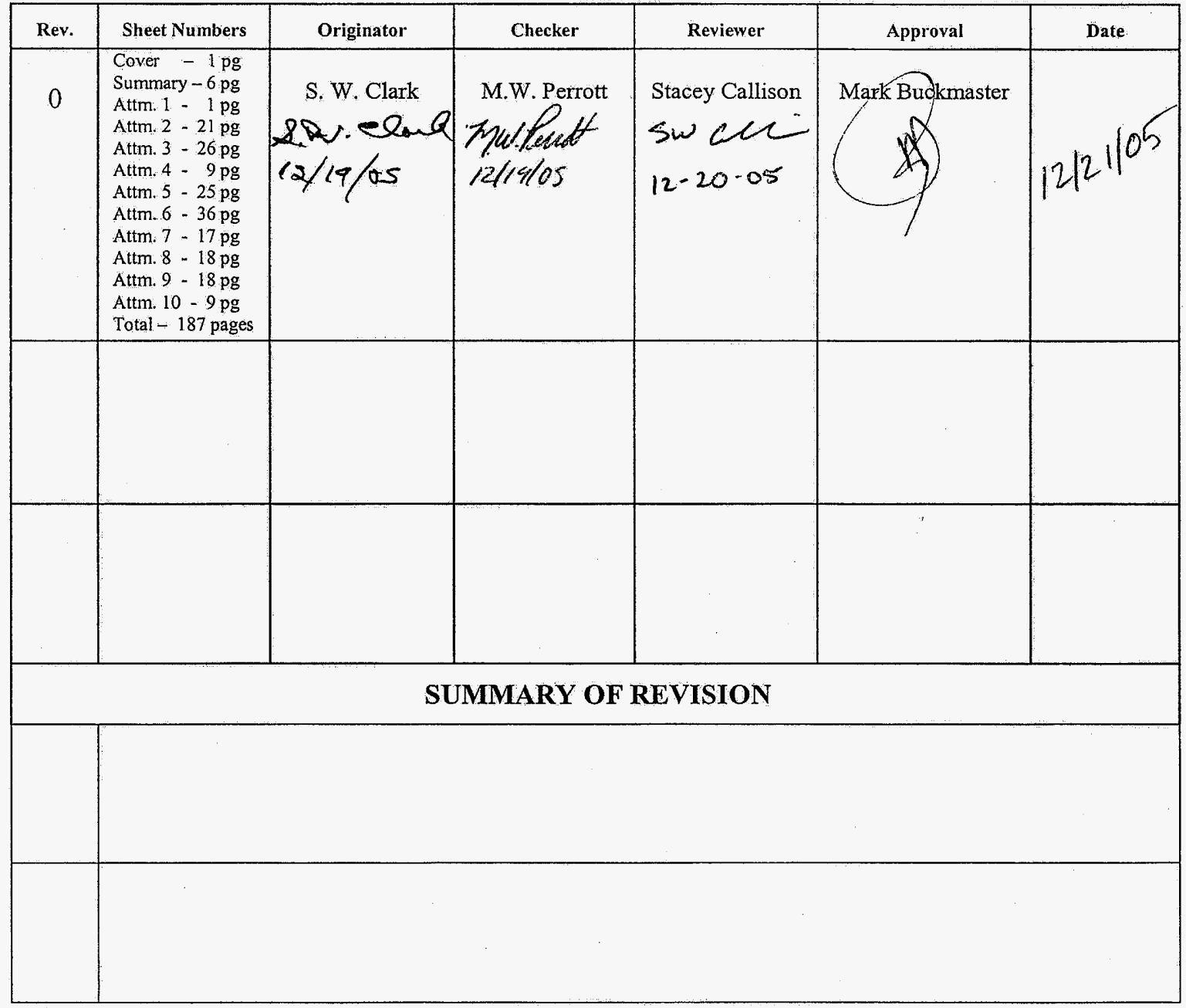

*Obtain Calc. No. from DIS

DE01-437.03 (12/09/2004) 


\begin{tabular}{|c|c|c|c|c|c|c|c|}
\hline Originator: & S. W. Clark \& \& Je & Date: & $10 / 19 / 05$ & Calc. No.: & $0100 \mathrm{~K}-\mathrm{CA}-\mathrm{V} 0063$ & Rev:: & 0 \\
\hline Project: & 100-K Field Remediation Project & Job No: & 14655 & Checked: & M.W. Perrott $M(\omega)$ & Date: & $12 / 19 / 05$ \\
\hline
\end{tabular}

\section{PURPOSE:}

Calculate the soil and groundwater concentrations, dose, and risk contributions from remaining radionuclide contaminants in the overburden and vadose zone over a period of 1,000 years.

\section{GIVEN/REFERENCES:}

1) Cleanup verification data from 116-K-2 Trench (West End) Cleanup Verification $95 \%$ UCL Calculations, Calculation No. 0100K-CA-V0061, Rev. 0, Bechtel Hanford, Inc., Richland, Washington.

2) Remedial Design Report/Remedial Action Work Plan for the 100 Area (RDR/RAWP), DOE/RL-96-17, Rev. 5, U.S. Department of Energy, Richland Operations Office, Richland, Washington.

3) Radioactive and nonradioactive contaminants of concern from the 100 Area Remedial Action Sampling and Analysis Plan (100 Area SAP), DOE/RL-96-22, Rev. 4, U.S. Department of Energy, Richland Operations Office, Richland, Washington. For the purpose of these RESRAD calculations, the radioactive contaminants of concern (COCs) are carbon-14, cesium-137, cobalt-60, europium-152, europium-154, nickel-63, plutonium-239/240, and strontium-90. Hexavalent chromium is the only nonradionuclide contaminant of concern.

4) Plutonium isotope ratios from Ratios of Plutonium Isotopes at 100 Areas Remedial Action Sites, Calculation No. 0100B-CA-V0013, Rev. 0, Bechtel Hanford, Inc., Richland, Washington.

5) RESidual RADioactivity (RESRAD) computer code, version 6.30, to calculate compliance with residual radioactivity guidelines, developed for the U.S. Department of Energy by the Environmental Assessment Division of Argonne National Laboratory, Argonne, Illinois.

6) Sample design data from the 116-K-2 Trench (West End) Sampling Plan, Calculation No. 0100K-CA-V0062, Rev. 0, Bechtel Hanford, Inc., Richland, Washington. For the purpose of these RESRAD calculations, the sampling areas identified in the sampling plan calculation brief are $14695.5 \mathrm{~m}^{2}$ for the shallow zone, $23046.93 \mathrm{~m}^{2}$ for the deep zone, and $8676 \mathrm{~m}^{2}$ for the overburden. Total sampling area of $37742 \mathrm{~m}^{2}$ was used for the combined shallow and deep zones for all RESRAD calculations.

7) Thickness of uncontaminated deep zone determined using analogous site data for the 116-C1 test pit from Cleanup Verification Package for the 116-C-1 Process Effluent Trench, CVP98-00006, Rev. 0, Bechtel Hanford, Inc., Richland, Washington.

8) Attainment of the river protection RAGs is based on comparison of results from the $100 \mathrm{~F}$ Area Soil Hexavalent Chromium and Carbon-14 Leachability Study Summary Report (Appendix D of the Cleanup Verification Package for the 100-F-19:1 North Pipelines, 100F-19:3 West Pipelines, 100-F-34 Biology Facility French Drain, and 116-F-12 French Drain, CVP-2001-00002, Rev. 0, Bechtel Hanford, Inc., Richland, Washington.), 100-H Area Soil Hexavalent Chromium Leachability Study Summary Report (Appendix D of the Cleanup Verification Package for the 116-H-7 Retention Basin, CVP-2000-00027, Rev. 0, Bechtel Hanford, Inc., Richland, Washington), and Estimation of Distribution Coefficients and Leachability of Hexavalent Chromium in 100-D Area Hanford Formation Sediments (Appendix D of the Cleanup Verification Package for the 116-D-7 Retention Basin, CVP-99-00007, Rev. 0, Bechtel Hanford, Inc., Richland, Washington). 


\begin{tabular}{|c|c|c|c|c|c|c|c|}
\hline Originator: & S. W. Clark \&Wx & Date: & la kar & Calc. No.: & $0100 \mathrm{~K}-\mathrm{CA}-\mathrm{V} 0063$ & Rev.: & 0 \\
\hline Project: & 100-K Field Remediation Project & Job No: & 14655 & Checked: & M.W. Perrott MlUP & Date: & $12 / 18 / 05$ \\
\hline Subject: & \multicolumn{5}{|c|}{ 116-K-2 Trench (West End) RESRAD Calculation } & \multicolumn{2}{|c|}{ Sheet No. 2 of 6} \\
\hline
\end{tabular}

1

\section{SOLUTION:}

1) Table 1 shows the waste site parameters used for RESRAD modeling. Based on the conservative assumption (discussed in the 100 Area RDR/RAWP) that residual contamination levels from the deep zone data set extend uniformly to groundwater, RESRAD predicted that the residual soil concentration of nickel-63 would result in groundwater concentrations that exceed the drinking water standard. Because this approach is too conservative, test pit data from the 116-C-1 Process Effluent Trench was used to include an uncontaminated deep zone between the contaminated deep zone and the groundwater to more accurately describe the 116-K-2 site for modeling using RESRAD.

2) Table 2 shows the radionuclide activities for each COC. Input factors for each RESRAD run are shown in the "Summary" section of the RESRAD "Mixture Sums and Single Radionuclide Guidelines" printouts in Attachments to this Calculation Summary.

3) The year where the peak dose (or concentration) occurs from each individual radionuclide COC was determined by a preliminary run. This year was then included in all final RESRAD runs. For the direct exposure pathway (i.e. soil ingestion and inhalation and external radiation), the peak year occurred at year zero (year 2005) for all COCs. For the water pathways (i.e., drinking water and food ingestion) the peak year also occurred at year zero (year 2005) for all COCs.

\begin{tabular}{|c|c|c|c|}
\hline Parameter & Units & Value & Comments \\
\hline Shallow Zone and Overburden Cover Depth & $\mathrm{m}$ & 0 & \\
\hline Area of Shallow and Deep Zone & $\mathrm{m}^{2}$ & 37742 & Based on Sample Design $^{2}$ \\
\hline Area of Overburden & $\mathrm{m}^{2}$ & 8676 & Based on Sample Design $^{2}$ \\
\hline Thickness: Shallow Zone and Overburden & $\mathrm{m}$ & 4.6 & Appendix B of 100 Area RDR/RAWP \\
\hline Elevation: Ground Surface & $\mathrm{m}$ & 133.5 & NAVD88 \\
\hline Elevation: Excavation Bottom & $\mathrm{m}$ & 125.9 & NAVD88 \\
\hline Elevation: Groundwater & $\mathrm{m}$ & 118.5 & NAVD 88 \\
\hline Thickness: Total Deep Zone & $\mathrm{m}$ & 10.4 & Vadose zone thickness minus shallow zone \\
\hline Thickness: Uncontaminated Deep Zone & $\mathrm{m}$ & 3.1 & Based on deep zone model from $116-\mathrm{C}-1$ test pit $^{\mathrm{b}}$ \\
\hline Thickness: Contaminated Deep Zone & $\mathrm{m}$ & 7.3 & Deep zone thickness minus uncontaminated deep zone \\
\hline \begin{tabular}{|l|l|} 
Length Parallel to Aquifer Flow \\
\end{tabular} & $\mathrm{m}$ & 319 & Based on Sample Design $^{\text {a }}$ \\
\hline \multicolumn{4}{|c|}{$\begin{array}{l}\text { Sample design data from the 116-K-2 Trench (West End) Sampling Plan, Calculation No. 0100K-CA-V0062, Rev. 0, Bechtel Hanford, Inc., } \\
\text { Richland, Washington. } \\
\text { b Two-layer deep zone model based on test pit data from Cleanup Verification Package for the 116-C-I Process Effluent Trench, CVP-98-00006, } \\
\text { Rev. 0, Bechtel Hanford, Inc., Richland, Washington. }\end{array}$} \\
\hline
\end{tabular}




\begin{tabular}{|c|c|c|c|c|c|c|c|}
\hline Originator: & S. W. Clark & Date: & $12 / 1960$ & Calc. No.: & $0100 \mathrm{~K}-\mathrm{CA}-\mathrm{V} 0063$ & Rev.: & 0 \\
\hline Project: & 100-K Field Remediation Project & Job No: & 14655 & Checked: & M.W. Perrott Miep & Date: & $12 / 19165$ \\
\hline Subject: & \multicolumn{5}{|c|}{ 116-K-2 Trench (West End) RESRAD Calculation } & \multicolumn{2}{|c|}{ Sheet No. 3 of 6} \\
\hline
\end{tabular}

\section{METHODOLOGY:}

1) Runs of RESRAD version 6.30 were completed for the shallow zone, deep zone, and overburden using the radionuclide concentrations shown in Table 2. RESRAD numerical output reports for dose, risk, and concentration for the shallow zone, deep zone, and overburden are presented in the Attachments to this calculation summary.

\begin{tabular}{|c|c|c|c|c|}
\hline \multicolumn{5}{|c|}{ Table 2. Cleanup Verification Data Set ${ }^{\text {a }}$} \\
\hline COCs & Shallow Zone & Deep Zone & Overburden & $\begin{array}{c}\text { Overburden Minus } \\
\text { Background }\end{array}$ \\
\hline \multicolumn{5}{|c|}{ Radionuclide Activity ( $p$ Ci/g) } \\
\hline $\mathrm{C}-14$ & 0.29 & 1.44 & $0.68 U^{b}$ & $0.68 \mathrm{U}^{\mathrm{b}}$ \\
\hline Cs-137 & 1.10 & 117 & 0.083 & $<0 U^{b}$ \\
\hline Co- 60 & $0.023 \mathrm{U}^{\mathrm{b}}$ & 4.23 & $0.020 \mathrm{U}^{\mathrm{b}}$ & $<0 U^{b}$ \\
\hline $\mathrm{Eu}-152$ & 0.626 & 76.0 & 0.17 & 0.17 \\
\hline Eu-154 & 0.129 & 7.2 & $0.068 \mathrm{U}^{\mathrm{b}}$ & $<0 \mathrm{U}^{\mathrm{b}}$ \\
\hline $\mathrm{Ni}-63$ & 1.77 & 650 & $0.689 \mathrm{U}^{\mathrm{b}}$ & $0.689 \mathrm{U}^{\mathrm{b}}$ \\
\hline $\mathrm{Pu}-239 / 240$ & $0.024 \mathrm{U}^{\mathrm{b}}$ & 4.7 & $0.031 \mathrm{U}^{\mathrm{b}}$ & $<0 U^{b}$ \\
\hline Sr-90 & 0.201 & 6.30 & $0.062 \mathrm{U}^{\mathrm{b}}$ & $<0 \mathrm{U}^{\mathrm{b}}$ \\
\hline \multicolumn{5}{|c|}{ Nonradionuclide Concentration $(\mathrm{mg} / \mathrm{Kg})$} \\
\hline $\mathrm{Cr}$ (VI) & 0.23 & 2.4 & 0.27 & 0.27 \\
\hline $\begin{array}{l}{ }^{\mathrm{a}} \text { Cleanup verificatio } \\
\text { 0100K-CA-Vo06 } \\
{ }^{b} \text { Value was not inp }\end{array}$ & rom the $116-K-2 T$ & (West End) Clec & cation $95 \%$ UC & lations, Calculation No. \\
\hline
\end{tabular}

2) $\mathrm{Pu}-239 / 240$ Conversion: The relative individual $\mathrm{Pu}-239$ and $\mathrm{Pu}-240$ activities used in the RESRAD model were calculated from the reported combined Pu-239/240 analytical results. The calculations were performed in accordance with Calculation No. 0100B-CA-V0013. The relative activities for $\mathrm{Pu}-239$ and $\mathrm{Pu}-240$ were calculated by multiplying the cleanup verification values for Pu-239/240 in each decision unit of the site by 0.807 and 0.193 , respectively. Table 3 shows the results of these calculations.

Table 3. Conversion of Pu-239/240 Activity to Relative Pu-239 and Pu-240 Activities

\begin{tabular}{|c|c|c|c|c|}
\hline $\begin{array}{c}\text { RESRAD } \\
\text { Run \# }\end{array}$ & $\begin{array}{c}\text { Vadose Zone } \\
\text { Horizon }\end{array}$ & $\begin{array}{c}\text { Pu-239/240 Activity } \\
(\mathbf{p C i} / \mathbf{g})\end{array}$ & $\begin{array}{c}\text { Pu-239, (pCi/g) } \\
(\mathbf{0 . 8 0 7} \text { multiplier) }\end{array}$ & $\begin{array}{c}\text { Pu-240, (pCi/g) } \\
(\mathbf{0 . 1 9 3} \text { multiplier) }\end{array}$ \\
\hline 1 & Shallow Zone & $0.024 \mathrm{U}$ & $0.019 \mathrm{U}$ & $0.005 \mathrm{U}$ \\
\hline 2 & Deep Zone & 4.7 & 3.8 & 0.9 \\
\hline 3 & Overburden & $0.062 \mathrm{U}$ & $0.050 \mathrm{U}$ & $0.012 \mathrm{U}$ \\
\hline
\end{tabular}

3) Protectiveness of residual hexavalent chromium soil concentrations: Shallow zone soil concentrations of hexavalent chromium represented by the $95 \%$ UCL value in Table 2 are less than the applicable RAGs (i.e., $2.1 \mathrm{mg} / \mathrm{kg}$ for direct exposure or $2.0 \mathrm{mg} / \mathrm{kg}$ for river protection). However, the deep zone hexavalent chromium soil concentration exceeds the applicable RAG of $2.0 \mathrm{mg} / \mathrm{kg}$ for river protection. Because this RAG was not met for the 
CVP-2006-00001

Rev. 0

Washington Closure Hanford

CALCULATION SHEET

\begin{tabular}{|c|c|c|c|c|c|c|c|}
\hline Originator: & S. W. Clark \& LNe & Date: & $12 / 18 / 05$ & Calc. No:: & $0100 \mathrm{~K}-\mathrm{CA}-\mathrm{V} 0063$ & Rev.: & 0 \\
\hline Project: & 100-K Field Remediation Project & Job No: & 94655 & Checked: & M.W. Perrott ZIaP & Date: & $12 / 19 / 05$ \\
\hline Subject: & \multicolumn{5}{|c|}{ 116-K-2 Trench (West End) RESRAD Calculation } & \multicolumn{2}{|c|}{ Sheet No. 4 of 6} \\
\hline
\end{tabular}

1

deep zone soils, a more detailed assessment of the potential impact of residual hexavalent chromium to groundwater was made using the 100-F, 100-H, and 100-D Area-specific hexavalent chromium leach studies (Appendix D of CVP-2001-00002, CVP-2000-00027, and CVP-99-00007). Using the simple and conservative approach of comparing the residual soil concentration of hexavalent chromium to the soil concentrations used in leach testing, and comparing the leachate concentrations from the leach tests to the surface water hexavalent chromium cleanup level, indicates that remaining hexavalent chromium in deep zone soil at the $116-\mathrm{K}-2$ site is protective of the river. The river protection soil RAG of 2.0 $\mathrm{mg} / \mathrm{kg}$ is based on the MTCA " 100 times dilution attenuation factor (DAF) times surface water quality criteria" rule. The near-shore river water hexavalent chromium cleanup level is $20 \mu \mathrm{g} / \mathrm{L}$ (surface water quality criteria of $10 \mu \mathrm{g} / \mathrm{L}$ times the dilution attenuation factor of 2 ). Based on the hexavalent chromium leach studies, the hexavalent chromium soil concentration at which the leachate did not exceed the surface water quality criteria was 6.8 $\mathrm{mg} / \mathrm{kg}$. Therefore, using the simple and conservative approach of comparing the hexavalent chromium deep zone soil concentration to the leach study hexavalent chromium soil concentrations demonstrates that cleanup of the deep zone hexavalent chromium is protective of the river (i.e., $2.4 \mathrm{mg} / \mathrm{kg}$ is less than $6.8 \mathrm{mg} / \mathrm{kg}$ ).

\section{RESULTS:}

1) Radionuclide "All Pathways" Dose Rate: The "all pathways" (maximum) dose rates are shown in Table 4. The maximum total all pathways dose rate for the shallow and deep zones is $7.77 \mathrm{mrem} / \mathrm{yr}$ which occurs at year zero (2005). The maximum all pathways dose rate for the overburden is $0.740 \mathrm{mrem} / \mathrm{yr}$ which occurs at year zero (2005).

\begin{tabular}{|c|c|c|c|c|c|c|c|c|}
\hline & & \multicolumn{7}{|c|}{ Table 4. All Pathway Dose Rate (mrem/yr) } \\
\hline \multirow{2}{*}{$\begin{array}{c}\text { Vadose Zone } \\
\text { Horizon }\end{array}$} & \multicolumn{8}{|c|}{ "All Pathways" Dose Contributions in mrem/yr at each time slice (yr) } \\
\hline & 0 & 1 & 3 & 13 & 30 & 100 & 300 & 1000 \\
\hline Shallow Zone & $7.77 \mathrm{E}+00$ & $6.96 \mathrm{E}+00$ & $6.29 \mathrm{E}+00$ & $4.33 \mathrm{E}+00$ & $2.45 \mathrm{E}+00$ & 3.67E-01 & $4.21 \mathrm{E}-03$ & 5.23E-06 \\
\hline Deep Zone & $1.70 \mathrm{E}-22$ & $1.53 \mathrm{E}-22$ & $1.24 \mathrm{E}-22$ & 4.61E-23 & $1.17 \mathrm{E}-23$ & $3.06 \mathrm{E}-06$ & $3.26 \mathrm{E}-04$ & 7.34E-04 \\
\hline $\begin{array}{c}\text { Total All } \\
\text { Pathways Dose } \\
\text { Rate (mrem/yr) }\end{array}$ & 7.77E+00 & $6.96 \mathrm{E}+00$ & $6.29 \mathrm{E}+00$ & $4.33 \mathrm{E}+00$ & $2.45 \mathrm{E}+00$ & 3.67E-01 & 4.54E-03. & $7.39 \mathrm{E}-04$ \\
\hline Overburden & $7.40 \mathrm{E}-01$ & 7.02E-01 & $6.33 \mathrm{E}-01$ & $3.76 \mathrm{E}-01$ & $1.55 \mathrm{E}-01$ & $4.06 \mathrm{E}-03$ & $1.22 \mathrm{E}-07$ & $2.61 \mathrm{E}-16$ \\
\hline
\end{tabular}

2) Radionuclide Excess Cancer Risk: The radionuclide excess lifetime cancer risk results are shown in Table 5. The maximum total excess lifetime cancer risk for the shallow and deep zones $\left(9.70 \times 10^{-5}\right)$ occurs at year zero $(2005)$. The maximum excess lifetime cancer risk for the overburden $\left(8.72 \times 10^{-6}\right)$ occurs at year zero $(2005)$. 
Washington Closure Hanford

CALCULATION SHEET

\begin{tabular}{|c|c|c|c|c|c|c|c|}
\hline Originator: & S.W. Clark Xewe & Date: & $12 / 17 /$ or & Calc. No.: & $0100 \mathrm{~K}-\mathrm{CA}-\mathrm{V} 0063$ & Rev.: & 0 \\
\hline Project: & 100-K Field Remediation Project & Job No: & 14655 & Checked: & M.W. Perrott JuWP & Date: & 121865 \\
\hline Subject: & \multicolumn{5}{|c|}{ 116-K-2 Trench (West End) RESRAD Calculation } & \multicolumn{2}{|c|}{ Sheet No. 5 of 6} \\
\hline
\end{tabular}

Table 5. Radionuclide Excess Lifetime Cancer Risk

\begin{tabular}{|c|c|c|c|c|c|c|c|c|}
\hline \multicolumn{7}{|c|}{ Table 5. Radionuclide Excess Lifetime Cancer Risk } \\
\hline $\begin{array}{c}\text { Vadose Zone } \\
\text { Horizon }\end{array}$ & 0 & 1 & 3 & 13 & 30 & 100 & 300 & 1000 \\
\cline { 2 - 9 } & 0 & 1 & 3 & Excess Cancer Risk at Each Time Slice (yr) \\
\hline Shallow Zone & $9.70 \mathrm{E}-05$ & $9.29 \mathrm{E}-05$ & $8.63 \mathrm{E}-05$ & $6.13 \mathrm{E}-05$ & $3.61 \mathrm{E}-05$ & $5.86 \mathrm{E}-06$ & $9.71 \mathrm{E}-08$ & $2.34 \mathrm{E}-10$ \\
\hline $\begin{array}{c}\text { Deep Zone } \\
\text { Total Excess } \\
\text { Cancer Risk }\end{array}$ & $1.30 \mathrm{E}-27$ & $1.18 \mathrm{E}-27$ & $9.73 \mathrm{E}-28$ & $3.91 \mathrm{E}-28$ & $9.40 \mathrm{E}-29$ & $9.34 \mathrm{E}-11$ & $2.75 \mathrm{E}-09$ & $5.80 \mathrm{E}-09$ \\
\hline Overburden & $8.72 \mathrm{E}-06$ & $8.27 \mathrm{E}-06$ & $7.46 \mathrm{E}-06$ & $4.43 \mathrm{E}-06$ & $1.83 \mathrm{E}-06$ & $4.78 \mathrm{E}-08$ & $1.44 \mathrm{E}-12$ & $1.78 \mathrm{E}-21$ \\
\hline
\end{tabular}

1

2

6

\section{Table 6. RESRAD Predicted Radionuclide Groundwater (Well Water) Concentrations}

\begin{tabular}{|c|c|c|c|c|c|c|c|c|c|c|}
\hline \multirow{2}{*}{$\begin{array}{l}\text { Radio- } \\
\text { nuclides }\end{array}$} & \multirow{2}{*}{$\begin{array}{c}\text { Vadose Zone } \\
\text { Horizon }\end{array}$} & \multicolumn{8}{|c|}{ Groundwater Concentrations in $\mathrm{pCi} / \mathrm{L}$ at Each Time Slice (yr) } & \multirow{2}{*}{$\begin{array}{c}\text { RAGs } \\
\text { From RDR }\end{array}$} \\
\hline & & 0 & 1 & 3 & 13 & 30 & 100 & 300 & 1000 & \\
\hline $\mathrm{C}-14$ & Total & 0 & 0 & 0 & 0 & 0 & 0 & 0 & 0 & 2000 \\
\hline $\mathrm{Co}-60$ & Total & 0 & 0 & 0 & 0 & 0 & 0 & 0 & 0 & 100 \\
\hline Cs-137 & Total & 0 & 0 & 0 & 0 & 0 & 0 & 0 & 0 & 60 \\
\hline Eu-152 & Total & 0 & 0 & 0 & 0 & 0 & 0 & 0 & 0 & 200 \\
\hline Eu-154 & Total & 0 & 0 & 0 & 0 & 0 & 0 & 0 & 0 & 60 \\
\hline $\mathrm{Ni}-63$ & Total & 0 & 0 & 0 & 0 & 0 & 0 & 0 & 0 & 50 \\
\hline $\mathrm{Sr}-90$ & Total & 0 & 0 & 0 & 0 & 0 & 0 & 0 & 0 & 8 \\
\hline
\end{tabular}

7

8

9

10

11

12

13

3) Radionuclide Groundwater Protection: The radionuclide concentrations in groundwater calculated by the RESRAD model are summarized in Table 6 . Because all concentrations presented here are zero no comparison to drinking water standards calculation was necessary.

\section{CONCLUSIONS:}

- The combined maximum all-pathways dose rate for the shallow and deep zones shown in Table 4 is $7.77 \mathrm{mrem} / \mathrm{yr}$ which occurs at year zero (2005).

- The maximum all-pathways dose rate for the overburden shown in Table 4 is $0.740 \mathrm{mrem} / \mathrm{yr}$ which occurs at year zero (2005).

- The dominant pathway for the dose rate is direct external exposure.

- The primary radionuclides contributing to the direct exposure pathway are cesium-137 and europium-152.

- The maximum combined shallow and deep zone excess lifetime cancer risk shown in Table 5 $\left(9.70 \times 10^{-5}\right)$ occurs at year zero $(2005)$.

- The maximum overburden excess lifetime cancer risk shown in Table $5\left(8.72 \times 10^{-6}\right)$ occurs at year zero (2005). 


\begin{tabular}{|r|l|l|l|l|l|l|l|}
\hline Originator: & S. W. Clark \&Le & Date: & $(2 / 19 /$ as & Calc. No.: & $0100 \mathrm{~K}$-CA-V0063 & Rev.: & 0 \\
\hline Project: & $100-$ K Field Remediation Project & Job No: & 14655 & Checked: & M.W. Perrott $/$ ha/ & Date: & $12 / 9 / 85$ \\
\hline Subject: & $116-K-2$ Trench (West End) RESRAD Calculation & & & Sheet No. 6 of 6 \\
\hline
\end{tabular}

- None of the radionuclide contaminants of concern are predicted to reach groundwater in the 1,000 years of the RESRAD model runs.

- None of the site COCs are projected to exceed remedial action goals (RAGs).

\section{ATTACHMENTS:}

1. Graphic showing 116-K-2 Cleanup Verification Model (1 page)

2. RESRAD Output: 116-K-2 Shallow Zone Radionuclides, Mixture Sums and Single Radionuclide Guidelines (21 pages)

3. RESRAD Output: 116-K-2 Shallow Zone Radionuclides, Intake Quantities and Health Risk Factors (26 pages)

4. RESRAD Output: 116-K-2 Shallow Zone Radionuclides, Concentration of Radionuclides, ( 9 pages)

5. RESRAD Output: $116-\mathrm{K}-2$ Deep Zone Radionuclides, Mixture Sums and Single Radionuclide Guidelines (25 pages)

6. RESRAD Output: 116-K-2 Deep Zone Radionuclides, Intake Quantities and Health Risk Factors (36 pages)

7. RESRAD Output: 116-K-2 Deep Zone Radionuclides, Concentration of Radionuclides, (17 pages)

8. RESRAD Output: 116-K-2 Overburden Radionuclides, Mixture Sums and Single Radionuclide Guidelines (18 pages)

9. RESRAD Output: 116-K-2 Overburden Radionuclides, Intake Quantities and Health Risk Factors (18 pages)

10. RESRAD Output: 116-K-2 Overburden Radionuclides, Concentration of Radionuclides, (9 pages) 
CVP-2006-00001

Rev. 0

\section{ATTACHMENT 1}

116-K-2 Trench Cleanup Verification Model

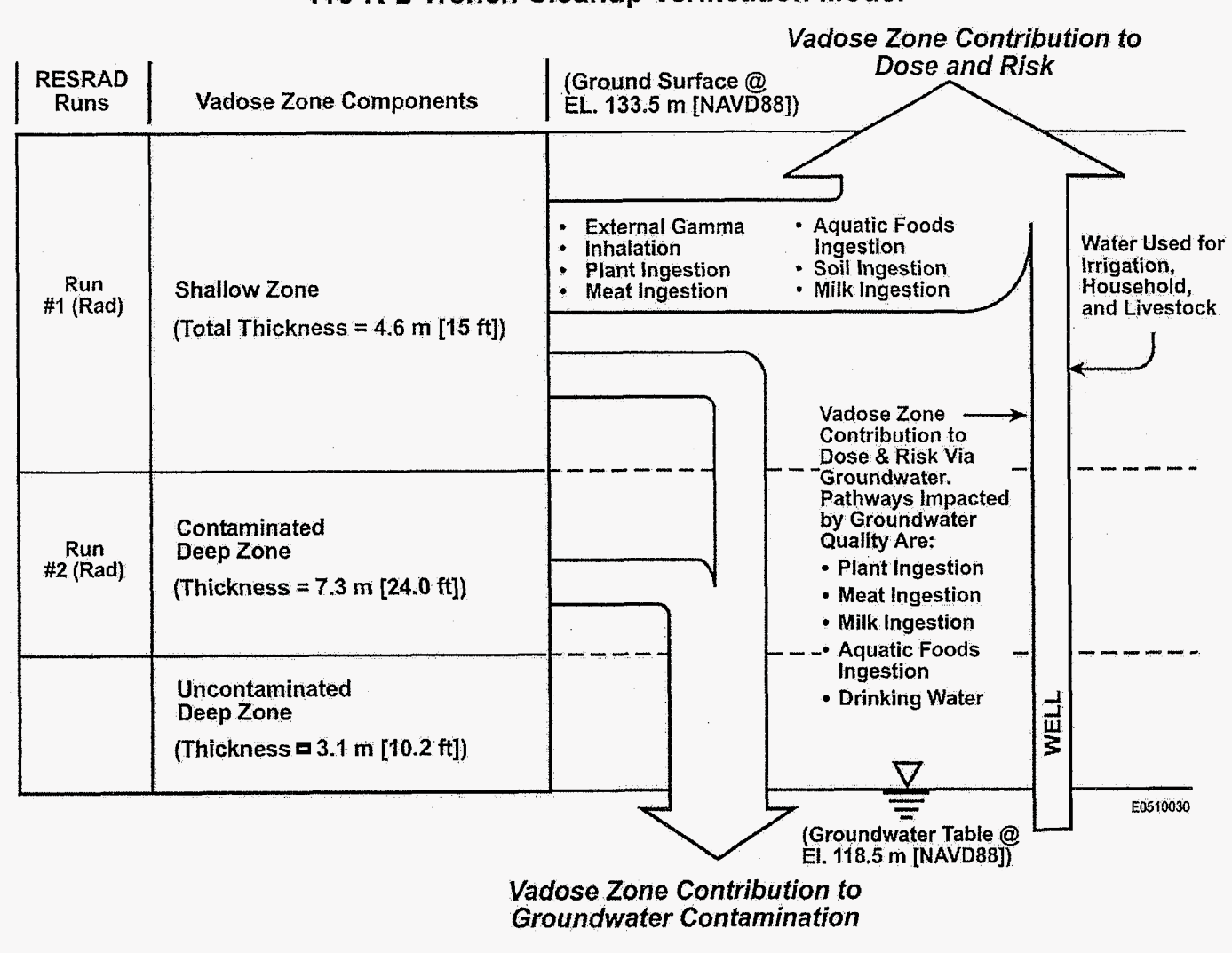

Attachment

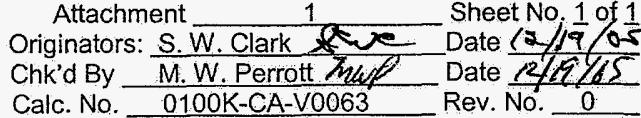




\section{DISTRIBUTION}

U.S. Department of Energy

Richland Operations Office

D. T. Evans (5)

A3-04

S. L. Sedgwick

A7-80

J. Zeisloft

A3-04

RL Correspondence Control

A5-10

DOE-RL Public Reading Room

$\mathrm{H} 2-53$

Fluor Hanford, Inc.

J. P. Shearer (WIDS)

A0-21

Washington Closure Hanford

K. A. Anselm

$\mathrm{H} 9-02$

M. A. Buckmaster

$\times 9-07$

S. W. Callison

$\mathrm{X} 9-07$

R. A. Carlson

$\mathrm{X} 0-17$

S. W. Clark

$\mathrm{H} 9-01$

J. S. Decker

$\mathrm{X} 0-17$

L. A. Dietz

$\mathrm{H} 0-23$

L. M. Dittmer

$\mathrm{H} 9-02$

L. R. Miller

X3-40

J. E. Thomson

$\mathrm{HO}-23$

Records and Document Control

$\mathrm{HO}-30$

Hanford Technical Library

P8-55 
CVP-2006-00001

Rev. 0

Distr-2 